\title{
Pathwise Uniqueness of the Stochastic Heat Equation with Hölder continuous diffusion coefficient and colored noise
}

\author{
Dissertation \\ zur Erlangung des mathematisch-naturwissenschaftlichen Doktorgrades \\ "Doctor rerum naturalium" \\ der Georg-August-Universität Göttingen \\ im Promotionsprogramm Mathematik \\ der Georg-August University School of Sciences (GAUSS) \\ vorgelegt von
}

Thomas Rippl

aus Bayreuth.

Göttingen, 2012 


\section{Betreuungsausschuss}

Prof. Dr. Anja Sturm, Institut für Math. Stochastik, Göttingen

Prof. Dr. Martin Schlather, Institut für Math. Stochastik, Göttingen

\section{Mitglieder der Prüfungskommission}

Referentin:

Prof. Dr. Anja Sturm, Institut für Math. Stochastik, Göttingen

Koreferentin:

Prof. Dr. Anita Winter, Fakultät für Mathematik, Universität Duisburg-Essen

Weitere Mitglieder der Prüfungskommission:

PD Dr. Ulf Fiebig, Institut für Math. Stochastik, Göttingen

Prof. Dr. Axel Munk, Institut für Math. Stochastik, Göttingen

Prof. Dr. Gerlind Plonka-Hoch, Institut für Num. und Ang. Mathematik, Göttingen

Prof. Dr. Ingo Witt, Mathematisches Institut, Göttingen

Tag der mündlichen Prüfung: 29. Oktober 2012 


\section{Abstract}

We consider the stochastic heat equation in $\mathbb{R}_{+} \times \mathbb{R}^{q}$ with multiplicative noise:

$$
\partial_{t} u(t, x)=\frac{1}{2} \Delta u(t, x)+b(t, x, u(t, x))+\sigma(t, x, u(t, x)) \dot{W}(t, x) .
$$

Here, $\dot{W}$ is a centered Gaussian noise which is white in time and colored in space with correlation kernel $k(x, y) \leq \operatorname{const}\left(|x-y|^{-\alpha}+1\right)$ for $x, y \in \mathbb{R}^{q}$ and $\alpha \in(0,2 \wedge$ $q): \mathbb{E}[\dot{W}(t, x) \dot{W}(s, y)]=\delta(s-t) k(x, y)$. Our main result states that if the noise coefficient $\sigma$ is Hölder-continuous of order $\gamma$ in the solution $u$ and satisfies $\alpha<$ $2(2 \gamma-1)$, then the equation has a pathwise unique solution. This was conjectured by Mytnik and Perkins in [MP11] and generalizes results in [MPS06]. Additionally, if $q=1$, we show that the compact support property holds for nonnegative solutions of the stochastic heat equation with $\sigma(t, x, u)=u^{\gamma}$ for all $\alpha, \gamma \in(0,1)$. 



\section{Abstract}

Wir betrachten die stochastische Wärmeleitungsgleichung in $\mathbb{R}_{+} \times \mathbb{R}^{q}$ mit farbigem Rauschen.

$$
\partial_{t} u(t, x)=\frac{1}{2} \Delta u(t, x)+b(t, x, u(t, x))+\sigma(t, x, u(t, x)) \dot{W}(t, x) .
$$

Hierbei ist $\dot{W}$ ein zentriertes Gaußsches Rauschen, welches weiß in der Zeit ist und farbig im Raum mit einem Korrelationskern $k(x, y) \leq \operatorname{const}\left(|x-y|^{-\alpha}+1\right)$ für $x, y \in$ $\mathbb{R}^{q}$ und $\alpha \in(0,2 \wedge q): E[\dot{W}(t, x) \dot{W}(s, y)]=\delta(s-t) k(x, y)$. Das Hauptresultat der Arbeit sagt, dass wenn der Koeffizient des Rauschens $\sigma$ Hölder-stetig von Ordnung $\gamma$ in der Lösung $u$ ist und es gilt, dass $\alpha<2(2 \gamma-1)$, dann hat die Gleichung eine pfadweise eindeutige Lösung. Diese Aussage wurde von Mytnik und Perkins 2011 vermutet. Zudem wird gezeigt, dass im Fall $q=1$ die sogenannte compact support property für nichtnegative Lösungen der stochastischen Wärmeleitungsgleichung mit $\sigma(t, x, u)=u^{\gamma}$ für alle $\alpha, \gamma \in(0,1)$ gilt. 


\section{Contents}

\begin{tabular}{lr}
\hline 1. Introduction & 8
\end{tabular}

\begin{tabular}{ll}
\hline I. Notation and Results & 13
\end{tabular}

\begin{tabular}{lr}
\hline 2. Partial Differential Equations & 14
\end{tabular}

2.1. Partial differential operators . . . . . . . . . . . . . . . . . . . . . . 14

2.2. Weak solutions, fundamental solutions and distributions . . . . . . . 16

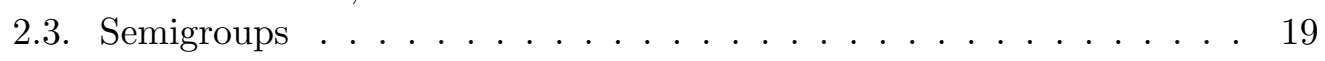

\begin{tabular}{ll}
\hline 3. Probability Essentials & 21
\end{tabular}

3.1. Stochastic processes . . . . . . . . . . . . . . . . . . . . . 21

3.2. Martingales and classical stochastic integration . . . . . . . . . . . . . 23

3.3. Gaussian processes and noises . . . . . . . . . . . . . . . 28

3.4. Generalized stochastic integration. . . . . . . . . . . . . . . . . . 38

3.5. Stochastic Partial Differential Equations . . . . . . . . . . . . . . 42

4. The Stochastic Heat Equation 46

4.1. The Stochastic Heat Equation with multiplicative noise . . . . . . . 46

4.2 . Known results on existence and regularity of solutions . . . . . . . . 49

4.3. Known results on uniqueness $\ldots \ldots \ldots . \ldots . \ldots . \ldots 51$

4.4. Known results on the compact support property . . . . . . . . . . 52

5. Main Results $\quad 54$

5.1. An abstract result on strong solutions . . . . . . . . . . . . . 55

$5.2 . \quad$ Existence and regularity of solutions . . . . . . . . . . . . . 55

5.3. Pathwise Uniqueness $\ldots \ldots \ldots \ldots$. . . . . . . . . . . 56

5.4. The compact support property . . . . . . . . . . . . . . 64

6. Outlook 66

6.1. Stable Motion . . . . . . . . . . . . . . . . . . 66

$6.2 . \quad$ Particle picture $\ldots \ldots \ldots \ldots \ldots$

$6.3 . \quad$ Non-Uniqueness . . . . . . . . . . . . . . . . . . . . . . . 73

6.4. The compact support property in higher dimensions . . . . . . . . . 74 
$\begin{array}{ll}\text { II. Proofs } & 76\end{array}$

\begin{tabular}{ll}
\hline 7. An abstract result on strong solutions & 77
\end{tabular}

\begin{tabular}{lr}
\hline 8. Existence and regularity & $\mathbf{8 2}$
\end{tabular}

8.1. Regularity results for solutions . . . . . . . . . . . . . . . 82

8.2. Tightness and construction of the solution . . . . . . . . . . . 85

\begin{tabular}{ll}
\hline 9. Pathwise Uniqueness & 93
\end{tabular}

9.1. Proof of Theorem 5.3.1 . . . . . . . . . . . . . . . . . . . . 93

9.2. Verification of the hypotheses of Proposition $9.1 .2 \ldots \ldots 9$

9.3. Heat kernel estimates . . . . . . . . . . . . . . . . . . . 108

9.4. Local bounds on the difference of two solutions . . . . . . . . . . 117

9.5. Proof of Proposition $9.2 .2 \ldots \ldots$. . . . . . . . . . . . . . . . . 149

9.6. Proof of Proposition 9.4 .16 . . . . . . . . . . . . . . . . . . 159

9.7. Proof of Proposition 9.4.18 . . . . . . . . . . . . . . . . 170

9.8. Lemma 5.7 from [MP11] in $q$ dimensions . . . . . . . . . . . . . . 179

9.9. Proof of Theorem|5.3.3 $\ldots \ldots \ldots \ldots$

9.10. Incorporation of drifts . . . . . . . . . . . . . . . . 189

9.11. An integral estimate . . . . . . . . . . . . . . . . 195

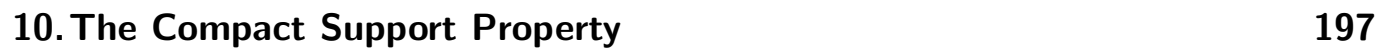

10.1. Proof of Theorem 15.4.1 . . . . . . . . . . . . . . . . . . . 197

10.2. Proof of Proposition 10.1.1 . . . . . . . . . . . . . . . . 199

10.3. Extinction of the process . . . . . . . . . . . . . . . . 206

10.4. Integral estimates . . . . . . . . . . . . . . . . . . . . . . . . 209

$\begin{array}{lr}\text { 11. Particle system } & 213\end{array}$

11.1. Offspring distributions . . . . . . . . . . . . . . . . . . 213

11.2. Proofs and proof ideas . . . . . . . . . . . . . . . 215

\begin{tabular}{lr}
\hline Index & 220
\end{tabular}

\begin{tabular}{ll}
\hline Bibliography & 221
\end{tabular} 


\section{Introduction}

In this dissertation we study a certain class of stochastic partial differential equations (SPDE). These kind of equations appeared first in the 1970s, amongst others in works of Dawson [Daw75, Pardoux [Par72 and Viot [Vio76]. One of the first manuscripts presenting a comprehensive theory was given by Walsh Wal86. As often in probability, the theory was motivated by possible applications which mostly stem from biological or physical models. We will also first provide motivation by considering such a model that can be described by these SPDEs. Rigorous definitions will be given in later chapters.

So, imagine one particle moving in the lattice $\mathbb{Z}^{q}, q \geq 1$. It starts at the origin $0 \in \mathbb{Z}^{q}$. At each time point in $\mathbb{Z}_{+}=\{0,1,2, \ldots\}$ it changes its position to a neighboring point, i.e. to a point where only one coordinate entry differs by the value 1 . All of the $2 q$ possible points are chosen with equal probability and each position change is independent of the other. Denote the position at time $n \in \mathbb{Z}_{+}$ by $Y_{n} \in \mathbb{Z}^{q}$ and call $Y=\left(Y_{n}\right)_{n \in \mathbb{Z}_{+}}$a random walk. If there are $N \in \mathbb{N}=\{1,2, \ldots\}$ particles, each moving independently, denote their random walks by $Y^{1}, \ldots, Y^{N}$. For $t \geq 0, N \in \mathbb{N}$, define the particles' (rescaled) empirical measure $X$ on $\mathbb{R}^{q}$ by

$$
X_{t}^{N}(\cdot)=\frac{1}{N} \sum_{k=1}^{N} \delta_{N^{-1 / 2} Y_{\lfloor N t\rfloor}^{k}}(\cdot) .
$$

It assigns $\frac{1}{N}$ times a Dirac-measure $\delta$ to positions, rescaled by $N^{-1 / 2}$, of the particles at time $\lfloor N t\rfloor$. Then for any $\phi \in C_{c}^{\infty}\left(\mathbb{R}^{q}\right)$, the compactly supported infinitely differentiable functions on $\mathbb{R}^{q}$, consider the integral $\left\langle\phi, X_{t}^{N}\right\rangle:=\int \phi(x) X_{t}^{N}(d x)$. As $N \rightarrow \infty$ the integral converges almost surely for any $t \geq 0$ to a constant depending on $\phi$. The constant of this large population, high density limit can be expressed as $\int_{\mathbb{R}^{q}} \phi(x) u(t, x) d x$ for a function $u: \mathbb{R}_{+} \times \mathbb{R}^{q} \rightarrow \mathbb{R}$, which is the same for any $\phi$. Furthermore, assuming all particles start at the origin, the function $u$ can be specified as the (unique) solution of the heat equation

$$
\partial_{t} u(t, x)=\frac{\Delta}{2} u(t, x), u(0, x)=\delta_{0}(x), t \geq 0, x \in \mathbb{R}^{q} .
$$

Here, $\Delta=\partial_{x_{1}}^{2}+\cdots+\partial_{x_{q}}^{2}$ is the Laplacian in $\mathbb{R}^{q}, \partial_{x_{i}}$ denotes partial derivative in $x_{i}$-direction and $\delta_{0}$ is the $\delta$-function with (singular) support in 0 . In fact, $u$ describes the density of particles in the limit. We sometimes abbreviate $u_{t}(x)=u(t, x)$. 
The heat equation is a deterministic equation. However, if particles not only move in the geographical space, but also branch after each position change (i.e. at times in $N^{-1} \mathbb{N}$ ), stochastic effects can be observed in the limit. Branching means that particles are erased and replaced with a random number of offspring at the same site. Consider the special case that this random number is either 0 or 2 , both with equal probability (critical binary branching). Any branching events are supposed to happen independently from the others. Doing the same rescaling as above, now summing over the random number of particles alive at a certain time, Dawson [Daw75] showed weak convergence of the empirical measure process $\left(X_{t}^{N}(d x)\right)_{t \geq 0}$ to a measure-valued process $\left(X_{t}(d x)\right)_{t \geq 0}$. To connect it with the above, that implies that the integrals $\left\langle\phi, X_{t}^{N}\right\rangle$ converge weakly for any $\phi$ as above, $t \geq 0$. Konno and Shiga [KS88] and Reimers [Rei89] showed that this process allows a density process $u(t, x)$ w.r.t. Lebesgue measure in dimension $q=1$, i.e. $X(t, d x)=u(t, x) d x$. This density $u$ is a nonnegative solution of the Dawson-Watanabe SPDE

$$
\partial_{t} u(t, x)=\frac{1}{2} \partial_{x}^{2} u(t, x)+\sqrt{u(t, x)} \dot{W}(t, x),
$$

where $\dot{W}$ is white noise on $\mathbb{R}_{+} \times \mathbb{R}$. Such a solution $u$ exists in a stochastically weak sense, meaning that there is a probability space with noise $\dot{W}$ and solution $u$ such that the SPDE holds almost surely. However, it would be more desirable to have the existence of stochastically strong solutions, meaning that for given $\dot{W}$ such a $u$ is a measurable function of $\dot{W}: u=F(\dot{W})$. Following a classical result of Yamada and Watanabe, it will be shown (Lemma 5.1.1) that this measurable function exists if pathwise uniqueness for the SPDE is satisfied. Pathwise uniqueness means that any two solutions $u^{1}$ and $u^{2}$, defined on the same space with probability measure $\mathbb{P}$ and the same noise $\dot{W}$, fulfill $\mathbb{P}\left(u^{1}=u^{2}\right)=1$. To obtain such pathwise uniqueness results will be the major task in this thesis.

Here, a more general version of a such a stochastic heat equation (SHE) is studied replacing the square root in (1.1) with a Hölder-continuous function $\sigma(u): \mathbb{R} \rightarrow \mathbb{R}:$

$$
\partial_{t} u(t, x)=\frac{\Delta}{2} u(t, x)+\sigma(u(t, x)) \dot{W}(t, x),
$$

with $t \geq 0, x \in \mathbb{R}^{q}$ and a suitable initial value $u(0, \cdot)=u_{0}(\cdot)$. For non-negative solutions, a special case of interest is the family $\sigma(u)=u^{\gamma}, \gamma \in(0,1]$. This family includes the two cases of (1.1) $(\gamma=1 / 2)$ and the parabolic Anderson model $(\gamma=1$, e.g. CM94). The latter yields a linear equation in $u$, which has been studied intensively in the last years, see GK05. Since $u^{\gamma}=u^{1 / 2} u^{\gamma-1 / 2}$ and $u^{1 / 2}$ is the term appearing in (1.1), general exponents $\gamma \in(0,1)$ could be understood as density dependent branching; see page 326 of [MP92] and Section 6.2 here.

The main reason for the existence of a density process $u$ only to hold for $q=1$ is the roughness of white noise in higher dimensions. To generalise that setting (primarily for the wave equation), different spatially smoother noises were considered 
more carefully, in particular by [Dal99]. There is a special focus on some of these so called colored noises, which are spatially correlated centered Gaussian noises with covariance given by:

$$
\mathbb{E}[\dot{W}(\phi) \dot{W}(\psi)]=\int_{\mathbb{R}^{1+2 q}} \phi(s, x) k(x, y) \psi(s, y) d x d y d s, \quad \phi, \psi \in C_{c}^{\infty}\left(\mathbb{R}_{+} \times \mathbb{R}^{q}\right),
$$

for a correlation kernel $k(x, y)=|x-y|^{-\alpha}, \alpha>0$. There are two reasons why considering this kind of colored noise is similar to white noise. The first is that for $\alpha \rightarrow q$ the kernel $|x-y|^{-\alpha}$ converges weakly to the white noise correlation kernel $\delta_{0}(x-y)$. Secondly, if in the above particle model the branching events are correlated in a sufficiently strong sense, then in a limit construction as above one can obtain SHEs with colored noise. For example, Mytnik Myt96 showed convergence of approximate densities of a certain particle system to an SHE with a mixture of white and colored noise. There have been results about SPDEs with this kind of colored noise in the last ten years, e.g. [Stu03, [FK10, [MPS06], [SSS02], [FSS06].

Going back to equation (1.2), let us note that the question of weak existence for continuous $\sigma$ was answered in Shi94 for white noise and in MPS06 for colored noise. However, the question of pathwise uniqueness in the white noise case was open for more than 20 years. To quote C. Mueller in [DKRA09]: "Almost sure uniqueness is an unsolved problem which has attracted the attention of many (...) probabilists and I have heard at least two false announcements of false proofs."

In 2006, Mytnik, Perkins and Sturm [MPS06] proved pathwise uniqueness provided that $\alpha<2 \gamma-1$ in the colored noise case. There were some indications that their result was not optimal. Extending that work, Mytnik and Perkins MP11 showed pathwise uniqueness in the white noise case if $\gamma>\frac{3}{4}$. They also provided the following conjecture:

Conjecture (Conjecture 1.6 of [MP11]). Pathwise uniqueness in $C\left(\mathbb{R}_{+}, C_{\text {tem }}\right)$ for (1.2) holds in the colored noise case if $\alpha<2(2 \gamma-1)$.

In this dissertation this conjecture is proved using a similar proof strategy as theirs. The function space $C_{\mathrm{tem}}$ is defined in 3.19).

In addition to existence and uniqueness there are a number of results which are known for white noise, but have not been transferred to the colored noise setting. Here, one of them is proved: the compact support property of nonnegative solutions to (1.2) with $\sigma(u)=u^{\gamma}, \gamma \in(0,1)$ and $\dot{W}$ colored noise. The compact support property means that $u(t, \cdot)$ has compact support, whenever the initial condition $u(0, \cdot)$ was compactly supported. In the white noise case, this was known to fail for $\gamma \geq 1$, Mue91 and known to hold if $\gamma<1$, MP92. In the proof here, it is also shown that $u$ gets extinct in finite time, meaning that there is an almost surely finite random variable $T<\infty$ with $u(T, \cdot) \equiv 0$. 
Let us put together some of the results that are known up to now and the gaps which are still to be filled for white noise and for colored noise. We will split up each table in two cases, real-valued solutions and nonnegative solutions. Sometimes, stronger results can be shown if one restricts attention to nonnegative solutions.

We use the abbreviations $W E$ for weak existence, $W U$ for weak uniqueness, $P U$ for pathwise uniqueness and CSP for compact support property. Some of the results require further restrictions on the solution spaces considered, e.g. the pathwise uniqueness is shown on a certain set $C\left(\mathbb{R}_{+}, C_{\text {tem }}\right)$ of functions. For the details we refer to the references given below the tables.

Consider solutions $u$ of 1.2 with $q=1$ and $\sigma(\cdot)=|u|^{\gamma}$, where $\gamma=1$ stands for $\sigma$ being Lipschitz.

\begin{tabular}{|l|c|c|c|c|c|c|c|}
\hline \multicolumn{9}{|c|}{ White Noise } \\
\hline \hline & \multicolumn{3}{|c|}{$u \in \mathbb{R}$} & \multicolumn{5}{c|}{$u \geq 0$} \\
& WE & WU & PU & WE & WU & PU & CSP \\
\hline$\gamma=1$ & $\checkmark$ a) & $\checkmark$ & $\checkmark$ a) & $\checkmark$ b) & $\checkmark$ & $\checkmark$ & no, c) \\
\hline$\gamma \in(0,1)$ & $\checkmark$ d) & no, $\gamma<\frac{3}{4}$ e) & $\left.\checkmark \gamma>\frac{3}{4}, \mathrm{f}\right)$ & $\checkmark$ g) & $\left.\checkmark \gamma \geq \frac{1}{2} \mathrm{~h}\right)$ & $?$ & $\checkmark$ i) \\
\hline
\end{tabular}

References:

a) Thm 3.5 in Wal86]; b) Thm 2.2 in [Shi94]; c) Thm 1 in [Mue91; d) Thm 1.1 in [MP11]; e) Thm 1.1 in [MMP12] f) Thm 1.2 in [MP11]; g) Thm 2.6 in [Shi94;

h) Thm 1.1 in Myt98; i) Thm 3.4 in [MP92, Thm 1.7 in Kry97.

Consider solutions $u$ of 1.2 with $\sigma(\cdot)=|u|^{\gamma}$, where $\gamma=1$ stands for $\sigma$ being Lipschitz and $\alpha \in[0, q)$ :

\begin{tabular}{|l|c|c|c|c|c|c|c|}
\hline \multicolumn{8}{|c|}{ Colored Noise $k(x, y)=|x-y|^{-\alpha}$} \\
\hline \hline & \multicolumn{3}{|c|}{$u \in \mathbb{R}$} & \multicolumn{5}{c|}{$u \geq 0$} \\
& WE & WU & PU & WE & WU & PU & CSP \\
\hline$\gamma=1$ & $\checkmark \alpha<2$ a) & $\checkmark$ & $\checkmark$ a $)$ & $\checkmark$ b) & $?$ & $?$ & $?$ \\
\hline$\gamma \in(0,1)$ & $\checkmark \alpha<2 \mathrm{c})$ & $?$ & $\alpha<2(2 \gamma-1) \mathrm{d})$ & $\checkmark$ & $?$ & $?$ & $\checkmark$ e) \\
\hline
\end{tabular}

References:

a) Thm 13 in [Dal99]; b) Thm 2.5 in [Kot92]; c) Thm 1.2 in [MPS06]; d) Thm 5.3.1 in this dissertation; e) Thm 5.4 .3 for $q=1$ in this dissertation.

One of the immediate impressions of these tables is that there are many more open questions in the colored noise setting. In this work at least two of them could be answered for $\gamma \in(0,1)$ : pathwise uniqueness and the compact support property, but still leaves a lot of room for future research.

Apart from these two results we show the auxiliary Lemma 5.1.1 and the weak 
existence of the solution of a colored noise equation including drift.

We end this introduction with an overview of the organization of this dissertation. It is divided into two parts.

The first part starts with the basic notions of PDE in Chapter 2 and stochastic processes including stochastic integration in Chapter 3. Proofs are mostly omitted, but many references are given; only the part containing Gaussian processes in Section 3.3 and the introduction to SPDE in Sections 3.4 and 3.5 are treated in more detail. Chapter 4 lists many of the known results for the stochastic heat equation and lays the basis of this work. The main results are presented in a separate chapter, Chapter 5. Finally, an outlook to future research is given in Chapter 6 .

The second part contains all of the proofs. The proof of Lemma 5.1.1 can be found in Chapter 7, the proof of weak existence can be found in Chapter 8. The main part of this work is contained in Chapter 9, the proof of pathwise uniqueness. The proof of compact support property is in Chapter 10. Finally, Chapter 11 contains some proofs and longer calculations used in the outlook.

A list of the notation used can be found in the index at the end of this work. 


\section{Part I.}

Notation and Results 


\section{Partial Differential Equations}

We will briefly recapitulate some facts about (deterministic) partial differential equations (PDE). As we are not aiming at a thorough treatment of that subject we refer the reader interested in that to [Eva10, [Fri76], Hör85], [Paz83] or [Rau91, from which we borrow most of what is contained within this section. As there are several ways to deal with PDE we will present various ideas and techniques as most of them will reappear later.

\subsection{Partial differential operators}

Partial differential equations are an extension of the concept of ordinary differential equations (ODE). In ODE we look for a function $u:[a, b] \rightarrow \mathbb{R}$, where $a<b \in$ $[-\infty, \infty]$ and we know that $u$ and its derivatives $u^{\prime}, u^{\prime \prime}, \ldots, u^{(m)}$ obey a certain set of restrictions

$$
F\left(t, u(t), u^{\prime}(t), \ldots, u^{(m)}(t)\right)=0 \text { for all } t \in[a, b]
$$

for some fixed $m \in \mathbb{N}$ and a function $F:[a, b] \times \mathbb{R}^{m+1} \rightarrow \mathbb{R}$. Additionally, we require an initial condition $u(a)=\bar{u} \in \mathbb{R}$.

One natural extension of this concept is to take into account real-valued functions $u: \Omega \rightarrow \mathbb{R}$, where $\Omega$ is a connected open subset of $\mathbb{R}^{q}, q \geq 1$. As then derivatives are partial derivatives we will use the symbol $\partial^{\alpha}:=\partial_{x_{1}^{\alpha_{1}}} \cdots \partial_{x_{q}^{\alpha_{q}}}$ for $\alpha=\left(\alpha_{1}, \ldots, \alpha_{q}\right) \in$ $\mathbb{Z}_{+}^{q}$ and $|\alpha|=\sum_{i=1}^{q} \alpha_{i}$, the order of the operator. Analogously to 2.1], we look for $u: \Omega \rightarrow \mathbb{R}$, s.t.

$$
F\left(x ;\left\{\partial^{\alpha} u(x),|\alpha| \leq m\right\}\right)=0, x \in \Omega,
$$

for some fixed $m \in \mathbb{N}, F: \mathbb{R}^{q} \times \mathbb{R}^{q^{m}} \rightarrow \mathbb{R}$. For ODE we had an initial value problem $(u(a)=\bar{u} \in \mathbb{R})$. In the PDE context the required/useful boundary conditions vary due to the properties of the equation, so we will leave that out for a moment.

Using the implicit function theorem, one can (at least locally) solve $(2.2)$ for one of the highest order derivatives appearing. This gives rise to some analytic existence and uniqueness results. Locally, nevertheless, the equation can then also be linearized and be written as

$$
P(x, \partial) u(x)=0, x \in \Omega,
$$


where

$$
P(x, \partial)=P\left(x, \partial_{x}\right)=\sum_{|\alpha| \leq m} a_{\alpha}(x) \partial^{\alpha} \text { and } a_{\alpha}=\frac{\partial}{\partial\left(\partial^{\alpha} u\right)} F\left(x,\left\{\partial^{\beta} u(x)\right\}\right): \Omega \rightarrow \mathbb{R}
$$

is an $m$-th order partial differential operator (PDO) for some $m \in \mathbb{N}$. The definition of the order came in here implicitly as the highest order appearing. Let us introduce some notation:

\section{Definition 2.1.1.}

(a) The main symbol $P_{m}: \Omega \times \mathbb{R}^{q} \rightarrow \mathbb{R}$ of a partial differential operator $P(x, \partial)$ is defined as

$$
P_{m}(x, \xi)=\sum_{|\alpha|=m} a_{\alpha}(x) \xi^{\alpha}, x \in \Omega, \xi \in \mathbb{R}^{q} .
$$

(b) For a $\operatorname{PDO} P\left(x, \partial_{x}\right)$ we say that $P$ is elliptic if $P_{m}(x, \xi) \neq 0$ for all $x \in \Omega$ and all $\xi \neq 0$.

(c) For a $\mathrm{PDO} Q((t, x), \partial)$ on $\Omega \subset \mathbb{R}_{+} \times \mathbb{R}^{q} \ni(t, x)$ we say that $Q$ is parabolic, if $Q((t, x), \partial)=\partial_{t}+P\left((t, x), \partial_{x}\right)$ and $P\left((t, x), \partial_{x}\right)$ is second-order (globally) and elliptic.

(d) If there is a $\theta>0$ s.t. $P_{m}(x, \xi) \geq \theta|\xi|^{2}$ for all $\xi \in \mathbb{R}^{q}$, then $P$ is called uniformly elliptic and $Q$ is called uniformly parabolic.

From now on we only use $\Omega=\mathbb{R}^{q}$. For parabolic equations it is sufficient to give initial data $u(0, \cdot)$ only, even though the hypersurface $\{t=0\} \times \mathbb{R}^{q}$ is characteristic. The most prominent example of such an equation is the heat equation

$$
\partial_{t} u(t, x)-\nu \Delta u(t, x)=0, t>0, x \in \mathbb{R}^{q},
$$

where $\nu>0$ and $P(x, \partial)=\Delta=\sum_{i=1}^{q} \partial_{x_{i}}^{2}$ is the Laplace(-Beltrami) operator on $\mathbb{R}^{q}$. Note that $P_{m}(x, \xi)=\sum_{i=1}^{q} \xi_{i}^{2}$ has only a trivial zero here, so we have a uniformly parabolic equation.

The two most basic questions for an equation such as (2.3) or 2.4 are that of existence and uniqueness of a solution. In the parabolic setup (which we will consider throughout this dissertation) the questions take the following form:

Given $f, g: \mathbb{R}^{q} \rightarrow \mathbb{R}$ fixed, the equation

$$
\left(\partial_{t}+A\right) u=f(t>0), u(0, \cdot)=g
$$

and a certain function space $U$,

(a) can we find a $u \in U$ satisfying the equation in some sense (existence) 
(b) and second, if there were two solutions $u_{1}, u_{2} \in U$, would they be equal in $U$ (uniqueness)?

We will ask these questions for the stochastic partial differential equations we introduce in the next chapters. Before that, we give some more definitions and notations for PDE to explain clearer what can be meant by "satisfying the equation in some sense."

\subsection{Weak solutions, fundamental solutions and distributions}

Often it is not possible to obtain solutions in a classical sense. For instance, due to the order of the differential operator, the solution $u$ might be required to be differentiable up to some order, but at the same time should obey a certain rough initial condition. In order to resolve that problem it is helpful to define function spaces allowing a different view of the PDE. We follow Chapter 2 of [Rau91].

We remind the reader that the space $L^{2}\left(\mathbb{R}^{q}, d x\right)$ where $d x$ is Lebesgue measure on $\mathbb{R}^{q}$ is a Hilbert-space with inner product $\langle f, g\rangle_{L^{2}}=\int f g d x$. The spaces $L^{p}\left(\mathbb{R}^{q}, d x\right)$ are Banach-spaces w.r.t. $\|f\|_{p}=\left(\int|f|^{p} d x\right)^{1 / p}$ and we can define the set of locally $p$-integrable functions

$$
L_{\mathrm{loc}}^{p}\left(\mathbb{R}^{q}\right)=\left\{f: \mathbb{R}^{q} \rightarrow \mathbb{R}: \int|f|^{p} \phi d x<\infty \forall \phi \in C_{c}^{\infty}\left(\mathbb{R}^{q}\right) .\right\}, p \geq 1 .
$$

Here, $C_{c}^{\infty}$ is the space of compactly supported, infinitely differentiable functions on $\mathbb{R}^{q}$.

We define certain function spaces which are well-known in the theory of partial differential equations.

\section{Definition 2.2.1.}

(a) The Fréchet space of tempered functions $\mathcal{S}\left(\mathbb{R}^{q}\right)$ is given by

$$
\mathcal{S}\left(\mathbb{R}^{q}\right)=\left\{u \in C^{\infty}\left(\mathbb{R}^{q}\right): \forall \alpha, \beta \in \mathbb{N}^{q}: \sup _{x \in \mathbb{R}^{q}}\left|x^{\alpha} \partial^{\beta} u(x)\right|<\infty\right\} .
$$

(b) The space of tempered distributions $\mathcal{S}^{\prime}\left(\mathbb{R}^{q}\right)$ is defined as the space of continuous linear functionals on $\mathcal{S}\left(\mathbb{R}^{q}\right)$.

(c) The space $\mathcal{D}^{\prime}\left(\mathbb{R}^{q}\right)$ of distributions is defined as the space of continuous linear functions on $\mathcal{D}\left(\mathbb{R}^{q}\right)=C_{c}^{\infty}\left(\mathbb{R}^{q}\right)$, the compactly supported, smooth functions on $\mathbb{R}^{q}$. Here $\mathcal{D}$ is equipped with the family of seminorms $\|u\|_{m, K}=$ $\sup _{x \in K,|\alpha| \leq m}\left|\partial^{\alpha} u(x)\right|$, where $m \in \mathbb{Z}_{+}, K$ a compact subset of $\mathbb{R}^{q}$. 
Clearly, $\mathcal{D}^{\prime} \supset \mathcal{S}^{\prime} \supset \mathcal{S} \supset \mathcal{D}$ and a prominent object in the first two spaces is the $\delta$-function $\delta_{x} \in \mathcal{S}^{\prime}\left(\mathbb{R}^{q}\right)$ for any fixed $x \in \mathbb{R}^{q}$, which maps $f \in \mathcal{S}\left(\mathbb{R}^{q}\right)$ to $f(x) \in \mathbb{R}$.

We can define the Fourier-transform for $f \in \mathcal{S}\left(\mathbb{R}^{q}\right)$ :

$$
(\mathcal{F} f)(\xi):=(2 \pi)^{-q / 2} \int_{\mathbb{R}^{q}} e^{-i x \xi} f(x) d x,
$$

where $x \xi$ is short for the Euclidean inner product $x^{t} \xi$ of $x$ and $\xi$. This transform $\mathcal{F}$ extends naturally to $\mathcal{S}^{\prime}\left(\mathbb{R}^{q}\right)$ by the usual pairing. There are several useful properties such as Plancherel's Theorem stating that $\|f\|_{L^{2}}=\|\mathcal{F} f\|_{L^{2}}$ for $f \in \mathcal{S}\left(\mathbb{R}^{q}\right)$ and

$$
(\phi * \psi)(x)=(2 \pi)^{q / 2} \mathcal{F}(\mathcal{F}(\phi) \mathcal{F}(\psi)),
$$

where $\phi, \psi \in \mathcal{S}\left(\mathbb{R}^{q}\right)$ and $\phi * \psi=\int \phi(\cdot-z) \psi(z) d z$ is the convolution. A useful property of the Fourier-transform is the following elementary identity for $f \in \mathcal{S}\left(\mathbb{R}^{q}\right)$, $\alpha \in \mathbb{Z}_{+}^{q}$ :

$$
\mathcal{F}\left(\partial_{x}^{\alpha} f\right)=(i \xi)^{\alpha} \mathcal{F} f
$$

Thus, it seems a good idea to define the so-called Sobolev-spaces

$$
H^{s}\left(\mathbb{R}^{q}\right)=\left\{u \in \mathcal{S}\left(\mathbb{R}^{q}\right):\langle\xi\rangle^{s}(\mathcal{F} u) \in L^{2}\left(\mathbb{R}^{q}, d x\right)\right\}, s \in \mathbb{R},
$$

where $\langle\xi\rangle=(1+|\xi|)$. This space is a Hilbert-space w.r.t. the inner product $\langle u, v\rangle=$ $\left\langle\mathcal{F} u,\langle\xi\rangle^{2 s} \mathcal{F} v\right\rangle_{L^{2}}$. Most often we will write

$$
\langle f, g\rangle=\langle f, g\rangle_{L^{2}}=\int_{\mathbb{R}^{q}} f(x) g(x) d x
$$

for the $L^{2}$-inner product if this is well-defined.

For the heat equation

$$
\left(\partial_{t}-\nu \Delta\right) u=0, u(0, \cdot)=\delta_{0}(\cdot) \in \mathcal{S}^{\prime}\left(\mathbb{R}^{q}\right),
$$

we can do the Fourier-transform w.r.t. the spatial variable $x$ to obtain

$$
\partial_{t}(\mathcal{F} u)(\xi)=-\nu|\xi|^{2}(\mathcal{F} u)(\xi), \mathcal{F}(u(0, \cdot))(\xi)=(2 \pi)^{-q / 2} .
$$

This is an ODE in $t$ and is solved by

$$
(\mathcal{F} u)(\xi)=(2 \pi)^{-q / 2} \exp \left(-\nu|\xi|^{2} t\right),
$$

which implies

$$
u(t, x)=(4 \pi \nu t)^{-q / 2} \exp \left(-\frac{|x|^{2}}{4 \nu t}\right) .
$$


This function, which we also denote by $\tilde{p}_{t}(x)=(4 \pi \nu t)^{-q / 2} \exp \left(-\frac{|x|^{2}}{4 \nu t}\right)$, is called the fundamental solution of the heat equation in $1+q$ dimensions. Elementary Fourier calculation and a variation of constants idea (Duhamel principle) allow to use $\tilde{p}$ for finding a solution of

$$
\left(\partial_{t}-\nu \Delta\right) u=f, u(0, \cdot)=g
$$

for $f \in C\left(\mathbb{R}_{+}, \mathcal{S}\left(\mathbb{R}^{q}\right)\right), g \in \mathcal{S}\left(\mathbb{R}^{q}\right)$ :

$$
u(t, x)=\int_{\mathbb{R}^{q}} \tilde{p}_{t}(y-x) g(y) d y+\int_{0}^{t} \int_{\mathbb{R}^{q}} \tilde{p}_{t-s}(y-x) f(s, y) d y d s .
$$

What have we done in the last few lines? We have found a formula (2.7) for $g \in \mathcal{S}\left(\mathbb{R}^{q}\right), f \in C\left(\mathbb{R}_{+}, \mathcal{S}\left(\mathbb{R}^{q}\right)\right)$. But the formula itself can also be applied to more general $f, g$. It turns out to be possible to use any $g \in \mathcal{S}^{\prime}$ and $f \in C\left(\mathbb{R}_{+}, \mathcal{S}^{\prime}\right)$. However, we might in return obtain a function $u=u(t, x)$ that does not have sufficient regularity for the differential operator being applied to it. Nevertheless, we call a function obtained as in (2.7) a mild solution to (2.6).

Another idea to construct solutions would be to consider (2.6) as an equation of elements in $\mathcal{D}^{\prime}\left(\mathbb{R}^{q}\right)$. Then, formally the equation would hold, if for all $\phi \in \mathcal{D}\left(\mathbb{R}^{q}\right)$ :

$$
\frac{d}{d t}\langle\phi, u(t, \cdot)\rangle=\langle u(t, \cdot), \nu \Delta \phi\rangle+\langle f(t, \cdot), \phi\rangle, t \geq 0 \text { and }\langle\phi, u(0, \cdot)\rangle=\langle\phi, g\rangle .
$$

Integrating this w.r.t. time variable $t$, we obtain

$$
\langle\phi, u(t, \cdot)\rangle=\langle\phi, u(0, \cdot)\rangle+\langle u(t, \cdot), \nu \Delta \phi\rangle+\langle f(t, \cdot), \phi\rangle .
$$

The last equation is called the weak formulation of 2.6) and any function $u \in$ $\mathcal{D}^{\prime}\left(\mathbb{R}^{q}\right)$ solving it for all $\phi \in \mathcal{D}\left(\mathbb{R}^{q}\right)$ is called a weak solution.

There is a relation between the two concepts:

Theorem 2.2.2 (Theorem 3.6.3 in [Rau91]). If $g \in H^{s}\left(\mathbb{R}^{q}\right), f \in C\left(\mathbb{R}_{+}, H^{s-2 q}\left(\mathbb{R}^{q}\right)\right)$ the following holds: $u \in C\left(\mathbb{R}_{+}, H^{s}\left(\mathbb{R}^{q}\right)\right.$ ) equivalently solves 2.7) or 2.8) for all $\phi \in \mathcal{D}\left(\mathbb{R}^{q}\right)$, where both equations hold in a classical pointwise sense.

Both concepts of solutions "mild" and "weak" will reappear in the stochastic setting.

Note that in 2.8 the operator $P(x, \partial)$ is applied to $\phi$ instead of $u$. Generally, one can shift the operators in the integral using Green's formula. As $\phi$ is of compact support there is generally no trouble with the boundary terms and we define the adjoint operator $P^{*}$ of $P$ as the unique operator, s.t.

$$
\left\langle P^{*} \phi, \psi\right\rangle=\langle\phi, P \psi\rangle, \quad \phi, \psi \in \mathcal{D}\left(\mathbb{R}^{q}\right) .
$$


Naturally, the heat equation is not a good example for the introduction of weak concepts for PDE, since the heat kernel $\tilde{p}$ smoothes the initial condition $g$ instantaneously. But as it will be the equation treated throughout the work it was used here.

Finally, let us remark that for the heat equation it holds that $\tilde{p}_{t}(x)=\tilde{p}_{1}\left(t^{-1 / 2} x\right)$, so $x$ and $\sqrt{t}$ are on the same scale, a fact reappearing also in the stochastic setup later. There are many more properties of the heat equation, but we refer the reader to Chapter 6 of [Eva10] for some overview.

\subsection{Semigroups}

We return to (2.7) in order to observe that the solution at time $t$ can be constructed using $\{u(s, \cdot): 0 \leq s<t\}$, which are the solutions up to time t. This phenomen appears frequently in parabolic (and also hyperbolic) equations and we formulate the PDE (2.6) in a more abstract function-valued setup:

$$
\begin{cases}\frac{d u(t)}{d t} & =A u(t)+f(t), t \geq 0 \\ u(0) & =g\end{cases}
$$

for a linear operator $A: D(A) \subset X \rightarrow X, f, g \in X$, where $X$ is a Banach space of functions with norm $\|\cdot\|$. The set $D(A)$ is called the domain of the operator $A$. This equation can abstractly be solved by

$$
u(t)=e^{t A} g+\int_{0}^{t} e^{(t-s) A} f(s) d s,
$$

which is really analogous to (2.7). The operator $e^{t A}$ is well-defined for bounded $A$ with $D(A)=X$. For general $A$ there is the concept of operator semigroups.

Definition 2.3.1. A family of operators $\left(T_{t}\right)_{t \geq 0} \subset L(X, X)$ is called a $C_{0}$-semigroup, if $T_{0}=\mathrm{Id}$, is the identity operator, $T_{t} T_{s}=T_{t+s}, t, s \geq 0$ and

$$
\lim _{t \rightarrow t_{0}}\left\|T_{t} x-T_{t_{0}} x\right\|=0
$$

for each $t_{0} \geq 0$ and $x \in X$. An operator $A: D(A) \subset X \rightarrow X$ is called the generator of the $C_{0}$-semigroup $\left(T_{t}\right)_{t \geq 0}$, if for all $x \in X$

$$
A x=\lim _{t \rightarrow 0} t^{-1}\left(T_{t} x-x\right) .
$$

We are interested in the converse of this definition as we are given the operator $A$ and look for the semigroup. These results can be obtained by the Hille-Yosida theorem or the Lumer-Phillips theorem for $C_{0}$-semigroups, see [Paz83]'s Chapter 1.

Theorem 2.3.2 (Lumer-Phillips, Theorem 1.4.3 of [Paz83]). A linear operator $A: D(A) \subset X \rightarrow X$ is the generator of a $C_{0}$-semigroup of contractions on $X$ if

(a) $D(A)$ is dense in $X$. 
(b) $A$ is dissipative, i.e. $\|(\lambda I d-A) x\| \geq \lambda\|x\|$ for all $x \in D(A), \lambda>0$.

(c) There is a $\lambda_{0}>0$, s.t. the range of the operator $\lambda I d-A$ is dense in $X$.

There is also an extension for noncontractive semigroups, which can be found in Theorem 1.3.1 in the same reference. We give an example where such a $C_{0^{-}}$ semigroup exists.

Example 2.3.3 (Theorem 2.1.43 in [Jac05]). Let $X=C_{0}\left(\mathbb{R}^{q}\right)$ be the continuous functions on $\mathbb{R}^{q}$ vanishing at $\infty$, equipped with the supremum-norm. Set

$$
A=\sum_{i, j=1}^{q} a_{i j}(x) \partial_{x_{i}} \partial_{x_{j}}+\sum_{i=1}^{q} b_{i}(x) \partial_{x_{i}}
$$

where $A$ is uniformly elliptic and the coefficients are bounded smooth functions. Then $A$ is the generator of a $C_{0}$-semigroup on $C_{0}\left(\mathbb{R}^{q}\right)$. 


\section{Probability Essentials}

Within this chapter a short introduction to the main notation related to stochastic processes, martingales and Gaussian processes is given. Most of the attention is given to the introduction of noises in the Gaussian process part, which is the only addendum to the standard theory. Good references on the first three sections are [Bre68, Kle08, Kal02] and RY91.

The last two sections contain the theory of stochastic integration in the multidimensional setting and the basic notions of stochastic partial differential equations.

\subsection{Stochastic processes}

Let $\Omega$ be the generic space, $\mathcal{P}(\Omega)$ be its power set and $\mathcal{F} \subset \mathcal{P}(\Omega)$ be a $\sigma$-field on $\Omega$. For a topological space $(\Omega, \tau)$ write $\mathcal{B}(\Omega)$ for the Borel $\sigma$-field generated by the open sets in $\tau$. If $\Omega$ has a canonical topology as the Euclidean space $\mathbb{R}^{q}$, we will assume that this topology is considered. A mapping $X: \Omega \rightarrow(E, \mathcal{E})$ into a measurable space $(E, \mathcal{E})$ is called measurable or a random variable, if $X^{-1} A \in \mathcal{F}$ for all $A \in \mathcal{E}$. If $E=\mathbb{R}$, we will call $X$ a real-valued random variable and it will be convenient to write $X \in \mathcal{F}$ in general.

Let $\mathbb{P}$ denote a probability measure on $(\Omega, \mathcal{F})$ and let $\mathbb{E}$ be its expectation functional. The pair $(\Omega, \mathcal{F})$ will be called a measurable space and the triple $(\Omega, \mathcal{F}, \mathbb{P})$ will be called a probability space. The set of probability measures on $\Omega$ will be denoted by $\mathcal{M}_{1}(\Omega)$; the set of finite measures by $\mathcal{M}_{f}(\Omega)$.

One often wants to consider a family of random variables indexed by a non-empty set $T$. For this, let $(\Omega, \mathcal{F}, \mathbb{P})$ be a probability space, $(E, \mathcal{E})$ be a Polish space and $\mathcal{E}^{T}$ the product- $\sigma$-field on $E^{T}$.

Definition 3.1.1. A family $\left(X_{t}\right)_{t \in T}$ of random variables $X_{t}: \Omega \rightarrow E, t \in T$, is called a stochastic process.

If $T \subset \mathbb{R}^{n}$ for a certain $n \in \mathbb{N}$, then we say that $\left(X_{t}\right)_{t \in T}$ is a random field.

Note that a stochastic process $\left(X_{t}\right)_{t \in T}$ induces a probability measure $\mathcal{L}[X]:=\tilde{\mathbb{P}}=$ $\mathbb{P} \circ X^{-1}$ on $\left(E^{T}, \mathcal{E}^{T}\right)$ via a consistency argument (Daniell-Kolmogorov Theorem, see Theorem 6.16 of [Kal02]). This probability measure is determined by its restriction to the finite subsets $\mathfrak{T} \subset \mathcal{P}(T)$ of $T$. Let $\pi_{S}^{T}: E^{T} \rightarrow E^{S},\left(x_{t}\right)_{t \in T} \mapsto\left(x_{t}\right)_{t \in S}$ be the projection down to a set $S \subset T$. We will call the collection

$$
\left(\tilde{\mathbb{P}} \circ\left(\pi_{S}^{T}\right)^{-1}\right)_{S \in \mathfrak{T}}
$$


the finite dimensional distributions (f.d.d.) of $X$.

Definition 3.1.2. Let $\left(X_{t}\right)_{t \in T}$ and $\left(Y_{t}\right)_{t \in T}$ be two stochastic processes on $T \neq \emptyset$.

(a) $X$ and $Y$ are called indistinguishable, if $\mathbb{P}\left(X_{t}=Y_{t}\right.$ for all $\left.t \in T\right)=1$.

(b) $X$ and $Y$ are called modifications, if $\mathbb{P}\left(X_{t}=Y_{t}\right)=1$ for all $t \in T$.

(c) If $\left(Z_{t}\right)_{t \in T}$ is another process on a different probability space $\left(\Omega^{\prime}, \mathcal{F}^{\prime}, \mathbb{P}^{\prime}\right)$ and $X$ and $Z$ have the same f.d.d., then $Z$ and $X$ are called versions of each other. Write $X \stackrel{\text { f.d.d. }}{=} Z$.

(d) If $T$ consists only of a single point, then we will write $X \stackrel{d}{=} Z$, if they are versions and we will say they have the same distribution.

In the case of countable $T$ the two first definitions coincide, whereas they differ if $T$ is uncountable. A modification of a process is also a version. Define the rectangle

$$
[[a, b]]:=\prod_{i=1}^{q}\left[a_{i}, b_{i}\right] \subset \mathbb{R}^{q} \text { for } a_{i}<b_{i} \in \mathbb{R} .
$$

In the case of $E=\mathbb{R}$, i.e. a real-valued process $X$, note that $X$ can be regarded as a random function $X: T \times \Omega \rightarrow \mathbb{R}$ on the set $T$. For fixed $\omega \in \Omega$, call $X(\omega): T \rightarrow \mathbb{R}$ the path of $X$. If $T \subset \mathbb{R}^{n}$ a natural question would be to ask if the process is continuous at a fixed point $t \in T$. However, using the Daniell-Kolmogorov construction via finite dimensional distributions, this question cannot be answered, generally. But at least the following well-known theorem holds:

Theorem 3.1.3 (Kolmogorov-Centsov; Theorem 3.23 in [Kal02]). Let $T \subset \mathbb{R}^{q}$. Suppose $\left(X_{t}\right)_{t \in T}$ is a stochastic process taking values in a complete metric space $(S, \rho)$ such that there are $C>0, p>0$ and $\gamma>0$

$$
\mathbb{E}\left[\rho\left(X_{t}, X_{s}\right)^{p}\right] \leq C|t-s|^{q+\gamma} \quad \forall s, t \in T .
$$

Then $X$ has a continuous version. Additionally, we have for $0 \leq \theta<\frac{\gamma}{p}$ :

$$
\mathbb{E}\left[\left(\sup _{s \neq t, \in T,|s-t| \leq 1} \frac{\rho\left(X_{t}, X_{s}\right)}{|t-s|^{\theta}}\right)^{p}\right]<\infty .
$$

We remind the reader that a function $f: \mathbb{R}^{q} \rightarrow \mathbb{R}$ is said to be Hölder-continuous of order $\gamma \in(0,1]$, if there is a constant $c<\infty$ such that

$$
\sup _{x \neq y \in \mathbb{R}^{q},|x-y| \leq 1} \frac{|f(x)-f(y)|}{|x-y|^{\gamma}} \leq c .
$$


In the case $\gamma=1$, the term Lipschitz-continuous is used more frequently. Thus, Theorem 3.1 .3 states that there is a version which almost surely has paths which are Hölder-continuous of order $\theta$.

Sometimes it is not possible to obtain such strong regularity results on the process. Therefore, we make the following definition for deterministic functions on the real line.

Definition 3.1.4. Let $(M, \mathcal{O})$ be a topological space. The function $f: \mathbb{R}_{+} \rightarrow M$ is called càdlàg, if we have that $\lim _{\varepsilon \rightarrow 0+} f(t-\varepsilon)$ exists for all $t>0$ and $\lim _{\varepsilon \rightarrow 0+} f(t+$ $\varepsilon)=f(t)$ for all $t \geq 0$. We write $D\left(\mathbb{R}_{+}, M\right)=\left\{f: \mathbb{R}_{+} \rightarrow M: f\right.$ càdlàg $\}$ and $C\left(\mathbb{R}_{+}, M\right)=\left\{f: \mathbb{R}_{+} \rightarrow M: f\right.$ continuous $\}$. The space $D\left(\mathbb{R}_{+}, M\right)$ is called Skorohod space.

Remember that if $M$ is a Polish space, then $D\left(\mathbb{R}_{+}, M\right)$ and $C\left(\mathbb{R}_{+}, M\right)$ are as well Polish space and that Theorem 3.1.3 is often used to establish that a process has a version with paths in $C\left(\mathbb{R}_{+}, M\right)$.

\subsection{Martingales and classical stochastic integration}

Now we concentrate on processes where $T=\mathbb{R}_{+}=[0, \infty)$ is the positive half-line. One can think of it as the time-axis. Assume that we are given a probability space $(\Omega, \mathcal{F}, \mathbb{P})$ throughout this section.

\section{Definition 3.2.1.}

(a) A filtration $\left(\mathcal{F}_{t}\right)_{t \geq 0}$ is an increasing family of sub- $\sigma$-fields of $\mathcal{F}$. That means $\mathcal{F}_{s} \subset \mathcal{F}$ and $\mathcal{F}_{s} \subset \mathcal{F}_{t}$ for all $0 \leq s \leq t<\infty$.

(b) The process $X$ is called adapted to the filtration, if $X_{t} \in \mathcal{F}_{t}$ for all $t \geq 0$.

(c) A random variable $\tau: \Omega \rightarrow[0, \infty]$ is called a stopping time, if $\{\tau \leq t\} \in \mathcal{F}_{t}$ for all $t \geq 0$.

(d) For such a stopping time $\tau$ define $\mathcal{F}_{\tau}=\left\{A \in \mathcal{F}: A \cap\{\tau \leq t\} \in \mathcal{F}_{t} \forall t \geq 0\right\}$.

(e) The filtration is said to satisfy the usual conditions, if all $\mathbb{P}$-null sets $\mathcal{N} \subset \mathcal{F}_{0}$ and $\mathcal{F}_{s}=\bigcap_{t>s} \mathcal{F}_{t}$.

Probability spaces with filtrations will be called filtered proability spaces and will be denoted by $\left(\Omega, \mathcal{F}, \mathcal{F}_{t}, \mathbb{P}\right)$.

There is much more that can be said about this abstract concept of filtrations and adapted processes, though we refer to Doob [Doo01], pp. 387 onwards, as a good reference. It is always possible to uniquely extend a filtration to one which 
satisfies the usual conditions. In this section we assume that the ususal conditions are always satisfied.

An important concept in the study of stochastic processes is that of a martingale.

Definition 3.2.2. An adapted process $X=\left(X_{t}\right)_{t \geq 0}$ is called a martingale, if $\mathbb{E}\left|X_{t}\right|<\infty$ for all $t \geq 0$ and

$$
\mathbb{E}\left[X_{t} \mid \mathcal{F}_{s}\right]=X_{s} \text { for all } 0 \leq s \leq t
$$

It is called a sub-(super-) martingale, if the last condition is replaced by $\geq(\leq)$.

If there exists an increasing sequence of stopping times $\tau_{n}<\infty$ with $\lim _{n \rightarrow \infty} \tau_{n}=\infty$ almost surely and for each $n \in \mathbb{N}$ the process $\left(X_{t \wedge \tau_{n}}\right)_{t \geq 0}$ is a martingale, then $X$ is called a local martingale.

A prominent example of a martingale is that of Brownian motion.

Definition 3.2.3. A real-valued, adapted process $\left(B_{t}\right)_{t \geq 0}$ on a filtered probability space $\left(\Omega, \mathcal{F}, \mathcal{F}_{t}, \mathbb{P}\right)$ is called a Brownian motion, if $B_{0}=0, B$ has almost surely continuous paths and

(a) the law $\mathcal{L}\left(B_{t}-B_{s}\right)$ is independent of $\mathcal{F}_{s}$

(b) and the law $\mathcal{L}\left(B_{t}-B_{s}\right)=N(0, t-s)$,

for any $0 \leq s<t$.

Here, $N(0, t-s)$ is a centered normal law with variance $t-s$, i.e. $N(0, t-s)(A)=$ $\int_{A}(2 \pi(t-s))^{-1 / 2} \exp \left(-(2(t-s))^{-1} x^{2}\right) d x$ for $A \in \mathcal{B}(\mathbb{R})$; see also Definition 3.3.1. One can show that $B$ is a martingale w.r.t. $\left(\mathcal{F}_{t}\right)_{t \geq 0}$ and also w.r.t. its own filtration $\mathcal{G}=\left(\mathcal{G}_{t}\right)_{t \geq 0}$, where $\mathcal{G}_{t}=\sigma\left(B_{s}: s \leq t\right), t \geq 0$. The adapted process $B$ is also a Brownian motion on $\left(\Omega, \mathcal{F}, \mathcal{G}_{t}, \mathbb{P}\right)$.

The general conditions for martingales already imply a lot of regularity on the paths, as shown in the following result taken from [KS00]:

Proposition 3.2.4 (Theorem 1.3.13 of [KS00]). A martingale $X$ has a modification s.t. its paths are càdlàg. Then we say that $X$ is a càdlàg martingale.

The next result will be used frequently later on without special reference to it. It is one of the reasons which make martingales such an important concept:

Proposition 3.2.5 (Theorem II.3.6 of [RY91]). On a filtered probability space let $\tau$ be a finite stopping time and $M$ be a càdlàg martingale. Then the stopped process $M^{\tau}=\left(M_{t}^{\tau}\right)_{t \geq 0}=\left(M_{\tau \wedge t}\right)$ is again a càdlàg martingale. 
Often it is helpful to restrict the class of martingales to square-integrable martingales as we will see for the construction of the Itô-integral at the end of this section.

Definition 3.2.6. The space of square-integrable martingales started in 0 is denoted by $\mathcal{M}^{2}$, its subset of continuous square-integrable martingales by $\mathcal{M}^{2, c}$. Set

$$
\|M\|_{t}:=\sqrt{\mathbb{E} M_{t}^{2}}, t \geq 0 ; \quad\|M\|=\sum_{n \in \mathbb{N}} 2^{-n}\left(1 \wedge\|M\|_{n}\right),
$$

for $M \in \mathcal{M}^{2}$. Let $d(M, N)=\|M-N\|$ for $M, N \in \mathcal{M}^{2, c}$.

Then the following result holds.

Proposition 3.2.7 (Proposition 1.5.23 of [KS00]). The metric space $\left(\mathcal{M}^{2}, d\right)$ is complete and $\mathcal{M}^{2, c}$ is closed in $\mathcal{M}^{2}$.

There are many interesting features for martingales and we refer the reader to [KS00] and [RY91] for a broader overview. Here, though, we present only a small selection of results including Itô-integration. For a martingale $M \in \mathcal{M}^{2, c}$ we define the quadratic variation

$$
\langle M\rangle_{t}, t \geq 0,
$$

as the unique, adapted and increasing process such that $M^{2}-\langle M\rangle$ is a martingale.

Now we want to give a short overview on Itô-integration for Brownian motion to motivate the definitions of the next two sections. One of the first reasons to study integration was to get an idea for solutions of stochastic differential equations, that means to look for a real-valued process $X=\left(X_{t}\right)_{t \geq 0}$, such that

$$
d X_{t}=b\left(t, X_{t}\right) d t+\sigma\left(t, X_{t}\right) d B_{t},
$$

in a certain sense. Here, $b, \sigma$ are real-valued functions and $B$ is a Brownian motion. If $\sigma=0$ the equation is an ODE and the theory is well-known. For nontrivial $\sigma$, however, we need to define an integral with respect to $d B_{t}$, which cannot be modeled as a Stieltjes-integral. We will give an idea here, but refer to Chapters 1 and 3 of [KS00] for a complete overview.

First we integrate elementary functions: For $0 \leq a<b$ and $X \in \mathcal{F}_{a}$ let $H_{t}=$ $X \mathbb{1}_{(a, b]}(t)$ be a real-valued stochastic process. Define its integral:

$$
(H \cdot B)_{t}(\omega)=X(\omega)\left(B_{t \wedge b}(\omega)-B_{t \wedge a}(\omega)\right), t \geq 0, \omega \in \Omega .
$$

One easily checks that $H \cdot B$ is a continuous martingale. Let $\mathbb{S}_{0}$ be the set of linear combinations of such functions $H$ :

$$
\begin{gathered}
\mathbb{S}_{0}=\left\{f: \mathbb{R}_{+} \times \Omega \rightarrow \mathbb{R} \mid \exists m \in \mathbb{N}, 0 \leq a_{i}<b_{i}, X_{i} \in \mathcal{F}_{a_{i}}, 1 \leq i \leq m,\right. \\
\left.f(t, \omega)=\sum_{i=1}^{m} X_{i}(\omega) \mathbb{1}_{\left(a_{i}, b_{i}\right]}\right\} .
\end{gathered}
$$


Extend the mapping $H \mapsto H \cdot B$ to $\mathbb{S}_{0}$ by linearity and observe that this is welldefined (independent of the partitions chosen). Again, $f \cdot B$ is a continuous martingale for all $f \in \mathbb{S}_{0}$ and the so-called Itô-isometry holds:

$$
\mathbb{E}\left[(f \cdot B)_{t}^{2}\right]=\mathbb{E}\left[\int_{0}^{t} f(s, \omega)^{2} d s\right] .
$$

For $f \in \mathbb{S}_{0}$ define $[f]_{T}=\left(\mathbb{E}\left[\int_{0}^{T} f(s, \omega)^{2} d s\right]\right)^{1 / 2}$ as well as the metric on $\mathbb{S}$ induced by

$$
[f]=\sum_{n=1}^{\infty} 2^{-n}\left(1 \wedge[f]_{n}\right) .
$$

The goal is to extend $f \mapsto f \cdot B$ to the following space of progressively measurable processes

$$
\begin{aligned}
\mathcal{L}^{*}=\left\{f: \mathbb{R}_{+} \times \Omega \rightarrow \mathbb{R} \mid[f]<\infty\right. \text { and } & \\
\forall t & \left.\geq 0: f:[0, t] \times \Omega \rightarrow \mathbb{R} \text { is } \mathcal{B}([0, t]) \otimes \mathcal{F}_{t} \text { measurable }\right\} .
\end{aligned}
$$

equipped with the metric induced by [·]. By Proposition 3.2 .8 of [KS00] it holds that $\mathbb{S}_{0}$ is dense in $\mathcal{L}^{*}$ w.r.t. [·].

Now, we want to extend the mapping $f \mapsto f \cdot B$ from $\mathbb{S}_{0}$ to $\mathcal{L}^{*}$. Let $f \in \mathcal{L}^{*}$ be approximated by the sequence $\left(f_{k}\right)_{k \in \mathbb{N}}$ with elements $f_{k} \in \mathbb{S}_{0}, k \in \mathbb{N}:\left[f_{k}-f\right] \rightarrow 0$. Then $f_{k}$ is a Cauchy-sequence in $\mathbb{S}_{0}, f_{k} \cdot B$ are in $\mathcal{M}^{2, c}$ and so for the distance $d\left(f_{k} \cdot B, f_{m} \cdot B\right)$, as defined in Definition 3.2 .6 we obtain

$$
\begin{aligned}
\left\|\left(f_{k} \cdot B\right)-\left(f_{m} \cdot B\right)\right\| & =\sum_{n \in \mathbb{N}} 2^{-n}\left(1 \wedge\left(\mathbb{E}\left[\left(\left(f_{k}-f_{m}\right) \cdot B\right)_{n}^{2}\right]\right)^{1 / 2}\right) \\
& =\sum_{n \in \mathbb{N}} 2^{-n}\left(1 \wedge\left(\left[f_{k}-f_{m}\right]_{n}\right)^{1 / 2}\right) \\
& =\left[f_{k}-f_{m}\right] .
\end{aligned}
$$

So, also $\left(f_{k} \cdot B\right)_{k}$ is a Cauchy-sequence in $\mathcal{M}^{2, c}$. By Proposition 3.2.7, we know that there is a unique limit element in $\mathcal{M}^{2, c}$ which we denote by

$$
f \cdot B=\left(\int_{0}^{t} f(s, \cdot) d B_{s}\right)_{t \geq 0} .
$$

One can show again that this definition does not depend on the approximating sequence. The key-role for the extension were the Itô-isometry (3.2) and the completeness of the space of continuous martingales, see Proposition 3.2.7. Two aspects we will reencounter in the multi-dimensional setup.

We conclude this section by citing two important theorems that will be used frequently later on. The first theorem says that for continuous square-integrable 
martingales there is a time-change, such that the time-changed martingale is a Brownian motion:

Theorem 3.2.8 (Dambis-Dubins-Schwarz, Thm V.1.6 [RY91]). Assume that $M \in$ $\mathcal{M}^{2, c}$ is such that $\langle M, M\rangle_{\infty}=\infty$. Set

$$
T_{t}=\inf \left\{s:\langle M, M\rangle_{s}>t\right\} .
$$

Then $B_{t}=M_{T_{t}}$ is an $\left(\mathcal{F}_{T_{t}}\right)$-Brownian motion.

The next theorem is an inequality which gives lower and upper bounds for the running supremum $M_{t}^{*}:=\sup _{s \leq t} M_{s}, t \leq \infty$ of a martingale via its quadratic variation:

Theorem 3.2.9 (BDG-inequality, Thm IV.4.1 [RY91]). For all $p \in(0, \infty)$, there exist two constants $c_{p}$ and $C_{p}$ such that, for all $M \in \mathcal{M}^{2, c}$,

$$
c_{p} \mathbb{E}\left[\langle M, M\rangle_{\infty}^{p / 2}\right] \leq \mathbb{E}\left[\left(M_{\infty}^{*}\right)^{p}\right] \leq C_{p} \mathbb{E}\left[\langle M, M\rangle_{\infty}^{p / 2}\right] .
$$

As mentioned before, one of the important uses of Itô-integration is the solution of stochastic differential equations, such as (3.1). We present the abstract setting for stochastic equations. The ideas are taken from Kur07]. Let $S_{1}, S_{2}$ be Polish spaces and $\Gamma: S_{1} \times S_{2} \rightarrow \mathbb{R}$. The framework of a stochastic equation often is the following: Let $Y$ be an $S_{2}$-valued random variable with law $\nu \in \mathcal{M}_{1}\left(S_{2}\right)$ fixed. Look for an $S_{1}$-valued random variable $X$, s.t.

$$
\Gamma(X, Y)=0
$$

holds. Usually, $Y$ is not given explicitly, but $\nu$ is. So we make the following definition.

Definition 3.2.10 (Stochastically weak and strong solutions). Any pair $(X, Y)$ of random variables on a probability space $(\Omega, \mathcal{F}, \mathbb{P})$ is called a stochastically weak solution to $(\Gamma, \nu)$, if $\Gamma(X, Y)=0$ holds $\mathbb{P}$-almost surely and $\mathbb{P}(Y \in \cdot)=\nu$.

A weak solution $(X, Y)$ is called a stochastically strong solution to $(\Gamma, \nu)$, if there is a Borel-measurable $F: S_{2} \rightarrow S_{1}$, such that $X=F(Y), \mathbb{P}$ almost surely.

This framework is also the right one for SDEs such as (3.1). There we are given the equation with the condition that $B$ is required to be a Brownian motion on a suitable filtered probability space. So we are given the law $\mathcal{L}[B]$ on $S_{2}=C\left(\mathbb{R}_{+}, \mathbb{R}\right)$, since $B$ has continuous paths. It remains to define a suitable space $S_{1}$ for the solution $X$. We will speak more on that and also the formulation of the SDE in such a setting in Chapter 7. Remark finally, that for a strong solution it is equivalent to say that $X$ is measurable w.r.t. the $\sigma$-field generated by $Y$. In the context of SDE this leads to saying that $X$ needs to be adapted to the filtration of the Brownian motion. 


\subsection{Gaussian processes and noises}

In this section we give some definitions and properties of Gaussian processes, partially taken from [DKRA09. Other good references are [Jan97, [HKPS93, and [HS08, especially for the second part. Gaussian processes are a first natural class of integrators, since Brownian motion (a Gaussian process as seen in Example 3.3.8) was a suitable process for the introduction of the one-dimensional stochastic integral in the previous section.

We start with the definition of a one-dimensional Gaussian random variable:

Definition 3.3.1. A real-valued random variable $X$ is a Gaussian random variable, if there exist $m \in \mathbb{R}$ and $\sigma^{2} \in \mathbb{R}_{+}=[0, \infty)$, s.t.

$$
\mathbb{P}(X \in A)=\int_{A}\left(2 \pi \sigma^{2}\right)^{-1 / 2} \exp \left(-\frac{(x-m)^{2}}{2 \sigma^{2}}\right) d x
$$

for any bounded Borel-set $A \subset \mathbb{R}$. If $\sigma^{2}=0$, this equation is to be understood in the sense that $\mathbb{P}(X \in A)=\mathbb{1}_{A}(m)$, i.e. $X=m$ almost surely.

A Gaussian random variable is also called a normal variable. The (unique) quantities $m$ and $\sigma^{2}$ are called mean and variance, respectively.

There is an extension to the multi-dimensional case. Let $q \in \mathbb{N}$.

Definition 3.3.2. An $\mathbb{R}^{q}$-valued random variable $g$ is a Gaussian random variable, if the real-valued random variable $t g$ is Gaussian for any $t \in \mathbb{R}^{q}$.

As in the one-dimensional case it is also possible to identify two quantities:

Proposition 3.3.3. An $\mathbb{R}^{q}$-valued random variable $g$ is a Gaussian random variable if and only if there exist $m \in \mathbb{R}^{q}$ and $C \in \mathbb{R}^{q \times q}$ symmetric and non-negative definite s.t. for all $t \in \mathbb{R}^{q}$ :

$$
H(t):=\mathbb{E}(\exp (i t \cdot g))=\exp \left(i t \cdot m-\frac{1}{2} t \cdot C t\right) .
$$

Our goal is to extend the notion of a Gaussian random variable to Gaussian processes on more general index sets $T$.

Definition 3.3.4. Let $(\Omega, \mathcal{F}, \mathbb{P})$ be a probability space and $T \neq \emptyset$ be a set. A stochastic process $G: \Omega \times T \rightarrow \mathbb{R}$ is called Gaussian, if for all $t_{1}, \ldots, t_{k} \in T, k \in \mathbb{N}$, the $\mathbb{R}^{k}$-valued random variable $\left(G\left(t_{1}\right), \ldots, G\left(t_{k}\right)\right)$ is Gaussian.

The easiest example one can think of is the discrete set $T=\{1, \ldots, q\}$, which leads us back to the definition of $\mathbb{R}^{q}$-valued Gaussian variables. The functions 
$C:\{1, \ldots, q\}^{2} \rightarrow \mathbb{R}$ in Proposition 3.3 .3 had the important property of positive definiteness we want to generalize.

Definition 3.3.5. A function $C: T^{2} \rightarrow \mathbb{C}$ is called positive definite, if

$$
\sum_{i, j=1}^{n} a_{i} a_{j} C\left(t_{i}, t_{j}\right) \geq 0 \quad \forall a_{i}, a_{j} \in \mathbb{R}, t_{i}, t_{j} \in T, 1 \leq i, j \leq n \in \mathbb{N} .
$$

If $(T,+)$ is a group, a function $H: T \rightarrow \mathbb{C}$ is called positive definite if $C(t, s):=$ $H(t-s), s, t \in T$, is positive definite.

Note that in Proposition 3.3 .3 we used the term "non-negative definite" to describe the same fact in the finite-dimensional (or matrix) setup. In the general setup, the expression "positive definite" is used more frequently, even though it is a bit misleading.

As in the finite-dimensional cases mentioned above, one can identify quantities describing the distribution of a Gaussian process. A trivial consequence of the Daniell-Kolmogorov extension theorem and Proposition 3.3 .3 is:

Proposition 3.3.6 (Lemma 13.1 in Kal02]). The distribution of a Gaussian variable $G$ is uniquely determined by the mean $m: T \rightarrow \mathbb{R}$ and the covariance $C$ : $T \times T \rightarrow \mathbb{R}$, where

$$
\begin{gathered}
m(t)=\mathbb{E}(G(t)) \text { and } C(s, t)=\operatorname{Cov}(G(s), G(t)), \\
H(t)=\mathbb{E}[\exp (i G(t))]=\exp \left(i m(t)-\frac{1}{2} C(t, t)\right), \quad s, t \in T .
\end{gathered}
$$

As often in probability, we write $\operatorname{Cov}(X, Y)=\mathbb{E}[X Y]-\mathbb{E}[X] \mathbb{E}[Y]$ for the covariance of two random variables $X$ and $Y$. One can show that $C$ is symmetric and positive definite. Similarly $H$ is positive definite if $T$ has a group structure (Theorem 3.2.2 of [BCR84]). Assuming these properties, the converse of the previous proposition holds true:

Theorem 3.3.7 (Theorem 3.1 in [Doo01]). Let $T \neq \emptyset$ be a set. For a function $m: T \rightarrow \mathbb{R}$ and a symmetric positive definite $C: T^{2} \rightarrow \mathbb{R}$, there is a Gaussian process, whose f.d.d. are explicitely given by $m$ and $C$.

It is time to provide the reader with some examples:

Example 3.3.8.

(a) Let $T=\mathbb{R}_{+}, m(t)=0, C(s, t)=s \wedge t=\min (s, t), s, t \in \mathbb{R}_{+}$. Then a continuous version of this Gaussian process is a standard Brownian motion, see Definition 3.2.3. 
(b) Let $T=[0,1], m(t)=0, C(s, t)=s \wedge t-s t, s, t \in[0,1]$. Then, one can show that $C$ is positive definite. By $\mathbb{E}\left(G(t)^{2}\right)=C(t, t)=t-t^{2}$ being equal to 0 for $t=0,1$, one gets the idea that $G$ might be a Brownian bridge, the process obtained by conditioning a Brownian motion on $\left\{B_{1}=0\right\}$ (p.253 in [Kal02]).

(c) Let $T=\mathbb{R}_{+}^{q}, m(t)=0, C(s, t)=\prod_{j=1}^{q}\left(s_{j} \wedge t_{j}\right), s, t \in \mathbb{R}_{+}^{q}$. Then $G$ is called a Brownian sheet. It has a continuous version; some more properties of it are given in Chapter 1 of [Wal86].

\section{Minlos' Theorem}

There is a more concrete setup, which helps if some structure of $T$ is given. We first give a general exposition leading to Minlos' Theorem, then specialize to the case of $T=\mathcal{S}\left(\mathbb{R}^{1+q}\right)$ and finally introduce the Gaussian noises $W^{k}$ and $W^{\delta}$ called colored noise and white noise, respectively.

Following [HKPS93, we want to construct a nuclear space $T$. Let $M$ be a vector space with a family of scalar products $(\cdot, \cdot)_{n}, n \in \mathbb{N}_{0}$. Denote by $T_{n}$ the completion of $M$ w.r.t. $|\cdot|_{n}:=(\cdot, \cdot)_{n}^{1 / 2}$. Assume that $|\cdot|_{n} \leq|\cdot|_{m}$ for all $n<m$, which implies $T_{n} \supset T_{m}$ for all $n<m$. Additionally, we require that if $\left(\xi_{k}\right)_{k \in \mathbb{N}} \subset M$ is a Cauchysequence w.r.t. $|\cdot|_{m}$ and $\left|\xi_{k}\right|_{n} \rightarrow 0(k \rightarrow \infty)$, then also $\left|\xi_{k}\right|_{m} \rightarrow 0(k \rightarrow \infty), n<m$. Let $\mathcal{N}=\bigcap_{n \in \mathbb{N}} T_{n}$ and equip $T$ with the projective limit topology $\tau_{p}$ given by $\xi_{k} \rightarrow \xi$ in $\tau_{p}$, iff $\xi_{k} \rightarrow \xi$ in all $\left(T_{n},|\cdot|_{n}\right), n \in \mathbb{N}$. One can show that $T$ is a Fréchet-space.

Assume additionally that $T$ is nuclear, that means that for all $n \in \mathbb{N}$, there is a $m \in \mathbb{N}, m \geq n$ such that the natural inclusion $i_{n}^{m}: T_{m} \rightarrow T_{n}$ is Hilbert-Schmidt, meaning that its spectrum is a square-integrable sequence. Let $T^{\prime}$ be the dual of $T$, i.e. $T^{\prime}=\left\{w: T \rightarrow \mathbb{R}\right.$ : bounded w.r.t. all $|\cdot|_{n}$ and linear $\}$ and similarly define the dual $T_{n}^{\prime}$ of $T_{n}$; define $T_{-n}:=T_{n}^{\prime}$. Write $\langle\cdot, \cdot\rangle$ for the pairing of $T$ and $T^{\prime}$ and $\mathcal{B}=\mathcal{B}\left(T^{\prime}\right)$. Consider a mapping:

$$
H: T \rightarrow \mathbb{R}, \phi \mapsto H(\phi) .
$$

Theorem 3.3.9 (Minlos' Theorem, Theorem 1.1 in [HKPS93]). Assume that $H$ is positive definite, $H(0)=1$ and $H$ is continuous on $T$. Then there exists a unique probability measure $\mu_{H}$ on the measurable space $\left(T^{\prime}, \mathcal{B}\right)$ such that

$$
H(\phi)=\int_{T^{\prime}} \exp (i\langle x, \phi\rangle) \mu_{H}(d x) .
$$

Moreover, if $H$ is continuous with respect to $|\cdot|_{m}, m \in \mathbb{N}$, and if $m>n$ is such that the injection $i_{n}^{m}: T_{m} \rightarrow T_{n}$ is of Hilbert-Schmidt type, then $\mu_{H}\left(T_{-n}\right)=1$.

Of course, this theorem is not limited to Gaussian processes, but we will only apply it in that setting here. To prepare this application, let us construct such a 
nuclear space $T$. For this construction we use the notation from Chapter A.5 in HKPS93.

Let $M=\mathcal{S}\left(\mathbb{R}^{1+q}\right)$ be equipped with a family of scalar products $(\cdot, \cdot)_{2, p}, p \in \mathbb{N}_{0}$ defined as

$$
(\phi, \psi)_{2, p}:=\left(\phi, J^{p} \psi\right)_{L^{2}}, \quad \phi, \psi \in \mathcal{S}\left(\mathbb{R}^{1+q}\right), p \in \mathbb{N}_{0} .
$$

Here, $J$ is the self-adjoint operator (for the $L^{2}$ scalar product) given by

$$
J \phi(u):=\left(-\Delta+\left(1+|u|^{2}\right)\right) \phi(u)
$$

and $J^{p}$ is its $p$-th power, $p \in \mathbb{N}_{0}$. The operator $J$ is the Hamiltonian of the harmonic oscillator in $q+1$ dimensions ( + the constant function 1 ) and has an orthogonal (w.r.t. the $L^{2}$-scalar product) eigenbasis in $\mathcal{S}\left(\mathbb{R}^{1+q}\right)$ given by the Hermite functions $h_{n}$ defined as

$$
\begin{gathered}
h_{n}(x)=h_{n_{1}}\left(x_{1}\right) \cdots h_{n_{1+q}}\left(x_{1+q}\right), \\
h_{n_{1}}\left(x_{1}\right)=c\left(n_{1}\right) e^{x_{1}^{2} / 2} \frac{\partial^{n_{1}}}{\partial x_{1}^{n_{1}}} e^{-x_{1}^{2}},
\end{gathered}
$$

for $n_{1}, \ldots, n_{1+q} \in \mathbb{Z}_{+}, x \in \mathbb{R}^{1+q}$ with

$$
J h_{n}=\lambda_{n} h_{n}=\left(2\left(n_{1}+\cdots+n_{1+q}\right)+q+2\right) h_{n}, n \in \mathbb{Z}_{+}^{q+1} .
$$

The Hermite functions can be normalized. Recall that they also constitute an eigenbasis for the Fourier-transform:

$$
\mathcal{F} h_{n}=(-i)^{|n|} h_{n}
$$

We write $\mathcal{S}_{p}\left(\mathbb{R}^{1+q}\right)$ for the completion of $M=\mathcal{S}\left(\mathbb{R}^{1+q}\right)$ w.r.t. the norms induced by $(\cdot, \cdot)_{p}, p \in \mathbb{N}_{0}$. Clearly, $\mathcal{S}_{0}=L^{2}$. One can show that the topological space

$$
\hat{\mathcal{S}}\left(\mathbb{R}^{1+q}\right):=\bigcap_{n \in \mathbb{N}_{0}} \mathcal{S}_{p}\left(\mathbb{R}^{1+q}\right)
$$

is topologically isomorphic to $\mathcal{S}\left(\mathbb{R}^{1+q}\right)$ and so we will identify both spaces. Moreover, considering the spectrum of $J$, it is true that $J^{-\left(1+\frac{q}{2}\right)}: L^{2} \rightarrow L^{2}$ is HilbertSchmidt. Since, $J^{p}: \mathcal{S}_{p} \rightarrow L^{2}$ is an isometry, we know that $I=J^{p} J^{-1+\frac{q}{2}} J^{-p}$ : $\mathcal{S}_{p} \rightarrow \mathcal{S}_{p}$ is Hilbert-Schmidt and the image is contained in $\mathcal{S}_{p+1+\frac{q}{2}}$. So, the injection

$$
i_{p+1+\frac{q}{2}}^{p}: \mathcal{S}_{p}\left(\mathbb{R}^{1+q}\right) \rightarrow \mathcal{S}_{p+1+\frac{q}{2}}\left(\mathbb{R}^{1+q}\right)
$$

is Hilbert-Schmidt. Hence, the space $\mathcal{S}\left(\mathbb{R}^{1+q}\right)$ is a nuclear space with

$$
\mathcal{S}\left(\mathbb{R}^{1+q}\right) \subset \cdots \subset \mathcal{S}_{1}\left(\mathbb{R}^{1+q}\right) \subset L^{2}\left(\mathbb{R}^{1+q}\right) \subset \mathcal{S}_{-1}\left(\mathbb{R}^{1+q}\right) \subset \cdots \subset \mathcal{S}^{\prime}\left(\mathbb{R}^{1+q}\right) .
$$

As $J$ is positive, one can also define scalar products $(\cdot, \cdot)_{2, p}$ for non-integer $p$. They embed clearly, within the previous chain and we will later use such spaces $\mathcal{S}_{p}$ for noninteger $p \in \mathbb{R}$. Finally, define $\mathcal{B}\left(\mathcal{S}^{\prime}\right)$ to be the Borel- $\sigma$-field given by the weak-*topology. 


\section{Gaussian Noises}

We want to introduce Gaussian processes on $\mathcal{S}\left(\mathbb{R}^{1+q}\right)$, which will be called Gaussian noises. They will play the role of the integrators, which was played by Brownian motion in the one-dimensional setting, see the end of the previous section. There will not be a treatment of Gaussian noises in full generality. We will only present Gaussian noises on $\mathbb{R}^{1+q}$, which are white in time and have a certain spatial dependence structure. Remember that the main goal of this thesis is to work with the heat equation, where "time" refers to a selected coordinate of the equation and "space" is represented by $\mathbb{R}^{q}$.

In order to obtain Gaussian noises, we want to apply Minlos' Theorem 3.3.9. So we need to construct characteristic functions $H$ on the nuclear space $\mathcal{S}\left(\mathbb{R}^{1+q}\right)$. Remembering the result of Proposition 3.3.6 we first define a covariance functional and give some regularity results:

Lemma 3.3.10. For $k \in L_{l o c}^{1}\left(\mathbb{R}^{2 q}\right)$, which is bounded by

$$
k(x, y) \leq c\left(|x-y|^{-\alpha}+1\right),
$$

for almost all $(x, y) \in \mathbb{R}^{2 q}$ for a constant $c<\infty$ and $\alpha \in[0, q)$, the mapping

$$
L_{k}:\left\{\begin{array}{l}
\left(\mathcal{S}\left(\mathbb{R}^{1+q}\right)\right)^{2} \rightarrow \mathbb{R} \\
(\phi, \psi) \mapsto \int_{0}^{t} \int_{\mathbb{R}^{q}} \int_{\mathbb{R}^{q}} \phi(s, x) k(x, y) \psi(s, y) d x d y d s
\end{array}\right.
$$

is continuous.

We will not give a proof here, as we will present a slightly stronger statement in the proof of Lemma 3.3.13. There is a special case, which is not covered in this lemma, but the same continuity statement holds for putting the Dirac- $\delta$-distribution $\delta=\delta_{0} \in \mathcal{S}^{\prime}\left(\mathbb{R}^{q}\right)$ instead of $k$ :

$$
L_{\delta}(\phi, \psi):=\int_{0}^{t} \int_{\mathbb{R}^{q}} \phi(s, x) \psi(s, x) d x d s .
$$

Define the mapping $H_{k}: \mathcal{S}\left(\mathbb{R}^{1+q}\right) \rightarrow \mathbb{R}$ by

$$
H_{k}(\phi):=\exp \left(-\frac{1}{2} L_{k}(\phi, \phi)\right) .
$$

Lemma 3.3.11. Assume that $k$ is bounded as in Lemma 3.3.10 and $L_{k}$ is positive definite and symmetric. There is a Gaussian measure $\mu_{k}$ on $\mathcal{B}\left(\mathcal{S}^{\prime}\left(\mathbb{R}^{1+q}\right)\right)$, such that for all $\phi \in \mathcal{S}\left(\mathbb{R}^{1+q}\right)$ :

$$
\int_{\mathcal{S}^{\prime}} \exp (i\langle x, \phi\rangle) \mu_{k}(d x)=H_{k}(\phi)
$$

A random variable $\dot{W}^{k}$ on $\mathcal{S}^{\prime}$ with law $\mu_{k}$ is a centered Gaussian process on $\mathcal{S}\left(\mathbb{R}^{1+q}\right)$ with covariance given by $L_{k}$. 
Proof. We want to apply Theorem 3.3.9. Clearly, $H_{k}(0)=1$ and by Lemma 3.3 .10 the mapping $L_{k}$ is continuous and so $H_{k}$ also is. By Theorem 3.2.2 of [BCR84], $H_{k}$ is positive definite since $L_{k}$ is.

For the second part, denote the expectation w.r.t. $\mu_{k}$ by $\mathbb{E}_{\mu_{k}}$ and write $\mathcal{S}=$ $\mathcal{S}\left(\mathbb{R}^{1+q}\right)$ and likewise for $\mathcal{S}^{\prime}$. Then, let $\dot{W}^{k}$ be a realization of a random variable with values in $\mathcal{S}^{\prime}$ and law $\mu_{k}$ :

$$
\dot{W}^{k}: \Omega \rightarrow \mathcal{S}^{\prime}=L(\mathcal{S}, \mathbb{R}),
$$

the space of bounded linear maps from $\mathcal{S}$ to $\mathbb{R}$. If for $\phi \in \mathcal{S}$ we define $\left(\dot{W}^{k}(\phi)\right)(\omega):=$ $\left(\dot{W}^{k}(\omega)\right)(\phi)$ observe that the mapping

$$
\dot{W}^{k}(\omega): \mathcal{S}\left(\mathbb{R}^{1+q}\right) \rightarrow \mathbb{R}
$$

is linear: $\dot{W}^{k}(a \phi+\psi)=a \dot{W}^{k}(\phi)+\dot{W}^{k}(\psi)$ for any $\phi, \psi \in \mathcal{S}, a \in \mathbb{R}$ almost surely. Moreover, $\dot{W}^{k}(\phi)$ is a centered real-valued Gaussian variable with

$$
\mathbb{E}_{\mu_{k}}\left[\dot{W}^{k}(\phi)^{2}\right]=-\left.2 \partial_{t}^{2} \mathbb{E}_{\mu_{k}}[H(t \phi)]\right|_{t=0}=L_{k}(\phi, \phi)
$$

and similarly using the symmetry of $L_{k}$,

$$
\mathbb{E}_{\mu_{k}}\left[\dot{W}^{k}(\phi) \dot{W}^{k}(\psi)\right]=L_{k}(\phi, \psi),
$$

for $\phi, \psi \in \mathcal{S}$.

Next, we provide some examples:

Example 3.3.12.

(a) Let $k(x, y)=\delta_{0}(x-y) \in H^{-q}\left(\mathbb{R}^{1+q}\right)$ the $\delta$-function in each coordinate. Of course, the reader will note that this is not in the setup as we presented it in Lemma 3.3.10. One can also prove 3.3 .11 for the functional $L_{\delta}$ as defined in (3.5). The process $\dot{W}^{\delta}$ is called white noise.

(b) Choosing $k(x, y)=k_{\alpha}(x-y)=|x-y|^{-\alpha}, x \neq y \in \mathbb{R}^{q}$ for $\alpha \in(0, q)$, the Riesz kernel, will be a classical example for a stationary Gaussian process. One can check, that $L_{k}$ is positive definite in that case. There is a measure $\mu_{k_{\alpha}}$ on $\mathcal{B}\left(\mathbb{R}^{q}\right)$, s.t. $\mu_{k_{\alpha}}=\mathcal{F} k_{\alpha}$. Here, $\mu_{k_{\alpha}}=c_{\alpha} k_{q-\alpha} d \lambda$ for a constanct $c_{\alpha}$ and Lebesgue measure $d \lambda$ (cf. Lemma V.1.2(a) in [Ste67]).

Let us define a space of distributions depending on $\alpha \in(0, q)$ :

$$
\begin{aligned}
L_{\alpha}\left(\mathbb{R}^{q}\right) & :=\left\{f \in \mathcal{S}^{\prime}\left(\mathbb{R}^{q}\right): \int_{\mathbb{R}^{q}}|z|^{-\alpha}(f * f)(z) d z<\infty\right\} \\
& =\left\{f \in \mathcal{S}^{\prime}\left(\mathbb{R}^{q}\right): \int_{\mathbb{R}^{q}}|\xi|^{-q+\alpha}(\mathcal{F} f(\xi))^{2} d \xi<\infty\right\} .
\end{aligned}
$$


It is equipped with the norm $\|f\|_{\alpha}:=\int_{\mathbb{R}^{q}}|z|^{-\alpha}(f * f)(z) d z, f \in L_{\alpha}\left(\mathbb{R}^{q}\right)$. Denote the completion of $L_{\alpha}$ w.r.t. $\|\cdot\|_{\alpha}$ by $L_{\alpha}\left(\mathbb{R}^{q}\right)$. Formally the space $\alpha=q$ corresponds to the space $L^{2}$. We define the product $M_{\alpha}$ of function spaces in the following way:

$$
M_{\alpha}:=\left\{f \cdot g: \mathbb{R}^{1+q} \rightarrow \mathbb{R}: f \in L^{2}(\mathbb{R}), g \in\left(L^{1}\left(\mathbb{R}^{q}\right) \cap L_{\alpha}\left(\mathbb{R}^{q}\right)\right), \alpha \in(0, q)\right\} .
$$

Continuing with the general setup, the following holds:

Lemma 3.3.13. The Gaussian process $\dot{W}^{k}$ defined in Lemma 3.3.11 can be extended to $M_{\alpha}$ in the sense that $\dot{W}: M_{\alpha} \rightarrow L^{2}(\Omega, \mathbb{P})$ is a Gaussian variable with covariance functional $L_{k}$ as in (3.6).

Proof. We take the proof idea from Theorem 2 of [Dal99]. Let $(s, x) \mapsto f(s, x)=$ $p(s) \phi(x) \in M_{\alpha}$. Let $p_{n} \rightarrow p$ in $L^{2}(\mathbb{R})$ and for $\psi \in \mathcal{D}\left(\mathbb{R}^{q}\right)$ with $\psi \geq 0, \int \psi d x=1$ and support of $\psi$ in the unit ball of $\mathbb{R}^{q}$ define the mollifier

$$
\psi_{n}(x)=n^{q} \psi(n x), x \in \mathbb{R}^{q} .
$$

If we set $\phi_{n}:=\phi * \psi_{n}$, then $\phi_{n} \in \mathcal{S}\left(\mathbb{R}^{q}\right)$ and

$$
\begin{aligned}
\left\|\phi_{n}-\phi\right\|_{\alpha} & =\int_{\mathbb{R}^{q}} d \xi|\xi|^{-q+\alpha}\left(\mathcal{F} \phi_{n}-\mathcal{F} \phi\right)^{2}(\xi) \\
& \leq \int_{\mathbb{R}^{q}} d \xi|\xi|^{-q+\alpha}\left|\mathcal{F} \psi_{n}-1\right|^{2}|\mathcal{F} \phi|^{2} .
\end{aligned}
$$

Since $\left|\mathcal{F} \psi_{n}-1\right|^{2} \leq 4$ and it converges pointwise to zero, the dominated convergence theorem tells us that $\phi_{n} \rightarrow \phi$ in $L_{\alpha}$. A similar argument holds for convergence in $L^{1}\left(\mathbb{R}^{q}\right)$.

The sequences $\phi_{n}$ and $p_{n}$ are Cauchy-sequences and so we can do the following estimate for $f_{n}=p_{n} \phi_{n}, n, m \in \mathbb{N}:$ By (3.6) and later (2.5),

$$
\begin{aligned}
& \mathbb{E}\left[\left(\dot{W}^{k}\left(f_{m}\right)-\dot{W}^{k}\left(f_{n}\right)\right)^{2}\right]=L_{k}\left(f_{m}-f_{n}, f_{m}-f_{n}\right) \\
& =\int_{0}^{\infty} d s \int_{\mathbb{R}^{q}} \int_{\mathbb{R}^{q}}\left(f_{n}(s, x)-f_{m}(s, x)\right) k(x, y)\left(f_{n}(s, y)-f_{m}(s, y)\right) d x d y \\
& =\int_{0}^{\infty}\left(p_{n}(s)-p_{m}(s)\right)^{2} d s \int_{\mathbb{R}^{q}} \int_{\mathbb{R}^{q}}\left(\phi_{n}(x)-\phi_{m}(x)\right) k(x, y)\left(\phi_{n}(y)-\phi_{m}(y)\right) d x d y \\
& \leq c\left\|p_{n}-p_{m}\right\|_{L^{2}(\mathbb{R})}^{2} \int_{\mathbb{R}^{q}} \int_{\mathbb{R}^{q}}\left|\phi_{n}(x)-\phi_{m}(x)\right|\left(1+|x-y|^{-\alpha}\right)\left|\phi_{n}(y)-\phi_{m}(y)\right| d x d y \\
& =c\left\|p_{n}-p_{m}\right\|_{L^{2}(\mathbb{R})}^{2}\left[\left(\int_{\mathbb{R}^{q}}\left|\phi_{n}(x)-\phi_{m}(x)\right| d x\right)^{2}\right. \\
& \left.\quad+\int_{\mathbb{R}^{q}} d z k_{\alpha}(z)\left(\left(\phi_{n}-\phi_{m}\right) *\left(\phi_{n}-\phi_{m}\right)\right)(z)\right] \\
& =c\left\|p_{n}-p_{m}\right\|_{L^{2}(\mathbb{R})}\left[\left\|\phi_{n}-\phi_{m}\right\|_{L^{1}\left(\mathbb{R}^{q}\right)}^{2}+\int_{\mathbb{R}^{q}} d z k_{\alpha}(z) \mathcal{F}\left(\left(\mathcal{F}\left(\phi_{n}-\phi_{m}\right)\right)^{2}\right)(z)\right] .
\end{aligned}
$$


So, we note that $\left(\dot{W}^{k}\left(f_{n}\right)\right)_{n \in \mathbb{N}}$ is a Cauchy-sequence in $L^{2}\left(\Omega, \mu_{k}\right)$ and we denote its limit by $\dot{W}^{k}(f)$.

Note that the indicator functions $\mathbb{1}_{A}: \mathbb{R}^{q} \rightarrow\{0,1\}$ for $A \in \mathcal{B}_{b}\left(\mathbb{R}^{q}\right)$, the bounded Borel sets of $\mathbb{R}^{q}$, are contained in $L_{\alpha}$ and $\mathbb{1}_{[0, t]} \in L^{2}(\mathbb{R})$ for $t \geq 0$.. So we can extend the defintion of $\dot{W}^{k}$ to these sets. Define $\dot{W}^{k}([0, t] \times A):=\dot{W}^{k}\left(\mathbb{1}_{[0, t]} \mathbb{1}_{A}\right)$. It holds that

$$
\dot{W}^{k}([0, t] \times(A \cup B))=\dot{W}^{k}([0, t] \times A)+\dot{W}^{k}([0, t] \times B),
$$

almost surely for disjoint $A, B \in \mathcal{B}_{b}\left(\mathbb{R}^{q}\right)$ since $\mathbb{1}_{A \cup B}=\mathbb{1}_{A}+\mathbb{1}_{B}$, recall the linearity of $\dot{W}^{k}$. By continuity we also have for disjoint $A_{1}, A_{2}, \cdots \in \mathcal{B}\left(\mathbb{R}^{q}\right)$, s.t. $A=\bigcup_{k \in \mathbb{N}} A_{k}$ is bounded:

$$
\dot{W}^{k}([0, t] \times A)=L^{2}-\lim _{n \rightarrow \infty} \sum_{k=1}^{n} \dot{W}^{k}\left([0, t] \times A_{k}\right) .
$$

It can be shown that this limit does not hold almost surely in general (see Example 1.3.16 of [DKRA09]). This refers to the fact that $\dot{W}^{k}: M_{\alpha} \rightarrow \mathbb{R}$ is not a continuous functional any more.

There is one more remark we want to make in the white noise case. Choosing $A_{1}=\prod_{i=1}^{q+1}\left[0, s_{i}\right]$ and $A_{2}=\prod_{i=1}^{q+1}\left[0, t_{i}\right]$ for $s_{i}, t_{i}>0$, gives

$$
\mathbb{E}\left(\dot{W}^{\delta}\left(A_{1}\right) \dot{W}^{\delta}\left(A_{2}\right)\right)=\prod_{i=1}^{q+1}\left(s_{i} \wedge t_{i}\right),
$$

which is nothing else than the covariance of the Brownian sheet in $q+1$ dimensions, see Example 3.3.8 (c). As Proposition 3.3.6 uniquely characterizes Gaussian processes, we can say that a Brownian sheet is "integrated white noise." Remember that Example 3.3.8 (c) provided that the Brownian sheet is continuous. So, if white noise is its "derivative", we should expect some regularity, at least in a certain Sobolev space.

As already indicated in the beginning we want to separate the first coordinate of $\mathbb{R}^{1+q}$ sometimes. Therefore, we consider the random linear functional $\dot{W}\left(\mathbb{1}_{[0, t]} \times \cdot\right)$ on $L^{1}\left(\mathbb{R}^{q}\right) \cap L_{\alpha}\left(\mathbb{R}^{q}\right)$ for $t \geq 0$. It will be convenient to write

$$
W_{t}(\phi)=\dot{W}\left(\mathbb{1}_{[0, t]} \times \phi\right)
$$

for $\phi \in \mathcal{S}\left(\mathbb{R}^{q}\right), t \geq 0$. We will always consider the extended version of the Gaussian processes $\dot{W}$. Encouraged by the regularity result on the Brownian sheet, we give the following regularity lemma for noises:

Lemma 3.3.14. The Gaussian process $\dot{W}^{k}$ as in Lemma 3.3.11 can be chosen such that $\left(\dot{W}^{k}([0, t] \times \cdot)\right)_{t \geq 0}$ has values in $C\left(\mathbb{R}_{+}, \mathcal{S}_{-q-1}\left(\mathbb{R}^{q}\right)\right)$. 
Proof. Consider the metric space $\mathcal{S}_{-q-1}\left(\mathbb{R}^{q}\right)$ with the metric given by the operator $J$ with eigenpairs $\left(\lambda_{n}, h_{n}\right)_{n \in \mathbb{Z}_{+}^{q}}$ for $p=-q-1$.

$$
\begin{aligned}
\mathbb{E}\left[\left\|W_{t}^{k}(\cdot)-W_{s}^{k}(\cdot)\right\|_{2, p}^{2}\right] & =\mathbb{E}\left[\left\|J^{p}\left(W_{t}^{k}(\cdot)-W_{s}^{k}(\cdot)\right)\right\|_{L^{2}\left(\mathbb{R}^{q}\right)}^{2}\right] \\
& =\mathbb{E}\left[\sum_{n \in \mathbb{N}_{0}^{q}}\left(J^{p}\left(W_{t}^{k}(\cdot)-W_{s}^{k}(\cdot)\right), h_{n}\right)_{L^{2}}\right] \\
& =\mathbb{E}\left[\sum_{n \in \mathbb{N}_{0}^{q}}\left(W_{t}^{k}(\cdot)-W_{s}^{k}(\cdot), J^{p} h_{n}\right)_{L^{2}}\right] \\
& =\sum_{n \in \mathbb{N}_{0}^{q}} \lambda_{n}^{p} \mathbb{E}\left[\left(W_{t}^{k}(\cdot)-W_{s}^{k}(\cdot), h_{n}\right)_{L^{2}}\right] \\
& =\sum_{n \in \mathbb{N}_{0}^{q}} \lambda_{n}^{p} \mathbb{E}\left[W^{k}\left(\mathbb{1}_{[s, t]} \times h_{n}\right)^{2}\right] \\
& =|t-s| \sum_{n \in \mathbb{N}_{0}^{q}} \lambda_{n}^{p} L_{k}\left(h_{n}, h_{n}\right) \\
& =c|t-s| \sum_{n \in \mathbb{N}_{0}^{q}}\left(2\left(n_{1}+\cdots+n_{q}\right)+q+1\right)^{p} \\
& \leq c|t-s|\left(\sum_{n_{1} \in \mathbb{N}_{0}}\left(1+n_{1}\right)^{-1-\frac{1}{q}}\right)^{q} \\
& \leq c|t-s| .
\end{aligned}
$$

since $L_{k}\left(h_{n}, h_{n}\right) \leq c\left\|h_{n}\right\|_{L^{2}}^{2}$ and $p$ is small enough such that series converges. Since $W^{k}$ is a Gaussian process, one can obtain estimates for the higher moments. These allow to use the Kolmogorov-Centsov Theorem 3.1 .3 to deduce continuity of the process.

The same proof also holds in the white noise setting, i.e. $k=\delta \in \mathcal{S}^{\prime}\left(\mathbb{R}^{q}\right)$, however in neither case do we think the result is optimal in the sense of regularity in the space variable. The lemma also implies that the paths of $\left(W_{t}\right)_{t \geq 0}$ can be chosen in $C\left(\mathbb{R}_{+}, \mathcal{S}^{\prime}\left(\mathbb{R}^{q}\right)\right)$, which will be sufficient for the results to come.

We will now give rigorous definitions of the Gaussian noises used in this dissertation. While the definition of white noise is standard, we will define colored noise depending on $k \in L_{\mathrm{loc}}^{1}\left(\mathbb{R}^{2 q}\right)$, which is surely not the most general form of colored noises which can be defined. However, we will always refer to these noises as colored noise. All of the colored noises defined here, will have the property that they are "white in time." 
Definition 3.3.15. Let $q \in \mathbb{Z}_{+}$.

(a) Let $\delta \in \mathcal{S}^{\prime}\left(\mathbb{R}^{q}\right)$ be the Dirac- $\delta$-distribution and $L_{\delta}$ as in (3.5). A white noise $\dot{W}=\dot{W}^{\delta}$ in $1+q$ dimensions is a centered Gaussian process on $\mathcal{S}\left(\mathbb{R}^{1+q}\right)$ with $\mathbb{E}\left[\dot{W}^{\delta}(\phi) \dot{W}^{\delta}(\phi)\right]=L_{\delta}(\phi, \psi), \phi, \psi \in \mathcal{S}\left(\mathbb{R}^{1+q}\right)$ and such that the extended process $\left(\dot{W}^{\delta}([0, t] \times \cdot)\right)_{t \geq 0}$ has values in $C\left(\mathbb{R}_{+}, \mathcal{S}^{\prime}\left(\mathbb{R}^{q}\right)\right)$ almost surely.

(b) Let $k \in L_{\text {loc }}^{1}\left(\mathbb{R}^{2 q}\right)$ be as in Lemma 3.3.11 and $L_{k}$ as in (3.4). A colored noise $\dot{W}=\dot{W}^{k}$ depending on $k$ in $1+q$ dimensions is a centered Gaussian process on $\mathcal{S}\left(\mathbb{R}^{1+q}\right)$ with $\mathbb{E}\left[\dot{W}^{k}(\phi) \dot{W}^{k}(\phi)\right]=L_{k}(\phi, \psi), \phi, \psi \in \mathcal{S}\left(\mathbb{R}^{1+q}\right)$ and such that the extended process $\left(\dot{W}^{k}([0, t] \times \cdot)\right)_{t \geq 0}$ has values in $C\left(\mathbb{R}_{+}, \mathcal{S}^{\prime}\left(\mathbb{R}^{q}\right)\right)$ almost surely.

(c) In both cases we will say $\dot{W}$ is white in time and we will write

$$
W_{t}(A)=\dot{W}([0, t] \times A),
$$

for $A \in \mathcal{B}_{b}\left(\mathbb{R}^{q}\right), t \geq 0$.

Let us finally give an example how to obtain general correlation kernels $k$ used in the previous definition. For a tempered distribution $f \in \mathcal{S}^{\prime}\left(\mathbb{R}^{q}\right)$ define the continuous mapping $L_{f}: \mathcal{S}\left(\mathbb{R}^{q}\right) \times \mathcal{S}\left(\mathbb{R}^{q}\right) \rightarrow \mathbb{R}, L_{f}(\phi, \psi):=\langle f, \phi * \psi\rangle$ for any $\phi, \psi \in \mathcal{S}\left(\mathbb{R}^{q}\right)$. Note that in the case of continuous $f$ we can write

$$
L_{f}(\phi, \psi)=\int_{\mathbb{R}^{q}} \int_{\mathbb{R}^{q}} f(x-y) \phi(x) \psi(y) d x d y .
$$

Relating to the definition of $L_{k}$ before, note that for $k(x, y):=f(x-y)$ the definitions of $L_{k}$ and $L_{f}$ coincide. Let us require that $|f(z)| \leq c\left(|z|^{-\alpha}+1\right)$, so that we are in the setting as in Lemma 3.3.10.

We say that $f$ is a distribution of positive type, if $L_{f}$ is a positive operator, i.e.

$$
L_{f}(\phi, \phi) \geq 0 \quad \forall \phi \in \mathcal{S}\left(\mathbb{R}^{q}\right) .
$$

Additionally, call a measure $\mu$ on $\left(\mathbb{R}^{q}, \mathcal{B}\left(\mathbb{R}^{q}\right)\right)$ slowly increasing if there exists a $p \in \mathbb{Z}$, s.t. $\left(1+|x|^{2}\right)^{-p}$ is integrable w.r.t. $\mu$. The Bochner-Schwartz Theorem (Theorem 7.2.1 of [BTA04]) states that any tempered distribution $f$ of positive type is the Fourier transform $\mathcal{F} \mu$ of a slowly increasing positive measure $\mu$ and vice versa.

Given such a slowly increasing measure $\mu$ we define $f=\mathcal{F} \mu \in \mathcal{S}^{\prime}\left(\mathbb{R}^{q}\right)$. Setting $k(x, y)=f(x-y)$, we can construct a centered Gaussian process $\dot{W}=\dot{W}^{k}$ on $\mathcal{S}\left(\mathbb{R}^{1+q}\right)$. In the general case without the bound on $|f|$, the path regularity results are not trivially transferable, but we will not consider that here. Instead, let us comment on the special features of the previous construction: Due to its definition, 
$L_{f}$ is a symmetric operator resulting in a spatially homogeneous Gaussian process in the sense that $\dot{W}^{f}(\cdot) \stackrel{d}{=} \dot{W}^{f}\left(\tau_{x} \cdot\right), x \in \mathbb{R}^{q}$. Here $\tau_{x}: \mathcal{S}\left(\mathbb{R}^{q}\right) \rightarrow \mathcal{S}\left(\mathbb{R}^{q}\right), f(\cdot) \mapsto f(\cdot+x)$ is the translation operator. These kind of spatially homogeneous noises were treated for example in [Dal99].

We conclude this section with a remark relating white and colored noise.

Remark 3.3.16. If we consider colored noise $\dot{W}^{k}$ for $k(x)=|x|^{-\alpha}$ and take the limit $\alpha \nearrow q$, then pointwise there is weak convergence: $\dot{W}^{k}(\phi) \Rightarrow \dot{W}^{\delta}(\phi), \phi \in \mathcal{S}\left(\mathbb{R}^{1+q}\right)$ (see Exercise 3.3 on page 52 of [DKRA09]). However, it is not immediately clear whether convergence in $C\left(\mathbb{R}_{+}, \mathcal{S}^{\prime}\right)$ holds.

\subsection{Generalized stochastic integration}

In Section 3.2 we presented a quick overview on Itô-integration in $\mathbb{R}^{1}$. A natural extension of that concept should be the integration w.r.t. a random field $\left(X_{t}\right)_{t \in T}$, where $T \subset \mathbb{R}^{1+q}$. As in the previous chapter we distinguish one variable the "time." When defining integration there are two natural questions: Which random fields can be chosen as good integrators? Which functions are good integrands?

Here, we will develop the classical theory of Walsh as in Wal86. First, we will speak about the integrators and give the definitions of martingale measures. We will show that the Gaussian noises defined in Definition 3.3.15 are martingale measures. Secondly, we will speak about the integrands and the definition of the integral. However, we will do this definition only in the case of the Gaussian noises presented in the previous section.

\section{Martingale Measures}

We will start with an abstract setting: let $E$ be a Polish space, $\mathcal{E}$ the Borel $\sigma$-field on $E$ and $\mathcal{A} \subset \mathcal{E}$ a certain subset.

Definition 3.4.1. Let $(\Omega, \mathcal{F}, \mathbb{P})$ be a probability space. A mapping $U: \Omega \times \mathcal{A} \rightarrow \mathbb{R}$ is called an $L^{2}(\mathbb{P})$-valued, $\sigma$-finite, signed measure on $\mathcal{A}$ if

(a) there exist $E_{n} \nearrow E$ with $\mathcal{E}_{n}=\mathcal{E}_{\mid E_{n}} \subset \mathcal{A}$, s.t. $U\left(E_{n}\right)<\infty$ a.s. for all $n \in \mathbb{N}$,

(b) for all $A \in \mathcal{A}$ the random variable $U(A)$ is in $L^{2}(\mathbb{P})$,

(c) for each $n \in \mathbb{N}$ the mapping $U$ is countably additive in an $L^{2}$-sense:

$$
U(A)+U(B)=U(A \cup B)(\text { a.s. }), \quad \lim _{j \rightarrow \infty} U\left(A_{j}\right) \stackrel{L^{2}}{\longrightarrow} 0
$$

for $A_{j} \searrow \emptyset$ and $A, B, A_{j} \in \mathcal{E}_{n}, j \in \mathbb{N}$ and $A, B$ disjoint. 
It is always possible to extend $U: \Omega \times \mathcal{A} \rightarrow \mathbb{R}$ to all of $\Omega \times \mathcal{E}$ by setting $U(A)=\lim _{n \rightarrow \infty} U\left(A \cap E_{n}\right)$ if this is a well-defined $L^{2}$-limit for a given $A \in \mathcal{E}$. Set the value to $\infty$ if this limit is not well-defined.

Let us show that the Gaussian noises from Definition 3.3.15 satisfy these conditions in the following sense:

Proposition 3.4.2. Let $\dot{W}$ be a Gaussian noise, which is white in time. Then for any $t \geq 0$ it is true that $W_{t}: \Omega \times \mathcal{B}_{b}\left(\mathbb{R}^{q}\right) \rightarrow \mathbb{R}$ is a $\sigma$-finite $L^{2}$-valued measure on $E$ and $E_{n}(t)$ can be chosen independently of $t \geq 0$.

Proof. Fix $t \geq 0$. Defining $E_{n}=B^{q}(0, n)=\left\{x \in \mathbb{R}^{q}:|x| \leq n\right\}$, observe

$$
\mathbb{E}\left[\left(W_{t}\left(E_{n}\right)\right)^{2}\right]=t L_{k}\left(\mathbb{1}_{E_{n}}, \mathbb{1}_{E_{n}}\right)<\infty,
$$

by $k \in L_{\text {loc }}^{1}\left(\mathbb{R}^{2 q}\right)$, which implies (a) of Definition 3.4.1. Finite additivity and countable $L^{2}$ additivity follow from Lemma 3.3 .13 . This shows (c). Then also (b) follows, since by finite additivity for any $A \in \mathcal{A}$ there is an $n \in \mathbb{N}$ with $A \subset E_{n}$, so $W_{t}(A) \leq W_{t}\left(E_{n}\right)$ and by the above $W_{t}\left(E_{n}\right) \in L^{2}$, so also $W_{t}(A) \in L^{2}$.

Remember that the $\mathbb{R}^{1}$-stochastic integral is defined for martingales. The concept of martingales also plays a crucial role here. We give the following definition.

Definition 3.4.3. Let $\left(\Omega, \mathcal{F}, \mathcal{F}_{t}, \mathbb{P}\right)$ be a filtered probability space. A random variable $M: \mathbb{R}_{+} \times \mathcal{A} \times \Omega \rightarrow \mathbb{R}$ is called an $L^{2}$-valued martingale measure, if

(a) $M_{0}(A)=0$ almost surely for all $A \in \mathcal{A}$.

(b) $\left(M_{t}(A)\right)_{t \geq 0}$ is a martingale for all bounded sets $A \in \mathcal{A}$ w.r.t. the filtration $\left(\mathcal{F}_{t}\right)_{t \geq 0}$.

(c) $M_{t}(\cdot)$ is an $L^{2}$-valued signed finite measure on $\mathcal{A}$ for all $t \geq 0$.

For the Gaussian noise $\dot{W}$ as in Definition 3.3 .15 we define for $t \geq 0$ :

$$
\tilde{\mathcal{F}}_{t}=\tilde{\mathcal{F}}_{t}^{W}=\sigma\left(W_{s}(A): 0 \leq s \leq t, A \in \mathcal{B}\left(\mathbb{R}^{q}\right)\right)
$$

as the natural filtration $\left(\tilde{\mathcal{F}}_{t}\right)_{t \geq 0}$. This filtration is not necessarily the one we want to work with. Call $\mathcal{N}$ the $\mathbb{P}$-null sets and define

$$
\mathcal{F}_{t}=\mathcal{F}_{t}^{W}=\bigcap_{u>t} \tilde{\mathcal{F}}_{u} \cup \mathcal{N}
$$

We call the filtration $\left(\mathcal{F}_{t}\right)_{t \geq 0}$ the natural filtration and note that it satisfies the usual conditions. Then we can define on the filtered probability space $\left(\Omega, \mathcal{F}_{\infty}, \mathcal{F}_{t}, \mathbb{P}\right)$ the random variable

$$
\dot{W}: \mathbb{R}_{+} \times \mathcal{B}_{b}\left(\mathbb{R}^{q}\right) \times \Omega \rightarrow \mathbb{R}
$$


by setting

$$
\dot{W}(t, A, \omega):=\dot{W}([0, t] \times A)(\omega) .
$$

For this random variable we can state the following lemma.

Lemma 3.4.4. The Gaussian noises $\dot{W}$ defined in Definition 3.3.15 are martingale measures with respect to their natural filtration and the martingales are continuous.

Proof. We want to check the three conditions of Definition 3.4.3. Proposition 3.4.2 gives (c); (a) is obvious and (b) is true, since $\dot{W}$ is Gaussian and

$$
\mathbb{E}\left[\left(W_{t}(A)-W_{s}(A)\right) W_{u}(B)\right]=0 \quad \forall u \leq s, B \in \mathcal{B}_{b}\left(\mathbb{R}^{q}\right) .
$$

The continuity of the martingales follows from Lemma 3.3.14.

This ends the paragraph on martingale measures, which will be the integrators and we can go on to the definition of the integrands and the integral itself.

\section{Stochastic Integral}

In this subsection we use the results of the previous paragraph and assume that $\left\{M_{t}(A), \mathcal{F}_{t}: t \geq 0, A \in \mathcal{B}_{b}\left(\mathbb{R}^{q}\right)\right\}$ is always a martingale measure obtained from a Gaussian noise from Definition 3.3.15. As in the Itô-theory first define the integral for a small class of functions.

\section{Definition 3.4.5.}

(a) A function $g: \mathbb{R}^{q} \times \mathbb{R}_{+} \times \Omega \rightarrow \mathbb{R}$ is called elementary if

$$
g(x, t, \omega)=X(\omega) \mathbb{1}_{(a, b]}(t) \mathbb{1}_{A}(x)
$$

where $X \in \mathcal{F}_{a}$ is bounded, $A \in \mathcal{B}\left(\mathbb{R}^{q}\right), 0 \leq a<b<\infty$.

(b) Linear combinations of elementary functions are called simple. The class of simple functions is denoted by $\mathbb{S}$. Let $\mathcal{P}=\sigma(\mathbb{S})$ be the predictable $\sigma$-field.

(c) Let $g$ be elementary as above and $M$ an $L^{2}$-valued martingale measure. The stochastic integral of $g$ w.r.t. $M$ is a martingale measure defined by

$$
(g \cdot M)_{t}(B)=\left(\int_{0}^{t} g d M\right)(B)(\omega):=X(\omega)\left[M_{t \wedge b}(A \cap B)-M_{t \wedge a}(A \cap B)\right](\omega),
$$

where $t \geq 0, B \in \mathcal{B}_{b}\left(\mathbb{R}^{q}\right), \omega \in \Omega$. 
Let us verify the claim in (c) that $(g \cdot M)$ is an $L^{2}$-valued martingale measure. Conditions (a) and (c) in Definition 3.4.3 are trivial, so concentrate on (b): $(g$. $M)(B)$ is the difference of two stopped martingales, so by Proposition 3.2 .5 it is a martingale. When checking the square-integrability, we have

$$
\begin{aligned}
\mathbb{E}\left[(g \cdot M)_{t}(B)^{2}\right] & =\mathbb{E}\left[X^{2}\left(\langle M .(A \cap B)\rangle_{t \wedge b}-\langle M .(A \cap B)\rangle_{t \wedge a}\right)\right] \\
& =\mathbb{E}\left[L_{k}\left(g \mathbb{1}_{[0, t] \times B}, g \mathbb{1}_{[0, t] \times B}\right)\right]
\end{aligned}
$$

where the brackets $\langle\cdot\rangle$ stand for the quadratic variation of the real martingale $\left(M_{s}(A \cap B)\right)_{s \geq 0}$. We can also show that the martingales are continuous. As in the classical Itô-theory, the notion of quadratic variation plays an important role for the extension of the integral. We define for $g \in \mathbb{S}$ the norm:

$$
\|g\|_{0, t}:=\mathbb{E}\left[L_{k}\left(g \mathbb{1}_{[0, t] \times \mathbb{R}^{q}}, g \mathbb{1}_{[0, t] \times \mathbb{R}^{q}}\right)\right]^{1 / 2}, \quad t \geq 0
$$

and the function

$$
\|g\|_{0}:=\sum_{n=1}^{\infty} 2^{-n}\left(1 \wedge\|g\|_{0, n}\right) .
$$

This function $\|\cdot\|_{0}$ induces a metric on $\mathbb{S}$ by $d_{0}(g, h):=\|g-h\|_{0}$; identifying $g, h \in \mathbb{S}$ with $\|g-h\|_{0}=0$, we observe that $\left(\mathbb{S}, d_{0}\right)$ is a linear space. By $(3.10)$, we have that the mapping

$$
I_{M}: \begin{cases}\left(\mathbb{S}, d_{0}\right) & \rightarrow\left(\mathcal{M}^{2, c},\|\cdot\|\right), \\ g & \mapsto\left((g \cdot M)_{t}\left(\mathbb{R}^{q}\right)\right)_{t \geq 0}\end{cases}
$$

is an isometry. Remember that $\mathcal{M}^{2, c}$ is complete (see Proposition 3.2.7).

Lemma 3.4.6. The mapping $I_{M}$ can be extended to the space of predictable, square integrable processes $\mathcal{P}^{M}$ on $\mathbb{R}_{+} \times \mathbb{R}^{q} \times \Omega$ :

$$
\mathcal{P}^{M}=\left\{f \in \mathcal{P}:\|f\|_{0, t}<\infty \forall t \geq 0\right\} .
$$

Proof. We only need to show that $\mathbb{S}$ is dense in $\mathcal{P}^{M}$ w.r.t. $d_{0}$. For this, see Theorem 2.5 in Wal86] or Theorem 2 in Dal99.

Definition 3.4.7 (Stochastic Integral). The extension of the mapping $I_{M}$ to the space $\mathcal{P}^{M}$ is called the stochastic integral. For $f \in \mathcal{P}^{M}, t \geq 0$ it is denoted by the process

$$
(f \cdot M)_{t}=\int_{0}^{t} \int_{\mathbb{R}^{q}} f(s, y, \omega) M(d s d y) .
$$

We list some properties of this generalized stochastic integral in the next lemma.

Lemma 3.4.8. For $g, h \in \mathcal{P}^{M}$ we have that

(a) $(\lambda g+h) \cdot M=\lambda(g \cdot M)+h \cdot M$, where $\lambda \in \mathbb{R}$, 
(b) $g \cdot M$ is again a martingale measure,

(c) $\langle(g \cdot M) \cdot(A),(h \cdot M) \cdot(B)\rangle_{t}=L_{k}\left(g \mathbb{1}_{[0, t] \times A}, h \mathbb{1}_{[0, t] \times B}\right)$.

Proof. The proof for $g, h \in \mathbb{S}$ was given before and in (3.10). The extension to $g, h \in \mathcal{P}^{M}$ is straightforward by approximation.

It might be worth noting that the definition of $d_{0}$ here and $\|\cdot\|_{\alpha}$ in (3.7) are similar. The extension of $\dot{W}$ was also obtained by a similar procedure. However, in the martingale approach we obtained that the stochastic integrals are again martingale measures and that integrands can be predictable. This is a result which was not attainable in the previous section.

For the generalized stochastic integrals there is also an analogue of the Fubini Theorem.

Proposition 3.4.9 (Stochastic Fubini Theorem). Let $M$ be a martingale measure and $(A, \mathcal{A}, \mu)$ be a finite measure space. Additionally, let $f: \mathbb{R}^{q} \times \mathbb{R}_{+} \times \Omega \times A \rightarrow \mathbb{R}$ be $\mathcal{P}^{M} \otimes \mathcal{A}$-measurable and for a $T>0$ assume

$$
\mathbb{E}_{\mathbb{P}}\left[\int_{\Omega \times \mathbb{R}^{q} \times \mathbb{R}^{q} \times[0, T] \times A}|f(x, t, \omega, u)||f(y, t, \omega, u)|\left|Q_{M}(d x d y d t)\right| \mu(d u)\right]<\infty .
$$

Then it holds $\mathbb{P}$-almost surely for any $0 \leq t \leq T$ :

$\int_{A}\left(\int_{\mathbb{R}^{q} \times[0, t]} f(x, s, \cdot, u) M(d s d x)\right) \mu(d u)=\int_{\mathbb{R}^{q} \times[0, t]}\left(\int_{A} f(x, s, \cdot, u) \mu(d u)\right) M(d s d x)$.

This result can be shown using elementary $f$ first and the full proof can be found on page 296 of [Wal86].

The reader may have noticed that for the Gaussian noises the letters $W$ and $\dot{W}$ are used both. The precise notation and distinction between the two is chosen according to the following heuristic. If $B$ is Brownian motion, we denote its temporal increments by $d B_{t}$. The noises defined in Definition 3.3 .15 are martingale measurese $W_{t}(d x)=W(t, d x)$. We could denote their spatial increment by $\partial_{t} W_{t}(d x)$, but using physics notation, it is more convenient to write $\dot{W}(t, d x)$. In a weak setting of an SPDE the notation $\dot{W}(t, x)$ will be used, but note that it is only a formal notation. What we have well-defined in this section is the integration w.r.t. time and space simultaneously and the notation will be $\int_{0}^{T} \int_{\mathbb{R}^{q}} W(d t d x)$.

\subsection{Stochastic Partial Differential Equations}

There is already quite a large body of introductory literature on SPDE and its integration theory. However, there are several ways to approach the topic. The 
first one, which we follow here is the approach of Walsh using martingales measures, as presented in Wal86]: it is a more analytic treatment of the subject using martingale measures. The second treatment based on a Hilbert-space approach is more functional analytic, formulating SPDE as Hilbert-space valued SDE. This is comparable to the abstract setting of PDE as in Section 2.3. Some good references are [DPZ08, [PZ00] and [PR07]. Another approach to SPDE theory is Kryloy's $L^{p}$-theory as presented in Kry96.

Before defining SPDE rigorously, let us consider another example (see [Wal86]). Imagine you left a guitar outside your house and a sandstorm came. What would be the melody the guitar strings are playing then? The amplitude $u$ of the guitar string can be modeled as a solution to the wave equation. The sandstorm can then be modeled as an added inhomogeneity $\dot{W}$ to the system:

$$
\left\{\begin{array}{l}
\partial_{t}^{2} u(t, x)=\kappa^{2} \partial_{x}^{2} u(t, x)+\dot{W}(t, x),(t, x) \in \mathbb{R}_{+} \times[0, L], \\
u(0, \cdot)=\partial_{t} u(0, \cdot)=0, u(t, 0)=u(t, L)=0
\end{array}\right.
$$

where $L$ is the length of the guitar string and $\kappa^{2}$ is the speed of wave propagation. We will make that more rigorous in a moment.

More abstractly, assume that $A$ and $C$ are partial differential operators (PDO) as in Section 2.1 on $\mathbb{R}_{+} \times \mathbb{R}^{q}$, then we look for solutions of

$$
A(u)=C(u) \dot{W}
$$

with specific initial conditions (suitable for the equation $A(u)=0$ ). As for PDE it is not possible to treat these equations in full generality if one wants to go to finer statements. We will single out one variable which will be called the "time" and call $\mathbb{R}^{q}$ "space". Consider the following evolution equation:

$$
\partial_{t} u(t, x)=A(t, x) u(t, x)+b(t, x, u(t, x))+C(t, x, u) \dot{W}(t, x), u(0, \cdot)=u_{0}(\cdot)
$$

where $A(t, x)$ only acts on spatial derivatives of $u ; b: \mathbb{R}_{+} \times \mathbb{R}^{q+m} \rightarrow \mathbb{R}^{m}$ and $u: \mathbb{R}_{+} \times \mathbb{R}^{q} \rightarrow \mathbb{R}^{m}$ for a certain $m \in \mathbb{N}$. Usually, $C$ can be chosen quite general (see e.g. (6.7) in [DPZ08]), but we restrict to $C(t, x, u)=\sigma(t, x, u)$ for a certain function $\sigma: \mathbb{R}_{+} \times \mathbb{R}^{q+m} \rightarrow \mathbb{R}:$

$$
\partial_{t} u(t, x)=A(t, x) u(t, x)+b(t, x, u(t, x))+\sigma(t, x, u) \dot{W}(t, x), u(0, \cdot)=u_{0}(\cdot)
$$

The last term in (3.14) will be called the noise term. We say that the equation has additive noise if $\sigma$ does not depend on $u$ and multiplicative noise otherwise. In the additive noise case, the use of the mild solutions below allows an explicit solution of (3.14) and thus is well understood. However, we will focus on the multiplicative noise case. 
Given the concepts developed in the last section we can now make the notion of a solution to (3.1) precise. Let us fix $A$ a PDO, $b, \sigma, u_{0}$ and $\mathcal{L}(\dot{W})$ and denote by $A^{*}$ the formal adjoint of $A$, see 2.10. Here $\dot{W}$ is a Gaussian noise with correlation functional $L_{k}$ as defined in Definition 3.3.15.

Definition 3.5.1. We say that a weak solution for (3.14) exists, if there is a filtered probability space $\left(\Omega, \mathcal{F}, \mathcal{F}_{t}, \mathbb{P}\right)$ supporting a noise $W$ and an adapted random field $u: \mathbb{R}_{+} \times \mathbb{R}^{q} \times \Omega \rightarrow \mathbb{R}^{m}$ s.t. for all $\phi \in C_{c}\left(\mathbb{R}^{q}\right)$ it $\mathbb{P}$-almost surely holds that

$$
\begin{gathered}
\mathbb{P}\left(\int_{0}^{t} \int_{\mathbb{R}^{q}} u(s, x) \phi(x) d x d s<\infty\right)=1 \\
\mathbb{P}\left(\int_{0}^{t} d s L_{k}(\phi(\cdot) \sigma(s, \cdot u(s, \cdot)), \phi(\cdot) \sigma(s, \cdot, u(s, \cdot)))<\infty\right)=1, \\
\int_{\mathbb{R}^{q}} \phi(x) u(t, x) d x=\int_{\mathbb{R}^{q}} \phi(x) u_{0}(x) d x+\int_{0}^{t} \int_{\mathbb{R}^{q}} A^{*} \phi(x)+b\left(s, x, u_{s}(x)\right) d x d s \\
\quad+\int_{0}^{t} \int_{\mathbb{R}^{q}} \phi(x) \sigma\left(s, x, u_{s}(x)\right) W(d s d x) \forall t>0 .
\end{gathered}
$$

The random field will $u$ will be called a weak solution.

From the discussion in Section 2.3 , we recall that there is also another important concept of solutions in the PDE setting, the concept of mild solutions. We will provide a description for this concept only in the following special case (for the general Hilbert-space setup see [DPZ08]): Assume that $A$ is the generator of a strongly continuous semigroup $(S(t))_{t \geq 0}$ on $C_{b}\left(\mathbb{R}^{q}\right)$ with density $s_{t}: \mathbb{R}^{q} \rightarrow \mathbb{R}^{m \times m}$, i.e. $S(t) f=\int_{\mathbb{R}^{q}} s_{t}(\cdot-y) f(y) d y, f \in C_{b}\left(\mathbb{R}^{q}\right)$.

Definition 3.5.2. We say that a mild solution for (3.14) exists, if there is a filtered probability space $\left(\Omega, \mathcal{F}, \mathcal{F}_{t}, \mathbb{P}\right)$ supporting a noise $W$ and an adapted random field $u: \mathbb{R}_{+} \times \mathbb{R}^{q} \times \Omega \rightarrow \mathbb{R}^{m}$ s.t. (3.15), (3.16) hold and for all $t>0, x \in \mathbb{R}^{q}, \mathbb{P}_{\text {-almost }}$ surely it holds that

$$
u(t, x)=S(t) u_{0}(x)+\int_{0}^{t} \int_{\mathbb{R}^{q}} s_{t-s}(x-y) \sigma(s, y, u(s, y)) W(d s d y) .
$$

The random field $u$ will be called a mild solution.

The following theorem relates these two notions:

Theorem 3.5.3 (Thm 6.5 of [DPZ08]). Any weak solution is a mild solution. Any mild solution satisfying

$$
\mathbb{E}\left[\int_{0}^{T} L_{k}(\sigma(s, \cdot, u(s, \cdot)), \sigma(s, \cdot, u(s, \cdot))) d s\right]<\infty
$$

is a weak solution. 
Both of these notions were stochastically weak concepts, see Definition 3.2 .10 . There is also a stochastically strong concept of solutions:

Definition 3.5.4. We say that a stochastically strong solution for (3.14) exists, if for any filtered probability space $\left(\Omega, \mathcal{F}, \mathcal{F}_{t}, \mathbb{P}\right)$ supporting a noise $W$ we have an adapted random field $u: \mathbb{R}_{+} \times \mathbb{R}^{q} \times \Omega \rightarrow \mathbb{R}^{m}$ s.t. (3.15), (3.16) and (3.17) holds $\mathbb{P}$-almost surely.

There is an essential difference between stochastically weak and stochastically strong solutions: in the latter case, $u$ needs to be adapted, independent of which probability space, including the filtration, is chosen. Choose a probability space supporting a noise $\dot{W}$ and its canonical filtration $\left(\mathcal{F}_{t}\right)_{t \geq 0}$ (see $(3.9)$ ). Then, $u_{t}$ is measurable w.r.t $\mathcal{F}_{t}$ and hence needs to be a function of the noise $\dot{W}$ up to time $t$ (see the considerations after Definition 3.2.10). Obviously, strong existence implies weak existence.

Oftentimes, it will be convenient that we restrict our attention to certain subclasses $\mathcal{U}$ of functions (continuous, vanishing at infinity, etc.) of functions. On these subclasses one can ask for uniqueness of solutions to (3.14). As for stochastic ordinary differential equations there are several notions of uniqueness:

Definition 3.5.5 (Uniqueness).

(a) We say that uniqueness in law in $\mathcal{U}$ holds for (3.14) if for any weak solution $u \in \mathcal{U}$ the f.d.d. of the random field $u$ are equal.

(b) We say that pathwise uniqueness (PU) in $\mathcal{U}$ holds for (3.14) if any weak solutions in $\mathcal{U}$ defined on the same probability space are indistinguishable.

A certain set of functions will be of special interest to us for the question of uniqueness. This class was first used in that context by Shiga in [Shi94]. Define for a real-valued function $u: \mathbb{R}^{q} \rightarrow \mathbb{R}$ :

$$
\|u\|_{\lambda, \infty}:=\sup _{x \in \mathbb{R}^{q}} e^{-\lambda|x|}|u(x)| .
$$

We consider the following functions spaces.

$$
\begin{aligned}
C_{\text {tem }} & =\left\{f: \mathbb{R}^{q} \rightarrow \mathbb{R}: f \text { continuous, }\|f\|_{\lambda, \infty}<\infty \forall \lambda>0\right\}, \\
C_{\text {rap }} & =\left\{f: \mathbb{R}^{q} \rightarrow \mathbb{R}: f \text { continuous, }\|f\|_{\lambda, \infty}<\infty \forall \lambda<0\right\} .
\end{aligned}
$$

We could say that functions are increasing subexponentially (tempered) or decreasing subexponentially (rapid), if they are in $C_{\text {tem }}$ or $C_{\text {rap }}$, respectively. Note that we write $C_{\text {tem }}^{+}$or $C_{\text {rap }}^{+}$if we restrict to non-negative functions. 


\section{The Stochastic Heat Equation}

This chapter contains a general overview on stochastic heat equations. In subsequent sections we give results which are already known in the literature.

\subsection{The Stochastic Heat Equation with multiplicative noise}

We will focus our attention to a specific class of equations, the stochastic heat equation in $\mathbb{R}_{+} \times \mathbb{R}^{q}$. In considering the general SPDE (3.14), this corresponds to $u$ being a real-valued function (i.e. $m=1$ ) and $A$ being a specific operator, (half) the Laplace-operator $\frac{1}{2} \Delta=\frac{1}{2}\left(\partial_{x_{1}}^{2}+\cdots+\partial_{x_{q}}^{2}\right)$ on $\mathbb{R}^{q}$. The PDE part of the equation is then a classical heat equation plus an inhomogeneity $b$, which we call the drift term in the following. Including the stochastic term (and assuming $\partial_{u} \sigma(u) \neq 0$ ), the SPDE reads then

$$
\frac{\partial}{\partial t} u(t, x)=\frac{1}{2} \Delta u(t, x)+b(t, x, u(t, x))+\sigma(t, x, u(t, x)) \dot{W}(t, x) .
$$

It is called a stochastic heat equation (SHE) with multiplicative noise. The noise $\dot{W}$ stays unspecified yet; it can be white noise $\dot{W}=\dot{W}^{\delta}$ or colored noise $\dot{W}=\dot{W}^{k}$.

In the previous chapter the concepts of weak and mild solutions were introduced. Let us quickly recall these concepts in the special setting considered here. Assume $u_{0}$ is a given initial value. We say that $u$ is a weak solution if for all $\phi \in C_{c}^{\infty}\left(\mathbb{R}^{q}\right)$ almost surely:

$$
\begin{aligned}
\int_{\mathbb{R}^{q}} u(t, x) \phi(x) d x= & \int_{\mathbb{R}^{q}} u_{0}(x) \phi(x) d x+\int_{0}^{t} \int_{\mathbb{R}^{q}} u(s, x) \frac{1}{2} \Delta \phi(x) d x d s \\
& +\int_{0}^{t} \int_{\mathbb{R}^{q}} b(s, x, u(s, x)) \phi(x) d x d s \\
& +\int_{0}^{t} \int_{\mathbb{R}^{q}} \sigma(s, x, u(s, x)) \phi(x) W(d s d x) \forall t \geq 0,
\end{aligned}
$$

compare with Definition 3.5.1. The other solution concept requires the semigroup related to the operator $\frac{1}{2} \Delta$ (or the fundamental solution of $\partial_{t}-\frac{1}{2} \Delta$ ). Let

$$
p_{t}(x)=(2 \pi t)^{-q / 2} \exp \left(-\frac{|x|^{2}}{2 t}\right), t>0, x \in \mathbb{R}^{q}
$$


denote the $q$-dimensional heat kernel. We call $\left(S_{t}\right)_{t \geq 0}$ the associated semigroup via $S_{t}(\psi)(\cdot)=\int p_{t}(\cdot-y) \psi(y) d y$ for $\psi \in C_{\text {tem }}$. We say that $u$ is a mild solution if for all $t \geq 0, x \in \mathbb{R}^{q}$ it almost surely holds that

$$
\begin{array}{r}
u(t, x)=\int_{\mathbb{R}^{q}} p_{t}(x-y) u_{0}(y) d y+\int_{0}^{t} \int_{\mathbb{R}^{q}} p_{t-s}(x-y) b(s, y, u(s, y)) d y d s \\
+\int_{0}^{t} \int_{\mathbb{R}^{q}} p_{t-s}(x-y) \sigma(s, y, u(s, y)) W(d s d y),
\end{array}
$$

compare with Definition 3.5.2. There are many physical situations and stochastic models in which equations such as (4.1) arise, see the references in the introduction for an overview. For example, they arise as high density, many particle limits of branching particle systems. We present such a particle system, mentioned in the introduction, in more detail. Its description is taken from Chapter 4 of [DMS93] and [Stu03]. The example underlines why colored noise models are interesting.

Example 4.1.1. Consider the following model of branching Brownian motion. At time $t=0$ consider a Poisson point process on $\mathbb{R}$ with intensity $n \mu$, where $\mu \in$ $\mathcal{M}_{f}(\mathbb{R})$ a finite Borel measure on $\mathbb{R}$ and $n \in \mathbb{N}$ is fixed. Label its points in an arbitrary fashion with $\left\{1,2, \ldots, n_{0}^{(n)}\right\}$ and denote the position of the points in $\mathbb{R}$ by $Y_{0}^{i}, 1 \leq i \leq n_{0}^{(n)}$, where $i$ is the label. Put $n_{0}^{(n)}$ particles at these points, so that we have exactly one particle at each point and label particle according to its position. Each particle moves independently on the real line as a Brownian motion until time $t=\frac{1}{n}$. At that time, each particle dies and is replaced by either 0 or 2 particles with equal probability. All branching events happen independently. Label the children of particle $i$ (if existent) by $i 0$ and $i 1 \in \mathbb{N} \times\{0,1\}$ and denote their positions by $Y_{\frac{1}{n}}^{\alpha}:=Y_{\frac{1}{n}-}^{\alpha_{0}}, \alpha=\left(\alpha_{0}, \alpha_{1}\right) \in \mathbb{N}_{0} \times\{0,1\}$. If $i$ has no children then $Y_{\frac{1}{n}}^{i 0}=Y_{\frac{1}{n}}^{i 1}=\Theta$, a cemetery state. Continue to let the particles move according to independent Brownian motions until the next branching event in $n^{-1} \mathbb{N}$ and so on. Let $\alpha \in \bigcup_{m} \mathbb{N} \times\{0,1\}^{m}$ denote the labels and define the measure-valued process

$$
X_{t}^{(n)}=\frac{1}{n} \sum_{\alpha \sim t} \delta_{Y_{t}^{\alpha}}, t \geq 0,
$$

where $\alpha \sim t$ means $\frac{|\alpha|}{n} \leq t<\frac{|\alpha|+1}{n}$. Here, $|\alpha|=\left|\left(\alpha_{0}, \ldots, \alpha_{n}\right)\right|=n$ is the length of the index $\alpha$ minus 1 . Then $\left(X_{t}^{(n)}\right)_{t \geq 0} \Rightarrow\left(X_{t}\right)_{t \geq 0}$ in $D\left(\mathbb{R}_{+}, \mathcal{M}_{f}\left(\mathbb{R}^{q}\right)\right)$ for $n \rightarrow \infty$, where $X$ is the Dawson-Watanabe superprocess (see Thm 4.6.2 in [DMS93]). By Theorem 2.1 of Konno and Shiga [KS88] it holds that a.s. there is a continuous process $u(t, x): \mathbb{R}_{+} \times \mathbb{R}^{q} \rightarrow \mathbb{R}$, s.t. $u(t, \cdot)$ is the density of $X_{t}$, namely

$$
\int u(t, x) \phi(x) d x=\int X_{t}(d x) \phi(x) \forall \phi \in C_{c}^{\infty}\left(\mathbb{R}^{q}\right) .
$$


They also state the following: The process $u$ is the unique in law, weak solution of

$$
\frac{\partial}{\partial t} u(t, x)=\frac{1}{2} \partial_{x}^{2} u(t, x)+\sqrt{u(t, x)} \dot{W}^{\delta}(t, x), t>0, x \in \mathbb{R} .
$$

Here, the noise $\dot{W}=\dot{W}^{\delta}$ is white noise, $\sigma(t, x, u)=\sqrt{u}$. The differential operator $A=\frac{1}{2} \partial_{x}^{2}$ is the one of the heat equation in one dimension; the drift $b \equiv 0$.

Now, consider a modification of that model given in [Stu03], see [Myt96 for a similar model. Consider a centered random field $\xi$ on $\mathbb{R}^{q}$ with correlation

$$
\mathbb{E}[\xi(x) \xi(y)]=\bar{k}(x, y), x, y \in \mathbb{R}^{q}
$$

for a bounded, continuous $\bar{k}: \mathbb{R}^{2 q} \rightarrow \mathbb{R}$. Assume finite third moment and define $\xi^{n}(x)=\left[\left(\xi^{n} \wedge \sqrt{n}\right) \vee(-\sqrt{n})\right]$. Consider an i.i.d. sequence of such random fields $\left(\xi_{i}\right)_{i \in \mathbb{N}}$. In the model above, the offspring $N^{\alpha, n}$ of particle $\alpha$ were given by

$$
\mathbb{P}\left(N^{\alpha, n}=0\right)=\mathbb{P}\left(N^{\alpha, n}=2\right)=\frac{1}{2}
$$

and all of the $N^{\alpha, n}$ are independent. Conditionally on $\xi$, redefine that to

$$
\begin{aligned}
& \mathbb{P}\left(\bar{N}^{\alpha, n}=0\right)=n^{-1 / 2} \xi_{|\alpha|,-}^{n}\left(Y_{\frac{|\alpha|+1}{n}}^{\alpha}\right), \\
& \mathbb{P}\left(\bar{N}^{\alpha, n}=1\right)=1-n^{-1 / 2}\left|\xi_{|\alpha|}^{n}\right|\left(Y_{\frac{\alpha \alpha+1}{n}}^{\alpha}\right), \\
& \mathbb{P}\left(\bar{N}^{\alpha, n}=2\right)=n^{-1 / 2} \xi_{|\alpha|,+}^{n}\left(Y_{\frac{|\alpha|+1}{n}}^{\alpha}\right),
\end{aligned}
$$

where $\xi_{+}=\xi \vee 0$ and $\xi_{-}=-(\xi \wedge 0)$. Now, branching events happening at the same time (same $\xi$ ) are dependent! In Theorem 2.2 of [Stu03] it is shown that the corresponding measure-valued process $X^{n}$ converges in $D\left(\mathbb{R}_{+}, \mathcal{M}_{f}\left(\mathbb{R}^{q}\right)\right)$. The limit process allows a density process $u$, which is the pathwise unique solution of

$$
\frac{\partial}{\partial t} u(t, x)=\frac{1}{2} \Delta u(t, x)+u(t, x) \dot{W}(t, x)
$$

where $\dot{W}=\dot{W}^{\bar{k}}$ is colored noise on $\mathbb{R}^{q}$ in the sense of Definition 3.3.15 with correlation kernel $\bar{k}$. To show such a convergence result for general noise coefficients $\sigma(u)$ is still an open question.

In the next three sections, we will list some results which were already known in the literature about the stochastic heat equation as in 4.1). This will prepare us for presenting the main results of this work in the following chapter. 


\subsection{Known results on existence and regularity of solutions}

In the beginning of the study of SHE, mostly white noise models were of interest. If $b, \sigma$ are uniformly Lipschitz-continuous (see below in (4.6)), then strong existence and uniqueness in $C\left(\mathbb{R}_{+}, C_{\text {tem }}\right)$ was shown in Theorem 2.2 of [Shi94] in dimension $q=1$. A result in higher dimension was not attainable, since solutions were not expected to be function-valued (see p. 119 of [DKRA09]).

To get results in higher dimensions, more regular noises were required. This was one of the motivations for studying SPDE with colored noise $\dot{W}=\dot{W}^{k}$, defined as in Definition 3.3.15. The results we give are either Gaussian white noise results $(\dot{W})$ or for this kind of Gaussian colored noise $\left(\dot{W}^{k}\right)$. We assume the kernel $k$ to be bounded by a Riesz-kernel in the following way for $\alpha \in(0, q)$,

$$
k(x, y) \leq 4 \frac{4.5}{4 .}\left(|x-y|^{-\alpha}+1\right),
$$

$x \neq y \in \mathbb{R}^{q}$, for a certain $44.5<\infty$. This assumption on $k$ will appear frequently in this work. Then as one of the first results, Dalang showed in [Dal99]:

Theorem 4.2.1 (Theorem 13 of [Dal99]). Let $q \in \mathbb{N}$ and $u_{0}$ be measurable and bounded. Assume that the functions $b, \sigma: \mathbb{R}_{+} \times \mathbb{R}^{q} \times \mathbb{R} \rightarrow \mathbb{R}$ satisfy

$$
|b(t, x, u)-b(t, x, v)|+|\sigma(t, x, u)-\sigma(t, x, v)| \leq c|u-v| \quad \forall t \geq 0, x \in \mathbb{R}^{q} .
$$

for a constant $c<\infty$. Assume that (4.5) holds and $\alpha \in(0,2 \wedge q)$. Then, there exists a pathwise unique, strong solution u to (4.1), where $\dot{W}=\dot{W}^{k}$ is colored noise. The process $u$ satisfies a uniform moment bound: For any $T>0$, and $p \in[1, \infty)$,

$$
\sup _{0 \leq t \leq T} \sup _{x \in \mathbb{R}^{q}} \mathbb{E}\left[|u(t, x)|^{p}\right]<\infty .
$$

Dalang's proof is based on Fourier techniques, which are apt for spatially homogeneous noise. However, his proof transfers to the general inhomogeneous case assuming 4.5 without any difficulties. This was already remarked after Theorem A.1 of [MPS06].

More results for the SHE with colored noise were obtained, such as the following regularity result:

Proposition 4.2.2 (Theorem 2.1 in [SSS02]). Assume that the initial condition $u_{0}$ is a bounded $\rho$-Hölder-continuous function for some $\rho \in(0,1)$. We also suppose that the coefficients $\sigma, b: \mathbb{R} \rightarrow \mathbb{R}$ are Lipschitz-continuous functions as in (4.6) and that $\dot{W}=\dot{W}^{k}$ is colored noise where the kernel obeys 4.5$), \alpha \in(0,2 \wedge q)$.

Then the trajectories of $u$ are a.s. $\beta_{1}$-Hölder continuous in $t$ and $\beta_{2}$-Hölder continuous in $x$ for any $\beta_{1} \in\left(0, \frac{1}{2}\left(\rho \wedge\left(1-\frac{\alpha}{2}\right)\right)\right), \beta_{2} \in\left(0, \rho \wedge\left(1-\frac{\alpha}{2}\right)\right)$. 
The proof of this result uses a deconvolution technique, called the factorization method, which was often of help for colored noise models. In the white noise case, Proposition 3.4 of [Wal86] stated a similar regularity result in dimension $q=1$ replacing $\alpha$ by 1 .

For both of the above results, however, the assumption on $\sigma$ to be a Lipschitzfunction was essential. However, this restriction excludes many prominent examples, such as the Dawson-Watanabe SPDE (1.1). In the white noise case, Theorem 2.6 in [Shi94] gave a positive answer to the question of existence (and a good proof strategy) for more relaxed conditions on $b$ and $\sigma$. His theorem applies to nonnegative solutions, but his proof does not require that assumption. Nevertheless, there is a drawback: the solutions, which are obtained in a limiting procedure, are not necessarily strong solutions anymore (see Remark 8.2.5).

In MPS06, Mytnik, Perkins and Sturm transfered Shiga's proof and the regularity result to the colored noise case, but with $b \equiv 0$. Additionally, they showed that solutions lie in a certain function space.

Theorem 4.2.3 (Theorem 1.2 and 1.8 of [MPS06]). Let $u_{0} \in C_{\text {tem }}, b \equiv 0$ and $\sigma$ be a continuous function satisfying the growth condition $\sigma(u) \leq c(1+|u|)$ for some $c<\infty$. Assume that (4.5) holds for $\alpha \in(0,2 \wedge q)$ and $\dot{W}=\dot{W}^{k}$ is colored noise.

Then there exists a (stochastically weak) mild solution to (4.1) with sample paths a.s. in $C\left(\mathbb{R}_{+}, C_{\text {tem }}\right)$. Any solution $u \in C\left(\mathbb{R}_{+}, C_{\text {tem }}\right)$ satisfies:

(a) For $T, \lambda, p>0$ :

$$
\mathbb{E}\left(\sup _{0 \leq t \leq T} \sup _{x \in \mathbb{R}^{q}}|u(t, x)|^{p} e^{-\lambda|x|}\right)<\infty .
$$

(b) For any $\xi \in(0,1-\alpha / 2)$ the process $u(\cdot, \cdot)$ is a.s. uniformly Hölder continuous on compact sets in $(0, \infty) \times \mathbb{R}^{q}$, and the process $Z(t, x) \equiv u(t, x)-S_{t} u_{0}(x)$ is uniformly Hölder continuous on compacts in $[0, \infty) \times \mathbb{R}^{q}$, both with Hölder coefficients $\frac{\xi}{2}$ in time and $\xi$ in space. For $K \geq 0$ the random variable

$$
H=H_{K}=\sup _{t \leq K} \sup _{|x| \vee\left|x^{\prime}\right| \leq K, x \neq x^{\prime}} \frac{\left|Z(t, x)-Z\left(t, x^{\prime}\right)\right|}{\left|x-x^{\prime}\right|^{\xi}}
$$

is p-integrable: $\mathbb{E}\left[H^{p}\right]<\infty$ for any $p \geq 2$.

We will repeat parts of their argument in the proof of Theorem 5.2.1. Regarding the regularity, this result also holds in the white noise case and can be found in Theorem 1.1 of [MP11] (no explicit proof is given there). 


\subsection{Known results on uniqueness}

In the previous theorem, weak existence of $u \in \mathcal{U}=C\left(\mathbb{R}_{+}, C_{\text {tem }}\right)$ was shown for continuous $\sigma$. But, pathwise uniqueness as in Theorem 4.2.1 could not be shown for general $\sigma$. To obtain uniqueness results was an open question for many years as mentioned in the introduction. We will address pathwise uniqueness for these kinds of SHE in the space $\mathcal{U}=C\left(\mathbb{R}_{+}, C_{\text {tem }}\right)$.

Note that in the case of Lipschitz $b$ and $\sigma$, Dalang's result Theorem 4.2.1 gave an answer on pathwise uniqueness. We first remark that it is unlikely to expect that the conditions on $b$ can be relaxed significantly (remember the classical ODE example $d u=u^{\beta} d t, \beta<1$ ). So let us focus our attention on $\sigma$.

In some cases where the SPDE (4.1) has a corresponding measure-valued process, uniqueness in law was shown using a duality argument. That was done for the Dawson-Watanabe SPDE (1.1] in [Daw75]. In the case of $\sigma(u)=u^{\gamma}, \gamma \in\left(\frac{1}{2}, 1\right), b=$ 0 , Mytnik in Myt98 applied an approximate duality in order to show uniqueness in law. For colored noise SPDE duality was used in Theorem 4.20 of Myt96. However, these techniques rely on a good understanding of a particle picture of the process and thus, are not very robust. To summarize, duality techniques will not help for general $\sigma: \mathbb{R}_{+} \times \mathbb{R}^{q} \times \mathbb{R} \rightarrow \mathbb{R}$.

Another idea comes from the study of ordinary SDE. There is a classical result proven by Yamada and Watanabe [YW71] for fairly general $\sigma$ :

Theorem 4.3.1 (Theorem 3.1 of [YW71]). Let $x_{0} \in \mathbb{R}$ and $B$ be a Brownian motion. Assume that $b, \sigma: \mathbb{R} \rightarrow \mathbb{R}$ satisfy $|b(x)-b(y)| \vee|\sigma(x)-\sigma(y)|^{2} \leq C|x-y|$ for all $x, y \in \mathbb{R}$ and a certain $C<\infty$. Then, pathwise uniqueness holds for the $S D E$

$$
d X_{t}=b\left(X_{t}\right) d t+\sigma\left(X_{t}\right) d B_{t}, X_{0}=x_{0} .
$$

Essentially, this tells us that the Hölder-regularity of the diffusion coefficient $\sigma$ plays an important role. In this zero-dimensional case (there is only time, but no space in our context), a Hölder-exponent of order $1 / 2$ suffices.

Of course, the hope was that this result could be transfered to SPDE, possibly using the same proof strategy. First, Viot [Vio76] succeeded in proving pathwise uniqueness on a bounded domain in $\mathbb{R}$ for $\sigma(u)=\sqrt{u(1-u)}$. Nevertheless, for many years it was not possible to use their SDE techniques in the general SPDE setting. In 2006, Mytnik, Perkins and Sturm [MPS06] applied Yamada and Watanabe's technique to colored noise and obtained

Theorem 4.3.2 (Theorem 1.4 of [MPS06]). Let $q \in \mathbb{N}$ and $\dot{W}=\dot{W}^{k}$ be colored noise. Assume that $b \equiv 0$, the function $\sigma: \mathbb{R} \rightarrow \mathbb{R}$ satisfies

$$
|\sigma(u)| \leq c(1+|u|),|\sigma(u)-\sigma(v)| \leq c|u-v|^{\gamma},
$$


for $u, v \in \mathbb{R}, c<\infty$ and $\gamma \in(0,1)$. For the kernel $k$ assume that equation (4.5) holds for some $\alpha \in(0,1), c<\infty$. If $\gamma \in\left(\frac{1+\alpha}{2}, 1\right]$, then pathwise uniqueness holds for solutions of (4.1) in $C\left(\mathbb{R}_{+}, C_{\mathrm{tem}}\right)$.

Few years later, Mytnik and Perkins in [MP11] proved an analogue of that result in the white noise case that used a refined proof technique:

Theorem 4.3.3 (Theorem 1.2 of [MP11]). Let $q=1$ and $\dot{W}=\dot{W}^{\delta}$ be white noise. Assume that $b \equiv 0$, the function $\sigma: \mathbb{R} \rightarrow \mathbb{R}$ satisfies (4.10). If $\gamma>\frac{3}{4}$, then pathwise uniqueness holds for solutions of (4.1) in $C\left(\mathbb{R}_{+}, C_{\mathrm{tem}}\right)$.

Their result was more general in the sense that it included Lipschitz drift $b$ and space and time dependence of $b$ and $\sigma$. We have mentioned in Remark 3.3 .16 that in dimension $q=1$, the Gaussian noises $\dot{W}^{k}$, colored noise, and $\dot{W}^{\delta}$, white noise, are related for $\alpha \rightarrow 1$. The condition for pathwise uniqueness in [MPS06] is $\gamma \in\left(\frac{1+\alpha}{2}, 1\right]$. For $\alpha \rightarrow 1$, this reduces to an empty set, whereas the [MP11] result says that $\gamma \in\left(\frac{3}{4}, 1\right]$ is sufficient for pathwise uniqueness. So there is a gap between the two results, which was believed to be closable. In [MP11, the following conjecture was stated, which we repeat:

Conjecture 1 (Conjecture 1.6 of [MP11]). Pathwise uniqueness in $C\left(\mathbb{R}_{+}, C_{\text {tem }}\right)$ for 1.2 holds in the colored noise case if $\alpha<2(2 \gamma-1)$.

To prove this conjecture is the main goal of this work. We will comment on the last two results and the proof strategy in more detail in Chapter 5 .

In 2012, Mueller, Mytnik and Perkins also showed the converse to Theorem 4.3.3 in a special case (see Theorem 1.1 of [MMP12 or Section 6.3). Within the last couple of years more results on pathwise uniqueness appeared. A very interesting one is that of Xiong in Xio12, who showed pathwise uniqueness for an SPDE related to the distribution function $v(t, y)=\int_{-\infty}^{y} u(t, x) d x$ of a one-dimensional SHE with white noise and $\gamma \geq \frac{1}{2}$.

\subsection{Known results on the compact support property}

Given a density process of a population, an interesting question is the following: How fast does the population spread from a given initial state and, even simpler, does it colonize the whole space immediately or does it stay within a compact set. As SHE are believed to be equations which hold for limit densities of branching particle systems as in Example 4.1.1, this is also a natural question to ask for solutions of SHE. As these densities are non-negative, we restrict to nonnegative solutions in this section. Let us make the following definition. 
Definition 4.4.1. (a) We define the support $\operatorname{supp}(f)$ of a function $f: \mathbb{R}^{q} \rightarrow \mathbb{R}$ to be the smallest closed set in $\mathbb{R}^{q}$ outside which $f$ vanishes. If $q=1$, we call the supremum of the support the point

$$
S(f):=\sup \operatorname{supp}(f) \in \mathbb{R} .
$$

(b) We say that a random field $X: \mathbb{R}_{+} \times \mathbb{R}^{q} \rightarrow \mathbb{R}_{+}$has the compact support property $(C S P)$, if whenever $X(0, \cdot)$ is deterministic and a function of compact support, then $\operatorname{supp}\left(X_{t}\right)$ is compact for all $t>0$ almost surely.

For PDE it is well-known that a solution of the heat equation (without drift) does not have the compact support property. Solutions cover the whole space instantaneously. Going back to the stochastic heat equation, the main results in the literature for the white noise case are the following:

Theorem 4.4.2. Let $u \in C\left(\mathbb{R}_{+}, C_{\mathrm{rap}}\right)$ be a nonnegative solution of

$$
\partial_{t} u(t, x)=\frac{1}{2} \partial_{x}^{2} u(t, x)+u^{\gamma} \dot{W}(t, x), t \geq 0, x \in \mathbb{R} .
$$

Then,

(a) for $\gamma=\frac{1}{2}$ the CSP holds ([Isc88] ),

(b) for $\gamma=1$ the CSP does not hold ([Mue91]]),

(c) for $\gamma \in(0,1)$ the CSP holds ([MP92]).

For (c) the proof was rather complicated and relied on the construction of a historical process, which was introduced in DP91. In the cases related to superprocesses, Laplace-transform techniques play an important role and have lead to many generalizations, e.g. including Lévy-noise. An interesting survey on that question is in Chapter 6 of Eng07. The linear case $\gamma=1$ is related to the parabolic Anderson model, where the phenomen of intermittency appears, see e.g. [FK10]. 


\section{Main Results}

In this section the main results of this work are presented. By far the most significant result is that of pathwise uniqueness as presented in Section 5.3 , followed by the result on compact support property in Section 5.4. All of these results are proven in Part II of this work; mathematically, the proof of the results presented in Section $5 . x$ is given in Chapter $6+x, x \in\{1,2,3,4\}$.

We rely on notation and definitions introduced in the previous chapters, but will shortly recall some: We consider the stochastic heat equation in $\mathbb{R}_{+} \times \mathbb{R}^{q}$ with multiplicative noise in the following form:

$$
\frac{\partial}{\partial t} u(t, x)=\frac{1}{2} \Delta u(t, x)+b(t, x, u(t, x))+\sigma(t, x, u(t, x)) \dot{W}(t, x) .
$$

We list a number of frequently used hypotheses on the jointly continuous functions $b, \sigma: \mathbb{R}_{+} \times \mathbb{R}^{q} \times \mathbb{R} \rightarrow \mathbb{R}$. The first is a linear growth condition, stating that there exists a constant 45.2 such that for all $(t, x, u) \in \mathbb{R}_{+} \times \mathbb{R}^{q+1}$,

$$
|\sigma(t, x, u)|+|b(t, x, u)| \leq q 5.2(1+|u|) .
$$

Next, we state a Hölder condition on $\sigma$ : For some $\gamma \in(0,1)$, there are $R_{1}, R_{2}>0$ and for all $T>0$ there is an $R_{0}(T)$ so that for all $t \in[0, T]$ and all $(x, u, v) \in \mathbb{R}^{q+2}$,

$$
|\sigma(t, x, u)-\sigma(t, x, v)| \leq R_{0}(T) e^{R_{1}|x|}(1+|u|+|v|)^{R_{2}}|u-v|^{\gamma} .
$$

We sometimes assume a Lipschitz condition for $b$ : There is a $B=B[5.4>0$ such that for all $(t, x, u, v) \in \mathbb{R}_{+} \times \mathbb{R}^{q+2}$,

$$
|b(t, x, u)-b(t, x, v)| \leq B[5.4|u-v| .
$$

In this chapter the noise $\dot{W}=\dot{W}^{k}$ is always a colored noise in the sense of Definiton 3.3.15. That means it is a centered Gaussian noise on $\mathbb{R}_{+} \times \mathbb{R}^{q}$, which is white in time and colored in space. It is uniquely characterized by

$$
\mathbb{E}[\dot{W}(\phi) \dot{W}(\psi)]=\int_{0}^{\infty} d s \int_{\mathbb{R}^{q}} d w \int_{\mathbb{R}^{q}} d z \phi(s, w) k(w, z) \psi(s, z)
$$

for $\phi, \psi \in \mathcal{S}\left(\mathbb{R}_{+} \times \mathbb{R}^{q}\right)$. The kernel $k \in L_{\text {loc }}^{1}\left(\mathbb{R}^{2 q}\right)$ is assumed to be bounded by a Riesz-kernel for $\alpha \in(0, q)$ in the following way:

$$
k(x, y) \leq q 5.5\left(|x-y|^{-\alpha}+1\right),
$$

for all $x \neq y \in \mathbb{R}^{q}$. 


\subsection{An abstract result on strong solutions}

In 1971, Yamada and Watanabe showed that in an SDE context we have the following statement:

$$
\text { weak existence }+ \text { pathwise uniqueness } \Rightarrow \text { strong existence. }
$$

They gave a rigorous proof which was technically demanding and based on regular conditional probabilities (see Section V.17 in [RW87]). Some years later, Jacod [Jac80] generalized their result to equations for semimartingales in the Skorohod space. Then in Kur07, Kurtz gave a more abstract statement of (5.6) for general stochastic equations. Applying his statement in the context of SPDE as suggested by his Example 3.9, leads to the following lemma.

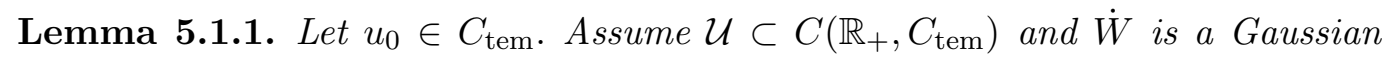
noise, which is white in time as in Definition 3.3.15. If the SPDE (3.14) admits a weak solution $u$ in $\mathcal{U}$ with

$$
\mathbb{E}\left[\sup _{t \geq 0, x \in K}|u(t, x)|^{2}\right]<\infty \text { for any compact } K \subset \mathbb{R}^{q}
$$

and pathwise uniqueness holds in $\mathcal{U}$, then there exists a strong solution in $\mathcal{U}$.

We give the proof in Chapter 7. It relies, as mentioned, on Kurtz's result. First, the SPDE is described by a set of (approximating) conditions. It is shown that solutions of SPDE equivalently satisfy these conditions. Then it is not difficult any more to check the compatibility conditions, which rely on $W$ having independent increments in time.

A result similar to Lemma 5.1.1 in a Banach-space setting can be found in Theorem E.1.8 of [PR07].

\subsection{Existence and regularity of solutions}

The first question related to an SPDE such as (5.1) is that of existence of a solution. Assuming rather mild conditions, we can prove a slight generalization of Theorem 4.2 .3 that suits the setting we use in this chapter.

Theorem 5.2.1. Let $u_{0} \in C_{\text {tem }}$ and let $b$ and $\sigma$ be continuous functions satisfying the growth condition (5.2). Assume that (5.5) holds for $k$ and that $\alpha \in(0,2 \wedge q)$. Then there exists a stochastically weak mild solution to (5.1) with sample paths a.s. in $C\left([0, \infty), C_{\text {tem }}\right)$. Any solution $u \in C\left([0, \infty), C_{\text {tem }}\right)$ satisfies:

(a) For $T, \lambda, p>0$ :

$$
\mathbb{E}\left(\sup _{0 \leq t \leq T} \sup _{x \in \mathbb{R}^{q}}|u(t, x)|^{p} e^{-\lambda|x|}\right)<\infty
$$


(b) For any $\xi \in(0,1-\alpha / 2)$ the process $u(\cdot, \cdot)$ is a.s. uniformly Hölder continuous on compact sets in $(0, \infty) \times \mathbb{R}^{q}$, and the process $Z(t, x) \equiv u(t, x)-S_{t} u_{0}(x)$ is uniformly Hölder continuous on compacts in $[0, \infty) \times \mathbb{R}^{q}$, both Höldercontinuities with Hölder coefficients $\frac{\xi}{2}$ in time and $\xi$ in space. For $K \geq 0$ the random variable

$$
H=H_{K}=\sup _{t \leq K} \sup _{|x| \vee\left|x^{\prime}\right| \leq K, x \neq x^{\prime}} \frac{\left|Z(t, x)-Z\left(t, x^{\prime}\right)\right|}{\left|x-x^{\prime}\right|^{\xi}},
$$

is p-integrable: $\mathbb{E}\left[H^{p}\right]<\infty$ for any $p \geq 2$.

It was remarked in [MPS06] (Remark 1.7) that the proof is only an extension of Theorem 4.2 .3 by standard arguments. We give the proof in Chapter 8 and quickly summarize the idea here:

Assuming the existence of solutions, show regularity properties as (a) and (b) by calculations as that of [SSS02]. For the existence, define Lipschitz-functions $\sigma^{n}$ and $b^{n}$ which approximate $\sigma$ and $b$. Consider the (strong) solutions $u^{n} \in C\left(\mathbb{R}_{+}, C_{\text {tem }}\right)$ obtained by Theorem 4.2.1 for (5.1), where $\sigma$ and $b$ are replaced by $\sigma^{n}$ and $b^{n}$. By the regularity properties from the beginning, show that the sequence $\left(u^{n}\right)_{n \in \mathbb{N}}$ is tight. Finally, by a Skorohod representation (introducing a new probability space) any weak limit point $u$ of that sequence solves (5.1) and has the required regularity properties. This last step is the point that leads to obtaining a stochastically weak instead of a stochastically strong solution, see Remark 8.2.5.

The reader familiar with heat equations will realize that also in the stochastic setting the different behavior of time $t$ and space $x$ appears, here in the regularity statement.

\subsection{Pathwise Uniqueness}

The central statement of this work is the following theorem:

Theorem 5.3.1. Let $u_{0} \in C_{\text {tem }}$ and assume that b, $\sigma: \mathbb{R}_{+} \times \mathbb{R}^{q} \times \mathbb{R} \rightarrow \mathbb{R}$ satisfy the linear growth bound (5.2), the Hölder-condition on $\sigma$ in (5.3) and the Lipschitzcondition (5.4) for $b$. Assume that (5.5) holds for $k$ for some $\alpha \in(0,2 \wedge q)$. Then, pathwise uniqueness for solutions of (5.1) holds in $C\left(\mathbb{R}_{+}, C_{\text {tem }}\right)$, if

$$
\alpha<2(2 \gamma-1) \text {. }
$$

Before giving a short explanation of the proof, let us note some of the consequences. First, using Lemma 5.1.1 and this theorem we obtain the following theorem as a corollary: 
Theorem 5.3.2. Assume that the assumptions of Theorem 5.3.1 above hold. Let $\left(\Omega, \mathcal{F}, \mathcal{F}_{t}, \mathbb{P}\right)$ be a filtered probability space with adapted colored noise $\dot{W}^{k}$ and let $u_{0} \in C_{\text {tem }}$ be $\mathcal{F}_{0}$-measurable. Then there exists a strong adapted solution u to (5.1) with respect to the prescribed $u_{0}$ and $\dot{W}$.

Proof. By Theorem 5.2.1 any solution $u$ with values in $\mathcal{U}=C\left(\mathbb{R}_{+}, C_{\text {tem }}\right)$ satisfies

$$
\mathbb{E}\left[\sup _{t \geq 0, x \in K}|u(t, x)|^{2}\right]<\infty \text { for any compact } K \subset \mathbb{R}^{q} .
$$

Pathwise uniqueness holds for solutions in $\mathcal{U}$, so applying Lemma 5.1.1 completes the proof.

Second, our uniqueness result is also an important ingredient for showing convergence of particle densities arising from discrete branching particle systems. The reason is that a classical weak convergence proof involves "tightness" and "uniqueness of the limit." If tightness is known and limits are known to be (stochastically weak) solutions of an SPDE, then our uniqueness result may help to obtain directly the desired weak convergence result. So far, except in some special cases no uniqueness results of this kind were available and no convergence results were attainable for colored noise equations.

Finally, Mytnik and Perkins stated the now proven Theorem 5.3.1 as a conjecture (see Conjecture 1 here). It related the results of their work [MP11] for white noise and that of [MPS06] for colored noise. If one considered the sufficient condition for pathwise uniqueness on $\gamma$ in the colored noise case of [MPS06]: $\gamma>\frac{1+\alpha}{2}$, it was clear that for $\alpha \rightarrow 1$ this condition was not consistent with $\gamma>\frac{3}{4}$, which was the result of [MP11]. So, there was a gap between the result of [MPS06] and the result of [MP11] for $\alpha=1$. This gap needed to be closed.

The proof of the theorem is the longest and technically most challenging part of this work and is a transfer of the idea of [MP11. However, the idea should be possible to be explained in the next pages. First, we give an idea why the result should hold and second, at page 62, why using this proof technique the result cannot be extended to less restrictive conditions on $\alpha$ and $\gamma$.

We do the heuristics in three steps: for ODE, SDE and SPDE. Assume the Hölder-condition (5.3) on $\sigma$ and $b \equiv 0$ throughout.

1) ODE: Recall the uniqueness proof for Lipschitz ODE: Consider the equation

$$
d u(t)=f(u) d t, t>0 ; u(0)=u_{0} \in \mathbb{R} .
$$

If $g: \mathbb{R} \rightarrow \mathbb{R}$ is absolutely continuous and $u^{1}$ and $u^{2}$ are two continuous solutions with $u^{1}(0)=u^{2}(0)$, then

$$
g\left(u^{1}(t)-u^{2}(t)\right)=g(0)+\int_{0}^{t} g^{\prime}\left(u^{1}(s)-u^{2}(s)\right)\left[f\left(u^{1}(s)\right)-f\left(u^{2}(s)\right)\right] d s .
$$


If $g(\cdot)=|\cdot|\left(g^{\prime}(0)\right.$ arbitrary) and $|f(u)-f(v)|<K|u-v|$ (Lipschitz), then

$$
\left|u^{1}(t)-u^{2}(t)\right| \leq K \int_{0}^{t}\left|u^{1}(s)-u^{2}(s)\right| d s
$$

and an application of Gronwall's inequality allows to deduce uniqueness: $u^{1}(t)=$ $u^{2}(t)$ for any $t \geq 0$.

2) SDE: In the stochastic setup of SDEs, similar ideas can be used. The Itôformula helps to estimate $g\left(u^{1}-u^{2}\right)$ for the SDE as in Theorem 4.3.1.

$$
\begin{aligned}
g\left(u^{1}(t)-u^{2}(t)\right)=\int_{0}^{t} g^{\prime}\left(u^{1}(s)-u^{2}(s)\right)\left(\sigma\left(u^{1}(s)\right)-\sigma\left(u^{2}(s)\right)\right) d B_{s} \\
\quad+\frac{1}{2} \int_{0}^{t} g^{\prime \prime}\left(u^{1}(s)-u^{2}(s)\right)\left(\sigma\left(u^{1}(s)\right)-\sigma\left(u^{2}(s)\right)\right)^{2} d s .
\end{aligned}
$$

Taking expectations, the stochastic integral vanishes (for details, see Chapter 5.2 in [KS00]). The important term is $\mathbb{E}\left[I_{1}\right]$, where

$$
I_{1}=\int_{0}^{t} g^{\prime \prime}\left(u^{1}(s)-u^{2}(s)\right)\left(\sigma\left(u^{1}(s)\right)-\sigma\left(u^{2}(s)\right)\right)^{2} d s .
$$

is the second-order/quadratic-variation term. Note that when approximating the modulus $g_{n} \rightarrow|\cdot|$, for example such that $g_{n}^{\prime \prime}(\cdot)=n \mathbb{1}_{\left[0, \frac{1}{n}\right)}(|\cdot|) \rightarrow \delta_{0}$ and using the Hölder-exponent $\gamma$ of $\sigma$ we can bound $I_{1}$ :

$$
I_{1} \leq n \int_{0}^{t} \mathbb{1}_{\left[0, \frac{1}{n}\right)}\left(\left|u^{1}(s)-u^{2}(s)\right|\right) c^{2 \gamma}\left|u^{1}(s)-u^{2}(s)\right|^{2 \gamma} d s \leq c^{2 \gamma} t n^{1-2 \gamma} .
$$

This goes to zero for $n \rightarrow \infty$, if $\gamma>\frac{1}{2}$; which is a part of [YW71]'s result as given in Theorem 4.3.1 a finer choice of $g_{n}$ leads to their full result including $\gamma=\frac{1}{2}$. Note that only small differences $\left|u^{1}-u^{2}\right|$ of the solutions played a role for $I_{1}$ and we obtained the result transfering that bound to $\left|u^{1}-u^{2}\right|^{\gamma}$.

3) SPDE: In the SPDE setting the estimates are more intricate. Let $u=u^{1}-u^{2}$ be the difference of two stochastically weak solutions defined on the same probability space. We will use the weak formulation 4.2 of the SHE. For $\Phi \in C_{c}^{\infty}\left(\mathbb{R}^{q}\right)$ the term $\int \Phi(y) u(t, y) d y$ is a semimartingale. Loosely define for any $n \in \mathbb{N}, x \in \mathbb{R}^{q}$,

$$
\Phi(\cdot)=\Phi_{x}^{n}(\cdot)=n^{\lambda_{0} q} \mathbb{1}\left(|\cdot-x| \leq n^{-\lambda_{0}}\right)
$$

for a certain $\lambda_{0}>0$. The function $\Phi_{x}^{n}(\cdot)$ converges weakly to a multiple of $\delta_{x}$ as $n \rightarrow$ $\infty$. For $g_{n}$ as before and $\Psi \in C_{c}^{\infty}\left(\mathbb{R}_{+} \times \mathbb{R}^{q}\right)$ apply Itô's formula to the semimartingale 
$\int d x \Psi(t, x) \int \Phi_{x}^{n}(y-x) u(t, y) d y$. The goal is to show that this integral is zero. The quadratic-variation term, similar to (5.9) will take the form

$$
\begin{aligned}
I_{n}(t)=n \int_{0}^{t} \int_{\mathbb{R}^{3 q}} \mathbb{1}\left(\left|\left\langle u_{s}, \Phi_{x}^{n}\right\rangle\right|<\frac{1}{n}\right)|u(s, w)|^{\gamma}|u(s, z)|^{\gamma} \\
\Phi_{x}^{n}(w) \Phi_{x}^{n}(z)\left(|w-z|^{-\alpha}+1\right) d w d z \Psi_{s}(x) d x d s .
\end{aligned}
$$

We need bounds for the values $|u(s, w)|^{\gamma}$ and $|u(s, z)|^{\gamma}$ similar to the ODE and SDE setting. Note, that the indicator function in the definition of $I^{n}(t)$ implies that there is an $\hat{x}_{0} \in B\left(x, n^{-\lambda_{0}}\right)$ such that $\left|u\left(s, \hat{x}_{0}\right)\right| \leq \frac{1}{n}$. If we could take $\hat{x}_{0}=w=z$, we could bound $I_{n}(t)$ by $C(t) n^{1-2 \gamma+\lambda_{0} \alpha}$ using that

$$
\int_{\mathbb{R}^{2 q}} d w d z \Phi_{x}^{n}(w) \Phi_{x}^{n}(z)\left(|w-z|^{-\alpha}+1\right) \leq c n^{\lambda_{0} \alpha},
$$

see Lemma 9.11.1. So $I_{n}(t)$ would go to zero for $\alpha<2(2 \gamma-1)$ if $\lambda_{0}=\frac{1}{2}$. However, choosing $|u(s, w)| \approx\left|u\left(s, \hat{x}_{0}\right)\right| \leq n^{-1}$ for all $w \in B\left(x, n^{-1 / 2}\right) \subset B\left(\hat{x}_{0}, 2 n^{-1 / 2}\right)$ is not justified, since there are no such strong regularity results on $u$. But, more formally, using the $1-\frac{\alpha}{2}-\varepsilon$-Hölder modulus of Theorem 5.2.1 gives for $w \in B\left(x, n^{-\lambda_{0}}\right) \subset$ $B\left(\hat{x}_{0}, 2 n^{-\lambda_{0}}\right)$ as before:

$$
\begin{aligned}
|u(s, w)| & \leq\left|u\left(s, \hat{x}_{0}\right)\right|+c\left|w-\hat{x}_{0}\right|^{1-\frac{\alpha}{2}-\varepsilon} \\
& \leq n^{-1}+c\left(2 n^{-\lambda_{0}}\right)^{1-\frac{\alpha}{2}-\varepsilon} \\
& \leq(1+2 c) n^{-1}
\end{aligned}
$$

if $n^{-\lambda_{0}} \approx n^{-\frac{2}{2-\alpha}}$, which is optimal balancing the terms. Using that estimate in (5.10), we obtain that $I_{n}$ goes to zero, if $\alpha<\left(1-\frac{\alpha}{2}\right)(2 \gamma-1)$. This is weaker than the result in Theorem 4.3.2, but yet a first step. We require better bounds on $|u(s, w)|$ for $w \in B\left(\hat{x}_{0}, n^{-\lambda_{0}}\right)$. In [MPS06], provided that $\alpha<2 \gamma-1$ the Hölder modulus near points where $u$ is small was improved to $1-\varepsilon$ for any $\varepsilon>0$. More precisely, let for $N, K \in \mathbb{N}$

$$
T_{K}=\inf \left\{s \geq 0: \sup _{y}\left(\left|u^{1}(s, y)\right| \vee\left|u^{2}(s, y)\right|\right) e^{-|y|}>K\right\} \wedge K
$$

a stopping time and define a metric on $\mathbb{R}_{+} \times \mathbb{R}^{q}: d((t, x),(s, y))=|t-s|^{1 / 2}+|x-y|$, appropriate for the study of the heat-equation. We define the following set of points $(t, x) \in \mathbb{R}_{+} \times \mathbb{R}^{q}$ which are close to a point $\left(\hat{t}_{0}, \hat{x}_{0}\right)$ with small value $u\left(\hat{t}_{0}, \hat{x}_{0}\right)$ :

$$
\begin{array}{r}
Z(N, K)(\omega)=\left\{(t, x) \in\left[0, T_{K}\right] \times[-K, K]^{q}: \text { there is a }\left(\hat{t}_{0}, \hat{x}_{0}\right) \in\left[0, T_{K}\right] \times \mathbb{R}^{q}\right. \text { s.t. } \\
\left.\left|u\left(\hat{t}_{0}, \hat{x}_{0}\right)\right| \leq 2^{-N} \text { and } d\left(\left(\hat{t}_{0}, \hat{x}_{0}\right),(t, x)\right) \leq 2^{-N}\right\} .
\end{array}
$$


Then the following analogue of Theorem 2.3 in [MP11] or Theorem 4.1 in [MPS06] holds:

Theorem 5.3.3. For each $K \in \mathbb{N}$ and $0<\xi<\frac{1-\frac{\alpha}{2}}{1-\gamma} \wedge 1$ there is an $N_{0}=$ $N_{0}(\xi, K, \omega) \in \mathbb{N}$ a.s. such that for all natural numbers $N \geq N_{0}$ and all $(t, x) \in$ $Z(N, K)$,

$$
d\left(\left(t^{\prime}, x^{\prime}\right),(t, x)\right) \leq 2^{-N} \text { and } t^{\prime} \leq T_{K} \text { implies }\left|u\left(t^{\prime}, x^{\prime}\right)-u(t, x)\right| \leq 2^{-N \xi} .
$$

We give a proof of this Theorem in Section 9.9. The theorem suggests that for a choice of $\alpha<2 \gamma-1$,

$$
u(t, \cdot) \text { is } \xi \text {-Hölder continuous near its zero set for } \xi<1
$$

Using a similar calculation as 5.12, now for $\lambda_{0}=\xi^{-1}$ (balancing terms), gives

$$
I^{n}(t) \leq C(t) n^{1-2 \gamma+\xi \alpha} .
$$

This allows us to derive the sufficiency of $\alpha<2 \gamma-1$ for pathwise uniqueness as it was shown in [MPS06].

To obtain an improved result we need to extend the range of $\xi$ beyond 1 . We will obtain a statement close to the following one:

$$
\nabla u(t, \cdot) \text { is } \xi \text {-Hölder on }\{x: u(t, x) \approx \nabla u(t, x) \approx 0\} \text { for } \xi<1,
$$

where $\nabla u$ denotes the spatial derivative (in a loose sense as $u$ is not differentiable). Actually, we will not be able to write down 5.15 formally, but some statements come close to it, e.g. Corollary 9.4.12 for $m=\bar{m}+1$.

Assuming (5.15) and $\alpha<2(2 \gamma-1)$, we give an idea how we will be able to derive that $I_{n}$ given in (5.10) goes to zero. First, choose $0=\beta_{0}<\beta_{1}<\cdots<\beta_{L}=\bar{\beta}<\infty$, a finite grid, and define

$$
\begin{aligned}
\hat{I}_{n, i}(t):=n \int_{0}^{t} \int & \mathbb{1}_{\hat{J}_{n, i}(s)}(x)|u(s, w)|^{\gamma}|u(s, z)|^{\gamma} \\
& \Phi_{x}^{n}(w) \Phi_{x}^{n}(z)\left(|w-z|^{-\alpha}+1\right) d w d z \Psi_{s}(x) d x d s,
\end{aligned}
$$

as $n \rightarrow \infty$ for all $i=0, \ldots, L$, where

$$
\hat{J}_{n, i}(s)=\left\{x \in \mathbb{R}^{d}:\left|\left\langle u_{s}, \Phi_{x}^{n}\right\rangle\right|<\frac{1}{n},|\nabla u(s, x)| \approx n^{-\beta_{i}}\right\}
$$

for $i<L$ and for $i=L$,

$$
\hat{J}_{n, L}(s)=\left\{x \in \mathbb{R}^{q}:\left|\left\langle u_{s}, \Phi_{x}^{n}\right\rangle\right|<n^{-1},|\nabla u(s, x)| \in\left[0, n^{-\beta_{L}}\right]\right\} .
$$


Since $I_{n}(t) \leq \sum_{i=0}^{L} \hat{I}_{n, i}(t)$, our goal of proving $I_{n}(t) \rightarrow 0$ will be attained, if we can show that

$$
\hat{I}_{n, i}(t) \rightarrow 0 \quad \text { for all } i=0, \ldots, L .
$$

Using the support of $\Phi_{x}^{n}$, note that for $x \in \hat{J}_{n, i}(s)$ there must be $\hat{x}_{n}(s) \in$ $B\left(x, n^{-\lambda_{0}}\right)$ such that $\left|u\left(s, \hat{x}_{n}(s)\right)\right|<\frac{1}{n}$. By a Taylor expansion, (5.15) (a calculation similar as in (5.12) ) we have for $w \in B\left(x, n^{-\lambda_{0}}\right), x \in \hat{J}_{n, i}(s)$ :

$$
\begin{aligned}
|u(s, w)| & \leq n^{-1}+\sup _{\tilde{w} \in\left[\hat{x}_{n}(s), w\right]} \nabla u(s, \tilde{w}) \cdot\left|\hat{x}_{n}(s)-w\right| \\
& \leq n^{-1}+\sup _{\tilde{w} \in\left[\hat{x}_{n}(s), w\right]}\left(|\nabla u(s, x)|+|\tilde{w}-x|^{\xi}\right)\left|\hat{x}_{n}(s)-w\right| \\
& \leq n^{-1}+\left(n^{-\beta_{i}}+2 n^{-\lambda_{0} \xi}\right) n^{-\lambda_{0}} \\
& \leq 7\left(n^{-1} \vee n^{-\beta_{i}-\frac{1}{2}}\right),
\end{aligned}
$$

if we choose $\lambda_{0}=\frac{1}{2}$, which is the smallest possible value for balancing terms. Similarly, $\beta_{i} \leq \frac{1}{2}$ is optimal in (5.17). If we put the estimate into (5.16), then we can bound $\hat{I}_{n, i}(t)$ by

$$
n\left(n^{-2 \gamma} \vee n^{-2 \gamma \beta_{i}-\gamma}\right) \int_{0}^{t} \int \mathbb{1}_{\hat{J}_{n, i}(s)}(x) \Phi_{x}^{n}(w) \Phi_{x}^{n}(z)\left(|w-z|^{-\alpha}+1\right) \Psi_{s}(x) d w d z d x d s
$$

and (5.11) with $\lambda_{0}=\frac{1}{2}$ leads to the bound

$$
n^{1+\frac{\alpha}{2}}\left(n^{-2 \gamma} \vee n^{-2 \gamma \beta_{i}-\gamma}\right) \int_{0}^{t} \int_{B\left(0, K_{1}\right)} \mathbb{1}_{\hat{J}_{n, i}(s)}(x) d x d s,
$$

for some $K_{1}>0$, since $\Psi$ is compactly supported. If $\beta_{i}$ is rather small, we find ourselves in the situation that the Hölder-estimate (5.17) is not that strong. With a choice of $\lambda_{0}=1$ we would just get back to the case $\alpha<2 \gamma-1$, since low $\beta_{i}$ refers to neglecting the estimate on derivatives. However, particularly in that case we can give a good estimate on $\left|\hat{J}_{n, i}(s)\right|$ the $q$-dimensional Lebesgue-measure of $\hat{J}_{n, i}(s)$.

Let us first consider $\beta=\beta_{L}$. Then, by the estimate in (5.18) we have

$$
\hat{I}_{n, L}(t) \leq c t\left(n^{-2 \gamma+1+\alpha / 2} \vee n^{-\left(2 \beta_{L}+1\right) \gamma+1+\alpha / 2}\right) \rightarrow 0
$$

as $n \rightarrow \infty$ as long as we require $\beta_{L} \geq 1 / 2$. From this and the considerations just after (5.17), we know that it should suffice to choose $\beta_{L}=1 / 2$, or more precisely, choosing $\beta_{L}$ lower will not lead to an optimal result, whereas $\beta_{L}>\frac{1}{2}$ will not improve the result.

We still need to check the convergence for $i=0, \ldots, L-1$ and write in order to simplify notation $\beta=\beta_{i}$ and $J_{n}=\hat{J}_{n, i}(s)$. From 5.15 we see that if $x \in J_{n}$, then 
there is a direction $\sigma_{x} \in S^{q-1}:=\left\{x \in \mathbb{R}^{q}:|x|=1\right\}$ with $\sigma_{x} \cdot \nabla u(s, y) \geq \frac{1}{2} n^{-\beta}$ if $|y-x| \leq L n^{-\beta / \xi}$ for an appropriate constant $L$ and $(y-x) \| \sigma_{x}$, meaning that $(y-x)$ is parallel to $\sigma_{x}$. Assuming for the moment that $u(s, x)>-\frac{1}{n}$ (which we only know precisely for a point $\hat{x}_{n}(s) \in B\left(x, n^{-1 / 2}\right)$ due to $\left.\left|\left\langle u_{s}, \Phi_{x}^{n^{-1 / 2}}\right\rangle\right|<\frac{1}{n}\right)$ we obtain because of the positive gradient for $y \in x+\mathbb{R}_{+} \sigma_{x}$ by the Fundamental Theorem of Calculus:

$$
u(s, y)>\frac{1}{n} \text { if } 4 n^{\beta-1}<|y-x| \leq L n^{-\beta / \xi} .
$$

Similarly, if $x, z \in J_{n}$ and $|x-z|<L n^{-\beta / \xi}$, we also have for $z^{\prime} \in z+\sigma_{x}$. $\left[4 n^{\beta-1} ; L n^{-\beta / \xi}\right]$ that $u\left(s, z^{\prime}\right)>\frac{1}{n}$ and thus $z^{\prime} \notin J_{n}$. So for $x \in J_{n}$, denoting by $\left\{x+\sigma_{x}^{\text {ortho }}\right\}$ the plane through $x$ orthogonal to $\sigma_{x}$, we have

$$
\begin{aligned}
\left|B\left(x, L a_{n}^{\beta / \xi}\right) \cap J_{n}\right| & \leq \int_{\left\{x+\sigma_{x}^{\text {ortho }} \cap \cap B\left(x, L n^{-\beta / \xi}\right)\right.} d z \int_{-L a_{n}^{\beta / \xi}}^{L n^{-\beta / \xi}} d z^{\prime} \mathbb{1}\left\{z+\sigma_{x} z^{\prime} \in J_{n}\right\} \\
& \leq c\left(n^{-\beta / \xi}\right)^{q-1} n^{\beta-1} .
\end{aligned}
$$

Covering the box $\left[-K_{1}, K_{1}\right]^{q}$ with finitely many balls of radius $\frac{L}{2} n^{-\beta / \xi}$ and using (5.19) we obtain $\left|J_{n}\right| \leq c\left(L, K_{1}\right) a_{n}^{1-\beta} n^{\beta / \xi}$. We can use this in 5.18) to get

$$
\hat{I}_{n, i}(t) \leq C t n^{1+\frac{\alpha}{2}-2 \gamma\left(1 \wedge\left(\beta_{i}+\frac{1}{2}\right)\right)-1+\beta_{i}+\beta_{i} / \xi} \leq C t n^{1+\frac{\alpha}{2}-2 \gamma\left(1 \wedge\left(\beta_{i}+\frac{1}{2}\right)\right)-1+\beta_{i}(1+1 / \xi)}
$$

for all $\beta_{i} \leq \bar{\beta}$. This tends to zero for all $\beta_{i} \leq \bar{\beta}$, if $-\frac{\alpha}{2}+2 \gamma\left(1 \wedge\left(\bar{\beta}+\frac{1}{2}\right)\right)-2 \bar{\beta}>0$, i.e.

$$
\gamma>\frac{1}{2}\left(1 \wedge\left(\bar{\beta}+\frac{1}{2}\right)\right)^{-1}\left(\frac{\alpha}{2}+2 \bar{\beta}\right) .
$$

The right-hand side is decreasing in $\bar{\beta}$ and attains its minimum value $\frac{1}{2}\left(1-\frac{\alpha}{2}\right)$ for $\bar{\beta}=1 / 2$. Then the estimate shows that: $\hat{I}_{n, i}(t)$ tends to zero for all $0 \leq \beta_{i} \leq \bar{\beta}$, if

$$
\alpha<2(2 \gamma-1) \text {. }
$$

This is what we wanted to show and ends the heuristic outline of the proof (for more about the heuristics we refer to Section 2 in [MP11]).

However, the real proof is somewhat more delicate and consists of splitting $u$ in two components, where the first follows more or less the heuristics and the second one can be shown to be small. The proof can be found in Chapter 9 .

Why should there not be a further improvement beyond the restriction $\alpha<$ $2(2 \gamma-1)$ using higher spatial derivatives on $u$ ? To show that heuristically, we make the case that for $\alpha>2(2 \gamma-1)$, we cannot expect the following slight strengthening of $(5.15)$ :

$$
u(t, \cdot) \text { is } C^{2} \text { on }\{x: u(t, x) \approx \nabla u(t, x) \approx 0\} .
$$


Assume that $(t, x)$ is such a point. Then for $s$ very close to $t$, we have by a Taylor series expansion in space,

$$
|u(s, y)| \approx|\operatorname{Hess} u(s, x)| \frac{|y-x|^{2}}{2} \approx c|y-x|^{2}
$$

for a certain $c>0$. Furthermore, a formal differentiation of (4.4) (for $b \equiv 0$ and $u$ is the difference of $u^{1}$ and $u^{2}$ ) with respect to the first coordinate $x_{1}$ gives

$$
\partial_{x_{1}}^{2} u(t, x)=\int_{0}^{t} \int \partial_{x_{1}}^{2} p_{t-s}(y-x)\left[\sigma\left(s, y, u^{1}(s, y)\right)-\sigma\left(s, y, u^{2}(s, y)\right)\right] W(d s, d y) .
$$

If $\sigma$ is a Weierstrass-type function that realizes its Hölder modulus at typical points, we have

$$
\left|\sigma\left(s, y, u^{1}(s, y)\right)-\sigma\left(s, y, u^{2}(s, y)\right)\right| \approx L|u(s, y)|^{\gamma}
$$

and we can check that the stochastic integral defining $\partial_{x_{1}}^{2} u(t, x)$ is not well-defined, i.e. has infinite quadratic variation. We can see this by giving a lower bound:

$$
\begin{gathered}
\mathbb{E}\left[\left(\partial_{x_{1}}^{2} u(t, x)\right)^{2}\right] \approx c^{2 \gamma} \int_{t-\delta}^{t} \int_{\mathbb{R}^{q}} \int_{\mathbb{R}^{q}} \partial_{x_{1}}^{2} p_{t-s}(w-x) \partial_{x_{1}}^{2} p_{t-s}(z-x)|w-x|^{2 \gamma}|z-x|^{2 \gamma} \\
\left(|w-z|^{-\alpha}+1\right) d w d z d s .
\end{gathered}
$$

After a longer calculation using the explicit value of the derivative

$$
\partial_{x_{1}}^{2} p_{t-s}(y-x)=(t-s)^{-1} p_{t-s}(y-x)\left(\frac{(y-x)_{1}^{2}}{t-s}+1\right),
$$

then restriction to $|w-x|>(t-s)^{1 / 2}$ and the change of variable $w^{\prime}=(t-s)^{-1 / 2}(w-$ $x)$ and $z^{\prime}=(t-s)^{-1 / 2}(z-x)$, obtain

$$
\mathbb{E}\left[\left(\partial_{x_{1}}^{2} u(t, x)\right)^{2}\right] \geq c \int_{t-\delta}^{t} d s(t-s)^{2 \gamma-2-\frac{\alpha}{2}}
$$

for some $\delta>0$. Thus, the quadratic variation is infinite if $2 \gamma-1-\alpha / 2 \leq 0$ which implies the necessity of $\alpha \leq 2(2 \gamma-1)$. Clearly, the proof techniques are not fine enough to make a comment on the case $\alpha=2(2 \gamma-1)$.

The main difficulty not allowing an easy proof as for SDE is the following: For fixed $x \in \mathbb{R}^{q}$ start with the mild formulation for two solutions $u^{1}(t, x)$ and $u^{2}(t, x)$. Their difference process $(u(t, x))_{t \geq 0}$ is a continuous process, but only Hölder-continuous of order $\frac{1}{2}\left(1-\frac{\alpha}{2}\right)<\frac{1}{2}$. So, this process cannot be a semimartingale for $\alpha>0$, since continuous semimartingales need to be Hölder-continuous up to order $\frac{1}{2}$. So Itô's formula cannot be applied directly.

Remark 5.3.4. In the previous heuristics it suffices to consider one direction of the gradient. This will be sufficient to obtain uniqueness for $\alpha<2(2 \gamma-1)$ rigorously. 
However, it is tempting to include further information on the gradient, e.g. $\nabla u \approx$ $\left(a_{n}^{\beta^{1}}, a_{n}^{\beta^{2}}, \ldots\right)$. We believe that no further improvement can be achieved, since 5.17) only requires the size of the principal component of the gradient.

\subsection{The compact support property}

In Section 4.4 the definitions and the known compact support property (CSP) results for white noise were summarized. Apart from the compact support property, there are also some results which hold about the growth of the support. For the Dawson-Watanabe process Dawson, Iscoe and Perkins [DIP89] showed, using the historical process, that the support grows within a certain bounded way. The historical process allows an analysis of the detailed structure of the support: they obtain path-regularity results jointly on the path of all particles simultaneously. We will not obtain such a strong result, but we can show that the supremum of the support grows in a bounded way, so we have control of "the rightmost particle."

Define $h(r) \equiv(r \log (1 / r))^{1 / 2}$ for any $r \in(0,1)$. Our main result holds in the colored noise case in dimension $q=1$ :

Theorem 5.4.1. Assume that $q=1, \sigma(u) \geq \sigma_{0} u^{\gamma}, u \geq 0, \gamma \in(0,1)$, for certain $\sigma_{0}>0$ and

$$
k(x, y)=|x-y|^{-\alpha} \text { for } \alpha \in(0,1) .
$$

Let $u \in C\left(\mathbb{R}_{+}, C_{\text {rap }}\right)$ be a nonnegative weak solution of (5.1) with $b \equiv 0$ and compact initial value $u_{0} \geq 0$, i.e. there is a compact set $K \subset \mathbb{R}$ with $\operatorname{supp}\left(u_{0}\right) \subset K$. Then, there is a constant $45.4 .1\left(\alpha,\|u\|_{\infty}\right)<\infty$ such that $\mathbb{P}$-almost surely there is a $\delta=$ $\delta(\omega)>0$ such that for all $0<s-r<\delta$ we have

$$
\sup \operatorname{supp}\left(u_{s}\right)-\sup \operatorname{supp}\left(u_{r}\right) \leq q 5.4 .1(1-\gamma)^{-1} h(s-r) \text {. }
$$

Existence of non-negative solutions should be possible to be established using a proof along the lines of that of Theorem 2.5 in Shi94.

Non-negative solutions are believed to be the limit of densities for branching particle systems. For branching processes, in general, the question of extinction is one of the first that poses itself. We show that exinction holds for a solution $u$ to (5.1) in the colored noise case: Let $U_{0}=\inf \{t \geq 0:\langle u(t, \cdot), 1\rangle=0\}$ denote the extinction time of the nonnegative solution $u$.

Proposition 5.4.2. If the assumptions of Theorem 5.4.1 hold and $\sigma(u) \leq \sigma_{1}(u+$ $\left.u^{\gamma}\right), u \geq 0$ for $\sigma_{1}>0$, then

$$
P\left(U_{0}<\infty\right)=1 \text { and } u\left(U_{0}+s, x\right)=0 \forall s>0, x \in \mathbb{R} \text { a.s. }
$$


The combination of the two previous results directly yields:

Theorem 5.4.3. If the assumptions of Theorem 5.4.1 hold, then the compact support property holds for non-negative solutions in $C\left(\mathbb{R}_{+}, C_{\text {rap }}\right)$.

The compact support property is of interest on its own. However, the remarkable result is that of Theorem 5.4.1. It states that no matter how correlated the noise is, the process behaves like the white noise driven SHE solution regarding its support. We hope that this result will help to establish a counterexample to Theorem 5.3.1 in the case where $\alpha>2(2 \gamma-1)$ as mentioned in Section 6.3

Let us give an outline of the proof of Theorem 5.4.1, which can be found in Chapter 10. The finite time extinction can be shown using standard methods for one-dimensional diffusions such as in [RW87] and is adapted from MP92.

For the growth of the support we use an idea of Krylov in Kry97. He also gave a nice heuristics for his result which is based on a lemma similar to our Lemma 10.2.1. Consider the following ODE

$$
u^{\prime \prime}(x)-u^{\gamma}(x)=0 \quad \text { in } \mathbb{R}_{+}
$$

with a certain boundary condition $u(0)=1, u^{\prime}(0)=0$ say. If $u$ is bounded (as we assume due to the fact solutions are in $C_{\text {rap }}$ ), say $\|u\|_{\infty} \leq K<\infty$, then $u^{\prime \prime}(x)-K^{\gamma-1} u \geq 0$ on $\mathbb{R}_{+}$. By the maximum principle (e.g. Theorem 6.4.2 in [Eva10] we get $u(x) \leq u(0) \exp \left(-K^{(\gamma-1) / 2} x\right)$. Then for $x \geq \mu_{1}:=K^{2 /(\gamma-1)}$ we have $u(x) \leq e^{-1}$. Using this new upper bound on the region $\left(\mu_{1}, \infty\right)$ obtain

$$
u^{\prime \prime}(x)-e^{1-\gamma} u(x) \geq 0, x \geq \mu_{1} .
$$

Iterating the procedure now, gives the bound $u(x) \leq e^{-n}$ for $x \geq K^{2 /(\gamma-1)}+$ $\sum_{k=1}^{n} e^{k(\gamma-1)}$. Finally, obtain:

$$
u(x)=0 \text { for any } x \geq K^{2 /(\gamma-1)}+\left(1-e^{\gamma-1}\right) .
$$

So a solution to the above ODE has compact support.

Clearly, the SPDE proof is a bit more complicated. The idea, however, is similar. Imagine again no mass at the right side of the origin, initially. If little "mass" flows over the point $r \in(0,1)$, then in a next time step little mass will flow over the point $r+r^{2}$ to the right, and so forth. The Itô-formula is a clever tool to formalize this idea in the martingale setup. Finally, an argument as that used for the proof of Kolmogorov-Centsov's Theorem 3.1 .3 establishes the "modulus of continuity". 


\section{Outlook}

In this final chapter of Part I, we give some ideas for the extension of some of the results obtained in the previous chapters. We list some conjectures and if there are proof ideas, we provide them.

\subsection{Stable Motion}

One of the classical generalizations of parabolic equations is to replace the Laplace operator by a certain pseudo-differential operator. Write $A^{\nu}=-(-\Delta)^{\nu / 2}$ short for the fractional Laplacian with $\nu \in(0,2)$. Thus, as a generalization of (5.1) we consider:

$$
\frac{\partial X}{\partial t}=\frac{1}{2} A^{\nu} X+\sigma(t, x, X) \dot{W}(t x)+b(t, x, X)
$$

Probabilistically, $A^{\nu}$ arises if one replaces 2 -stable motion (Brownian motion) by $\nu$-stable motion in the underlying particle model. Then, the following holds.

Lemma 6.1.1. If $b$ and $\sigma$ are both Lipschitz-continuous as in Theorem 4.2.1 and $\dot{W}^{k}$ is colored noise as in Definition 3.3.15 with $k(x, y)=|x-y|^{-\alpha}, \alpha>0$, then there exists a strong unique solution to (6.1) if $\alpha \in(0, \nu \wedge q)$.

Proof. The condition that $\alpha<q$ was required in Lemma 3.3.10 for the existence of the Gaussian colored noise $\dot{W}$. The Fourier transform of the correlation kernel $k$ is given by $\mu(d \xi)=|\xi|^{-q+\alpha} d \xi$. Let $\left(S_{t}\right)_{t \geq 0}$ denote the fundamental solution for $\left(\partial_{t}-A^{\nu}\right) u=0$. Its Fourier-transform is given by $\mathcal{F} S_{t}(\xi)=\exp \left(-t|\xi|^{\nu}\right)$. We need to check Condition (26) of [Dal99]. Let $T>0$,

$$
\begin{aligned}
\int_{0}^{T} d t \int_{\mathbb{R}^{q}} & \mu(d \xi)\left|\mathcal{F} S_{t}(\xi)\right|^{2}=\int_{0}^{T} d t \int_{\mathbb{R}^{q}} \mu(d \xi) \exp \left(-2 t|\xi|^{\nu}\right) \\
& \leq \int_{0}^{T} d t \int_{\mathbb{R}^{q}} d \xi|\xi|^{\alpha-q} \exp \left(-2 t|\xi|^{\nu}\right) \\
& =\int_{0}^{T} d t \int_{\mathbb{R}^{q}} t^{-q / \nu} d \eta t^{(q-\alpha) / \nu}|\eta|^{\alpha-q} \exp \left(-2|\eta|^{\nu}\right) \quad\left(\eta=t^{1 / \nu} \xi\right) \\
& \leq c \int_{0}^{T} d t t^{-\alpha / \nu}\left(\int_{\mathbb{R}^{q}} d \eta|\eta|^{\alpha-q} \exp \left(-2|\eta|^{\nu}\right)\right) \\
& <\infty
\end{aligned}
$$


if and only if $\alpha<\nu$. By Theorem 13 of [Dal99] the finiteness of the integral ensures the existence of a strong unique solution.

We believe that the following result holds for colored noise:

Conjecture 2. The existence result as Theorem 5.2.1 should hold for $\alpha<\nu \wedge q$, when $\Delta$ is replaced by $A^{\nu}$ in (5.1).

Surely, the question of pathwise uniqueness is also of interest in that case. We give some model calculations for the white noise case (so $q=1$ will be used). Of course, one could repeat the calculations of [SSS02], [MPS06] and of [MP11] - a task probably to ambitious for this thesis. So we give some ideas which lead to a conjecture in the end.

The $\nu$-stable heat kernel $p^{(\nu)}$, required in the mild formulation (4.4), allows the following decomposition:

$$
p_{t}^{(\nu)}(x)=\int_{0}^{\infty} d s q_{t}^{\nu / 2}(s) p_{s}^{(2)}(x), \quad t>0, x \in \mathbb{R}
$$

where $q_{t}^{\nu / 2}$ is the density of the positive $\nu / 2$-stable subordinator (there is only an explicit formula for $\nu \in\{1,2\}$, but its Laplace-transform is explicitely known, e.g. page 7 of [Ber99]). As before, $p_{s}^{(2)}(x)=(2 \pi s)^{-q / 2} \exp \left(-\frac{x^{2}}{2 s}\right), s>0, x \in \mathbb{R}^{q}$, is the ordinary heat kernel, which we also sometimes write as $p_{s}(x)$ with an abuse of notation. The formula itself holds by the subordination formula as in Theorem 8.6 of Ber99. Additionally, $p_{t}^{(\nu)}$ also has the following self-similarity property:

$$
p_{t}^{(\nu)}(x)=t^{-1 / \nu} p_{1}^{(\nu)}\left(t^{-1 / \nu} x\right)
$$

We present some estimates which are much in the spirit of the technical lemmas in Section 9.3 needed for the proof of pathwise uniqueness.

Lemma 6.1.2. Let $\nu \in(1,2]$. Then there is a $c>0$ such that for all $t>0, x \in \mathbb{R}$,

$$
\left|\partial_{x} p_{t}^{(\nu)}(x)\right| \leq c t^{-1 / \nu} p_{2 t}^{(\nu)}(x)
$$


Proof. We use self-similarity and the subordination formula to obtain

$$
\begin{aligned}
\left|\partial_{x} p_{t}^{(\nu)}(x)\right| & =\left|\partial_{x} t^{-1 / \nu} \int_{0}^{\infty} d s q_{1}^{\nu / 2}(s) p_{s}^{(2)}\left(t^{-1 / \nu} x\right)\right| \\
& =\left|t^{-1 / \nu} \int_{0}^{\infty} d s q_{1}^{\nu / 2}(s) \frac{-x}{\sqrt{s} t^{2 / \nu}} p_{s}^{(2)}\left(t^{-1 / \nu} x\right)\right| \\
& =\left|t^{-2 / \nu} \int_{0}^{\infty} d s q_{1}^{\nu / 2}(s) \frac{t^{-1 / \nu} x}{\sqrt{s}} p_{s}^{(2)}\left(t^{-1 / \nu} x\right)\right| \\
& \leq c\left|t^{-2 / \nu} \int_{0}^{\infty} d s s^{-\frac{1}{2}} q_{1}^{\nu / 2}(s) p_{2 s}^{(2)}\left(t^{-1 / \nu} x\right)\right| \\
& =\frac{c}{\sqrt{2}}\left|t^{-2 / \nu} \int_{0}^{\infty} d s s^{-\frac{1}{2}} q_{1}^{\nu / 2}(s) p_{s}^{(2)}\left(\frac{t^{-1 / \nu}}{\sqrt{2}} x\right)\right| \\
& =c\left|t^{-1 / \nu} t^{-1 / \nu} p_{1}^{(\nu)}\left(\frac{t^{-1 / \nu}}{\sqrt{2}} x\right)\right| \\
& =c t^{-1 / \nu} p_{2^{\nu / 2} t}^{(\nu)}(x) \\
& \leq c t^{-1 / \nu} p_{2 t}^{(\nu)}(x) .
\end{aligned}
$$

In the end we used Lemma 1 of [Haw71.

Lemma 6.1.3. For every $\delta \in(0,1]$, there is a $c=c(\delta, \nu)<\infty$, s.t. for $t>0, x, x^{\prime} \in$ $\mathbb{R}:$

$$
\int_{\mathbb{R}} d y\left(p_{t}^{(\nu)}(x-y)-p_{t}^{(\nu)}\left(x^{\prime}-y\right)\right)^{2} \leq c t^{-1 / \nu}\left(t^{-2 \delta / \nu}\left|x-x^{\prime}\right|^{2 \delta} \wedge 1\right) .
$$

Proof. Applying the proof strategy of Lemma 4.3 in [MP11 and using self-similarity we have for the second part of the minimum:

$$
\begin{aligned}
\int d y & \left(p_{t}^{(\nu)}(x-y)-p_{t}^{(\nu)}\left(x^{\prime}-y\right)\right)^{2} \\
& =\int d y t^{-2 / \nu}\left(p_{1}^{(\nu)}\left(t^{-1 / \nu}\left(y-x^{\prime}\right)\right)-p_{1}^{(\nu)}\left(t^{-1 / \nu}(y-x)\right)\right)^{2} \\
& \leq t^{-1 / \nu} \int \frac{d y}{t^{1 / \nu}}\left(p_{1}^{(\nu)}\left(\frac{y-x^{\prime}}{t^{1 / \nu}}\right)+p_{1}^{(\nu)}\left(\frac{y-x}{t^{1 / \nu}}\right)\right)^{2} .
\end{aligned}
$$

And for the first part we obtain by Lemma 2.1 of [FMW10] for $\delta \in(0,1]$ :

$$
\begin{aligned}
\int d y\left(p_{t}^{(\nu)}(x-y)-\right. & \left.p_{t}^{(\nu)}\left(x^{\prime}-y\right)\right)^{2} \leq c \int d y \frac{\left|x-x^{\prime}\right|^{2 \delta}}{t^{2 \delta / \nu}}\left(p_{t}^{(\nu)}\left(\frac{x-y}{2}\right)+p_{t}^{(\nu)}\left(\frac{x^{\prime}-y}{2}\right)\right)^{2} \\
& =c \frac{\left|x-x^{\prime}\right|^{2 \delta}}{t^{2 \delta / \nu}} \int \frac{d y}{t^{1 / \nu}} t^{-1 / \nu}\left(p_{1}^{(\nu)}\left(\frac{y-x}{2 t^{1 / \nu}}\right)+p_{1}^{(\nu)}\left(\frac{y-x^{\prime}}{2 t^{1 / \nu}}\right)\right)^{2} \\
& \leq c t^{-(2 \delta+1) / \nu}\left|x-x^{\prime}\right|^{\delta} .
\end{aligned}
$$


We state another lemma, which can be proved in a similar way to the previous one by applying the bound on the derivative.

Lemma 6.1.4. There is a $c=c(\nu)<\infty$, s.t. for $t>0, x, x^{\prime} \in \mathbb{R}$

$$
\int d y\left(\partial_{x} p_{t}^{(\nu)}(x-y)-\partial_{x^{\prime}} p_{t}^{(\nu)}\left(x^{\prime}-y\right)\right)^{2} \leq c t^{-3 / \nu}\left(t^{-2 \delta / \nu}\left|x-x^{\prime}\right|^{\delta} \wedge 1\right) .
$$

If one compares the spirit of these lemmas with Lemmas 9.6 .2 of Section 9.6 and 9.3.4 of Section 9.3, one sees the 2 there is replaced by $\nu$ (setting $\alpha=1$, since we consider colored noise here). We do not know how many of the lemmas we see in Chapter 9, especially in Section 9.3. can be transferred to the stable setting, in colored or white noise. However one might guess that the following conjecture holds:

Conjecture 3. Pathwise uniqueness in $C\left(\mathbb{R}_{+}, C_{\text {tem }}\right)$ holds for 6.1), if $\alpha<\nu(2 \gamma-$ $1)$.

\subsection{Particle picture}

As already mentioned in Example 4.1.1, the large population limits for densities of branching particle systems are known to approach solutions of stochastic heat equations (5.1); [Daw75] or [Blo96] are two references. A similar convergence result holds true for certain interacting particle systems, called long-range voter models; [MT94 or Kli11 are two references. However, all of these results are limited to the noise coefficient being of the following form: $\sigma(u)=c \sqrt{u}, \sigma(u)=c u$ or $\sigma(u)=c \sqrt{u(1-u)}$ for a constant $c$. So, many of the SHE described by the class (5.1) do not have a so-called "particle picture", i.e. there is no weakly converging density of a particle system. However, on page 326 of [MP92] an idea for densitydependent branching is given.

A classical weak convergence proof includes the two steps 1) Tightness; 2) Uniqueness of the limit. Even if tightness results were possible, the lack of a uniqueness result for the SHE made it difficult to obtain such convergence results. Now we have two such results: Theorem 4.3 .3 for white noise and Theorem 5.3 .1 for colored noise. So, it seems possible to attain weak convergence results as we describe in the following.

We will present a model which we conjecture to converge to a solution of (5.1) with diffusion coefficient $\sigma(u)=u^{\gamma}$ and white noise. In order to describe the model, we need a certain class of offspring distributions $\nu_{\gamma^{\prime}}(u, \cdot) \in \mathcal{M}_{1}\left(\mathbb{Z}_{+}\right), u \in \mathbb{R}_{+}, \gamma^{\prime} \in \mathbb{R}$, whose heuristic derivation we present in Chapter 11 . 
The model describes density-dependent branching with independent branching events. This will lead to a white noise SHE. Surely, it would be desirable to extend that to spatially correlated Gaussian noise (colored noise) as well, as the present work gives the required uniqueness result, but it is technically more demanding. So far, there are few convergence results (for example [Myt96] or [Stu03]) available for colored noise as in Definition 3.3.15.

For the description of the model we borrow notation from [Per02] and it is similar to Example 4.1.1.

\section{Definition of the model}

There are some properties of the class of offspring distributions. Let $N$ be distributed according to $\nu_{\gamma^{\prime}}(u, \cdot) \in \mathcal{M}_{1}\left(\mathbb{Z}_{+}\right)$for fixed $u \in \mathbb{R}_{+}, \gamma^{\prime} \in \mathbb{R}$. Then

$$
\mathbb{E}[N]=1, \quad \mathbb{E}\left[(N-1)^{2}\right]=u^{\gamma^{\prime}} .
$$

Let $n \in \mathbb{N}$ be the renormalization constant which is kept fixed throughout this definition.

We want to consider a population model in discrete time, where each individual gets a label $\alpha \in I=\mathbb{Z}_{+}^{*}=\bigcup_{n \in \mathbb{Z}_{+}} \mathbb{N}^{n}$. If $\alpha=\alpha_{0} \alpha_{1} \cdots \alpha_{n} \in I$, then we write $|\alpha|=n$ for the generation of individual $\alpha$. For $i \in\{0,1, \ldots,|\alpha|\}$ we can write $\left.\alpha\right|_{i}=\alpha_{0} \ldots \alpha_{i}$ if we restrict $\alpha$ to its first $i+1$ elements. This gives a natural ancestral relation: Write $\alpha \prec \beta$, whenever $\alpha=\left.\beta\right|_{i}$ holds for a certain $i \in\{0, \ldots,|\beta|\}$. Then $\alpha$ is called an ancestor of $\beta$.

Our population inhabits a space $E$, which we suppose from now on to be $\mathbb{R}$. Either we can think of $E$ as a geographical space or a certain trait space of the individuals. Let $A$ be the generator of a Feller semigroup on $C_{b}(E)$ describing the motion of the individuals. Here we will specialize to the case $A=\frac{1}{2} \Delta$. The corresponding semigroup (here the heat semigroup) is denoted by $\left(S_{t}\right)_{t \geq 0}$.

Let $M$ be a Poisson point process on $\mathbb{R}$ with intensity $n \mu$, where $\mu \in \mathcal{M}_{f}(\mathbb{R})$ a finite Borel measure on $\mathbb{R}$. Label its points in an arbitrary fashion with $\left\{1,2, \ldots, k_{n}\right\}$ and denote the position of the points in $\mathbb{R}$ by $x_{\alpha_{0}}, 1 \leq \alpha_{0} \leq k_{n}$, where $\alpha_{0}$ is the label.

The population to be considered will evolve in time and the $k_{n}$ initial individuals labeled $\left\{1, \ldots, k_{n}\right\}$ start at position $x_{\alpha_{0}}$ for $\alpha_{0} \in\left\{1,2, \ldots, k_{n}\right\}$. The evolution of the individuals is characterized by two meachanisms: First, motion in space $E$ and second, resampling after discrete time steps of length $n^{-1}$.

Let therefore $\left\{Y^{\alpha, n}: \alpha_{0} \leq k_{n}, \alpha \in I\right\}$ be a collection of Feller-processes, with generator $A$, started at $x_{\alpha_{0}}$ and stopped at time $(|\alpha|+1) n^{-1}$ each. We require that individuals share a common path up to their most recent common ancestor

$$
Y^{\alpha, n}(t)=Y^{\left.\alpha\right|_{|\alpha|-1}, n}(t), 0 \leq t \leq \frac{|\alpha|}{n}
$$


and that $\left\{Y^{\alpha, n}:|\alpha|=k\right\}$ are independent given $\sigma\left(Y^{\beta, n}:|\beta|<k\right)$ for a fixed $k \in \mathbb{N}$. Note that the first requirement gives a tree structure.

Recursively, we define variables $\tau$ and $N$. The $n^{-1} \mathbb{Z}_{+}$-valued random variables $\tau^{\alpha, n}$ will stand for the first point in time when an ancestor of $\alpha$ had no offspring or, even, there were not enough initial individuals. Then $\alpha$ will be called "dead." The $\mathbb{Z}_{+}$-valued random variables $N^{\alpha, N}$ stand for the number of offspring of individual $\alpha$.

Let $\bar{\alpha} \in I$ with $|\bar{\alpha}|=k \in \mathbb{Z}_{+}$be fixed. Assume that

$$
\left(\tau^{\beta, n}, N^{\beta, n}\right) \text { for } \beta \in I,|\beta|<k
$$

are given (this includes the case $k=0$ ). Then let

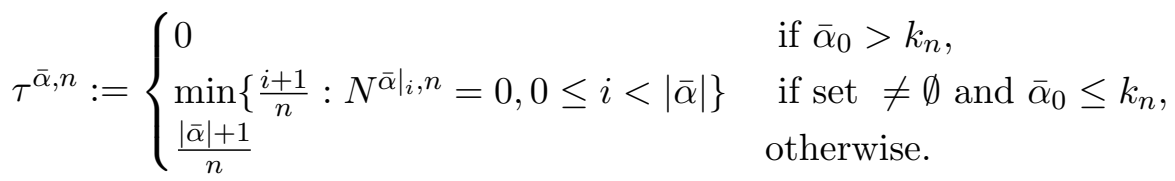

For $t \in\left[0, \frac{k+1}{n}\right)=\left[0, \frac{|\bar{\alpha}|+1}{n}\right.$ ) we define the following (random) relation

$$
\bar{\alpha} \sim_{n} t \Leftrightarrow t \in\left[\frac{|\bar{\alpha}|}{n}, \frac{|\bar{\alpha}|+1}{n}\right) \text { and } t<\tau^{\bar{\alpha}, n},
$$

indicating up to which time the direct ancestor of individual $\bar{\alpha}$ was alive. For $B \in \mathcal{B}(\mathbb{R})$ define for $0 \leq t<\frac{|\bar{\alpha}|+1}{n}$ the renormalized number of alive individuals in the region $B$ by

$$
X_{t}^{n}(B)=\frac{1}{n} \#\left\{\beta: \beta \sim_{n} t, Y^{\beta, n}(t) \in B\right\}=\frac{1}{n} \sum_{\beta \sim_{n} t} \delta_{Y^{\beta, n}(t)}(B)
$$

For any $y \in \mathbb{R}$ define:

$$
u^{n}(t, y):=\frac{1}{n} \sum_{\beta \sim_{n} t} p_{1 / n}\left(Y^{\beta, n}(t)-y\right)
$$

where $p_{1 / n}(\cdot)$ is the 1-dimensional heat kernel at time $n^{-1}$. The non-negative function $u^{n}$ describes an approximate density of individuals close to $y$.

Now, we have defined what is happening up to time $t=\frac{|\bar{\alpha}|+1}{n}-$ with individual $\bar{\alpha}$. At time $\frac{|\bar{\alpha}|+1}{n}$ a branching event happens with a certain offspring we define now. Consider the $\mathbb{Z}_{+}$-valued random variable $N^{\bar{\alpha}, n}$ with distribution

$$
\begin{array}{r}
\mathbb{P}\left(N^{\bar{\alpha}, n}=\cdot \mid\left\{Y^{\beta, n}\left(\frac{|\beta|+1}{n}\right),|\beta|=|\bar{\alpha}|, \tau^{\beta, n}=\frac{|\beta|+1}{n}\right\}\right) \\
=\nu_{2 \gamma-1}\left(u^{n}\left(\left(\frac{|\bar{\alpha}|+1}{n}\right)_{-}, Y^{\bar{\alpha}, n}\left(\frac{|\bar{\alpha}|+1}{n}\right)\right), \cdot\right)
\end{array}
$$


in the case $\tau^{\bar{\alpha}, n}=\frac{k+1}{n}$. Here, $\nu_{\gamma^{\prime}}$ is a probability law on $\mathbb{Z}_{+}$with the properties in (6.2); the definition is given in 11.2 for $\gamma^{\prime} \in \mathbb{R}$. We assume that conditionally on $\mathcal{F}_{(k+1) n^{-1}}^{n}=\sigma\left(Y^{\beta, n}(s),|\beta| \leq k+1, s \leq(k+1) n^{-1}, N^{\beta^{\prime}, n},\left|\beta^{\prime}\right| \leq k\right)$ the collection $\left\{N^{\alpha, n}:|\alpha|=k+1\right\}$ is independent. This completes the iterative description.

\section{Convergence conjecture}

Let us write $\langle f, g\rangle=\int_{\mathbb{R}} f(x) g(x) d x$ for the $L^{2}$-scalar product. For the density $u^{N}$ as defined above, we conjecture the following fact:

Conjecture 4. Assume $q=1, \gamma \in(1 / 2,1)$ and assume that $u_{0}^{n}, u_{0} \in C_{\text {tem }}$ are deterministic for $n \in \mathbb{N}$. If $u_{0}^{n} \rightarrow u_{0}$ in $C_{\text {tem }}$, then $u^{n}$ is relatively compact in $D\left(\mathbb{R}_{+}, C_{\text {tem }}\right)$. Each weak limit $u$ is defined on a filtered probability space which also supports a white noise $W$ such that for each $\phi \in C_{c}^{\infty}(\mathbb{R})$ we have almost surely for all $t \geq 0$

$$
\langle u(t, \cdot), \phi\rangle=\langle u(0, \cdot), \phi\rangle+\int_{0}^{t}\left\langle u(s, \cdot), \frac{1}{2} \phi^{\prime \prime}\right\rangle d s+\int_{0}^{t} \int_{\mathbb{R}} \sqrt{\lambda} u(s, y)^{\gamma} \phi(y) W(d s d y) .
$$

If $\gamma \in(3 / 4,1)$, then $u^{n}$ converges weakly to $u$.

In order to show such a result, there are two things to do: 1) Tightness, 2) Identification of the limit.

Our idea of proof is based on the classic ways which one can use for measure-valued processes or densities, carried out e.g. in [MT94, [Blo96] or [Per02]. We give a more precise description of the idea in Section 11.2. Here, we will only consider part of what belongs to "identification of the limit:"

We will follow Perkins' notation again and only write down the "branching martingale" (defined in (11.4)), leading to the diffusion term in the SPDE:

$$
M_{\underline{\underline{t}}}^{b, n}(\phi)=\frac{1}{n} \sum_{\underline{s}<\underline{t}} \sum_{\alpha \sim n \underline{s}} \phi\left(Y_{\underline{s}+n^{-1}}^{\alpha, n}\right)\left(N^{\alpha, n}-1\right)
$$

for $\underline{t} \in n^{-1} \mathbb{N}$. This is a discrete time martingale $(\mathbb{E}[N]=1)$ and we calculate its quadratic variation:

$$
\begin{aligned}
\mathbb{E} & {\left[\left(M_{\underline{s}+n^{-1}}^{b, n}(\phi)-M_{\underline{s}}^{b, n}(\phi)\right)^{2} \mid \overline{\mathcal{F}}_{\underline{s}}\right] } \\
& =n^{-2} \sum_{\alpha \sim n \underline{s}} \phi\left(Y_{\underline{s}+n^{-1}}^{\alpha, n}\right)^{2} \mathbb{E}\left[\left(N^{\alpha, n}-1\right)^{2} \mid \overline{\mathcal{F}}_{\underline{s}}\right] \\
& =n^{-2} \sum_{\alpha \sim n \underline{s}} \phi\left(Y_{\underline{s}+n^{-1}}^{\alpha, n}\right)^{2} \lambda u^{n}\left(\underline{s}+n^{-1}, Y^{\alpha, n}\left(\underline{s}+n^{-1}\right)\right)^{2 \gamma-1} .
\end{aligned}
$$


Therefore summing up over $\underline{s}<\underline{t}$, we get

$$
\begin{aligned}
\left\langle M^{b, n}(\phi)\right\rangle_{\underline{t}}= & \frac{1}{n} \sum_{\underline{s}<\underline{t}} \frac{1}{n} \sum_{\alpha \sim_{n} \underline{s}} \phi\left(Y_{\underline{s}}^{\alpha, n}\right)^{2} \lambda u^{n}\left(\underline{s}, Y^{\alpha, n}(\underline{s})\right)^{2 \gamma-1} \\
& +\varepsilon_{\underline{t}, 1}^{n}(\phi),
\end{aligned}
$$

where

$$
\begin{array}{r}
\varepsilon_{\underline{t}, 1}^{n}(\phi)=n^{-2} \sum_{\underline{s}<\underline{t}} \sum_{\alpha \sim n \underline{s}}\left[\phi\left(Y_{\underline{s}+n^{-1}}^{\alpha, n}\right)^{2} \lambda u^{n}\left(\underline{s}+n^{-1}, Y^{\alpha, n}\left(\underline{s}+n^{-1}\right)\right)^{2 \gamma-1}\right. \\
\left.-\phi\left(Y_{\underline{s}}^{\alpha, n}\right)^{2} \lambda u^{n}\left(\underline{s}, Y^{\alpha, n}(\underline{s})\right)^{2 \gamma-1}\right] .
\end{array}
$$

Suppose that $\sup _{t \leq K}\left|\varepsilon_{t, 1}^{n}(\phi)\right| \stackrel{L^{1}}{\rightarrow} 0$ as $n \rightarrow \infty$ for all $K>0$. Hence, neglecting $\varepsilon_{t, n}^{n}$ in the forthcoming we can write:

$$
\begin{aligned}
\left\langle M^{b, n}(\phi)\right\rangle_{\underline{t}} & =\frac{1}{n} \sum_{\underline{s}<\underline{t}} \frac{1}{n} \sum_{\alpha \sim n \underline{s}} \int_{\mathbb{R}^{q}} \delta_{Y_{\underline{s}}^{\alpha}}(d y)\left(\phi(y)^{2} \lambda u^{n}(s, y)^{2 \gamma-1}\right) \\
= & \frac{1}{n} \sum_{\underline{s}<\underline{t}} \int_{\mathbb{R}^{q}} d y u^{n}(s, y) \phi(y)^{2} \lambda u^{n}(s, y)^{2 \gamma-1} \\
& +\frac{1}{n} \sum_{\underline{s}<\underline{t}} \frac{1}{n} \sum_{\alpha \sim n \underline{s}} \int_{\mathbb{R}^{q}}\left(\delta_{Y_{\underline{s}}^{\alpha}}(d y)-p_{1 / n}\left(Y^{\alpha, n}(\underline{s})-y\right) d y\right)\left(\phi(y)^{2} \lambda u^{n}(s, y)^{2 \gamma-1}\right) \\
= & \int_{0}^{\underline{t}} d s \int_{\mathbb{R}^{q}} d y u^{n}(s, y)^{2 \gamma} \lambda \phi(y)^{2}+\varepsilon_{\underline{t}, 2}^{n}(\phi) .
\end{aligned}
$$

Suppose that $\sup _{t \leq K}\left|\varepsilon_{t, 2}^{n}(\phi)\right| \stackrel{L^{1}}{\rightarrow} 0$ as $n \rightarrow \infty$ for all $K>0$. If we calculate the quadratic variation of the stochastic integral in Conjecture 4 we get:

$$
\left\langle\int_{0} \int_{\mathbb{R}} \sqrt{\lambda} u(s, y)^{\gamma} \phi(y) W(d s d y)\right\rangle_{t}=\int_{0}^{t} \int_{\mathbb{R}} \lambda u(s, y)^{2 \gamma} \phi(y)^{2} d y d s .
$$

As this expression is analogous to (6.4), we believe that the model described here is a good candidate for Conjecture 4 to hold. More explanations and calculations are given in Section 11.2 .

\subsection{Non-Uniqueness}

The main result, Theorem 5.3.1 indirectly raises another question: Is the result sharp? In the case of white noise, Mueller, Mytnik and Perkins [MMP12] could 
find an SPDE which does not satisfy pathwise uniqueness (not even uniqueness in law), if the sufficient conditions of Theorem 4.3.3 are not fulfilled:

Theorem 6.3.1 (Theorem 1.1 in [MMP12]). Consider

$$
\partial_{t} u(t, x)=\frac{1}{2} \partial_{x}^{2} u(t, x)+|u|^{\gamma} \dot{W}(t, x), t>0, x \in \mathbb{R} ; u(0, x)=0, x \in \mathbb{R} .
$$

If $\dot{W}$ is white noise and $0<\gamma<3 / 4$, there is a $C_{\mathrm{rap}}$-valued solution $u(t, x)$ such that with positive probability $u$ is not identically zero. In particular, uniqueness in law and pathwise uniqueness fail.

One might believe that this result also holds in the colored noise case, at least in dimension $q=1$. It was not possible yet to transfer their result, but the first steps in that direction were already taken: the compact support property, finite time extinction and additionally, the growth behavior of the support were given in Theorem 5.4.1 and Proposition 5.4.2.

The most difficult part of transfering their proof techniques seems to be the lower bound on the growth of the emerging cluster in their Lemma 4.1. There is a heuristic (personal communication, Leonid Mytnik) explaining their Lemma 4.1 in the colored noise setting requiring (spatial) Hölder-continuity of order 2 at points where the solution is small. However, we have not yet been able to make this rigorous.

In [MMP12, real-valued solutions were considered. Also Theorem 5.3.1 and Theorem 4.3.3 deal with real-valued solutions. If we restrict the SHE to nonnegative solutions, it might be true that less strict conditions on $\gamma$ are sufficient for pathwise uniqueness. A first indication towards that is the result in Myt98 which says that uniqueness in law holds for (6.5) if solutions are required to be non-negative. Whether the uniqueness in law result holds in the case of general Hölder-continuous $\sigma$ for nonnegative solutions remains unclear. Considering the convergence conjecture of the previous section, it is true that particle densities of a branching particle system are always non-negative. If rescaled particle densities are tight and their limit points solve a SHE, such as (6.5) then the limit points are also non-negative solutions of the SHE. So, to obtain strict conditions for uniqueness results (pathwise or in law) for the SHE with non-negative solutions is a highly interesting question.

\subsection{The compact support property in higher dimensions}

For the white noise case the compact support result is limited to one spatial dimension as otherwise solutions will not exist (as functions). However, the superprocess results on compact support go beyond one dimensional results. For the colored 
noise case the dimension restriction, $q=1$, does not exist (except $\alpha<q$ ), so it is natural to ask whether the compact support property holds in higher dimensions.

The main trick in our argument which relied on some spatial structure of $\mathbb{R}^{1}$ is done in (10.6), where the Itô-formula is applied to the function $\zeta(x)=(x \vee 0)$. The function $\zeta$ is a harmonic function (w.r.t. $\Delta$ ) outside the support $(-\infty, 0]$ of $u_{0}$. In higher dimensions imagine that the support of $u_{0}$ is contained in $B(0,1) \subset \mathbb{R}^{q}$. Then we need a harmonic function $v$, which will not grow too fast:

$$
\left\{\begin{array}{lll}
\Delta v(x) & =0, \quad x \in \mathbb{R}^{q} \backslash B(0,1), \\
v(x) & =0, \quad x \in \partial B(0,1), \\
\lim \sup _{|x| \rightarrow \infty} \frac{v(x)}{|x|^{p}} & =0, &
\end{array}\right.
$$

for some $p>0$. The last criterion is used in order to ensure finiteness of the integral $\int_{\mathbb{R}^{q}} v(x) u(s, x) d x$. Nontrivial solutions for this problem are well-known:

$$
v(x)= \begin{cases}\log |x|, & q=2, \\ 1-|x|^{q-2} & q \geq 3 .\end{cases}
$$

So we are led to the following conjecture:

Conjecture 5. Assume $\sigma(u)=\sigma_{0} u^{\gamma}, \gamma \in(0,1)$ and $\sigma_{0}>0$. Then, the compact support property holds for nonnegative solutions $u \in C\left(\mathbb{R}_{+}, C_{\text {rap }}\right)$ of (5.1) with $b \equiv 0$ and $\dot{W}=\dot{W}^{k}, k(x, y)=|x-y|^{-\alpha}, \alpha \in(0,2 \wedge q)$.

There are surely other minor modifications of equation (4.11) which might still lead to compact support property. The lower bound on $\sigma(u) \geq \sigma_{0} u^{\gamma}$ should be possible to be relaxed to one which only holds close to zero. This would be helpful to include cases such as Fisher-Wright noise $\sigma(u)=\sqrt{u(1-u)}$ or similar models.

Another minor modification should be the relaxation of the diffusion operator $\Delta$ in a similar way as in Krylov's work [Kry97], where he used a uniformly elliptic operator $L=a \partial^{2}+b \partial+c$. In fact, this would allow a (small) nonnegative drift. A very interesting other modification would be the use of this technique in the case of Lévy-noise driven SPDE. 
Part II.

Proofs 


\section{An abstract result on strong solutions}

We start this chapter with a lemma characterising (weak) solutions of SPDE via an approximation procedure. The proof of Lemma 5.1.1 is done after that.

Lemma 7.1.1. Let $\left(\Omega, \mathcal{F}, \mathcal{F}_{t}, \mathbb{P}\right)$ be a filtered probability space with a noise $\dot{W}$, which is white in time (see Definition 3.3.15). Let $u: \mathbb{R}_{+} \times \mathbb{R}^{q} \rightarrow \mathbb{R}$ be a jointly continuous random field such that for any compact set $K \subset \mathbb{R}^{q}$ the following holds: $\mathbb{E}\left[\sup _{s \leq t} \sup _{x \in K}|u(s, x)|^{2}\right]<\infty$. Let $b: \mathbb{R} \rightarrow \mathbb{R}$ and $\sigma: \mathbb{R}_{+} \times \mathbb{R}^{q} \times \mathbb{R}$ be continuous with

$$
b(t, x, u)+\sigma(t, x, u) \leq C(T, K)(1+|u|) \forall t \leq T, x \in K
$$

for a certain constant $C(T, K)$, where $T>0$ and $K \subset \mathbb{R}^{q}$ compact. Let $A$ be a partial differential operator with adjoint $A^{*}$. If $\phi \in C_{c}^{\infty}\left(\mathbb{R}^{q}\right)$ then the two following conditions are equivalent:

(a)

$$
\begin{aligned}
\int_{\mathbb{R}^{q}} \phi(x) u(t, x) d x=\int_{\mathbb{R}^{q}} \phi(x) u_{0}(x) d x+\int_{0}^{t} \int_{\mathbb{R}^{q}} u_{s}(x) A^{*} \phi(x)+b\left(u_{s}(x)\right) \phi(x) d x d t \\
+\int_{0}^{t} \int_{\mathbb{R}^{q}} \phi(x) \sigma\left(s, x, u_{s}(x)\right) W(d s d x) \forall t>0 \quad \text { a.s. }
\end{aligned}
$$

(b) For any $T \geq 0$ :

$$
\begin{aligned}
\limsup _{n \rightarrow \infty} & \mathbb{E}\left[\sup _{t \leq T} \mid\left\langle u_{t}-u_{0}, \phi\right\rangle-\sum_{k=1}^{\lfloor n t\rfloor} \sum_{l \in \mathbb{Z}^{q}} n^{-(q+1)} u\left(\frac{k}{n}, \frac{l}{n}\right) A^{*} \phi\left(\frac{l}{n}\right)+b\left(u\left(\frac{k}{n}, \frac{l}{n}\right)\right) \phi\left(\frac{l}{n}\right)\right. \\
+ & \left.\left.\sum_{k=1}^{\lfloor n t\rfloor} \sum_{l \in \mathbb{Z}^{q}} \sigma\left(\frac{k}{n}, \frac{l}{n}, u\left(\frac{k}{n}, \frac{l}{n}\right)\right) \phi\left(\frac{l}{n}\right) \dot{W}\left(\left[\frac{k}{n}, \frac{k+1}{n}\right] \times\left[\frac{l}{n}, \frac{l+\mathbb{1}}{n}\right]\right)\right|^{2}\right]=0,
\end{aligned}
$$

where $\mathbb{1}=(1, \ldots, 1) \in \mathbb{R}^{q}$.

Proof. Assume that $\phi$ is supported within the compact set $K \subset \mathbb{R}^{q}$ and for simplicity that $b$ and $\sigma$ only depend on $u$; the proof can also be done more general, 
but notations get lengthier.

(a) $\Rightarrow$ (b): Let $T \geq 0$ and $\mathbb{1}=(1, \ldots, 1) \in \mathbb{R}^{q}$. Use (a) to get

$$
\begin{gathered}
\mathbb{E}\left[\sup _{t \leq T} \mid\left\langle u_{t}-u_{0}, \phi\right\rangle-\sum_{k=1}^{\lfloor n t\rfloor} \sum_{l \in \mathbb{Z}^{q}} n^{-(q+1)} u\left(\frac{k}{n}, \frac{l}{n}\right) A^{*} \phi\left(\frac{l}{n}\right)+b\left(u\left(\frac{k}{n}, \frac{l}{n}\right)\right) \phi\left(\frac{l}{n}\right)\right. \\
\left.\quad+\left.\sum_{k=1}^{\lfloor n t\rfloor} \sum_{l \in \mathbb{Z}^{q}} n^{-1} \sigma\left(u\left(\frac{k}{n}, \frac{l}{n}\right)\right) \phi\left(\frac{l}{n}\right) \dot{W}\left(\left[\frac{k}{n}, \frac{k+1}{n}\right] \times\left[\frac{l}{n}, \frac{l+\mathbb{1}}{n}\right]\right)\right|^{2}\right] \\
\leq \mathbb{E}\left[\sup _{t \leq T} \mid \int_{0}^{t} \int_{\mathbb{R}^{q}} u_{s}(x) A^{*} \phi(x)+b\left(u_{s}(x)\right) \phi(x) d x d s\right. \\
\quad-\sum_{k=1}^{\lfloor n t\rfloor} \sum_{l \in \mathbb{Z}^{q}} n^{-(q+1)} u\left(\frac{k}{n}, \frac{l}{n}\right) A^{*} \phi\left(\frac{l}{n}\right)+\left.b\left(u\left(\frac{k}{n}, \frac{l}{n}\right)\right) \phi\left(\frac{l}{n}\right)\right|^{2} \\
+\mid \int_{0}^{t} \int_{\mathbb{R}^{q}} \sigma(s, x, u) \phi(x) \dot{W}(d x d s) \\
\left.\quad-\left.\sum_{k=1}^{\lfloor n t\rfloor} \sum_{l \in \mathbb{Z}^{q}} \sigma\left(u\left(\frac{k}{n}, \frac{l}{n}\right)\right) \phi\left(\frac{l}{n}\right) \dot{W}\left(\left[\frac{k}{n}, \frac{k+1}{n}\right] \times\left[\frac{l}{n}, \frac{l+\mathbb{1}}{n}\right]\right)\right|^{2}\right] \\
=I_{1}+I_{2} . \quad
\end{gathered}
$$

As $I_{2}$ is the sum of two martingales we can use Doob's inequality to obtain:

$$
\begin{aligned}
I_{2} & \leq 2 \mathbb{E}\left[\left|\int_{0}^{t} \int_{\mathbb{R}^{q}}\left(\sigma\left(u_{s}(x)\right) \phi(x)-\sigma\left(u\left(\frac{\lfloor n s\rfloor}{n}, \frac{\lfloor n x\rfloor}{n}\right)\right) \phi\left(\frac{\lfloor n x\rfloor}{n}\right)\right) W(d s d x)\right|^{2}\right] \\
& \leq 2 \mathbb{E}\left[\int _ { 0 } ^ { t } d s \int _ { \mathbb { R } ^ { q } } \int _ { \mathbb { R } ^ { q } } d w d z \left(\left(\sigma\left(u_{s}(w)\right) \phi(w)-\sigma\left(u\left(\frac{\lfloor n s\rfloor}{n}, \frac{\lfloor n w\rfloor}{n}\right)\right) \phi\left(\frac{\lfloor n w\rfloor}{n}\right)\right)\right.\right. \\
& =o(1),
\end{aligned}
$$

by dominated convergence, since $k$ is locally integrable, $\phi$ has compact support and $\mathbb{E}\left[\sup _{s, z \in K}|u(s, z)|^{2}\right]<\infty$ on the one hand and continuity of $u(\cdot, \cdot)$ on the other hand.

Treating $I_{1}$ is even easier. We use the bound on $b$ and that $\left\|A^{*} \phi\right\|_{\infty} \leq C_{1}$, since 
$A^{*}$ is a bounded operator on $\mathcal{D}=C_{c}^{\infty}$. Then

$$
\begin{aligned}
I_{1} \leq \mathbb{E}\left[\sup _{t \leq T} \mid \int_{0}^{t} \int_{\mathbb{R}^{q}}\left(u_{s}(x) A^{*} \phi(x)+b\left(u_{s}(x)\right) \phi(x)\right)\right. \\
\left.\quad-\left.\left(u\left(\frac{\lfloor n s\rfloor}{n}, \frac{\lfloor n x\rfloor}{n}\right) A^{*} \phi(x)+b\left(u\left(\frac{\lfloor n s\rfloor}{n}, \frac{\lfloor n x\rfloor}{n}\right)\right) \phi(x)\right) d x d s\right|^{2}\right] \\
+\mathbb{E}\left[\sup _{t \leq T} \mid \int_{0}^{t} \int_{\mathbb{R}^{q}}\left(u\left(\frac{\lfloor n s\rfloor}{n}, \frac{\lfloor n x\rfloor}{n}\right) A^{*} \phi(x)-u\left(\frac{\lfloor n s\rfloor}{n}, \frac{\lfloor n x\rfloor}{n}\right) A^{*} \phi\left(\frac{\lfloor n x\rfloor}{n}\right)\right.\right. \\
\left.\left.+b\left(u\left(\frac{\lfloor n s\rfloor}{n}, \frac{\lfloor n x\rfloor}{n}\right)\right) \phi(x)-b\left(u\left(\frac{\lfloor n s\rfloor}{n}, \frac{\lfloor n x\rfloor}{n}\right)\right) \phi\left(\frac{\lfloor n x\rfloor}{n}\right)\right)\left.d x d s\right|^{2}\right] \\
\leq C_{1} \mathbb{E}\left[\sup _{s \leq t, x \in K}\left|u(s, x)-u\left(\frac{\lfloor n s\rfloor}{n}, \frac{\lfloor n x\rfloor}{n}\right)\right|^{2}\right] \\
+\mathbb{E}\left[\sup _{s \leq t, x \in K} 2|u(s, x)|^{2}\right] t \operatorname{Leb}(K) \sup _{x \in K}\left|A^{*} \phi(x)-A^{*} \phi\left(\frac{\lfloor n x\rfloor}{n}\right)\right|^{2} \\
=o(1) .
\end{aligned}
$$

by the dominated convergence theorem and continuity of $u$.

(b) $\Rightarrow$ (a): By definition of the Riemann integral and by the assumption on $u$ being continuous, we have

$$
\begin{aligned}
\left|J_{c}-J_{d}\right|:= & \mid \int_{0}^{t} \int_{\mathbb{R}^{q}}\left(u_{s}(x) A^{*} \phi(x)+b\left(u_{s}(x)\right) \phi(x)\right) d x d s \\
& -\sum_{k=1}^{\lfloor n t\rfloor} \sum_{l \in \mathbb{Z}^{q}} n^{-(q+1)} u\left(\frac{k}{n}, \frac{l}{n}\right) A^{*} \phi\left(\frac{l}{n}\right)+b\left(u\left(\frac{k}{n}, \frac{l}{n}\right)\right) \phi\left(\frac{l}{n}\right) \mid=o(1)(n \rightarrow \infty),
\end{aligned}
$$

pointwise and hence in $L^{2}$ by square-integrability of the limit. With a similar argument as that for $I_{2}$, we obtain for $M_{c}$ and $M_{d}$ defined in the following obvious manner:

$$
\begin{aligned}
\mathbb{E}\left[\left|M_{c}-M_{d}\right|^{2}\right]=\mathbb{E}\left[\sup _{t \leq T} \mid \int_{0}^{t} \int_{\mathbb{R}^{q}} \sigma(u(s, x)) \phi(x) W(d s d x)\right. \\
\left.-\left.\sum_{k=1}^{\lfloor n t\rfloor} \sum_{l \in \mathbb{Z}^{q}} \sigma\left(u\left(\frac{k}{n}, \frac{l}{n}\right)\right) \phi\left(\frac{l}{n}\right) \dot{W}\left(\left[\frac{k}{n}, \frac{k+1}{n}\right] \times\left[\frac{l}{n}, \frac{l+1}{n}\right]\right)\right|^{2}\right]=o(1) .
\end{aligned}
$$


Use (b) and these two observations in the following:

$$
\begin{aligned}
& \mathbb{E}\left[\sup _{t \leq T} \mid\left\langle\phi, u_{t}-u_{0}\right\rangle-\int_{0}^{t} \int_{\mathbb{R}^{q}} u_{s}(x) A^{*} \phi(x)+b\left(u_{s}(x)\right) \phi(x) d x d s\right. \\
& \left.\quad-\left.\int_{0}^{t} \int_{\mathbb{R}^{q}} \phi(x) \sigma(s, x, u) W(d s d x)\right|^{2}\right] \\
& \quad \leq \mathbb{E}\left[\sup _{t \leq T}\left|\int_{\mathbb{R}^{q}} \phi(x)\left(u(t, x)-u_{0}(x)\right) d x-J_{d}-M_{d}\right|^{2}+\left|M_{d}-M_{c}\right|^{2}+\left|J_{d}-J_{c}\right|^{2}\right] \\
& \quad=o(1) \quad(n \rightarrow \infty) .
\end{aligned}
$$

This completes the proof.

Remark 7.1.2. The assumption "white noise" in time is essential for the martingale techniques to apply. The requirement having a.s. continuous solutions might be possible to be relaxed to the case of continuity in probability.

Then we can do the proof of Lemma 5.1.1.

Proof of Proposition 5.1.1. Assume that we are given a filtered probability space $\left(\Omega, \mathcal{F}, \mathcal{F}_{t}, \mathbb{P}\right)$ with adapted weak solution $u$ and noise $\dot{W}$. The latter implies $\mathcal{F}_{t}^{u, W} \subset$ $\mathcal{F}_{t}$. We want to apply Proposition $3.14 \mathrm{a}$ ) $\Rightarrow$ b) in [Kur07. Some of his notation is required here.

Let $S_{1}=\mathcal{U}$ and $S_{2} \in C\left([0, \infty), \mathcal{S}^{\prime}\left(\mathbb{R}^{q}\right)\right)$, both Polish spaces. By Definition 3.3.15 we know that $W$ takes values in $S_{2}$. First, we check that $u$ is compatible with $W$ with respect to the compatibility structure $C=\left\{\left(\mathcal{F}_{t}^{\mathcal{U}}, \mathcal{F}_{t}^{\mathcal{S}^{\prime}}\right): t \in \mathbb{R}_{+}\right\}$. Since $\mathcal{B}\left(\mathcal{S}^{\prime}\right)$ is generated by $\mathcal{S}$ it suffices to check compatibility for all $\phi \in \mathcal{S}\left(\mathbb{R}^{1+q}\right)$. Let $t>0$ fixed and define $\phi_{1}(s, x)=\mathbb{1}_{[0, t]}(s) \phi(s, x)$ and $\phi_{2}=\phi-\phi_{1}$, both in $M_{\alpha}$. So, by Lemma 3.3 .13 we can use linearity

$$
\begin{aligned}
\mathbb{E}\left[\dot{W}(\phi) \mid \mathcal{F}_{t}^{W} \vee \mathcal{F}_{t}^{u}\right] & =\mathbb{E}\left[\mathbb{E}\left[\dot{W}\left(\phi_{1}\right)+\dot{W}\left(\phi_{2}\right) \mid \mathcal{F}_{t}\right] \mid \mathcal{F}_{t}^{W} \vee \mathcal{F}_{t}^{u}\right] \\
& =\mathbb{E}\left[\left(\dot{W}\left(\phi_{1}\right)+0\right) \mid \mathcal{F}_{t}^{W} \vee \mathcal{F}_{t}^{u}\right] \\
& =\dot{W}\left(\phi_{1}\right) \\
& =\mathbb{E}\left[\dot{W}(\phi) \mid \mathcal{F}_{t}^{W}\right]
\end{aligned}
$$

since $\dot{W}$ is a (centred) noise white in time w.r.t. the filtration $\left(\mathcal{F}_{t}\right)_{t \geq 0}$. By the usual induction, this result extends to all bounded measurable functions on $\mathcal{S}^{\prime}\left(\mathbb{R}^{1+q}\right)$. So $u$ is compatible to $\dot{W}$.

By construction, $\nu=\mathcal{L}[\dot{W}]$ on $C\left(\mathbb{R}_{+} \times H^{-q}\left(\mathbb{R}^{q}\right)\right.$. Finally, the set of convex restrictions $\Gamma$ needs to be given. Let $\left\{\phi^{m} \in \mathcal{D}\left(\mathbb{R}^{q}\right): m \in \mathbb{N}\right\}$ be a dense subset of 
$\mathcal{D}\left(\mathbb{R}^{q}\right)$ (separable!). Let for $m, n \in \mathbb{N}$ :

$$
\begin{aligned}
h_{m, n, t}(u, \dot{W})= & \mid\left\langle u_{t}-u_{0}, \phi^{m}\right\rangle-\sum_{k=1}^{\lfloor n t\rfloor} \sum_{l \in \mathbb{Z}^{q}} n^{-(q+1)} u\left(\frac{k}{n}, \frac{l}{n}\right) A^{*} \phi^{m}\left(\frac{l}{n}\right)+b\left(u\left(\frac{k}{n}, \frac{l}{n}\right)\right) \phi^{m}\left(\frac{l}{n}\right) \\
& +\sum_{k=1}^{\lfloor n t\rfloor} \sum_{l \in \mathbb{Z}^{q}} \sigma\left(\frac{k}{n}, \frac{l}{n}, u\left(\frac{k}{n}, \frac{l}{n}\right)\right) \phi^{m}\left(\frac{l}{n}\right) \dot{W}\left(\left[\frac{k}{n}, \frac{k+1}{n}\right] \times\left[\frac{l}{n}, \frac{l+\mathbb{1}}{n}\right]\right) \mid \wedge 1
\end{aligned}
$$

and

$$
\begin{aligned}
g_{n}(u)=\sum_{k=1}^{\lceil n t\rceil} \frac{1}{n} \sum_{l \in \mathbb{Z} q,|l|_{\infty} \leq n}(2 n+1)^{-q} & \\
& {\left[\left|u_{k / n}\left(\frac{l}{n}\right)\right|+\sum_{j \in \mathbb{Z}^{q},|j|_{\infty} \leq n}(2 n+1)^{-q}\left|k\left(\frac{l}{n}, \frac{j}{n}\right) u_{k / n}\left(\frac{l}{n}\right) u_{k / n}\left(\frac{m}{n}\right)\right|\right] . }
\end{aligned}
$$

Clearly the conditions $\lim \sup _{n \in \mathbb{N}} \mathbb{E}\left[\sup _{t \leq T} h_{m, n, t}^{2}\right]=0$ and $g_{n}<\infty$ a.s. are convex constraints on $\mathcal{M}_{1}\left(\mathcal{U} \times \mathcal{S}^{\prime}\left(\mathbb{R}^{1+q}\right)\right)$. Let

$$
\Gamma=\left\{\limsup _{n \rightarrow \infty} g_{n}<\infty \text { a.s., } \sup _{m \in \mathbb{N}} \limsup _{n \rightarrow \infty} \mathbb{E}\left[\sup _{t \leq T} h_{m, n, t}^{2}\right]=0\right\}
$$

be the convex constraints. Since there is a weak solution, by Lemma 7.1.1 the law $\mu:=\mathbb{P} \circ(u, \dot{W})^{-1}$ lies in $S_{\Gamma, C, \nu}$. By pointwise uniqueness (which is just pathwise uniqueness, where joint compatibitility follows as in the compatibility proof before) we know that there exists a strong compatible solution, i.e. $u=F(\dot{W})$. 


\section{Existence and regularity}

In this chapter we want to prove the existence and regularity result Theorem 5.2.1. Right after the theorem there was an overview of the proof. However, we quickly repeat the important steps in a more technical description.

The proof will follow closely the one given in the appendix of [MPS06] and is decomposed into several lemmas. First, we quote the classical existence result for Lipschitz coefficients. Then we derive uniform regularity results for an approximating sequence of coefficients $\sigma^{n} \rightarrow \sigma$ for general $\sigma$. This regularity allows to get a certain tightness result; one of the limit points is then shown to be a mild solution. The proofs will be less explicit in the first steps, but more explicit in the last step, the "convergence" argument from Theorem 2.6 of [Shi94].

\subsection{Regularity results for solutions}

First, write down the analogues of Theorem A.1, Lemma A.3, Lemma A.5 and Lemma A.4 from [MPS06] (we changed the order). The modifications to include the inhomogeneity $b$ into the proof are given in the proof sketches. The first theorem is taken from Theorem 13 of [Dal99] and was written here in Theorem 4.2.1;

Theorem 8.1.1 (Theorem A.1). Let $q \in \mathbb{N}$ and $u_{0}$ be measurable and bounded. Assume that $b, \sigma: \mathbb{R}_{+} \times \mathbb{R}^{q} \times \mathbb{R} \rightarrow \mathbb{R}$ satisfy: For any $T>0, n \in \mathbb{N}$ there is a constant $c<\infty$ such that

$$
|b(t, x, u)-b(t, x, v)|+|\sigma(t, x, u)-\sigma(t, x, v)| \leq c|u-v| \quad \forall 0 \leq t, x \in \mathbb{R}^{q} .
$$

Assume that the bound (5.5) on the kernel $k$ holds with $\alpha \in(0,2 \wedge q)$. Then, there exists a pathwise unique strong solution $u$ to (5.1). The process $u$ satisfies a uniform moment bound: For any $T>0$, and $p \in[1, \infty)$,

$$
\sup _{0 \leq t \leq T} \sup _{x \in \mathbb{R}^{q}} \mathbb{E}\left[|u(t, x)|^{p}\right]<\infty .
$$

Dalang's theorem requires spatially homogeneous noise. However his proof carries over if we require $k$ to be bounded such as in (5.5) as remarked in [MPS06].

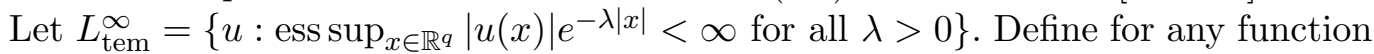
$v: \mathbb{R}_{+} \times \mathbb{R}^{q} \rightarrow \mathbb{R}, \lambda, p>0$ and a stopping time $\tau$ :

$$
G_{\lambda, p}^{\tau} v(t, x):=\mathbb{E}\left[|v(t, x)|^{p} \mathbb{1}(t \leq \tau) e^{-\lambda|x|}\right]
$$


and write $G_{\lambda, p}=G_{\lambda, p}^{\infty}$ if $\tau=\infty$.

From now on drop the assumption of $\sigma$ being Lipschitz. The function $b$, however, will still be Lipschitz. Even in that more general setup it is possible to deduce several properties of solutions (assuming their existence).

Lemma 8.1.2 (Lemma A.3). Let $u_{0} \in L_{\text {tem }}^{\infty}$ and let $\sigma, b$ be as in Theorem 5.2.1. If $u$ is any solution to (5.1) such that

$$
\sup _{0 \leq t \leq T} \sup _{x \in \mathbb{R}^{q}} \mathbb{E}\left(|u(t, x)|^{p} e^{-\lambda|x|}\right)<\infty
$$

for some $T>0, p>0, \lambda>0$, then there exists $\tilde{p} \geq p$ such that

$$
\begin{aligned}
\mathbb{E}\left(\sup _{0 \leq t \leq T} \sup _{x \in \mathbb{Q}^{q}}|u(t, x)|^{p} e^{-\lambda|x|}\right) & \leq C_{T, \lambda, p}\left(c,\left\|u_{0}\right\|_{\lambda / p, \infty}\right) \\
& \times\left(1+\sup _{0 \leq t \leq T} \sup _{x \in \mathbb{R}^{q}} G_{\lambda / 2, \tilde{p}} u(t, x)\right),
\end{aligned}
$$

where $C_{T, \lambda, p}(\cdot, \cdot)$ is bounded on the compacts of $\mathbb{R}_{+}^{2}$.

Proof. First assume that $p \geq 1+q$ and directly start with equation (119) in [MPS06]. The constant $C$ depends on $p$ only.

$$
\begin{aligned}
& \mathbb{E}\left(\sup _{0 \leq t \leq T} \sup _{x \in \mathbb{Q}^{q}}|u(t, x)|^{p} e^{-\lambda|x|}\right) \\
& \leq C \mathbb{E}\left(\sup _{0 \leq t \leq T} \sup _{x \in \mathbb{R}^{q}}\left|\int_{\mathbb{R}^{q}} p_{t}(x-y) u_{0}(y) d y\right|^{p} e^{-\lambda|x|}\right) \\
&+C \mathbb{E}\left(\sup _{0 \leq t \leq T} \sup _{x \in \mathbb{Q}^{q}}\left|\int_{0}^{t} \int_{\mathbb{R}^{q}} p_{t-s}(x-y) \sigma(s, x, u(s, y)) W(d s d y)\right|^{p} e^{-\lambda|x|}\right) \\
&+C \mathbb{E}\left(\sup _{0 \leq t \leq T} \sup _{x \in \mathbb{R}^{q}}\left|\int_{0}^{t} \int_{\mathbb{R}^{q}} p_{t-s}(x-y) b(s, y, u(s, y)) d y d s\right|^{p} e^{-\lambda|x|}\right) .
\end{aligned}
$$

Only the last term will get attention here as the others can be treated with the methods of [MPS06]. Using the growth bound on $b$ and Hölder-inequality with $a=1+q^{-1}$.

$$
\begin{aligned}
& \int_{\mathbb{R}^{q}} p_{t-s}(x-y)|b(s, y, u(s, y))| d y \leq \\
& \quad \leq q 5.2\left(\int_{\mathbb{R}^{q}}\left(p_{t-s}(x-y) e^{\lambda|y|}\right)^{a} d y\right)^{1 / a}\left(\int_{\mathbb{R}^{q}}\left((1+|u(s, y)|) e^{-\lambda|y|}\right)^{q+1} d y\right)^{1 /(1+q)} \\
& \quad \leq c(t-s)^{-1 / 2} e^{\frac{\lambda}{p}|x|}\left(\int_{\mathbb{R}^{q}}\left(1+|u(s, y)|^{q+1}\right) e^{-\frac{\lambda}{p}|y|} e^{-\frac{\lambda}{p} q|y|} d y\right)^{1 /(1+q)} .
\end{aligned}
$$


We used Lemma 6.2 of [Shi94]. Since $p \geq 1+q \geq 2$, obtain by using Hölderinequalities,

$$
\begin{aligned}
\mathbb{E}\left(\sup _{0 \leq t \leq T} \sup _{x \in \mathbb{R}^{q}}\left|\int_{0}^{t} \int_{\mathbb{R}^{q}} p_{t-s}(x-y) b(s, y, u(s, y)) d y\right|^{p} e^{-\lambda|x|}\right) \\
\leq c \mathbb{E}\left[\left(\int_{0}^{T} d s(T-s)^{-1 / 2}\left(\int_{\mathbb{R}^{q}}(1+|u(s, y)|)^{q+1} e^{-\frac{\lambda}{p}|y|} e^{-\frac{\lambda}{p} q|y|} d y\right)^{1 /(1+q)}\right)^{p}\right] \\
\leq c \mathbb{E}\left[\left(\int_{0}^{T} d s(T-s)^{-p /(2(p-1))}\right)^{p-1}\left(\int_{0}^{T} d s\left(\int_{\mathbb{R}^{q}} \ldots d y\right)^{\frac{p}{1+q}}\right)^{p / p}\right] \\
\leq c \int_{0}^{T} d s \mathbb{E}\left[\left(\int_{\mathbb{R}^{q}} d y e^{-\lambda|y| /(p-1-q)}\right)^{p-1-q / p}\left(\int_{\mathbb{R}^{q}} d y(1+|u(s, y)|)^{p} e^{-\lambda \frac{q}{1+q}|y|}\right)^{1}\right] \\
\leq c T \sup _{0 \leq s \leq T} \int_{\mathbb{R}^{q}} d y \mathbb{E}\left[(1+|u(s, y)|)^{p} e^{-\lambda \frac{q}{q+1}|y|}\right] \\
\leq c\left(1+\sup _{0 \leq s \leq T} \sup _{y \in \mathbb{R}^{q}} G_{\tilde{\lambda}, p} u(s, y)\right),
\end{aligned}
$$

where $\tilde{\lambda}=\lambda \frac{q}{q+1} \geq \frac{\lambda}{2}$.

If $p \leq 1+q$ set $\tilde{p}=1+q$ and after using the estimate $u^{p} \leq c(p)\left(1+u^{\tilde{p}}\right)$ we can apply the calculations from above.

One can improve that result to

Lemma 8.1.3 (Lemma A.5). Let $u_{0} \in C_{\text {tem }}$ and the conditions of Lemma 8.1.2 be satisfied. If $u \in C\left(\mathbb{R}_{+}, C_{\text {tem }}\right)$ a solution to (5.1), then it satisfies the following moment bound:

For any $T>0$ and $p \geq 1$,

$$
\sup _{0 \leq t \leq T} \sup _{x \in \mathbb{R}^{q}} \mathbb{E}\left[|u(t, x)|^{p} e^{-\lambda|x|}\right] \leq C_{T, \lambda, p}\left(q_{5.2},\|u\|_{\lambda / p, \infty}\right),
$$

where $C_{T, \lambda, p}(\cdot, \cdot)$ is bounded on the compacts of $\mathbb{R}_{+}^{2}$.

The proof of this result is just an application of Gronwall's lemma to a similar statement as that of Lemma 8.1.2 (leave the time-integral unevaluated) and we refer to Lemma A.5 of [MPS06] for the details. Finally, there is a lemma about the regularity: 
Lemma 8.1.4 (Lemma A.4). Let $u$ be a solution to (5.1) satisfying the conditions of the previous lemma. Define

$$
\begin{aligned}
Z(t, x)=\int_{0}^{t} & \int_{\mathbb{R}^{q}} p_{t-s}(x-y) \sigma(s, y, u(s, y)) W(d s d y) \\
& +\int_{0}^{t} \int_{\mathbb{R}^{q}} p_{t-s}(x-y) b(s, y, u(s, y)) d y d s
\end{aligned}
$$

for $t \geq 0, x \in \mathbb{R}^{q}$. Then, for $T, R>0$ and $0 \leq t \leq t^{\prime} \leq T, x, x^{\prime} \in \mathbb{R}^{q}$ such that $\left|x-x^{\prime}\right| \leq R$, as well as $p \in[2, \infty)$ and $\xi \in(0,1-\alpha / 2)$,

$$
\begin{aligned}
& \mathbb{E}\left[\left|Z(t, x)-Z\left(t^{\prime}, x^{\prime}\right)\right|^{p} e^{-\lambda|x|}\right] \\
& \leq C(T, \lambda, p)\left(1+\sup _{0 \leq t \leq T} \sup _{z \in \mathbb{R}^{q}} G_{\lambda /(p+1), p} u(s, z)\right) \\
& \quad \times\left(\left|t-t^{\prime}\right|^{\xi p / 2}+\left|x-x^{\prime}\right|^{\xi p}\right) .
\end{aligned}
$$

Proof. The difficult part of the proof can be found in [MPS06]. We content ourselves here with the statement that Lemma 8.1.2 and Lemma 5.2 (a) of [MPS06], combined with the growth bound on $b$ give sufficient estimates for

$$
Y(t, x)=\int_{0}^{t} \int_{\mathbb{R}^{q}} p_{t-s}(x-y) b(s, y, u(s, y)) d y d s .
$$

One could remark that as there is a Riemann integral it is not surprising to have very high Hölder-regularity, even better than the one proposed in the lemma for this integral.

\subsection{Tightness and construction of the solution}

We give the proof of Theorem 5.2.1 and decompose it into several steps. First we define a family of approximate mild solutions to (5.1), show that it has a limit point (at least one) and then show that any limit point is a mild solution. Finally, the specific filtration chosen is considered.

Step 1: Family of approximate solutions

Let $\left(\sigma^{n}\right)_{n}$ be a sequence of Lipschitz-continuous functions with

- $\left|\sigma^{n}(u)\right| \leq q 5.2(1+|u|)$ and

- $\sigma^{n} \rightarrow \sigma$ uniformly on compacta. 
An example of such a sequence is constructed in [MP11] (proof of Theorem 1.1). Furthermore define for any $x \in \mathbb{R}^{q}$ :

$$
u_{0}^{m}(x):= \begin{cases}-m & \text { if } u_{0}(x)<-m \\ u_{0}(x) & \text { if }\left|u_{0}(x)\right|<m \\ m & \text { if } u_{0}(x)>m\end{cases}
$$

Then $u_{0}^{m} \in C_{b}\left(\mathbb{R}^{d}\right)$ and

$$
\sup _{m} \sup _{x \in \mathbb{R}^{d}}\left|u_{0}^{m}(x)\right| e^{-\lambda|x|} \leq \sup _{m} \sup _{x \in \mathbb{R}^{d}}\left|u_{0}(x)\right| e^{-\lambda|x|}<\infty
$$

since $u_{0} \in C_{\text {tem }}$. If we fix $m$ and $n$ then we can use Theorem 8.1.1 and obtain unique solutions $u^{m, n}$, i.e. for given $t \geq 0$ it almost surely holds, that for any $x \in \mathbb{R}^{q}$

$$
u^{m, n}(t, x)=\int_{\mathbb{R}^{d}} p_{t}(x-y) u_{0}^{m}(y) d y+\int_{0}^{t} \int_{\mathbb{R}^{d}} p_{t-s}(x-y) \sigma^{n}\left(u^{m, n}(s, y)\right) W(d s d y) .
$$

Step 2: Tightness of $u^{m, n}$

Tightness in $C\left(\mathbb{R}_{+}, C_{\text {tem }}\right)$ can be shown using Lemma 6.3 (ii) of [Shi94]. The estimates obtained in Lemma 8.1.4 do not depend on the specific value of the Lipschitzconstant for $\sigma^{n}$ only on the constant of the growth bound.

\section{Step 3: The limit points are mild solutions}

By Skorohod's representation theorem (cf. Theorem 3.1.8 of [EK86]) extract an a.s. converging subsequence on a joint probability space. Let $u$ be an almost sure limit point of $u^{m, n}$ on $(\Omega, \mathcal{F}, \mathbb{P})$, where $\mathcal{F}$ is assumed to be $\mathbb{P}$-complete. That means there is a sequence $(n(l), m(l))_{l}$ s.t. both $m(l)$ and $n(l)$ tend to infinity as $l \rightarrow \infty$ and

$$
u^{m(l), n(l)} \rightarrow u \text { in } C\left(\mathbb{R}_{+}, C_{\text {tem }}\right) \text { almost sure. }
$$

In order to simplify notation we will write $u^{l}:=u^{m(l), n(l)}$.

Lemma 8.2.1. Then $u$ is a mild solution of the limiting equation:

$$
\begin{aligned}
u(t, x)=\int_{\mathbb{R}^{d}} p_{t}(x-y) u_{0}(y) d y & +\int_{0}^{t} \int_{\mathbb{R}^{q}} p_{t-s}(x-y) b(s, y, u(s, y)) d y d s \\
& +\int_{0}^{t} \int_{\mathbb{R}^{d}} p_{t-s}(x-y) \sigma(s, y, u(s, y)) W(d s d y)
\end{aligned}
$$


for any $x \in \mathbb{R}^{q}$ almost surely for all $t>0$. Additionally $\dot{W}$ is a noise, white in time w.r.t. $\mathcal{F}_{t}$, where

$$
\mathcal{F}_{t}=\bigvee_{n, m} \mathcal{F}_{t}^{n, m}, \mathcal{F}_{t}^{n, m}=\sigma\left(u_{s}^{m, n}, W_{s}, s \leq t^{+}\right)^{\text {complete }} .
$$

The superscript stands for completion with respect to $\mathbb{P}$.

The last point is shown in Step 4. Before giving a proof we state some lemmas.

Lemma 8.2.2. For any $\lambda>0, T>0$ and solutions $u^{l}, u$ to the SPDE with diffusion coefficient $\sigma^{l}, \sigma$, respectively, the following holds

(a) $\sup _{l \geq 1} \sup _{t \leq T} \sup _{x \in \mathbb{R}^{d}} e^{-\lambda|x|} \mathbb{E}\left[\left|u^{l}(t, x)\right|^{k}\right]<c(\lambda, T)<\infty, k \in \mathbb{N}$

(b) $\sup _{t \leq T} \sup _{x \in \mathbb{R}^{d}} e^{-\lambda|x|} \mathbb{E}\left[|u(t, x)|^{k}\right]<\infty, k \in \mathbb{N}$

(c) The family $\left(e^{-\lambda|x|}\left|u^{l}(t, x)\right|^{k}\right)_{t \leq T, x \in \mathbb{R}^{d}, l \in \mathbb{N}}$ is uniformly integrable for any $k \in \mathbb{N}$.

Proof. The first line (a) follows by Lemma 8.1.3, since we never used the Lipschitzcoefficient in the proof (consider line (131) in [MPS06] for the explicit statement). The proof of (b) is taken from Lemma 6.19 of [Zäh04]:

$$
\begin{aligned}
\sup _{t \leq T} \sup _{x \in \mathbb{R}^{d}} e^{-\lambda|x|} \mathbb{E}[u(t, x)] & \left.=\sup _{t \leq T} \sup _{x \in \mathbb{R}^{d}} e^{-\lambda|x|} \mathbb{E}\left[\lim _{N \rightarrow \infty} N \wedge|u(t, x)|\right)\right] \\
& \leq \sup _{t \leq T} \sup _{x \in \mathbb{R}^{d}} e^{-\lambda|x|} \liminf _{N \rightarrow \infty} \mathbb{E}[N \wedge|u(t, x)|] \\
& \leq \sup _{t \leq T} \sup _{x \in \mathbb{R}^{d}} e^{-\lambda|x|} \liminf _{N \rightarrow \infty} \lim _{l \rightarrow \infty} \mathbb{E}\left[N \wedge\left|u^{l}(t, x)\right|\right] \\
& \leq \sup _{t \leq T} \sup _{x \in \mathbb{R}^{d}} \sup _{l \geq 1} e^{-\lambda|x|} \mathbb{E}\left[\left|u^{l}(t, x)\right|\right] \\
& \leq \sup _{l \geq 1}\left\|\mathbb{E}\left[\left|u^{l}(\cdot, \cdot)\right|\right]\right\|_{\lambda, \infty, T} \\
& <c(\lambda, T) .
\end{aligned}
$$

The last assertion (c) follows by the uniform bound on higher moments of order $2 k$ and using (a), when replacing $\lambda$ by $\lambda / 2$ there.

Define the cut-off functions $F_{N}: \mathbb{R} \rightarrow \mathbb{R}$ for any natural number $N \in \mathbb{N}$ :

$$
F_{N}(x)=-N, x \leq-N ; F_{N}(x)=x,-N<x<N ; F_{N}(x)=N, x \geq N .
$$

Then 


\section{Lemma 8.2.3.}

$$
\sup _{l \geq 1} \mathbb{E}\left[\left|\int_{0}^{t} \int_{\mathbb{R}^{d}} p_{t-s}(x-y)\left(\sigma^{n(l)}\left(F_{N} u^{l}(s, y)\right)-\sigma^{n(l)}\left(u^{l}(s, y)\right)\right) W(d s d y)\right|^{2}\right] \rightarrow 0
$$

for $N \rightarrow \infty$. The same holds true for $\sigma$ and $u$ without the superscripts.

Proof. Write $A^{N}$ for the expression inside the modulus in the lemma.

$$
\begin{aligned}
& \mathbb{E}\left[\left|A^{N}\right|^{2}\right] \leq \mathbb{E}\left[\int_{0}^{t} \int_{\mathbb{R}^{2 d}}\left|p_{t-s}(x-y) p_{t-s}(x-z)\right|\left(\sigma^{n(l)}\left(F_{N} u^{l}(s, y)\right)-\sigma^{n(l)}\left(u^{l}(s, y)\right)\right)\right. \\
& \quad \times\left(\sigma^{n(l)}\left(F_{N} u^{l}(s, z)\right)-\sigma^{n(l)}\left(u^{l}(s, z)\right) \mid k(y, z) d y d z d s\right] \\
& \leq q_{5.2}^{2} \int_{0}^{t} \int_{\mathbb{R}^{2 d}}\left|p_{t-s}(x-y) p_{t-s}(x-z)\right| e^{\lambda(|z|+|y|)} k(y, z) \\
& \quad \times\left(1+\mathbb{E}\left[\left|u^{l}(s, y)\right|\right] e^{-\lambda|y|}\right) \mathbb{1}_{\left|u^{l}(s, y)\right|>N}\left(1+\mathbb{E}\left[\left|u^{l}(s, z)\right|\right] e^{-\lambda|z|}\right) \mathbb{1}_{\left|u^{l}(s, z)\right|>N} d y d z d s \\
& \leq q_{5.2}^{2} \int_{0}^{t} \int_{\mathbb{R}^{2 d}}\left|p_{t-s}(x-y) p_{t-s}(x-z)\right| e^{\lambda(|z|+|y|)} k(y, z)\left(\left(1+\mathbb{E}\left[\left|u^{l}(s, y)\right|\right] e^{-\lambda|y|}\right)^{2}\right. \\
& \left.\quad \times \mathbb{1}_{\left|u^{l}(s, y)\right|>N}+\left(1+\mathbb{E}\left[\left|u^{l}(s, z)\right|\right] e^{-\lambda|z|}\right)^{2} \mathbb{1}_{\left|u^{l}(s, y)\right|>N}\right) d y d z d s
\end{aligned}
$$

By Lemma 5.1 in MPS06] it is true that

$$
\int_{0}^{t} \int_{\mathbb{R}^{2 d}}\left|p_{t-s}(x-y) p_{t-s}(x-z)\right| e^{\lambda(|z|+|y|)} k(y, z) d y d z d s
$$

constitutes a finite measure on $\mathbb{R}_{+} \times \mathbb{R}^{2 q}$. Using Lemma 8.2 .2 we also know that

$$
\sup _{l} \mathbb{1}\left\{u^{l}(s, y)>N\right\} \rightarrow 0 \text { a.s. as } N \rightarrow \infty \text {. }
$$

Hence, we can use a standard argument for uniformly integrable random variables (e.g. Kle08, Thm 6.24(iii)]) and see that

$$
\mathbb{E}\left[\left|A^{N}\right|^{2}\right] \rightarrow 0 \text { as } N \rightarrow \infty .
$$

The fact that the same holds true in the case without the superscripts $l$ can be shown just the same way.

Now we try to put things together to show that $u$ is a mild solution of the SPDE. We need to show (8.6). We do that by showing that the second moment 
of the differences from left and right hand side is zero. Using that $u^{m(l)}$ are mild solutions, consider

$$
\begin{aligned}
& \mathbb{E}\left[\left(u(t, x)-\int_{\mathbb{R}^{d}} p_{t}(x-y) u_{0}(y) d y-\int_{0}^{t} \int_{\mathbb{R}^{d}} p_{t-s}(x-y) \sigma(u(s, y)) W(d y d s)\right.\right. \\
& \left.\left.\quad-\int_{0}^{t} \int_{\mathbb{R}^{q}} p_{t-s}(x-y) b(s, y, u(s, y)) d y d s\right)^{2}\right] \\
& \leq \mathbb{E}\left[\left|u(t, x)-u^{m(l)}(t, x)\right|^{2}\right] \\
& +\mathbb{E}\left[\left|\int_{\mathbb{R}^{d}} p_{t}(x-y) u_{0}(y) d y-\int_{\mathbb{R}^{d}} p_{t}(x-y) u_{0}^{m(l)}(y) d y\right|^{2}\right] \\
& +\mathbb{E}\left[\left|\int_{0}^{t} \int_{\mathbb{R}^{d}} p_{t-s}(x-y)\left(\sigma^{n(l)}\left(u^{m(l)}(s, y)\right)-\sigma^{n(l)}\left(F_{N} u^{m(l)}(s, y)\right)\right) W(d s d y)\right|^{2}\right] \\
& \quad+\mathbb{E}\left[\left|\int_{0}^{t} \int_{\mathbb{R}^{d}} p_{t-s}(x-y)\left(\sigma^{n(l)}\left(F_{N} u^{m(l)}(s, y)\right)-\sigma\left(F_{N} u^{m(l)}(s, y)\right)\right) W(d s d y)\right|^{2}\right] \\
& +\mathbb{E}\left[\left|\int_{0}^{t} \int_{\mathbb{R}^{d}} p_{t-s}(x-y)\left(\sigma\left(F_{N} u^{m(l)}(s, y)\right)-\sigma\left(F_{N} u(s, y)\right)\right) W(d s d y)\right|^{2}\right] \\
& \quad+\mathbb{E}\left[\left|\int_{0}^{t} \int_{\mathbb{R}^{d}} p_{t-s}(x-y)\left(\sigma\left(F_{N} u(s, y)\right)-\sigma(u(s, y))\right) W(d s d y)\right|^{2}\right] \\
& \quad+\mathbb{E}\left[\left|\int_{0}^{t} \int_{\mathbb{R}^{q}} p_{t-s}(x-y)\left(b(s, y, u(s, y))-b\left(s, y, u^{m(l)}(s, y)\right)\right) d y d s\right|^{2}\right] \\
& =I_{1}+I_{2}+I_{3}+I_{4}+I_{5}+I_{6}+I_{7},
\end{aligned}
$$

where $F_{N}$ was the cut-off function defined above. If for $l \rightarrow \infty$ all expressions vanish, we know that $u$ is a mild solution. This looks complicated, but reduces to two problems since

- $I_{1}$ goes to zero since $\left(\left(u^{m}(t, x)\right)^{2}\right)_{m}$ is a uniformly integrable family (Lemma 8.2.2 and combined with its almost sure convergence we have $L^{2}$-convergence $($ as $l \rightarrow \infty)$.

- $I_{2}$ goes to zero by an estimate on $\int_{|y| \geq m(l)} p_{t}(x-y) e^{\lambda|y|} d y($ as $l \rightarrow \infty)$.

- $I_{3}$ and $I_{6}$ go to zero by Lemma 8.2 .3 (as $N \rightarrow \infty$ ).

- $I_{7}$ vanishes in the limit, since $\int_{0}^{t} d s \int_{\mathbb{R}^{q}} d y p_{t-s}^{2}(\cdot-y)$ is a finite measure on $\mathbb{R}_{+} \times \mathbb{R}^{q}$ and $\left(e^{-|\cdot|} u^{m}\right)_{m}^{2}$ is uniformly integrable by Lemma 8.2 .2 .

Therefore given an $\epsilon>0$ we can bound each of the previous $I_{i}$ by $\frac{\epsilon}{7}$ when choosing $l$ sufficiently large, say $l \geq L_{1}$ and $N$ sufficiently large, say $N \geq N_{1}$ (keep that $N$ 
fixed). We have to deal with $I_{4}$ and $I_{5}$.

Before going ahead we define two integrals

$$
\begin{aligned}
M_{1}(t, x) & :=\int_{0}^{t} d s \int_{\mathbb{R}^{2 d}} d x d y p_{t-s}(x-y) p_{t-s}(x-z) k(z, y), \\
M_{2}(k, t, x) & :=\int_{0}^{t} d s \int_{y \in \mathbb{R}^{d}} d y \int_{|z|>k} d z p_{t-s}(x-y) p_{t-s}(x-z) k(z, y) .
\end{aligned}
$$

To obtain a bound on $I_{4}$ choose $\epsilon_{1}<\sqrt{\frac{\epsilon}{7\left(M_{1}(t, x)\right)}}$ and $L_{2}$ so large that (for the fixed $N)$

$$
\sup _{|u|<N}\left|\sigma(u)-\sigma^{n(l)}(u)\right|<\epsilon_{1} \quad \forall l \geq L_{2} .
$$

Then we get that

$$
I_{4} \leq \epsilon_{1}^{2} \int_{0}^{t} \int_{\mathbb{R}^{2 d}} p_{t-s}(x-y) p_{t-s}(x-z) k(z, y) d y d z d s<\frac{\epsilon}{7} \quad \forall l \geq L_{2} .
$$

So it remains to work with $I_{5}$. First we take a $K_{1}$ so large that

$$
q_{5.2}^{2}(1+N)^{2} M_{2}(k, t, x) \leq \frac{\epsilon}{18} \quad \forall k \geq K_{1} .
$$

The trick somehow needs to be that we have some information about the closeness of $\sigma\left(u^{m(l)}\right)$ and $\sigma(u)$. As $\sigma$ is a continuous function it is uniformly continuous on the compactum $[-N, N]$ and for any $\epsilon_{4}>0$ we can find a $\delta_{4}\left(\epsilon_{4}\right)>0$ such that

$$
u, u^{\prime} \in[-N, N] \text { and }\left|u-u^{\prime}\right|<\delta_{4} \Rightarrow\left|\sigma(u)-\sigma\left(u^{\prime}\right)\right|<\epsilon_{4} .
$$

Let us take $\epsilon_{4}^{2} \in\left(0, \frac{\epsilon}{36 q_{5.2}(1+N) M_{1}}\right)$.

Hence, the only thing which is required is that we need to know about the closeness of $u$ and $u^{m(l)}$. As we have almost sure convergence in the path space with values in $C_{\text {tem }}$ we also have almost sure convergence on any compact set, e.g. $\left\{(t, x): t \leq T,|x| \leq K_{1}\right\}$, where $K_{1}$ is the constant from above. Hence, let an $\epsilon_{2} \in\left(0, \delta_{4}\left(\epsilon_{4}\right) \wedge \frac{\epsilon}{18 M_{1} q_{[.2]}^{2}(1+N)^{2}}\right)$ be given. Choose for given $\lambda>0$ an $L_{3}\left(\epsilon_{2}, \lambda\right)$ so large that

$$
\mathbb{P}\left[\left\|u-u^{m(l)}\right\|_{\lambda, \infty, T}<\epsilon_{2}\right]>1-\epsilon_{2} \quad \forall l \geq L_{3} .
$$

We can take $\lambda=K_{1}^{-1}$. Then we get

$$
\mathbb{P}\left[\sup _{s \leq T} \sup _{|y|<K_{1}}\left|F_{N} u(s, y)-F_{N} u^{l}(s, y)\right|<\epsilon_{2} e^{1}\right]>1-\epsilon_{2} \quad \forall l \geq L_{3} .
$$


Then we we can start putting the things together

$$
\begin{aligned}
\mathbb{E} & {\left[\left|\int_{0}^{t} \int_{\mathbb{R}^{d}} p_{t-s}(x-y)\left(\sigma\left(F_{N} u^{m(l)}(s, y)\right)-\sigma\left(F_{N} u(s, y)\right)\right) W(d s d y)\right|^{2}\right] } \\
\leq & \mathbb{E}\left[\left|\int_{0}^{t} \int_{\mathbb{R}^{2 d}} p_{t-s}(x-y) p_{t-s}(x-z)\right| \sigma\left(F_{N} u^{m(l)}(s, y)\right)-\sigma\left(F_{N} u(s, y)\right) \mid\right. \\
& \left.\left.\times\left|\sigma\left(F_{N} u^{m(l)}(s, z)\right)-\sigma\left(F_{N} u(s, z)\right)\right| k(z, y) d y d z d s\right)\right] \\
\leq & \mathbb{E} \int_{0}^{t} d s \int_{y \in \mathbb{R}^{d}} \int_{|z|<K_{1}} \cdots+\int_{0}^{t} d s \int_{|y|<K_{1}} \int_{z \in \mathbb{R}^{d}} \cdots+\int_{0}^{t} d s \int_{|y|>K_{1}} \int_{|z|>K_{1}} \cdots
\end{aligned}
$$

The first two summands are similar and we will only consider the first one:

$$
\begin{aligned}
& \mathbb{E}\left[\left|\int_{0}^{t} \int_{y \in \mathbb{R}^{d}} \int_{|z|<K_{1}} p_{t-s}(x-y) p_{t-s}(x-z)\right| \sigma\left(F_{N} u^{m(l)}(s, y)\right)-\sigma\left(F_{N} u(s, y)\right) \mid\right. \\
& \left.\left.\quad \times\left|\sigma\left(F_{N} u^{m(l)}(s, z)\right)-\sigma\left(F_{N} u(s, z)\right)\right| k(z, y) d y d z d s\right)\right] \\
& \leq \mathbb{E}\left[\left|\int_{0}^{t} \int_{y \in \mathbb{R}^{d}} \int_{|z|<K_{1}} p_{t-s}(x-y) p_{t-s}(x-z) k(z, y)\right| \sigma\left(F_{N} u^{m(l)}(s, y)\right)-\sigma\left(F_{N} u(s, y)\right) \mid\right. \\
& \left.\left.\times\left|\sigma\left(F_{N} u^{m(l)}(s, z)\right)-\sigma\left(F_{N} u(s, z)\right)\right| \mathbb{1}_{\left\{\sup _{s \leq T} \sup _{|y|<K_{1}}\left|F_{N} u(s, y)-F_{N} u^{l}(s, y)\right|<\epsilon_{2}\right\}} d y d z d s\right)\right] \\
& +\mathbb{E}\left[\left|\int_{0}^{t} \int_{y \in \mathbb{R}^{d}} \int_{|z|<K_{1}} p_{t-s}(x-y) p_{t-s}(x-z) k(z, y)\right| \sigma\left(F_{N} u^{m(l)}(s, y)\right)-\sigma\left(F_{N} u(s, y)\right) \mid\right. \\
& \left.\left.\times\left|\sigma\left(F_{N} u^{m(l)}(s, z)\right)-\sigma\left(F_{N} u(s, z)\right)\right| \mathbb{1}_{\left\{\sup _{s \leq T} \sup _{|y|<K_{1}}\left|F_{N} u(s, y)-F_{N} u^{l}(s, y)\right| \geq \epsilon_{2}\right\}} d y d z d s\right)\right],
\end{aligned}
$$

now use 8.18 and 8.15 to bound that by

$$
\begin{aligned}
& \leq \epsilon_{4}^{2} q_{5.2}(1+N) M_{1}(t, x)+\epsilon_{2} M_{1}(t, x) q_{5.2}^{2}(1+N)^{2} \\
& <\frac{\epsilon}{9} \quad \forall l \geq L_{3} .
\end{aligned}
$$

So, we are left to deal with the last integral in 8.19 :

$$
\begin{aligned}
\mathbb{E}\left[\mid \int_{0}^{t}\right. & \int_{|y|>K_{1}} \int_{|z|>K_{1}} p_{t-s}(x-y) p_{t-s}(x-z)\left|\sigma\left(F_{N} u^{m(l)}(s, y)\right)-\sigma\left(F_{N} u(s, y)\right)\right| \\
& \left.\left.\times\left|\sigma\left(F_{N} u^{m(l)}(s, z)\right)-\sigma\left(F_{N} u(s, z)\right)\right| d y d z d s\right)\right] \\
\leq & q_{5.2}^{2}(1+N)^{2} M_{2}\left(K_{1}, t, x\right)<\frac{\epsilon}{18} .
\end{aligned}
$$

where again we used 8.15). Hence, the sum of the three integrals in 8.19 is at most $\epsilon / 6$. 
Therefore we have shown that for the given $\epsilon$ we could choose (in a cascade of many $\epsilon_{i}$ ) an $l \geq L_{1} \vee L_{2} \vee L_{3}$ such that the expression in (8.10) is bounded by $\epsilon$ and we see that $u$ is a mild solution to the SPDE.

\section{Step 4: $u$ is adapted}

It remains to show that $u$ is adapted to the filtration chosen. We provide the following proposition:

Proposition 8.2.4. Let $X^{n}, W, X$ be stochastic processes with values in a Polish space $(E, \mathcal{E})$, s.t. $\mathcal{F}_{t}^{n}=\bigcap_{s>t} \sigma\left(W_{r}, X_{r}^{n}: r \leq s\right)$ is the right-continuous filtration of the pair $\left(X^{n}, W\right), n \in \mathbb{N}, t \geq 0$. Assume $\left(X^{n}, W\right) \rightarrow(X, W)$ P-almost surely on a complete probability space $(\Omega, \mathcal{F}, P)$ and $W$ is an $\mathcal{F}_{t}^{n}$-Brownian Motion for each $n \in \mathbb{N}$. Define $\mathcal{F}_{t}=\bigvee_{n} \mathcal{F}_{t}^{n} \vee \overline{\mathcal{F}}_{0}, t \geq 0$. Then,

(a) $X_{t} \in \mathcal{F}_{t}, t \geq 0$.

(b) $\left(W_{t}\right)$ is an $\mathcal{F}_{t}$-Brownian Motion, i.e. $W_{t} \in \mathcal{F}_{t}, W_{t+u}-W_{t}$ is independent of $\mathcal{F}_{t}$ (and the distribution is Gaussian).

Proof. (a) Let $A \in \mathcal{E}$. Then,

$$
\begin{aligned}
\left\{X_{t} \in A\right\} & =\left\{X_{t} \in A, \lim X^{n}=X\right\} \cup\left\{X_{t} \in A, \lim X^{n} \neq X\right\} \\
& =\left\{\lim X_{t}^{n} \in A, \lim X^{n}=X\right\} \cup C \\
& =\left(\left\{\lim X_{t}^{n} \in A\right\} \cap C^{c}\right) \cup C \\
& =\left\{\lim X_{t}^{n} \in A\right\} \cup C \in \bigvee_{n} \mathcal{F}_{t}^{n} \vee \overline{\mathcal{F}}_{0}=\mathcal{F}_{t},
\end{aligned}
$$

with obvious notation for $C$.

(b) $W_{t} \in \mathcal{F}_{t}$ by definition. And for measurable functions $f, g: E \rightarrow \mathbb{R}$.

$$
\mathbb{E}\left[f\left(W_{t+u}-W_{t}\right) g\left(X_{t}^{n}\right)\right]=\mathbb{E}\left[\mathbb{E}\left[f\left(W_{t+u}-W_{t}\right) \mid \mathcal{F}_{t}^{n}\right] g\left(X_{t}^{n}\right)\right]=0,
$$

for all $n \in \mathbb{N}, t, u \geq 0$, since we require $W$ to be a $\mathcal{F}_{t}^{n}$-Brownian Motion.

Remark 8.2.5. The proof of Proposition 8.2.4 suggests, that the limit point $X$ is adapted to $\mathcal{F}_{t}$, a filtration which can be chosen to be the one of the noise $\dot{W}$, if $X^{n}$ are strong solutions. But keep in mind, that after having used the Skorohod representation the probability space has changed. So we do not have a strong solution in general. 


\section{Pathwise Uniqueness}

The proof of Theorem 5.3.1 is inspired by the idea of Yamada-Watanabe [YW71] that was already used in [MPS06] and [MP11]. The heuristics was given right after Theorem 5.3.1. Most parts of the proof follow directly [MP11], however they are extensions to a multi-dimensional setup. One improvement to the results of [MP11] is given in Proposition 9.5.8, which also allows a better bound in the white noise case.

As the proof is extremely long one might look for a proof overview. We will give it at the end of Section 9.1. First, we will say something more about the regularity conditions on $\sigma$ stated in (5.3):

(a) When (5.2) holds, it suffices to assume (5.3) for $|u-v| \leq 1$.

(b) Condition (5.3) implies the following local Hölder condition: For all $K>0$ there is an $L_{K}$ so that for all $t \in[0, K]$ and $x \in B^{q}(0, K), u, v \in[-K, K]$,

$$
|\sigma(t, x, u)-\sigma(t, x, v)| \leq L_{K}|u-v|^{\gamma}
$$

\subsection{Proof of Theorem 5.3.1}

We closely follow Chapter 2 in MP11 as most of the ideas can be translated from white to colored noise. For notational convenience replace the name of solutions $u$ to (5.1) by $X$ (we will, analogously to [MP11 in Section 9.2 define $u_{1}$ and $u_{2}$, so we do not want to use the terms $u^{1}, u^{2}$ yet).

Now, consider Theorem 5.3.1 and assume its hypotheses throughout. Let $\left(\Omega, \mathcal{F}, \mathcal{F}_{t}, \mathbb{P}\right)$ be a filtered probability space supporting a colored noise $\dot{W}$, and $X^{1}$ and $X^{2}$, two mild solutions of (5.1) on with sample paths in $C\left(\mathbb{R}_{+}, C_{\text {tem }}\right)$ a.s., with the same initial condition, $X_{0}^{1}=X_{0}^{2}=X_{0} \in C_{\text {tem. }}$. Since $X^{i} \in C\left(\mathbb{R}_{+}, C_{\text {tem }}\right)$ for $i=1,2$, they satisfy (4.2), the weak form of (5.1): For $\Phi \in C_{c}^{\infty}(\mathbb{R})$ we have that

$$
\begin{aligned}
\int_{\mathbb{R}^{q}} X^{i}(t, x) \Phi(x) d x= & \int_{\mathbb{R}^{q}} X_{0}^{i}(x) \Phi(x) d x+\int_{0}^{t} \int_{\mathbb{R}^{q}} X^{i}(s, x) \frac{1}{2} \Delta \Phi(x) d x d s \\
& +\int_{0}^{t} \int_{\mathbb{R}^{q}} \sigma\left(s, x, X^{i}(s, x)\right) \Phi(x) W(d s d x) \\
& +\int_{0}^{t} \int_{\mathbb{R}^{q}} b\left(s, x, X^{i}(s, x)\right) \Phi(x) d x d s \quad \forall t \geq 0 \quad \text { a.s. }
\end{aligned}
$$


Remember the definition of $T_{K}$ in (5.13):

$$
T_{K}=\inf \left\{s \geq 0: \sup _{y}\left(\left|X^{1}(s, y)\right| \vee\left|X^{2}(s, y)\right|\right) e^{-|y|}>K\right\} \wedge K .
$$

We will only show Theorem 5.3 .1 for $\sigma$ such that for some $1>\gamma>\frac{1}{2}+\frac{\alpha}{4}$ there are $R_{0}, R_{1} \geq 1$ so that for all $t \geq 0$ and all $\left(x, X, X^{\prime}\right) \in \mathbb{R}^{q+2}$,

$$
\left|\sigma(t, x, X)-\sigma\left(t, x, X^{\prime}\right)\right| \leq R_{0} e^{R_{1}|x|}\left|X-X^{\prime}\right|^{\gamma} .
$$

This is sufficient since we can easily extend the result from compact time intervals to all of $\mathbb{R}_{+}$and since we will consider solutions stopped at time $T_{K}$ and $T_{K} \rightarrow \infty$ almost surely. In order apply an argument similar to that of Yamada and Watanabe, we set for any $n \in \mathbb{N}$ as in [MP11]

$$
a_{n}=\exp \{-n(n+1) / 2\}
$$

and fix a positive function $\psi_{n} \in C^{\infty}\left(\mathbb{R}, \mathbb{R}_{+}\right)$with $\operatorname{supp} \psi_{n} \subset\left(a_{n}, a_{n-1}\right), \psi_{n}(x) \leq \frac{2}{n x}$ and

$$
\int_{a_{n}}^{a_{n-1}} \psi_{n}(x) d x=1 .
$$

As this function converges to $\delta_{0} \in \mathcal{S}^{\prime}(\mathbb{R})$, we define

$$
\phi_{n}(x):=\int_{0}^{|x|} d y \int_{0}^{y} d z \psi_{n}(z), \quad x \in \mathbb{R}
$$

which then approximates the modulus. More precisely, we have

- $\phi_{n}(x) \rightarrow|x|$ uniformly in $x \in \mathbb{R}$,

- $\left|\phi_{n}^{\prime}(x)\right| \leq 1$ and

- $\left|\phi_{n}^{\prime \prime}(x)\right| \leq \frac{2}{n x}$ for all $x \neq 0$.

Recall that in the heuristics of Chapter 5 we had used a simpler function $g_{n}$ instead of $\phi_{n}$. Next, we fix a point $x \in \mathbb{R}^{q}, t_{0}>0$ and a positive function $\Phi \in C_{c}^{\infty}\left(\mathbb{R}^{q}, \mathbb{R}_{+}\right)$ such that $\operatorname{supp} \Phi \subset B^{q}(0,1)$ and $\int \Phi(y) d y=1$. Let $\Phi_{x}^{m}(y)=m^{q} \Phi(m(y-x))$ for $m>0$. Define the difference of the solutions

$$
u:=X^{1}-X^{2}
$$

and note that we can write down an equation of the form 9.2 for $u$. Let $\langle\cdot, \cdot\rangle$ denote the scalar product on $L^{2}\left(\mathbb{R}^{q}\right)$ and assume $t \in\left(0, t_{0}\right)$. We apply the Itôformula for the semimartingales $\left\langle\Phi_{x}^{m}(\cdot), u_{t}(\cdot)\right\rangle$, which is the difference of the two 
semimartingales given in $(9.2)$, and $\phi_{n}$ as in 9.5 in order to obtain

$$
\begin{aligned}
\phi_{n} & \left(\left\langle u_{t}, \Phi_{x}^{m}\right\rangle\right) \\
= & \int_{0}^{t} \int_{\mathbb{R}^{q}} \phi_{n}^{\prime}\left(\left\langle u_{s}, \Phi_{x}^{m}\right\rangle\right)\left(\sigma\left(s, y, X^{1}(s, y)\right)-\sigma\left(s, y, X^{2}(s, y)\right)\right) \Phi_{x}^{m}(y) W(d s d y) \\
& +\int_{0}^{t} \phi_{n}^{\prime}\left(\left\langle u_{s}, \Phi_{x}^{m}\right\rangle\right)\left\langle u_{s}, \frac{1}{2} \Delta \Phi_{x}^{m}\right\rangle d s \\
& +\frac{1}{2} \int_{0}^{t} d s \int_{\mathbb{R}^{q}} d w \int_{\mathbb{R}^{q}} d z \psi_{n}\left(\mid\left\langle u_{s}, \Phi_{x}^{m}\right\rangle\right) \Phi_{x}^{m}(w) \Phi_{x}^{m}(z) k(w, z) \\
& \times\left(\sigma\left(s, w, X^{1}(s, w)\right)-\sigma\left(s, w, X^{2}(s, w)\right)\right)\left(\sigma\left(s, z, X^{1}(s, z)\right)-\sigma\left(s, z, X^{2}(s, z)\right)\right) \\
& +\int_{0}^{t} \int_{\mathbb{R}^{q}} \phi_{n}^{\prime}\left(\left\langle u_{s}, \Phi_{x}^{m}\right\rangle\right)\left(b\left(s, y, X^{1}(s, y)\right)-b\left(s, y, X^{2}(s, y)\right)\right) \Phi_{x}^{m}(y) d y d s .
\end{aligned}
$$

We integrate this function of $x$ against another non-negative test function $\Psi \in$ $C_{c}^{\infty}\left(\left[0, t_{0}\right] \times \mathbb{R}^{q}\right)$. Choose $K_{1} \in \mathbb{N}$ large so that for $\lambda=1$,

$$
\left\|X_{0}\right\|_{\lambda, \infty}<K_{1} \text { and } \Gamma \equiv\left\{x: \exists s \leq t_{0} \text { with } \Psi_{s}(x)>0\right\} \subset B^{q}\left(0, K_{1}\right) .
$$

We then apply the classical and stochastic versions of Fubini's Theorem, here in Proposition 3.4 .9 for the latter. Condition 3.12 can be verified using localization and the stopping times $\left\{T_{K}\right\}$. Arguing as in the proof of Proposition II.5.7 of Per02 to handle the time dependence in $\Psi$, we then obtain that for any $t \in\left[0, t_{0}\right]$,

$$
\begin{aligned}
\left\langle\phi_{n}\left(\left\langle u_{t}, \Phi_{\cdot}^{m}\right\rangle\right), \Psi_{t}(\cdot)\right\rangle & \\
= & \int_{0}^{t} \int_{\mathbb{R}^{q}}\left\langle\phi_{n}^{\prime}\left(\left\langle u_{s}, \Phi_{\cdot}^{m}\right\rangle\right) \Phi_{\cdot}^{m}(y), \Psi_{s}\right\rangle\left(\sigma\left(s, y, X^{1}(s, y)\right)-\sigma\left(s, y, X^{2}(s, y)\right)\right) W(d s d y) \\
& +\int_{0}^{t}\left\langle\phi_{n}^{\prime}\left(\left\langle u_{s}, \Phi_{\cdot}^{m}\right\rangle\right)\left\langle u_{s}, \frac{1}{2} \Delta \Phi_{\cdot}^{m}\right\rangle, \Psi_{s}\right\rangle d s \\
& +\frac{1}{2} \int_{0}^{t} d s \int_{\mathbb{R}^{3 q}} d x d w d z \Psi_{s}(x) \psi_{n}\left(\left|\left\langle u_{s}, \Phi_{x}^{m}\right\rangle\right|\right) \Phi_{x}^{m}(w) \Phi_{x}^{m}(z) k(w, z) \\
& \times\left(\sigma\left(s, w, X^{1}(s, w)\right)-\sigma\left(s, w, X^{2}(s, w)\right)\right)\left(\sigma\left(s, z, X^{1}(s, z)\right)-\sigma\left(s, z, X^{2}(s, z)\right)\right) \\
& +\int_{0}^{t}\left\langle\phi_{n}\left(\left\langle u_{s}, \Phi_{\cdot}^{m}\right\rangle\right), \dot{\Psi}_{s}\right\rangle d s \\
& +\int_{0}^{t} \int_{\mathbb{R}^{q}}\left\langle\phi_{n}^{\prime}\left(\left\langle u_{s}, \Phi_{\cdot}^{m}\right\rangle\right) \Phi_{\cdot}^{m}(y), \Psi_{s}\right\rangle\left(b\left(s, y, X^{1}(s, y)\right)-b\left(s, y, X^{2}(s, y)\right)\right) d y d s \\
\equiv & I_{1}^{m, n}(t)+I_{2}^{m, n}(t)+I_{3}^{m, n}(t)+I_{4}^{m, n}(t)+I_{5}^{m, n}(t) .
\end{aligned}
$$

Now, set $m_{n}=a_{n-1}^{-1 / 2}=\exp \{(n-1) n / 4\}$ for $n \in \mathbb{N}$ (this choice is where the improvement to [MPS06] is made, see their Lemma 4.3).

We quote Lemma 2.2 from MPS06] and add a last point treating $I_{5}^{m_{n}, n}(t)$ : 
Lemma 9.1.1. [Lemma 2.2 in [MPS06]] For any stopping time $T$ and constant $t \geq 0$ we have:

(a)

$$
\mathbb{E}\left(I_{1}^{m_{n}, n}(t \wedge T)\right)=0 \text { for all } n
$$

(b)

$$
\limsup _{n \rightarrow \infty} \mathbb{E}\left(I_{2}^{m_{n}, n}(t \wedge T)\right) \leq \mathbb{E}\left(\int_{0}^{t \wedge T} \int_{\mathbb{R}}|u(s, x)| \frac{1}{2} \Delta \Psi_{s}(x) d x d s\right) .
$$

(c)

$$
\lim _{n \rightarrow \infty} \mathbb{E}\left(I_{4}^{m_{n}, n}(t \wedge T)\right)=\mathbb{E}\left(\int_{0}^{t \wedge T} \int_{\mathbb{R}^{q}}|u(s, x)| \dot{\Psi}_{s}(x) d x d s\right) .
$$

(d)

$$
\left.\lim _{n \rightarrow \infty} \mathbb{E}\left(I_{5}^{m_{n}, n}(t \wedge T)\right) \leq B \mathbb{E}\left(\int_{0}^{t \wedge T} \int_{\mathbb{R}^{q}}|u(s, x)| \Psi_{s}(x) d x d s\right) \text { with } B \text { as in } 5.4\right) .
$$

Proof. We only need to show the last point, where we follow (2.48) of [MP11]. Since $\left|\phi_{n}^{\prime}\right| \leq 1,5.4$ implies that for a stopping time $T$,

$$
I_{5}^{m_{n}, n}(t \wedge T) \leq B \int_{0}^{t \wedge T} \iint|u(s, y)| \Phi_{x}^{m_{n}}(y) \Psi_{s}(x) d y d x d s=: B \tilde{I}_{5}^{n}(t \wedge T) .
$$

It follows using (5.7) to obtain an integrable bound for the integrand and Lebesgue's Dominated Convergence Theorem that as $n \rightarrow \infty$,

$$
\tilde{I}_{5}^{n}(t \wedge T) \rightarrow \int_{0}^{t \wedge T} \int|u(s, x)| \Psi_{s}(x) d x d s \text { a.s. }
$$

and hence in $L^{1}$ since, again by (5.7), $\left\{\tilde{I}_{5}^{n}\left(t_{0}\right): n \in \mathbb{N}\right\}$ is $L^{2}$-bounded.

It will be $I_{3}^{m_{n+1}, n+1}$ which will mostly concern us for the rest of the work. In its integral definition we may assume $|x| \leq K_{1}$ by (9.6) and so $|w| \vee|z| \leq K_{1}+1$. If $K \geq K_{1}, s \leq T_{K}$ and $|w| \leq K_{1}+1$ we have

$$
\left|X^{i}(s, w)\right| \leq K e^{|w|} \leq K e^{\left(K_{1}+1\right)} \text { for } i=1,2
$$


Therefore, (5.5), 9.1), and the fact that $\psi_{n}(x) \leq \frac{2}{n x} \mathbb{1}\left\{a_{n+1}<x<a_{n}\right\}$ show that if $K^{\prime}=K e^{\left(K_{1}+1\right)}\left(\geq K_{1}+1\right)$, then for all $t \in\left[0, t_{0}\right]$,

$$
\begin{aligned}
& I_{3}^{m_{n+1}, n+1}\left(t \wedge T_{K}\right) \\
& \leq \frac{q 5.5}{2} \int_{0}^{t \wedge T_{K}} \int_{\mathbb{R}^{3 q}} 2(n+1)^{-1}\left|\left\langle u_{s}, \Phi_{x}^{m_{n+1}}\right\rangle\right|^{-1} \mathbb{1}\left\{a_{n+1}<\left|\left\langle u_{s}, \Phi_{x}^{m_{n+1}}\right\rangle\right|<a_{n}\right\} \\
& \quad \times L_{K^{\prime}}^{2}|u(s, w)|^{\gamma}|u(s, z)|^{\gamma} \Phi_{x}^{m_{n+1}}(w) \Phi_{x}^{m_{n+1}}(z)\left(|w-z|^{-\alpha}+1\right) \Psi_{s}(x) d w d z d x d s \\
& \leq q 5.5 L_{K^{\prime}}^{2} a_{n+1}^{-1} \int_{0}^{t \wedge T_{K}} \int_{\mathbb{R}^{3 q}} \mathbb{1}\left\{a_{n+1}<\left|\left\langle u_{s}, \Phi_{x}^{m_{n+1}}\right\rangle\right|<a_{n}\right\}|u(s, w)|^{\gamma}|u(s, z)|^{\gamma} \\
& \quad \times \Phi_{x}^{m_{n+1}}(w) \Phi_{x}^{m_{n+1}}(z)\left(|w-z|^{-\alpha}+1\right) \Psi_{s}(x) d w d z d x d s .
\end{aligned}
$$

We note that $a_{n+1}^{-1}=a_{n}^{-1-2 / n}$. Thus, as the quantity of interest, we define

$$
\begin{array}{r}
I^{n}(t)=a_{n}^{-1-2 / n} \int_{0}^{t} \int_{\mathbb{R}^{3 q}} 1\left(\left|\left\langle u_{s}, \Phi_{x}^{m_{n+1}}\right\rangle\right|<a_{n}\right)|u(s, w)|^{\gamma}|u(s, z)|^{\gamma} \\
\Phi_{x}^{m_{n+1}}(w) \Phi_{x}^{m_{n+1}}(z)\left(|w-z|^{-\alpha}+1\right) \Psi_{s}(x) d w d z d x d s .
\end{array}
$$

The following result holds:

Proposition 9.1.2. Suppose $\left\{U_{M, n, K}: M, n, K \in \mathbb{N}, K \geq K_{1}\right\}$ are $\mathcal{F}_{t}$-stopping times such that for each $K \in \mathbb{N} \geq K_{1}$,

$$
\begin{gathered}
U_{M, n, K} \leq T_{K} \text { for all } M, n \in \mathbb{N}, \\
U_{M, n, K} \nearrow T_{K} \text { as } M \rightarrow \infty \text { for all } n \in \mathbb{N}, \\
\lim _{M \rightarrow \infty} \sup _{n \in \mathbb{N}} \mathbb{P}\left(U_{M, n, K}<T_{K}\right)=0,
\end{gathered}
$$

and

$$
\lim _{n \rightarrow \infty} E\left(I^{n}\left(t_{0} \wedge U_{M, n, K}\right)\right)=0 \text { for all } M \in \mathbb{N}
$$

are satisfied. Then, the conclusion of Theorem 5.3.1 holds.

The proof of this proposition is exactly the same as the proof of Proposition 2.1 in [MP11, here using Lemma 9.1.1. So we omit it.

We observe that all that is left is the construction of the stopping times $U_{M, n, K}$ and the verification of $\left(H_{1}\right)$ and $\left(H_{2}\right)$. This will take the rest of this work. The reader who has not taken a look at the heuristics after the statement of Theorem 5.3.1 is encouraged to do so before proceeding. The others might note that the quadratic variation term 9.15 is in fact quite similar to 5.10 . We remark the differences of the heuristics to the preceding quickly:

Remark 9.1.3. Here, $a_{n}$ is used instead of $n^{-1}$, but the argument should also work with $n^{-1}$ instead. The $g_{n}$ from the heuristics are called $\phi_{n}$ here and finally $\Phi_{x}^{m}$ is really chosen as a $C^{\infty}$-function, whereas there it was an indicator function. 
It is now time for the overview of this chapter. Section 9.2 contains the verification of the hypotheses of Proposition 9.1.2. The proof there also relies on another result, Proposition 9.2.2, which we can prove in Section 9.5. The rest of the work is devoted to provide results for this section. In Section 9.3 some heat kernel estimates are given, which are used in Section 9.4 to give in fact the proof of the "Hölder-regularity up to order 2", stated in Proposition 9.4.2. For this section, the results from Sections 9.9, 9.6 and Section 9.7 are needed. Section 9.9 contains the extension of the proof of [MPS06]'s Theorem 4.1. This chapter contains three more sections: Section 9.8 contains a Kolmogorov-Centsov result apt for the proofs here, Section 9.10 contains the necessary modifications to include in drift component in all of the sections and finally, Section 9.11 contains an integral estimate.

\subsection{Verification of the hypotheses of Proposition 9.1.2}

We follow Chapter 3 in [MP11. First, recall the canonical distance for the heatequation:

Notation. For $t, t^{\prime} \geq 0$ and $x, x^{\prime} \in \mathbb{R}^{q}$ let $d\left((t, x),\left(t^{\prime}, x^{\prime}\right)\right)=\sqrt{\left|t^{\prime}-t\right|}+\left|x^{\prime}-x\right|$.

Assume the setting of the beginning of the last chapter. That means that $X^{1}$ and $X^{2}$ are two solutions of the SPDE (5.1) with the same noise $\dot{W}$ and $u:=X^{1}-X^{2}$ is the difference of the two, i.e.

$$
u(t, x)=\int_{0}^{t} \int_{\mathbb{R}^{q}} p_{t-s}(y-x) D(s, y) W(d s d y) \text { a.s. for all } t \geq 0, x \in \mathbb{R}^{q},
$$

where $p_{t}(x)$ is the $q$-dimensional heat kernel and $D(s, y)=\sigma\left(X^{1}(s, y)\right)-\sigma\left(X^{2}(s, y)\right)$, which by 9.4 obeys

$$
|D(s, y)| \leq R_{0} e^{R_{1}|y|}|u(s, y)|^{\gamma} .
$$

Let $\left(P_{t}\right)_{t \geq 0}$ be the heat-semigroup acting on $C_{\text {tem }}$. To verify $\left(H_{2}\right)$ of Propositon 9.1.2 we need to consider (9.15) and hence need to know what happens close to $(t, x) \in \mathbb{R}_{+} \times \mathbb{R}^{q}$ where $|u(t, x)| \approx a_{n}$. It will be a key idea to split up $u$ into a smooth part $u_{1}$ and the difference $u_{2}=u-u_{1}$. The smooth part $u_{1}$ will allow to be differentiated and follow more or less the heuristics given in Chapter 5 . Therefore, for $\delta \geq 0$ set

$$
u_{1, \delta}(t, x):=P_{\delta}\left(u\left((t-\delta)^{+}, \cdot\right)\right)(x), \quad u_{2, \delta}:=u-u_{1, \delta} .
$$

We will show that for $u_{1, \delta}$ there is more or less a Hölder-continuous derivative in the space-coordinate and for $u_{2, \delta}$ we can find good estimates for small $\delta$. By uniform continuity of the heat kernel $P_{\delta}: C_{\text {tem }} \rightarrow C_{\text {tem }}$ it is true that both $u_{1, \delta}$ and so $u_{2, \delta}$ have sample paths in $C\left(\mathbb{R}_{+}, C_{\text {tem }}\right)$. By definition, we have

$$
u_{1, \delta}(t, x)=\int_{\mathbb{R}^{q}} \int_{0}^{(t-\delta)^{+}} \int_{\mathbb{R}^{q}} p_{(t-\delta)^{+-s}}(y-z) D(s, y) W(d s d y) p_{\delta}(z-x) d z
$$


and with the help of the Stochastic-Fubini-Formula (Proposition 3.4.9, where 5.7 , is used for the expectation condition) reformulate that for $\delta \leq t$ to

$$
u_{1, \delta}(t, x)=\int_{0}^{(t-\delta)^{+}} \int_{\mathbb{R}^{q}} p_{t-s}(y-x) D(s, y) W(d s d y) .
$$

We define the following functions

$$
G_{\delta}(s, t, x)=P_{(t-s)^{+}+\delta}\left(u_{(s-\delta)^{+}}\right)(x), \quad F_{\delta, l}(s, t, x)=-\partial_{x_{l}} G_{\delta}(s, t, x),
$$

$1 \leq l \leq q$, for which we easily have $u_{1, \delta}(t, x)=G_{\delta}(t, t, x)$. We denote by

$$
p_{t, l}(x)=\partial_{x_{l}} p_{t}(x), 1 \leq l \leq q,
$$

the spatial derivative of the heat kernel. The following result holds, which is analogous to Lemma 3.1 in [MP11] and has essentially the same proof:

Lemma 9.2.1. The random fields $G_{\delta}$ and $F_{\delta, l}$ are jointly continuous in $(s, t, x) \in$ $\mathbb{R}_{+}^{2} \times \mathbb{R}^{q}$ and

$$
\begin{aligned}
G_{\delta}(s, t, x) & =\int_{0}^{(s-\delta)^{+}} \int p_{(t \vee s)-r}(y-x) D(r, y) W(d r d y) \\
F_{\delta, l}(s, t, x) & =\int_{0}^{(s-\delta)^{+}} \int p_{(t \vee s)-r, l}(y-x) D(r, y) W(d r d y), \text { where } 1 \leq l \leq q .
\end{aligned}
$$

Note that for the special choice of $s=t$ in the previous lemma:

$$
\partial_{x_{l}} u_{1, \delta}(t, x)=\int_{0}^{(t-\delta)^{+}} \int p_{t-r, l}(y-x) D(r, y) W(d r d y)=F_{\delta, l}(t, t, x) .
$$

For $(t, x) \in \mathbb{R}_{+} \times \mathbb{R}^{q}$ and $n \in \mathbb{N}$ let

$$
B_{n}(t, x):=\left\{y \in \mathbb{R}^{q}:|y-x| \leq \sqrt{a_{n}},|u(t, y)|=\inf \left\{|u(t, z)|:|z-x| \leq \sqrt{a_{n}}\right\}\right\}
$$

be the set of points with the smallest $u$-values in a certain neighborhood close to $x$ and let

$$
\hat{x}_{n}(t, x)
$$

be a measurable choice of a point in $B_{n}(t, x)$ (e.g. with the smallest first coordinate, if this does not suffice to uniquely select a point, take the smallest second coordinate and so on). Let us fix two positive but very small constants $\varepsilon_{0}, \varepsilon_{1}$ throughout the paper

$$
\varepsilon_{1} \in\left(0, \frac{1}{32}(2(2 \gamma-1)-\alpha)\right), \varepsilon_{0} \in\left(0, \frac{1}{4}(1-\gamma) \varepsilon_{1}\right) .
$$


Let $L=L\left(\varepsilon_{0}, \varepsilon_{1}\right)=\left\lfloor\varepsilon_{0}^{-1}\left(1 / 2-6 \varepsilon_{1}\right)\right\rfloor \in \mathbb{N}$ and set for $i=0, \ldots, L$

$$
\beta_{i}=i \varepsilon_{0} \in\left[0, \frac{1}{2}-6 \varepsilon_{1}\right], \quad \lambda_{i}=2\left(\beta_{i}+\varepsilon_{1}\right) \in[0,1]
$$

and $\beta_{L+1}=\frac{1}{2}-\varepsilon_{1}$. So alltogether for $i=0, \ldots, L+1$ :

$$
\beta_{i} \in\left[0, \frac{1}{2}-\varepsilon_{1}\right]
$$

We define the following subsets of $\mathbb{R}^{q}$ :

$$
\begin{aligned}
& \left.J_{n, 0}(s):=\left\{x \in \mathbb{R}^{q}:|x| \leq K_{0},\left|\left\langle u_{s}, \Phi_{x}^{m_{n+1}}\right\rangle\right| \leq a_{n},\left|\nabla u_{1, a_{n}}\left(s, \hat{x}_{n}(s, x)\right)\right| \geq \frac{a_{n}^{\varepsilon_{0}}}{4}\right]\right\}, \\
& \left.J_{n, L}(s):=\left\{x \in \mathbb{R}^{q}:|x| \leq K_{0},\left|\left\langle u_{s}, \Phi_{x}^{m_{n+1}}\right\rangle\right| \leq a_{n},\left|\nabla u_{1, a_{n}}\left(s, \hat{x}_{n}(s, x)\right)\right| \leq \frac{a_{n}^{\beta_{L}}}{4}\right]\right\}
\end{aligned}
$$

and for $i=1, \ldots, L-1$ :

$J_{n, i}(s):=\left\{x \in \mathbb{R}^{q}:|x| \leq K_{0},\left|\left\langle u_{s}, \Phi_{x}^{m_{n+1}}\right\rangle\right| \leq a_{n},\left|\nabla u_{1, a_{n}}\left(s, \hat{x}_{n}(s, x)\right)\right| \in\left[\frac{a_{n}^{\beta_{i+1}}}{4}, \frac{a_{n}^{\beta_{i}}}{4}\right]\right\}$.

Recall 9.15) and observe that for $t \geq 0, n \in \mathbb{N}$ :

$$
\begin{aligned}
I^{n}(t) \leq & a_{n}^{-1-2 / n} \sum_{i=0}^{L\left(\varepsilon_{0}, \varepsilon_{1}\right)} \int_{0}^{t} d s \int_{\mathbb{R}^{3 q}} d x d w d z \mathbb{1}_{J_{n, i}(s)}(x)|u(s, w)|^{\gamma}|u(s, z)|^{\gamma} \\
& \times \Phi_{x}^{m_{n+1}}(w) \Phi_{x}^{m_{n+1}}(z)\left(|w-z|^{-\alpha}+1\right) \Psi_{s}(x) \\
= & : \sum_{i=0}^{L\left(\varepsilon_{0}, \varepsilon_{1}\right)} I_{n, i}(t) .
\end{aligned}
$$

To verify the hypotheses of Proposition 9.1.2, it suffices to show the existence of stopping times $U_{M, n, K}$ satisfying $\left(H_{1}\right)$ as well as for $i=0, \ldots, L$,

$$
\left(H_{2, i}\right) \quad \text { for all } M, K \in \mathbb{N} \text { with } K \geq K_{1} \quad \lim _{n \rightarrow \infty} E\left(I_{n, i}\left(t_{0} \wedge U_{M, n, K}\right)\right)=0 \text {. }
$$

We will get to the definition of these stopping times in Chapter 9.5. We now define

$$
\sigma_{x}:=\sigma_{x}(n, s):=\nabla u_{1, a_{n}}\left(s, \hat{x}_{n}(s, x)\right)\left(\left|\nabla u_{1, a_{n}}\left(s, \hat{x}_{n}(s, x)\right)\right|\right)^{-1}
$$

as the direction of the gradient $\nabla u_{1, a_{n}}$ at the point $\hat{x}_{n}(s, x)$ close to $x$. We also set

$$
\bar{l}_{n}\left(\beta_{i}\right)=a_{n}^{\beta_{i}+5 \varepsilon_{1}},
$$

where dependence on $\beta_{i}$ is not written out explicitly if there are no disambiguities. 
To get $\left(H_{2, i}\right)$ we want to derive some properties of points in $J_{n, i}$. Therefore, set

$$
\begin{aligned}
& \tilde{J}_{n, 0}(s):=\left\{x \in \mathbb{R}^{q}:|x| \leq K_{0},\left|\left\langle u_{s}, \Phi_{x}^{m_{n+1}}\right\rangle\right| \leq a_{n},\right. \\
& \sigma_{x} \cdot \nabla u_{1, a_{n}^{2 \varepsilon_{0}}}\left(s, x^{\prime}\right) \geq a_{n}^{\varepsilon_{0}} / 16 \text { for all } x^{\prime} \in \mathbb{R}^{q} \text { s. t. }\left|x^{\prime}-x\right| \leq 5 \bar{l}_{n}\left(\beta_{0}\right) \\
& \text { and }\left|u_{2, a_{n}^{\lambda_{0}}}\left(s, x^{\prime}\right)-u_{2, a_{n}^{\lambda_{0}}}\left(s, x^{\prime \prime}\right)\right| \leq 2^{-75} a_{n}^{\beta_{1}}\left(\left|x^{\prime}-x^{\prime \prime}\right| \vee a_{n}^{\frac{2}{\alpha}\left(\gamma-\beta_{1}-\varepsilon_{1}\right)} \vee a_{n}\right) \\
& \quad \text { for all } x^{\prime}, x^{\prime \prime} \in \mathbb{R}^{q} \text { s.t. }\left|x^{\prime}-x\right| \leq 4 \sqrt{a_{n}},\left|x^{\prime \prime}-x^{\prime}\right| \leq \bar{l}_{n}\left(\beta_{0}\right) \\
& \text { and } \left.\left|u\left(s, x^{\prime}\right)\right| \leq 3 a_{n}^{\left(1-\varepsilon_{0}\right) / 2} \text { for all } x^{\prime} \in \mathbb{R}^{q} \text { s.t. }\left|x^{\prime}-x\right| \leq \sqrt{a_{n}}\right\}, \\
& \tilde{J}_{n, L}(s):=\left\{\begin{array}{l}
\left\{x \in \mathbb{R}^{q}:|x| \leq K_{0},\left|\left\langle u_{s}, \Phi_{x}^{m_{n+1}}\right\rangle\right| \leq a_{n},\right. \\
\left|\nabla u_{1, a_{n}^{\lambda_{L}}}\left(s, x^{\prime}\right)\right| \leq a_{n}^{\beta_{L}} \text { for all } x^{\prime} \in \mathbb{R}^{q} \text { s.t. }\left|x^{\prime}-x\right| \leq 5 \bar{l}_{n}\left(\beta_{L}\right) \\
\text { and }\left|u_{2, a_{n}^{\lambda_{L}}}\left(s, x^{\prime}\right)-u_{2, a_{n}^{\lambda_{L}}}\left(s, x^{\prime \prime}\right)\right| \leq 2^{-75} a_{n}^{\beta_{L+1}}\left(\left|x^{\prime}-x^{\prime \prime}\right| \vee a_{n}^{\frac{2}{\alpha}}\left(\gamma-\beta_{L}-\varepsilon_{1}\right) \vee a_{n}\right) \\
\text { for all } \left.x^{\prime}, x^{\prime \prime} \in \mathbb{R}^{q} \text { s.t. }\left|x^{\prime}-x\right| \leq 4 \sqrt{a_{n}},\left|x^{\prime \prime}-x^{\prime}\right| \leq \bar{l}_{n}\left(\beta_{L}\right)\right\}
\end{array}\right.
\end{aligned}
$$

and for $i=1, \ldots, L-1$ :

$$
\begin{aligned}
& \tilde{J}_{n, i}(s):=\left\{x \in \mathbb{R}^{q}:|x| \leq K_{0},\left|\left\langle u_{s}, \Phi_{x}^{m_{n+1}}\right\rangle\right| \leq a_{n},\right. \\
& \left|\nabla u_{1, a_{n}^{\lambda_{L}}}\left(s, x^{\prime}\right)\right| \leq a_{n}^{\beta_{L}} \text { and } \sigma_{x} \cdot \nabla u_{1, a_{n}^{\lambda_{i}}}\left(s, x^{\prime}\right) \geq a_{n}^{\beta_{i+1}} / 16 \\
& \text { for all } x^{\prime} \in \mathbb{R}^{q} \text { s.t. }\left|x^{\prime}-x\right| \leq 5 \bar{l}_{n}\left(\beta_{i}\right) \\
& \text { and }\left|u_{2, a_{n}^{\lambda_{i}}}\left(s, x^{\prime}\right)-u_{2, a_{n}^{\lambda_{i}}}\left(s, x^{\prime \prime}\right)\right| \leq 2^{-75} a_{n}^{\beta_{i+1}}\left(\left|x^{\prime}-x^{\prime \prime}\right| \vee a_{n}^{\frac{2}{\alpha}\left(\gamma-\beta_{i+1}-\varepsilon_{1}\right)} \vee a_{n}\right) \\
& \text { for all } \left.x^{\prime}, x^{\prime \prime} \in \mathbb{R}^{q} \text { s.t. }\left|x^{\prime}-x\right| \leq 4 \sqrt{a_{n}},\left|x^{\prime \prime}-x^{\prime}\right| \leq \bar{l}_{n}\left(\beta_{i}\right)\right\} \text {. }
\end{aligned}
$$

We also define two deterministic constants

$n_{M}\left(\varepsilon_{1}\right)=\inf \left\{n \geq 1: a_{n}^{\varepsilon_{1}} \leq 2^{-M-8}\right\}, n_{0}\left(\varepsilon_{0}, \varepsilon_{1}\right)=\sup \left\{n \in \mathbb{N}: \sqrt{a_{n}}<2^{-a_{n}^{-\varepsilon_{0} \varepsilon_{1} / 4}}\right\}$

and will from now on always assume that

$$
n>n_{M}\left(\varepsilon_{1}\right) \vee n_{0}\left(\varepsilon_{0}, \varepsilon_{1}\right) .
$$

The next proposition shows that we can ultimately estimate the size of the sets $\tilde{J}_{n, i}(s)$ instead of that of $J_{n, i}(s)$ :

Proposition 9.2.2. $\tilde{J}_{n, i}(s)$ is a compact set for all $s \geq 0, i \in\{0, \ldots, L\}$. There exist stopping times $U_{M, n, K}$ satisfying $\left(H_{1}\right)$ such that for all $n \geq n_{M}, i \in\{0, \ldots, L\}$ and $s \leq U_{M, n, K}$ :

$$
J_{n, i}(s) \subset \tilde{J}_{n, i}(s) .
$$


The proof of this proposition can be found in Chapter 9.5. We will use this proposition to show $\left(H_{2, i}\right)$ at the end of this chapter. We need the following notation for $i \in\{0, \ldots, L\}$ :

$$
l_{n}\left(\beta_{i}\right):=\left(129 a_{n}^{1-\beta_{i+1}}\right) \vee a_{n}^{\frac{2}{\alpha}\left(\gamma-\beta_{i+1}-\varepsilon_{1}\right)},
$$

where we omit the dependence on $\beta_{i}$ if there are no disambiguities and obtain:

Lemma 9.2.3. If $i \in\{0, \ldots, L\}$ and $n>n_{M}\left(\varepsilon_{1}\right)$, then

$$
l_{n}\left(\beta_{i}\right)<\sqrt{a_{n}}<\frac{1}{2} \bar{l}_{n}\left(\beta_{i}\right) .
$$

Proof.

$$
\begin{aligned}
l_{n}\left(\beta_{i}\right) a_{n}^{-1 / 2} & =\left(129 a_{n}^{\frac{1}{2}-\beta_{i+1}}\right) \vee a_{n}^{\frac{2}{\alpha}\left(\gamma-\beta_{i+1}-\varepsilon_{1}\right)-\frac{1}{2}} \\
& \leq\left(129 a_{n}^{\varepsilon_{1}}\right) \vee a_{n}^{\frac{1}{2 \alpha}\left(4 \gamma-4 \beta_{i}-4 \varepsilon_{0}-\varepsilon_{1}-\alpha\right)} \\
& <1
\end{aligned}
$$

by $9.20,(9.22)$ and because $a_{n}^{\varepsilon_{1}}<2^{-8}$ by $(9.24)$. This gives the first inequality. For the second one, use $\beta_{i} \leq \frac{1}{2}-6 \varepsilon_{1}$ and $(9.24)$ to see that

$$
\sqrt{a_{n}} \bar{l}_{n}\left(\beta_{i}\right)^{-1}=a_{n}^{\frac{1}{2}-\beta_{i}-5 \varepsilon_{1}} \leq a_{n}^{\varepsilon_{1}}<1 / 2 .
$$

We give some elementary properties of the sets $\tilde{J}_{n, i}(s)$.

Lemma 9.2.4. Assume $s \geq 0, i \in\{0, \ldots, L\}, x \in \tilde{J}_{n, i}(s), x^{\prime} \in \mathbb{R}^{q}$ and $\left|x^{\prime}-x\right| \leq$ $4 \sqrt{a_{n}}$.

(a) If $i>0, x^{\prime \prime} \in \mathbb{R}^{q}$ s. t. $\left|x^{\prime \prime}-x^{\prime}\right| \leq \bar{l}_{n}\left(\beta_{i}\right)$, then $\left|u\left(s, x^{\prime \prime}\right)-u\left(s, x^{\prime}\right)\right| \leq$ $2 a_{n}^{\beta_{i}}\left(\left|x^{\prime}-x^{\prime \prime}\right| \vee a_{n}^{\frac{2}{\alpha}\left(\gamma-\beta_{i+1}-\varepsilon_{1}\right)} \vee a_{n}\right)$.

(b) If $i<L, x^{\prime \prime} \in \mathbb{R}^{q}$ s.t. $\left(x^{\prime \prime}-x^{\prime}\right) \| \sigma_{x}$ and $a_{n}^{\frac{2}{\alpha}\left(\gamma-\beta_{i+1}-\varepsilon_{1}\right)} \vee a_{n} \leq\left|x^{\prime}-x^{\prime \prime}\right| \leq \bar{l}_{n}\left(\beta_{i}\right)$, then

$$
u\left(s, x^{\prime \prime}\right)-u\left(s, x^{\prime}\right)\left\{\begin{array}{l}
\geq 2^{-5} a_{n}^{\beta_{i+1}}\left(x^{\prime \prime}-x^{\prime}\right) \cdot \sigma_{x} \text { if }\left(x^{\prime \prime}-x^{\prime}\right) \cdot \sigma_{x} \geq 0 \\
\leq 2^{-5} a_{n}^{\beta_{i+1}}\left(x^{\prime \prime}-x^{\prime}\right) \cdot \sigma_{x} \text { if }\left(x^{\prime \prime}-x^{\prime}\right) \cdot \sigma_{x}<0
\end{array}\right.
$$

(c) Let $y \in \tilde{J}_{n, i}(s),|x-y| \leq \bar{l}_{n}\left(\beta_{i}\right)$. Additionally let $y^{\prime}, y^{\prime \prime} \in \mathbb{R}^{q}$, s.t $\left|y-y^{\prime}\right| \leq$ $\sqrt{a_{n}},\left(y^{\prime \prime}-y^{\prime}\right) \| \sigma_{x}$ and $\left|y^{\prime \prime}-y^{\prime}\right| \in\left(a_{n}^{\frac{2}{\alpha}\left(\gamma-\beta_{i+1}-\varepsilon_{1}\right)} \vee a_{n}, \bar{l}_{n}\left(\beta_{i}\right)\right)$. Then

$$
u\left(s, y^{\prime \prime}\right)-u\left(s, y^{\prime}\right)\left\{\begin{array}{l}
\geq 2^{-5} a_{n}^{\beta_{i+1}}\left(y^{\prime \prime}-y^{\prime}\right) \cdot \sigma_{x} \text { if }\left(y^{\prime \prime}-y^{\prime}\right) \cdot \sigma_{x} \geq 0, \\
\leq 2^{-5} a_{n}^{\beta_{i+1}}\left(y^{\prime \prime}-y^{\prime}\right) \cdot \sigma_{x} \text { if }\left(y^{\prime \prime}-y^{\prime}\right) \cdot \sigma_{x}<0 .
\end{array}\right.
$$


(d) If $i>0$, then for $|w-x|<\sqrt{a_{n}}$,

$$
|u(s, w)| \leq 5 a_{n}^{\beta_{i}+1 / 2} .
$$

Proof. To prove (a) let $n, i, s, x, x^{\prime}, x^{\prime \prime}$ be as above. Since

$$
\left|x^{\prime}-x\right| \vee\left|x^{\prime \prime}-x\right| \leq 4 \sqrt{a_{n}} \vee\left(4 \sqrt{a_{n}}+\bar{l}_{n}\left(\beta_{i}\right)\right) \leq 5 \bar{l}_{n}\left(\beta_{i}\right)
$$

the distance to $x$ of any point on the line between $x^{\prime}$ and $x^{\prime \prime}$ is bounded from above by $5 \bar{l}_{n}\left(\beta_{i}\right)$. By the Mean Value Theorem and the definition of $\tilde{J}_{n, i}(s)$, we get

$$
\begin{aligned}
\left|u\left(s, x^{\prime \prime}\right)-u\left(s, x^{\prime}\right)\right| & \leq\left|u_{1, a_{n}^{\lambda_{i}}}\left(s, x^{\prime \prime}\right)-u_{1, a_{n}^{\lambda_{i}}}\left(s, x^{\prime}\right)\right|+\left|u_{2, a_{n}^{\lambda_{i}}}\left(s, x^{\prime \prime}\right)-u_{2, a_{n}^{\lambda_{i}}}\left(s, x^{\prime}\right)\right| \\
& \leq a_{n}^{\beta_{i}}\left|x^{\prime \prime}-x^{\prime}\right|+2^{-75} a_{n}^{\beta_{i+1}}\left(\left|x^{\prime \prime}-x^{\prime}\right| \vee a_{n}^{\frac{2}{\alpha}\left(\gamma-\beta_{i+1}-\varepsilon_{1}\right)} \vee a_{n}\right) \\
& \leq 2 a_{n}^{\beta_{i}}\left(\left|x^{\prime \prime}-x^{\prime}\right| \vee a_{n}^{\frac{2}{\alpha}\left(\gamma-\beta_{i+1}-\varepsilon_{1}\right)} \vee a_{n}\right) .
\end{aligned}
$$

To prove (b) w.l.o.g. consider $\left(x^{\prime \prime}-x^{\prime}\right) \cdot \sigma_{x} \geq 0$ and do the same estimate as just done:

$$
\begin{aligned}
u\left(s, x^{\prime \prime}\right)-u\left(s, x^{\prime}\right) & \geq \inf _{y \in\left[x^{\prime}, x^{\prime \prime}\right]}\left[\nabla u_{1, a_{n}^{\lambda_{i}}}(s, y) \cdot\left(x^{\prime \prime}-x^{\prime}\right)\right]-\left|u_{2, a_{n}^{\lambda_{i}}}\left(s, x^{\prime \prime}\right)-u_{2, a_{n}^{\lambda_{i}}}\left(s, x^{\prime}\right)\right| \\
& \geq\left(a_{n}^{\beta_{i+1}} / 16\right) \sigma_{x} \cdot\left(x^{\prime \prime}-x^{\prime}\right)-2^{-75} a_{n}^{\beta_{i+1}}\left|x^{\prime \prime}-x^{\prime}\right| \\
& \geq\left(a_{n}^{\beta_{i+1}} / 32\right)\left(x^{\prime \prime}-x^{\prime}\right) \cdot \sigma_{x} .
\end{aligned}
$$

Next, we prove (c) using that $\left|y^{\prime}-x\right| \vee\left|y^{\prime \prime}-x\right|<\sqrt{a_{n}}+\bar{l}_{n}\left(\beta_{i}\right)+\bar{l}_{n}\left(\beta_{i}\right) \leq 5 \bar{l}_{n}\left(\beta_{i}\right)$.

$$
\begin{aligned}
u\left(s, y^{\prime \prime}\right)-u\left(s, y^{\prime}\right) & \geq \inf _{z \in\left[y^{\prime}, y^{\prime \prime}\right]}\left[\nabla u_{1, a_{n}^{\lambda_{i}}}(s, z) \cdot\left(y^{\prime \prime}-y^{\prime}\right)\right]-\left|u_{2, a_{n}^{\lambda_{i}}}\left(s, y^{\prime \prime}\right)-u_{2, a_{n}^{\lambda_{i}}}\left(s, y^{\prime}\right)\right| \\
& \geq\left(a_{n}^{\beta_{i+1}} / 16\right)\left(y^{\prime \prime}-y^{\prime}\right) \cdot \sigma_{x}-2^{-75} a_{n}^{\beta_{i+1}}\left|y^{\prime \prime}-y^{\prime}\right| \\
& \geq\left(a_{n}^{\beta_{i+1}} / 32\right)\left(y^{\prime \prime}-y^{\prime}\right) \cdot \sigma_{x},
\end{aligned}
$$

where, in the next to last inequality, we used that $x \in \tilde{J}_{n, i}(s)$ for the $\nabla u_{1, a_{n}^{\lambda_{i}}}$-part and $y \in \tilde{J}_{n, i}(s)$ for the $u_{2, a_{n}^{\lambda_{i}}}$-part.

Finally, prove (d) much in the same way as the previous claims: We have $\left|\hat{x}_{n}(s, x)-w\right|<\left|\hat{x}_{n}(s, x)-x\right|+|x-w| \leq 2 \sqrt{a_{n}} \leq \bar{l}_{n}\left(\beta_{i}\right)$ by Lemma 9.2.3. So we can apply (a) for $x^{\prime}=\hat{x}_{n}(s, x)$ and $x^{\prime \prime}=w$ to obtain

$$
\begin{aligned}
|u(s, w)| & \leq\left|u\left(s, \hat{x}_{n}(s, x)\right)\right|+\left|u\left(s, \hat{x}_{n}(s, x)\right)-u(s, w)\right| \\
& \leq a_{n}+2 a_{n}^{\beta_{i}}\left(\left|w-\hat{x}_{n}(s, x)\right| \vee a_{n}^{\frac{2}{\alpha}\left(\gamma-\beta_{i+1}-\varepsilon_{1}\right)} \vee a_{n}\right) \\
& \leq 5 a_{n}^{\beta_{i}+1 / 2}
\end{aligned}
$$

since $a_{n}^{\frac{2}{\alpha}\left(\gamma-\beta_{i+1}+\varepsilon_{1}\right)}<\sqrt{a_{n}}$, again by Lemma 9.2.3. 
Now, define

$$
F_{n}(s, x):=\left\langle\Phi_{x}^{m_{n+1}}, u_{s}\right\rangle=\int_{B\left(0, \sqrt{a_{n}}\right)} \Phi^{m_{n+1}}(z) u(s, x+z) d z
$$

and recall that we write $x \| y$ if $x, y \in \mathbb{R}^{q}$ are collinear.

Lemma 9.2.5. Assume $i \in\{0, \ldots, L-1\}, s \in \mathbb{R}_{+}$.

(a) If $x \in \tilde{J}_{n, i}(s), \tilde{x} \in \mathbb{R}^{q}$ with $(\tilde{x}-x) \| \sigma_{x}$ and $l_{n}\left(\beta_{i}\right)<\left|(\tilde{x}-x) \cdot \sigma_{x}\right| \leq \bar{l}_{n}\left(\beta_{i}\right)$, then

$$
F_{n}(s, \tilde{x})-F_{n}(s, x)\left\{\begin{array}{l}
\geq 2^{-5} a_{n}^{\beta_{i+1}}|\tilde{x}-x|, \text { if }(\tilde{x}-x) \cdot \sigma_{x} \geq 0 \\
\leq-2^{-5} a_{n}^{\beta_{i+1}}|\tilde{x}-x|, \text { if }(\tilde{x}-x) \cdot \sigma_{x}<0 .
\end{array}\right.
$$

(b) If $x, y \in \tilde{J}_{n, i}(s),|x-y| \leq \bar{l}_{n}\left(\beta_{i}\right)$. Then for $\tilde{y} \in \mathbb{R}^{q}$, such that $(y-\tilde{y}) \| \sigma_{x}$ and $l_{n}\left(\beta_{i}\right)<|y-\tilde{y}|<\bar{l}_{n}\left(\beta_{i}\right)$ it holds that

$$
\tilde{y} \notin \tilde{J}_{n, i}(s) .
$$

(c) If $x \in \tilde{J}_{n, i}(s), z \in \mathbb{R}^{q}$ and $|x-z| \leq \bar{l}_{n}\left(\beta_{i}\right) / 2$, then

$$
\int_{\left(-\bar{l}_{n} / 2, \bar{l}_{n} / 2\right)} d b \mathbb{1}\left\{z+\sigma_{x} b \in \tilde{J}_{n, i}(s) \cap B\left(x, \bar{l}_{n} / 2\right)\right\} \leq 2 l_{n}\left(\beta_{i}\right) .
$$

Proof. For (a) assume $(\tilde{x}-x) \cdot \sigma_{x} \in\left[l_{n}\left(\beta_{i}\right), \bar{l}_{n}\left(\beta_{i}\right)\right]$. Then

$$
F_{n}(s, \tilde{x})-F_{n}(s, x)=\int_{B^{q}\left(0, \sqrt{a_{n}}\right)} \Phi^{m_{n+1}}(z)(u(s, \tilde{x}+z)-u(s, x+z)) d z .
$$

Clearly, $|z| \leq \sqrt{a_{n}}$ and for $x^{\prime \prime}=\tilde{x}+z, x^{\prime}=x+z$, we have

$$
\left|x^{\prime}-x\right| \leq \sqrt{a_{n}},\left(x^{\prime \prime}-x^{\prime}\right)=(\tilde{x}-x) \| \sigma_{x},\left|x^{\prime}-x^{\prime \prime}\right| \in\left[l_{n}\left(\beta_{i}\right), \bar{l}_{n}\left(\beta_{i}\right)\right] .
$$

Therefore, we can apply Lemma 9.2.4 (b) to obtain:

$$
\begin{aligned}
F_{n}(s, \tilde{x})-F_{n}(s, x) & \geq \int_{B^{q}\left(0, \sqrt{a_{n}}\right)} \Phi^{m_{n+1}}(z)|\tilde{x}-x| 2^{-5} a_{n}^{\beta_{i+1}} d z \\
& \geq 2^{-5} a_{n}^{\beta_{i+1}}|\tilde{x}-x| .
\end{aligned}
$$

The same can be done in the case $(\tilde{x}-x) \cdot \sigma_{x}<0$. 
To do (b) use the same ideas as before, where Lemma 9.2.4 (b) is replaced by Lemma 9.2 .4 (c), to obtain

$$
\begin{aligned}
\left|F_{n}(s, \tilde{y})\right| & \geq\left|F_{n}(s, \tilde{y})-F_{n}(s, y)\right|-\left|F_{n}(s, y)\right| \\
& \geq 2^{-5} a_{n}^{\beta_{i+1}} l_{n}\left(\beta_{i}\right)-a_{n} \\
& \geq \frac{97}{32} a_{n} .
\end{aligned}
$$

Hence, $\tilde{y} \notin \tilde{J}_{n, i}(s)$.

For (c) assume $y=z+\sigma_{x} b \in \tilde{J}_{n, i}(s)$ for a certain $b \in\left[-\bar{l}_{n} / 2, \bar{l}_{n} / 2\right]$ (otherwise the integral is 0 anyway). Observe that

$$
|y-x| \leq|y-z|+|b| \leq \bar{l}_{n} .
$$

So, we can apply (b) for $x, y \in \tilde{J}_{n, i}(s)$ to obtain that

$$
\begin{aligned}
\int_{\left(-\bar{l}_{n} / 2, \bar{l}_{n} / 2\right)} d b \mathbb{1}\left\{z+\sigma_{x} b \in \tilde{J}_{n, i}(s) \cap B\left(x, \bar{l}_{n} / 2\right)\right\} \\
\leq \int_{\left(-\bar{l}_{n}, \bar{l}_{n}\right)} d b \mathbb{1}\left\{y+\sigma_{x} b \in \tilde{J}_{n, i}(s)\right\} \leq 2 l_{n}\left(\beta_{i}\right) .
\end{aligned}
$$

Let $\Sigma_{x}$ be a $q \times(q-1)$ dimensional matrix consisting of an orthonormal basis of the orthogonal space $\sigma_{x}^{\text {ortho }}=\left\{y \in \mathbb{R}^{q}: \sigma_{x} \cdot y=0\right\}$ and let $|A|$ denote the Lebesgue measure of a measurable set $A \subset \mathbb{R}^{q}$.

Lemma 9.2.6. For $i \in\{0, \ldots, L-1\}$ and $s \geq 0, n \in \mathbb{N}$ there is a constant q9.2.6 $=9.2 .6(q)$ such that

$$
\left|\tilde{J}_{n, i}(s)\right| \leq \Phi_{9.2 .6} K_{0}^{q} l_{n}\left(\beta_{i}\right) \bar{l}_{n}\left(\beta_{i}\right)^{-1} .
$$

Proof. Set $B_{x}=B^{q}\left(x, \bar{l}_{n}\left(\beta_{i}\right) / 4\right)$ and cover the compact set $\tilde{J}_{n, i}(s)$ with a finite number of these balls, say $B_{x^{\prime 1}}, \ldots, B_{x^{\prime} Q^{\prime}}$. If $\left|x^{\prime j}-x^{\prime k}\right| \leq \bar{l}_{n}\left(\beta_{i}\right) / 4$, then $B_{x^{\prime j}} \subset$ $B^{q}\left(x^{\prime k}, \bar{l}_{n}\left(\beta_{i}\right) / 2\right)$. So, if we increase the radius of the balls around $x^{\prime 1}, \ldots, x^{\prime Q^{\prime}}$ to $\bar{l}_{n}\left(\beta_{i}\right) / 2$, it suffices to use those balls whose centers have at least distance $\bar{l}_{n}\left(\beta_{i}\right) / 4$, which we denote by $x^{1}, \ldots, x^{Q}$. If we consider $B^{q}\left(x^{k}, \bar{l}_{n}\left(\beta_{i}\right) / 8\right), k=1, \ldots, Q$, then all of these balls are disjoint. Thus, we have

$$
Q \leq K_{0}^{q}\left(\bar{l}_{n}\left(\beta_{i}\right) / 8\right)^{-q}
$$

and also

$$
\tilde{J}_{n, i}(s) \subset \bigcup_{k=1}^{Q} B^{q}\left(x^{k}, \bar{l}_{n}\left(\beta_{i}\right) / 2\right) \cap \tilde{J}_{n, i}(s) .
$$


Next we want to consider the Lebesgue measure of the sets on the right-handside using some kind of Cavalieri decomposition and Lemma 9.2.5 (c). Fix $k \in$ $\{1, \ldots, Q\}$ and denote by $c(q)$ the volume of the $q$-dimensional Euclidean ball. We have

$$
\begin{aligned}
& \left|B^{q}\left(x^{k}, \bar{l}_{n}\left(\beta_{i}\right) / 2\right) \cap \tilde{J}_{n, i}(s)\right|=\int_{B^{q}\left(0, \bar{l}_{n} / 2\right)} d z \mathbb{1}\left\{x^{k}+z \in \tilde{J}_{n, i}(s)\right\} \\
& \quad \leq \int_{B^{q-1}\left(0, \bar{l}_{n} / 2\right)} d z \int_{\left(-\bar{l}_{n} / 2, \bar{l}_{n} / 2\right)} d b \mathbb{1}\left\{x^{k}+\Sigma_{x^{k}} z+\sigma_{x^{k}} b \in \tilde{J}_{n, i}(s)\right\} \\
& \quad \leq \int_{B^{q-1}\left(0, \bar{l}_{n} / 2\right)} d z 2 l_{n}\left(\beta_{i}\right) \\
& \quad=2 c(q-1)\left(\bar{l}_{n}\left(\beta_{i}\right) / 2\right)^{q-1} l_{n}\left(\beta_{i}\right),
\end{aligned}
$$

since $\left|x^{k}+\Sigma_{x} z-x^{k}\right|=\left|\Sigma_{x} z\right|=|z| \leq \bar{l}_{n} / 2$. And therefore, by (9.26) and (9.27) for q9.2.6 $=4 \cdot 4^{q} c(q-1)$ :

$$
\left|\tilde{J}_{n, i}(s)\right| \leq \Phi 9.2 .6 K_{0}^{q} l_{n}\left(\beta_{i}\right)\left(\bar{l}_{n}\left(\beta_{i}\right)\right)^{-1}
$$

We are now in the position to complete the

Verification of the Hypothese $\left(H_{2}\right)$ in Proposition 9.1.2

Let $n \in \mathbb{N}, t>0$ and $M \in \mathbb{N}$ fixed.

First, consider $i=0$. For $x \in J_{n, 0}(s)$ and $|y-x| \leq \sqrt{a_{n}}$ we have $|u(s, y)| \leq$ $3 a_{n}^{\left(1-\varepsilon_{0}\right) / 2}$ due to Proposition 9.2.2. So, we obtain in 9.23 for $n$ large enough so that $\varepsilon_{1}>\frac{2}{n}$ :

$$
\begin{aligned}
& I_{0}^{n}\left(t_{0} \wedge U_{M, n, K}\right) \leq a_{n}^{-1-2 / n} 3^{2 \gamma} a_{n}^{\gamma\left(1-\varepsilon_{0}\right)} \int_{0}^{t_{0} \wedge U_{M, n, K}} d s \int_{\mathbb{R}^{q}} d x \Psi_{s}(x) \mathbb{1}_{J_{n, 0}(s)}(x) \\
& \quad \int_{\mathbb{R}^{q}} d w \int_{\mathbb{R}^{q}} d z \Phi_{x}^{m_{n+1}}(w) \Phi_{x}^{m_{n+1}}(z)\left(|w-z|^{-\alpha}+1\right) \\
& \leq C_{1}\left(\|\Psi\|_{\infty},\|\Phi\|_{\infty}\right) a_{n}^{-1-\varepsilon_{1}} a_{n}^{\gamma\left(1-\varepsilon_{0}\right)} \\
& \quad a_{n}^{-\alpha / 2} t_{0} K_{0}^{q} l_{n}\left(\beta_{0}\right) \bar{l}_{n}\left(\beta_{0}\right)^{-1}(\text { by } \underline{(9.24)} \text { and Lemma 9.2.6 } \\
& \leq C_{1}^{\prime} t_{0} K_{0}^{q} a_{n}^{-1-\alpha / 2+\gamma\left(1-\varepsilon_{0}\right)}\left(a_{n}^{1-\varepsilon_{0}-5 \varepsilon_{1}} \vee a_{n}^{\frac{2}{\alpha}\left(\gamma-\varepsilon_{1}-\varepsilon_{0}\right)-5 \varepsilon_{1}}\right)
\end{aligned}
$$

And this expression tends to zero as $n \rightarrow \infty$ since, using that $\varepsilon_{0}<\varepsilon_{1}$ by 9.20 ,

$$
-1-\alpha / 2+\gamma\left(1-\varepsilon_{0}\right)+1-\varepsilon_{0}-5 \varepsilon_{1} \geq \gamma-\alpha / 2-7 \varepsilon_{1}>9 \varepsilon_{1}>0
$$


also by 9.20 and

$$
\begin{aligned}
-1-\alpha / 2+\gamma\left(1-\varepsilon_{0}\right)+\frac{2}{\alpha}\left(\gamma-\varepsilon_{1}-\varepsilon_{0}\right)-5 \varepsilon_{1} & \geq \gamma-1-\alpha / 2+\left(\gamma-\varepsilon_{1}-\varepsilon_{0}\right)-6 \varepsilon_{1} \\
& \geq 2 \gamma-1-\alpha / 2-8 \varepsilon_{1}>24 \varepsilon_{1}>0,
\end{aligned}
$$

again by $(9.20)$.

Next, let $i \in\{1, \ldots, L\}$ and assume $x \in \tilde{J}_{n, i}(s), y \in \mathbb{R}^{q},|y-x| \leq \sqrt{a_{n}}$. So, we can use Lemma 9.2.4 (d) to get that

$$
|u(s, y)| \leq 5 a_{n}^{\beta_{i}+\frac{1}{2}} .
$$

Put that into 9.23 for $y=w$ and $y=z$ to obtain that

$$
\begin{aligned}
I_{i}^{n}\left(t_{0} \wedge U_{M, n, K}\right) \leq 5^{2 \gamma} & a_{n}^{-1-2 / n} a_{n}^{2 \beta_{i} \gamma+\gamma} \int_{0}^{t} d s \int_{\mathbb{R}^{q}} d x 1_{J_{n, i}(s)}(x) \Psi_{s}(x) \\
& \times \int_{\mathbb{R}^{2 q}} d w d z \Phi_{x}^{m_{n+1}}(w) \Phi_{x}^{m_{n+1}}(z)\left(|w-z|^{-\alpha}+1\right) .
\end{aligned}
$$

To treat the integral in $w$ and $z$, we use Lemma 9.11.1 to obtain:

$$
\begin{aligned}
I_{i}^{n}\left(t_{0} \wedge U_{M, n, K}\right) & \leq 25 c a_{n}^{-1-2 / n} a_{n}^{2 \beta_{i} \gamma+\gamma} m_{n+1}^{\alpha} \int_{0}^{t_{0} \wedge U_{M, n, K}} \int_{\mathbb{R}^{q}} \mathbb{1}_{J_{n, i}(s)}(x) \Psi_{s}(x) d x d s \\
& \leq C_{1}\left(\alpha, d,\|\Psi\|_{\infty},\|\Phi\|_{\infty}\right) a_{n}^{-1-\varepsilon_{1}} a_{n}^{2 \beta_{i} \gamma+\gamma} a_{n}^{-\frac{\alpha}{2}} \int_{0}^{t_{0} \wedge U_{M, n, K}}\left|\tilde{J}_{n, i}(s)\right| d s .
\end{aligned}
$$

Next, we use Lemma 9.2.6 in the case $i \in\{1, \ldots, L-1\}$ :

$$
\begin{aligned}
I_{i}^{n}\left(t_{0} \wedge U_{M, n, K}\right) \leq & C_{1} a_{n}^{-1-\varepsilon_{1}} a_{n}^{2 \beta_{i} \gamma+\gamma} a_{n}^{-\frac{\alpha}{2}} t_{0} c(q) K_{0}^{q} l_{n}\left(\beta_{i}\right) \bar{l}_{n}\left(\beta_{i}\right)^{-1} \\
\leq & C_{2} t_{0} a_{n}^{-1-\frac{\alpha}{2}+2 \beta_{i} \gamma+\gamma-\varepsilon_{1}}\left(a_{n}^{1-\beta_{i+1}} \vee a_{n}^{\frac{2}{\alpha}\left(\gamma-\beta_{i+1}-\varepsilon_{1}\right)}\right) a_{n}^{-\beta_{i}-5 \varepsilon_{1}} \\
= & C_{2} t_{0}\left(a_{n}^{-1-\frac{\alpha}{2}+2 \beta_{i} \gamma+\gamma+1-\beta_{i+1}-\beta_{i}-6 \varepsilon_{1}}\right. \\
& \left.\vee a_{n}^{-1-\frac{\alpha}{2}+2 \beta_{i} \gamma+\gamma+\frac{2}{\alpha}\left(\gamma-\beta_{i+1}-\varepsilon_{1}\right)-\beta_{i}-6 \varepsilon_{1}}\right) \\
= & C_{2} t_{0}\left[a_{n}^{\rho_{1, i}} \vee a_{n}^{\rho_{2, i}}\right] .
\end{aligned}
$$

Hence, it suffices to check for positivity of $\rho_{1, i}$ and $\rho_{2, i}$ to obtain the desired result.

$$
\begin{aligned}
\rho_{1, i} & =-1-\frac{\alpha}{2}+2 \beta_{i} \gamma+\gamma+1-\beta_{i+1}-\beta_{i}-6 \varepsilon_{1} \\
& =-\frac{\alpha}{2}+\gamma+2 \gamma \beta_{i}-2 \beta_{i}-6 \varepsilon_{1}-\varepsilon_{0} \\
& \geq \frac{1}{2}(2(2 \gamma-1)-\alpha)+1-\gamma-2 \beta_{i}(1-\gamma)-7 \varepsilon_{1} \\
& >8 \varepsilon_{1}+(1-\gamma)\left(1-2 \beta_{i}\right)-7 \varepsilon_{1}>\varepsilon_{1}>0
\end{aligned}
$$


by 9.20 . Additionally, note that by 9.20 ,

$$
\begin{aligned}
\frac{2}{\alpha}\left(\gamma-\beta_{i+1}-\varepsilon_{1}\right) & =\frac{2 \gamma-1}{\alpha}+\frac{1-2 \beta_{i+1}-2 \varepsilon_{1}}{\alpha} \\
& \geq \frac{1}{2}+\frac{1}{2}\left(1-2 \beta_{i}-4 \varepsilon_{1}\right) \\
& =1-\beta_{i}-2 \varepsilon_{1} .
\end{aligned}
$$

So we can calculate

$$
\begin{aligned}
\rho_{2, i} & =-1-\frac{\alpha}{2}+2 \beta_{i} \gamma+\gamma+\frac{2}{\alpha}\left(\gamma-\beta_{i+1}-\varepsilon_{1}\right)-\beta_{i}-6 \varepsilon_{1} \\
& \geq-1-\frac{\alpha}{2}+2 \beta_{i} \gamma+\gamma+1-\beta_{i}-2 \varepsilon_{1}-\beta_{i}-6 \varepsilon_{1} \\
& =2 \gamma-1-\frac{\alpha}{2}-8 \varepsilon_{1}>\varepsilon_{1}>0
\end{aligned}
$$

by $(9.20)$.

To finish the proof, we note that in the case $i=L$ it suffices to use a trivial bound on the integral in 9.31) and obtain with $\beta_{L} \geq \frac{1}{2}-6 \varepsilon_{1}-\varepsilon_{0} \geq \frac{1}{2}-7 \varepsilon_{1}$ from 9.20):

$$
\begin{aligned}
I_{L}^{n}\left(t_{0} \wedge U_{M, n, K}\right) & \leq C_{3} a_{n}^{-1-\varepsilon_{1}} a_{n}^{2 \beta_{L} \gamma+\gamma} a_{n}^{-\frac{\alpha}{2}} t_{0} K_{0}^{q} \\
& \leq C_{4} a_{n}^{\gamma-1-\alpha / 2+2\left(\frac{1}{2}-7 \varepsilon_{1}\right) \gamma-\varepsilon_{1}} \\
& =C_{4} a_{n}^{2 \gamma-1-\alpha / 2-15 \varepsilon_{1}} \leq C_{4} a_{n}^{\varepsilon_{1}}
\end{aligned}
$$

by 9.20 . And so, we are done with the proof of Propostion 9.1.2.

\subsection{Heat kernel estimates}

Remember that $C, c>0$ denote constants that may change its values from line to line. As necessary we will highlight the dependence of $c$ on various quantities. This section will be concerned with estimates for the heat kernel in $\mathbb{R}^{q}$ defined by

$$
p_{t}(x)=(2 \pi t)^{-\frac{q}{2}} \exp \left(-\frac{|x|^{2}}{2 t}\right)
$$

and its derivative in space

$$
p_{t, l}(x):=\partial_{x_{l}} p_{t}(x)=-\frac{x_{l}}{t} p_{t}(x), 1 \leq l \leq q,
$$

for $x \in \mathbb{R}^{q}, t>0$. There are already a number of results in Section 5 of [MPS06] regarding bounds on heat kernels, in particular when they are connected by a 
correlation kernel as well as in Section 4 of [MP11] regarding the derivatives of heat kernels.

Here, we will combine the techniques used for those results in order to obtain bounds on integrals of the derivatives $p_{t, l}$ that are connected by a correlation kernel related to colored noise.

This first simple lemma will be used frequently later on:

Lemma 9.3.1. Let $0<r_{0} \leq r_{1}$. Then there is a constant $C=C\left(r_{0}, r_{1}\right)>0$ such that for all $r \in\left[r_{0}, r_{1}\right]$ and $a \geq 0, u>0$,

$$
a \leq C u^{1 / r} \exp \left(\frac{a^{r}}{u}\right) \leq C u^{1 / r_{0}} \exp \left(\frac{a^{r}}{u}\right) .
$$

Proof. Consider for $r, u>0$ the function

$$
f(a)=a \exp \left(-\frac{a^{r}}{u}\right), a \geq 0,
$$

which attains its maximal value $u^{1 / r}\left(\frac{1}{r}\right)^{1 / r} \exp (-1 / r)$ at $a=\left(\frac{u}{r}\right)^{1 / r}$. Hence, choose $C\left(r_{0}, r_{1}\right)=\max _{r \in\left[r_{0}, r_{1}\right]}\left(\frac{1}{r}\right)^{1 / r} \exp (-1 / r)$ to obtain the result.

We apply that lemma to get the following basic estimate:

Lemma 9.3.2. For the heat kernel in $\mathbb{R}^{q}$ there is a uniform constant $C>0$ such that for all $l=1, \ldots, q, t>0, x \in \mathbb{R}^{q}$ :

$$
\left|p_{t, l}(x)\right| \leq C \frac{1}{\sqrt{t}} p_{2 t}(x)
$$

Proof. We have by Lemma 9.3.1 applied with $a=\frac{|x|}{2 \sqrt{t}}, u=1, r=2$,

$$
\left|p_{t, l}(x)\right| \leq \frac{1}{\sqrt{t}} \frac{|x|}{\sqrt{t}}(2 \pi t)^{-q / 2} \exp \left(-\frac{|x|^{2}}{2 t}\right) \leq C \frac{1}{\sqrt{t}}(4 \pi t)^{-q / 2} \exp \left(-\frac{|x|^{2}}{4 t}\right),
$$

which proves the result.

Next, we state a lemma about the spatial and temporal distances of heat kernel derivatives:

Lemma 9.3.3. There is a uniform constant $C>0$ such that for any $0<t<t^{\prime}$, $w, v \in \mathbb{R}^{q}$ the following holds for $l=1, \ldots, q$ :

(a) Setting $\hat{v}_{0}:=0$ and $\hat{v}_{i}:=\hat{v}_{i-1}+v_{i} e_{i}, 1 \leq i \leq q$, where $e_{i}$ is the $i$-th unit vectors in $\mathbb{R}^{q}$, we have for the spatial differences

$$
\left|p_{t, l}(w+v)-p_{t, l}(w)\right| \leq C t^{-1} \sum_{i=1}^{q} \int_{0}^{\left|v_{i}\right|} d r_{i} p_{2 t}\left(w+\hat{v}_{i-1}+r_{i} e_{i}\right) .
$$


(b) We obtain for the time differences

$$
\left|p_{t, l}(w)-p_{t^{\prime}, l}(w)\right| \leq C\left|t-t^{\prime}\right|^{\frac{1}{2}} t^{-\frac{1}{2}}\left(t^{-1 / 2} p_{2 t}(w)+t^{\prime-1 / 2} p_{4 t^{\prime}}(w)\right)
$$

Proof. We follow [MPS06, page 1932]. Without loss of generality we can assume that $l=1$. Then we consider for (a):

$$
\begin{aligned}
\left|\frac{w_{1}}{t} \exp \left(-\frac{|w|^{2}}{2 t}\right)-\frac{w_{1}+v_{1}}{t} \exp \left(-\frac{|w+v|^{2}}{2 t}\right)\right| \\
\leq\left|\frac{w_{1}}{t} \exp \left(-\frac{|w|^{2}}{2 t}\right)-\frac{w_{1}+v_{1}}{t} \exp \left(-\frac{\left|w+\hat{v}_{1}\right|^{2}}{2 t}\right)\right|+ \\
\quad+\sum_{i=2}^{q}\left|\frac{w_{1}+v_{1}}{t}\right|\left|\exp \left(-\frac{\left|w+\hat{v}_{i-1}\right|^{2}}{2 t}\right)-\exp \left(-\frac{\left|w+\hat{v}_{i}\right|^{2}}{2 t}\right)\right|
\end{aligned}
$$

Now, observe that

$$
\partial_{x_{1}}\left(x_{1} / t \exp \left(-|x|^{2} /(2 t)\right)\right)=t^{-1} \exp \left(-|x|^{2} /(2 t)\right)-\left(x_{1} / t\right)^{2} \exp \left(-|x|^{2} /(2 t)\right)
$$

and $\partial_{x_{1}} \exp \left(-|x|^{2} /(2 t)\right)=-\left(x_{1} / t\right) \exp \left(-|x|^{2} /(2 t)\right)$. Hence, the above is bounded by

$$
\begin{gathered}
\left|\int_{0}^{\left|v_{1}\right|} d r_{1}\left[t^{-1} \exp \left(-\frac{\left|w+r_{1} e_{1}\right|^{2}}{2 t}\right)-\left(\frac{w_{1}+r_{1}}{t}\right)^{2} \exp \left(-\frac{\left|w+r_{1} e_{1}\right|^{2}}{2 t}\right)\right]\right| \\
+\frac{\left|w_{1}+v_{1}\right|}{t} \sum_{i=2}^{q}\left|\int_{0}^{\left|v_{i}\right|} d r_{i} \frac{w_{i}+r_{i}}{t} \exp \left(-\frac{\left|w+\hat{v}_{i-1}+r_{i} e_{i}\right|^{2}}{2 t}\right)\right| .
\end{gathered}
$$

Now use 9.32 twice with $a=\frac{w_{i}+r_{i}}{\sqrt{t}}, u=4$ and $r=1$ respectively $r=2$ to bound this further by

$$
\begin{aligned}
& t^{-1} \int_{0}^{\left|v_{1}\right|} d r_{1}\left(\exp \left(-\frac{\left|w+r_{1} e_{1}\right|^{2}}{2 t}\right)+C \exp \left(-\frac{\left|w+r_{1} e_{1}\right|^{2}}{2 t}+\frac{\left|w_{1}+r_{1}\right|^{2}}{4 t}\right)\right) \\
& \quad+C \sum_{i=2}^{q} \int_{0}^{\left|v_{i}\right|} d r_{i} \exp \left(-\frac{\left|w+\hat{v}_{i-1}+r_{i} e_{i}\right|^{2}}{2 t}+\frac{\left|w_{i}+r_{i}\right|^{2}}{4 t}+\frac{\left|w_{1}+v_{1}\right|^{2}}{4 t}\right) \\
& \leq c t^{-1} \int_{0}^{\left|v_{1}\right|} d r_{1} \exp \left(-\frac{\left|w+r_{1} e_{1}\right|^{2}}{4 t}\right)+c t^{-1} \sum_{i=2}^{q} \int_{0}^{\left|v_{i}\right|} d r_{i} \exp \left(-\frac{\left|w+\hat{v}_{i-1}+r_{i} e_{i}\right|^{2}}{4 t}\right) .
\end{aligned}
$$

And the result follows by multiplication with $(2 \pi t)^{-q / 2}$.

To prove (b) we consider the time differences, following (52) in [MPS06]. First, 
rewriting and then using the Mean Value Theorem we get

$$
\begin{aligned}
\left|p_{t, 1}(w)-p_{t^{\prime}, 1}(w)\right|= & (2 \pi)^{-q / 2}\left|\frac{w_{1}}{t} t^{-q / 2} \exp \left(-\frac{|w|^{2}}{2 t}\right)-\frac{w_{1}}{t^{\prime}} t^{\prime-q / 2} \exp \left(-\frac{|w|^{2}}{2 t^{\prime}}\right)\right| \\
\leq & (2 \pi)^{-q / 2}\left|\left(t^{1 / 2}\right)^{-q-2}-\left(t^{\prime / 2}\right)^{-q-2}\right||w| \exp \left(-\frac{|w|^{2}}{2 t}\right) \\
& +(2 \pi)^{-q / 2}|w| t^{\prime-q / 2-1}\left|\exp \left(-\frac{|w|^{2}}{2 t}\right)-\exp \left(-\frac{|w|^{2}}{2 t^{\prime}}\right)\right| \\
\leq & (2 \pi)^{-q / 2}(q+2)|w|\left|t^{1 / 2}-t^{\prime 1 / 2}\right|\left(t^{1 / 2}\right)^{-q-3} \exp \left(-\frac{|w|^{2}}{2 t}\right)+ \\
& +(2 \pi)^{-q / 2}|w| t^{\prime-1-q / 2} \int_{t^{1 / 2}}^{t^{\prime 1 / 2}} \exp \left(-\frac{|w|^{2}}{2 s^{2}}\right) \frac{|w|^{2}}{s^{3}} d s
\end{aligned}
$$

Using $a \leq \exp (a)$ for $a=|w|^{2} /\left(4 s^{2}\right)$, we have

$\int_{t^{1 / 2}}^{t^{\prime / 2}} \exp \left(-\frac{|w|^{2}}{2 s}\right) \frac{|w|^{2}}{s^{3}} d s \leq \int_{t^{1 / 2}}^{t^{\prime / 2}} \frac{4}{s} \exp \left(-\frac{|w|^{2}}{4 s^{2}}\right) d s \leq\left|t^{1 / 2}-t^{\prime 1 / 2}\right| \frac{4}{t^{1 / 2}} \exp \left(-\frac{|w|^{2}}{4 t^{\prime}}\right)$.

Using further 9.32 in both lines of the above expression, we can bound it by

$$
\begin{aligned}
\left|p_{t, 1}(w)-p_{t^{\prime}, 1}(w)\right| \leq & (2 \pi)^{-q / 2} c t^{-1-q / 2}\left|t^{1 / 2}-t^{\prime 1 / 2}\right| \exp \left(-\frac{|w|^{2}}{4 t}\right) \\
& +(2 \pi)^{-q / 2} c t^{\prime-1 / 2-q / 2}\left|t^{1 / 2}-t^{\prime 1 / 2}\right| t^{-1 / 2} \exp \left(-\frac{|w|^{2}}{8 t^{\prime}}\right) \\
\leq & c\left|t^{1 / 2}-t^{\prime 1 / 2}\right| t^{-1 / 2}\left(t^{-1 / 2} p_{2 t}(w)+t^{\prime-1 / 2} p_{4 t^{\prime}}(w)\right) .
\end{aligned}
$$

Now, we can state a lemma concerning the distances of heat kernel derivatives:

Lemma 9.3.4. For $\alpha \in(0, q), K \geq 0$, there is a positive constant $C=C(\alpha, q, K)<$ $\infty$ such that for any $x, x^{\prime} \in \mathbb{R}^{q}, 0<t \leq t^{\prime} \leq K$

$$
\begin{gathered}
\int_{\mathbb{R}^{q}} \int_{\mathbb{R}^{q}}\left|\left(p_{t, l}(w-x)-p_{t^{\prime}, l}\left(w-x^{\prime}\right)\right)\left(p_{t, l}(z-x)-p_{t^{\prime}, l}\left(z-x^{\prime}\right)\right)\right|\left(|w-z|^{-\alpha}+1\right) d w d z \\
\leq C t^{-1-\alpha / 2}\left(1 \wedge \frac{\left|x-x^{\prime}\right|^{2}+\left|t-t^{\prime}\right|}{t}\right) .
\end{gathered}
$$

Proof. There are two estimates to make, one for each part of the $\wedge$. 
First, let us consider the left part. Expanding the product in the integral gives

$$
\begin{aligned}
\int_{\mathbb{R}^{q}} \int_{\mathbb{R}^{q}} \mid & \left(p_{t, l}(w-x)-p_{t^{\prime}, l}\left(w-x^{\prime}\right)\right)\left(p_{t, l}(z-x)-p_{t^{\prime}, l}\left(z-x^{\prime}\right)\right) \mid\left(|w-z|^{-\alpha}+1\right) d w d z \\
\leq & \int_{\mathbb{R}^{q}} \int_{\mathbb{R}^{q}}\left|p_{t, l}(w-x) p_{t, l}(z-x)\right|\left(|w-z|^{-\alpha}+1\right) d w d z \\
& +\int_{\mathbb{R}^{q}} \int_{\mathbb{R}^{q}}\left|p_{t^{\prime}, l}\left(w-x^{\prime}\right) p_{t^{\prime}, l}\left(z-x^{\prime}\right)\right|\left(|w-z|^{-\alpha}+1\right) d w d z \\
& +\int_{\mathbb{R}^{q}} \int_{\mathbb{R}^{q}}\left|p_{t, l}(w-x) p_{t^{\prime}, l}\left(z-x^{\prime}\right)\right|\left(|w-z|^{-\alpha}+1\right) d w d z \\
& +\int_{\mathbb{R}^{q}} \int_{\mathbb{R}^{q}}\left|p_{t^{\prime}, l}\left(w-x^{\prime}\right) p_{t, l}(z-x)\right|\left(|w-z|^{-\alpha}+1\right) d w d z
\end{aligned}
$$

Note that by a change of variables (and $|w|=|-w|$ ) the last two lines coincide. The same is true for the first two lines except that $t$ and $t^{\prime}$ differ. Thus, expression (9.35) is equal to

$$
\begin{aligned}
& \int_{\mathbb{R}^{q}} \int_{\mathbb{R}^{q}}\left|p_{t, l}(w) p_{t, l}(z)\right|\left(|w-z|^{-\alpha}+1\right) d w d z \\
& +\int_{\mathbb{R}^{q}} \int_{\mathbb{R}^{q}}\left|p_{t^{\prime}, l}(w) p_{t^{\prime}, l}(z)\right|\left(|w-z|^{-\alpha}+1\right) d w d z \\
& +2 \int_{\mathbb{R}^{q}} \int_{\mathbb{R}^{q}}\left|p_{t, l}\left(w-\left(x-x^{\prime}\right)\right) p_{t^{\prime}, l}(z)\right|\left(|w-z|^{-\alpha}+1\right) d w d z .
\end{aligned}
$$

For the first line of $(9.36)$ we write, using $\left|w_{l}\right| \leq|w|$ and $(9.32)$ :

$$
\begin{aligned}
& t^{-1} \int_{\mathbb{R}^{q}} \int_{\mathbb{R}^{q}}(2 \pi t)^{-q} \frac{\left|w_{l}\right|}{\sqrt{t}} \exp \left(-\frac{|w|^{2}}{2 t}\right) \frac{\left|z_{l}\right|}{\sqrt{t}} \exp \left(-\frac{|z|^{2}}{2 t}\right)\left(|w-z|^{-\alpha}+1\right) d w d z \\
& \leq c t^{-1} \int_{\mathbb{R}^{q}} \int_{\mathbb{R}^{q}}(2 \pi t)^{-q} \exp \left(-\frac{|w|^{2}}{4 t}\right) \exp \left(-\frac{|z|^{2}}{4 t}\right)\left(|w-z|^{-\alpha}+1\right) d w d z, \\
& \leq c(\alpha, q)\left(t^{-\alpha / 2-1}+t^{-1}\right)
\end{aligned}
$$

by an application of Lemma 5.1 in [MPS06] and the fact that $t \leq t^{\prime}$. For the second line (with $t^{\prime}$ ) we can do exactly the same and obtain the same even with $t$ instead of $t^{\prime}$, since $t \leq t^{\prime}$. 
For the third line of 9.36 the same reasoning leads to the bound

$$
\begin{aligned}
& \quad 2\left(t t^{\prime}\right)^{-1 / 2} \int_{\mathbb{R}^{q}} \int_{\mathbb{R}^{q}}(2 \pi)^{-q}\left(t t^{\prime}\right)^{-q / 2} \frac{\left|\left(w-\left(x-x^{\prime}\right)\right)_{l}\right|}{\sqrt{t}} \exp \left(-\frac{\left|w-\left(x-x^{\prime}\right)\right|^{2}}{2 t}\right) \\
& \frac{|z| \mid}{\sqrt{t^{\prime}}} \exp \left(-\frac{|z|^{2}}{2 t^{\prime}}\right)\left(|w-z|^{-\alpha}+1\right) d w d z \\
& \leq c t^{-1} \int_{\mathbb{R}^{q}} \int_{\mathbb{R}^{q}}(2 \pi t)^{-q} \exp \left(-\frac{\left|w-\left(x-x^{\prime}\right)\right|^{2}}{4 t}\right) \exp \left(-\frac{|z|^{2}}{4 t^{\prime}}\right)\left(|w-z|^{-\alpha}+1\right) d w d z, \\
& \leq c(\alpha, q)\left(t^{-\alpha / 2-1}+t^{-1}\right)
\end{aligned}
$$

by an application of Lemma 5.1 in [MPS06] and $t \leq t^{\prime} \leq K$.

So this was the first part of the $\wedge$. To consider the second estimate, we start with a decomposition:

$$
\begin{aligned}
\mid\left(p_{t, l}(w-x)-\right. & \left.p_{t^{\prime}, l}\left(w-x^{\prime}\right)\right)\left(p_{t, l}(z-x)-p_{t^{\prime}, l}\left(z-x^{\prime}\right)\right) \mid \\
\leq & \left|\left(p_{t, l}(w-x)-p_{t, l}\left(w-x^{\prime}\right)\right)\left(p_{t, l}(z-x)-p_{t, l}\left(z-x^{\prime}\right)\right)\right| \\
& +\left|\left(p_{t, l}(w-x)-p_{t, l}\left(w-x^{\prime}\right)\right)\left(p_{t, l}\left(z-x^{\prime}\right)-p_{t^{\prime}, l}\left(z-x^{\prime}\right)\right)\right| \\
& +\left|\left(p_{t, l}\left(w-x^{\prime}\right)-p_{t^{\prime}, l}\left(w-x^{\prime}\right)\right)\left(p_{t, l}(z-x)-p_{t, l}\left(z-x^{\prime}\right)\right)\right| \\
& +\left|\left(p_{t, l}\left(w-x^{\prime}\right)-p_{t^{\prime}, l}\left(w-x^{\prime}\right)\right)\left(p_{t, l}\left(z-x^{\prime}\right)-p_{t^{\prime}, l}\left(z-x^{\prime}\right)\right)\right| .
\end{aligned}
$$

We start with the simplest case in (9.37):

$$
\int_{\mathbb{R}^{q}} \int_{\mathbb{R}^{q}}\left|\left(p_{t, l}(w-x)-p_{t, l}\left(w-x^{\prime}\right)\right)\left(p_{t, l}(z-x)-p_{t, l}\left(z-x^{\prime}\right)\right)\right|\left(|w-z|^{-\alpha}+1\right) d w d z .
$$

Changing variables, setting $v=x-x^{\prime}$ and using Lemma 9.3.3, we bound this by

$$
\begin{gathered}
c \int_{\mathbb{R}^{q}} \int_{\mathbb{R}^{q}}\left[t^{-1} \sum_{i=1}^{q} \int_{0}^{\left|v_{i}\right|} d r_{i} p_{2 t}\left(w+\hat{v}_{i-1}+r_{i} e_{i}\right)\right]\left[t^{-1} \sum_{j=1}^{q} \int_{0}^{\left|v_{j}\right|} d \tilde{r}_{j} p_{2 t}\left(z+\hat{v}_{j-1}+\tilde{r}_{j} e_{j}\right)\right] \\
=c t^{-2} \sum_{i=1}^{q} \int_{0}^{\left|v_{i}\right|} d r_{i} \sum_{j=1}^{q} \int_{0}^{\left|v_{j}\right|} d \tilde{r}_{j} \\
\quad \int_{\mathbb{R}^{q}} \int_{\mathbb{R}^{q}} p_{2 t}\left(w+\hat{v}_{i-1}+r_{i} e_{i}\right) p_{2 t}\left(z+\hat{v}_{j-1}+\tilde{r}_{j} e_{j}\right)\left(|w-z|^{-\alpha}+1\right) d w d z \\
\leq c t^{-2}\left(t^{-\alpha / 2}+1\right) \max _{i, j}\left|v_{i} v_{j}\right| \leq c t^{-2}\left(t^{-\alpha / 2}+1\right)|v|_{2}^{2}=c t^{-2}\left(t^{-\alpha / 2}+1\right)\left|x-x^{\prime}\right|_{2}^{2} .
\end{gathered}
$$

using Lemma 5.1 (a) of [MPS06] in the last step (compare this with Lemma 5.2 (b) in [MPS06]). 
Now, we consider the temporal distances in (9.37), i.e. the last line. There we get by Lemma 9.3 .3 and Lemma 5.1 (a) of [MPS06]:

$$
\begin{aligned}
& \int_{\mathbb{R}^{q}} \int_{\mathbb{R}^{q}}\left|\left(p_{t, l}\left(w-x^{\prime}\right)-p_{t^{\prime}, l}\left(w-x^{\prime}\right)\right)\left(p_{t, l}\left(z-x^{\prime}\right)-p_{t^{\prime}, l}\left(z-x^{\prime}\right)\right)\right|\left(|w-z|^{-\alpha}+1\right) d w d z \\
& \leq c\left|t-t^{\prime}\right| t^{-2}\left(t^{-\alpha / 2}+1\right)
\end{aligned}
$$

and this is the next part of the proposition - similar to Lemma 5.3 in [MPS06. The mixed parts in (9.37) just lie in between the two extreme cases (use $2 a b \leq$ $\left.a^{2}+b^{2}\right)$.

Now we give two technical lemmas required in the next chapters:

Lemma 9.3.5. For $R>0$ there is a constant $C=C(R)$ such that for any $y, \tilde{y} \in \mathbb{R}^{q}$, $0<t \leq t^{\prime}$ and $\eta_{0} \in(1 / R, 1 / 2)$ the following holds for $l=1, \ldots, q$ :

(a) $\mathbb{1}\left\{|\tilde{y}|>t^{\prime 1 / 2-\eta_{0}} \vee 2|y-\tilde{y}|\right\}\left|p_{t, l}(y)\right| \leq C \exp \left(-\frac{1}{64} t^{-2 \eta_{0}}\right) p_{4 t}(y)$.

(b) $\mathbb{1}\left\{|\tilde{y}|>t^{\prime 1 / 2-\eta_{0}} \vee 2|y-\tilde{y}|\right\}\left|p_{t, l}(y)\right| \leq 2^{q} C \exp \left(-\frac{1}{64} t^{-2 \eta_{0}}\right) p_{16 t}(\tilde{y})$.

Proof. Let us write $A:=\left\{|\tilde{y}|>t^{\prime 1 / 2-\eta_{0}} \vee 2|y-\tilde{y}|\right\}$, then on that event it holds that

$$
|y| \geq|\tilde{y}|-|y-\tilde{y}|>\frac{|\tilde{y}|}{2}>\frac{t^{\prime 1 / 2-\eta_{0}}}{2} \geq \frac{t^{1 / 2-\eta_{0}}}{2} \text {, thus } \frac{|y|^{2}}{t} \geq \frac{t^{-2 \eta_{0}}}{4} .
$$

Using this and $(9.32)$ twice, we calculate

$$
\begin{aligned}
\mathbb{1}_{A}\left|p_{t, l}(y)\right| & =\mathbb{1}_{A} \frac{\left|y_{l}\right|}{t}(2 \pi t)^{-q / 2} \exp \left(-\frac{|y|^{2}}{2 t}\right) \\
& \leq \mathbb{1}_{A} \frac{|y|}{t}(2 \pi t)^{-q / 2} \exp \left(-\frac{|y|^{2}}{2 t}\right) \\
& \leq \mathbb{1}_{A} C t^{-1 / 2}(2 \pi t)^{-q / 2} \exp \left(-\frac{|y|^{2}}{4 t}\right) \\
& =\mathbb{1}_{A} C t^{-1 / 2} \exp \left(-\frac{|y|^{2}}{8 t}\right)(2 \pi t)^{-q / 2} \exp \left(-\frac{|y|^{2}}{8 t}\right) \\
& \leq \mathbb{1}_{A} C t^{-1 / 2} \exp \left(-\frac{1}{32} t^{-2 \eta_{0}}\right)(2 \pi t)^{-q / 2} \exp \left(-\frac{|y|^{2}}{8 t}\right) \\
& \leq \mathbb{1}_{A} C(R) \exp \left(-\frac{1}{64} t^{-2 \eta_{0}}\right) p_{4 t}(y) .
\end{aligned}
$$

Given that on the set $A$ we have

$$
|\tilde{y}|<2|y|, \text { thus }|y|^{2} \geq \frac{|\tilde{y}|^{2}}{4}
$$

we can bound this further by

$$
\mathbb{1}_{A}\left|p_{t, l}(y)\right| \leq C(R) \exp \left(-\frac{1}{64} t^{-2 \eta_{0}}\right) p_{16 t}(\tilde{y}) .
$$


Lemma 9.3.6. For $0<t \leq t^{\prime}, 0 \leq r_{1}, r_{2}, r_{3} \leq R$, there is a constant $C=C(R)$ such that

$$
\begin{array}{r}
\int_{\mathbb{R}^{q}} \int_{\mathbb{R}^{q}} p_{t}(w) p_{t^{\prime}}(z)|w|^{r_{1}}|z|^{r_{2}} e^{r_{3}(|w|+|z|)}\left(|w-z|^{-\alpha}+1\right) d w d z \\
\leq C e^{2 r_{3}^{2} t^{\prime}} t^{r_{1} / 2} t^{\prime r_{2} / 2}\left(t^{-\alpha / 2}+1\right)
\end{array}
$$

and for $x, y \in[-K, K]^{q}$ there is a constant $C=C(K, R)$

$$
\begin{array}{r}
\int_{\mathbb{R}^{q}} \int_{\mathbb{R}^{q}} p_{t}(x-w) p_{t^{\prime}}(y-z)|w|^{r_{1}}|z|^{r_{2}} e^{r_{3}(|w|+|z|)}\left(|w-z|^{-\alpha}+1\right) d w d z \\
\leq C e^{2 r_{3}^{2} t^{\prime}}\left(t^{r_{1} / 2}+1\right)\left(t^{\prime r_{2} / 2}+1\right)\left(t^{-\alpha / 2}+1\right) .
\end{array}
$$

Proof. By 9.32 we bound

$$
|w|^{r_{1}} p_{t}(w) \leq 4^{r_{1} / 2+q / 2} t^{r_{1} / 2} p_{2 t}(w) \quad \text { and } \quad|z|^{r_{2}} p_{t^{\prime}}(z) \leq 4^{r_{2} / 2+q / 2} t^{\prime r_{2} / 2} p_{2 t^{\prime}}(z) .
$$

Next apply Lemma 5.1 (b) of [MPS06] if $r_{3}>0$ and their Lemma 5.1 (a) if $r_{3}=0$, to get the estimate.

For the second estimate note that by $(9.32)$ and $|x| \leq \sqrt{q} K$

$$
\begin{aligned}
p_{t}(x-w)|w|^{r_{1}} & \leq p_{t}(x-w) 2^{r_{1}}\left(|w-x|^{r_{1}}+|x|^{r_{1}}\right) \\
& \leq 2^{R}(4 t)^{r_{1} / 2} p_{2 t}(x-w)+(2 \sqrt{q} K)^{r_{1}} p_{t}(x-w) \\
& \leq c(K, R) p_{2 t}(x-w)\left(t^{r_{1} / 2}+1\right)
\end{aligned}
$$

and obtain the result by the first part.

Lemma 9.3.7. For all $R>2, K>0$, there is a constant $C=C(K, R)$ such that for all $0 \leq p, r \leq R, \eta_{0}, \eta_{1} \in(1 / R, 1 / 2), l=1, \ldots, q, 0 \leq s<t \leq t^{\prime}<K$ and $x, x^{\prime} \in[-K, K]^{q}:$

$$
\begin{aligned}
\int_{\mathbb{R}^{q}} \int_{\mathbb{R}^{q}} & |w-x|^{p}|z-x|^{p}\left(p_{t-s, l}(w-x)-p_{t^{\prime}-s, l}\left(w-x^{\prime}\right)\right)\left(p_{t-s, l}(z-x)-p_{t^{\prime}-s, l}\left(z-x^{\prime}\right)\right) \\
& \times \mathbb{1}\left\{|w-x|>\left(t^{\prime}-s\right)^{1 / 2-\eta_{0}} \vee 2\left|x-x^{\prime}\right|\right\} e^{r|w-x|+r|z-x|}\left(|w-z|^{-\alpha}+1\right) d w d z \\
& \leq C(t-s)^{-1-\alpha / 2} \exp \left(-\eta_{1}\left(t^{\prime}-s\right)^{-2 \eta_{0}} / 256\right)\left[1 \wedge\left(\frac{\left|x-x^{\prime}\right|^{2}+\left|t-t^{\prime}\right|}{t-s}\right)\right]^{1-\eta_{1} / 2}
\end{aligned}
$$


Proof. By Hölder's Inequality we can bound the left hand side in $(9.38)$ by

$$
\begin{aligned}
& {\left[\int_{\mathbb{R}^{q}} \int_{\mathbb{R}^{q}}\left|\left(p_{t-s, l}(w-x)-p_{t^{\prime}-s, l}\left(w-x^{\prime}\right)\right)\left(p_{t-s, l}(z-x)-p_{t^{\prime}-s, l}\left(z-x^{\prime}\right)\right)\right|\right.} \\
&\left.\left(|w-z|^{-\alpha}+1\right) d w d z\right]^{1-\eta_{1} / 2} \\
& \times {\left[\int_{\mathbb{R}^{q}} \int_{\mathbb{R}^{q}}\left|\left(p_{t-s, l}(w-x)-p_{t^{\prime}-s, l}\left(w-x^{\prime}\right)\right)\left(p_{t-s, l}(z-x)-p_{t^{\prime}-s, l}\left(z-x^{\prime}\right)\right)\right|\right.} \\
&|w-x|^{2 p / \eta_{1}}|z-x|^{2 p / \eta_{1}} \mathbb{1}\left\{|w-x|>\left(t^{\prime}-s\right)^{1 / 2-\eta_{0}} \vee 2\left|x-x^{\prime}\right|\right\} \\
&\left.e^{2 r / \eta_{1}(|w-x|+|z-x|)}\left(|w-z|^{-\alpha}+1\right) d w d z\right]^{\eta_{1} / 2} .
\end{aligned}
$$

Now estimate the first integral using Lemma 9.3.4 and expand the second one to obtain the bound for 9.38

$$
\begin{aligned}
& c(R)(t-s)^{-(1+\alpha / 2)\left(1-\eta_{1} / 2\right)}\left[1 \wedge\left(\frac{\left|x-x^{\prime}\right|^{2}+\left|t-t^{\prime}\right|}{t-s}\right)\right]^{1-\eta_{1} / 2} \\
& \times\left[\int_{\mathbb{R}^{q}} \int_{\mathbb{R}^{q}}\left|p_{t-s, l}(w-x) p_{t-s, l}(z-x)\right| L\left(x, x^{\prime}, w, z, s, t^{\prime}\right) d w d z\right. \\
& \quad+\int_{\mathbb{R}^{q}} \int_{\mathbb{R}^{q}}\left|p_{t^{\prime}-s, l}\left(w-x^{\prime}\right) p_{t^{\prime}-s, l}\left(z-x^{\prime}\right)\right| L\left(x, x^{\prime}, w, z, s, t^{\prime}\right) d w d z \\
& \quad+\int_{\mathbb{R}^{q}} \int_{\mathbb{R}^{q}}\left|p_{t-s, l}(w-x) p_{t^{\prime}-s, l}\left(z-x^{\prime}\right)\right| L\left(x, x^{\prime}, w, z, s, t^{\prime}\right) d w d z \\
& \left.\quad+\int_{\mathbb{R}^{q}} \int_{\mathbb{R}^{q}}\left|p_{t^{\prime}-s, l}\left(w-x^{\prime}\right) p_{t-s, l}(z-x)\right| L\left(x, x^{\prime}, w, z, s, t^{\prime}\right) d w d z\right]^{\eta_{1} / 2},
\end{aligned}
$$

where

$$
\begin{aligned}
L\left(x, x^{\prime}, w, z, s, t^{\prime}\right):= & |w-x|^{2 p / \eta_{1}}|z-x|^{2 p / \eta_{1}} \mathbb{1}\left\{|w-x|>\left(t^{\prime}-s\right)^{1 / 2-\eta_{0}} \vee 2\left|x-x^{\prime}\right|\right\} \\
& e^{2 r / \eta_{1}(|w-x|+|z-x|)}\left(|w-z|^{-\alpha}+1\right) .
\end{aligned}
$$

Since all of the four summands in the end are similar, we only consider the last one which is the worst with respect to $(t-s)$-asymptotics. Use Lemma 9.3.2, replace 
$\tilde{w}=w-x, \tilde{z}=z-x$ and then use Lemma 9.3.5 (b) to obtain

$$
\begin{aligned}
& \int_{\mathbb{R}^{q}} \int_{\mathbb{R}^{q}}\left|p_{t^{\prime}-s, l}\left(w-x^{\prime}\right) p_{t-s, l}(z-x)\right||w-x|^{2 p / \eta_{1}}|z-x|^{2 p / \eta_{1}} \\
& \times \mathbb{1}\left\{|w-x|>\left(t^{\prime}-s\right)^{1 / 2-\eta_{0}} \vee 2\left|x-x^{\prime}\right|\right\} e^{2 r / \eta_{1}(|w-x|+|z-x|)}\left(|w-z|^{-\alpha}+1\right) d w d z \\
& \leq c \int_{\mathbb{R}^{q}} \int_{\mathbb{R}^{q}}\left|p_{t^{\prime}-s, l}\left(\tilde{w}+x-x^{\prime}\right)\right|(t-s)^{-1 / 2} p_{2(t-s)}(\tilde{z})|\tilde{w}|^{2 p / \eta_{1}}|\tilde{z}|^{2 p / \eta_{1}} \\
& \times \mathbb{1}\left\{|\tilde{w}|>\left(t^{\prime}-s\right)^{1 / 2-\eta_{0}} \vee 2\left|x-x^{\prime}\right|\right\} e^{2 r / \eta_{1}(|\tilde{w}|+|\tilde{z}|)}\left(|\tilde{w}-\tilde{z}|^{-\alpha}+1\right) d \tilde{w} d \tilde{z} \\
& \leq c(R) \int_{\mathbb{R}^{q}} \int_{\mathbb{R}^{q}} \exp \left(-\frac{1}{64}\left(t^{\prime}-s\right)^{-2 \eta_{0}}\right) p_{16\left(t^{\prime}-s\right)}(\tilde{w}) p_{2(t-s)}(\tilde{z}) \\
& \times|\tilde{w}|^{2 p / \eta_{1}}|\tilde{z}|^{2 p / \eta_{1}}(t-s)^{-1 / 2} e^{2 r / \eta_{1}(|\tilde{w}|+|\tilde{z}|)}\left(|\tilde{w}-\tilde{z}|^{-\alpha}+1\right) d \tilde{w} d \tilde{z} . \\
& \leq c\left(R, \eta_{1}, K\right)(t-s)^{-1 / 2} \exp \left(-\frac{1}{64}\left(t^{\prime}-s\right)^{-2 \eta_{0}}\right) \\
& e^{32 r^{2} \eta_{1}^{-2}\left(t^{\prime}-s\right)}\left(t^{\prime}-s\right)^{p \eta_{1}^{-1}}(t-s)^{p \eta_{1}^{-1}}\left(\left(t^{\prime}-s\right)^{-\frac{\alpha}{2}}+1\right) . \\
& \leq c(R, K)(t-s)^{-1 / 2} \exp \left(-\frac{1}{128}\left(t^{\prime}-s\right)^{-2 \eta_{0}}\right),
\end{aligned}
$$

where we used Lemma 9.3.6. first part, in the next to last line and $\left(t^{\prime}-s\right) \leq K$. The other summands are similar, we use Lemma 9.3.5 (with $t=t^{\prime}$ for lines 1 and 3) and can use the exponential of $t^{\prime}-s(t-s$ in lines 1 and 3$)$ to control all of the negative exponents. Putting this back in $(9.39)$ gives the result, since $(1+\alpha / 2)\left(1-\eta_{1} / 2\right)+(1 / 2)\left(\eta_{1} / 2\right) \leq 1+\alpha / 2$.

\subsection{Local bounds on the difference of two solutions}

Within this section we present the extension of Theorem 5.3.3, i.e. the results showing "Hölder-continuity of order 2". This chapter is very similar in its ideas to Chapter 5 of [MP11].

Let us recall that for $n \in \mathbb{N}_{0}$,

$$
a_{n}=\exp (-n(n+1) / 2)
$$

and for $(t, x),\left(t^{\prime}, x^{\prime}\right) \in \mathbb{R}_{+} \times \mathbb{R}^{q}$ :

$$
d\left((t, x),\left(t^{\prime}, x^{\prime}\right)\right)=\sqrt{\left|t-t^{\prime}\right|}+\left|x-x^{\prime}\right| \quad(|\cdot| \text { Euclidean norm }) .
$$

Define for $N, K, n \in \mathbb{N}, \beta \in[0,1 / 2]$ the random set

$$
\begin{aligned}
& Z(N, n, K, \beta)(\omega)=\left\{(t, x) \in\left[0, T_{K}\right] \times[-K, K]^{q} \subset \mathbb{R}_{+} \times \mathbb{R}^{q}:\right. \text { there is a } \\
& \left(\hat{t}_{0}, \hat{x}_{0}\right) \in\left[0, T_{K}\right] \times \mathbb{R} \text { such that } d\left((t, x),\left(\hat{t}_{0}, \hat{x}_{0}\right)\right) \leq 2^{-N}, \\
& \left.\left|u\left(\hat{t}_{0}, \hat{x}_{0}\right)\right| \leq a_{n} \wedge\left(\sqrt{a_{n}} 2^{-N}\right), \text { and }\left|\nabla u_{1, a_{n}}\left(\hat{t}_{0}, \hat{x}_{0}\right)\right| \leq a_{n}^{\beta}\right\},
\end{aligned}
$$


For $\beta=0$ define $Z(N, n, K, 0)=Z(N, n, K)$ as above, but with the condition on $\nabla u_{1, a_{n}}$ omitted.

Note that $(t, x) \in Z(N, n, K, \beta)$ always implies $t \leq K$. For $\gamma<1$ define recursively $\gamma_{0}=1$ and

$$
\gamma_{m+1}=\gamma \gamma_{m}+1-\frac{\alpha}{2}
$$

This gives the explicit formula

$$
\gamma_{m}=1+\frac{(\gamma-\alpha / 2)\left(1-\gamma^{m}\right)}{1-\gamma}
$$

Since $\alpha<2(2 \gamma-1)$ we have $\gamma_{\infty}=\frac{1-\alpha / 2}{1-\gamma}>2$ so there will be an $\bar{m} \in \mathbb{N}$ such that $\gamma_{\bar{m}+1}>2 \geq \gamma_{\bar{m}}$. Set $\tilde{\gamma}_{m}:=\gamma_{m} \wedge 2,0 \leq m \leq \bar{m}+1$.

Definition 9.4.1. A collection of $[0, \infty]$-valued random variables $\{N(\alpha): \alpha \in A\}$ will be called stochastically bounded uniformly in $\alpha$, iff

$$
\lim _{M \rightarrow \infty} \sup _{\alpha \in A} \mathbb{P}[N(\alpha) \geq M]=0 .
$$

For $m \in \mathbb{Z}_{+}$, we will let $\left(P_{m}\right)$ denote the following property:

Property $\left(P_{m}\right)$. For any $n \in \mathbb{N}, \xi, \varepsilon_{0} \in(0,1), K \in \mathbb{N} \geq K_{1}$ and $\beta \in[0,1 / 2]$, there is an $N_{1}(\omega)=N_{1}\left(m, n, \xi, \varepsilon_{0}, K, \beta\right) \in \mathbb{N}$ a.s., such that for all $N \geq N_{1}$, if $(t, x) \in Z(N, n, K, \beta), t^{\prime} \leq T_{K}$ and $d\left((t, x),\left(t^{\prime}, x^{\prime}\right)\right) \leq 2^{-N}$, then

$$
\left|u\left(t^{\prime}, x^{\prime}\right)\right| \leq a_{n}^{-\varepsilon_{0}} 2^{-N \xi}\left[\left(\sqrt{a_{n}} \vee 2^{-N}\right)^{\gamma_{m}-1}+a_{n}^{\beta} \mathbb{1}\{m>0\}\right] .
$$

Moreover, $N_{1}$ is stochastically bounded uniformly in $(n, \beta)$.

Proposition 9.4.2. Property $\left(P_{m}\right)$ holds for any $m \leq \bar{m}+1$.

We first give a proof of the induction start, meaning that we prove $\left(P_{0}\right)$ :

Proof of Proposition 9.4.2, first part. We apply Theorem 5.3.3. Set $Z(N, K):=$ $Z(N, 0, K, 0)$, meaning that $a_{0}=1$. Let $\xi \in(0,1)$ and $\xi^{\prime}:=(1+\xi) / 2$. If $(t, x) \in$ $Z(N, n, K, \beta)$ then $(t, x) \in Z(N, K)$. Theorem 5.3.3 gives that there exists an $N_{0}\left(\xi^{\prime}, K+1\right)$ a.s. such that for $N \geq N_{0}\left(\xi^{\prime}, K+1\right)$ we have $\left|u(t, x)-u\left(\hat{t}_{0}, \hat{x}_{0}\right)\right| \leq$ $2^{-N \xi^{\prime}}$. Setting $N_{1}(0, \xi, K):=N_{0}\left(\xi^{\prime}(\xi), K+1\right) \vee 4(1-\xi)^{-1}$, we obtain for $N \geq$ $N_{1}(0, \xi, K)$,

$$
|u(t, x)| \leq 2^{-N \xi^{\prime}}+\left|u\left(\hat{t}_{0}, \hat{x}_{0}\right)\right| \leq 2^{-N \xi^{\prime}}+2^{-N} \leq 2^{1-N \xi^{\prime}} .
$$

And therefore, for $\left(t^{\prime}, x^{\prime}\right)$ with $t^{\prime} \leq T_{K}$ and $d\left((t, x),\left(t^{\prime}, x^{\prime}\right)\right) \leq 2^{-N}$ we obtain

$$
\left|u\left(t^{\prime}, x^{\prime}\right)\right| \leq\left|u\left(t^{\prime}, x^{\prime}\right)-u(t, x)\right|+|u(t, x)| \leq 2^{-N \xi^{\prime}}+2^{1-N \xi^{\prime}} \leq 2^{2-N \xi^{\prime}} \leq 2^{-N \xi}
$$

where the last inequality holds since $N \geq 4(1-\xi)^{-1}$. 
The induction step from $\left(P_{m}\right)$ to $\left(P_{m+1}\right)$ is a bit more technical and needs some preparation. It will be completed at the end of this section on page 146 .

To get there we first write down a lemma, which tells us what we can get out of Property $\left(P_{m}\right)$ :

Lemma 9.4.3. Let $0 \leq m \leq \bar{m}+1$. Assume that $\left(P_{m}\right)$ holds. Let $\eta, \xi, \varepsilon_{0}, K, \beta$ as in $\left(P_{m}\right)$. If $\bar{d}_{N}=2^{-N} \vee d((s, y),(t, x))$ and $C_{9.4 .3}(\omega)=\left(4 a_{n}^{-\varepsilon_{0}}+2^{2 N_{1}(\omega)} 2 K e^{K}\right)^{2}$, then for any fixed $N \in \mathbb{N}$. On the event

$$
\left\{\omega: N \geq N_{1}\left(m, n, \xi, \varepsilon_{0}, K, \beta\right),(t, x) \in Z(N, n, K, \beta)\right\},
$$

we have

$$
\begin{aligned}
|u(s, y)| \leq & \sqrt{C_{9.4 .3} e^{|y-x|}} \bar{d}_{N}^{\xi} \\
& \times\left[\left(\sqrt{a_{n}} \vee \bar{d}_{N}\right)^{\gamma_{m}-1}+\mathbb{1}\{m>0\} a_{n}^{\beta}\right]
\end{aligned}
$$

for all $s<T_{K}$ and $y \in \mathbb{R}^{q}$.

Proof. There are two cases to consider.

Case 1: $d=d((s, y),(t, x)) \leq 2^{-N_{1}}$.

Choose an $N^{\prime} \in\left\{N_{1}, \ldots, N\right\}$ such that

$$
\begin{aligned}
& \text { if } d>2^{-N}: 2^{-N^{\prime}-1}<d \leq 2^{-N^{\prime}}, \\
& \text { if } d \leq 2^{-N}: N^{\prime}:=N .
\end{aligned}
$$

Then, $(t, x) \in Z\left(N^{\prime}, n, K, \beta\right)$ and $d \leq 2^{-N^{\prime}} \leq 2 d \vee 2^{-N} \leq 2 \bar{d}_{N}$ and hence, Property $\left(P_{m}\right)$ yields:

$$
\begin{aligned}
|u(s, y)| & \leq a_{n}^{-\varepsilon_{0}} 2^{-N^{\prime} \xi}\left[\left(\sqrt{a_{n}} \vee 2^{-N^{\prime}}\right)^{\gamma_{m}-1}+a_{n}^{\beta} \mathbb{1}\{m>0\}\right] \\
& \leq 2 a_{n}^{-\varepsilon_{0}} \bar{d}_{N}^{\xi}\left[2\left(\sqrt{a_{n}} \vee \bar{d}_{N}\right)^{\gamma_{m}-1}+a_{n}^{\beta} \mathbb{1}\{m>0\}\right] .
\end{aligned}
$$

Case 2: $d>2^{-N_{1}}$.

If $K \geq K_{1}$ and $s \leq T_{K}$ :

$$
\begin{aligned}
|u(s, y)| & \leq 2 K e^{|y|} \leq 2 K e^{|y|}\left(d 2^{N_{1}}\right)^{\xi+\gamma_{m}-1} \\
& \leq 2 K e^{K} e^{|y-x|} 2^{2 N_{1}} \bar{d}_{N}^{\xi+\gamma_{m}-1} .
\end{aligned}
$$

This lemma gives control on $u(s, \cdot)$ spatially close to points in $Z(N, n, K, \beta)$. To do the induction step we want to use this control in $|D(r, w)| \leq R_{0} e^{R_{1}|w|}|u(r, w)|^{\gamma}$, which played a role for

$$
F_{\delta, l}(s, t, x)=\int_{0}^{(s-\delta)^{+}} p_{t-r, l}(w-x) D(r, w) W(d r d w),
$$


$\delta>0,0 \leq s \leq t, x \in \mathbb{R}^{q}$ and for $1 \leq l \leq q$. This was the derivative of $u_{1, \delta}$, as given in Lemma 9.2.1. This will lead to an even better bound on $u_{1, \delta}$ and this iterative procedure will lead to the induction step. Later, we will also give estimates for $u_{2, \delta}$. To estimate $F_{\delta, l}$ we use the following decomposition for $s \leq t \leq t^{\prime}, s^{\prime} \leq t^{\prime}$ :

$$
\begin{aligned}
\mid F_{\delta, l}(s, t, x)- & F_{\delta, l}\left(s^{\prime}, t^{\prime}, x^{\prime}\right) \mid \\
\leq & \left|F_{\delta, l}(s, t, x)-F_{\delta, l}\left(s, t, x^{\prime}\right)\right|+ \\
& +\left|F_{\delta, l}\left(s, t, x^{\prime}\right)-F_{\delta, l}\left(s, t^{\prime}, x^{\prime}\right)\right| \\
& +\left|F_{\delta, l}\left(s, t^{\prime}, x^{\prime}\right)-F_{\delta, l}\left(s^{\prime}, t^{\prime}, x^{\prime}\right)\right| \\
= & \left|\int_{0}^{(s-\delta)^{+}}\left(p_{t-r, l}\left(w-x^{\prime}\right)-p_{t-r, l}(w-x)\right) D(r, w) W(d r d w)\right| \\
& +\left|\int_{0}^{(s-\delta)^{+}}\left(p_{t-r, l}\left(w-x^{\prime}\right)-p_{t^{\prime}-r, l}\left(w-x^{\prime}\right)\right) D(r, w) W(d r d w)\right| \\
& +\left|\int_{\left(s \wedge s^{\prime}-\delta\right)^{+}}^{\left(s \vee s^{\prime}-\delta\right)^{+}} p_{t^{\prime}-r, l}\left(w-x^{\prime}\right) D(r, w) W(d r d w)\right|, 1 \leq l \leq q .
\end{aligned}
$$

All of these three expressions are martingales in the upper integral bound, where the rest of the values $x, x^{\prime}, t, t^{\prime},\left(s \wedge s^{\prime}-\delta\right)^{+}$stay fixed. We want to consider the quadratic variations of these martingales and use the Dubins-Schwarz theorem.

Remark 9.4.4. As for any spatial dimension $1 \leq l \leq q$ we would execute the same calculations we restrict ourselves now to $l=1$ for the estimates on $F_{\delta, l}$.

Dependence of constants on the universal constants $\alpha, q, \gamma, R_{0}$ and $R_{1}$ will not be mentioned in the following lemmas.

In order to calculate the first two of these quadratic variations, we need to introduce the following partition of $\mathbb{R}^{q}$ (for fixed values of $x, x^{\prime}, \eta_{0}$ ):

$$
\begin{aligned}
& A_{1}^{\eta_{0}}(r, t)=\mathbb{1}\left\{y \in \mathbb{R}^{q}:|y-x| \leq(t-r)^{1 / 2-\eta_{0}} \vee 2\left|x-x^{\prime}\right|\right\}, \\
& A_{2}^{\eta_{0}}(r, t)=\mathbb{1}\left\{y \in \mathbb{R}^{q}:|y-x|>(t-r)^{1 / 2-\eta_{0}} \vee 2\left|x-x^{\prime}\right|\right\},
\end{aligned}
$$

whenever $0 \leq r<t$. Most of the times we will just write $A_{1}$ and $A_{2}$ instead of $A_{1}(r, t), A_{2}(r, t)$ if the values of $r, t$ are clear from the context. Following (9.46), we introduce the following square functions for $i, j \in\{1,2\}$ :

$$
\begin{gathered}
Q_{X, \delta, \eta_{0}}^{i, j}\left(s, t, x, t, x^{\prime}\right)=\int_{0}^{(s-\delta)^{+}} d r \int_{A_{i}^{\eta_{0}}(r, t)} d w \int_{A_{j}^{\eta_{0}}(r, t)} d z \\
\left|\left(p_{t-r, 1}\left(w-x^{\prime}\right)-p_{t-r, 1}(w-x)\right)\left(p_{t-r, 1}\left(z-x^{\prime}\right)-p_{t-r, 1}(z-x)\right)\right| \\
R_{0}^{2} e^{R_{1}(|w|+|z|)}|u(r, w)|^{\gamma}|u(r, z)|^{\gamma}\left(|w-z|^{-\alpha}+1\right),
\end{gathered}
$$




$$
\begin{gathered}
Q_{T, \delta, \eta_{0}}^{i, j}\left(s, t, x^{\prime}, t^{\prime}, x^{\prime}\right)=\int_{0}^{(s-\delta)^{+}} d r \int_{A_{i}^{\eta_{0}}\left(r, t^{\prime}\right)} d w \int_{A_{j}^{\eta_{0}}\left(r, t^{\prime}\right)} d z \\
\left|\left(p_{t-r, 1}\left(w-x^{\prime}\right)-p_{t^{\prime}-r, 1}\left(w-x^{\prime}\right)\right)\left(p_{t-r, 1}\left(z-x^{\prime}\right)-p_{t^{\prime}-r, 1}\left(z-x^{\prime}\right)\right)\right| \\
R_{0}^{2} e^{R_{1}(|w|+|z|)}|u(r, w)|^{\gamma}|u(r, z)|^{\gamma}\left(|w-z|^{-\alpha}+1\right)
\end{gathered}
$$

and

$$
\begin{gathered}
Q_{S, \delta}\left(s, s^{\prime}, t^{\prime}, x^{\prime}\right)=\int_{\left(s \wedge s^{\prime}-\delta\right)^{+}}^{\left(s \vee s^{\prime}-\delta\right)^{+}} d r \int_{\mathbb{R}^{q}} d w \int_{\mathbb{R}^{q}} d z\left|p_{t^{\prime}-r, 1}\left(w-x^{\prime}\right) p_{t^{\prime}-r, 1}\left(z-x^{\prime}\right)\right| \\
R_{0}^{2} e^{R_{1}(|w|+|z|)|u(r, w)|^{\gamma}|u(r, z)|^{\gamma}\left(|w-z|^{-\alpha}+1\right) .}
\end{gathered}
$$

Now, we want to establish an upper bound for

$$
\begin{gathered}
Q_{\delta, \eta_{0}}^{\mathrm{tot}}\left(s, t, x, s^{\prime}, t^{\prime}, x^{\prime}\right)=Q_{S, \delta}\left(s, s^{\prime}, t^{\prime}, x^{\prime}\right)+\sum_{i, j=1}^{2}\left(Q_{T, \delta, \eta_{0}}^{i, j}\left(s, t, x^{\prime}, t^{\prime}, x^{\prime}\right)\right. \\
\left.+Q_{X, \delta, \eta_{0}}^{i, j}\left(s, t, x, t, x^{\prime}\right)\right)
\end{gathered}
$$

when $s, t, x, s^{\prime}, t^{\prime}, x^{\prime}$ are subject to some restrictions. Then, (9.47) is clearly an upper bound itself for the quadratic variation of each of the three martingales in 9.46.

We first consider the cases $(i, j)=(1,2),(2,1)$ or $(2,2)$, so $i+j \geq 3$. We start with a spatial estimate.

Lemma 9.4.5. For all $K \in \mathbb{N} \geq K_{1}, R>2$ there exist $49.4 .5(K, R), N_{9.4 .5}(K, \omega)$ almost surely such that $\forall \eta_{0}, \eta_{1} \in(1 / R, 1 / 2), \delta \in(0,1], \beta \in[0,1 / 2], N, n \in \mathbb{N},(t, x) \in$ $\mathbb{R}_{+} \times \mathbb{R}^{q}$ the following holds for $i+j \geq 3$ : For $\omega \in\{(t, x) \in Z(N, n, K, \beta), N \geq$ $N[9.4 .5\}$, we have

$$
Q_{X, \delta, \eta_{0}}^{i, j}\left(s, t, x, t, x^{\prime}\right) \leq q 9.4 .52^{4 \Gamma[9.4 .5}\left|x-x^{\prime}\right|^{2-\eta_{1}}
$$

for all $0 \leq s \leq t,\left|x^{\prime}\right| \leq K+1$.

Proof. We will just give the proof for $i=2$ without taking into account $j$, i.e. the restriction on $z$. This suffices by symmetry.

Use the estimate on $D$, take $\xi=3 / 4$ and set $N_{9.4 .5}(K, \omega)=N_{1}(0,3 / 4, K)$ and w.l.o.g. $\delta<s$. Then, in Lemma 9.4 .3 for the case $m=0$ we can take $\varepsilon_{0}=0$ 
$\left(C_{9.4 .3}=c(K) 2^{N_{1}(0,3 / 4, K)}\right)$ and obtain

$$
\begin{aligned}
Q_{X, \delta, \eta_{0}}^{i, j}\left(s, t, x, t, x^{\prime}\right) \leq & G_{9.4 .3} \int_{0}^{s-\delta} d r \int_{\mathbb{R}^{q}} \int_{\mathbb{R}^{q}} \mathbb{1}\left\{|w-x|>(t-r)^{1 / 2-\eta_{0}} \vee 2\left|x-x^{\prime}\right|\right\} \\
& \left(p_{t-r, 1}\left(w-x^{\prime}\right)-p_{t-r, 1}(w-x)\right)\left(p_{t-r, 1}\left(z-x^{\prime}\right)-p_{t-r, 1}(z-x)\right) \\
& e^{R_{1}(|w|+|z|)} e^{\gamma(|w-x|+|z-x|)} R_{0}^{2}\left(|w-z|^{-\alpha}+1\right) \\
& {\left[2^{-N} \vee d((r, w),(t, x))\right]^{3 \gamma / 4}\left[2^{-N} \vee d((r, z),(t, x))\right]^{3 \gamma / 4} d w d z }
\end{aligned}
$$

Using $d((r, w),(t, x))^{\gamma}=(\sqrt{t-r}+|w-x|)^{\gamma} \leq 2\left((t-r)^{\gamma / 2}+|w-x|^{\gamma}\right)$ and $t, r \leq K$ we bound this by

$$
\begin{aligned}
& \leq q_{9.4 .3} R_{0}^{2} \int_{0}^{(s-\delta)^{+}} d r \int_{\mathbb{R}^{q}} \int_{\mathbb{R}^{q}} \mathbb{1}\left\{|w-x|>(t-r)^{1 / 2-\eta_{0}} \vee 2\left|x-x^{\prime}\right|\right\} \\
& \left(p_{t-r, 1}\left(w-x^{\prime}\right)-p_{t-r, 1}(w-x)\right)\left(p_{t-r, 1}\left(z-x^{\prime}\right)-p_{t-r, 1}(z-x)\right) \\
& e^{2 R_{1}|x|} e^{\left(\gamma+R_{1}\right)(|w-x|+|z-x|)} \\
& 2\left(K^{3 \gamma / 8}+|w-x|^{3 \gamma / 4}\right) 2\left(K^{3 \gamma / 8}+|z-x|^{3 \gamma / 4}\right)\left(|w-z|^{-\alpha}+1\right) d w d z .
\end{aligned}
$$

With the help of Lemma 9.3 .7 for $t=t^{\prime} \leq K$ bound this by

$$
\begin{aligned}
& \leq C_{9.4 .3} c_{1}(K, R) \int_{0}^{s-\delta} d r(t-r)^{-1-\alpha / 2} \exp \left(-\frac{\eta_{1}(t-r)^{-2 \eta_{0}}}{256}\right)\left[1 \wedge \frac{\left|x-x^{\prime}\right|^{2}}{t-r}\right]^{1-\eta_{1} / 2} \\
& \leq C_{9.4 .3} c_{1}(K, R)(256 R)^{R} \int_{0}^{s-\delta} d r\left[1 \wedge \frac{\left|x-x^{\prime}\right|^{2}}{t-r}\right]^{1-\eta_{1} / 2} \\
& \leq C{ }_{9.4 .3} c_{2}(K, R)\left|x-x^{\prime}\right|^{2-\eta_{1}} \int_{0}^{t} d r(t-r)^{\eta_{1} / 2-1} \\
& \leq C C_{9.4 .3} c_{3}(K, R)\left|x-x^{\prime}\right|^{2-\eta_{1}},
\end{aligned}
$$

where we used Lemma 9.3.1 in the next to last line.

Slightly more difficult are the time estimates:

Lemma 9.4.6. For all $K \in \mathbb{N} \geq K_{1}, R>2$ there exist q9.4.6 $(K, R), \quad{ }_{\overline{9.4 .6}}(K, \omega)$ almost surely such that $\forall \eta_{0}, \eta_{1} \in(1 / R, 1 / 2), \delta \in(0,1], \beta \in[0,1 / 2], N, n \in \mathbb{N}$, $(t, x) \in \mathbb{R}_{+} \times \mathbb{R}^{q}$ the following holds for $i+j \geq 3$ :

For $\omega \in\{(t, x) \in Z(N, n, K, \beta), N \geq N[9.4 .6\}$

$$
\begin{array}{r}
Q_{T, \delta, \eta_{0}}^{i, j}\left(s, t, x^{\prime}, t^{\prime}, x^{\prime}\right) \leq \underset{9.4 .6}{4} 2^{4 \times[.4 .6}\left[\left|t-t^{\prime}\right|^{1-\eta_{1} / 2}+\left|t-t^{\prime}\right|^{1-\eta_{1} / 2} \delta^{-1-\alpha / 2}\left(\left|t-t^{\prime}\right| \wedge 1\right)^{4 \gamma}\right] \\
\forall 0 \leq s \leq t,\left|x^{\prime}\right| \leq K+1 .
\end{array}
$$


Proof. As in the previous lemma we only consider the $i=2$ case.

We use just the same proof as in Lemma 9.4.5, i.e. $\varepsilon_{0}=0, \xi=3 / 4$ and $N$ 9.4.6 $(K, \omega)=$ $N_{1}(0,3 / 4, K)$. The only difference is that we get time-differences instead of space differences (and when using Lemma 9.6 .2 with $t \leq t^{\prime}$ ):

$$
\begin{aligned}
& Q_{T, \delta, \eta_{0}}^{i, j}\left(s, t, x^{\prime}, t^{\prime}, x^{\prime}\right) \\
& \quad \leq C_{9.4 .3} c_{0}(K, R) \int_{0}^{s-\delta} d r(t-r)^{-1-\alpha / 2} \exp \left(-\frac{\eta_{1}\left(t^{\prime}-r\right)^{-2 \eta_{0}}}{256}\right)\left[1 \wedge \frac{\left|t-t^{\prime}\right|}{t-r}\right]^{1-\eta_{1} / 2} .
\end{aligned}
$$

Now, we use the trivial inequality

$$
\exp \left(-\frac{\eta_{1}\left(t^{\prime}-r\right)^{-2 \eta_{0}}}{256}\right) \leq \exp \left(-\frac{\eta_{1}\left(t^{\prime}-t\right)^{-2 \eta_{0}}}{512}\right)+\exp \left(-\frac{\eta_{1}(t-r)^{-2 \eta_{0}}}{512}\right)
$$

and hence, have to consider two summands:

$$
\begin{aligned}
Q_{T, \delta, \eta_{0}}^{i, j}\left(s, t, x^{\prime}, t^{\prime}, x^{\prime}\right) & \\
& =C_{9.4 .3} c_{0}(K, R)\left(I_{1} \exp \left(-\frac{\eta_{1}\left(t^{\prime}-t\right)^{-2 \eta_{0}}}{512}\right)+I_{2}\right) .
\end{aligned}
$$

We bound the first one by using Lemma 4.1 of [MP11]:

$$
\begin{aligned}
I_{1} & =\int_{0}^{s-\delta} d r(t-r)^{-1-\alpha / 2}\left[1 \wedge \frac{\left|t-t^{\prime}\right|}{t-r}\right]^{1-\eta_{1} / 2} \\
& \leq \frac{2}{2 / \alpha}\left(\left|t-t^{\prime}\right| \wedge \delta\right)^{1-\eta_{1} / 2} \delta^{-\alpha / 2-1+\eta_{1} / 2} \\
& \leq c\left(\left|t-t^{\prime}\right| \wedge \delta\right)^{1 / 2-\eta_{1} / 2} \delta^{-1-\alpha / 2}
\end{aligned}
$$

and by Lemma 9.3.1

$$
\exp \left(-\frac{\eta_{1}\left(t^{\prime}-t\right)^{-2 \eta_{0}}}{512}\right) \leq c(R)(512 R)^{R}\left(\left|t-t^{\prime}\right|^{1 / 2} \wedge 1\right)^{4 \gamma} .
$$

The second one

$$
\begin{aligned}
I_{2} & \leq \int_{0}^{s-\delta} d r(t-r)^{-1-\alpha / 2} \exp \left(-\frac{\eta_{1}(t-r)^{-2 \eta_{0}}}{512}\right)\left[1 \wedge \frac{\left|t-t^{\prime}\right|}{t-r}\right]^{1-\eta_{1} / 2} \\
& \leq\left|t-t^{\prime}\right|^{1-\eta_{1} / 2} \sup _{r \leq t}\left[(t-r)^{-2-\alpha / 2+\eta_{1} / 2} \exp \left(-\frac{\eta_{1}(t-r)^{-2 \eta_{0}}}{512}\right)\right](s-\delta) \\
& \leq\left|t-t^{\prime}\right|^{1-\eta_{1} / 2}(512 R)^{4 R} K .
\end{aligned}
$$

Setting $C_{9.4 .6}(K, R)=C_{\overline{9.4 .3} c_{0}}(K, R)\left(c c(R)(512 R)^{R}+(512 R)^{4 R} K\right)$ gives the result. 
Next we need to consider the distances for the cases $i=j=1$. We start again with the distance in space:

Lemma 9.4.7. Let $0 \leq m \leq \bar{m}+1$ and assume that $\left(P_{m}\right)$ holds. For all $K \in$ $\mathbb{N} \geq K_{1}, R>2, n \in \mathbb{N}, \beta \in[0,1 / 2], \varepsilon_{0} \in(0,1)$, there exist q.4.7 $(K, R)$ and $N$ 9.4.7 $\left(m, n, R, \varepsilon_{0}, K, \beta\right)(\omega) \in \mathbb{N}$ almost surely such that for all $\eta_{1} \in(1 / R, 1 / 2)$, $\eta_{0} \in\left(0, \eta_{1} / 32\right), \delta \in\left[a_{n}, 1\right], N \in \mathbb{N},(t, x) \in \mathbb{R}_{+} \times \mathbb{R}^{q}$ the following holds.

$$
\begin{aligned}
& \text { For } \omega \in\{(t, x) \in Z(N, n, K, \beta), N \geq N \overline{[9.4 .6}]: \\
& \begin{aligned}
& Q_{X, \delta, \eta_{0}}^{1,1}\left(s, t, x, t, x^{\prime}\right) \leq \overline{9.4 .7}\left[a_{n}^{-2 \varepsilon_{0}}+2^{4 N[\overline{9.4 .7}]}\right. \\
& \times\left[\left|x-x^{\prime}\right|^{2-\eta_{1}}\left(\bar{\delta}_{N}^{\left(\gamma \gamma_{m}-1-\alpha / 2\right) \wedge 0}+a_{n}^{2 \beta \gamma} \bar{\delta}_{N}^{(\gamma-1-\alpha / 2) \wedge 0}\right)\right. \\
&\left.+(d \wedge \sqrt{\delta})^{2-\eta_{1}} \delta^{-1-\alpha / 2}\left[\bar{d}_{N}^{2 \gamma \gamma_{m}}+a_{n}^{2 \beta \gamma} \bar{d}_{N}^{2 \gamma}\right]\right] \\
& \forall 0 \leq s \leq t,\left|x^{\prime}\right| \leq K+1 .
\end{aligned}
\end{aligned}
$$

Here $\bar{d}_{N}=\left|x-x^{\prime}\right| \vee 2^{-N}$ and $\bar{\delta}_{N}=\delta \vee \bar{d}_{N}^{2}$. Moreover, $N_{9.4 .7}$ is stochastically bounded uniformly in $(n, \beta)$.

Proof. Let $\xi=1-(8 R)^{-1} \in(15 / 16,1)$ and set $N_{9.4 .7}=N_{1}\left(m, n, \xi, \varepsilon_{0}, K, \beta\right)$. We can assume that $s>\delta$ and therefore, we always have $d((r, w),(t, x)) \wedge d((r, z),(t, x)) \geq$ $\sqrt{a_{n}}$ in the integral. A use of Lemma 9.4 .3 and the bound on $|w-x|,|z-x|$ respectively, gives

$$
\begin{aligned}
Q_{X, \delta, \eta_{0}}^{1,1}\left(s, t, x, t, x^{\prime}\right) \leq & C_{9.4 .3} \int_{0}^{s-\delta} d r \int_{\mathbb{R}^{q}} d w \int_{\mathbb{R}^{q}} d z \\
& \left(p_{t-r, 1}\left(w-x^{\prime}\right)-p_{t-r, 1}(w-x)\right)\left(p_{t-r, 1}\left(z-x^{\prime}\right)-p_{t-r, 1}(z-x)\right) \\
& e^{4 R_{1} K} e^{4 \gamma K} R_{0}^{2 \gamma} \\
& {\left[2^{-N} \vee\left((t-r)^{1 / 2}+(t-r)^{1 / 2-\eta_{0}} \vee 2\left|x-x^{\prime}\right|\right)\right]^{2 \gamma \xi} } \\
& \left\{\left[2^{-N} \vee\left((t-r)^{1 / 2}+(t-r)^{1 / 2-\eta_{0}} \vee 2\left|x-x^{\prime}\right|\right)\right]^{\gamma_{m}-1}+a_{n}^{\beta}\right\}^{2 \gamma} \\
& \left(|w-z|^{-\alpha}+1\right) .
\end{aligned}
$$

Let $\gamma^{\prime}=\gamma\left(1-2 \eta_{0}\right)$ and observe the trivial inequalities

$$
\begin{aligned}
\sqrt{t-r} & \leq K^{\eta_{0}}(t-r)^{1 / 2-\eta_{0}} \\
\left|x-x^{\prime}\right| & \leq c(q) K\left|x-x^{\prime}\right|^{1-2 \eta_{0}} .
\end{aligned}
$$

Then, Lemma 9.3 .4 allows the following bound

$$
\begin{aligned}
Q_{X, \delta, \eta_{0}}^{1,1}\left(s, t, x, t, x^{\prime}\right) \leq & C_{\left[\overline{9.4 .3}_{1}\right.} c_{1}(K) \int_{0}^{s-\delta} d r(t-r)^{-1-\alpha / 2}\left[1 \wedge \frac{\left|x-x^{\prime}\right|^{2}}{t-r}\right] \\
& {\left[2^{-2 N \gamma} \vee(t-r)^{\gamma^{\prime}} \vee\left|x-x^{\prime}\right|^{2 \gamma^{\prime}}\right]^{\xi} } \\
& {\left[2^{-N \gamma^{\prime}\left(\gamma_{m}-1\right)} \vee(t-r)^{\gamma^{\prime}\left(\gamma_{m}-1\right)} \vee\left|x-x^{\prime}\right|^{2 \gamma\left(\gamma_{m}-1\right)}+a_{n}^{2 \beta \gamma}\right] . }
\end{aligned}
$$


Using

$$
\begin{aligned}
2^{-2 N \gamma} \vee(t-r)^{\gamma^{\prime}} \vee\left|x-x^{\prime}\right|^{2 \gamma^{\prime}} & \leq 2^{-2 N \gamma^{\prime}} \vee\left|x-x^{\prime}\right|^{2 \gamma^{\prime}}+(t-r)^{\gamma^{\prime}} \\
& \leq 2\left[\bar{d}_{N}^{2 \gamma^{\prime}} \vee(t-r)^{\gamma^{\prime}}\right],
\end{aligned}
$$

we can bound $Q_{X, \delta, \eta_{0}}^{1,1}\left(s, t, x, t, x^{\prime}\right)$ by

$$
\begin{aligned}
& C_{90.4 .3} c_{1}(K) \int_{0}^{s-\delta} d r(t-r)^{-1-\alpha / 2}\left[1 \wedge \frac{\left|x-x^{\prime}\right|^{2}}{t-r}\right] 2^{\xi}\left(\vec{d}_{N}^{2 \gamma^{\prime} \xi} \vee(t-r)^{\gamma^{\prime} \xi}\right) \\
& 2^{\gamma_{m}-1}\left[\left(\bar{d}_{N}^{2} \vee(t-r)\right)^{\gamma^{\prime}\left(\gamma_{m}-1\right)}+a_{n}^{2 \beta \gamma}\right] \\
& \leq 4 C_{\overline{9.4 .3} c_{1}}(K) \int_{0}^{s-\delta} d r \mathbb{1}\left\{t-r \geq \bar{d}_{N}^{2}\right\}(t-r)^{-1-\alpha / 2+\gamma^{\prime} \xi}\left[1 \wedge \frac{\left|x-x^{\prime}\right|^{2}}{t-r}\right] \\
& {\left[(t-r)^{\gamma^{\prime}\left(\gamma_{m}-1\right)}+a_{n}^{2 \beta \gamma}\right]} \\
& +4 C_{9.4 .3} c_{1}(K) \int_{0}^{s-\delta} d r \mathbb{1}\left\{t-r<\vec{d}_{N}^{2}\right\}(t-r)^{-1-\alpha / 2}\left[1 \wedge \frac{\left|x-x^{\prime}\right|^{2}}{t-r}\right] \vec{d}_{N}^{2 \gamma^{\prime} \xi} \\
& {\left[\vec{d}_{N}^{2 \gamma^{\prime}\left(\gamma_{m}-1\right)}+a_{n}^{2 \beta \gamma}\right]} \\
& =C_{9.4 .3} c_{1}(K)\left(I_{1}+I_{2}\right) \text {. }
\end{aligned}
$$

We start with an estimate on $I_{1}$. If $r \leq s-\delta$ and $t-r \geq \bar{d}_{N}^{2}$ then

$$
r \leq t-\bar{d}_{N}^{2} \wedge s-\delta \leq t-\bar{d}_{N}^{2} \wedge t-\delta=t-\bar{\delta}_{N} .
$$

Use that to start with

$$
\begin{aligned}
I_{1} \leq & \int_{0}^{t-\bar{\delta}_{N}} d r(t-r)^{-1-\alpha / 2+\gamma^{\prime} \xi+\gamma^{\prime}\left(\gamma_{m}-1\right)}\left[1 \wedge \frac{\left|x-x^{\prime}\right|^{2}}{t-r}\right] \\
& +(t-r)^{-1-\alpha / 2+\gamma^{\prime} \xi}\left[1 \wedge \frac{\left|x-x^{\prime}\right|^{2}}{t-r}\right] a_{n}^{2 \beta \gamma} .
\end{aligned}
$$

We want to drop the minimum with 1 to consider

$$
\left|x-x^{\prime}\right|^{2} \int_{\bar{\delta}_{N}}^{t} d u\left(u^{-2-\alpha / 2+\gamma^{\prime} \xi+\gamma\left(\gamma_{m}-1\right)}+u^{-2-\alpha / 2+\gamma^{\prime} \xi} a_{n}^{2 \beta \gamma}\right)
$$

and then face three cases for the exponents: $<-1,=-1,>-1$. In the first and third case use the following inequality for $p \in(-1,1), p \neq 0,0<a<b$ :

$$
\int_{a}^{b} u^{p-1} d u=\frac{1}{|p|}\left|a^{p}-b^{p}\right| \leq \log (b / a)\left(a^{p}+b^{p}\right)
$$

which is true, since $1-x \leq-\log x, x \geq 0$.

In the -1 -case the integral is bounded by $\log K+\log \left(1 / \bar{\delta}_{N}\right)$. Hence, using that 
$t \leq K$ (therefore $\left.t^{0 \vee\left(-1-\alpha / 2+\gamma^{\prime} \xi+\gamma^{\prime}\left(\gamma_{m}-1\right)\right)} \leq K^{1}\right)$ in any of the cases there is a constant $c(K)$, such that :

$$
I_{1} \leq K^{1}\left|x-x^{\prime}\right|^{2} \log \left(K / \bar{\delta}_{N}\right)\left(\bar{\delta}_{N}^{\left(-1-\alpha / 2+\gamma^{\prime} \xi+\gamma^{\prime}\left(\gamma_{m}-1\right)\right) \wedge 0}+\bar{\delta}_{N}^{\left(-1-\alpha / 2+\gamma^{\prime} \xi\right) \wedge 0} a_{n}^{2 \beta \gamma}\right) .
$$

The log-term is bounded by $c(K, R)\left|x-x^{\prime}\right|^{-\eta_{1} / 2}$ (use Lemma 9.3.1).

Moreover, by Lemma 4.1(c) in [MP11] we bound

$$
I_{2} \leq \frac{2}{2 / \alpha}\left(\delta \wedge\left|x-x^{\prime}\right|^{2}\right) \delta^{-1-\alpha / 2} \vec{d}_{N}^{2 \gamma^{\prime} \xi}\left[\vec{d}_{N}^{2 \gamma^{\prime}\left(\gamma_{m}-1\right)}+a_{n}^{2 \beta \gamma}\right]
$$

Therefore,

$$
\begin{aligned}
Q_{X, \delta, \eta_{0}}^{1,1}\left(s, t, x, t, x^{\prime}\right) \leq & \\
& {[\mid x-4.3} \\
c & c_{3}(K, R) \\
& +\left(\delta \wedge | x - x ^ { \prime } | ^ { 2 - \eta _ { 1 } / 2 } \left(\bar{\delta}_{N}^{\left(-1-\alpha / 2+\gamma^{\prime}\left(\gamma_{m}+\xi-1\right)\right) \wedge 0}+\bar{\delta}_{N}^{(-1-\alpha / 2} \vec{d}_{N}^{2 \gamma^{\prime} \xi}\left[\vec{d}_{N}^{2 \gamma^{\prime}\left(\gamma_{m}-1\right)}+a_{n}^{2 \beta \gamma}\right]\right.\right.
\end{aligned}
$$

To finish the proof, replace $\xi=1-(8 R)^{-1}$ by 1 and $\gamma^{\prime}=\gamma\left(1-2 \eta_{0}\right)$ by $\gamma$ at the cost of multiplying by $d^{-\eta_{1} / 2} \geq \bar{\delta}_{N}^{-\eta_{1} / 4}$, since:

$$
\xi \gamma^{\prime}=\geq \gamma\left(1-\eta_{1} / 4\right), \text { hence } \xi \gamma^{\prime}-\gamma \geq-\gamma \eta_{1} / 4 \geq-\eta_{1} / 4
$$

and

$$
\gamma^{\prime}\left(\gamma_{m}+\xi-1\right)=\gamma\left(1-2 \eta_{0}\right)\left(\gamma_{m}-\frac{1}{8 R}\right) \geq \gamma \gamma_{m}-\frac{\eta_{1}}{4}
$$

This holds, since $\eta_{1} \geq 32 \eta_{0}$ gives

$$
\begin{aligned}
\gamma\left(1-2 \eta_{0}\right)\left(1-(8 R)^{-1}\right) & \geq \gamma\left(1-\frac{\eta_{1}}{16}\right)\left(\gamma_{m}-\frac{1}{8 R}\right) \\
& \geq \gamma \gamma_{m}-\gamma \frac{1}{8 R}-\gamma \gamma_{m} \frac{\eta_{1}}{16} \\
& \geq \gamma \gamma_{m}-\frac{1}{8 R}-2 \frac{\eta_{1}}{16} \\
& \geq \gamma \gamma_{m}-\frac{\eta_{1}}{8}-\frac{\eta_{1}}{8} \quad\left(\text { using } \eta_{1}>R^{-1}\right) \\
& \geq \gamma \gamma_{m}-\frac{\eta_{1}}{4} .
\end{aligned}
$$

A similar result can be obtained for the temporal distances. 
Lemma 9.4.8. Let $0 \leq m \leq \bar{m}+1$ and assume that $\left(P_{m}\right)$ holds. For all $K \in$ $\mathbb{N} \geq K_{1}, R>2, n \in \mathbb{N}, \beta \in[0,1 / 2], \varepsilon_{0} \in(0,1)$, there exist $q 9.4 .8(K, R)$ and $N$ 9.4.8 $\left(m, n, R, \varepsilon_{0}, K, \beta\right)(\omega) \in \mathbb{N}$ almost surely such that

$\forall \eta_{1} \in(1 / R, 1 / 2), \eta_{0} \in\left(0, \eta_{1} / 32\right), \delta \in\left[a_{n}, 1\right], N \in \mathbb{N},(t, x) \in \mathbb{R}_{+} \times \mathbb{R}^{q}$ the following holds.

$$
\begin{aligned}
& \text { For } \omega \in\left\{(t, x) \in Z(N, n, K, \beta), N \geq N_{9.4 .8}\right\} \\
& Q_{T, \delta, \eta_{0}}^{1,1}\left(s, t, x^{\prime}, t^{\prime}, x^{\prime}\right) \leq 9.4 .8\left[a_{n}^{-2 \varepsilon_{0}}+2^{4 N[9.4 .8}\right] \\
& {\left[\left|t-t^{\prime}\right|^{1-\eta_{1} / 2}\left(\bar{\delta}_{N}^{\left(\gamma \gamma_{m}-1-\alpha / 2\right) \wedge 0}+a_{n}^{2 \beta \gamma} \bar{\delta}_{N}^{\gamma-1-\alpha / 2}\right)\right.} \\
& \left.+\left(\left|t-t^{\prime}\right| \wedge \delta\right)^{1-\eta_{1} / 2} \delta^{-1-\alpha / 2}\left[\vec{d}_{N}^{2 \gamma \gamma_{m}}+a_{n}^{2 \beta \gamma} \vec{d}_{N}^{2 \gamma}\right]\right] \\
& \forall 0 \leq s \leq t,\left|x^{\prime}\right| \leq K+1 \text {. }
\end{aligned}
$$

Moreover, $N_{9.4 .8}$ is stochastically bounded uniformly in $(n, \beta)$.

Proof. Choose $\xi=1-(8 R)^{-1}$.

We do just the same proof as before and use

$$
\begin{aligned}
\sqrt{t-r} & \leq K^{\eta_{0}}\left(t^{\prime}-r\right)^{1 / 2-\eta_{0}} \\
2^{-N \gamma} \vee\left(t^{\prime}-r\right)^{\gamma^{\prime} / 2} & \leq 2^{-N \gamma^{\prime}}+\left(t^{\prime}-t\right)^{\gamma^{\prime} / 2}+(t-r)^{\gamma^{\prime} / 2} \\
& \leq 2\left[\bar{d}_{N}^{\gamma^{\prime}} \vee(t-r)^{\gamma^{\prime} / 2}\right]
\end{aligned}
$$

to get:

$$
\begin{aligned}
Q_{T, \delta, \eta_{0}}^{1,1}\left(s, t, x^{\prime}, t^{\prime}, x^{\prime}\right) \leq{ }_{9.4 .3} c_{1}(K) \int_{0}^{s-\delta} d r(t-r)^{-1-\alpha / 2}\left[1 \wedge \frac{\left|t-t^{\prime}\right|}{t-r}\right] \\
2^{\xi}\left(\vec{d}_{N}^{2 \gamma^{\prime} \xi} \vee(t-r)^{\gamma^{\prime} \xi}\right) \\
2^{\gamma_{m}-1}\left[\left(\bar{d}_{N}^{2} \vee(t-r)\right)^{\gamma^{\prime}\left(\gamma_{m}-1\right)}+a_{n}^{2 \beta \gamma}\right] \\
={ }_{9.4 .3} c_{2}(K)\left[I_{1}+I_{2} \vec{d}_{N}^{2 \gamma^{\prime} \xi}\left(\vec{d}_{N}^{2 \gamma^{\prime}\left(\gamma_{m}-1\right)}+a_{n}^{2 \beta \gamma}\right)\right]
\end{aligned}
$$

This means we are just in the same situation as in $(9.52)$ and we can continue the proof as we did there.

The next lemma describes the quadratic variation of the last martingale in 9.46 :

Lemma 9.4.9. Let $0 \leq m \leq \bar{m}+1$ and assume that $\left(P_{m}\right)$ holds. For all $K \in$ $\mathbb{N} \geq K_{1}, R>2, n \in \mathbb{N}, \beta \in[0,1 / 2], \varepsilon_{0} \in(0,1)$, there exist $q 9.4 .9(K, R, \gamma)$ and $N \overline{9.4 .9}\left(m, n, R, \varepsilon_{0}, K, \beta\right)(\omega) \in \mathbb{N}$ almost surely such that 
$\forall \eta_{1} \in(1 / R, 1 / 2), \delta \in\left[a_{n}, 1\right], N \in \mathbb{N},(t, x) \in \mathbb{R}_{+} \times \mathbb{R}^{q}$ the following holds.

$$
\begin{aligned}
& \text { For } \omega \in\{(t, x) \in Z(N, n, K, \beta), N \geq N \text { [9.4.9] } \\
& Q_{S, \delta}\left(s, s^{\prime}, t^{\prime}, x^{\prime}\right) \leq 99.4 .9\left[a_{n}^{-2 \varepsilon_{0}}+2^{4 N[9.4 .9}\right] \\
& \left|s-s^{\prime}\right|^{1-\eta_{1} / 2}\left(\bar{\delta}_{N}^{\left(\gamma \gamma_{m}-1-\alpha / 2\right) \wedge 0}+a_{n}^{2 \beta \gamma} \bar{\delta}_{N}^{(\gamma-1-\alpha / 2) \wedge 0}\right) \\
& \left.+\left(\left|s^{\prime}-s\right| \wedge \delta\right)^{1-\eta_{1} / 2} \mathbb{1}\left\{\delta<\bar{d}_{N}^{2}\right\} \delta^{-1-\alpha / 2}\left[\vec{d}_{N}^{2 \gamma \gamma_{m}}+a_{n}^{2 \beta \gamma} \vec{d}_{N}^{2 \gamma}\right]\right] \\
& \forall 0 \leq s \leq t, s^{\prime} \leq t^{\prime},\left|x^{\prime}\right| \leq K+1 .
\end{aligned}
$$

Here $\bar{d}_{N}=\left(\left|t-t^{\prime}\right|^{1 / 2}+\left|x-x^{\prime}\right|\right) \vee 2^{-N}$ and $\bar{\delta}_{N}=\delta \vee \bar{d}_{N}^{2}$. Moreover, $N_{9.4 .9}$ is stochastically bounded uniformly in $(n, \beta)$.

Proof. Choose $\xi=\left(3 / 2-(2 \gamma)^{-1}\right) \vee\left(1-(4 \gamma R)^{-1}\right)$.

Choose $N$ 9.4.9 $=N_{1}\left(m, n, \xi, \varepsilon_{0}, K, \beta\right)$. We have for $r \leq s \vee s^{\prime}-\delta: \sqrt{a_{n}}<\sqrt{\delta}<$ $\sqrt{s-r}<\sqrt{t-r}$, thus

$$
\begin{aligned}
2^{-N} \vee\left(\left(t^{\prime}-r\right)^{1 / 2}+|w-x|\right) & \leq 2^{-N} \vee\left|x-x^{\prime}\right|+\left(t^{\prime}-r\right)^{1 / 2}+\left|w-x^{\prime}\right| \\
& \leq \bar{d}_{N}+\left(t^{\prime}-r\right)^{1 / 2}+\left|w-x^{\prime}\right|
\end{aligned}
$$

This bound, Lemmas 9.3.2, 9.4.3 and 9.3.6 give:

$$
\begin{aligned}
& Q_{S, \delta, \eta_{0}}\left(s, s^{\prime}, t^{\prime}, x^{\prime}\right) \leq c_{0}(K, R)\left[a_{n}^{-2 \varepsilon_{0}}+2^{\left.4 N_{\text {[9.4.9. }}\right] \times}\right. \\
& \int_{\left(s \wedge s^{\prime}-\delta\right)^{+}}^{\left(s \vee s^{\prime}-\delta\right)^{+}} d r\left(t^{\prime}-r\right)^{-1-\alpha / 2}\left[\vec{d}_{N}^{2 \xi \gamma}+\left(t^{\prime}-r\right)^{\xi \gamma}\right]\left[\vec{d}_{N}^{2 \gamma\left(\gamma_{m}-1\right)}+\left(t^{\prime}-r\right)^{\gamma\left(\gamma_{m}-1\right)}+a_{n}^{2 \beta \gamma}\right] .
\end{aligned}
$$

And we split this up in two integrals

$$
\begin{aligned}
& \leq 4 c_{0}(K, R)\left[a_{n}^{-2 \varepsilon_{0}}+2^{4}\right. \text { [9.4.9] } \\
& \quad\left\{\int_{\left(s \wedge s^{\prime}-\delta\right)^{+}}^{\left(s \vee s^{\prime}-\delta\right)^{+}} \mathbb{1}\left\{r \leq t^{\prime}-\vec{d}_{N}^{2}\right\}\left(t^{\prime}-r\right)^{-1-\alpha / 2+\xi \gamma}\left[\left(t^{\prime}-r\right)^{\gamma\left(\gamma_{m}-1\right)}+a_{n}^{2 \beta \gamma}\right] d r\right. \\
& \left.\quad+\int_{\left(s \wedge s^{\prime}-\delta\right)^{+}}^{\left(s \vee s^{\prime}-\delta\right)^{+}} \mathbb{1}\left\{r>t^{\prime}-\bar{d}_{N}^{2}\right\}\left(t^{\prime}-r\right)^{-1-\alpha / 2} d r \vec{d}_{N}^{2 \xi \gamma}\left[\vec{d}_{N}^{2 \gamma\left(\gamma_{m}-1\right)}+a_{n}^{2 \beta \gamma}\right]\right\} \\
& =c\left(J_{1}+J_{2}\right) .
\end{aligned}
$$

For $J_{2}$, the same estimate as in the proof of Lemma 5.6 in [MP11] holds:

$$
J_{2} \leq c(\alpha) \mathbb{1}\left\{\delta<\bar{d}_{N}^{2}\right\} \delta^{-1-\alpha / 2}\left(\left|s^{\prime}-s\right| \wedge \delta\right)^{1-\eta_{1} / 2}\left[\bar{d}_{N}^{2 \gamma \gamma_{m}}+a_{n}^{2 \beta \gamma} \bar{d}_{N}^{2 \gamma}\right] .
$$

For the other integral we note that since

$$
-1-\alpha / 2+\xi \gamma>-1-(2 \gamma-1)+\left(\frac{3}{2}-\frac{1}{2 \gamma}\right) \gamma=-\frac{1+\gamma}{2}>-1,
$$


the integral is well-defined. Set $p=\gamma\left(\gamma_{m}+\xi-1\right)-1-\alpha / 2$ or $\gamma \xi-1-\alpha / 2$. By (9.56), $p \in(-(1+\gamma) / 2,1)$. If $p^{\prime}=0 \wedge p$ and $\varepsilon \in\left[0,-p^{\prime}\right]$, then:

$$
\begin{aligned}
I(p) & :=\int_{\left(s \wedge s^{\prime}-\delta\right)^{+}}^{\left(s \vee s^{\prime}-\delta\right)^{+}} \mathbb{1}\left\{r \leq t^{\prime}-\bar{d}_{N}^{2}\right\}\left(t^{\prime}-r\right)^{p} d r \\
& \leq \int_{\left(s \wedge s^{\prime}-\delta\right)^{+}}^{\left(s \vee s^{\prime}-\delta\right)^{+}} \mathbb{1}\left\{r \leq t^{\prime}-\bar{d}_{N}^{2}\right\} K\left(t^{\prime}-r\right)^{p^{\prime}} d r \\
& \leq K \min \left(\left|s^{\prime}-s\right| \bar{\delta}_{N}^{p^{\prime}}, \int_{0}^{\left|s^{\prime}-s\right|} u^{p^{\prime}} d u\right)(\text { compare }(9.53)) \\
& \left.\leq \frac{2}{1-\gamma} K\left|s^{\prime}-s\right|^{p^{\prime}+1} \min \left(\left(\frac{\left|s^{\prime}-s\right|}{\bar{\delta}_{N}}\right)^{-p^{\prime}}, 1\right) \text { (using (9.56) }\right) \\
& \leq \frac{2}{1-\gamma} K\left|s^{\prime}-s\right|^{p^{\prime}+1}\left(\frac{\left|s^{\prime}-s\right|}{\bar{\delta}_{N}}\right)^{-p^{\prime}-\varepsilon} \\
& \leq C(\gamma, K)\left|s^{\prime}-s\right|^{1-\varepsilon} \bar{\delta}_{N}^{\varepsilon+p^{\prime}} .
\end{aligned}
$$

Then, there can be two cases for $v=p+\gamma(1-\xi)$, such that $v=\gamma \gamma_{m}-1-\frac{\alpha}{2}$ or $v=\gamma-1-\frac{\alpha}{2}$.

Case 1: $v<0$.

Then $p^{\prime}=p<0$. Choose $\varepsilon=\gamma(1-\xi) \leq(4 R)^{-1}<\eta_{1} / 4$, then $\varepsilon+p^{\prime}=v \leq 0$. Therefore,

$$
I(p) \leq C(\gamma, K)\left|s^{\prime}-s\right|^{1-\eta_{1} / 4} \bar{\delta}_{N}^{v} .
$$

Case 2: $v \geq 0$.

Then $p^{\prime}=(v-\gamma(1-\xi)) \wedge 0 \geq-\gamma(1-\xi)$. Choose $\varepsilon=-p^{\prime} \leq \gamma(1-\xi)<\eta_{1} / 4$. Thus, $1-\varepsilon \geq 1-\frac{\eta_{1}}{4}$ and

$$
I(p) \leq C(K)\left|s^{\prime}-s\right|^{1-\varepsilon} \bar{\delta}_{N}^{0}<C_{1}(\gamma, K)\left|s^{\prime}-s\right|^{1-\eta_{1} / 4} .
$$

In either case $I(p) \leq C_{1}(K)\left|s^{\prime}-s\right|^{1-\eta_{1} / 2} \bar{\delta}_{N}^{v \wedge 0}$. Hence,

$$
J_{1} \leq c_{4}(K)\left|s^{\prime}-s\right|^{1-\eta_{1} / 2}\left[\bar{\delta}_{N}^{\left(\gamma \gamma_{m}-1-\alpha / 2\right) \wedge 0}+a_{n}^{2 \beta \gamma} \bar{\delta}_{N}^{\gamma-1-\alpha / 2}\right] .
$$

And this completes the proof.

Notation: $\quad \tilde{d}\left((s, t, x),\left(s^{\prime}, t^{\prime}, x^{\prime}\right)\right):=\sqrt{\left|s-s^{\prime}\right|}+\sqrt{\left|t-t^{\prime}\right|}+\left|x-x^{\prime}\right|, s, t, s^{\prime}, t^{\prime} \in$ $\mathbb{R}_{+}, x, x^{\prime} \in \mathbb{R}^{q}$.

As a corollary of all the previous calculations, we get a bound on $Q_{\delta, \eta_{0}}^{\text {tot }}$ as defined in (9.47).

Corollary 9.4.10. Let $0 \leq m \leq \bar{m}+1$ and assume that $\left(P_{m}\right)$ holds. For all $K \in \mathbb{N} \geq K_{1}, R>2, n \in \mathbb{N}, \beta \in[0,1 / 2], \varepsilon_{0} \in(0,1)$, there exist 49.4.10 $(K, R)$, 
$N_{9.4 .10}\left(m, n, R, \varepsilon_{0}, K, \beta\right)(\omega) \in \mathbb{N}$ almost surely such that

$\forall \eta_{1} \in(1 / R, 1 / 2), \eta_{0} \in\left(1 / R, \eta_{1} / 32\right) \delta \in\left[a_{n}, 1\right], N \in \mathbb{N},(t, x) \in \mathbb{R}_{+} \times \mathbb{R}^{q}$ the following holds.

$$
\begin{aligned}
& \text { For } \omega \in\{(t, x) \in Z(N, n, K, \beta), N \geq N \overline{9.4 .10}\} \\
& \begin{aligned}
Q_{\delta, \eta_{0}}^{\text {tot }}\left(s, t, x, s^{\prime}, t^{\prime}, x^{\prime}\right) \leq \overline{9.4 .10}\left(a_{n}^{-2 \varepsilon_{0}}+2^{4 N \overline{9.4 .10}}\right) \tilde{d}^{2-\eta_{1}} \\
{\left[\delta^{-1-\alpha / 2} \bar{d}_{N}^{\gamma \gamma_{m}}+\delta^{-1-\alpha / 2} a_{n}^{2 \beta \gamma} \bar{d}_{N}^{2 \gamma}+\bar{\delta}_{N}^{\left(\gamma \gamma_{m}-1-\alpha / 2\right) \wedge 0}+a_{n}^{2 \beta \gamma} \bar{\delta}_{N}^{\gamma-1-\alpha / 2}\right] } \\
\forall 0 \leq s \leq t \leq t^{\prime} \leq T_{K},\left|x^{\prime}\right| \leq K+1 .
\end{aligned}
\end{aligned}
$$

Here $\tilde{d}=\tilde{d}\left((s, t, x),\left(s^{\prime}, t^{\prime}, x^{\prime}\right)\right), \bar{d}_{N}=d\left((t, x),\left(t^{\prime}, x^{\prime}\right)\right) \vee 2^{-N}$ and $\bar{\delta}_{N}=\delta \vee \bar{d}_{N}$. Moreover, $N$ [9.4.10 is stochastically bounded uniformly in $(n, \beta)$.

Proof. The proof simply consists of putting together the last lemmas. Let $N_{9.4 .10}=$ $N_{\overline{9.4 .5}} \vee N_{9.4 .6} \vee N_{9.4 .7} \vee N_{[9.4 .8} \vee N_{9.4 .9}$, which is then clearly uniformly bounded in $(n, \beta)$ and $c_{0}=q 9.4 .5 \vee q 9.4 .6 \vee q 9.4 .7 \vee q 9.4 .8 \vee 99.4 .9$. Then,

$$
\begin{aligned}
& Q_{\delta, \eta_{0}}^{\text {tot }}\left(s, t, x, s^{\prime}, t^{\prime}, x^{\prime}\right) \leq 5 c_{0}\left(a_{n}^{-2 \varepsilon_{0}}+2^{4 N \overline{9.4 .10}}\right)\left\{\tilde{d}^{2-\eta_{1}}\left[\bar{\delta}_{N}^{\left(\gamma \gamma_{m}-1-\alpha / 2\right) \wedge 0}+a_{n}^{2 \beta \gamma} \bar{\delta}_{N}^{(\gamma-1-\alpha / 2) \wedge 0}\right]\right. \\
& \left.+\tilde{d}_{N}^{2-\eta_{1}} \delta^{-1-\alpha / 2}\left[\vec{d}_{N}^{2 \gamma \gamma_{m}}+a_{n}^{2 \beta \gamma} d_{N}^{2 \gamma}+\left(\left|t-t^{\prime}\right|^{1 / 2} \wedge 1\right)^{4 \gamma}\right]\right\} \\
& \leq \text { q9.4.10 }\left(a_{n}^{-2 \varepsilon_{0}}+2^{4 \sqrt{0.4 .10}}\right) \tilde{d}^{2-\eta_{1}}\left[\delta^{-1-\alpha / 2} \bar{d}_{N}^{\gamma \gamma_{m}}+\delta^{-1-\alpha / 2} a_{n}^{2 \beta \gamma} \bar{d}_{N}^{2 \gamma}\right. \\
& \left.+\bar{\delta}_{N}^{\left(\gamma \gamma_{m}-1-\alpha / 2\right) \wedge 0}+a_{n}^{2 \beta \gamma} \bar{\delta}_{N}^{\gamma-1-\alpha / 2}\right] \text {. }
\end{aligned}
$$

Notation: In the following, the $\alpha$ of [MP11] is replaced by $\lambda \in[0,1]$. Introduce

$$
\begin{gathered}
\bar{\Delta}_{u_{1}^{\prime}}\left(m, n, \lambda, \varepsilon_{0}, 2^{-N}\right)=a_{n}^{-\varepsilon_{0}}\left[a_{n}^{-\lambda / 2(1+\alpha / 2)} 2^{-N \gamma \gamma_{m}}+\left(a_{n}^{\lambda / 2} \vee 2^{-N}\right)^{\left(\gamma \gamma_{m}-1-\alpha / 2\right) \wedge 0}\right. \\
\left.+a_{n}^{-\lambda / 2(1+\alpha / 2)+\beta \gamma}\left(a_{n}^{\lambda / 2} \vee 2^{-N}\right)^{\gamma}\right] .
\end{gathered}
$$

Proposition 9.4.11. Let $0 \leq m \leq \bar{m}+1$ and assume that $\left(P_{m}\right)$ holds. Then, for all $n \in \mathbb{N}, \eta_{1} \in(0,1 / 2], \varepsilon_{0} \in(0,1), K \in \mathbb{N} \geq K_{1}, \lambda \in[0,1], \beta \in[0,1 / 2]$ there is an $N_{9.4 .11}=N$ 9.4.11 $\left(m, n, \eta_{1}, \varepsilon_{0}, K, \lambda, \beta\right)(\omega) \in \mathbb{N} \geq 2$ almost surely such that for all $N \geq$ $N$ 9.4.11, $(t, x) \in Z(N, n, K, \beta), s \leq t \leq t^{\prime}, s^{\prime} \leq t^{\prime} \leq T_{K}$ and $d\left((s, t, x)\left(s^{\prime}, t^{\prime}, x^{\prime}\right)\right)<$ $2^{-N}$, it holds that

$$
\left|F_{a_{n}^{\lambda}, l}(s, t, x)-F_{a_{n}^{\lambda}, l}\left(s^{\prime}, t^{\prime}, x^{\prime}\right)\right| \leq 2^{-86} q^{-4} \tilde{d}^{1-\eta_{1}} \bar{\Delta}_{u_{1}^{\prime}}\left(m, n, \lambda, \varepsilon_{0}, 2^{-N}\right) \quad, l=1, \ldots, q .
$$

Moreover, $N \overline{9.4 .11}$ is stochastically bounded uniformly in $(n, \lambda, \beta)$. 
Proof. We do the proof for $l=1$ only, see Remark 9.4.4. Let $R=33 \eta_{1}^{-1}, \eta_{0} \in$ $\left(R^{-1}, \eta_{1} / 32\right)$ and consider the case $s \leq t$ in the beginning only. Set

$$
\begin{aligned}
d & =d\left((t, x),\left(t^{\prime}, x^{\prime}\right)\right), \\
\tilde{d} & =\sqrt{\left|s^{\prime}-s\right|}+d, \\
\bar{d}_{N} & =d \vee 2^{-N}, \\
\bar{\delta}_{n, N} & =a_{n}^{\lambda} \vee \bar{d}_{N}^{2} .
\end{aligned}
$$

By Corollary 9.4 .10 for $(t, x) \in Z(N, n, K, \beta), N \geq N[9.4 .10$ it holds that:

$$
\begin{aligned}
& Q_{a_{n}^{\lambda}, \eta_{0}}^{\text {tot }}\left(s, t, x, s^{\prime}, t^{\prime}, x^{\prime}\right)^{1 / 2} \leq q .61\left(K, \eta_{1}\right)\left(a_{n}^{-\varepsilon_{0}}+2^{2 N[.4 .10}\right) \tilde{d}^{1-\eta_{1} / 2} \\
& {\left[\left(a_{n}^{\lambda}\right)^{-1 / 2(1+\alpha / 2)}\left[\bar{d}_{N}^{\gamma \gamma_{m}}+a_{n}^{\beta \gamma} \bar{d}_{N}^{\gamma}\right]\right.} \\
& \left.+\bar{\delta}_{n, N}^{\left(\gamma \gamma_{m}-1-\alpha / 2\right) / 2 \wedge 0}+a_{n}^{\beta \gamma} \bar{\delta}_{n, N}^{(\gamma-1-\alpha / 2) / 2}\right] \\
& \forall s \leq t \leq t^{\prime}, s^{\prime} \leq t^{\prime} \leq T_{K},\left|x^{\prime}\right| \leq K+2 \text {. }
\end{aligned}
$$

Therefore, define

$$
\begin{aligned}
\Delta\left(m, n, \bar{d}_{N}\right)= & 2^{-96} a_{n}^{-\varepsilon_{0}}\left\{a_{n}^{-\lambda / 2(1+\alpha / 2)}\left[\bar{d}_{N}^{\gamma \gamma_{m}}+a_{n}^{\beta \gamma} \bar{d}_{N}^{\gamma}\right]\right. \\
& \left.+\left(\sqrt{\bar{\delta}_{n, N}}\right)^{\left(\gamma \gamma_{m}-1-\alpha / 2\right) \wedge 0}+a_{n}^{\beta \gamma}\left(\sqrt{\bar{\delta}_{n, N}}\right)^{\gamma-1-\alpha / 2}\right\}\left(q^{5} 4^{c(q)}\right)^{-1},
\end{aligned}
$$

which satisfies

$$
\Delta\left(m, n, 2^{-N+1}\right) \leq\left(2^{\gamma \gamma_{m}} \vee 2^{\gamma} \vee 2^{0} \vee 2^{\gamma-1-\alpha / 2}\right) \Delta\left(m, n, 2^{-N}\right) \leq 4 \Delta\left(m, n, 2^{-N}\right) .
$$

Choose $N_{3}=\frac{33}{\eta_{1}}\left[N\left[\overline{9.4 .10}+N_{4}\left(K, \eta_{1}\right)\right]++\frac{4}{\eta_{1}}(8+10 \log q)\right.$, where $N_{4}$ is chosen in such a way that

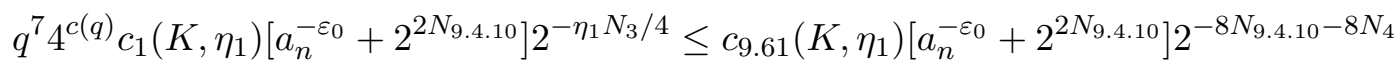

$$
\begin{aligned}
& \leq a_{n}^{-\varepsilon_{0}} 2^{-100} \text {, }
\end{aligned}
$$

i.e. $N_{4}=N_{4}\left(a_{n}, \varepsilon_{0}, N_{\overline{9.4 .10},}, \overline{9.61}\right)$ and hence, $N_{3}=N_{3}\left(n, \varepsilon_{0}, N_{9.4 .10}, K, \eta_{1}\right)$, which is stochastically uniformly bounded in $(n, \lambda, \beta)$.

Let $N^{\prime} \in \mathbb{N}$ be such that $\tilde{d} \leq 2^{-N^{\prime}}$, which implies $\tilde{d}^{1-\eta_{1} / 2} \leq 2^{-N^{\prime} \eta_{1} / 4} \tilde{d}^{1-3 \eta_{1} / 4}$. Then, it is true that on the event

$$
\left\{\omega:(t, x) \in Z(N, n, K+1, \beta), N \geq N_{3}, N^{\prime} \geq N_{3}\right\},
$$

we have that

$$
\begin{aligned}
Q_{a_{n}^{\lambda}, \eta_{0}}^{\mathrm{tot}}\left(s, t, x, s^{\prime}, t^{\prime}, x^{\prime}\right)^{1 / 2} \leq & c_{1}\left(K, \eta_{1}\right)\left(a_{n}^{-\varepsilon_{0}}+2^{2 N[.4 .10}\right) \\
& 2^{-N^{\prime} \eta_{1} / 4} \tilde{d}^{1-3 \eta_{1} / 4} 2^{100} a_{n}^{\varepsilon_{0}} \Delta\left(m, n, \bar{d}_{N}\right)\left(q^{7} 4^{c(q)}\right) \\
\leq & \tilde{d}^{1-3 \eta_{1} / 4} \frac{1}{16} \Delta\left(m, n, \bar{d}_{N}\right)
\end{aligned}
$$


whenever $s \leq t \leq t^{\prime}, s^{\prime} \leq t^{\prime} \leq T_{k},\left|x^{\prime}\right| \leq K+2$. Remembering the decomposition of $F_{\delta, 1}$ in (9.46) into the sum of 3 martingales and applying the Dubins-SchwarzTheorem (Theorem 3.2.8 we can write as long as $s \leq t \leq t^{\prime}, s^{\prime} \leq t^{\prime}$ and $\tilde{d} \leq 2^{-N}$ :

$$
\begin{aligned}
& \mathbb{P}\left[\left|F_{a_{n}^{\lambda}, 1}(s, t, x)-F_{a_{n}^{\lambda}, 1}\left(s^{\prime}, t^{\prime}, x^{\prime}\right)\right| \geq d\left((s, t, x)\left(s^{\prime}, t^{\prime}, x^{\prime}\right)\right)^{1-\eta_{1}} \Delta\left(m, n, \bar{d}_{N}\right)\right. \\
& \quad \leq 3 \mathbb{P}\left[\sup _{u \leq \tilde{d}^{2-3 \eta_{1} / 2}\left(\Delta\left(m, n, \bar{d}_{N}\right) / 16\right)^{2}}|B(u)| \geq \tilde{d}^{1-\eta_{1}} \Delta\left(m, n, \bar{d}_{N}\right) / 3\right] \\
& \quad \leq 3 \mathbb{P}\left[\sup _{u \leq 1}|B(u)| \geq \tilde{d}^{-\eta_{1} / 4}\right] \\
& \quad \leq c \int_{\tilde{d}^{-\eta_{1} / 4}}^{\infty} \exp \left(-y^{2} / 2\right) d y \\
& \quad \leq c_{0} \exp \left(-\tilde{d}^{-\eta_{1} / 2} / 2\right),
\end{aligned}
$$

where we used the Reflection Principle in the next to last inequality.

Next, apply Lemma 9.8.1. where we should make clear what the parameters are. We take

$$
\begin{aligned}
& q_{1}=q_{2}=1, q_{3}=q, r=3, E=\mathbb{R}_{+}^{2} \times \mathbb{R}^{q}, \\
& \bar{q}=q+2, v_{1}=v_{2}=2, v_{3}=1, v_{0}=0, \\
& \hat{n}=(m, n, \lambda, \beta), S=\mathbb{N}^{2} \times[0,1 / 2] \times(0,1), \\
& \Sigma(N, K, \hat{n})=Z(N, n, K, \beta), \Sigma^{\prime}(N)=E \cap\{0\} \times\left\{0 \leq t \leq T_{K}\right\} \times \mathbb{R}^{q}, \\
& s=1, \alpha_{1}=1, \Delta_{1}\left(\hat{n}, 2^{-N}\right)=\Delta\left(m, n, 2^{-N}\right), k_{1}=4, c\left(\alpha_{1}\right) \leq 4, \eta=\eta_{1}, \\
& Y_{\hat{n}}(y)=F_{a_{n}^{\lambda}, 1}(s, t, x) \text { with } y=(s, t, x), N_{0}(\eta, K, \hat{n})=N_{3}\left(n, \varepsilon_{0}, N \text { 9.4.10, } K, \eta_{1}\right) .
\end{aligned}
$$

Note that the $N_{0}$ is uniformly bounded in $\hat{n}$. Then, we obtain for $N \geq N$ 9.4.11 $:=$ $N_{1}(\eta, K, \hat{n})$ and $(t, x) \in Z(N, n, K, \beta)(\omega), \tilde{d}=d\left((s, t, x),\left(s^{\prime}, t^{\prime}, x^{\prime}\right)\right) \leq 2^{-N}, s \leq K$ and $s \leq t, s^{\prime} \leq t^{\prime} \leq T_{K}$ :

$$
\left|F_{a_{n}^{\lambda}, 1}(s, t, x)-F_{a_{n}^{\lambda}, 1}\left(s^{\prime}, t^{\prime}, x^{\prime}\right)\right| \leq 32(q+2) 4^{c(q)+1} \Delta\left(m, n, 2^{-N}\right) \tilde{d}^{1-\eta_{1}} .
$$

Thus,

$$
\left|F_{a_{n}^{\lambda}, 1}(s, t, x)-F_{a_{n}^{\lambda}, 1}\left(s^{\prime}, t^{\prime}, x^{\prime}\right)\right| \leq 2^{-88} q^{-4} \tilde{d}^{1-\eta_{1}} \bar{\Delta}_{u_{1}^{\prime}}\left(m, n, \lambda, \varepsilon_{0}, 2^{-N}\right) .
$$

However, if $t^{\prime} \leq t$, then $\left(t^{\prime}, x^{\prime}\right) \in Z(N-1, n, K+1, \beta)$ interchange $(s, t, x)$ with $\left(s^{\prime}, t^{\prime}, x^{\prime}\right)$ and we give the same estimate as 9.61) to bound

$$
Q_{a_{n}^{\lambda}}^{t o t}\left(s^{\prime}, t^{\prime}, x^{\prime}, s, t, x\right) \leq 4 \mathrm{RHS} \text { of 9.61), }
$$

as always "RHS" stands for "the right hand side of". Proceeding as in the case $t \leq t^{\prime}$, we end up with (9.64) replaced by:

$$
\left|F_{a_{n}^{\lambda}, 1}\left(s^{\prime}, t^{\prime}, x^{\prime}\right)-F_{a_{n}^{\lambda}, 1}(s, t, x)\right| \leq 2^{-86} q^{-4} \tilde{d}^{1-\eta_{1}} \bar{\Delta}_{u_{1}^{\prime}}\left(m, n, \lambda, \varepsilon_{0}, 2^{-N}\right) .
$$


This does the proof for the first coordinate and clearly the constant 9.4 .11 and $N \overline{9.4 .11}$ can be chosen such that the result holds uniformly for all dimensions $1 \leq$ $l \leq q$.

So, applying Proposition 9.4.11 for any dimension $l=1, \ldots, q$, we get for the gradient $\nabla u_{1, \delta}(t, x)=\left(F_{\delta, l}(t, t, x)\right)_{1 \leq l \leq q}($ remember the Stochastic Fubini Theorem):

Corollary 9.4.12. Let $0 \leq m \leq \bar{m}+1$ and assume that $\left(P_{m}\right)$ holds. Let $n, \eta_{1}, \varepsilon_{0}$, $K, \lambda$ and $\beta$ be as in Proposition 9.4.11. For all $N \geq N_{\text {9.4.11, }}(t, x) \in Z(N, n, K, \beta)$, $x^{\prime} \in \mathbb{R}^{q}$ and $t^{\prime} \leq T_{K}$ :

$$
\begin{aligned}
& d\left((t, x),\left(t^{\prime}, x^{\prime}\right)\right) \leq 2^{-N} \text { implies that } \\
& \left|\nabla u_{1, a_{n}^{\lambda}}(t, x)-\nabla u_{1, a_{n}^{\lambda}}\left(t^{\prime}, x^{\prime}\right)\right| \leq 2^{-85} q^{-2} d\left((t, x),\left(t^{\prime}, x^{\prime}\right)\right)^{1-\eta_{1}} \bar{\Delta}_{u_{1}^{\prime}}\left(m, n, \lambda, \varepsilon_{0}, 2^{-N}\right) .
\end{aligned}
$$

This result gives us something like a Hölder regularity of the gradient $\nabla u_{1, \delta}$ with $\delta=a_{n}^{\lambda}$. This will be helpful later.

Recalling the definition of $J_{n, i}$, however, we just "know" the range of the gradients of $u_{1, \delta}$ for $\delta=a_{n}$. But it will be helpful to find a result relating this range to the gradients of $u_{1, \delta}$ for $\delta=a_{n}^{\lambda}$. The definition of $F_{\delta, l}$ allows us to relate these two gradients, since for $\delta \geq a_{n}$ and $s=t-\delta+a_{n}$ :

$$
\begin{aligned}
\partial_{x_{l}} u_{1, \delta}(t, x)=\partial_{x_{l}} P_{\delta}\left(u_{(t-\delta)^{+}}\right)(x) & =\partial_{x_{l}} P_{t-s+a_{n}}\left(u_{\left(s-a_{n}\right)^{+}}\right)(x) \\
& =-F_{a_{n}, l}(s, t, x) \\
& =-F_{a_{n}, l}\left(t-\delta+a_{n}, t, x\right) .
\end{aligned}
$$

Note the last equality holds for any $t, \delta, a_{n} \geq 0$, where they are trivial if $t-\delta \leq 0$. Again we will give the proof for $l=1$ only, see Remark 9.4.4.

Lemma 9.4.13. Let $0 \leq m \leq \bar{m}+1$ and assume that $\left(P_{m}\right)$ holds. For all $K \in \mathbb{N} \geq K_{1}, R>2, n \in \mathbb{N}, \beta \in[0,1 / 2], \varepsilon_{0} \in(0,1)$, there exist 90.4.13 $(K)$, $N N_{9.4 .13}\left(m, n, R, \varepsilon_{0}, K, \beta\right)(\omega) \in \mathbb{N}$ almost surely such that $\forall \eta_{1} \in(1 / R, 1 / 2), N \in \mathbb{N}$, $(t, x) \in \mathbb{R}_{+} \times \mathbb{R}^{q}$ the following holds.

$$
\begin{aligned}
& \text { For } \omega \in\{(t, x) \in Z(N, n, K, \beta), N \geq N \overline{9.4 .13}\} \\
& Q_{S, a_{n}}(s, t, t, x) \leq 9.9 .13(K)\left[a_{n}^{-2 \varepsilon_{0}}+2^{4 N[.4 .13}\right] \\
& {\left[|t-s|^{1-\eta_{1} / 4}\left(\left((t-s) \vee a_{n}\right)^{\left(\gamma \gamma_{m}-1-\alpha / 2\right)}+a_{n}^{2 \beta \gamma}\left((t-s) \vee a_{n}\right)^{(\gamma-1-\alpha / 2)}\right)\right.} \\
& \left.+\mathbb{1}\left\{a_{n}<2^{-2 N}\right\}\left((t-s) \wedge a_{n}\right) a_{n}^{-1-\alpha / 2} 2^{N \eta_{1} / 2}\left[2^{-2 N \gamma \gamma_{m}}+a_{n}^{2 \beta \gamma} 2^{-2 N \gamma}\right]\right] \\
& \forall 0 \leq s \leq t .
\end{aligned}
$$


Proof. Let $\xi=\left(1-(4 \gamma R)^{-1}\right) \vee\left(3 / 2-(2 \gamma)^{-1}\right)$ and set $N_{\overline{9.4 .13}}=N_{1}\left(m, n, \xi(R), \varepsilon_{0}, K, \beta\right)$. We can assume that $t \geq a_{n}$ and hence for all $0 \leq r \leq t-a_{n}$ :

$$
|t-r|^{1 / 2} \geq\left|t-\left(t-a_{n}\right)\right|^{1 / 2}=a_{n}^{1 / 2}
$$

and

$$
2^{-N} \vee d((r, w),(t, x)) \geq a_{n}^{1 / 2}
$$

Use this in Lemma 9.4 .3 to obtain

$$
\begin{aligned}
& Q_{S, a_{n}}(s, t, t, x)=\int_{(s-\delta)^{+}}^{t-\delta} d r \int_{\mathbb{R}^{q}} d w \int_{\mathbb{R}^{q}} d z p_{t^{\prime}-r, 1}\left(w-x^{\prime}\right) p_{t^{\prime}-r, 1}\left(z-x^{\prime}\right) \\
& R_{0}^{2 \gamma} e^{R_{1}(|w|+|z|)}|u(r, w)|^{\gamma}|u(r, z)|^{\gamma}\left(|w-z|^{-\alpha}+1\right) \\
& \leq G_{9.4 .3} \int_{(s-\delta)^{+}}^{t-\delta} \int_{\mathbb{R}^{q}} d w \int_{\mathbb{R}^{q}} d z p_{t-r, 1}(w-x) p_{t-r, 1}(z-x) e^{\left(R_{1}+\gamma\right)(|w-x|+|z-x|)} \\
& R_{0}^{2 \gamma} e^{2 R_{1}|x|}\left(2^{-N} \vee(t-r)^{1 / 2}+|x-w|\right)^{\xi \gamma}\left(2^{-N} \vee(t-r)^{1 / 2}+|x-z|\right)^{\xi \gamma} \\
& {\left[\left(2^{-N} \vee(t-r)^{1 / 2}+|x-w|\right)^{\gamma_{m}-1}+a_{n}^{\beta}\right]^{\gamma}} \\
& {\left[\left(2^{-N} \vee(t-r)^{1 / 2}+|x-z|\right)^{\gamma_{m}-1}+a_{n}^{\beta}\right]^{\gamma}\left(|w-z|^{-\alpha}+1\right) .}
\end{aligned}
$$

By Lemma 9.3.6, we can evaluate the spatial integrals

$$
\begin{aligned}
& Q_{S, a_{n}}(s, t, t, x) \leq C_{9.4 .3} c(K) \int_{\left(s-a_{n}\right)^{+}}^{t-a_{n}} d r(t-r)^{-1-\alpha / 2} \\
& \left(2^{-N} \vee(t-r)^{1 / 2}\right)^{2 \xi \gamma}\left[\left(2^{-N} \vee(t-r)^{1 / 2}\right)^{2 \gamma\left(\gamma_{m}-1\right)}+a_{n}^{2 \beta \gamma}\right] \\
& =C_{9.4 .3} c(K) \\
& {\left[\int_{\left(s-a_{n}\right)^{+}}^{t-a_{n}} d r(t-r)^{-1-\alpha / 2+\gamma \xi+\gamma\left(\gamma_{m}-1\right)}+a_{n}^{2 \beta \gamma}(t-r)^{-1-\alpha / 2+\gamma \xi}\right.} \\
& \left.+2^{-2 N \gamma \xi}\left[2^{-2 N \gamma\left(\gamma_{m}-1\right)}+a_{n}^{2 \beta \gamma}\right] \int_{\left(s-a_{n}\right)^{+}}^{t-a_{n}} \mathbb{1}\left\{r \geq t-2^{-2 N}\right\}(t-r)^{-1-\alpha / 2} d r\right] \\
& =c\left(J_{1}+J_{2}\right) .
\end{aligned}
$$

As in Lemma 9.4 .9 there are two integrals to consider now. For $J_{2}$ we do

$$
\begin{aligned}
J_{2} & =2^{-2 N \gamma \xi}\left[2^{-2 N \gamma\left(\gamma_{m}-1\right)}+a_{n}^{2 \beta \gamma}\right] \int_{\left(s-a_{n}\right)^{+}}^{t-a_{n}} \mathbb{1}\left\{r \geq t-2^{-2 N}\right\}(t-r)^{-1-\alpha / 2} d r \\
& \leq 2^{-2 N \gamma \xi}\left[2^{-2 N \gamma\left(\gamma_{m}-1\right)}+a_{n}^{2 \beta \gamma}\right] \mathbb{1}\left\{a_{n} \leq 2^{-2 N}\right\}\left[a_{n}^{-1-\alpha / 2}|t-s| \wedge \frac{2}{\alpha} a_{n}^{-\alpha / 2}\right] \\
& \leq 2^{-2 N \gamma \xi}\left[2^{-2 N \gamma\left(\gamma_{m}-1\right)}+a_{n}^{2 \beta \gamma}\right] \mathbb{1}\left\{a_{n} \leq 2^{-2 N}\right\} \frac{2}{\alpha} a_{n}^{-1-\alpha / 2}\left[|t-s| \wedge a_{n}\right] .
\end{aligned}
$$


And for $J_{1}$ we first refer to the estimate (9.56) showing that all exponents in the integrands stay above -1 . Before we start, note that for $p \in(-(1+\gamma) / 2,1)$.

$$
\begin{aligned}
\int_{\left(s-a_{n}\right)^{+}}^{t-a_{n}}(t-r)^{p} d r & \leq \frac{2}{1-\gamma}\left[\left(t-\left(s-a_{n}\right)\right)^{p+1}-a_{n}^{p+1}\right] \\
& \leq \frac{4}{1-\gamma}(t-s)\left[(t-s) \vee a_{n}\right]^{p}
\end{aligned}
$$

by the Mean Value Theorem and $a+b \leq 2(a \vee b)$. Then, we get for $J_{1}$ :

$$
\begin{aligned}
J_{1}= & C_{9.4 .3} c\left(R_{1}, K, \gamma\right) \int_{\left(s-a_{n}\right)^{+}}^{t-a_{n}} d r(t-r)^{-1-\alpha / 2+\gamma \xi+\gamma\left(\gamma_{m}-1\right)}+a_{n}^{2 \beta \gamma}(t-r)^{-1-\alpha / 2+\gamma \xi} \\
\leq & \frac{4}{1-\gamma}(t-s)\left[\left((t-s) \vee a_{n}\right)^{\gamma\left(\gamma_{m}+\xi-1\right)-1-\alpha / 2}+a_{n}^{2 \beta \gamma}\left((t-s) \vee a_{n}\right)^{\xi \gamma-1-\alpha / 2}\right] \\
\leq & C \overline{9.4 .3} c_{1}\left(\alpha, d, R_{1}, K, \gamma\right)(t-s)^{1-\gamma(1-\xi)} \\
& {\left[\left((t-s) \vee a_{n}\right)^{\gamma \gamma_{m}-1-\alpha / 2}+a_{n}^{2 \beta \gamma}\left((t-s) \vee a_{n}\right)^{\gamma-1-\alpha / 2}\right] . }
\end{aligned}
$$

In the last line we just used that for $p<q<1$ it holds that $(a \vee b)^{p}=(a \vee b)^{q}(a \vee$ $b)^{p-q} \leq(a \vee b)^{q} a^{p-q}$.

Putting all things together and using $\gamma(1-\xi) \leq(4 R)^{-1}<\eta_{1} / 4$, the proof ends.

Proposition 9.4.14. Let $0 \leq m \leq \bar{m}+1$ and assume that $\left(P_{m}\right)$ holds. Then for all $n \in \mathbb{N}, \eta_{1} \in(0,1 / 2], \varepsilon_{0} \in(0,1), K \in \mathbb{N} \geq K_{1}, \beta \in[0,1 / 2]$ there is an $N_{9.4 .14}=$ $N_{9.4 .14}\left(m, n, \eta_{1}, \varepsilon_{0}, K, \beta\right)(\omega) \in \mathbb{N} \geq 2$ almost surely such that $\forall N \geq N \overline{9.4 .14},(t, x) \in$ $Z(N, n, K, \beta)$ and $0 \leq t-s \leq N^{-8 / \eta_{1}}$ it holds that for $l=1, \ldots, q$ :

$$
\begin{aligned}
& \left|F_{a_{n}, l}(s, t, x)-F_{a_{n}, l}(t, t, x)\right| \leq 2^{-78} q^{-1} a_{n}^{-\varepsilon_{0}}\left[2^{-N\left(1-\eta_{1}\right)}\left(a_{n}^{1 / 2} \vee 2^{-N}\right)^{\left(\gamma \gamma_{m}-1-\alpha / 2\right) \wedge 0}\right. \\
& \quad+2^{N \eta_{1}} a_{n}^{-\alpha / 4}\left(\frac{2^{-N}}{\sqrt{a_{n}}}+1\right)\left(2^{-N \gamma \gamma_{m}}+a_{n}^{\beta \gamma}\left(\sqrt{a_{n}} \vee 2^{-N}\right)^{\gamma}\right) \\
& \left.\quad+q^{-1}(t-s)^{\left(1-\eta_{1}\right) / 2}\left(\left(\sqrt{t-s} \vee \sqrt{a_{n}}\right)^{\gamma \gamma_{m}-1-\alpha / 2}+a_{n}^{\beta \gamma}\left(\sqrt{t-s} \vee \sqrt{a_{n}}\right)^{\gamma-1-\alpha / 2}\right)\right] .
\end{aligned}
$$

Moreover, $N$ is.4.14 stochastically bounded uniformly in $(n, \beta)$.

Proof. Let $R=2 / \eta_{1}$. We give a proof for $l=1$ only. Then, Lemma 9.4 .13 implies that

$$
\begin{aligned}
& Q_{S, a_{n}}(s, t, t, x)^{1 / 2} \leq \sqrt{q_{9.4 .13}}(K)\left[a_{n}^{-\varepsilon_{0}}+2^{2 N \overline{9.4 .13}]}\right. \\
& \quad\left[\sqrt{t-s} \eta^{1 / 4} \sqrt{t-s}{ }^{1-\eta_{1} / 2}\left(\left(\sqrt{t-s} \vee \sqrt{a_{n}}\right)^{\gamma \gamma_{m}-1-\alpha / 2}+a_{n}^{\beta \gamma}\left(\sqrt{t-s} \vee \sqrt{a_{n}}\right)^{\gamma-1-\alpha / 2}\right)\right. \\
& \left.\quad+2^{-N \eta_{1} / 4} \mathbb{1}\left\{a_{n}<2^{-2 N}\right\}\left((t-s) \wedge a_{n}\right)^{1 / 2} a_{n}^{-(1+\alpha / 2) / 2} 2^{N \eta_{1} / 2}\left[2^{-N \gamma \gamma_{m}}+a_{n}^{\beta \gamma} 2^{-N \gamma}\right]\right] \\
& \leq c_{1}(K)\left[a_{n}^{-\varepsilon_{0}}+2^{2 N[9.4 .13}\right]\left[\sqrt{t-s} \eta_{1 / 4} \sqrt{t-s}^{1-\eta_{1} / 2}\right. \\
& \quad\left(\left(\sqrt{t-s} \vee \sqrt{a_{n}}\right)^{\gamma \gamma \gamma_{m}-1-\alpha / 2}+a_{n}^{\beta \gamma}\left(\sqrt{t-s} \vee \sqrt{a_{n}}\right)^{\gamma-1-\alpha / 2}\right) \\
& \left.\quad+2^{-N \eta_{1} / 4} a_{n}^{-\alpha / 4} 2^{N \eta_{1} / 2}\left[2^{-N \gamma \gamma_{m}}+a_{n}^{\beta \gamma} 2^{-N \gamma}\right]\right]
\end{aligned}
$$


Now, we proceed similarly as in the proof of Proposition 9.4.11. Choose $N_{0}=$ $N_{0}(K)$ such that

$$
c_{1}(K)\left[a_{n}^{-\varepsilon_{0}}+2^{2 N[9.4 .13]}\right] 2^{-2 N\left[\frac{9.4 .13}{2}-2 N_{0}\right.} \leq 2^{-100} a_{n}^{-\varepsilon_{0}}
$$

and then observe that for $N_{2}\left(m, n, \eta_{1}, \varepsilon_{0}, K, \beta\right)=\frac{8}{\eta_{1}}\left[N\left[\overline{9.4 .13}+N_{0}(K)+3 \log (q+\right.\right.$ $\left.2)(\log 2)^{-1}\right]$ :

$$
\begin{aligned}
(q+2)^{3} c_{1}\left(K, R_{0}\right)\left[a_{n}^{-\varepsilon_{0}}+2^{2 N\left[\frac{9.4 .13}{6}\right]} 2^{-N_{2} \eta_{1} / 4}\right. & \leq c_{1}\left(K, R_{0}\right)\left[a_{n}^{-\varepsilon_{0}}+2^{2 N\left[\frac{9.4 .13}{10}\right]} 2^{-2 N\left[\frac{9.4 .13}{-2 N_{0}}\right.}\right. \\
& \leq 2^{-100} a_{n}^{-\varepsilon_{0}} .
\end{aligned}
$$

In the case $\sqrt{t-s} \leq 2^{-N_{2}}$, we clearly have $(t-s)^{\eta_{1} / 8} \leq 2^{-\eta_{1} N_{2} / 4}$. For $N \geq N_{2}$ it follows that:

$$
\begin{aligned}
Q_{S, a_{n}}(s, t, t, x)^{1 / 2} \leq(q+2)^{-3} 2^{-100} a_{n}^{-\varepsilon_{0}}\left\{\sqrt { t - s } ^ { 1 - \eta _ { 1 } / 2 } \left[\left(\sqrt{t-s} \vee \sqrt{a_{n}}\right)^{\gamma \gamma_{m}-1-\alpha / 2}\right.\right. \\
\left.+a_{n}^{\beta \gamma}\left(\sqrt{t-s} \vee \sqrt{a_{n}}\right)^{\gamma-1-\alpha / 2}\right] \\
\left.+a_{n}^{-\alpha / 4} 2^{N \eta_{1} / 2}\left[2^{-N \gamma \gamma_{m}}+a_{n}^{\beta \gamma} 2^{-N \gamma}\right]\right\} \\
=: \sqrt{t-s}^{1-\eta_{1} / 2} \Delta_{1}\left(m, n, \sqrt{t-s} \vee \sqrt{a_{n}}\right)+2^{N \eta_{1} / 2} \Delta_{2}\left(m, n, 2^{-N}\right) .
\end{aligned}
$$

With the Dubins-Schwarz Theorem (Theorem 3.2.8) we obtain

$$
\begin{aligned}
\mathbb{P}\left[\mid F_{a_{n}, 1}(s,\right. & t, x)-F_{a_{n}, 1}(t, t, x) \mid \geq \sqrt{t-s}{ }^{1-\eta_{1}} \Delta_{1}\left(m, n, \sqrt{t-s} \vee \sqrt{a_{n}}\right) \\
& \left.+2^{N \eta_{1}} \Delta_{2}\left(m, n, 2^{-N}\right),(t, x) \in Z(N, n, K, \beta), N \geq N_{2}, \sqrt{t-s} \leq 2^{-N_{2}}\right] \\
\leq & \mathbb{P}\left[\sup _{u \leq 1}|B(u)| \geq(t-s)^{-\eta_{1} / 4} \wedge 2^{N \eta_{1} / 2}\right] \mathbb{1}\{t-s \leq 1\} \\
\leq & c_{0} \exp \left(-\frac{1}{2}\left((t-s)^{-\eta_{1} / 2} \wedge 2^{N \eta_{1}}\right)\right) .
\end{aligned}
$$

Let

$$
\begin{aligned}
\ell_{N} & =2^{2(N+c(q)+1)} N^{-8 / \eta_{1}}, \text { where } \\
c(q) & =(\log 2)^{-1} \log (q+2) \leq q+1 .
\end{aligned}
$$

If $N \geq N_{3}:=2^{N_{2}}$ that easily leads to

$$
\begin{array}{r}
N \geq 2^{N_{2}} \Rightarrow N^{-4 / \eta_{1}} \leq 2^{-4 N_{2} / \eta_{1}} \leq 2^{-8 N_{2}} \leq 2^{-N_{2}-1} \text { and } \\
\sqrt{\ell_{N}} 2^{-N-c(q)}=2^{N+c(q)+1} N^{-4 / \eta_{1}} 2^{-N-c(q)} \leq 2 N^{-4 / \eta_{1}}\left(\leq 2^{-N_{2}}\right) .
\end{array}
$$

We are going to consider

$$
\begin{aligned}
& M_{N}=\max \{ \\
& \frac{\left|F_{a_{n}, 1}\left(i 2^{-2(N+c(q))}, j 2^{-2(N+c(q))}, k 2^{-(N+c(q))}\right)-F_{a_{n}, 1}\left(j 2^{-2(N+c(q))}, j 2^{-2(N+c(q))}, k 2^{-(N+c(q))}\right)\right|}{\left(\sqrt{j-i} 2^{-(N+c(q))}\right)^{1-\eta_{1}} \Delta_{1}\left(m, n, \sqrt{j-i} 2^{-(N+c(q))} \vee \sqrt{a_{n}}\right)+2^{N \eta_{1} \Delta_{2}\left(m, n, 2^{-N}\right)},} \\
& \left.\quad 0 \leq j-i \leq \ell_{N},\left(j 2^{-2(N+c(q))}, k 2^{-(N+c(q))}\right) \in Z(N, n, K, \beta), i, j \in \mathbb{Z}_{+}, k \in \mathbb{Z}^{q}\right\}
\end{aligned}
$$


for which it holds by decomposing into the different possible choices for $i, j$ and $k$ and (9.68):

$$
\begin{aligned}
& \mathbb{P}\left[M_{N} \geq 1, N \geq N_{3}\right] \\
& \leq(K+1)^{2} 2^{4(N+c(q))}(2 K+1)^{q} 2^{q(N+c(q))} \\
& \quad c_{0} \exp \left(-\frac{1}{2}\left(\ell_{N} 2^{-2(N+c(q))}\right)^{-\eta_{1} / 2} \wedge 2^{N \eta_{1}}\right) \mathbb{1}\left\{\ell_{N} \geq 1\right\} \\
& =c_{2}(q, K) 2^{(4+q) N} \exp \left(-\frac{1}{2}\left(\sqrt{\ell_{N}} 2^{-(N+c(q))}\right)^{-\eta_{1}} \wedge\left(2^{(N+c(q)) \eta_{1}} 2^{-c(q) \eta_{1}}\right)\right) \mathbb{1}\left\{\ell_{N} \geq 1\right\} \\
& \leq c_{2}(q, K) 2^{(4+q) N} \exp \left(-\frac{1}{2} 2^{-c(q) \eta_{1}}\left(\sqrt{\ell_{N}} 2^{-(N+c(q))}\right)^{-\eta_{1}} \wedge\left(2^{-(N+c(q))}\right)^{-\eta_{1}}\right) \mathbb{1}\left\{\ell_{N} \geq 1\right\} \\
& \leq c_{2}(q, K) 2^{(4+q) N} \exp \left(-2^{-1-c(q) \eta_{1}}\left(\sqrt{\ell_{N}} 2^{-(N+c(q))}\right)^{-\eta_{1}}\right) \\
& \left.\leq c_{2}(q, K) 2^{(4+q) N} \exp \left(-2^{-2-q / 2} N^{4}\right)(\text { by } 9.70,09.72) \text { and } \eta_{1} \leq 1 / 2\right) .
\end{aligned}
$$

Set

$$
A_{N}=\left\{M_{N} \geq 1, N \geq N_{3}\right\}
$$

and

$$
N_{4}=\min \left\{N: \omega \in \bigcap_{N^{\prime}=N}^{\infty} A_{N^{\prime}}^{c}\right\} .
$$

Then, we have for $N \geq N_{5}=(\log 2)^{1 / 3} 2^{1+q / 6}(q+4)^{1 / 3}$ that

$$
\begin{aligned}
P\left[N_{4} \geq N\right] & =P\left[\bigcup_{N^{\prime}=N}^{\infty} A_{N^{\prime}}\right] \leq c_{2}(q, K) \sum_{N^{\prime}=N}^{\infty} 2^{(4+q) N^{\prime}} \exp \left(-2^{-2-q / 2} N^{\prime 4}\right) \\
& \leq c_{3}(q, K) \exp \left(-2^{-3-q / 2} N^{4}\right) .
\end{aligned}
$$

Next, choose $N_{6}$ such that $2^{1-N} \leq N^{-4 / \eta_{1}}$ for all $N \geq N_{6}$. Then set

$$
N_{9.4 .14}:=\left(N \overline{9.4 .11} \vee N_{3} \vee N_{4} \vee N_{5} \vee N_{6}\right)+c(q),
$$

which is uniformly bounded in $(n, \beta)$ as all of the components are.

From now on, consider the event

$$
N \geq N \overline{\text { 9.4.14, }}(t, x) \in Z(N, n, K, \beta), s \leq t, \sqrt{t-s} \leq N^{-4 / \eta_{1}} .
$$

Then, there are two cases:

Case 1: $t-s \geq 2^{2(1-N)}$

As $N \geq N \overline{9.4 .14}$ we have $N-c(q) \geq N_{4}$, i.e. $\omega \in A_{N-c(q)}^{c}$. Additionally, $N-c(q) \geq$ $N_{3}$ and therefore

$$
M_{N-c(q)}<1 .
$$


To use this result we need to introduce discrete versions of $s, t$ and $x$. We set $s_{\ell}=$ $\left\lfloor 2^{2 \ell} s\right\rfloor 2^{-2 \ell}, t_{\ell}=\left\lfloor 2^{2 \ell} t\right\rfloor 2^{-2 \ell}, x_{\ell}=\sum_{k=1}^{q} \operatorname{sgn}\left(x_{k}\right)\left\lfloor 2^{\ell}\left|x_{k}\right|\right\rfloor 2^{-\ell} e_{k}$, where $\left(e_{k}\right)$ are the standard unit vectors in $\mathbb{R}^{q}$. Then $d\left(\left(t_{N}, x_{N}\right),\left(\hat{t}_{0}, \hat{x}_{0}\right)\right) \leq 2^{-N}+d\left(\left(t_{N}, x_{N}\right),(t, x)\right) \leq$ $2^{-(N-c(q))}$. Hence due to the choice of $c(q)$ in $(9.70)$ :

$$
\left(t_{N}, x_{N}\right) \in Z(N-c(q), n, K, \beta) .
$$

Now, split the expressions

$$
\begin{aligned}
\mid F_{a_{n}, 1}(s, & t, x)-F_{a_{n}, 1}(t, t, x) \mid \\
\leq & {\left[\left|F_{a_{n}, 1}(s, t, x)-F_{a_{n}, 1}\left(s_{N}, t_{N}, x_{N}\right)\right|+\left|F_{a_{n}, 1}\left(t_{N}, t_{N}, x_{N}\right)-F_{a_{n}, 1}(t, t, x)\right|\right] } \\
& +\left|F_{a_{n}, 1}\left(s_{N}, t_{N}, x_{N}\right)-F_{a_{n}, 1}\left(t_{N}, t_{N}, x_{N}\right)\right| \\
= & T_{1}+T_{2} .
\end{aligned}
$$

Since $(t, x) \in Z(N, n, K, \beta), \tilde{d}\left((t, t, x),\left(t_{N}, t_{N}, x_{N}\right)\right) \vee \tilde{d}\left((s, t, x),\left(s_{N}, t_{N}, x_{N}\right)\right) \leq(2+$ q) $2^{-N}=2^{-(N-c(q))}$ and as $N-c(q) \geq N \sqrt{9.4 .11}$ we can apply Proposition 9.4.11 for both terms in $T_{1}$ and obtain

$$
\begin{aligned}
T_{1} & \leq 2^{-85} 2^{-(N-c(q))\left(1-\eta_{1}\right)} \bar{\Delta}_{u_{1}^{\prime}}\left(m, n, 1, \varepsilon_{0}, 2^{-(N-c(q))}\right) q^{-4} \\
& \leq 2^{-85} 2^{c(q)} 4^{c(q)} q^{-4} 2^{-N\left(1-\eta_{1}\right)} \bar{\Delta}_{u_{1}^{\prime}}\left(m, n, 1, \varepsilon_{0}, 2^{-N}\right) \\
& \leq 2^{-85} q^{-4} q^{3} 3^{3} 2^{-N\left(1-\eta_{1}\right)} \bar{\Delta}_{u_{1}^{\prime}}\left(m, n, 1, \varepsilon_{0}, 2^{-N}\right) \\
& \leq 2^{-80} q^{-1} 2^{-N\left(1-\eta_{1}\right)} \bar{\Delta}_{u_{1}^{\prime}}\left(m, n, 1, \varepsilon_{0}, 2^{-N}\right),
\end{aligned}
$$

where we used $\bar{\Delta}_{u_{1}^{\prime}}\left(\ldots, 2^{-N+c(q)}\right) \leq 4^{c(q)} \bar{\Delta}_{u_{1}^{\prime}}\left(\ldots, 2^{-N}\right)$, which can be seen from 9.59. For $T_{2}$ we use $N \geq N_{6}$ :

$$
\begin{aligned}
\sqrt{\left\lfloor 2^{2 N} t\right\rfloor-\left\lfloor 2^{2 N} s\right\rfloor 2^{-N} \leq \sqrt{t_{N}-s_{N}}} & \leq 2 \cdot 2^{-N}+\sqrt{t-s} \\
& \leq 2 N^{-4 / \eta_{1}} \\
& =2^{N+1} N^{-4 / \eta_{1}} 2^{-N} \\
& \leq 2^{N+1}(N-c(q))^{-4 / \eta_{1}} 2^{-N} \\
& =\sqrt{\ell_{N-c(q)}} 2^{-N}(\text { recall }(9.69)), \\
\text { hence }\left\lfloor 2^{2 N} t\right\rfloor-\left\lfloor 2^{2 N} s\right\rfloor & \leq \ell_{N-c(q)} .
\end{aligned}
$$

Therefore, by 9.74), 9.75) and the definition of $M_{N-c(q)}$ :

$$
\begin{aligned}
T_{2} \leq & M_{N-c(q)}\left[\left(\sqrt{t_{N}-s_{N}}\right)^{1-\eta_{1}} \Delta_{1}\left(m, n, \sqrt{t_{N}-s_{N}} \vee \sqrt{a_{n}}\right)\right. \\
& \left.+2^{(N-c(q)) \eta_{1}} \Delta_{2}\left(m, n, 2^{-(N-c(q))}\right)\right] \\
\leq & {\sqrt{t_{N}-s_{N}}}^{1-\eta_{1}} \Delta_{1}\left(m, n, \sqrt{t_{N}-s_{N}} \vee \sqrt{a_{n}}\right)+2^{(N-c(q)) \eta_{1}} \Delta_{2}\left(m, n, 2^{-(N-c(q))}\right) .
\end{aligned}
$$


Since $t-s \geq 2^{2-2 N}$, we can bound $t-s$ by

$$
\left(t-t_{N}\right)+\left(t_{N}-s_{N}\right)+\left(s_{N}-s\right) \leq 2^{1-2 N}+\left(t_{N}-s_{N}\right) \leq \frac{1}{2}(t-s)+\left(t_{N}-s_{N}\right),
$$

and hence,

$$
\frac{1}{2}(t-s) \leq\left(t_{N}-s_{N}\right)
$$

and similarly,

$$
\left(t_{N}-s_{N}\right) \leq t-s+2^{-2 N+1} \leq 2(t-s) .
$$

Therefore, we get by adding up $T_{1}$ and $T_{2}$ and writing out the definition of the several $\Delta$ 's, defined in 9.59) and 9.67):

$$
\begin{aligned}
& \left|F_{a_{n}, 1}(s, t, x)-F_{a_{n}, 1}(t, t, x)\right| \leq 2^{-80} q^{-1} 2^{-N\left(1-\eta_{1}\right)} \bar{\Delta}_{u_{1}^{\prime}}\left(m, n, 1, \varepsilon_{0}, 2^{-N}\right) \\
& +2^{-99} a_{n}^{-\varepsilon_{0}} \sqrt{t-s}{ }^{1-\eta_{1}}\left[\left(\sqrt{t-s} \vee \sqrt{a_{n}}\right)^{\gamma \gamma_{m}-1-\alpha / 2}+a_{n}^{\beta \gamma}\left(\sqrt{t-s} \vee \sqrt{a_{n}}\right)^{\gamma-1-\alpha / 2}\right](q+2)^{-3} \\
& +2^{-100} a_{n}^{-\varepsilon_{0}} a_{n}^{-\alpha / 4} 2^{(N-c(q)) \eta_{1}}\left[2^{-(N-c(q)) \gamma \gamma_{m}}+a_{n}^{\beta \gamma} 2^{-(N-c(q)) \gamma}\right](q+2)^{-3} \\
& \leq 2^{-80} q^{-1} 2^{-N\left(1-\eta_{1}\right)} a_{n}^{-\varepsilon_{0}}\left[a_{n}^{-1 / 2(1+\alpha / 2)} 2^{-N \gamma \gamma_{m}}+\left(a_{n}^{1 / 2} \vee 2^{-N}\right)^{\left(\gamma \gamma_{m}-1-\alpha / 2\right) \wedge 0}\right. \\
& \left.\quad+a_{n}^{-1 / 2(1+\alpha / 2)+\beta \gamma}\left(a_{n}^{1 / 2} \vee 2^{-N}\right)^{\gamma}\right] \\
& +2^{-99}(q+2)^{-3} a_{n}^{-\varepsilon_{0}} \sqrt{t-s}^{1-\eta_{1}}\left[\left(\sqrt{t-s} \vee \sqrt{a_{n}}\right)^{\gamma \gamma_{m}-1-\alpha / 2}+a_{n}^{\beta \gamma}\left(\sqrt{t-s} \vee \sqrt{a_{n}}\right)^{\gamma-1-\alpha / 2}\right] \\
& +2^{-100}(q+2)^{-1} a_{n}^{-\varepsilon_{0}} a_{n}^{-\alpha / 4} 2^{N \eta_{1}}\left[2^{-N \gamma \gamma_{m}}+a_{n}^{\beta \gamma} 2^{-N \gamma}\right] .
\end{aligned}
$$

We can put together the first and third term in the first bracket, and the last bracket using

$$
2^{-N\left(1-\eta_{1}\right)} a_{n}^{-(1+\alpha / 2) / 2}+2^{N \eta_{1}} a_{n}^{-\alpha / 4}=a_{n}^{-\alpha / 4} 2^{N \eta_{1}}\left[\frac{2^{-N}}{\sqrt{a_{n}}}+1\right]
$$

This gives,

$$
\begin{aligned}
& \left|F_{a_{n}, 1}(s, t, x)-F_{a_{n}, 1}(t, t, x)\right| \\
& \leq 2^{-78} q^{-1} a_{n}^{-\varepsilon_{0}}\left\{a_{n}^{-\frac{\alpha}{4}} 2^{N \eta_{1}}\left[\frac{2^{-N}}{\sqrt{a_{n}}}+1\right]\left[2^{-N \gamma \gamma_{m}}+a_{n}^{\beta \gamma}\left(\sqrt{a_{n}} \vee 2^{-N}\right)^{\gamma}\right]\right. \\
& \left.+\left(\sqrt{a_{n}} \vee 2^{-N}\right)^{\left(\gamma \gamma_{m}-1-\alpha / 2\right) \wedge 0} 2^{-N\left(1-\eta_{1}\right)}\right\} \\
& +2^{-99} q^{-1} a_{n}^{-\varepsilon_{0}} \sqrt{t-s}^{1-\eta_{1}}\left[\left(\sqrt{t-s} \vee \sqrt{a_{n}}\right)^{\left(\gamma \gamma_{m}-1-\alpha / 2\right)}+a_{n}^{\beta \gamma}\left(\sqrt{t-s} \vee \sqrt{a_{n}}\right)^{\gamma-1-\alpha / 2}\right] .
\end{aligned}
$$


Case 2. $t-s \leq 2^{2(1-N)}$

Our assumptions 9.73) imply that $(t, x) \in Z(N-1, n, K, \beta), s \leq t \leq K, N-1 \geq$ $N \overline{9.4 .11}$ and (in this case)

$$
\tilde{d}((s, t, x),(t, t, x)) \leq 2^{-(N-1)} .
$$

Now, just use Proposition 9.4.11 and obtain

$$
\begin{aligned}
& \left|F_{a_{n}, 1}(s, t, x)-F_{a_{n}, 1}(t, t, x)\right| \leq 2^{-86} q^{-4}(\sqrt{t-s})^{1-\eta_{1}} \bar{\Delta}_{u_{1}^{\prime}}\left(m, n, 1, \varepsilon_{0}, 2^{-(N-1)}\right) \\
& \leq 2^{-83} q^{-1} 2^{-N\left(1-\eta_{1}\right)} a_{n}^{-\varepsilon_{0}} \\
& {\left[a_{n}^{-\alpha / 4} 2^{-N \gamma \gamma_{m}}+\left(a_{n}^{1 / 2} \vee 2^{-N}\right)^{\left(\gamma \gamma_{m}-1-\alpha / 2\right) \wedge 0}+a_{n}^{\beta \gamma} a_{n}^{-(1+\alpha / 2) / 2}\left(2^{-N} \vee a_{n}^{1 / 2}\right)^{\gamma}\right]} \\
& \leq 2^{-83} q^{-1} a_{n}^{-\varepsilon_{0}}\left\{a_{n}^{-\alpha / 4} 2^{N \eta_{1}}\left(\frac{2^{-N}}{\sqrt{a_{n}}}\right)\left(2^{-N \gamma \gamma_{m}}+a_{n}^{\beta \gamma}\left(2^{-N} \vee a_{n}^{1 / 2}\right)^{\gamma}\right)\right. \\
& \left.+2^{-N\left(1-\eta_{1}\right)}\left(a_{n}^{1 / 2} \vee 2^{-N}\right)^{\left(\gamma \gamma_{m}-1-\alpha / 2\right) \wedge 0}\right\} .
\end{aligned}
$$

And this term is bounded by the first terms on the right-hand side of $(9.79)$.

Remark 9.4.15. We could have tried to obtain a more general version of Lemma 9.8.1 to be applied in the previous proof, but note that we used Proposition 9.4.11 just right after (9.75). So there needed to be some a-priori regularity assumption in Lemma 9.8.1 which made it quite more complicated.

There is a similar result for $G_{\delta}$ in a special case, which will be needed later:

Proposition 9.4.16. Let $0 \leq m \leq \bar{m}+1$ and assume that $\left(P_{m}\right)$ holds. For any $n \in \mathbb{N}, \eta_{1} \in(0,1 / 2), \varepsilon_{0} \in(0,1), K \in \mathbb{N} \geq K_{1}, \lambda \in[0,1]$ and $\beta \in[0,1 / 2]$, there is

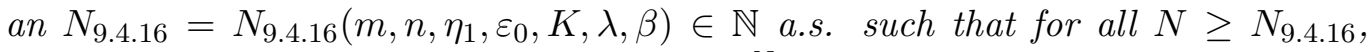
$(t, x) \in Z(N, n, K, \beta), s \leq t$ and $\sqrt{t-s} \leq 2^{-N}$,

$$
\begin{aligned}
\left|G_{a_{n}^{\lambda}}(s, t, x)-G_{a_{n}^{\lambda}}(t, t, x)\right| \leq 2^{-95} a_{n}^{-\varepsilon_{0}}(t-s)^{\left(1-\eta_{1}\right) / 2} a_{n}^{-\lambda \alpha / 4} & \\
& {\left[\left(a_{n}^{\lambda / 2} \vee 2^{-N}\right)^{\gamma \gamma_{m}}+a_{n}^{\beta \gamma}\left(\sqrt{a_{n}} \vee 2^{-N}\right)^{\gamma}\right] . }
\end{aligned}
$$

Moreover, $N_{9.4 .16}$ is stochastically bounded uniformly in $(n, \lambda, \beta)$.

The proof of this Proposition is put in Chapter 9.6. Most of the calculations are similar and even easier than the ones before: we have to deal directly with the heat kernels instead of their derivatives. Recall that the goal of this chapter was to do the induction step of $\left(P_{m}\right)$, i.e. to get good Hölder-estimates on $u=u_{1, \delta}+u_{2, \delta}$. We require some notation to give a result for $u_{1, \delta}$ :

Notation:

$$
\begin{aligned}
\bar{\Delta}_{u_{1}}\left(m, n, \lambda, \varepsilon_{0}, 2^{-N}\right)=a_{n}^{-\varepsilon_{0}-\lambda(1+\alpha / 2) / 2}[ & a_{n}^{\beta+\lambda(1+\alpha / 2) / 2}+\mathbb{1}(m \geq \bar{m})\left(a_{n}^{\lambda / 2} \vee 2^{-N}\right) \\
& \left.+\left(a_{n}^{\lambda / 2} \vee 2^{-N}\right)^{\gamma \gamma \gamma_{m}+1}+a_{n}^{\beta \gamma}\left(a_{n}^{\lambda / 2} \vee 2^{-N}\right)^{\gamma+1}\right]
\end{aligned}
$$


and

$$
N_{9.4 .17}^{\prime}(\eta)=\min \left\{N \in \mathbb{N}: 2^{1-N} \leq N^{-8 / \eta}\right\}
$$

for a constant $\eta>0$.

Proposition 9.4.17. Let $0 \leq m \leq \bar{m}+1$ and assume that $\left(P_{m}\right)$ holds. Then for all $n \in \mathbb{N}, \eta_{1} \in(0,1 / 2 \wedge(2-\alpha) / 2), \varepsilon_{0}, \varepsilon_{1} \in(0,1), K \in \mathbb{N} \geq K_{1}, \lambda \in\left[\varepsilon_{1}, 1-\varepsilon_{1}\right], \beta \in[0,1 / 2]$ there is an $N_{9.4 .17}=N \overline{9.4 .17}\left(m, n, \eta_{1}, \varepsilon_{0}, K, \lambda, \beta\right)(\omega) \in \mathbb{N} \geq 2$ almost surely such that $\forall N \geq N \overline{9.4 .17}$ and $n, \lambda$ that satisfy

$$
a_{n} \leq 2^{-2\left(N \frac{0.4 .14}{+1)}\right.} \wedge 2^{-2\left(N_{9.4 .17}^{\prime}\left(\eta_{1} \varepsilon_{1}\right)+1\right)} \text { and } \lambda \geq \varepsilon_{1},
$$

and $(t, x) \in Z(N, n, K, \beta), t^{\prime} \leq T_{K}, x^{\prime} \in \mathbb{R}^{q}$ s.t. $d\left((t, x),\left(t^{\prime}, x^{\prime}\right)\right) \leq 2^{-N}$ it holds that

$$
\left|u_{1, a_{n}^{\lambda}}(t, x)-u_{1, a_{n}^{\lambda}}\left(t^{\prime}, x^{\prime}\right)\right| \leq 2^{-90} d\left((t, x),\left(t^{\prime}, x^{\prime}\right)\right)^{1-\eta_{1}} \bar{\Delta}_{u_{1}}\left(m, n, \lambda, \varepsilon_{0}, 2^{-N}\right) .
$$

Moreover, $N_{9.4 .17}$ is stochastically bounded uniformly in $(n, \lambda, \beta)$.

Proof. Let

$N_{9.4 .17}^{\prime \prime}=2 N \overline{9.4 .11}\left(m, n, \eta_{1} / 2, \varepsilon_{0}, K+1, \lambda, \beta\right) \vee N \overline{9.4 .16}\left(m, n, \eta_{1} / 2, \varepsilon_{0}, K+1, \lambda, \beta\right)+1$, which is uniformly bounded in $(n, \lambda, \beta)$. Assume

$$
N \geq N \frac{N_{9.4 .17}^{\prime \prime}}{}(t, x) \in Z(N, n, K, \beta), t^{\prime} \leq T_{K}, d\left((t, x),\left(t^{\prime}, x^{\prime}\right)\right) \leq 2^{-N}
$$

and that 9.80 holds. Then $\left(t^{\prime}, x^{\prime}\right) \in Z(N-1, n, K+1, \beta)$ and without loss of generality $t^{\prime} \leq t$ (otherwise, we interchange the role of $t^{\prime}$ and $t$ in the following). We have by definition

$$
G_{a_{n}^{\lambda}}\left(t^{\prime}, t, x\right)=P_{t-t^{\prime}+a_{n}^{\lambda}}\left(u\left(\left(t^{\prime}-a_{n}^{\lambda}\right)^{+}, \cdot\right)\right)(x)=P_{t-t^{\prime}}\left(u_{1, a_{n}^{\lambda}}\left(t^{\prime}, \cdot\right)\right)(x) .
$$

And therefore, we can write

$$
\begin{aligned}
\left|u_{1, a_{n}^{\lambda}}\left(t^{\prime}, x^{\prime}\right)-u_{1, a_{n}^{\lambda}}(t, x)\right| \leq & \left|u_{1, a_{n}^{\lambda}}\left(t^{\prime}, x^{\prime}\right)-u_{1, a_{n}^{\lambda}}\left(t^{\prime}, x\right)\right| \\
& +\left|u_{1, a_{n}^{\lambda}}\left(t^{\prime}, x\right)-P_{t-t^{\prime}}\left(u_{1, a_{n}^{\lambda}}\left(t^{\prime}, \cdot\right)\right)(x)\right| \\
& +\left|G_{a_{n}^{\lambda}}\left(t^{\prime}, t, x\right)-G_{a_{n}^{\lambda}}(t, t, x)\right| \\
\equiv & T_{1}+T_{2}+T_{3} .
\end{aligned}
$$

We start with $T_{1}$. If $y \in\left[\left[x, x^{\prime}\right]\right]=\times_{i=1}^{q}\left[x_{i} \wedge x_{i}^{\prime}, x_{i} \vee x_{i}^{\prime}\right]$, then by Corollary 9.4.12 with $\eta_{1} / 2$ instead of $\eta_{1}$ for $1 \leq l \leq q$ and 9.65:

$$
\begin{aligned}
\left|\nabla u_{1, a_{n}^{\lambda}}\left(t^{\prime}, y\right)\right| \leq & \left|\nabla u_{1, a_{n}^{\lambda}}\left(t^{\prime}, y\right)-\nabla u_{1, a_{n}^{\lambda}}(t, x)\right|+\left|\nabla u_{1, a_{n}^{\lambda}}(t, x)-\nabla u_{1, a_{n}^{\lambda}}\left(\hat{t}_{0}, \hat{x}_{0}\right)\right| \\
& +\left|\nabla u_{1, a_{n}^{\lambda}}\left(\hat{t}_{0}, \hat{x}_{0}\right)-\nabla u_{1, a_{n}}\left(\hat{t}_{0}, \hat{x}_{0}\right)\right|+a_{n}^{\beta} . \\
\leq & 2^{-84} q^{-2} 2^{-N\left(1-\eta_{1} / 2\right)} \bar{\Delta}_{u_{1}^{\prime}}\left(m, n, \lambda, \varepsilon_{0}, 2^{-N}\right) \\
& +q \max _{l=1, \ldots, q}\left(\left|F_{a_{n}, l}\left(\hat{t}_{0}-a_{n}^{\lambda}+a_{n}, \hat{t}_{0}, \hat{x}_{0}\right)-F_{a_{n}, l}\left(\hat{t}_{0}, \hat{t}_{0}, \hat{x}_{0}\right)\right|\right) \\
& +a_{n}^{\beta} .
\end{aligned}
$$


Choose $N^{\prime}=N^{\prime}(n)$ such that $2^{-N^{\prime}-1} \leq \sqrt{a_{n}} \leq 2^{-N^{\prime}}$. By 9.80 we have $N^{\prime} \geq$ $N$ 9.4.14 and $N^{\prime} \geq N_{9.4 .17}^{\prime}\left(\eta_{1} \varepsilon_{1}\right)$, hence

$$
a_{n}^{\lambda / 2} \leq 2^{-N^{\prime} \lambda} \leq 2^{-N^{\prime} \varepsilon_{1}} \leq N^{\prime-\frac{8 \varepsilon_{1}}{\eta_{1} \varepsilon_{1}}}=N^{\prime-8 / \eta_{1}}
$$

and

$$
\left|u\left(\hat{t}_{0}, \hat{x}_{0}\right)\right| \leq a_{n}=a_{n} \wedge\left(\sqrt{a_{n}} 2^{-N^{\prime}}\right) .
$$

Therefore $\left(\hat{t}_{0}, \hat{x}_{0}\right) \in Z\left(N^{\prime}, n, K, \beta\right)$, and we apply Proposition 9.4.14 with $N^{\prime}$ instead of $N,\left(\hat{t}_{0}, \hat{x}_{0}\right)$ instead of $(t, x), \eta_{1} / 2$ instead of $\eta_{1}$ and $s=\hat{t}_{0}-a_{n}^{\lambda}+a_{n}$ :

$$
\begin{aligned}
& \left|F_{a_{n}, l}\left(\hat{t}_{0}-a_{n}^{\lambda}+a_{n}, \hat{t}_{0}, \hat{x}_{0}\right)-F_{a_{n}, l}\left(\hat{t}_{0}, \hat{t}_{0}, \hat{x}_{0}\right)\right| \\
& \leq 2^{-78} q^{-1} a_{n}^{-\varepsilon_{0}}\left[2{\sqrt{a_{n}}}^{1-\eta_{1} / 2} 2 \sqrt{a_{n}}\left(\gamma \gamma_{m}-1-\alpha / 2\right) \wedge 0+3 a_{n}^{-\frac{\alpha+\eta_{1}}{4}}\left(4 a_{n}^{\gamma \gamma_{m} / 2}+2 a_{n}^{\beta \gamma} a_{n}^{\gamma / 2}\right)\right. \\
& \left.\quad+a_{n}^{\lambda\left(1-\eta_{1} / 2\right) / 2}\left(a_{n}^{\lambda\left(\gamma \gamma_{m}-1-\alpha / 2\right) / 2}+a_{n}^{\beta \gamma} a_{n}^{\lambda(\gamma-1-\alpha / 2) / 2}\right)\right] .
\end{aligned}
$$

The second term can now be bounded by the third one, since $\sqrt{a_{n}} \leq a_{n}^{\lambda / 2}$ :

$$
\begin{aligned}
& \left|F_{a_{n}, l}\left(\hat{t}_{0}-a_{n}^{\lambda}+a_{n}, \hat{t}_{0}, \hat{x}_{0}\right)-F_{a_{n}, l}\left(\hat{t}_{0}, \hat{t}_{0}, \hat{x}_{0}\right)\right| \\
& \leq 2^{-75} q^{-1} a_{n}^{-\varepsilon_{0}}[ \\
& { }_{a_{n}}^{1-\eta_{1} / 2} \sqrt{a_{n}}\left(\gamma \gamma_{m}-1-\alpha / 2\right) \wedge 0 \\
& \left.\quad+a_{n}^{\lambda\left(1-\eta_{1} / 2\right) / 2}\left(a_{n}^{\lambda\left(\gamma \gamma_{m}-1-\alpha / 2\right) / 2}+a_{n}^{\beta \gamma} a_{n}^{\lambda(\gamma-1-\alpha / 2) / 2}\right)\right] .
\end{aligned}
$$

Now, use that

$$
\begin{aligned}
1-\frac{\eta_{1}}{2}+\gamma \gamma_{m}-1-\frac{\alpha}{2} & >-\frac{\eta_{1}}{2}+\left(\frac{1}{2}+\frac{\alpha}{4}\right) \gamma_{m}-\frac{\alpha}{2} \\
& \geq-\frac{\eta_{1}}{2}+\frac{1}{2}-\frac{\alpha}{4}>0, \text { as long as } \eta_{1}<(2-\alpha) / 2
\end{aligned}
$$

to estimate

$$
{\sqrt{a_{n}}}^{1-\eta_{1} / 2}{\sqrt{a_{n}}}^{\left(\gamma \gamma_{m}-1-\alpha / 2\right) \wedge 0} \leq\left(a_{n}^{\lambda / 2} \vee 2^{-N}\right)^{1-\frac{\eta_{1}}{2}+\left(\gamma \gamma_{m}-1-\alpha / 2\right)} .
$$

Now we put together the estimate for 9.83 :

$$
\begin{aligned}
\left|\nabla u_{1, a_{n}^{\lambda}}\left(t^{\prime}, y\right)\right| \leq 2^{-74} a_{n}^{-\varepsilon_{0}}\left\{2^{-N\left(1-\eta_{1} / 2\right)}\left(a_{n}^{\lambda / 2} \vee 2^{-N}\right)^{\left(\gamma \gamma_{m}-1-\alpha / 2\right) \wedge 0}\right. & \\
& +\left(2^{-N}\right)^{1-\frac{\eta_{1}}{2}} a_{n}^{-\lambda(1+\alpha / 2) / 2}\left[2^{-N \gamma \gamma}+\left(a_{n}^{\lambda / 2} \vee 2^{-N}\right)^{\gamma} a_{n}^{\beta \gamma}\right] \\
& +\left(a_{n}^{\lambda / 2} \vee 2^{-N}\right)^{1-\frac{\eta_{1}}{2}+\left(\gamma \gamma_{m}-1-\alpha / 2\right)} \\
& \left.+a_{n}^{\lambda\left(1-\eta_{1} / 2\right) / 2} a_{n}^{-\lambda(1+\alpha / 2) / 2}\left(a_{n}^{\frac{\lambda}{2} \gamma \gamma_{m}}+a_{n}^{\beta \gamma+\frac{\lambda}{2} \gamma}\right)\right\} \\
+ & a_{n}^{\beta} \\
\leq & a_{n}^{\beta}+2^{-73} a_{n}^{-\varepsilon_{0}}\left(a_{n}^{\lambda / 2} \vee 2^{-N}\right)^{1-\frac{\eta_{1}}{2}} \\
& \quad\left(a_{n}^{\lambda / 2} \vee 2^{-N}\right)^{\left(\gamma \gamma_{m}-1-\alpha / 2\right) \wedge 0}+a_{n}^{-\lambda(1+\alpha / 2) / 2}\left(a_{n}^{\lambda / 2} \vee 2^{-N}\right)^{\gamma \gamma_{m}} \\
& \left.+a_{n}^{-\lambda(1+\alpha / 2) / 2} a_{n}^{\beta \gamma}\left(a_{n}^{\lambda / 2} \vee 2^{-N}\right)^{\gamma}\right] \\
=: & a_{n}^{\beta}+2^{-73} \tilde{\Delta}_{u_{1}}\left(m, n, \lambda, \varepsilon_{0}, \eta_{1}, a_{n}^{\lambda / 2} \vee 2^{-N}\right) .
\end{aligned}
$$


With this estimate and the Mean Value Theorem in $\mathbb{R}^{q}$ we obtain

$$
T_{1} \leq\left|x-x^{\prime}\right|\left(a_{n}^{\beta}+2^{-73} \tilde{\Delta}_{u_{1}}\left(m, n, \lambda, \varepsilon_{0}, \eta_{1}, a_{n}^{\lambda / 2} \vee 2^{-N}\right)\right) .
$$

Additionally, Proposition 9.4 .16 gives

$$
T_{3} \leq 2^{-92}\left|t-t^{\prime}\right|^{\frac{1}{2}\left(1-\eta_{1}\right)} a_{n}^{-\varepsilon_{0}-\frac{\lambda \alpha}{4}}\left[\left(a_{n}^{\lambda / 2} \vee 2^{-N}\right)^{\gamma \gamma_{m}}+\left(a_{n}^{\lambda / 2} \vee 2^{-N}\right)^{\gamma} a_{n}^{\beta \gamma}\right] .
$$

For $T_{2}=\left|u_{1, a_{n}^{\lambda}}\left(t^{\prime}, x\right)-P_{t-t^{\prime}}\left(u_{1, a_{n}^{\lambda}}\left(t^{\prime}, \cdot\right)\right)(x)\right|$ a bit more work is required. Let $(B(s): s \geq 0)$ be a $q$-dimensional Brownian Motion started in $x \in \mathbb{R}^{q}$ under the law $\mathbb{P}_{x}$.

First, assume that

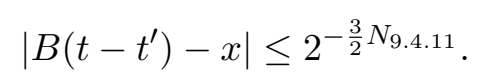

Then,

$$
\begin{aligned}
& \sqrt{t-t^{\prime}} \leq D:=d\left(\left(t^{\prime}, B\left(t-t^{\prime}\right)\right),(t, x)\right) \leq \sqrt{t-t^{\prime}}+2^{-\frac{3}{2} N \sqrt{9.4 .11}} \\
& \leq 2^{-N}+2^{-\frac{3}{2} N}
\end{aligned}
$$

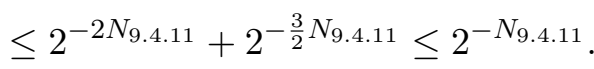

Next, choose $N^{\prime} \in\left\{N_{9.4 .11}, \ldots, N\right\}$ in such a way that

(a) if $D \leq 2^{-N}$ then $N^{\prime}:=N$

(b) if $D>2^{-N}$ then $2^{-N^{\prime}-1}<D \leq 2^{-N^{\prime}}$.

In Case (b) we have

$$
2^{-N^{\prime}-1} \leq 2^{-N}+\left|B\left(t-t^{\prime}\right)-x\right| \text {, hence } 2^{-N^{\prime}} \leq 2^{1-N}+2\left|B\left(t-t^{\prime}\right)-x\right|
$$

and trivially in Case (a):

$$
2^{-N^{\prime}}=2^{-N} \leq 2^{1-N}+2\left|B\left(t-t^{\prime}\right)-x\right| .
$$

If $y \in \mathbb{R}^{q}$ such that $|y-x| \leq\left|B\left(t-t^{\prime}\right)-x\right|$, then for $(t, x) \in Z\left(N^{\prime}, n, K, \beta\right)$ do the estimate of 9.86 to obtain:

$$
\begin{gathered}
\left|\nabla u_{1, a_{n}^{\lambda}}\left(t^{\prime}, y\right)\right| \leq 2^{-73} a_{n}^{-\varepsilon_{0}}\left(a_{n}^{\lambda / 2} \vee 2^{-N^{\prime}}\right)^{1-\frac{\eta_{1}}{2}} \\
{\left[\left(a_{n}^{\lambda / 2} \vee 2^{-N^{\prime}}\right)^{\left(\gamma \gamma_{m}-1-\alpha / 2\right) \wedge 0}+a_{n}^{-\lambda(1+\alpha / 2) / 2}\left(a_{n}^{\lambda / 2} \vee 2^{-N^{\prime}}\right)^{\gamma \gamma_{m}}\right.} \\
\left.\quad+a_{n}^{-\lambda(1+\alpha / 2) / 2} a_{n}^{\beta \gamma}\left(a_{n}^{\lambda / 2} \vee 2^{-N^{\prime}}\right)^{\gamma}\right]+a_{n}^{\beta} \\
\leq 2^{-70} a_{n}^{-\varepsilon_{0}}\left(a_{n}^{\lambda / 2}+2^{-N}+\left|B\left(t-t^{\prime}\right)-x\right|\right)^{1-\frac{\eta_{1}}{2}} \\
{\left[\left(a_{n}^{\lambda / 2} \vee 2^{-N}\right)^{\left(\gamma \gamma_{m}-1-\alpha / 2\right) \wedge 0}\right.} \\
\quad+a_{n}^{-\lambda(1+\alpha / 2) / 2}\left(a_{n}^{\lambda / 2}+2^{-N}+\left|B\left(t-t^{\prime}\right)-x\right|\right)^{\gamma \gamma_{m}} \\
\left.+a_{n}^{-\lambda(1+\alpha / 2) / 2} a_{n}^{\beta \gamma}\left(a_{n}^{\lambda / 2}+2^{-N}+\left|B\left(t-t^{\prime}\right)-x\right|\right)^{\gamma}\right]+a_{n}^{\beta} .
\end{gathered}
$$


If we use the multidimensional Mean Value Theorem we can write where expectation is taken over $B$ alone:

$$
\begin{aligned}
& \mathbb{E}_{x}\left[\mathbb{1}\left\{\left|B\left(t-t^{\prime}\right)-x\right| \leq 2^{-\frac{3}{2} N[9.411}\right\}\left|u_{1, a_{n}^{\lambda}}\left(t^{\prime}, x\right)-u_{1, a_{n}^{\lambda}}\left(t^{\prime}, B\left(t-t^{\prime}\right)\right)\right|\right] \\
& \leq \mathbb{E}_{0}\left[| B ( t - t ^ { \prime } ) | \left\{a_{n}^{-\varepsilon_{0}}\left(a_{n}^{\lambda / 2}+2^{-N}+\left|B\left(t-t^{\prime}\right)\right|\right)^{1-\frac{\eta_{1}}{2}}\right.\right. \\
& \quad\left[\left(a_{n}^{\lambda / 2} \vee 2^{-N} \mid\right)^{\left(\gamma \gamma_{m}-1-\alpha / 2\right) \wedge 0}+a_{n}^{-\lambda(1+\alpha / 2) / 2}\left(a_{n}^{\lambda / 2}+2^{-N}+\left|B\left(t-t^{\prime}\right)\right|\right)^{\gamma \gamma_{m}}\right. \\
& \left.\left.\left.\quad+a_{n}^{-\lambda(1+\alpha / 2) / 2} a_{n}^{\beta \gamma}\left(a_{n}^{\lambda / 2}+2^{-N}+\left|B\left(t-t^{\prime}\right)\right|\right)^{\gamma}\right]+a_{n}^{\beta}\right\}\right] \\
& \leq c_{1} \sqrt{t-t^{\prime}}\left\{a_{n}^{-\varepsilon_{0}}\left(a_{n}^{\lambda / 2}+2^{-N}+\sqrt{t-t^{\prime}}\right)^{1-\frac{\eta_{1}}{2}}\right. \\
& {\left[\left(a_{n}^{\lambda / 2} \vee 2^{-N}\right)^{\left(\gamma \gamma_{m}-1-\alpha / 2\right) \wedge 0}+a_{n}^{-\lambda(1+\alpha / 2) / 2}\left(a_{n}^{\lambda / 2}+2^{-N}+\sqrt{t-t^{\prime}}\right)^{\gamma \gamma_{m}}\right.} \\
& \left.\left.+a_{n}^{-\lambda(1+\alpha / 2) / 2} a_{n}^{\beta \gamma}\left(a_{n}^{\lambda / 2}+2^{-N}+\sqrt{t-t^{\prime}}\right)^{\gamma}\right]+a_{n}^{\beta}\right\}
\end{aligned}
$$

using $\left|t-t^{\prime}\right| \leq 2^{-2 N}$ bound this by

$$
\begin{aligned}
& \leq c_{2} \sqrt{t-t^{\prime}}\left\{a_{n}^{-\varepsilon_{0}}\left(a_{n}^{\lambda / 2}+2^{-N}\right)^{1-\frac{\eta_{1}}{2}}\right. \\
& \quad\left[\left(a_{n}^{\lambda / 2} \vee 2^{-N}\right)^{\left(\gamma \gamma_{m}-1-\alpha / 2\right) \wedge 0}+a_{n}^{-\lambda(1+\alpha / 2) / 2}\left(a_{n}^{\lambda / 2}+2^{-N}\right)^{\gamma \gamma_{m}}\right. \\
& \left.\left.\quad+a_{n}^{-\lambda(1+\alpha / 2) / 2} a_{n}^{\beta \gamma}\left(a_{n}^{\lambda / 2}+2^{-N}\right)^{\gamma}\right]+a_{n}^{\beta}\right\} \\
& \leq c_{3} \sqrt{t-t^{\prime}}\left\{a_{n}^{\beta}+a_{n}^{-\varepsilon_{0}}\left(a_{n}^{\lambda / 2} \vee 2^{-N}\right)^{1-\frac{\eta_{1}}{2}}\right. \\
& \quad\left[a_{n}^{-\lambda(1+\alpha / 2) / 2}\left(a_{n}^{\lambda / 2} \vee 2^{-N}\right)^{\gamma \gamma_{m}}+\left(a_{n}^{\lambda / 2} \vee 2^{-N}\right)^{\left(\gamma \gamma_{m}-1-\alpha / 2\right) \wedge 0}+\right. \\
& \left.\left.\quad a_{n}^{\beta \gamma-\lambda(1+\alpha / 2) / 2}\left(a_{n}^{\lambda / 2} \vee 2^{-N}\right)^{\gamma}\right]\right\} \\
& =: \\
& c_{3} \sqrt{t-t^{\prime}}\left\{a_{n}^{\beta}+\tilde{\Delta}_{u_{1}}\left(m, n, \lambda, \varepsilon_{0}, \eta_{1}, a_{n}^{\lambda / 2} \vee 2^{-N}\right)\right\} .
\end{aligned}
$$

This was the estimate for the "good" $B$. Turning to the "bad" $B$ we observe that for $K \geq K_{1}$ and $t^{\prime} \leq T_{K}$ it is always true that

$$
\left|u_{1, a_{n}^{\lambda}}\left(t^{\prime}, y\right)\right| \leq \mathbb{E}_{y}\left[\left|u\left(\left(t^{\prime}-a_{n}^{\lambda}\right)^{+}, B\left(a_{n}^{\lambda}\right)\right)\right|\right] \leq 2 K \mathbb{E}_{y}\left[e^{\left|B\left(a_{n}^{\lambda}\right)\right|}\right] \leq 2 K e^{1+|y|} .
$$

Since $t-t^{\prime} \leq 2^{-4 N} \sqrt{9.4 .11}$ it is true that

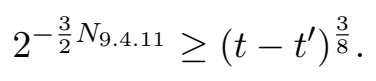

Therefore,

$$
\begin{aligned}
& \mathbb{E}_{x}\left[\mathbb{1}\left\{\left|B\left(t-t^{\prime}\right)-x\right|>2^{\left.-\frac{3}{2} N \overline{9.4 .11}\right\}}\left|u_{1, a_{n}^{\lambda}}(t, x)-u_{1, a_{n}^{\lambda}}\left(t^{\prime}, B\left(t-t^{\prime}\right)\right)\right|\right]\right. \\
& \leq \mathbb{P}_{0}\left[\left|B\left(t-t^{\prime}\right)-x\right|>2^{-\frac{3}{2} N[0.4 .11}\right]^{1 / 2} 8 e K\left(\mathbb{E}_{x}\left[e^{2\left|B\left(t-t^{\prime}\right)\right|}+e^{2|x|}\right]\right)^{1 / 2} \\
& \leq \mathbb{P}_{0}\left[B(1)>\left(t-t^{\prime}\right)^{-1 / 2}\left(t-t^{\prime}\right)^{3 / 8}\right]^{1 / 2} c(K) \\
& \leq c\left(\left(t-t^{\prime}\right)^{16 / 8} \mathbb{E}_{0}\left[|B(1)|^{16}\right]\right)^{1 / 2} \\
& \leq c\left(t-t^{\prime}\right) \leq c \sqrt{t-t^{\prime}} \tilde{\Delta}_{u_{1}} \text {. }
\end{aligned}
$$


Hence we can conclude that:

$$
T_{2} \leq c \sqrt{t-t^{\prime}}\left\{a_{n}^{\beta}+\tilde{\Delta}_{u_{1}}\left(m, n, \lambda, \varepsilon_{0}, \eta_{1}, a_{n}^{\lambda / 2} \vee 2^{-N}\right)\right\} .
$$

Now, put 9.87, 9.88) and 9.90 together to obtain

$$
\begin{aligned}
& \left|u_{1, a_{n}^{\lambda}}\left(t^{\prime}, x^{\prime}\right)-u_{1, a_{n}^{\lambda}}(t, x)\right| \\
& \leq c d\left((t, x),\left(t^{\prime}, x^{\prime}\right)\right)\left[a_{n}^{\beta}+\tilde{\Delta}_{u_{1}}\left(m, n, \lambda, \varepsilon_{0}, \eta_{1}, a_{n}^{\lambda / 2} \vee 2^{-N}\right)\right] \\
& \quad+2^{-92}\left|t-t^{\prime}\right| \frac{1}{2}\left(1-\eta_{1}\right) a_{n}^{-\varepsilon_{0}-\frac{\lambda \alpha}{4}}\left[\left(a_{n}^{\lambda / 2} \vee 2^{-N}\right)^{\gamma \gamma_{m}}+\left(a_{n}^{\lambda / 2} \vee 2^{-N}\right)^{\gamma} a_{n}^{\beta \gamma}\right] . \\
& =c d\left((t, x),\left(t^{\prime}, x^{\prime}\right)\right)\left\{a_{n}^{\beta}+a_{n}^{-\varepsilon_{0}}\left(a_{n}^{\lambda / 2} \vee 2^{-N}\right)^{1-\eta_{1} / 2} a_{n}^{-\frac{\lambda}{2}(1+\alpha / 2)}\right. \\
& \left.\quad \quad\left[\left(a_{n}^{\lambda / 2} \vee 2^{-N}\right)^{\gamma \gamma_{m}}+a_{n}^{\frac{\lambda}{2}(1+\alpha / 2)}\left(a_{n}^{\lambda / 2} \vee 2^{-N}\right)^{\left(\gamma \gamma_{m}-1-\alpha / 2\right) \wedge 0}+a_{n}^{\beta \gamma}\left(a_{n}^{\lambda / 2} \vee 2^{-N}\right)^{\gamma}\right]\right\} \\
& \quad+2^{-92} d\left((t, x),\left(t^{\prime}, x^{\prime}\right)\right)^{1-\eta_{1}} a_{n}^{-\varepsilon_{0}} a_{n}^{-\frac{\lambda}{2}(1+\alpha / 2)} a_{n}^{\frac{\lambda}{2}}\left[\left(a_{n}^{\lambda / 2} \vee 2^{-N}\right)^{\gamma \gamma_{m}}+a_{n}^{\beta \gamma}\left(a_{n}^{\lambda / 2} \vee 2^{-N}\right)^{\gamma}\right] .
\end{aligned}
$$

Next use $d\left((t, x),\left(t^{\prime}, x^{\prime}\right)\right) \leq 2^{-N}$, such that $d^{\eta_{1} / 2}\left(a_{n}^{\lambda / 2} \vee 2^{-N}\right)^{-\eta_{1} / 2} \leq\left(2^{-N}\right)^{0}=1$ and $a_{n}^{\varepsilon_{0}} \leq 1$ to bound the above by:

$$
\begin{aligned}
\leq & \left(c 2^{-N \frac{\eta_{1}}{2}}+2^{-92}\right) d\left((t, x),\left(t^{\prime}, x^{\prime}\right)\right)^{1-\eta_{1}} a_{n}^{-\varepsilon_{0}-\lambda(1+\alpha / 2) / 2} \\
& \left\{a_{n}^{\beta} a_{n}^{\lambda(1+\alpha / 2) / 2}+\left(a_{n}^{\lambda / 2} \vee 2^{-N}\right)\left[\left(a_{n}^{\lambda / 2} \vee 2^{-N}\right)^{\gamma \gamma_{m}}+a_{n}^{\beta \gamma}\left(a_{n}^{\lambda / 2} \vee 2^{-N}\right)^{\gamma}\right.\right. \\
& \left.\left.+a_{n}^{\lambda(1+\alpha / 2) / 2}\left(a_{n}^{\lambda / 2} \vee 2^{-N}\right)^{\left(\gamma \gamma_{m}-1-\alpha / 2\right) \wedge 0}\right]\right\} .
\end{aligned}
$$

Choose $N_{1}\left(K, \eta_{1}\right)$ so large that $2^{-\frac{\eta_{1}}{2} N_{1}} c \leq 2^{-92}$, and $N_{9.4 .17}=N_{9.4 .17}^{\prime \prime} \vee N_{1}$ which then clearly is stochastically bounded in $n, \lambda, \beta$. For $N \geq N \overline{9.4 .17}$ and $m<\bar{m}$ we have

$$
\begin{aligned}
a_{n}^{\lambda(1+\alpha / 2) / 2}\left(a_{n}^{\lambda / 2} \vee 2^{-N}\right)^{\left(\gamma \gamma_{m}-1-\alpha / 2\right) \wedge 0} & \leq\left(a_{n}^{\lambda / 2} \vee 2^{-N}\right)^{1+\alpha / 2}\left(a_{n}^{\lambda / 2} \vee 2^{-N}\right)^{\left(\gamma \gamma_{m}-1-\alpha / 2\right) \wedge 0} \\
& \leq\left(a_{n}^{\lambda / 2} \vee 2^{-N}\right)^{\gamma \gamma_{m}}
\end{aligned}
$$

If $m \geq \bar{m}$ the left hand side is bounded by $a_{n}^{\lambda / 2(1+\alpha / 2)}$ Therefore, we can write

$$
\begin{aligned}
& \left|u_{1, a_{n}^{\lambda}}\left(t^{\prime}, x^{\prime}\right)-u_{1, a_{n}^{\lambda}}(t, x)\right| \leq \\
& \quad \leq 2^{-90} d^{1-\eta_{1}} a_{n}^{-\varepsilon_{0}} a_{n}^{-\lambda(1+\alpha / 2) / 2}+\mathbb{1}(m \geq \bar{m})\left(a_{n}^{\lambda / 2} \vee 2^{-N}\right) \\
& \quad\left[a_{n}^{\beta} a_{n}^{\lambda(1+\alpha / 2) / 2}+\left(\left(a_{n}^{\lambda / 2} \vee 2^{-N}\right)^{\gamma \gamma_{m}+1}+a_{n}^{\beta \gamma}\left(a_{n}^{\lambda / 2} \vee 2^{-N}\right)^{\gamma+1}\right]\right. \\
& \quad=2^{-90} d\left((t, x),\left(t^{\prime}, x^{\prime}\right)\right)^{1-\eta_{1}} \bar{\Delta}_{u_{1}}\left(m, n, \lambda, \varepsilon_{0}, 2^{-N}\right) .
\end{aligned}
$$

And this is what we wanted to show. 
We also would like to obtain a similar result for $u_{2, \delta}$. We postpone its proof which is simpler than the previous calculations to Section 9.7 .

Notation:

$$
\begin{aligned}
\bar{\Delta}_{1, u_{2}}\left(m, n, \varepsilon_{0}, 2^{-N}\right) & =a_{n}^{-\varepsilon_{0}} 2^{-N \gamma}\left[\left(a_{n}^{1 / 2} \vee 2^{-N}\right)^{\gamma\left(\gamma_{m}-1\right)}+a_{n}^{\beta \gamma}\right], \\
\bar{\Delta}_{2, u_{2}}\left(m, n, \lambda, \varepsilon_{0}\right) & =a_{n}^{-\varepsilon_{0}}\left[a_{n}^{\frac{\lambda}{2}\left(\tilde{\gamma}_{m+1}-1\right)}+a_{n}^{\beta \gamma} a_{n}^{\frac{\lambda}{2}(\gamma-\alpha / 2)}\right] .
\end{aligned}
$$

Proposition 9.4.18. Let $0 \leq m \leq \bar{m}+1$ and assume that $\left(P_{m}\right)$ holds. Then for all $n \in \mathbb{N}, \eta_{1} \in(0, \alpha / 4 \wedge(2-\alpha) / 2), \varepsilon_{0} \in(0,1), K \in \mathbb{N} \geq K_{1}, \lambda \in[0,1], \beta \in[0,1 / 2]$ there is an $N_{9.4 .18}=N \overline{9.4 .18}\left(m, n, \eta_{1}, \varepsilon_{0}, K, \lambda, \beta\right)(\omega) \in \mathbb{N}$ almost surely such that $\forall N \geq N \overline{9.4 .18},(t, x) \in Z(N, n, K, \beta), t^{\prime} \leq T_{K}$

$$
\begin{aligned}
& d:=d\left((t, x),\left(t^{\prime}, x^{\prime}\right)\right) \leq 2^{-N} \text { implies that } \\
& \left|u_{2, a_{n}^{\lambda}}(t, x)-u_{2, a_{n}^{\lambda}}\left(t^{\prime}, x^{\prime}\right)\right| \leq 2^{-94}\left(d^{(1-\alpha / 2)\left(1-\eta_{1}\right)} \bar{\Delta}_{1, u_{2}}+d^{1-\eta_{1}} \bar{\Delta}_{2, u_{2}}\right) .
\end{aligned}
$$

Moreover, $N$ 9.4.18 is stochastically bounded uniformly in $(n, \lambda, \beta)$.

Now, we are ready to finish the chapter, that means to do the induction step in the proof of Proposition 9.4.2.

Proof of Proposition 9.4.2.

Let $0 \leq m \leq \bar{m}$ and assume that $\left(P_{m}\right)$ holds. We want to show $\left(P_{m+1}\right)$. Let therefore $\varepsilon_{0} \in(0,1), M=\left\lceil\frac{2}{\varepsilon_{0}}\right\rceil, \varepsilon_{1}=\frac{1}{M} \leq \varepsilon_{0} / 2$ and $\lambda_{i}=i \varepsilon_{1}$ for $i=1, \ldots, M$. Then, clearly $\lambda_{i} \in\left[\varepsilon_{1}, 1\right]$ for all $i=1, \ldots, M$.

Let $n, \xi, K, \beta$ be as in $\left(P_{m}\right)$ and w.l.o.g. $\xi$ is sufficiently large, such that for $\eta_{1}:=1-\xi$ we have $\eta_{1}<\frac{\alpha}{4} \wedge\left(1-\frac{\alpha}{2}\right)$. Set $\xi^{\prime}=(1+\xi) / 2 \in(\xi, 1)$ and

$$
\begin{aligned}
N_{2}\left(m, n, \xi, \varepsilon_{0}, K, \beta\right)(\omega) & =\bigvee_{i=1}^{M} N_{9.4 .17}\left(m, n, \eta_{1}, \varepsilon_{0} / 2, K+1, \lambda_{i}, \beta\right) . \\
N_{3}\left(m, n, \xi, \varepsilon_{0}, K, \beta\right)(\omega) & =\bigvee_{i=1}^{M} N_{9.4 .18}\left(m, n, \eta_{1}, \varepsilon_{0} / 2, K+1, \lambda_{i}, \beta\right) . \\
N_{4}\left(m, n, \xi, \varepsilon_{0}, K, \beta\right)(\omega) & =\left\lceil\frac{2}{1-\xi}\right\rceil\left(N_{9.4 .14} \vee N_{9.4 .17}+1\right) \\
& =: \frac{1}{1-\xi} N_{5} . \\
N_{1} & =N_{2} \vee N_{3} \vee N_{4} \vee N_{0}\left(\xi^{\prime}, K\right)+1 \in \mathbb{N},
\end{aligned}
$$

where $N_{0}\left(\xi^{\prime}, K\right)$ is the constant we obtained from Theorem 5.3.3. By the results on each of the single constants we know that $N_{1}$ is then stochastically bounded uniformly in $(n, \beta)$. Let now

$$
N \geq N_{1},(t, x) \in Z(N, n, K, \beta), t^{\prime} \leq T_{K} \text { and } d:=d\left((t, x),\left(t^{\prime}, x^{\prime}\right)\right) \leq 2^{-N} .
$$


There are two cases for the values of $n$ to consider.

We start with the case of small $n$ :

$$
a_{n}>2^{-N_{5}\left(m, n, \eta_{1}, \varepsilon_{0}, K, \beta\right)},
$$

which implies ${\sqrt{a_{n}}}^{\tilde{\gamma}_{m+1}-1} \geq a_{n}^{1 / 2} \geq 2^{-N_{5} / 2}$. As $N \geq N_{0}\left(\xi^{\prime}, K\right)$ we get by $\left(P_{0}\right)$ in the case $\varepsilon_{0}=0$ :

$$
\begin{aligned}
\left|u\left(t^{\prime}, x^{\prime}\right)\right| & \leq 2^{-N \xi^{\prime}} \\
& \leq 2^{-N \xi^{\prime}}\left[\left(\sqrt{a_{n}} \vee 2^{-N}\right)^{\tilde{\gamma}_{m}+1}\right] 2^{N_{5} / 2} \\
& \leq 2^{-N(1-\xi) / 2} 2^{N_{5} / 2} 2^{-N \xi}\left[\left(\sqrt{a_{n}} \vee 2^{-N}\right)^{\tilde{\gamma}_{m}+1}+a_{n}^{\beta}\right] \\
& \leq 2^{-N \xi}\left[\left(\sqrt{a_{n}} \vee 2^{-N}\right)^{\tilde{\gamma}_{m}+1}+a_{n}^{\beta}\right] .
\end{aligned}
$$

This already completes the first case.

For large $n$, i.e.:

$$
a_{n} \leq 2^{-N_{5}}
$$

let $N^{\prime}=N-1 \geq N_{2} \vee N_{3}$, which gives $\left(t^{\prime}, x^{\prime}\right) \in Z\left(N^{\prime}, n, K+1, \beta\right)$ by triangle inequality. As (9.94) holds we can apply Proposition 9.4.17 with $\left(\varepsilon_{0} / 2, K+1\right)$ instead of $\left(\varepsilon_{0}, K\right)$.

Additionally one can also use Proposition 9.4.18. So we can estimate $\mid u\left(\hat{t}_{0}, \hat{x}_{0}\right)-$ $u\left(t^{\prime}, x^{\prime}\right) \mid$. Before doing so we have to choose which partition of $u$ to take in the sense of (9.18) and then give some estimates. Therefore, select $i \in\{1, \ldots, M\}$ such that

(a) if $2^{-N}>\sqrt{a_{n}}$, then $a_{n}^{\lambda_{i} / 2}<2^{-N^{\prime}} \leq a_{n}^{\lambda_{i-1} / 2}=a_{n}^{\lambda_{i} / 2} a_{n}^{-\varepsilon_{1} / 2}$.

(b) if $2^{-N} \leq \sqrt{a_{n}}$, then $i=M$ and hence $a_{n}^{\lambda_{i} / 2}=\sqrt{a_{n}} \geq 2^{-N^{\prime}}$.

Then, in both cases

$$
a_{n}^{\lambda_{i} / 2} \vee 2^{-N^{\prime}} \leq \sqrt{a_{n}} \vee 2^{-N^{\prime}}
$$

and writing $\lambda=\lambda_{i}$ :

$$
\begin{gathered}
a_{n}^{-\frac{\lambda}{2}(1+\alpha / 2)}\left(\sqrt{a_{n}} \vee 2^{-N^{\prime}}\right)^{1+\alpha / 2} \leq\left(a_{n}^{-\frac{\lambda}{2}}\left(\sqrt{a_{n}} \vee 2^{-N^{\prime}}\right)\right)^{1+\alpha / 2} . \\
\text { Case } \mathrm{a}, \leq\left(a_{n}^{-\lambda / 2} 2^{-N^{\prime}}\right)^{1+\alpha / 2} \leq a_{n}^{-\frac{\varepsilon_{1}}{2}(1+\alpha / 2)}, \\
\text { Case } \mathrm{b} \leq\left(a_{n}^{-\lambda / 2} a_{n}^{1 / 2}\right)^{1+\alpha / 2} \leq 1 \leq a_{n}^{-\frac{\varepsilon_{1}}{2}(1+\alpha / 2)} . \\
\text { Hence, in both cases: } \quad a_{n}^{-\frac{\lambda}{2}(1+\alpha / 2)}\left(\sqrt{a_{n}} \vee 2^{-N^{\prime}}\right)^{1+\alpha / 2} \leq a_{n}^{-\frac{\varepsilon_{1}}{2}(1+\alpha / 2)} .
\end{gathered}
$$


Furthermore, we have by $\xi-\alpha / 2 \leq(2-\alpha) \frac{\xi}{2}$

$$
\begin{aligned}
2^{-N^{\prime}(2-\alpha) \frac{\xi}{2}} & \leq 2^{-N^{\prime} \xi} 2^{N^{\prime} \frac{\alpha}{2}} \text { and } \\
2^{-N^{\prime}\left((2-\alpha) \frac{\xi}{2}+\gamma\right)} & =2^{-N^{\prime} \xi} 2^{-N^{\prime}(\gamma-\alpha / 2)} \\
& \leq 2^{-N^{\prime} \xi}\left(a_{n}^{1 / 2} \vee 2^{-N^{\prime}}\right)^{\gamma-\alpha / 2} \quad(\gamma>\alpha / 2) .
\end{aligned}
$$

Using the aforementioned propositions, the special $i, 9.95$ and the just previous lines, we get:

$$
\begin{aligned}
& \left|u\left(\hat{t}_{0}, \hat{x}_{0}\right)-u\left(t^{\prime}, x^{\prime}\right)\right| \leq\left|u_{1, a_{n}^{\lambda_{i}}}\left(\hat{t}_{0}, \hat{x}_{0}\right)-u_{1, a_{n}^{\lambda_{i}}}(t, x)\right|+\left|u_{2, a_{n}^{\lambda_{i}}}\left(\hat{t}_{0}, \hat{x}_{0}\right)-u_{2, a_{n}^{\lambda_{i}}}(t, x)\right| \\
& \leq 2^{-90} a_{n}^{-\varepsilon_{0} / 2} 2^{-N^{\prime} \xi}\left[a_{n}^{\beta}+a_{n}^{-\lambda(1+\alpha / 2) / 2} a_{n}^{\beta \gamma}\left(\left(a_{n}^{\lambda / 2} \vee 2^{-N^{\prime}}\right)^{\gamma+1}+\mathbb{1}(m \geq \bar{m})\left(a_{n}^{\lambda / 2} \vee 2^{-N}\right)\right.\right. \\
& \left.\quad+a_{n}^{-\lambda(1+\alpha / 2) / 2}\left(a_{n}^{\lambda / 2} \vee 2^{-N^{\prime}}\right)^{\gamma \gamma_{m}+1}\right] \\
& \quad+2^{-94} a_{n}^{-\varepsilon_{0} / 2}\left[2^{-N^{\prime}\left((2-\alpha) \frac{\xi}{2}+\gamma\right)}\left(\left(a_{n}^{1 / 2} \vee 2^{-N^{\prime}}\right)^{\gamma\left(\gamma_{m}-1\right)}+a_{n}^{\beta \gamma}\right)\right. \\
& \left.\quad 2^{-N^{\prime} \xi}\left(\left(a_{n}^{1 / 2} \vee 2^{-N^{\prime}}\right)^{\tilde{\gamma} m+1}+a_{n}^{\beta \gamma}\left(a_{n}^{1 / 2} \vee 2^{-N^{\prime}}\right)^{\gamma-\alpha / 2}\right)\right] . \\
& \leq 2^{-89} a_{n}^{-\varepsilon_{0} / 2} 2^{-N^{\prime} \xi}\left[a_{n}^{\beta}+a_{n}^{-\lambda(1+\alpha / 2) / 2} a_{n}^{\beta \gamma}\left(a_{n}^{1 / 2} \vee 2^{-N}\right)^{1+\alpha / 2}\left(a_{n}^{1 / 2} \vee 2^{-N}\right)^{\gamma-\alpha / 2}\right. \\
& \quad+\mathbb{1}(m \geq \bar{m})\left(\sqrt{a_{n}} \vee 2^{-N}\right) \\
& \quad+a_{n}^{-\lambda(1+\alpha / 2) / 2}\left(a_{n}^{1 / 2} \vee 2^{-N}\right)^{1+\alpha / 2}\left(a_{n}^{1 / 2} \vee 2^{-N}\right)^{\tilde{\gamma}_{m+1}-1} \\
& \quad+\left(a_{n} \vee 2^{-N^{\prime}}\right)^{\gamma-\alpha / 2}\left(\left(a_{n}^{1 / 2} \vee 2^{-N^{\prime}}\right)^{\gamma\left(\gamma_{m}-1\right)}+a_{n}^{\beta \gamma}\right) \\
& \left.\quad+\left(\left(a_{n}^{1 / 2} \vee 2^{-N^{\prime}}\right)^{\tilde{\gamma}_{m+1}-1}+a_{n}^{\beta \gamma}\left(a_{n}^{1 / 2} \vee 2^{-N^{\prime}}\right)^{\gamma-\alpha / 2}\right)\right] .
\end{aligned}
$$

Now, apply 9.96 to bound this by

$$
\begin{array}{r}
\leq 2^{-89} a_{n}^{-\frac{\varepsilon_{0}}{2}} a_{n}^{-\frac{\varepsilon_{1}}{2}(1+\alpha / 2)} 2^{-N^{\prime} \xi}\left[a_{n}^{\beta} a_{n}^{\frac{\varepsilon_{1}}{2}(1+\alpha / 2)}+a_{n}^{\beta \gamma}\left(\sqrt{a_{n}} \vee 2^{-N}\right)^{\gamma-\alpha / 2}\left(2 a_{n}^{\frac{\varepsilon_{1}}{2}(1+\alpha / 2)}+1\right)\right. \\
\left.+\left(\sqrt{a_{n}} \vee 2^{-N}\right)^{\gamma \gamma_{m}-\alpha / 2}\left(1+2 a_{n}^{\frac{\varepsilon_{1}}{2}(1+\alpha / 2)}\right)+\mathbb{1}(m \geq \bar{m})\left(\sqrt{a_{n}} \vee 2^{-N}\right)\right],
\end{array}
$$

since $\varepsilon_{0} \leq \varepsilon_{1} / 2$ and $\varepsilon_{1} \geq 0$. Trivially,

$$
\left(\sqrt{a_{n}} \vee 2^{-N}\right)^{\tilde{\gamma}_{m+1}-1}+\mathbb{1}(m \geq \bar{m})\left(\sqrt{a_{n}} \vee 2^{-N}\right) \leq 2\left(\sqrt{a_{n}} \vee 2^{-N}\right)^{\tilde{\gamma}_{m+1}-1} .
$$

So we obtain

$$
\begin{aligned}
\left|u\left(\hat{t}_{0}, \hat{x}_{0}\right)-u\left(t^{\prime}, x^{\prime}\right)\right| \leq 2^{-87} 2^{-N^{\prime} \xi} a_{n}^{-\varepsilon_{0}}[ & a_{n}^{\beta}+a_{n}^{\beta \gamma}\left(\sqrt{a_{n}} \vee 2^{-N}\right)^{\gamma-\alpha / 2} \\
& \left.+\left(\sqrt{a_{n}} \vee 2^{-N}\right)^{\gamma \gamma_{m}-\alpha / 2}\right] .
\end{aligned}
$$

Now, we can proceed to the last step of this statement and obtain

$$
\begin{aligned}
& \left|u\left(t^{\prime}, x^{\prime}\right)\right| \leq\left|u\left(\hat{t}_{0}, \hat{x}_{0}\right)\right|+\left|u\left(\hat{t}_{0}, \hat{x}_{0}\right)-u\left(t^{\prime}, x^{\prime}\right)\right| \\
& \quad \leq \sqrt{a_{n}} 2^{-N}+2^{-85} 2^{-N \xi} a_{n}^{-\varepsilon_{0}}\left[a_{n}^{\beta}+a_{n}^{\beta \gamma}\left(\sqrt{a_{n}} \vee 2^{-N}\right)^{\gamma-\alpha / 2}+\left(\sqrt{a_{n}} \vee 2^{-N}\right)^{\gamma \gamma_{m}-\alpha / 2}\right] \\
& \leq a_{n}^{-\varepsilon_{0}} 2^{-N \xi}\left[\sqrt{a_{n}} 2^{-N(1-\xi)}\right. \\
& \quad+2^{-85}\left(a_{n}^{\beta}+a_{n}^{\beta \gamma}\left(2^{-N} \vee \sqrt{a_{n}}\right)^{\gamma-\alpha / 2}+\left(2^{-N} \vee \sqrt{a_{n}}\right)^{\gamma \gamma_{m}-\alpha / 2}\right] .
\end{aligned}
$$


Clearly, $\sqrt{a_{n}} 2^{-N(1-\xi)} \leq \sqrt{a_{n}} / 2 \leq a_{n}^{\beta} / 2$ and by 9.94 and an easy calculation (Lemma 5.15 in [MP11]):

$$
a_{n}^{\beta \gamma}\left(2^{-N} \vee \sqrt{a_{n}}\right)^{\gamma-\alpha / 2} \leq a_{n}^{\beta} \vee 2^{-N} \leq a_{n}^{\beta}+\left(2^{-N} \vee \sqrt{a_{n}}\right)^{\tilde{\gamma}_{m+1}-1} .
$$

Therefore, we can write

$$
\begin{aligned}
\left|u\left(t^{\prime}, x^{\prime}\right)\right| & \leq a_{n}^{-\varepsilon_{0}} 2^{-N \xi}\left(\frac{a_{n}^{\beta}}{2}+2^{-84} a_{n}^{\beta}+2^{-84}\left(2^{-N} \vee \sqrt{a_{n}}\right)^{\tilde{\gamma}_{m+1}-1}\right) \\
& \leq a_{n}^{-\varepsilon_{0}} 2^{-N \xi}\left(a_{n}^{\beta}+\left(2^{-N} \vee \sqrt{a_{n}}\right)^{\tilde{\gamma}_{m+1}-1}\right),
\end{aligned}
$$

since

$$
\tilde{\gamma}_{m+1}=\left(\gamma \gamma_{m}+1-\frac{\alpha}{2}\right) \wedge 2
$$

\subsection{Proof of Proposition 9.2.2}

Fix $K_{0} \in \mathbb{N} \geq K_{1}, \varepsilon_{0}, \varepsilon_{1} \in(0,1)$ as in the definition 9.20 and for $M, n \in \mathbb{N}, 0<\beta \leq$ $1-\varepsilon_{1}$ define

$$
\lambda(\beta):=2\left(\beta+\varepsilon_{1}\right) \in[0,1] .
$$

We define the following random time

$$
\begin{gathered}
U_{M, n, \beta}^{(1)}=\inf \left\{t \geq 0: \exists \varepsilon \in\left[0,2^{-M}\right],|x| \leq K_{0}+1, \hat{x}_{0}, x^{\prime} \in \mathbb{R}^{q}, \text { such that }\left|x-x^{\prime}\right| \leq 2^{-M},\right. \\
\left|\hat{x}_{0}-x\right| \leq \varepsilon,\left|u\left(t, \hat{x}_{0}\right)\right| \leq a_{n} \wedge\left(\sqrt{a_{n}} \varepsilon\right),\left|\nabla u_{1, a_{n}}\left(t, \hat{x}_{0}\right)\right| \leq a_{n}^{\beta} \text { and } \\
\left|\nabla u_{1, a_{n}^{\lambda}}(t, x)-\nabla u_{1, a_{n}^{\lambda}}\left(t, x^{\prime}\right)\right|>2^{-82} a_{n}^{-\varepsilon_{0}-\varepsilon_{1}(1+\alpha / 2)}\left|x-x^{\prime}\right|^{1-\varepsilon_{0}} \\
{\left[a_{n}^{-\beta(1+\alpha / 2)}\left(\varepsilon \vee\left|x-x^{\prime}\right|\right)^{2 \gamma}+1+a_{n}^{\beta(\gamma-1-\alpha / 2)}\left(\varepsilon \vee\left|x^{\prime}-x\right|\right)^{\gamma}\right]} \\
\wedge T_{K_{0}},
\end{gathered}
$$

whenever $\beta>0$. We define $U_{M, n, 0}^{(1)}$ in the same way, omitting the condition on $\nabla u_{1, a_{n}}\left(t, \hat{x}_{0}\right)$. These random times are actually stopping times by Theorem IV.T.52 of Mey66].

Lemma 9.5.1. For all $n \in \mathbb{N}, \beta$ as in 9.22 it holds that $U_{M, n, \beta}^{(1)} \nearrow T_{K_{0}}$ almost surely as $M \rightarrow \infty$ and

$$
\lim _{M \rightarrow \infty} \sup _{n, 0 \leq \beta \leq 1 / 2-\varepsilon_{1}} \mathbb{P}\left[U_{M, n, \beta}^{(1)}<T_{K_{0}}\right]=0 .
$$

Proof. The almost sure convergence follows from the second statement by monotonicity of the probabilities in $M$. For the second statement use Corollary 9.4.12 with $m=\bar{m}+1, \eta_{1}=\varepsilon_{0}, K=K_{0}+1$. Then there is a $N_{0}=N_{0}\left(n, \varepsilon_{0}, \varepsilon_{1}, K_{0}+1, \beta\right)$ 
stochastically bounded uniformly in $n$ and $\beta$ such that for all $N \geq N_{0},(t, x) \in$ $Z\left(N, n, K_{0}+1, \beta\right)$ and $\left|x-x^{\prime}\right| \leq 2^{-N}$ it holds that

$$
\begin{aligned}
& \left|\nabla u_{1, a_{n}^{\lambda}}(t, x)-\nabla u_{1, a_{n}^{\lambda}}\left(t, x^{\prime}\right)\right| \\
& \leq 2^{-85}\left|x-x^{\prime}\right|^{1-\varepsilon_{0}} a_{n}^{-\varepsilon_{0}}\left[a_{n}^{-\left(\beta+\varepsilon_{1}\right)(1+\alpha / 2)} 2^{-2 N \gamma}+\left(a_{n}^{\beta+\varepsilon_{1}} \vee 2^{-N}\right)^{0}\right. \\
& \left.\quad+a_{n}^{-\left(\beta+\varepsilon_{1}\right)(1+\alpha / 2)} a_{n}^{\beta \gamma}\left(a_{n}^{\beta+\varepsilon_{1}} \vee 2^{-N}\right)^{\gamma}\right] \\
& \leq 2^{-85}\left|x-x^{\prime}\right|^{1-\varepsilon_{0}} a_{n}^{-\varepsilon_{0}-\varepsilon_{1}(1+\alpha / 2)} \\
& \quad\left[a_{n}^{-\beta(1+\alpha / 2)} 2^{-2 N \gamma}+1+a_{n}^{\beta(\gamma-1-\alpha / 2)}\left(a_{n}^{\beta+\varepsilon_{1}} \vee 2^{-N}\right)^{\gamma}\right] .
\end{aligned}
$$

Note that by $\beta(\gamma-1-\alpha / 2)+\beta \gamma+\left(\beta+\varepsilon_{1}\right) \gamma=\beta(2 \gamma-\alpha / 2-1)+\varepsilon_{1} \gamma>0$ :

$$
\begin{aligned}
\left|\nabla u_{1, a_{n}^{\lambda}}(t, x)-\nabla u_{1, a_{n}^{\lambda}}\left(t, x^{\prime}\right)\right| \leq 2^{-84} a_{n}^{-\varepsilon_{0}-\varepsilon_{1}(1+\alpha / 2)}\left|x-x^{\prime}\right|^{1-\varepsilon_{0}} \\
\\
{\left[a_{n}^{-\beta(1+\alpha / 2)} 2^{-2 N \gamma}+1+a_{n}^{\beta(\gamma-1-\alpha / 2)} 2^{-N \gamma}\right] . }
\end{aligned}
$$

We only do the case $\beta>0$. Assume $M>N_{0}$ and there is a $t<T_{K_{0}}, \varepsilon \in\left[0,2^{-M}\right]$, $|x| \leq K_{0}+1, \hat{x}_{0}, x^{\prime} \in \mathbb{R}^{q}$ with $\left|x-x^{\prime}\right| \leq 2^{-M},\left|\hat{x}_{0}-x\right| \leq \varepsilon,\left|u\left(t, \hat{x}_{0}\right)\right| \leq a_{n} \wedge\left(\sqrt{a_{n}} \varepsilon\right)$ and

$$
\left|\nabla u_{1, a_{n}}\left(t, \hat{x}_{0}\right)\right| \leq a_{n}^{\beta} .
$$

If $x \neq x^{\prime}$, then there is a $N \geq N_{0}$ such that $2^{-N-1}<\left|x-x^{\prime}\right| \vee \varepsilon \leq 2^{-N} \leq 2^{-N_{0}}$. Then $(t, x) \in Z\left(N, n, K_{0}+1, \beta_{i}\right)$ and we can use the previous estimate. Hence,

$$
\begin{aligned}
\left|\nabla u_{1, a_{n}^{\lambda}}(t, x)-\nabla u_{1, a_{n}^{\lambda}}\left(t, x^{\prime}\right)\right| \leq 2^{-82} a_{n}^{-\varepsilon_{0}-\varepsilon_{1}(1+\alpha / 2)}\left|x-x^{\prime}\right|^{1-\varepsilon_{0}} \\
{\left[a_{n}^{-\beta(1+\alpha / 2)}\left(\varepsilon \vee\left|x-x^{\prime}\right|\right)^{2 \gamma}+1+a_{n}^{\beta(\gamma-1-\alpha / 2)}\left(\varepsilon \vee\left|x-x^{\prime}\right|\right)^{\gamma}\right] . }
\end{aligned}
$$

Therefore, $U_{M, n, \beta}^{(1)}=T_{K_{0}}$. As $N_{0}$ is stochastically bounded uniformly in $(n, \beta)$ the assertion follows.

Remark 9.5.2. The previous lemma is where the fact that we consider splitting at $\delta=a_{n}^{\lambda}$ rather than $\delta=a_{n}$ is suggested.

Let us do the same again, this time with $u_{2}$. For $0<\beta \leq 1 / 2-\varepsilon_{1}$ set

$$
\begin{gathered}
U_{M, n, \beta}^{(2)}=\inf \left\{t \geq 0: \exists \varepsilon \in\left[0,2^{-M}\right],|x| \leq K_{0}+1, \hat{x}_{0}, x^{\prime} \in \mathbb{R}^{q}, \text { such that }\left|x-x^{\prime}\right| \leq 2^{-M},\right. \\
\left|\hat{x}_{0}-x\right| \leq \varepsilon,\left|u\left(t, \hat{x}_{0}\right)\right| \leq a_{n} \wedge\left(\sqrt{a_{n}} \varepsilon\right),\left|\nabla u_{1, a_{n}}\left(t, \hat{x}_{0}\right)\right| \leq a_{n}^{\beta} \text { and } \\
\left|u_{2, a_{n}^{\lambda}}(t, x)-u_{2, a_{n}^{\lambda}}\left(t, x^{\prime}\right)\right|>2^{-87} a_{n}^{-\varepsilon_{0}}\left[\left|x-x^{\prime}\right|^{\left(1-\varepsilon_{0}\right)(1-\alpha / 2)}\right. \\
{\left[\left(\sqrt{a_{n}} \vee \varepsilon \vee\left|x^{\prime}-x\right|\right)^{2 \gamma}+a_{n}^{\beta \gamma}\left(\varepsilon \vee\left|x^{\prime}-x\right|\right)^{\gamma}\right]} \\
\left.\left.+\left|x-x^{\prime}\right|^{1-\varepsilon_{0}} a_{n}^{\beta+\varepsilon_{1}(1-\gamma)}\right]\right\} \wedge T_{K_{0}} .
\end{gathered}
$$

And in the case $\beta=0$, we make the same definition but without the condition on $\nabla u_{1, a_{n}}\left(t, \hat{x}_{0}\right)$. Then, we get 
Lemma 9.5.3. For all $n \in \mathbb{N}, \beta$ as in 9.22 it holds that $U_{M, n, \beta}^{(2)} \nearrow T_{K_{0}}$ almost surely as $M \rightarrow \infty$ and

$$
\lim _{M \rightarrow \infty} \sup _{n, 0 \leq \beta \leq 1 / 2-\varepsilon_{1}} \mathbb{P}\left[U_{M, n, \beta}^{(2)}<T_{K_{0}}\right]=0 .
$$

Proof. Use Proposition 9.4.18 with $m=\bar{m}+1, \eta_{1}=\varepsilon_{0}, K=K_{0}+1, \lambda(\beta)=$ $2\left(\beta+\varepsilon_{1}\right), \beta$ as in 9.22 . Then there is a $N_{0}=N_{0}\left(n, \varepsilon_{0}, \varepsilon_{1}, K_{0}+1, \beta\right)$ almost surely stochastically bounded uniformly in $(n, \beta)$ such that for any $N \geq N_{0},(t, x) \in$ $Z\left(N, n, K_{0}+1, \beta\right)$ and $\left|x-x^{\prime}\right| \leq 2^{-N}$ it holds that:

$$
\begin{array}{r}
\left|u_{2, a_{n}^{\lambda}}(t, x)-u_{2, a_{n}^{\lambda}}\left(t, x^{\prime}\right)\right| \leq 2^{-91} a_{n}^{-\varepsilon_{0}}\left\{\left|x-x^{\prime}\right|^{\left(1-\varepsilon_{0}\right)(1-\alpha / 2)} 2^{-N \gamma}\left[\left(\sqrt{a_{n}} \vee 2^{-N}\right)^{\gamma}+a_{n}^{\beta \gamma}\right]\right. \\
\left.+\left|x-x^{\prime}\right|^{1-\varepsilon_{0}}\left[a_{n}^{\left(\beta+\varepsilon_{1}\right)(2 \gamma-\alpha / 2)}+a_{n}^{\beta \gamma} a_{n}^{\left(\beta+\varepsilon_{1}\right)(\gamma-\alpha / 2)}\right]\right\} .
\end{array}
$$

We apply the following inequalities

$$
a_{n}^{\left(\beta+\varepsilon_{1}\right)(2 \gamma-\alpha / 2)} \leq a_{n}^{\beta+\varepsilon_{1}} \text { and } a_{n}^{\beta \gamma} a_{n}^{\left(\beta+\varepsilon_{1}\right)(\gamma-\alpha / 2)} \leq a_{n}^{\beta+\varepsilon_{1}-\varepsilon_{1} \gamma}
$$

to obtain the bound:

$$
\begin{gathered}
\left|u_{2, a_{n}^{\lambda}}(t, x)-u_{2, a_{n}^{\lambda}}\left(t, x^{\prime}\right)\right| \leq 2^{-89} a_{n}^{-\varepsilon_{0}}\left\{\left|x-x^{\prime}\right|^{\left(1-\varepsilon_{0}\right)(1-\alpha / 2)} 2^{-N \gamma}\left[\left(\sqrt{a_{n}} \vee 2^{-N}\right)^{\gamma}+a_{n}^{\beta \gamma}\right]\right. \\
\left.+\left|x-x^{\prime}\right|^{1-\varepsilon_{0}} a_{n}^{\beta+\varepsilon_{1}(1-\gamma)}\right\} .
\end{gathered}
$$

Now, use the same arguments as in the proof of Lemma 9.5.1 to finish the proof.

Define

$$
\tilde{\Delta}_{u_{1}^{\prime}}\left(n, \varepsilon_{1}, \varepsilon_{0}, \beta\right)=a_{n}^{-\varepsilon_{0}} \varepsilon^{-\varepsilon_{0}}\left\{\varepsilon+a_{n}^{-\alpha / 4}\left(\varepsilon a_{n}^{-1 / 2}+1\right)\left(\varepsilon^{2 \gamma}+a_{n}^{\beta \gamma}\left(\varepsilon \vee \sqrt{a_{n}}\right)^{\gamma}\right)\right\} .
$$

And for $0<\beta \leq 1 / 2-\varepsilon_{1}$ set

$$
\begin{aligned}
& U_{M, n, \beta}^{(3)}=\inf \left\{t \geq 0: \exists \varepsilon \in\left[2^{-a_{n}^{-\left(\beta+\varepsilon_{1}\right) \varepsilon_{0} / 4}}, 2^{-M}\right],|x| \leq K_{0}+1, \hat{x}_{0} \in \mathbb{R}^{q},\right. \\
& \left|\hat{x}_{0}-x\right| \leq \varepsilon,\left|u\left(t, \hat{x}_{0}\right)\right| \leq a_{n} \wedge\left(\sqrt{a_{n}} \varepsilon\right),\left|\nabla u_{1, a_{n}}\left(t, \hat{x}_{0}\right)\right| \leq a_{n}^{\beta} \text { and } \\
& \left.\left|\nabla u_{1, a_{n}^{\lambda}}(t, x)-\nabla u_{1, a_{n}}(t, x)\right|>2^{-74}\left(\tilde{\Delta}_{u_{1}^{\prime}}\left(n, \varepsilon_{1}, \varepsilon_{0}, \beta\right)+a_{n}^{\beta+\varepsilon_{1}(1-\gamma) / 4}\right)\right\} \\
& \wedge T_{K_{0}} \text {. }
\end{aligned}
$$

The same definition for $\beta=0$ but without the condition on $\nabla u_{1, a_{n}}\left(t, \hat{x}_{0}\right)$. Again, that's a stopping time.

Lemma 9.5.4. For all $n \in \mathbb{N}, \beta$ as in 9.22 it holds that $U_{M, n, \beta}^{(3)} \nearrow T_{K_{0}}$ almost surely as $M \rightarrow \infty$ and

$$
\lim _{M \rightarrow \infty} \sup _{n, 0 \leq \beta \leq 1 / 2-\varepsilon_{1}} \mathbb{P}\left[U_{M, n, \beta}^{(3)}<T_{K_{0}}\right]=0 .
$$


Proof. Let $s=t-a_{n}^{\lambda}+a_{n}<t$. Then

$$
\sqrt{t-s}=\sqrt{a_{n}^{\lambda}-a_{n}} \leq a_{n}^{\lambda / 2}=a_{n}^{\beta+\varepsilon_{1}}
$$

and for any $N \in \mathbb{N}$

$$
a_{n}^{\beta+\varepsilon_{1}} \leq N^{-4 / \varepsilon_{0}} \Leftrightarrow 2^{-N} \geq 2^{-a_{n}^{-\left(\beta+\varepsilon_{1}\right) \varepsilon_{0} / 4}} .
$$

Using Proposition 9.4.14 with $m=\bar{m}+1, \eta_{1}=\varepsilon_{0}, K=K_{0}+1, \lambda(\beta)=2\left(\beta+\varepsilon_{1}\right), \beta$ as in 9.22$)$. Then there is a $N_{0}=N_{0}\left(n, \varepsilon_{0}, \varepsilon_{1}, K_{0}+1, \beta\right)$ almost surely stochastically bounded uniformly in $(n, \beta)$. Assume $(t, x) \in Z\left(N, n, K_{0}+1, \beta\right)$ we get for $N \geq$ $N$ 9.4.14 such that additionally, $2^{-N} \geq 2^{-a_{n}^{-\left(\beta+\varepsilon_{1}\right) \varepsilon_{0} / 4}}$ :

$$
\begin{aligned}
\mid \nabla u_{1, a_{n}^{\lambda}}(t, x)-\nabla u_{1, a_{n}}( & t, x)\left|\leq \max _{l=1, \ldots, q}\right| F_{a_{n}, l}\left(t-a_{n}^{\lambda}+a_{n}, t, x\right)-F_{a_{n}, l}(t, t, x) \mid \\
\leq & 2^{-78} a_{n}^{-\varepsilon_{0}}\left\{2^{-N\left(1-\varepsilon_{0}\right)}\left(\sqrt{a_{n}} \vee 2^{-N}\right)^{0}\right. \\
& +2^{N \varepsilon_{0}} a_{n}^{-\alpha / 4}\left(2^{-N} a_{n}^{-1 / 2}+1\right)\left(2^{-2 N \gamma}+a_{n}^{\beta \gamma}\left(\sqrt{a_{n}} \vee 2^{-N}\right)^{\gamma}\right) \\
& +\sqrt{t-s} 1-\varepsilon_{0}\left[\left(\sqrt{t-s} \vee \sqrt{a_{n}}\right)^{2 \gamma-1-\alpha / 2}\right. \\
& \left.\left.+a_{n}^{\beta \gamma}\left(\left(\sqrt{t-s} \vee \sqrt{a_{n}}\right)^{\gamma-1-\alpha / 2}\right)\right]\right\} .
\end{aligned}
$$

Since $a_{n}^{\beta+\varepsilon_{1}} \geq \sqrt{a_{n}} \vee \sqrt{t-s}$, we consider

$$
\begin{aligned}
a_{n}^{-\varepsilon_{0}} a_{n}^{\left(\beta+\varepsilon_{1}\right)\left(1-\varepsilon_{1}\right)}\left[a_{n}^{\left(\beta+\varepsilon_{1}\right)(2 \gamma-1-\alpha / 2)}\right. & \left.+a_{n}^{\left(\beta+\varepsilon_{1}\right)(\gamma-1-\alpha / 2)}\right] \\
& \leq 2 a_{n}^{-\varepsilon_{0}} a_{n}^{\left(\beta+\varepsilon_{1}\right)\left(1-\varepsilon_{0}\right)+\beta \gamma+\left(\beta+\varepsilon_{1}\right)(\gamma-1-\alpha / 2)} \\
& =2 a_{n}^{\beta\left(2 \gamma-\alpha / 2-\varepsilon_{0}\right)+\varepsilon_{1}\left(\gamma-\alpha / 2-\varepsilon_{0}\right)-\varepsilon_{0}} .
\end{aligned}
$$

Note that by $\alpha<2(2 \gamma-1)$ and $\varepsilon_{0} \leq \frac{1}{2}(2(2 \gamma-1)-\alpha)$ it is true that

$$
2 \gamma-\alpha / 2-\varepsilon_{0} \geq 1 \text { and } \gamma-\alpha / 2-\varepsilon_{0}>(1-\gamma) \text {. }
$$

Therefore,

$$
\left|\nabla u_{1, a_{n}^{\lambda}}(t, x)-\nabla u_{1, a_{n}}(t, x)\right| \leq 2^{-77}\left[\tilde{\Delta}_{u_{1}^{\prime}}\left(n, \varepsilon_{1}, \varepsilon_{0}, \beta\right)+a_{n}^{\beta+\varepsilon_{1}(1-\gamma) / 2}\right] .
$$

Now, keep on doing the proof as in Lemma 9.5.1. We do the case $\beta>0$ again only. Assume $M>N_{0}$ and that there exist $t<T_{K_{0}}, \varepsilon \in\left[2^{-a_{n}^{-\left(\beta+\varepsilon_{1}\right) \varepsilon_{0} / 4}}, 2^{-M}\right],|x| \leq$ $K_{0}+1, \hat{x}_{0} \in \mathbb{R}^{q}$ with

$$
\left|x-\hat{x}_{0}\right|<\varepsilon,\left|u\left(t, \hat{x}_{0}\right)\right| \leq a_{n} \wedge\left(\sqrt{a_{n}} \varepsilon\right) \text { and }\left|\nabla u_{1, a_{n}}\left(t, \hat{x}_{0}\right)\right| \leq a_{n}^{\beta} .
$$


We have for $s=t-a_{n}^{\lambda}+a_{n}$ that $\sqrt{t-s} \leq a_{n}^{\beta+\varepsilon_{1}} \leq a_{n}^{\varepsilon_{1}} \leq M^{-4 / \varepsilon_{0}}$. If we choose $N \geq M$ such that $2^{-N-1}<\varepsilon \leq 2^{-N}$, then

$$
(t, x) \in Z\left(N, n, K_{0}+1, \beta\right) \text { and } 2^{-N} \geq \varepsilon \geq 2^{-a_{n}^{-\left(\beta+\varepsilon_{1}\right) \varepsilon_{0} / 4}} .
$$

So, we can use (9.101) and obtain

$$
\begin{gathered}
\left|\nabla u_{1, a_{n}^{\lambda}}(t, x)-\nabla u_{1, a_{n}}(t, x)\right| \leq 2^{-77}\left[\tilde{\Delta}_{u_{1}^{\prime}}\left(n, 2 \varepsilon, \varepsilon_{0}, \beta\right)+a_{n}^{\beta+\varepsilon_{1}(1-\gamma) / 4}\right] \\
2^{-74}\left[\tilde{\Delta}_{u_{1}^{\prime}}\left(n, \varepsilon, \varepsilon_{0}, \beta\right)+a_{n}^{\beta+\varepsilon_{1}(1-\gamma) / 4}\right] .
\end{gathered}
$$

And finally set

$$
\begin{gathered}
U_{M}^{(4)}=\inf \left\{t \geq 0: \exists \varepsilon \in\left[0,2^{-M}\right],|x| \leq K_{0}+1, \hat{x}_{0}, x^{\prime} \in \mathbb{R}^{q},\left|x-x^{\prime}\right| \leq 2^{-M},\left|x-\hat{x}_{0}\right|<\varepsilon\right. \\
\left.\left|u\left(t, \hat{x}_{0}\right)\right| \leq \varepsilon,\left|u(t, x)-u\left(t, x^{\prime}\right)\right|>\left(\varepsilon \vee\left|x^{\prime}-x\right|\right)^{1-\varepsilon_{0}}\right\} \wedge T_{K_{0}} .
\end{gathered}
$$

Lemma 9.5.5. Almost surely $U_{M}^{(4)} \nearrow T_{K_{0}}$ as $M \rightarrow \infty$.

Proof. Let $\xi=1-\varepsilon_{0} / 2$ and consider $N_{\xi}\left(K_{0}+1\right)$ from Theorem 4.1 and Corollary 4.2 of [MPS06]. Their result tells us that for $N \geq N_{\xi}\left(K_{0}+1\right),(t, x) \in Z_{K_{0}+1, N, \xi}$ and $\left(t^{\prime}, x^{\prime}\right) \in \mathbb{R}_{+} \times \mathbb{R}^{q}$ with $d\left(\left(t^{\prime}, x^{\prime}\right),(t, x)\right) \leq 2^{-N}, t^{\prime} \leq T_{K_{0}}$

$$
\left|u\left(t^{\prime}, x^{\prime}\right)-u(t, x)\right| \leq 2^{-N \xi}
$$

Assume now that we have $M \geq N_{\xi}\left(K_{0}+1\right) \vee 6 \varepsilon_{0}^{-1}, x, \hat{x}_{0}, x^{\prime} \in \mathbb{R}^{q},|x| \leq K_{0}+1$ and $\varepsilon \in\left[0,2^{-M}\right]$ with

$$
\left|x-\hat{x}_{0}\right| \leq \varepsilon,\left|u\left(t, \hat{x}_{0}\right)\right| \leq \varepsilon \text { and }\left|x-x^{\prime}\right| \leq \varepsilon .
$$

We choose $N \geq M$ so large such that $2^{-N-1}<\varepsilon \leq 2^{-N}$. Then, we have trivially that $\left(t, \hat{x}_{0}\right) \in Z_{K_{0}+1, N, \xi}$ and

$$
d\left((t, x),\left(t, \hat{x}_{0}\right)\right) \leq \varepsilon \leq 2^{-N} \leq 2^{-N^{\prime}}, d\left(\left(t, x^{\prime}\right),\left(t, \hat{x}_{0}\right)\right) \leq 2 \varepsilon \leq 2^{-N+1} \leq 2^{-N_{0}} .
$$

By $(9.102)$ we get

$$
\begin{aligned}
\left|u(t, x)-u\left(t, x^{\prime}\right)\right| & \leq 2^{-N \xi}+2^{(-N+1) \xi} \leq 2^{N \xi / 2}\left(1+2^{\xi}\right) \\
& \leq 4\left(2^{-N}\right)^{\varepsilon_{0} / 2}\left(2^{-N}\right)^{1-\varepsilon_{0}} \\
& \leq 2^{3-\varepsilon_{0}}\left(2^{-N}\right)^{\varepsilon_{0} / 2}\left(\varepsilon \vee\left|x-x^{\prime}\right|\right)^{1-\varepsilon_{0}} \leq\left(\varepsilon \vee\left|x-x^{\prime}\right|\right)^{1-\varepsilon_{0}},
\end{aligned}
$$

since $N \geq 6 \varepsilon_{0}^{-1}$. Therefore, $U_{M}^{(4)}=T_{K_{0}}$ in that case, i.e.

$$
\mathbb{P}\left[U_{M}^{(4)}<T_{K_{0}}\right] \leq \mathbb{P}\left[M \leq N_{0}\right] .
$$

And since $N_{0}$ is stochastically bounded uniformly in $(n, \beta)$ the assertion follows. 
Next, set

$$
\begin{aligned}
U_{M, n, \beta} & =\bigvee_{j=1}^{3} U_{M, n, \beta}^{(j)} \\
U_{M, n} & =\left(\bigvee_{i=0}^{L\left(\varepsilon_{0}, \varepsilon_{1}\right)} U_{M, n, \beta_{i}}\right) \wedge U_{M}^{(4)} .
\end{aligned}
$$

By Lemmas 9.5.1, 9.5.3, 9.5.4, 9.5.5 we have that $U_{M . n}$ fulfills $\left(H_{1}\right)$. So, there is not much left to complete the proof of Proposition 9.2.2 compactness of $\tilde{J}_{n, i}(s)$ and $\tilde{J}_{n, i}(s) \supset J_{n, i}(s)$ for all $s<U_{M, n}$. We will be mostly concerned with $\tilde{J}_{n, i}(s) \supset$ $J_{n, i}(s)$, show that in several steps and assume (9.24) throughout the rest of the section, i.e.

$$
a_{n}^{\varepsilon_{1}} \leq 2^{-M-4} \text { and } \sqrt{a_{n}} \geq 2^{-a_{n}^{-\varepsilon_{0} \varepsilon_{1} / 4}} .
$$

First, we give an important estimate:

Lemma 9.5.6. When $i \in\{0, \ldots, L\}, 0 \leq s<U_{M, n}, x \in J_{n, i}(s)$, then

(a) $\left|\nabla u_{1, a_{n}}\left(s, \hat{x}_{n}(s, x)\right)-\nabla u_{1, a_{n}^{\lambda_{i}}}\left(s, \hat{x}_{n}(s, x)\right)\right| \leq 2^{-71} a_{n}^{\beta_{i}+\varepsilon_{1}(1-\gamma) / 2}$.

(b) $\nabla u_{1, a_{n}^{\lambda_{i}}}\left(s, \hat{x}_{n}(s, x)\right) \cdot \sigma_{x} \leq\left|u_{1, a_{n}^{\lambda_{i}}}\left(s, \hat{x}_{n}(s, x)\right)\right| \leq a_{n}^{\beta_{i}} / 2 \quad(i>0)$.

(c) $\nabla u_{1, a_{n}^{\lambda_{i}}}\left(s, \hat{x}_{n}(s, x)\right) \cdot \sigma_{x} \geq a_{n}^{\beta_{i+1}} / 8 \quad(i<L)$.

Proof. Let us do (a) first and let $(n, i, s, x)$ as above. Set $\varepsilon=\sqrt{a_{n}}$. We have $\left|\left\langle u_{s}, \Phi_{x}^{m_{n+1}}\right\rangle\right| \leq a_{n}$ and $\operatorname{supp}\left(\Phi_{x}^{m_{n+1}}\right) \subset B^{q}\left(x, \sqrt{a_{n}}\right)$. Therefore, by continuity of $u$ it is

$$
\begin{gathered}
\left|u\left(s, \hat{x}_{n}(s, x)\right)\right| \leq a_{n}=a_{n} \wedge\left(\sqrt{a_{n}} \varepsilon\right) \text { and }\left|\hat{x}_{n}(s, x)-x\right| \leq \varepsilon . \\
\left|\nabla u_{1, a_{n}}\left(s, \hat{x}_{n}(s, x)\right)\right| \leq a_{n}^{\beta_{i}} / 4 \quad(\text { for } i>0) .
\end{gathered}
$$

As $x \in J_{n, i}(s)$, because of $9.103,(9.104)$ and 9.105 we can use $U_{M, n, \beta_{i}}^{(3)}$ for $x=$ $\hat{x}_{0}=\hat{x}_{n}(s, x)$ to obtain for $s<U_{M, n}(99.104)$ is not used for $\left.i=0\right)$ :

$\left|\nabla u_{1, a_{n}}\left(s, \hat{x}_{n}(s, x)\right)-\nabla u_{1, a_{n}^{\lambda_{i}}}\left(s, \hat{x}_{n}(s, x)\right)\right| \leq 2^{-74}\left[\tilde{\Delta}_{u_{1}^{\prime}}\left(u, \sqrt{a_{n}}, \varepsilon_{0}, \beta_{i}\right)+a_{n}^{\beta_{i}+\varepsilon_{1}(1-\gamma) / 2}\right]$.

for all $i=0, \ldots, L$. It is

$$
\begin{aligned}
\tilde{\Delta}_{u_{1}^{\prime}}\left(n, \sqrt{a_{n}}, \varepsilon_{0}, \beta_{i}\right) & =a_{n}^{-\varepsilon_{0}} a_{n}^{-\varepsilon_{0} / 2}\left[\sqrt{a_{n}}+a_{n}^{-\alpha / 4}(1+1)\left(a_{n}^{\gamma}+a_{n}^{\beta_{i} \gamma} a_{n}^{\gamma / 2}\right)\right] \\
& \leq 4 a_{n}^{-3 \varepsilon_{0} / 2}\left[a_{n}^{1 / 2}+a_{n}^{\gamma\left(\beta_{i}+1 / 2\right)-\alpha / 4}\right]\left(\beta_{i}<1 / 2\right) \\
& =4\left[a_{n}^{\frac{1-3 \varepsilon_{0}}{2}}+a_{n}^{\gamma\left(\beta_{i}+1 / 2\right)-\alpha / 4-3 \varepsilon_{0} / 2}\right] \\
& \leq 4\left[a_{n}^{\frac{1-3 \varepsilon_{0}}{2}}+a_{n}^{\beta_{i}+\frac{3 \varepsilon_{1}}{2}-\frac{3 \varepsilon_{0}}{2}}\right] \\
& \leq 4 a_{n}^{\beta_{i}+\frac{\varepsilon_{1}}{2}}
\end{aligned}
$$


The next to last inequality holds, since

$$
\begin{array}{r}
\varepsilon_{1}<\frac{1}{3}(\gamma-\alpha / 2-(1-\gamma)) \\
\text { implies }-\frac{3 \varepsilon_{1}}{2}>-\frac{\gamma}{2}+\frac{\alpha}{4}+\frac{1-\gamma}{2} \\
\text { implies } \frac{\gamma}{2}-\frac{\alpha}{4}-\frac{3 \varepsilon_{1}}{2}>\frac{1-\gamma}{2}>\beta_{i}(1-\gamma) \\
\text { thus } \beta_{i} \gamma+\gamma / 2-\alpha / 4>\beta_{i}+3 \varepsilon_{1} / 2
\end{array}
$$

and the last one since

$$
\beta_{i}+\frac{3 \varepsilon_{1}}{2} \leq \frac{1}{2}-6 \varepsilon_{1}+\frac{3 \varepsilon_{1}}{2} \leq \frac{1}{2} \text { and } \varepsilon_{0}<\varepsilon_{1} .
$$

So we are done with (a), since $\varepsilon_{1} / 2>(1-\gamma) \varepsilon_{1} / 2$.

For (b), observe that by $x \in J_{n, i}(s)$, triangle inequality and (a), it suffices to show

$$
2^{-71} a_{n}^{\beta_{i}+(1-\gamma) \varepsilon_{1} / 2}+a_{n}^{\beta_{i}} / 4 \leq a_{n}^{\beta_{i}} / 2 .
$$

For (c) observe that $a_{n}^{\beta_{i}+(1-\gamma) \varepsilon_{1} / 2} \leq a_{n}^{\beta_{i+1}}$ by $\varepsilon_{0} \leq \frac{1-\gamma}{2} \varepsilon_{1}$. Hence, $x \in J_{n, i}(s)$, triangle inequality and the result of (a) give

$$
\nabla u_{1, a_{n}^{\lambda_{i}}}\left(t, \hat{x}_{n}(s, x)\right) \cdot \sigma_{x} \geq a_{n}^{\beta_{i+1}} / 4-2^{-71} a_{n}^{\beta_{i}+\varepsilon_{1} / 8} \geq a_{n}^{\beta_{i+1}} / 8 .
$$

Next, consider the derivatives of $u_{1, a_{n}^{\lambda}}$.

Lemma 9.5.7. Let $0 \leq s<U_{M, n}, i \in\{0, \ldots, L\}, x \in J_{n, i}(s)$ and $\left|x-x^{\prime}\right| \leq 5 \bar{\ell}_{n}\left(\beta_{i}\right)$. Then,

(a) For $i>0:\left|\nabla u_{1, a_{n}^{\lambda_{i}}}\left(s, x^{\prime}\right)\right| \leq a_{n}^{\beta_{i}}$.

(b) For $i<L: \nabla u_{1, a_{n}^{\lambda_{i}}}\left(s, x^{\prime}\right) \cdot \sigma_{x} \geq a_{n}^{\beta_{i+1}} / 16$.

Proof. Let $\varepsilon=\left|x-x^{\prime}\right|+\sqrt{a_{n}} \geq \sqrt{a_{n}}$. Then, we have 9.104 and 9.105 and

$$
\varepsilon \leq 5 a_{n}^{5 \varepsilon_{1}}+\sqrt{a_{n}} \leq 2^{4} a_{n}^{\varepsilon_{1}} \leq 2^{-M}
$$

and

$$
\left|x^{\prime}-\hat{x}_{n}(s, x)\right| \leq \varepsilon \leq 2^{-M}, \quad\left|\hat{x}_{n}(s, x)\right| \leq K_{0}+1 .
$$


Since $s<U_{M, n, \beta_{i}}^{(1)}$, we have

$$
\begin{aligned}
& \left|\nabla u_{1, a_{n}^{\lambda_{i}}}\left(s, x^{\prime}\right)-\nabla u_{1, a_{n}^{\lambda_{i}}}\left(s, \hat{x}_{n}(s, x)\right)\right| \\
& \leq 2^{-82} a_{n}^{-\varepsilon_{1}(1+\alpha / 2)-\varepsilon_{0}}\left(\left|x-x^{\prime}\right|+\sqrt{a_{n}}\right)^{1-\varepsilon_{0}} \\
& \quad\left[a_{n}^{-\beta_{i}(1+\alpha / 2)}\left(\sqrt{a_{n}}+\left|x-x^{\prime}\right|\right)^{2 \gamma}+1+a_{n}^{\beta_{i}(\gamma-1-\alpha / 2)}\left(\sqrt{a_{n}}+\left|x-x^{\prime}\right|\right)^{\gamma}\right] .
\end{aligned}
$$

As $\beta_{i}+5 \varepsilon_{1} \leq 1 / 2$, we get

$$
\left|x-x^{\prime}\right|+\sqrt{a_{n}} \leq 6 a_{n}^{\beta_{i}+5 \varepsilon_{1}} \leq a_{n}^{\beta_{i}} \quad(n>n \underline{9.24)} .
$$

Therefore,

$$
\begin{aligned}
2^{-79} a_{n}^{-\varepsilon_{1}(1+\alpha / 2)-\varepsilon_{0}} a_{n}^{\left(\beta_{i}+5 \varepsilon_{1}\right)\left(1-\varepsilon_{0}\right)} & \\
& {\left[a_{n}^{\beta_{i}(2 \gamma-1-\alpha / 2)}+1+a_{n}^{\beta_{i}(2 \gamma-1-\alpha / 2)}\right] } \\
\leq & 2^{-77} a_{n}^{-\varepsilon_{1}(1+\alpha / 2)-\varepsilon_{0}} a_{n}^{\left(\beta_{i}+5 \varepsilon_{1}\right)\left(1-\varepsilon_{0}\right)} \\
\quad \leq & (2 \gamma-1-\alpha / 2>0) \\
\leq & 2^{-77} a_{n}^{\beta_{i+1}}
\end{aligned}
$$

since

$\beta_{i+1}=\beta_{i}+\varepsilon_{0} \leq \beta_{i}+\varepsilon_{0}-8 \varepsilon_{0}+3 \varepsilon_{1} \leq \beta_{i}+5 \varepsilon_{1}-(1+\alpha / 2) \varepsilon_{1}-\beta_{i} \varepsilon_{0}-5 \varepsilon_{1} \varepsilon_{0}-\varepsilon_{0}$.

Now, we can apply Lemma 9.5.6 (b) and (c) and get the result.

To finish things we only need a similar result for the $u_{2}$ expressions:

Lemma 9.5.8. If $0 \leq i \leq L, 0 \leq s<U_{M, n}, x \in J_{n, i}(s), x^{\prime}, x^{\prime \prime} \in \mathbb{R}^{q}$ and $\left|x-x^{\prime}\right| \leq$ $4 \sqrt{a_{n}}$, then

$$
\left|u_{2, a_{n}^{\lambda_{i}}}\left(s, x^{\prime}\right)-u_{2, a_{n}^{\lambda_{i}}}\left(s, x^{\prime \prime}\right)\right| \leq 2^{-75} a_{n}^{\beta_{i+1}}\left(\left|x^{\prime}-x^{\prime \prime}\right| \vee a_{n}^{\frac{2}{\alpha}\left(\gamma-\beta_{i+1}-\varepsilon_{1}\right)} \vee a_{n}\right)
$$

as long as $\left|x^{\prime}-x^{\prime \prime}\right| \leq \bar{\ell}_{n}\left(\beta_{i}\right)$.

Proof. Let $\left(i, n, s, x, x^{\prime}\right)$ as above and $\varepsilon=5 \sqrt{a_{n}} \leq 2^{-M}$ by (9.24). Then

$$
\begin{array}{r}
\left|x^{\prime}-\hat{x}_{n}(s, x)\right| \leq\left|x^{\prime}-x\right|+\sqrt{a_{n}} \leq \varepsilon,\left|x^{\prime}\right| \leq K_{0}+1 \text { and } \\
\left|u\left(s, \hat{x}_{n}(s, x)\right)\right| \leq a_{n}=a_{n} \wedge\left(\sqrt{a_{n}} \varepsilon\right) .
\end{array}
$$

Since $x \in J_{n, i}(s):\left|\nabla u_{1, a_{n}}\left(s, \hat{x}_{n}(s, x)\right)\right| \leq a_{n}^{\beta_{i}} / 4 \leq a_{n}^{\beta_{i}}$. Let

$$
Q\left(n, \varepsilon_{0}, \beta_{i}, r\right):=a_{n}^{-\varepsilon_{0}} r^{\left(1-\varepsilon_{0}\right)(1-\alpha / 2)}\left(\left(\sqrt{a_{n}} \vee r\right)^{2 \gamma}+a_{n}^{\beta_{i} \gamma} r^{\gamma}\right) .
$$


Assume that $\left|x^{\prime}-x^{\prime \prime}\right| \leq \bar{\ell}_{n}\left(\beta_{i}\right)\left(\leq 2^{-M}\right)$. Since $s<U_{M, n, \beta_{i}}^{(2)}$, it holds that

$$
\begin{aligned}
\mid u_{2, a_{n}^{\lambda_{i}}}\left(s, x^{\prime}\right)- & u_{2, a_{n}^{\lambda_{i}}}\left(s, x^{\prime \prime}\right) \mid \\
& \leq 2^{-87}\left[Q\left(n, \varepsilon_{0}, \beta_{i}, \varepsilon \vee\left|x^{\prime}-x^{\prime \prime}\right|\right)+a_{n}^{-\varepsilon_{0}}\left|x^{\prime}-x^{\prime \prime}\right|^{1-\varepsilon_{0}} a_{n}^{\beta_{i}+\varepsilon_{1}(1-\gamma)}\right] \\
& \leq 2^{-80}\left[Q\left(n, \varepsilon_{0}, \beta_{i},\left|x^{\prime}-x^{\prime \prime}\right|\right)+\left|x^{\prime}-x^{\prime \prime}\right|^{1-\varepsilon_{0}} a_{n}^{\beta_{i}+\varepsilon_{1}(1-\gamma)-\varepsilon_{0}}\right]
\end{aligned}
$$

Claim: $Q\left(n, \varepsilon_{0}, \beta_{i}, r\right) \leq 2 a_{n}^{\beta_{i+1}}\left(r \vee a_{n}^{\left.\frac{2}{\alpha} \gamma-\beta_{i+1}-\varepsilon_{1}\right)} \vee a_{n}\right)$ if $0 \leq r \leq \bar{\ell}_{n}\left(\beta_{i}\right)$.

We split the proof into several cases:

Case 1: $\sqrt{a_{n}} \leq r \leq \bar{\ell}_{n}\left(\beta_{i}\right)=a_{n}^{\beta_{i}+5 \varepsilon_{1}}$

In this case we only need the $r$ in the claim and it is shown if we can show that

$$
r^{\left(1-\varepsilon_{0}\right)(1-\alpha / 2)+2 \gamma-1} \leq a_{n}^{\beta_{i+1}+\varepsilon_{0}}
$$

and

$$
r^{\gamma-1+\left(1-\varepsilon_{0}\right)(1-\alpha / 2)} \leq a_{n}^{\beta_{i+1}+\varepsilon_{0}-\beta_{i} \gamma}
$$

Note that

$$
\left(1-\varepsilon_{0}\right)(1-\alpha / 2)-1+2 \gamma \geq 2 \gamma-\alpha / 2-\varepsilon_{0} \geq 1(\text { by } 9.21) \text {. }
$$

Therefore, 9.110 already follows (additionally using $5 \varepsilon_{1}>2 \varepsilon_{0}$ ). For the other inequality, by $r \leq a_{n}^{\beta_{i}+5 \varepsilon_{1}}$ it suffices to show

$\beta_{i}(1-\gamma)+2 \varepsilon_{0}-\beta_{i}\left[\gamma-1+\left(1-\varepsilon_{0}\right)(1-\alpha / 2)\right]-5 \varepsilon_{1}\left[\gamma-1+\left(1-\varepsilon_{0}\right)(1-\alpha / 2)\right] \leq 0$

This already holds, when

$$
\beta_{i}\left(2 \gamma-1-\alpha / 2+\varepsilon_{0}(1-\alpha / 2)\right)-2 \varepsilon_{0}+5 \varepsilon_{1}(\gamma-\alpha / 2) \geq 0
$$

And this holds since $\alpha<2(2 \gamma-1)$ and $2 \varepsilon_{0}<5 \varepsilon_{1}$. So, we are done with the first case.

Case 2: $a_{n}^{\frac{2}{\alpha}\left(\gamma-\beta_{i+1}-\varepsilon_{1}\right)} \leq r<\sqrt{a_{n}}$.

In this situation:

$$
Q\left(n, \varepsilon_{0}, \beta_{i}, r\right)=a_{n}^{-\varepsilon_{0}} r^{\left(1-\varepsilon_{0}\right)(1-\alpha / 2)}\left[a_{n}^{\gamma}+a_{n}^{\gamma \beta_{i}} r^{\gamma}\right] .
$$

We need to show things for both summands. First,

$$
r^{\left(1-\varepsilon_{0}\right)(1-\alpha / 2)} a_{n}^{\gamma-\varepsilon_{0}} \leq r a_{n}^{\beta_{i+1}}
$$


is true by the lower bound on $r$ and the fact that $\varepsilon_{0}<\varepsilon_{1}\left(1+\frac{\alpha}{2}\right)^{-1}$. The second summand satisfies

$$
a_{n}^{-\varepsilon_{0}} r^{\left(1-\varepsilon_{0}\right)(1-\alpha / 2)} a_{n}^{\gamma \beta_{i}} r^{\gamma} \leq r a_{n}^{\beta_{i+1}},
$$

since

$$
\begin{aligned}
r^{\gamma-\alpha / 2-\varepsilon_{0}(1-\alpha / 2)} & \leq{\sqrt{a_{n}}}^{\gamma-\alpha / 2-\varepsilon_{0}} \\
& \leq{\sqrt{a_{n}}}^{1-\gamma+5 \varepsilon_{0}} \leq a_{n}^{\beta_{i}(1-\gamma)} a_{n}^{2 \varepsilon_{0}}
\end{aligned}
$$

by $3 \varepsilon_{0}<\varepsilon_{1}<\frac{1}{2}\left(2 \gamma-1-\frac{\alpha}{2}\right)$.

Case 3: $r<a_{n}^{\frac{2}{\alpha}\left(\gamma-\beta_{i+1}-\varepsilon_{1}\right)}$

This follows from montonicity in $r$ and the fact that Case 2 is not based on a vacuous condition. This is not the case since

$$
\frac{2}{\alpha}\left(\gamma-\beta_{i+1}-\varepsilon_{1}\right) \geq \frac{2}{\alpha}\left(\gamma-\frac{1}{2}\right)>\frac{\alpha / 2}{\alpha}=\frac{1}{2} .
$$

Hence, the claim is shown.

Next consider the other term in 9.109 to finish the proof. In the case $r \geq a_{n}$ we have:

$$
\begin{aligned}
r^{1-\varepsilon_{0}} a_{n}^{\beta_{i}+\varepsilon_{1}(1-\gamma)-\varepsilon_{0}}\left(a_{n}^{\beta_{i+1}} r\right)^{-1} & =r^{-\varepsilon_{0}} a_{n}^{-2 \varepsilon_{0}+\varepsilon_{1}(1-\gamma)} \\
& \leq a_{n}^{-3 \varepsilon_{0}+\varepsilon_{1}(1-\gamma)}<1
\end{aligned}
$$

since $\varepsilon_{0}<\frac{1-\gamma}{3} \varepsilon_{1}$. So, for any $r>0$ (when using the previous estimate with $\left(r \vee a_{n}\right)$ in place of $r$ ):

$$
\begin{aligned}
r^{1-\varepsilon_{0}} a_{n}^{\beta_{i}+\varepsilon_{1}(1-\gamma)-\varepsilon_{0}} & \leq a_{n}^{\beta_{i+1}}\left(r \vee a_{n}\right) \\
& \leq a_{n}^{\beta_{i+1}}\left(r \vee a_{n}^{\frac{2}{\alpha}\left(\gamma-\beta_{i+1}-\varepsilon_{1}\right)} \vee a_{n}\right)
\end{aligned}
$$

Putting things together we get the statement of the lemma.

Lemma 9.5.9. If $0 \leq s<U_{M, n}$ and $x \in J_{n, 0}(s)$, then

$$
\left|u(s, x)-u\left(s, x^{\prime}\right)\right| \leq\left(\sqrt{a_{n}} \vee\left|x-x^{\prime}\right|\right)^{1-\varepsilon_{0}} \text { if } x^{\prime} \text { such that }\left|x^{\prime}-x\right| \leq 2^{-M} .
$$

and

$$
\left|u\left(s, x^{\prime}\right)\right| \leq 3\left(\sqrt{a_{n}}\right)^{1-\varepsilon_{0}} \quad \text { if }\left|x^{\prime}-x\right| \leq \sqrt{a_{n}} .
$$

This lemma just has the same proof as Lemma 6.8 in MP11, so we omit it.

Proof of Proposition 9.2.2.

The compactness of $\tilde{J}_{n, i}(s)$ follows from the continuity of all the functions involved and the inclusion $J_{n, i}(s) \subset \tilde{J}_{n, i}(s)$ follows from Lemmas 9.5.7, 9.5.8 and 9.5.9. 


\subsection{Proof of Proposition 9.4.16}

Using the stochastic Fubini Formula we obtain

$$
G_{\delta}(s, t, x)=\int_{\mathbb{R}^{q}} \int_{0}^{(s-\delta)^{+}} p_{t \vee s-r}(x-z) D(r, z) W(d r d z) .
$$

We have

$$
\begin{aligned}
\left|G_{\delta}(s, t, x)-G_{\delta}\left(s^{\prime}, t^{\prime}, x^{\prime}\right)\right| \leq & \left|G_{\delta}(s, t, x)-G_{\delta}\left(s, t, x^{\prime}\right)\right|+\left|G_{\delta}\left(s, t, x^{\prime}\right)-G_{\delta}\left(s, t^{\prime}, x^{\prime}\right)\right| \\
& +\left|G_{\delta}\left(s, t^{\prime}, x^{\prime}\right)-G_{\delta}\left(s^{\prime}, t^{\prime}, x^{\prime}\right)\right| .
\end{aligned}
$$

Hence, we define the following square functions for $i, j \in\{1,2\}$ :

$$
\begin{aligned}
\check{Q}_{X, \delta, \eta_{0}}^{i, j}\left(s, t, x, t, x^{\prime}\right)= & \int_{0}^{(s-\delta)^{+}} d r \int_{A_{i}(r, t)} d w \int_{A_{j}(r, t)} d z \\
& \left|\left(p_{t-r}\left(w-x^{\prime}\right)-p_{t-r}(w-x)\right)\left(p_{t-r}\left(z-x^{\prime}\right)-p_{t-r}(z-x)\right)\right| \\
& R_{0}^{2 \gamma} e^{R_{1}(|w|+|z|)}|u(r, w)|^{\gamma}|u(r, z)|^{\gamma} \\
& \left(|w-z|^{-\alpha}+1\right),
\end{aligned}
$$

$$
\begin{aligned}
\check{Q}_{X, \delta, \eta_{0}}^{i, j}\left(s, t, x^{\prime}, t^{\prime}, x^{\prime}\right)= & \int_{0}^{(s-\delta)^{+}} d r \int_{A_{i}\left(r, t^{\prime}\right)} d w \int_{A_{j}\left(r, t^{\prime}\right)} d z \\
& \left|\left(p_{t^{\prime}-r}\left(w-x^{\prime}\right)-p_{t-r}\left(w-x^{\prime}\right)\right)\left(p_{t^{\prime}-r}\left(z-x^{\prime}\right)-p_{t-r}\left(z-x^{\prime}\right)\right)\right| \\
& R_{0}^{2 \gamma} e^{R_{1}(|w|+|z|)}|u(r, w)|^{\gamma}|u(r, z)|^{\gamma} \\
& \left(|w-z|^{-\alpha}+1\right)
\end{aligned}
$$

and

$$
\begin{aligned}
\check{Q}_{S, \delta}\left(s, s^{\prime}, t^{\prime}, x^{\prime}\right)= & \int_{\left(s \wedge s^{\prime}-\delta\right)^{+}}^{\left(s \vee s^{\prime}-\delta\right)^{+}} d r \int_{\mathbb{R}^{q}} d w \int_{\mathbb{R}^{q}} d z\left(|w-z|^{-\alpha}+1\right) \\
& \left|p_{t^{\prime}-r}\left(w-x^{\prime}\right) p_{t^{\prime}-r}\left(z-x^{\prime}\right)\right| R_{0}^{2 \gamma} e^{R_{1}(|w|+|z|)}|u(r, w)|^{\gamma}|u(r, z)|^{\gamma} .
\end{aligned}
$$

The sum of these estimates of square functions is denoted by

$$
\check{Q}_{\delta}^{\text {tot }}=\sum_{i, j=1}^{2}\left(\check{Q}_{X, \delta, \eta_{0}}^{i, j}\left(s, t, x, t, x^{\prime}\right)+\check{Q}_{X, \delta, \eta_{0}}^{i, j}\left(s, t, x^{\prime}, t^{\prime}, x^{\prime}\right)\right)+\check{Q}_{S, \delta}\left(s, s^{\prime}, t^{\prime}, x^{\prime}\right) .
$$


All of the proofs are similar to the ones in Chapter 9.4. First, we present some analogues of the bounds on the heat kernels.

Lemma 9.6.1. There is a positive constant $C>0$, such that for $0<t<t^{\prime}$, $w, v \in \mathbb{R}^{q}$ the following holds:

(a) Setting $\hat{v}_{0}:=0$ and $\hat{v}_{i}:=\hat{v}_{i-1}+v_{i} e_{i}, 1 \leq i \leq q$, where $e_{i}$ is the $i$-th unit vector in $\mathbb{R}^{q}$, we have for the spatial differences

$$
\left|p_{t}(w+v)-p_{t}(w)\right| \leq C t^{-\frac{1}{2}} \sum_{i=1}^{q} \int_{0}^{\left|v_{i}\right|} d r_{i} p_{2 t}\left(w+\hat{v}_{i-1}+r_{i} e_{i}\right) .
$$

(b) We obtain for the time differences

$$
\left|p_{t}(w)-p_{t^{\prime}}(w)\right| \leq C\left|t-t^{\prime}\right|^{\frac{1}{2}} t^{-\frac{1}{2}}\left(p_{t}(w)+p_{2 t^{\prime}}(w)\right) .
$$

And as in Lemma 9.3.4 we can derive:

Lemma 9.6.2. There is a positive constant $C=C(\alpha, q)<\infty$ such that for any $x, x^{\prime} \in \mathbb{R}^{q}, 0<t \leq t^{\prime}:$

$$
\begin{gathered}
\int_{\mathbb{R}^{q}} \int_{\mathbb{R}^{q}}\left|\left(p_{t}(w-x)-p_{t^{\prime}}\left(w-x^{\prime}\right)\right)\left(p_{t}(z-x)-p_{t^{\prime}}\left(z-x^{\prime}\right)\right)\right|\left(|w-z|^{-\alpha}+1\right) d w d z \\
\leq C t^{-\alpha / 2}\left(1 \wedge \frac{\left|x-x^{\prime}\right|^{2}+\left|t-t^{\prime}\right|}{t}\right) .
\end{gathered}
$$

Both of these lemmas can also be found in a slightly different way (the temporal exponents differ) in Lemma 5.3 of [MPS06], so we omit their proof here.

Lemma 9.6.3. Let $R>2$ and $\eta_{0} \in(1 / R, 1 / 2)$. Then there is a constant $C=C\left(\eta_{0}\right)$ such that for $y, \tilde{y} \in \mathbb{R}^{q}$ and $0<t \leq t^{\prime}$ :

(a) $\mathbb{1}\left\{|\tilde{y}|>t^{\prime 1 / 2-\eta_{0}} \vee 2|y-\tilde{y}|\right\}\left|p_{t}(y)\right| \leq C \exp \left(-\frac{1}{16} t^{-2 \eta_{0}}\right) p_{2 t}(y)$.

(b) $\mathbb{1}\left\{|\tilde{y}|>t^{\prime 1 / 2-\eta_{0}} \vee 2|y-\tilde{y}|\right\}\left|p_{t}(y)\right| \leq 2^{q} C \exp \left(-\frac{1}{16} t^{-2 \eta_{0}}\right) p_{8 t}(\tilde{y})$.

The proof of this lemma is almost the same as the proof of Lemma 9.3 .5 and we omit it. 
Lemma 9.6.4. For all $R>2$, there is a constant $C=C(K, R)$ such that for all $0 \leq p, r \leq R, \eta_{0}, \eta_{1} \in(1 / R, 1 / 2), 0 \leq s<t \leq t^{\prime}<R$ and $x, x^{\prime} \in[-K, K]^{q}:$

$$
\begin{aligned}
\int_{\mathbb{R}^{q}} \int_{\mathbb{R}^{q}} \mid w- & \left.x\right|^{p}|z-x|^{p}\left(p_{t-s}(w-x)-p_{t^{\prime}-s}\left(w-x^{\prime}\right)\right)\left(p_{t-s}(z-x)-p_{t^{\prime}-s}\left(z-x^{\prime}\right)\right) \\
& \quad \times \mathbb{1}\left\{|w-x|>\left(t^{\prime}-s\right)^{1 / 2-\eta_{0}} \vee 2\left|x-x^{\prime}\right|\right\} \\
& \quad \times e^{r|w-x|+r|z-x|}\left(|w-z|^{-\alpha}+1\right) d w d z \\
\leq & C(t-s)^{-\alpha / 2} \exp \left(-\eta_{1}\left(t^{\prime}-s\right)^{-2 \eta_{0}} / 64\right)\left[1 \wedge\left(\frac{\left|x-x^{\prime}\right|^{2}+\left|t-t^{\prime}\right|}{t-s}\right)\right]^{1-\eta_{1} / 4}
\end{aligned}
$$

This lemma can be proven in the same way as Lemma 9.3.7

Lemma 9.6.5. For all $K \in \mathbb{N} \geq K_{1}, R>2$ there exist $\overline{q .6 .5}(K, R), N_{[9.6 .5}(K, \omega)$ almost surely such that

$\forall \eta_{0}, \eta_{1} \in(1 / R, 1 / 2), \delta \in(0,1], \beta \in[0,1 / 2], N, n \in \mathbb{N},(t, x) \in \mathbb{R}_{+} \times \mathbb{R}^{q}$ the following holds for $i+j \geq 3$ :

$$
\begin{aligned}
& \text { For } \omega \in\{(t, x) \in Z(N, n, K, \beta), N \geq N \overline{9.6 .5}\} \\
& \check{Q}_{X, \delta, \eta_{0}}^{i, j}\left(s, t, x, t^{\prime}, x^{\prime}\right) \leq \overline{9.4 .5}^{4{ }^{9.6 .5}[}\left[(d \wedge \sqrt{\delta})^{2-\eta_{1} / 2} \delta^{-\alpha / 2}(d \wedge 1)^{2 \gamma}+d^{2-\eta_{1} / 2}\right] \\
& \forall 0 \leq s \leq t \leq t^{\prime}, x^{\prime} \in \mathbb{R}^{q},
\end{aligned}
$$

where $d=d\left((t, x),\left(t^{\prime}, x^{\prime}\right)\right)$.

This lemma has almost the same proof as Lemma 9.4.5.

Lemma 9.6.6. For all $K \in \mathbb{N} \geq K_{1}, R>2$ there exist $9.9 .6 .6(K, R), N_{9.6 .6}(K, \omega)$ almost surely such that $\forall \eta_{0}, \eta_{1} \in(1 / R, 1 / 2), \delta \in(0,1], \beta \in[0,1 / 2], N, n \in \mathbb{N}$, $(t, x) \in \mathbb{R}_{+} \times \mathbb{R}^{q}$ the following holds for $i+j \geq 3$ :

$$
\begin{aligned}
& \text { For } \omega \in\left\{(t, x) \in Z(N, n, K, \beta), N \geq N \overline{\left.9_{9.6 .6}\right\}}\right. \\
& \check{Q}_{T, \delta, \eta_{0}}^{i, j}\left(s, t, x^{\prime}, t^{\prime}, x^{\prime}\right) \leq \overline{9.6 .6} 2^{4 N \overline{9.6 .6}\left[\left|t-t^{\prime}\right|^{1-\eta_{1} / 2}+\left|t-t^{\prime}\right|^{1-\eta_{1} / 2} \delta^{-\alpha / 2}\left(\left|t-t^{\prime}\right| \wedge 1\right)^{\gamma}\right]} \\
& \forall 0 \leq s \leq t, x^{\prime} \in \mathbb{R}^{q} .
\end{aligned}
$$

This lemma has almost the same proof as Lemma 9.4.6. The next lemma gets endowed with a proof, as an example for the one of the proofs, but also for some technical differences to Lemma 9.4.7.

Lemma 9.6.7. Let $0 \leq m \leq \bar{m}+1$ and assume that $\left(P_{m}\right)$ holds. For all $K \in$ $\mathbb{N} \geq K_{1}, R>2, n \in \mathbb{N}, \beta \in[0,1 / 2], \varepsilon_{0} \in(0,1)$, there exist $q 9.6 .7(K, R)$ and 
$N \overline{9.6 .7}\left(m, n, R, \varepsilon_{0}, K, \beta\right)(\omega) \in \mathbb{N}$ almost surely such that

$\forall \eta_{1} \in(1 / R, 1 / 2), \eta_{0} \in\left(0, \eta_{1} / 32\right), \delta \in\left[a_{n}, 1\right], N \in \mathbb{N},(t, x) \in \mathbb{R}_{+} \times \mathbb{R}^{q}$ the following holds.

$$
\begin{aligned}
& \text { For } \omega \in\left\{(t, x) \in Z(N, n, K, \beta), N \geq N_{9.6 .7}\right\} \\
& \check{Q}_{X, \delta, \eta_{0}}^{1,1}\left(s, t, x, t^{\prime}, x^{\prime}\right) \leq q_{9.6 .7}\left[a_{n}^{-2 \varepsilon_{0}}+2^{4 N[\overline{9.6 .7]}]}\left[d^{2-\eta_{1} / 2}\right.\right. \\
& \left.+d^{2-\eta_{1} / 2} \delta^{-\alpha / 2}\left[\vec{d}_{N}^{2 \gamma \gamma_{m}}+a_{n}^{2 \beta \gamma} \vec{d}_{N}^{2 \gamma}\right]\right] \\
& \forall 0 \leq s \leq t \leq t^{\prime}, x^{\prime} \in \mathbb{R}^{q} \text {. }
\end{aligned}
$$

Here $\bar{d}_{N}=\left|x-x^{\prime}\right|+\sqrt{t^{\prime}-t} \vee 2^{-N}$ and $\bar{\delta}_{N}=\delta \vee \bar{d}_{N}^{2}$. Moreover, $N_{9.6 .7}$ is stochastically bounded uniformly in $(n, \beta)$.

Proof. Let $\xi=1-(8 R)^{-1} \in(15 / 16,1)$ and set $N_{9.6 .7}=N_{1}\left(m, n, \xi, \varepsilon_{0}, K, \beta\right)$. We can assume that $s>\delta$ and therefore, we have always $d((r, w),(t, x)) \geq \sqrt{a_{n}}$ in the integral. A use of Lemma 9.4.3 and the bound on $|w-x|,|z-x|$ respectively, gives

$$
\begin{aligned}
\check{Q}_{X, \delta, \eta_{0}}^{1,1}\left(s, t, x, t, x^{\prime}\right) \leq & G_{9.4 .3} \int_{0}^{s-\delta} d r \int_{\mathbb{R}^{q}} d w \int_{\mathbb{R}^{q}} d z \\
& \left(p_{t-r}\left(w-x^{\prime}\right)-p_{t-r}(w-x)\right)\left(p_{t-r}\left(z-x^{\prime}\right)-p_{t-r}(z-x)\right) \\
& R_{0}^{2 \gamma} e^{4 R_{1} K} e^{4 \gamma K} \\
& {\left[2^{-N} \vee\left((t-r)^{1 / 2}+(t-r)^{1 / 2-\eta_{0}} \vee 2\left|x-x^{\prime}\right|\right)\right]^{2 \gamma \xi} } \\
& \left\{\left[2^{-N} \vee\left((t-r)^{1 / 2}+(t-r)^{1 / 2-\eta_{0}} \vee 2\left|x-x^{\prime}\right|\right)\right]^{\gamma_{m}-1}+a_{n}^{\beta}\right\}^{2 \gamma} \\
& \left(|w-z|^{-\alpha}+1\right) .
\end{aligned}
$$

Let $\gamma^{\prime}=\gamma\left(1-2 \eta_{0}\right)$ and observe the trivial inequalities

$$
\begin{aligned}
\sqrt{t-r} & \leq K^{\eta_{0} / 2}(t-r)^{1 / 2-\eta_{0}}, \\
\left|x-x^{\prime}\right| & \leq c(q) K\left|x-x^{\prime}\right|^{1-2 \eta_{0}} .
\end{aligned}
$$

Then, Lemma 9.3.4 allows the following bound

$$
\begin{aligned}
\check{Q}_{X, \delta, \eta_{0}}^{1,1}\left(s, t, x, t, x^{\prime}\right) \leq & C_{[.4 .3} c_{1}(K) \int_{0}^{s-\delta} d r(t-r)^{-\alpha / 2}\left[1 \wedge \frac{\left|x-x^{\prime}\right|^{2}}{t-r}\right] \\
& {\left[2^{-2 N \gamma} \vee(t-r)^{\gamma^{\prime}} \vee\left|x-x^{\prime}\right|^{2 \gamma^{\prime}}\right]^{\xi} } \\
& {\left[2^{-N \gamma^{\prime}\left(\gamma_{m}-1\right)} \vee(t-r)^{\gamma^{\prime}\left(\gamma_{m}-1\right)} \vee\left|x-x^{\prime}\right|^{2 \gamma\left(\gamma_{m}-1\right)}+a_{n}^{2 \beta \gamma}\right] . }
\end{aligned}
$$

Using

$$
\begin{aligned}
2^{-2 N \gamma} \vee(t-r)^{\gamma^{\prime}} \vee\left|x-x^{\prime}\right|^{2 \gamma^{\prime}} & \leq 2^{-2 N \gamma^{\prime}} \vee\left|x-x^{\prime}\right|^{2 \gamma^{\prime}}+(t-r)^{\gamma^{\prime}} \\
& \leq 2\left[\vec{d}_{N}^{2 \gamma^{\prime}} \vee(t-r)^{\gamma^{\prime}}\right]
\end{aligned}
$$


we can bound the above by

$$
\begin{gathered}
\check{Q}_{X, \delta, \eta_{0}}^{1,1}\left(s, t, x, t, x^{\prime}\right) \leq C_{\overline{9.4 .3}} c_{1}(K) \int_{0}^{s-\delta} d r(t-r)^{-\alpha / 2}\left[1 \wedge \frac{\left|x-x^{\prime}\right|^{2}}{t-r}\right] 2^{\xi}\left(\vec{d}_{N}^{2 \gamma^{\prime} \xi} \vee(t-r)^{\gamma^{\prime} \xi}\right) \\
2^{\gamma_{m}-1}\left[\left(\bar{d}_{N}^{2} \vee(t-r)\right)^{\gamma^{\prime}\left(\gamma_{m}-1\right)}+a_{n}^{2 \beta \gamma}\right] \\
\leq C_{9.4 .3} c_{2}(K) \int_{0}^{s-\delta} d r \mathbb{1}\left\{t-r \geq \bar{d}_{N}^{2}\right\}(t-r)^{-\alpha / 2+\gamma^{\prime} \xi}\left[1 \wedge \frac{\left|x-x^{\prime}\right|^{2}}{t-r}\right] \\
\quad\left[(t-r)^{\gamma^{\prime}\left(\gamma_{m}-1\right)}+a_{n}^{2 \beta \gamma}\right] \\
+C_{9.4 .3} c_{2}(K) \int_{0}^{s-\delta} d r \mathbb{1}\left\{t-r<\bar{d}_{N}^{2}\right\}(t-r)^{-\alpha / 2}\left[1 \wedge \frac{\left|x-x^{\prime}\right|^{2}}{t-r}\right] \vec{d}_{N}^{2 \gamma^{\prime} \xi} \\
{\left[\vec{d}_{N}^{2 \gamma^{\prime}\left(\gamma_{m}-1\right)}+a_{n}^{2 \beta \gamma}\right]} \\
=C_{9.4 .3} c_{2}(K)\left(I_{1}+I_{2}\right) .
\end{gathered}
$$

We start with an estimate on $I_{1}$. If $r \leq s-\delta$ and $t-r \geq \bar{d}_{N}^{2}$, then

$$
r \leq t-\bar{d}_{N}^{2} \wedge s-\delta \leq t-\vec{d}_{N}^{2} \wedge t-\delta=t-\bar{\delta}_{N}
$$

Use that to start with

$$
\begin{aligned}
I_{1} \leq \int_{0}^{t-\bar{\delta}_{N}} d r(t-r)^{-\alpha / 2+\gamma^{\prime} \xi+\gamma^{\prime}\left(\gamma_{m}-1\right)}\left[1 \wedge \frac{\left|x-x^{\prime}\right|^{2}}{t-r}\right] \\
\quad+(t-r)^{-\alpha / 2+\gamma^{\prime} \xi}\left[1 \wedge \frac{\left|x-x^{\prime}\right|^{2}}{t-r}\right] a_{n}^{2 \beta \gamma} .
\end{aligned}
$$

We want to drop the minimum with 1 to consider

$$
\left|x-x^{\prime}\right|^{2} \int_{\bar{\delta}_{N}}^{t} d u u^{-2-\alpha / 2+\gamma^{\prime} \xi+\gamma^{\prime}\left(\gamma_{m}-1\right)}+u^{-2-\alpha / 2+\gamma^{\prime} \xi} a_{n}^{2 \beta \gamma}
$$

and then face three cases for the exponents: $<-1,=-1,>-1$. In the first and third case use the following inequality for $p \in(-1,1), p \neq 0,0<a<b$ :

$$
\int_{a}^{b} u^{p-1} d u=\frac{1}{|p|}\left|a^{p}-b^{p}\right| \leq \log (b / a)\left(a^{p}+b^{p}\right) .
$$

This is true, since $1-x \leq-\log x$, for $x \geq 0$ with $x=(b / a)^{p}$ or $x=(a / b)^{p}$.

In the -1 -case the integral is bounded by $\log K+\log \left(1 / \bar{\delta}_{N}\right)$. Hence, using that $t \leq K$ (therefore $\left.t^{0 \vee\left(-1-\alpha / 2+\gamma^{\prime} \xi+\gamma^{\prime}\left(\gamma_{m}-1\right)\right)} \leq K^{1}\right)$, in any of the cases there is a constant $c(K)$ such that :

$$
I_{1} \leq K^{1}\left|x-x^{\prime}\right|^{2} \log \left(K / \bar{\delta}_{N}\right)\left(\bar{\delta}_{N}^{\left(-\alpha / 2+\gamma^{\prime} \xi+\gamma^{\prime}\left(\gamma_{m}-1\right)\right) \wedge 0}+\bar{\delta}_{N}^{\left(-\alpha / 2+\gamma^{\prime} \xi\right) \wedge 0} a_{n}^{2 \beta \gamma}\right),
$$


The log-term is bounded by $c(K, R)\left|x-x^{\prime}\right|^{-\eta_{1} / 2}$ using Lemma 9.3 .1 .

Moreover, by Lemma 4.1(b) in [MP11] we bound

$$
I_{2} \leq c(\alpha)\left|x-x^{\prime}\right|^{2}\left(\left|x-x^{\prime}\right|^{2} \vee \delta\right)^{-\alpha / 2} \vec{d}_{N}^{2 \gamma^{\prime} \xi}\left[\vec{d}_{N}^{2 \gamma^{\prime}\left(\gamma_{m}-1\right)}+a_{n}^{2 \beta \gamma}\right] .
$$

Therefore, we can bound $\check{Q}_{X, \delta, \eta_{0}}^{1,1}\left(s, t, x, t, x^{\prime}\right)$ by

$$
\begin{gathered}
\text { q9.4.3 } c_{3}(K, R)\left[\left|x-x^{\prime}\right|^{2-\eta_{1} / 2}\left(\bar{\delta}_{N}^{\left(-\alpha / 2+\gamma^{\prime}\left(\gamma_{m}+\xi-1\right)\right) \wedge 0}+\bar{\delta}_{N}^{\left(-\alpha / 2+\gamma^{\prime} \xi \wedge 0\right)} a_{n}^{2 \beta \gamma}\right)\right. \\
+\left(\delta \wedge\left|x-x^{\prime}\right|^{2}\right) \delta^{-\alpha / 2} \vec{d}_{N}^{2 \gamma^{\prime} \xi}\left[\vec{d}_{N}^{2 \gamma^{\prime}\left(\gamma_{m}-1\right)}+a_{n}^{2 \beta \gamma}\right]
\end{gathered}
$$

Now we can replace $\xi=1-(8 R)^{-1}$ by 1 and $\gamma^{\prime}=\gamma\left(1-2 \eta_{0}\right)$ by $\gamma$ at the cost of multiplying by $d^{-\eta_{1} / 2} \geq \bar{\delta}_{N}^{-\eta_{1} / 4}$. This is true, since

$$
\xi \gamma^{\prime}=\gamma\left(1-2 \eta_{0}\right)\left(1-(8 R)^{-1}\right) \geq \gamma\left(1-\eta_{1} / 4\right), \text { hence } \xi \gamma^{\prime}-\gamma \geq-\gamma \eta_{1} / 4 \geq-\eta_{1} / 4
$$

and

$$
\begin{aligned}
\gamma^{\prime}\left(\gamma_{m}+\xi-1\right) & =\gamma\left(1-2 \eta_{0}\right)\left(\gamma_{m}-\frac{1}{8 R}\right) x \\
& \geq \gamma\left(1-\frac{\eta_{1}}{16}\right)\left(\gamma_{m}-\frac{1}{8 R}\right)\left(\text { by } \eta_{1} \geq 32 \eta_{0}\right) \\
& \geq \gamma \gamma_{m}-\gamma \frac{1}{8 R}-\gamma \gamma_{m} \frac{\eta_{1}}{16} \\
& \geq \gamma \gamma_{m}-\frac{1}{8 R}-2 \frac{\eta_{1}}{16} \\
& \geq \gamma \gamma_{m}-\frac{\eta_{1}}{8}-\frac{\eta_{1}}{8}\left(\text { by } \eta_{1}>R^{-1}\right) \\
& \geq \gamma \gamma_{m}-\frac{\eta_{1}}{4} .
\end{aligned}
$$

Use

$$
\gamma \gamma_{m}-\alpha / 2 \geq \gamma-\alpha / 2>1-\gamma>0
$$

to put all things together to

$$
\begin{aligned}
\check{Q}_{X, \delta, \eta_{0}}^{1,1}\left(s, t, x, t, x^{\prime}\right) \leq & \underset{9.4 .3}{ } c_{3}(K, R)\left[\left|x-x^{\prime}\right|^{2-\eta_{1} / 2}\right. \\
& +\left(\sqrt{\delta} \wedge\left|x-x^{\prime}\right|\right)^{2-\eta_{1} / 2} \delta^{-1-\alpha / 2}\left[\vec{d}_{N}^{2 \gamma \gamma_{m}}+\vec{d}_{N}^{2 \gamma} a_{n}^{2 \beta \gamma}\right]
\end{aligned}
$$

Lemma 9.6.8. Let $0 \leq m \leq \bar{m}+1$ and assume that $\left(P_{m}\right)$ holds. For all $K \in$ $\mathbb{N} \geq K_{1}, R>2, n \in \mathbb{N}, \beta \in[0,1 / 2], \varepsilon_{0} \in(0,1)$, there exist q9.6.8 $(K, R)$ and $N \overline{9_{0.6 .8}}\left(m, n, R, \varepsilon_{0}, K, \beta\right)(\omega) \in \mathbb{N}$ almost surely such that 
$\forall \eta_{1} \in(1 / R, 1 / 2), \eta_{0} \in\left(0, \eta_{1} / 32\right), \delta \in\left[a_{n}, 1\right], N \in \mathbb{N},(t, x) \in \mathbb{R}_{+} \times \mathbb{R}^{q}$ the following holds.

$$
\begin{aligned}
& \text { For } \omega \in\left\{(t, x) \in Z(N, n, K, \beta), N \geq N \overline{\left.9_{0.6 .8}\right\}}\right\} \\
& \qquad \begin{aligned}
\check{Q}_{T, \delta, \eta_{0}}^{1,1}\left(s, t, x^{\prime}, t^{\prime}, x^{\prime}\right) \leq \frac{q 9.6 .8}{\left[\left|t-t^{\prime}\right|^{1-\eta_{1} / 2}\right.} \\
\left.+\left|t-t^{\prime}\right|^{1-\eta_{1} / 2} \delta^{-\alpha / 2}\left[\vec{d}_{N}^{2 \gamma \gamma_{m}}+a_{n}^{2 \beta \gamma} \vec{d}_{N}^{2 \gamma}\right]\right] \\
\forall 0 \leq s \leq t, x^{\prime} \in \mathbb{R}^{q} .
\end{aligned}
\end{aligned}
$$

Here $\bar{d}_{N}=\left|x-x^{\prime}\right|+\sqrt{t^{\prime}-t} \vee 2^{-N}$ and $\bar{\delta}_{N}=\delta \vee \bar{d}_{N}^{2}$. Moreover, $N_{9.6 .8}$ is stochastically bounded uniformly in $(n, \beta)$.

For this lemma, the proof would just be the same as Lemma 9.4.8 using some ideas of the proof just before. And finally, we state a lemma about the distance in the $s$-variable:

Lemma 9.6.9. Let $0 \leq m \leq \bar{m}+1$ and assume that $\left(P_{m}\right)$ holds. For all $K \in$ $\mathbb{N} \geq K_{1}, R>2, n \in \mathbb{N}, \beta \in[0,1 / 2], \varepsilon_{0} \in(0,1)$, there exist $9.6 .9(K, R, \gamma)$ and $N\left[\overline{9.6 .9}\left(m, n, R, \varepsilon_{0}, K, \beta\right)(\omega) \in \mathbb{N}\right.$ almost surely such that $\forall \eta_{1} \in(1 / R, 1 / 2), \delta \in$ $\left[a_{n}, 1\right], N \in \mathbb{N},(t, x) \in \mathbb{R}_{+} \times \mathbb{R}^{q}$ the following holds.

$$
\begin{aligned}
& \text { For } \omega \in\{(t, x) \in Z(N, n, K, \beta), N \geq N \overline{\text { [9.6.9 }}\}
\end{aligned}
$$

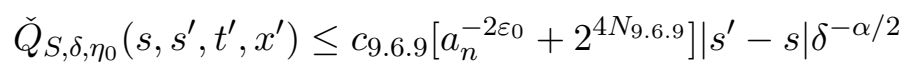

$$
\begin{aligned}
& \left\{| s ^ { \prime } - s | ^ { - \eta _ { 1 } / 2 } \left[\left(\left|t^{\prime}-t\right| \vee(t-s) \vee\left(t^{\prime}-s^{\prime}\right) \vee \delta\right)^{\gamma \gamma_{m}}\right.\right. \\
& \left.+a_{n}^{2 \beta \gamma}\left(\left|t^{\prime}-t\right| \vee(t-s) \vee\left(t^{\prime}-s^{\prime}\right) \vee \delta\right)^{\gamma}\right] \\
& \left.+2^{N \eta_{1}}\left[\vec{d}_{N}^{2 \gamma\left(\gamma_{m}-1\right)}+a_{n}^{2 \beta \gamma} \vec{d}_{N}^{2 \gamma}\right]\right\} \\
& \forall 0 \leq s \leq t, x^{\prime} \in \mathbb{R}^{q} .
\end{aligned}
$$

Here $\bar{d}_{N}=\left(\left|t-t^{\prime}\right|^{1 / 2}+\left|x-x^{\prime}\right|\right) \vee 2^{-N}$ and $\bar{\delta}_{N}=\delta \vee \bar{d}_{N}^{2}$. Moreover, N $N_{9.6 .9}$ is stochastically bounded uniformly in $(n, \beta)$.

Proof. Choose $\xi=1-(2 \gamma R)^{-1}$.

Choose $N \overline{9.6 .9}=N_{1}\left(m, n, \xi, \varepsilon_{0}, K, \beta\right)$. It is for $r \leq s \vee s^{\prime}-\delta: \sqrt{a_{n}}<\sqrt{\delta}<\sqrt{s-r}<$ $\sqrt{t-r}$, thus

$$
\begin{aligned}
2^{-N} \vee\left(\left(t^{\prime}-r\right)^{1 / 2}+|w-x|\right) & \leq 2^{-N} \vee\left|x-x^{\prime}\right|+\left(t^{\prime}-r\right)^{1 / 2}+\left|w-x^{\prime}\right| \\
& \leq \bar{d}_{N}^{2}+\left(t^{\prime}-r\right)^{1 / 2}+\left|w-x^{\prime}\right|
\end{aligned}
$$


This bound, Lemmas 9.6.2, 9.4.3 and 9.3.6 give:

$$
\begin{aligned}
& \check{Q}_{S, \delta, \eta_{0}}\left(s, s^{\prime}, t^{\prime}, x^{\prime}\right) \leq c_{0}(K, R)\left[a_{n}^{-2 \varepsilon_{0}}+2^{4 N[\overline{9.6 .9}]}\right. \\
& \quad \int_{\left(s \wedge s^{\prime}-\delta\right)^{+}}^{\left(s \vee s^{\prime}-\delta\right)^{+}} d r\left(t^{\prime}-r\right)^{-\alpha / 2}\left[\vec{d}_{N}^{2 \xi \gamma}+\left(t^{\prime}-r\right)^{\xi \gamma}\right]\left[\vec{d}_{N}^{2 \gamma\left(\gamma_{m}-1\right)}+\left(t^{\prime}-r\right)^{\gamma\left(\gamma_{m}-1\right)}+a_{n}^{2 \beta \gamma}\right] \\
& \leq 4 c_{0}(K, R)\left[a_{n}^{-2 \varepsilon_{0}}+2^{4 N\left[\frac{9.6 .9}{}\right]}\{\right. \\
& \quad \int_{\left(s \wedge s^{\prime}-\delta\right)^{+}}^{\left(s \vee s^{\prime}-\delta\right)^{+}} \mathbb{1}\left\{r \leq t^{\prime}-\vec{d}_{N}^{2}\right\}\left(t^{\prime}-r\right)^{-\alpha / 2+\xi \gamma}\left[\left(t^{\prime}-r\right)^{\gamma\left(\gamma_{m}-1\right)}+a_{n}^{2 \beta \gamma}\right] d r \\
& \left.\quad+\int_{\left(s \wedge s^{\prime}-\delta\right)^{+}}^{\left(s \vee s^{\prime}-\delta\right)^{+}} \mathbb{1}\left\{r>t^{\prime}-\vec{d}_{N}^{2}\right\}\left(t^{\prime}-r\right)^{-\alpha / 2} d r \vec{d}_{N}^{2 \xi \gamma}\left[\vec{d}_{N}^{2 \gamma\left(\gamma_{m}-1\right)}+a_{n}^{2 \beta \gamma}\right]\right\} \\
& =c\left(J_{1}+J_{2}\right) .
\end{aligned}
$$

Both integrals are bounded by integral length times the maximal integrand:

$$
\begin{aligned}
& J_{1} \leq\left|s^{\prime}-s\right|\left[\left(t^{\prime}-(\underline{s}-\delta)^{+}\right)^{\gamma\left(\gamma_{m}+\xi-1\right)-\alpha / 2}+a_{n}^{2 \beta \gamma}\left(t^{\prime}-(\underline{s}-\delta)^{+}\right)^{\gamma \xi-\alpha / 2}\right. \\
& J_{2} \leq\left|s^{\prime}-s\right|\left(t^{\prime}-(\bar{s}-\delta)\right)^{-\alpha / 2}\left[\vec{d}_{N}^{2 \gamma\left(\gamma_{m}-1\right)}+a_{n}^{2 \beta \gamma}\right] .
\end{aligned}
$$

Observe the following estimates

$$
\begin{aligned}
\left(t^{\prime}-(\bar{s}-\delta)^{+}\right) & \geq\left(t^{\prime}-\left(t^{\prime}-\delta\right)^{+}\right) \geq \delta, \\
\left(t^{\prime}-(\bar{s}-\delta)^{+}\right)^{-\alpha / 2} & \leq \delta^{-\alpha / 2}, \\
t^{\prime}-(\underline{s}-\delta)^{+} & \leq\left|t^{\prime}-t\right|+\left((t-s) \vee\left(t^{\prime}-s^{\prime}\right)\right)+\delta, \\
\left(t^{\prime}-(\underline{s}-\delta)^{+}\right)^{\gamma \gamma_{m}-\alpha / 2} & \leq 8 \delta^{-\alpha / 2}\left(\left|t^{\prime}-t\right| \vee(t-s) \vee\left(t^{\prime}-s^{\prime}\right) \vee \delta\right)^{\gamma \gamma_{m}} \\
\vec{d}_{N}^{2 \gamma(\xi-1)} & =\left(2^{-N} \vee d\right)^{-1 / R} \leq 2^{N \eta_{1}}, \\
\left|s^{\prime}-s\right| & =|\bar{s}-\underline{s}| \leq\left|t^{\prime}-\underline{s}\right| \leq\left|t^{\prime}-(\underline{s}-\delta)^{+}\right|, \\
\left(t^{\prime}-(\bar{s}-\delta)^{+}\right)^{\gamma(\xi-1)} & \leq\left(t^{\prime}-(\bar{s}-\delta)^{+}\right)^{-(2 R)^{-1}} \leq\left|s^{\prime}-s\right|^{-(2 R)^{-1}} \leq\left(\left|s^{\prime}-s\right| \wedge 1\right)^{-\eta_{1} / 2} .
\end{aligned}
$$

With these estimates one can easily obtain:

$$
\begin{aligned}
\check{Q}_{S, \delta, \eta_{0}}\left(s, s^{\prime}, t^{\prime}, x^{\prime}\right) \leq c_{1}(K, R)\left[a_{n}^{-2 \varepsilon_{0}}+2^{4 N[9.6 .9]}\left|s^{\prime}-s\right| \delta^{-\alpha / 2}\right. \\
\left\{\left|s^{\prime}-s\right|^{-\eta_{1} / 2}\left(\left|t^{\prime}-t\right| \vee(t-s) \vee\left(t^{\prime}-s^{\prime}\right) \vee \delta\right)^{\gamma \gamma_{m}}\right. \\
\left.\quad+a_{n}^{2 \beta \gamma}\left(\left|t^{\prime}-t\right| \vee(t-s) \vee\left(t^{\prime}-s^{\prime}\right) \vee \delta\right)^{\gamma}+2^{N \eta_{1}} \delta^{-\alpha / 2}\left[\vec{d}_{N}^{2 \gamma\left(\gamma_{m}-1\right)}+a_{n}^{2 \beta \gamma}\right]\right\}
\end{aligned}
$$

Notation: $\check{d}\left((s, t, x),\left(s^{\prime}, t^{\prime}, x^{\prime}\right)\right):=\left|s-s^{\prime}\right|^{1 / 2}+\left|t-t^{\prime}\right|^{1 / 6}+\left|x-x^{\prime}\right|^{1 / 3}$.

The definition of this metric seems rather strange. The reason for doing so is that 
we only need a result for $\left|t-t^{\prime}\right|+\left|x-x^{\prime}\right|$ very small (equal 0 , in fact). The reader may already have noticed that however, without the derivatives on the heat kernels, the local bounds get better in the exponent by +1 . Nevertheless, in Lemma 9.4 .7 there is a $\wedge 0$ which allows us not to be better than $d^{2} \sim\left|x-x^{\prime}\right|^{2}$. So the only way to get better there is to punish large distances in $d=\left|x-x^{\prime}\right|$. By setting $\check{d} \approx d^{1 / 3}$, we have in Lemma 9.6.7 (the analogue of Lemma 9.4.7)

$$
d^{2}=d^{1 / 3} d^{2 / 3} \leq d^{1 / 3} d^{\gamma \gamma_{m} / 3}=\check{d}\left(\check{d}^{\gamma \gamma_{m}}\right) .
$$

Remark 9.6.10. There is a subtle point why we did not prescribe $(j+f) 2^{-6 \ell}-$ $(i+e) 2^{-2 \ell} \leq 2^{-2 \ell}$ instead of $\leq 2^{-2 N}$. The reason is that $2^{-\ell} \approx \hat{d}$ is the asymptotics only for the first factor. The expression in brackets is governed by the $2^{-N}$ expression, which is determined by the $Z(N, n, K, \beta)$ expression, i.e. the distance $d\left((t, x),\left(\hat{t}_{0}, \hat{x}_{0}\right)\right)$.

Next, define

$$
\bar{\Delta}_{u_{1}}\left(m, n, \lambda, \varepsilon_{0}, 2^{-N}\right)=a_{n}^{-\varepsilon_{0}} a_{n}^{-\lambda \alpha / 4}\left[\left(a_{n}^{\lambda / 2} \vee 2^{-N}\right)^{\gamma \gamma_{m}}+a_{n}^{\beta \gamma}\left(a_{n}^{\lambda / 2} \vee 2^{-N}\right)^{\gamma}\right]
$$

with $\bar{\Delta}_{u_{1}}\left(\ldots, 2^{-N+1}\right) \leq 2 \bar{\Delta}_{u_{1}}\left(\ldots, 2^{-N}\right)$. Then, we put together the various estimates on the quadratic variations for $G_{\delta}$ :

Corollary 9.6.11. Let $0 \leq m \leq \bar{m}+1$ and assume that $\left(P_{m}\right)$ holds. For all $K \in \mathbb{N} \geq K_{1}, R>2, n \in \mathbb{N}, \beta \in[0,1 / 2], \varepsilon_{0} \in(0,1)$, there exist q9.6.11 $(K, R)$, $N \overline{9.6 .11}\left(m, n, R, \varepsilon_{0}, K, \beta\right)(\omega) \in \mathbb{N}$ almost surely such that

$\forall \eta_{1} \in(1 / R, 1 / 2), \eta_{0} \in\left(1 / R, \eta_{1} / 32\right) \delta \in\left[a_{n}, 1\right], N \in \mathbb{N},(t, x) \in \mathbb{R}_{+} \times \mathbb{R}^{q}$ and $\check{d}=\check{d}\left((s, t, x),\left(s^{\prime}, t^{\prime}, x^{\prime}\right)\right) \leq 2^{-N},|t-s| \vee\left|t^{\prime}-s^{\prime}\right| \leq 2^{-2 N}$ the following holds:

$$
\begin{aligned}
& \text { For } \omega \in\{(t, x) \in Z(N, n, K, \beta), N \geq N \overline{9.4 .10]}\} \\
& \check{Q}_{\delta}^{t o t}\left(s, t, x, s^{\prime}, t^{\prime}, x^{\prime}\right) \leq \overline{9.6 .11}\left(a_{n}^{-2 \varepsilon_{0}}+2^{4 \hat{9.6 .11})} \check{d}^{2-\frac{3}{2} \eta_{1}} \delta^{-\alpha / 2}\right. \\
& {\left[\left(\delta \vee 2^{-N}\right)^{\gamma \gamma_{m}}+a_{n}^{\beta \gamma}\left(\delta \vee 2^{-N}\right)^{\gamma}\right]^{2}} \\
& \forall 0 \leq s \leq t \leq t^{\prime}, x^{\prime} \in \mathbb{R}^{q} \text {. }
\end{aligned}
$$

Moreover, $N \overline{9.6 .11}$ is stochastically bounded uniformly in $(n, \beta)$.

Proof. The proof simply consists of putting together the last lemmas. Let $N \overline{9.6 .11}=$ $N N_{9.6 .5} \vee N=N_{\overline{9.6 .6}} \vee N \overline{9.6 .7} \vee N \overline{9.6 .8} \vee N \overline{9.6 .9}$, which is then clearly uniformly bounded in $(n, \beta)$ and

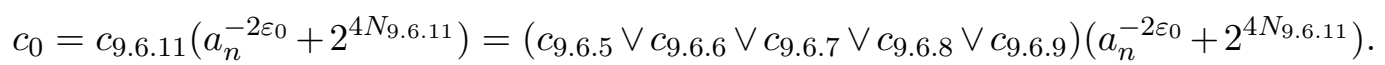


Then, we get for $\breve{d}=\check{d}\left((s, t, x),\left(s^{\prime}, t^{\prime}, x^{\prime}\right)\right) \leq 2^{-N}$ :

$$
\begin{aligned}
d=d\left((t, x),\left(t^{\prime}, x^{\prime}\right)\right) & \leq 2^{-3 N} \\
\bar{d}_{N} & =2^{-N} \\
\left|t-t^{\prime}\right| \vee(t-s) \vee\left(t^{\prime}-s^{\prime}\right) & \leq 2^{-6 N} \vee 2^{-2 N}=2^{-2 N} \\
\check{d}^{4} \leq 2^{-4 N} & \leq 2^{-2 N \gamma \gamma_{m}} \\
\left(\check{d}^{-\frac{3}{2} \eta_{1}}+2^{N \eta_{1}}\right) & \leq\left(\check{d}^{-\frac{3}{2} \eta_{1}}+\check{d}^{-\eta_{1}}\right) \leq 2 \check{d}^{-\frac{3}{2} \eta_{1}}
\end{aligned}
$$

and thus,

$$
\begin{aligned}
& \check{Q}_{\delta}^{\text {tot }}\left(s, t, x, s^{\prime}, t^{\prime}, x^{\prime}\right) \leq 3 c_{0}\left\{\left(\check{d}^{3} \wedge \sqrt{\delta}\right)^{2-\eta_{1} / 2} \delta^{-\alpha / 2}\left(\check{d}^{3} \wedge 1\right)^{2 \gamma}+\check{d}^{6-\frac{3}{2} \eta_{1}}\right. \\
& +\check{d}^{6-\frac{3}{2} \eta_{1}}+\check{d}^{6-\frac{3}{2} \eta_{1}} \delta^{-\alpha / 2}\left[2^{-2 N \gamma \gamma_{m}}+a_{n}^{2 \beta \gamma} 2^{-2 N \gamma}\right] \\
& +\left|s^{\prime}-s\right|^{1-\eta_{1} / 2} \delta^{-\alpha / 2}\left(\left|t^{\prime}-t\right| \vee(t-s) \vee\left(t^{\prime}-s^{\prime}\right) \vee \delta\right)^{\gamma \gamma_{m}} \\
& +a_{n}^{2 \beta \gamma}\left(\left|t^{\prime}-t\right| \vee(t-s) \vee\left(t^{\prime}-s^{\prime}\right) \vee \delta\right)^{\gamma} \\
& \left.+\left|s^{\prime}-s\right| 2^{N \eta_{1}} \delta^{-\alpha / 2}\left[\vec{d}_{N}^{2 \gamma\left(\gamma_{m}-1\right)}+a_{n}^{2 \beta \gamma}\right]\right\} \text {. } \\
& \leq 3 c_{0}\left\{\check{d}^{2} \delta^{-\alpha / 2}\left(2^{-2 N \gamma \gamma_{m}}+a_{n}^{2 \beta \gamma}\right)\left(\check{d}^{-\frac{3}{2} \eta_{1}}+2^{N \eta_{1}}\right)\right. \\
& +\breve{d}^{2-\frac{3}{2} \eta_{1}} \delta^{-\alpha / 2}\left[2 \breve{d}^{4}+\left(2^{-2 N} \vee \delta\right)^{\gamma \gamma_{m}}+a_{n}^{2 \beta \gamma}\left(2^{-2 N} \vee \delta\right)^{\gamma}\right] \\
& \leq 6 c_{0} \check{d}^{2-\frac{3}{2} \eta_{1}} \delta^{-\alpha / 2}\left(2^{-2 N \gamma \gamma_{m}}+a_{n}^{2 \beta \gamma}\right) \\
& +\check{d}^{2-\frac{3}{2} \eta_{1}} \delta^{-\alpha / 2}\left[2^{-4 N+1}+\left(2^{-2 N} \vee \delta\right)^{\gamma \gamma_{m}}+a_{n}^{2 \beta \gamma}\left(2^{-2 N} \vee \delta\right)^{\gamma}\right] \\
& \leq 9 c_{0} \check{d}^{2-\frac{3}{2} \eta_{1}} \delta^{-\alpha / 2}\left[\left(2^{-N} \vee \sqrt{\delta}\right)^{2 \gamma \gamma_{m}}+a_{n}^{2 \beta \gamma}\left(2^{-N} \vee \sqrt{\delta}\right)^{2 \gamma}\right] \text {. }
\end{aligned}
$$

Finally, we can do the proof of Proposition 9.4.16 just in the same way as Proposition 9.4.11.

Proof of Proposition 9.4.16; Let $R=33 \eta_{1}^{-1}, \eta_{0} \in\left(R^{-1}, \eta_{1} / 32\right)$ and consider the case $s \leq t$ in the beginning only. Set

$$
\check{d}=\sqrt{\left|s^{\prime}-s\right|}+\left|x-x^{\prime}\right|^{1 / 3}+\left|t-t^{\prime}\right|^{1 / 6} .
$$

By Corollary 9.6.11 for $(t, x) \in Z(N, n, K, \beta), N \geq N \overline{9.6 .11}$ it holds that:

$$
\begin{gathered}
\check{Q}_{a_{n}^{\lambda}}^{\text {tot }}\left(s, t, x, s^{\prime}, t^{\prime}, x^{\prime}\right)^{1 / 2} \leq \underset{9.6 .11}{\left(a_{n}^{-\varepsilon_{0}}+2^{2 N \overline{9.6 .11}}\right)} \check{d}^{\eta_{1} / 8} \breve{d}^{1-7 \eta_{1} / 8}\left[\bar{\Delta}_{u_{1}}\left(m, n, \lambda, \varepsilon_{0}, 2^{-N}\right)\right] \\
\forall s \leq t \leq t^{\prime}, s^{\prime} \leq t^{\prime} \leq T_{K},\left|x^{\prime}\right| \leq K+2,
\end{gathered}
$$


if $\check{d}\left((s, t, x),\left(s^{\prime}, t^{\prime}, x^{\prime}\right)\right) \leq 2^{-N}$ and $\left|t^{\prime}-s^{\prime}\right| \vee|t-s| \leq 2^{-2 N}$.

Choose $N_{3}=\frac{33}{\eta_{1}}\left[N\left[{ }_{9.6 .11}+N_{4}\left(K, \eta_{1}\right)\right]+c_{5}(q)\right.$, where $N_{4}$ is chosen in such a way that

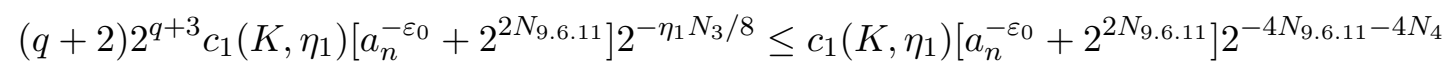

$$
\begin{aligned}
& \leq a_{n}^{-\varepsilon_{0}} 2^{-102}
\end{aligned}
$$

i.e. $N_{4}=N_{4}\left(a_{n}, \varepsilon_{0}, N_{\overline{9.6 .11}}, c_{1}\left(K, \eta_{1}\right)\right)$ and hence $N_{3}=N_{3}\left(n, \varepsilon_{0}, N_{\overline{9.6 .11},}, K, \eta_{1}\right)$. Set

$$
\left.\Delta\left(m, n, \bar{d}_{N}\right):=(q+2) 2^{q+3}\right)^{-1} a_{n}^{-\varepsilon_{0}} 2^{-100} \bar{\Delta}_{u_{1}}\left(m, n, \lambda, \varepsilon_{0}, 2^{-N}\right)
$$

and let $N^{\prime} \in \mathbb{N}$ such that $\check{d} \leq 2^{-N^{\prime}}$. Then it holds on

$$
\left\{\omega:(t, x) \in Z(N, n, K+1, \beta), N \geq N_{3}, N^{\prime} \geq N_{3}\right\}
$$

that

$$
\check{Q}_{a_{n}^{\lambda}}^{\text {tot }}\left(s, t, x, s^{\prime}, t^{\prime}, x^{\prime}\right)^{1 / 2} \leq \check{d}^{1-\left(7 \eta_{1} / 8\right)} 2^{-2} \Delta\left(m, n, \bar{d}_{N}\right) .
$$

Remembering the decomposition of $G_{\delta}$ in (9.114) into the sum of three martingales and applying the Dubins-Schwarz-Theorem (Theorem 3.2.8), we can write as long as $s \leq t \leq t^{\prime}, s^{\prime} \leq t^{\prime}$ and $\check{d} \leq 2^{-N}$ :

$$
\begin{aligned}
& \mathbb{P}\left[\left|G_{a_{n}^{\lambda}}(s, t, x)-G_{a_{n}^{\lambda}}\left(s^{\prime}, t^{\prime}, x^{\prime}\right)\right| \geq d\left((s, t, x)\left(s^{\prime}, t^{\prime}, x^{\prime}\right)\right)^{1-\eta_{1}} \Delta\left(m, n, \bar{d}_{N}\right)\right. \\
& \left.\quad(t, x) \in Z(N, n, K+1, \beta), N^{\prime} \wedge N \geq N_{3}, t^{\prime} \leq T_{K}\right] \\
& \quad \leq 3 \mathbb{P}\left[\sup _{u \leq \tilde{d}^{2-7 \eta_{1} / 4}\left(\Delta\left(m, n, \bar{d}_{N}\right) / 4\right)^{2}}|B(u)| \geq \tilde{d}^{1-\eta_{1}} \Delta\left(m, n, \bar{d}_{N}\right) / 3\right] \\
& \quad \leq 3 \mathbb{P}\left[\sup _{u \leq 1}|B(u)| \geq \tilde{d}^{-\eta_{1} / 8}\right] \\
& \quad \leq c \int_{\tilde{d}^{-\eta_{1} / 8}}^{\infty} \exp \left(-y^{2} / 2\right) d y \\
& \quad \leq c_{0} \exp \left(-\tilde{d}^{-\eta_{1} / 4} / 2\right),
\end{aligned}
$$

where we used the Reflection Principle in the second last inequality.

Next, apply Lemma 9.8.1, where we should make clear what the parameters are. We take

$$
\begin{aligned}
& q_{1}=q_{2}=1, q_{3}=q, r=3, E=\mathbb{R}_{+}^{2} \times \mathbb{R}^{q}, \\
& \bar{q}=q+2, v_{1}=v_{2}=2, v_{3}=1, v_{0}=1, \\
& \hat{n}=(m, n, \lambda, \beta), S=\mathbb{N}^{2} \times[0,1 / 2] \times(0,1), \\
& \Sigma(N, K, \hat{n})=Z(N, n, K, \beta), \Sigma^{\prime}(N)=\{0\} \times\left\{0 \leq t \leq T_{K}\right\} \times \mathbb{R}^{q}, \\
& s=1, \alpha_{1}=1, \Delta_{1}\left(\hat{n}, 2^{-N}\right)=\Delta\left(m, n, 2^{-N}\right), k_{1}=2, c\left(\alpha_{1}\right) \leq 4, \eta=\eta_{1}, \\
& Y_{\hat{n}}(y)=G_{a_{n}^{\lambda}}(s, t, x) \text { with } y=(s, t, x), N_{0}(\eta, K, \hat{n})=N_{3} .
\end{aligned}
$$


Note that the $N_{0}$ is uniformly bounded in $\hat{n}=(n, \lambda, \beta)$. By Lemma 9.8.1 we know there exists a $N \overline{9.4 .16}$ stochastically bounded uniformly in $(n, \beta, \lambda)$, for which we obtain for $s \leq t \leq t^{\prime}, s^{\prime} \leq t^{\prime},(t, x) \in Z(N, n, K, \beta), N \geq N \overline{\text { 9.4.16, }},|t-s| \vee\left|t^{\prime}-s^{\prime}\right| \leq$ $2^{-2 N}$ and $\check{d} \leq 2^{-N}$ that

$$
\begin{aligned}
\left|G_{a_{n}^{\lambda}}(s, t, x)-G_{a_{n}^{\lambda}}\left(s^{\prime}, t^{\prime}, x^{\prime}\right)\right| & \leq 2^{5}(q+2) \check{d}^{1-\eta_{1}} \Delta\left(m, n, \bar{d}_{N}\right) \\
& \leq 2^{-95} \breve{d}^{1-\eta_{1}} \bar{\Delta}_{u_{1}}\left(m, n, \lambda, \varepsilon_{0}, 2^{-N}\right) .
\end{aligned}
$$

\subsection{Proof of Proposition 9.4.18}

In this chapter we consider spatial and temporal distances of $u_{2, \delta}(t, x)$. We will always assume that $0 \leq t \leq t^{\prime}$.

$$
\begin{aligned}
\left|u_{2, \delta}\left(t^{\prime}, x^{\prime}\right)-u_{2, \delta}(t, x)\right| & \\
= & \left|\int_{t^{\prime}-\delta}^{t^{\prime}} \int_{\mathbb{R}^{q}} p_{t^{\prime}-s}\left(y-x^{\prime}\right) u(s, y) W(d s d y)-\int_{t-\delta}^{t} \int_{\mathbb{R}^{q}} p_{t-s}(y-x) u(s, y) W(d s d y)\right| \\
\leq & \left|\int_{(t-\delta)^{+}}^{t \wedge\left(t^{\prime}-\delta\right)} \int_{\mathbb{R}^{q}} p_{t-s}(y-x) u(s, y) W(d s d y)\right| \\
& +\left|\int_{\left(t^{\prime}-\delta\right)^{+} \vee}^{t^{\prime}} \int_{\mathbb{R}^{q}} p_{t^{\prime}-s}\left(y-x^{\prime}\right) u(s, y) W(d s d y)\right| \\
& +\mathbb{1}\left\{t^{\prime}-t<\delta\right\}\left|\int_{\left(t^{\prime}-\delta\right)^{+}}^{t} \int_{\mathbb{R}^{q}}\left(p_{t^{\prime}-s}\left(y-x^{\prime}\right)-p_{t-s}(y-x)\right) u(s, y) W(d s d y)\right| .
\end{aligned}
$$

Therefore, it seems reasonable to introduce the following square functions

$$
\begin{aligned}
& \hat{Q}_{T, 1, \delta}\left(t, t^{\prime}, x\right)=\int_{(t-\delta)^{+}}^{t \wedge\left(t^{\prime}-\delta\right)} \int_{\mathbb{R}^{q}} \int_{\mathbb{R}^{q}} p_{t-s}(w-x) p_{t-s}(z-x) \\
& R_{0}^{2 \gamma} e^{2 R_{1}(|w|+|z|)}|u(s, w)|^{\gamma}|u(s, z)|^{\gamma}\left(|w-z|^{-\alpha}+1\right) d w d z d s, \\
& \hat{Q}_{T, 2, \delta}\left(t, t^{\prime}, x^{\prime}\right)=\int_{\left(t^{\prime}-\delta\right)+\vee t}^{t^{\prime}} \int_{\mathbb{R}^{q}} \int_{\mathbb{R}^{q}} p_{t^{\prime}-s}\left(w-x^{\prime}\right) p_{t^{\prime}-s}\left(z-x^{\prime}\right) \\
& R_{0}^{2 \gamma} e^{2 R_{1}(|w|+|z|)}|u(s, w)|^{\gamma}|u(s, z)|^{\gamma}\left(|w-z|^{-\alpha}+1\right) d w d z d s, \\
& \hat{Q}_{X, \delta, \eta_{0}}^{i, j}\left(t, x, t^{\prime}, x^{\prime}\right)=\mathbb{1}\left\{t^{\prime}-t<\delta\right\} \int_{\left(t^{\prime}-\delta\right)^{+}}^{t} d s \int_{A_{i}(s, t)} d w \int_{A_{j}(s, t)} d z \\
& \left|\left(p_{t^{\prime}-s}\left(w-x^{\prime}\right)-p_{t-s}(w-x)\right)\left(p_{t^{\prime}-s}\left(z-x^{\prime}\right)-p_{t-s}(z-x)\right)\right| \\
& e^{2 R_{1}(|w|+|z|)} R_{0}^{2 \gamma}|u(s, w)|^{\gamma}|u(s, z)|^{\gamma}\left(|w-z|^{-\alpha}+1\right) d s,
\end{aligned}
$$


for $i, j=1,2$. Remember the definition of $A_{i}(t, \hat{t})$ in Chapter 9.4 .

Lemma 9.7.1. For all $N \in \mathbb{N} \geq K_{1}, R>2$, there is 9 9.7.1 $(K, R)$ and a $N_{9.7 .1}$ almost surely such that $\forall \eta_{0}, \eta_{1} \in(1 / R, \alpha / 4), \delta \in(0,1], N, n \in \mathbb{N}, \beta \in[0,1 / 2],(t, x) \in$ $\mathbb{R}_{+} \times \mathbb{R}^{q}$ and $i+j \geq 3$ it holds on the set

$$
\begin{aligned}
\{\omega:(t, x) & \in Z(N, n, K, \beta), N \geq N \overline{9.7 .1}\} \text { that: } \\
\hat{Q}_{X, \delta, \eta_{0}}^{i, j}\left(t, x, t, x^{\prime}\right) & \leq q 9.7 .1(K, R) 2^{N \sqrt{9.7 .1}(d \wedge \sqrt{\delta})^{2-\eta_{1}} \delta^{2-\alpha / 2}} \\
& \text { for all } 0 \leq t \leq t^{\prime} \leq K,\left|x^{\prime}\right| \leq K+1,
\end{aligned}
$$

where $d=d\left((t, x),\left(t^{\prime}, x^{\prime}\right)\right)$.

Proof. We just do the $i=2$ case. Compare the proof of Lemma 9.4.5.

Let $d=d\left((t, x),\left(t^{\prime}, x^{\prime}\right)\right), \xi=3 / 4$ and $N_{9.7 .1}=N_{3 / 4}(K)$ of Theorem 4.1 in [MPS06].

Without loss of generality $t^{\prime}-t<\delta$. With Lemma 9.4.3 and the help of Lemma 9.6.4, we get

$$
\begin{aligned}
\hat{Q}_{X, \delta, \eta_{0}}^{i, j}\left(t, x, t^{\prime}, x^{\prime}\right) \leq C_{9.4 .3} \int_{\left(t^{\prime}-\delta\right)^{+}}^{t} \int_{\mathbb{R}^{q}} \int_{\mathbb{R}^{q}} \mathbb{1}\left\{|w-x|>(t-s)^{1 / 2-\eta_{0}} \vee 2\left|x-x^{\prime}\right|\right\} \\
\quad\left(p_{t-s}(w-x)-p_{t-s}\left(w-x^{\prime}\right)\right)\left(p_{t-s}(z-x)-p_{t-s}\left(z-x^{\prime}\right)\right) \\
\quad e^{2 R_{1}(|w|+|z|)+|w-x|+|z-x|}\left(2^{-N} \vee \sqrt{t-s}+|w-x|\right)^{3 \gamma / 4} R_{0}^{2 \gamma} \\
\quad\left(2^{-N} \vee \sqrt{t-s}+|z-x|\right)^{3 \gamma / 4} \\
\quad\left(|w-z|^{-\alpha}+1\right) d w d z d s \\
\leq c_{1}(K, R) C_{[9.4 .3} \int_{\left(t^{\prime}-\delta\right)^{+}}^{t} d s(t-s)^{-\alpha / 2} \exp \left(-\frac{\eta_{1}}{64}\left(t^{\prime}-s\right)^{-2 \eta_{0}}\right)\left[1 \wedge \frac{d^{2}}{t-s}\right]^{1-\eta_{1} / 4} \\
\leq c_{1}(K, R) C_{9.4 .3} \int_{\left(t^{\prime}-\delta\right)^{+}}^{t} d s(t-s)^{-\alpha / 2} \exp \left(-\frac{\eta_{1}}{128}(t-s)^{-2 \eta_{0}}\right)\left[1 \wedge \frac{d^{2}}{t-s}\right]^{1-\eta_{1} / 4} \\
+c_{1}(K, R) C_{9.4 .3} \int_{\left(t^{\prime}-\delta\right)^{+}}^{t} d s(t-s)^{-\alpha / 2} \exp \left(-\frac{\eta_{1}}{128}\left(t^{\prime}-t\right)^{-2 \eta_{0}}\right)\left[1 \wedge \frac{d^{2}}{t-s}\right]^{1-\eta_{1} / 4} \\
\leq c_{2}(K, R) C_{[9.4 .3} \int_{\left(t^{\prime}-\delta\right)^{+}}^{t} d s(t-s)^{2-\alpha / 2}\left[1 \wedge \frac{d^{2}}{t-s}\right]^{1-\eta_{1} / 4} \\
+c_{2}(K, R) C_{9.4 .3]} t^{\prime}-\left.t\right|^{2} \int_{\left(t^{\prime}-\delta\right)^{+}}^{t} d s(t-s)^{-\alpha / 2}\left[1 \wedge \frac{d^{2}}{t-s}\right]^{1-\eta_{1} / 4} .
\end{aligned}
$$

Next, use Lemma 4.1 (a) and (b) of [MP11] and the facts that $\left|t^{\prime}-t\right| \leq \delta$ and 
$2 \alpha-\eta_{1} \geq 0$ to obtain:

$$
\begin{aligned}
\hat{Q}_{X, \delta, \eta_{0}}^{i, j}\left(t, x, t, x^{\prime}\right) \leq & c_{3}(K, R) C_{9.4 .3}\left(d^{2} \wedge \delta\right)^{1-\eta_{1} / 4} \delta^{2-\alpha / 2+\eta_{1} / 4} \\
& +c_{3}(K, R) C_{9.4 .3} d^{2}\left(d^{2} \wedge \delta\right)^{1-\alpha / 2} \\
\leq & c_{4}(K, R) C_{9.4 .3}\left(d^{2} \wedge \delta\right)^{1-\eta_{1} / 2} \delta^{2-\alpha / 2}
\end{aligned}
$$

Let

$$
R_{\overline{9.137}}(\gamma):=\frac{4}{1-\gamma}
$$

Lemma 9.7.2. Let $0 \leq m \leq \bar{m}+1$ and assume that $\left(P_{m}\right)$ holds. For any $K \in$ $\mathbb{N} \geq K_{1}, R>R_{9.137}, n \in \mathbb{N}, \varepsilon_{0} \in(0,1)$ and $\beta \in[0,1 / 2]$ there is a q9.7.2 and a $N_{9.7 .2} \in$ $\mathbb{N}$ almost surely such that for any $\eta_{1} \in(1 / R, 1 / 2(2-\alpha)), \eta_{0} \in\left(0,(1 \wedge(2-\alpha)) \eta_{1} / 32\right)$, $\delta \in\left[a_{n}, 1\right], N \in \mathbb{N}$ and $(t, x) \in \mathbb{R}_{+} \times \mathbb{R}^{q}$, on

$$
\begin{aligned}
& \{\omega:(t, x) \in Z(N, n, K, \beta), N \geq N \overline{9.7 .2}\} \text { that: } \\
& \hat{Q}_{X, \delta, \eta_{0}}^{1,1}\left(t, x, t^{\prime}, x^{\prime}\right) \leq \\
& \text { q9.7.2 }(d \wedge \sqrt{\delta})^{(2-\alpha)\left(1-\eta_{1} / 2\right)}\left(2^{-N} \vee d\right)^{2 \gamma}\left[\left(\sqrt{a_{n}} \vee 2^{-N} \vee d\right)^{2 \gamma\left(\gamma_{m}-1\right)}+a_{n}^{2 \beta \gamma}\right] \\
& +\left(d^{2} \wedge \delta\right)^{1-\eta_{1} / 2} \delta^{-\alpha / 2+\gamma}\left(\delta^{\gamma\left(\gamma_{m}-1\right)}+a_{n}^{2 \beta \gamma}\right)\left[\left(\gamma^{\prime} \xi-\alpha / 2\right)^{-1}+\right] \\
& \text { for } 0 \leq t \leq t^{\prime} \leq K+1,\left|x^{\prime}\right| \leq K+1 \text {, }
\end{aligned}
$$

where $d=d\left((t, x),\left(t^{\prime}, x^{\prime}\right)\right)$. Moreover, $N$ 9.7.2 is stochastically bounded uniformly in $n, \lambda, \beta$.

Proof. Let $\xi=\left(1-\frac{2-\alpha}{16 R \gamma}\right) \vee \frac{2 \alpha}{2+\alpha}\left(1-\frac{2-\alpha}{31}\right)^{-1} \in(3 / 4,1)$. Set $\gamma^{\prime}=\gamma\left(1-2 \eta_{0}\right)$.

By Lemma 9.4 .3 and Lemma 9.6.1

$$
\begin{aligned}
\hat{Q}_{X, \delta, \eta_{0}}^{1,1}\left(t, x, t, x^{\prime}\right) \leq & C_{9.4 .3} c_{1}(K) \mathbb{1}_{\left\{t^{\prime}-t \leq \delta\right\}} \int_{\left(t^{\prime}-\delta\right)^{+}}^{t} d s(t-s)^{-\alpha / 2}\left[1 \wedge \frac{d^{2}}{t-s}\right] \\
& \left(2^{-N} \vee d^{1-2 \eta_{0}}+(t-s)^{1 / 2-\eta_{0}}\right)^{2 \gamma \xi} \\
& {\left[\left(\left(\sqrt{a_{n}} \vee 2^{-N} \vee d^{1-\eta_{0}}\right)+(t-s)^{1 / 2-\eta_{0}}\right)^{2 \gamma\left(\gamma_{m}-1\right)}+a_{n}^{2 \beta \gamma}\right] }
\end{aligned}
$$

Now, there are three cases to consider

$$
\begin{array}{r}
(t-s)^{1 / 2-\eta_{0}}<2^{-N} \vee d^{1-2 \eta_{0}},(t-s)^{1 / 2-\eta_{0}} \geq \sqrt{a_{n}} \vee 2^{-N} \vee d^{1-2 \eta_{0}} \\
\text { and } 2^{-N} \vee d^{1-2 \eta_{0}} \leq(t-s)^{1 / 2-\eta_{0}}<\sqrt{a_{n}} \vee 2^{-N} \vee d^{1-2 \eta_{0}}
\end{array}
$$


Note that since $\eta_{0}<\eta_{1} / 32<(2-\alpha) / 64$ and the definition of $\xi$ we obtain for a certain $c(\alpha)>0$ :

$$
\begin{aligned}
2 \gamma\left(1-2 \eta_{0}\right) \xi & >\left(1+\frac{\alpha}{2}\right)\left(1-\frac{2-\alpha}{32}\right) \frac{2 \alpha}{2+\alpha}\left(1-\frac{2-\alpha}{31}\right)^{-1} \\
& =\alpha+c(\alpha)>\alpha .
\end{aligned}
$$

This tells us that always $\gamma^{\prime} \xi-\alpha / 2>c(\alpha)>0$ and additionally, $\gamma_{m}-1 \geq 0$ allows us to use (4.2) and (4.1) (twice) of [MP11]:

$$
\begin{aligned}
& \hat{Q}_{X, \delta, \eta_{0}}^{1,1}(t, x,\left.t, x^{\prime}\right) \leq C_{9.4 .3} c_{2}\left(\alpha, K, R_{1}\right) \mathbb{1}_{\left\{t^{\prime}-t \leq \delta\right\}}\left\{\left(2^{-N} \vee d^{1-2 \eta_{0}}\right)^{2 \gamma \xi}\right. \\
&\left(\left(\sqrt{a_{n}} \vee 2^{-N} \vee d^{1-2 \eta_{0}}\right)^{2 \gamma\left(\gamma_{m}-1\right)}+a_{n}^{2 \beta \gamma}\right) \int_{\left(t^{\prime}-\delta\right)^{+}}^{t} d s(t-s)^{-\alpha / 2}\left[1 \wedge \frac{d^{2}}{t-s}\right] \\
& \quad+\int_{\left(t^{\prime}-\delta\right)^{+}}^{t} d s\left((t-s)^{-\alpha / 2+\gamma^{\prime}\left(\gamma_{m}-1+\xi\right)}+(t-s)^{-\alpha / 2+\gamma^{\prime} \xi} a_{n}^{2 \beta \gamma}\right)\left[1 \wedge \frac{d^{2}}{t-s}\right] \\
&\left.\quad+\left[a_{n}^{\gamma\left(\gamma_{m}-1\right)}+a_{n}^{2 \beta \gamma}\right] \int_{\left(t^{\prime}-\delta\right)^{+}}^{t} d s(t-s)^{-\alpha / 2+\gamma^{\prime} \xi}\left[1 \wedge \frac{d^{2}}{t-s}\right]\right\} \\
& \leq C_{9.4 .3} c_{2}\left(\alpha, K, R_{1}\right) \mathbb{1}_{\left\{t^{\prime}-t \leq \delta\right\}} \\
&\left\{\left(2^{-N} \vee d\right)^{2 \gamma^{\prime} \xi}\left(\left(\sqrt{a_{n}} \vee 2^{-N} \vee d\right)^{2 \gamma^{\prime}\left(\gamma_{m}-1\right)}+a_{n}^{2 \beta \gamma}\right)\left(d^{2} \wedge\left(t-t^{\prime}+\delta\right)\right)^{1-\alpha / 2}\right. \\
& \quad+2 c(\alpha)^{-1}\left(d^{2} \wedge \delta\right) \delta^{-\alpha / 2+\gamma^{\prime}\left(\gamma_{m}+\xi-1\right)}+2 c(\alpha)^{-1}\left(d^{2} \wedge \delta\right) \delta^{-\alpha / 2+\gamma^{\prime} \xi} a_{n}^{2 \beta \gamma} \\
&\left.\quad+2 c(\alpha)^{-1}\left(d^{2} \wedge \delta\right) \delta^{-\alpha / 2+\gamma^{\prime} \xi}\left(a_{n}^{\gamma\left(\gamma_{m}-1\right)}+a_{n}^{2 \beta \gamma}\right)\right\} .
\end{aligned}
$$

As $1>\delta>a_{n}$, we see that the last summand is bounded by the ones just before. Additionally,

$$
\begin{aligned}
& \left(d^{2} \wedge \delta\right)^{1-\alpha / 2} \\
= & (d \wedge \sqrt{\delta})^{(2-\alpha)\left(1-\eta_{1} / 2\right)}(d \wedge \sqrt{\delta})^{(2-\alpha) \eta_{1} / 4}(d \wedge \sqrt{\delta})^{(2-\alpha) \eta_{1} / 4} \\
\leq & (d \wedge \sqrt{\delta})^{(2-\alpha)\left(1-\eta_{1} / 2\right)}\left(d \vee 2^{-N}\right)^{(2-\alpha) \eta_{1} / 4}\left(\sqrt{a_{n}} \vee 2^{-N} \vee d\right)^{(2-\alpha) \eta_{1} / 4},
\end{aligned}
$$

and

$$
\begin{aligned}
\left(d^{2} \wedge \delta\right) \delta^{-\alpha / 2+\gamma^{\prime}\left(\gamma_{m}+\xi-1\right)} & \leq\left(d^{2} \wedge \delta\right)^{1-\eta_{1} / 2} \delta^{-\alpha / 2+\gamma^{\prime}\left(\gamma_{m}+\xi-1\right)+\eta_{1} / 2} \text { and } \\
\left(d^{2} \wedge \delta\right) \delta^{-\alpha / 2+\gamma^{\prime} \xi} & \leq\left(d^{2} \wedge \delta\right)^{1-\eta_{1} / 2} \delta^{-\alpha / 2+\gamma^{\prime} \xi+\eta_{1} / 2} .
\end{aligned}
$$

Therefore,

$$
\begin{aligned}
& \hat{Q}_{X, \delta, \eta_{0}}^{1,1}\left(t, x, t, x^{\prime}\right) \leq \\
& C_{9.4 .3} c_{3}(K)\left\{(d \wedge \sqrt{\delta})^{(2-\alpha)\left(1-\eta_{1} / 2\right)}\left(2^{-N} \vee d\right)^{2 \gamma^{\prime} \xi+(2-\alpha) \eta_{1} / 4}\right. \\
& {\left[\left(\sqrt{a_{n}} \vee 2^{-N} \vee d\right)^{2 \gamma^{\prime}\left(\gamma_{m}-1\right)+(2-\alpha) \eta_{1} / 4}+a_{n}^{2 \beta \gamma}\right]} \\
& \left.+\left(d^{2} \wedge \delta\right)^{1-\eta_{1} / 2} \delta^{-\alpha / 2+\gamma^{\prime} \xi+\eta_{1} / 2}\left(\delta^{\gamma^{\prime}\left(\gamma_{m}-1\right)}+a_{n}^{2 \beta \gamma}\right)\right\}
\end{aligned}
$$


Now, we have to do some estimates to get rid of $\eta_{0}$ : Since $\xi>1-(2-\alpha)(16 R \gamma)^{-1}>$ $1-(2-\alpha) \eta_{1}(16 \gamma)^{-1}$ and $32 \gamma \xi \eta_{0}<\eta_{1}(2-\alpha)$ we have

$$
\begin{aligned}
\gamma \xi>-\frac{1}{16}(2-\alpha) \eta_{1}+\gamma & >-\frac{1}{8}(2-\alpha) \eta_{1}+2 \gamma \xi \eta_{0}+\gamma, \text { thus } \\
2 \gamma \xi\left(1-2 \eta_{0}\right)+\frac{\eta_{1}}{4}(2-\alpha) & >2 \gamma .
\end{aligned}
$$

Secondly,

$$
\begin{gathered}
\left(1-2 \eta_{0}\right) 2 \gamma\left(\gamma_{m}-1\right)+\eta_{1} / 4 \stackrel{!}{\geq} 2 \gamma\left(\gamma_{m}-1\right), \text { since } \\
2 \gamma\left(\gamma_{m}-1\right) 2 \eta_{0} \leq 2 \cdot 1 \cdot 1 \cdot 2 \eta_{0} \leq \eta_{1} / 4 .
\end{gathered}
$$

Thirdly, by $\xi>1-\eta_{1}(2-\alpha)(16 \gamma)^{-1}>1-\eta_{1}(8 \gamma)^{-1}$ we have

$$
\begin{aligned}
-2 \eta_{1} / 8+\eta_{1} / 2+\gamma(\xi-1) & \geq 0, \text { therefore } \\
-4 \eta_{0}+\eta_{1} / 2+\left(1-\eta_{1} / 16\right) \gamma(\xi-1) & \geq 0, \text { and hence } \\
-4 \eta_{0}+\eta_{1} / 2+\left(1-2 \eta_{0}\right) \gamma(\xi-1)+\gamma \gamma_{m} & \geq \gamma \gamma_{m}, \text { and hence } \\
\left(1-2 \eta_{0}\right) \gamma\left(\gamma_{m}-1+\xi\right)+\eta_{1} / 2 & \geq \gamma \gamma_{m}
\end{aligned}
$$

and similarly as the first inequality

$$
\left(1-2 \eta_{0}\right) \gamma \xi+\eta_{1} / 2 \geq \gamma
$$

Use these estimates to complete the proof:

$$
\begin{aligned}
& \hat{Q}_{X, \delta, \eta_{0}}^{1,1}\left(t, x, t, x^{\prime}\right) \leq \overline{q 9.7 .2}((\wedge \sqrt{\delta})^{(2-\alpha)\left(1-\eta_{1} / 2\right)}\left(2^{-N} \vee d\right)^{2 \gamma} \\
& {\left[\left(\sqrt{a_{n}} \vee 2^{-N} \vee d\right)^{2 \gamma\left(\gamma_{m}-1\right)}+a_{n}^{2 \beta \gamma}\left(\sqrt{a_{n}} \vee 2^{-N} \vee d\right)^{\eta_{1} / 2}\right] } \\
&+\left(d^{2} \wedge \delta\right)^{1-\eta_{1} / 2} \delta^{-\alpha / 2+\gamma}\left(\delta^{\gamma\left(\gamma_{m}-1\right)}+a_{n}^{2 \beta \gamma}\right) .
\end{aligned}
$$

Lemma 9.7.3. Let $0 \leq m \leq \bar{m}+1$ and assume that $\left(P_{m}\right)$ holds. For any $K \in$ $\mathbb{N} \geq K_{1}, R>2, n \in \mathbb{N}, \varepsilon_{0} \in(0,1)$ and $\beta \in[0,1 / 2]$ there is a 99.7 .3 and a $N \overline{9.7 .3} \in \mathbb{N}$ almost surely such that for any $\eta_{1} \in(1 / R, 1 / 2), \delta \in\left[a_{n}, 1\right], N \in \mathbb{N}$ and $(t, x) \in$ $\mathbb{R}_{+} \times \mathbb{R}^{q}$, on

$$
\begin{gathered}
\{\omega:(t, x) \in Z(N, n, K, \beta), N \geq N[\overline{9.7 .3}\} \text { that: } \\
\hat{Q}_{T, 1, \delta}\left(t, t^{\prime}, x\right) \leq C_{9.4 .3}(\omega) q_{9.7 .3}\left(\vec{d}_{N}^{2 \gamma}\left(a_{n}^{2 \beta \gamma}+\vec{d}_{n, N}^{2 \gamma\left(\gamma_{m}-1\right)}\right)\left(\left|t^{\prime}-t\right| \wedge \delta\right)^{(1-\alpha / 2)\left(1-\eta_{1} / 2\right)}\right. \\
\left.+\left(\left|t^{\prime}-t\right| \wedge \delta\right)^{1-\eta_{1} / 2}\left(a_{n}^{2 \beta \gamma} \delta^{\gamma-\alpha / 2}+\delta^{\gamma \gamma_{m}-\alpha / 2}\right)\right) .
\end{gathered}
$$

and

$$
\hat{Q}_{T, 2, \delta}\left(t, t^{\prime}, x^{\prime}\right) \leq C_{\overline{9.4 .3}}(\omega) q_{9.7 .3}\left(\sqrt{t^{\prime}-t} \wedge \sqrt{\delta}\right)^{(2-\alpha)\left(1-\eta_{1} / 2\right)} \vec{d}_{N}^{2 \gamma}\left(a_{n}^{2 \beta \gamma}+\vec{d}_{n, N}^{2 \gamma\left(\gamma_{m}-1\right)}\right),
$$

where $\bar{d}_{N}=2^{-N} \vee d$ and $\bar{d}_{n, N}=\sqrt{a_{n}} \vee \bar{d}_{N}$. 


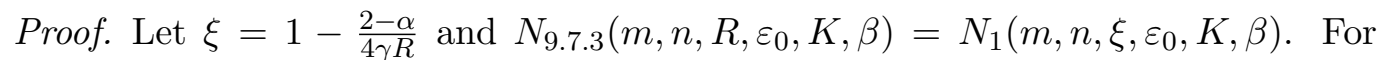
$\omega, t, t^{\prime}, x, x^{\prime}$ as in 9.141, by Lemma 9.4 .3 we get

$$
\begin{aligned}
\hat{Q}_{T, 2, \delta}\left(t, t^{\prime}, x^{\prime}\right) \leq & C_{9.4 .3}(\omega) \int_{t^{\prime}-\left(\delta \wedge\left(t^{\prime}-t\right)\right)}^{t^{\prime}} d s \int_{\mathbb{R}^{q}} \int_{\mathbb{R}^{q}} p_{t^{\prime}-s}\left(w-x^{\prime}\right) p_{t^{\prime}-s}\left(z-x^{\prime}\right) \\
& e^{2 R_{1} K+\left(R_{1}+1\right)\left(\left|w-x^{\prime}\right|+\left|z-x^{\prime}\right|\right)+2\left(R_{1}+1\right)(2 K+1)} R_{0}^{2 \gamma} \\
& \left(2^{-N} \vee\left|x-x^{\prime}\right|+\sqrt{t^{\prime}-s}+\left|w-x^{\prime}\right|\right)^{\gamma \xi} \\
& \left(2^{-N} \vee\left|x-x^{\prime}\right|+\sqrt{t^{\prime}-s}+\left|z-x^{\prime}\right|\right)^{\gamma \xi} \\
& {\left[\left(\sqrt{a_{n}} \vee 2^{-N} \vee\left|x-x^{\prime}\right|+\sqrt{t^{\prime}-s}+\left|w-x^{\prime}\right|\right)^{\gamma\left(\gamma_{m}-1\right)}+a_{n}^{\beta \gamma}\right] } \\
& {\left[\left(\sqrt{a_{n}} \vee 2^{-N} \vee\left|x-x^{\prime}\right|+\sqrt{t^{\prime}-s}+\left|z-x^{\prime}\right|\right)^{\gamma\left(\gamma_{m}-1\right)}+a_{n}^{\beta \gamma}\right] } \\
& \left(|w-z|^{-\alpha}+1\right) d w d z .
\end{aligned}
$$

Next, use the inequality $(a+b)^{p} \leq c(p)\left(a^{p}+b^{p}\right)$ for $a, b, p \geq 0$ and apply Lemma 5.1 of [MPS06 and our Lemma 9.3.1.

$$
\begin{aligned}
\hat{Q}_{T, 2, \delta}\left(t, t^{\prime}, x^{\prime}\right) \leq & C_{[9.4 .3}(\omega) c_{1}(K) \int_{t^{\prime}-\left(\delta \wedge\left(t^{\prime}-t\right)\right)}^{t^{\prime}} d s\left(t^{\prime}-s\right)^{-\alpha / 2} \\
& \left(\left(2^{-N} \vee\left|x-x^{\prime}\right|\right)^{2 \gamma \xi}+4\left(t^{\prime}-s\right)^{\gamma \xi}\right) \\
& \left\{a_{n}^{2 \beta \gamma}+2 a_{n}^{\beta \gamma}\left[\left(\sqrt{a_{n}} \vee 2^{-N} \vee\left|x-x^{\prime}\right|\right)^{\gamma\left(\gamma_{m}-1\right)}+2\left(t^{\prime}-s\right)^{\gamma / 2\left(\gamma_{m}-1\right)}\right]\right. \\
& \left.\quad+\left(\sqrt{a_{n}} \vee 2^{-N} \vee\left|x-x^{\prime}\right|\right)^{2 \gamma\left(\gamma_{m}-1\right)}+4\left(t^{\prime}-s\right)^{\gamma\left(\gamma_{m}-1\right)}\right\} .
\end{aligned}
$$

Now, use that for $y, y^{\prime}, z \in \mathbb{R}_{+}$:

$$
z^{2}+2 z\left(y+y^{\prime}\right)+y^{2}+y^{\prime 2} \leq 3\left(z^{2}+\left(y+y^{\prime}\right)^{2}\right)
$$

and the fact that $t^{\prime}-s \leq t^{\prime}-t$ under the integral to obtain

$$
\begin{aligned}
\hat{Q}_{T, 2, \delta} & \left(t, t^{\prime}, x^{\prime}\right) \leq 192 C_{9.4 .3}(\omega) c_{1} \vec{d}_{N}^{2 \gamma \xi}\left(a_{n}^{2 \beta \gamma}+\vec{d}_{n, N}^{2 \gamma\left(\gamma_{m}-1\right)}\right) \int_{t^{\prime}-\left(\delta \wedge\left(t^{\prime}-t\right)\right)}^{t^{\prime}} d s\left(t^{\prime}-s\right)^{-\alpha / 2} \\
& \leq C_{9.4 .3}(\omega) c_{2}(K, \alpha) \vec{d}_{N}^{2 \gamma \xi}\left(a_{n}^{2 \beta \gamma}+\vec{d}_{n, N}^{2 \gamma\left(\gamma_{m}-1\right)}\right)\left(\delta \wedge\left|t^{\prime}-t\right|\right)^{1-\alpha / 2} \\
& \leq C_{9.4 .3}(\omega) c_{2} \vec{d}_{N}^{2 \gamma \xi+(2-\alpha) \eta_{1} / 2}\left(a_{n}^{2 \beta \gamma}+\vec{d}_{n, N}^{2 \gamma\left(\gamma_{m}-1\right)}\right)\left(\sqrt{\delta} \wedge \sqrt{t^{\prime}-t}\right)^{(2-\alpha)\left(1-\eta_{1} / 2\right)} \\
& \leq C_{9.4 .3}(\omega) c_{2}\left(\sqrt{\delta} \wedge \sqrt{t^{\prime}-t}\right)^{(2-\alpha)\left(1-\eta_{1} / 2\right)} \vec{d}_{N}^{2 \gamma}\left(a_{n}^{2 \beta \gamma}+\vec{d}_{n, N}^{2 \gamma\left(\gamma_{m}-1\right)}\right),
\end{aligned}
$$

where the last inequality is true since

$$
(2-\alpha) \frac{\eta_{1}}{2}+2 \gamma \xi=2 \gamma+2 \gamma(\xi-1)+(2-\alpha) \frac{\eta_{1}}{2} \geq 2 \gamma,
$$

by the choice of $\xi$ and $\eta_{1}>R^{-1}$.

Next we have to deal with $\hat{Q}_{T, 1, \delta}$. Assume that $\omega, t, t^{\prime}, x, x^{\prime}$ are as in 9.141) and 
w.l.o.g it is $t^{\prime}>\delta$, since otherwise there is nothing to show. Then, we can again use Lemma 9.4 .3 and do the same steps as above

$$
\begin{aligned}
\hat{Q}_{T, 1, \delta}\left(t, t^{\prime}, x\right) \leq & C_{9.4 .3}(\omega) \int_{(t-\delta)^{+}}^{t \wedge\left(t^{\prime}-\delta\right)} d s \int_{\mathbb{R}^{q}} \int_{\mathbb{R}^{q}} p_{t-s}(w-x) p_{t-s}(z-x) \\
& e^{2 R_{1} K+\left(R_{1}+1\right)(|w-x|+|z-x|)+4 K\left(R_{1}+1\right)} R_{0}^{2 \gamma} \\
& \left(2^{-N}+\sqrt{t-s}+|w-x|\right)^{\gamma \xi}\left(2^{-N}+\sqrt{t-s}+|z-x|\right)^{\gamma \xi} \\
& {\left[\left(\sqrt{a_{n}} \vee 2^{-N}+\sqrt{t-s}+|w-x|\right)^{\gamma\left(\gamma_{m}-1\right)}+a_{n}^{\beta \gamma}\right] } \\
& {\left[\left(\sqrt{a_{n}} \vee 2^{-N}+\sqrt{t-s}+|z-x|\right)^{\gamma\left(\gamma_{m}-1\right)}+a_{n}^{\beta \gamma}\right] } \\
& \left(|w-z|^{-\alpha}+1\right) d w d z \\
\leq & C \overline{9.4 .3}(\omega) c_{3} \int_{(t-\delta)^{+}}^{t \wedge\left(t^{\prime}-\delta\right)} d s(t-s)^{-\alpha / 2}\left(2^{-N}\right)^{2 \gamma \xi}+4(t-s)^{\gamma \xi} \\
& {\left[a_{n}^{2 \beta \gamma}+a_{n}^{\beta \gamma} 2\left(\left(\sqrt{a_{n}} \vee 2^{-N}\right)^{\gamma\left(\gamma_{m}-1\right)}+2(t-s)^{\gamma / 2\left(\gamma_{m}-1\right)}\right)\right.} \\
& \left.+\left(\sqrt{a_{n}} \vee 2^{-N}\right)^{2 \gamma\left(\gamma_{m}-1\right)}+4(t-s)^{\gamma\left(\gamma_{m}-1\right)}\right] .
\end{aligned}
$$

This leads to the following bound

$$
\begin{aligned}
\hat{Q}_{T, 1, \delta}\left(t, t^{\prime}, x\right) \leq & G_{9.4 .3}(\omega) c_{4} \int_{(t-\delta)^{+}}^{t \wedge\left(t^{\prime}-\delta\right)} d s(t-s)^{-\alpha / 2} \\
& \left(2^{-2 N \gamma \xi}+(t-s)^{\gamma \xi}\right)\left(a_{n}^{2 \beta \gamma}+\left(\sqrt{a_{n}} \vee 2^{-N}\right)^{2 \gamma\left(\gamma_{m}-1\right)}+(t-s)^{\gamma\left(\gamma_{m}-1\right)}\right) .
\end{aligned}
$$

Note that $-\alpha / 2+\gamma \xi>0$ by the choice of $\xi$ and the restriction (9.137) on $R$. Now, we split up in three cases

$$
\begin{aligned}
& \text { (i) } \sqrt{t-s} \leq 2^{-N},(\text { ii }) \sqrt{t-s} \geq \sqrt{a_{n}} \vee 2^{-N} \\
& \text { (iii) } 2^{-N} \leq \sqrt{t-s} \leq \sqrt{a_{n}} \vee 2^{-N}
\end{aligned}
$$

Therefore, we have to deal with different integrals for which we use either of the following estimates:

$$
\begin{gathered}
\int_{(t-\delta)^{+}}^{t \wedge\left(t^{\prime}-\delta\right)}(t-s)^{-\alpha / 2} d s=c(\alpha)\left(\delta \wedge\left|t^{\prime}-t\right|\right)^{1-\alpha / 2} \\
\int_{(t-\delta)^{+}}^{t \wedge\left(t^{\prime}-\delta\right)}(t-s)^{p} d s \leq \delta^{p}\left(\delta \wedge\left|t^{\prime}-t\right|\right) \quad(p \geq 0) .
\end{gathered}
$$

Then, we get

$$
\begin{gathered}
\hat{Q}_{T, 1, \delta}\left(t, t^{\prime}, x\right) \leq C_{9.4 .3}(\omega) c_{5}(K)\left\{2^{-2 N \gamma \xi}\left(a_{n}^{2 \beta \gamma}+\left(\sqrt{a_{n}} \vee 2^{-N}\right)^{2 \gamma\left(\gamma_{m}-1\right)}\right)\left(\delta \wedge\left|t^{\prime}-t\right|\right)^{1-\alpha / 2}\right. \\
+a_{n}^{2 \beta \gamma} \delta^{\gamma \xi-\alpha / 2}\left(\left|t^{\prime}-t\right| \wedge \delta\right)+\delta^{\gamma\left(\gamma_{m}+\xi-1\right)-\alpha / 2}\left(\left|t^{\prime}-t\right| \wedge \delta\right) \\
\left(a_{n}^{2 \beta \gamma}+a_{n}^{\gamma\left(\gamma_{m}-1\right)} \delta^{\gamma \xi-\alpha / 2}\left(\left|t^{\prime}-t\right| \wedge \delta\right)\right\} .
\end{gathered}
$$


Since $1>\delta>a_{n}$, the last line is bounded by the two lines just before. Using the definitions of $\bar{d}_{N}$ and $\bar{d}_{n, N}$ gives

$$
\begin{aligned}
& \hat{Q}_{T, 1, \delta}\left(t, t^{\prime}, x\right) \leq C_{9.4 .3}(\omega) c_{6}(K)\left(\vec{d}_{N}^{2 \gamma \xi+(2-\alpha) \eta_{1} / 2}\left(a_{n}^{2 \beta \gamma}+\vec{d}_{n, N}^{2 \gamma\left(\gamma_{m}-1\right)}\right)\right. \\
&\left(\delta \wedge\left|t^{\prime}-t\right|\right)^{(1-\alpha / 2)\left(1-\eta_{1} / 2\right)} \\
&\left.+\left(\left|t^{\prime}-t\right| \wedge \delta\right)^{1-\eta_{1} / 2}\left(a_{n}^{2 \beta \gamma} \delta^{\gamma \xi-\alpha / 2+\eta_{1} / 2}+\delta^{\gamma\left(\gamma_{m}+\xi-1\right)-\alpha / 2+\eta_{1} / 2}\right)\right) .
\end{aligned}
$$

Using the definition of $\xi$ and $\alpha>0$ gives

$$
2 \gamma(\xi-1)+(2-\alpha) \frac{\eta_{1}}{2}>0
$$

and

$$
\gamma(\xi-1)+(2-\alpha) \frac{\eta_{1}}{2}>0
$$

This gives the estimate

$$
\begin{gathered}
\hat{Q}_{T, 1, \delta}\left(t, t^{\prime}, x\right) \leq C_{9.4 .3}(\omega) c_{6}\left(\vec{d}_{N}^{2 \gamma}\left(a_{n}^{2 \beta \gamma}+\vec{d}_{n, N}^{2 \gamma\left(\gamma_{m}-1\right)}\right)\left(\delta \wedge\left|t^{\prime}-t\right|\right)^{(1-\alpha / 2)\left(1-\eta_{1} / 2\right)}\right. \\
\left.+\left(\left|t^{\prime}-t\right| \wedge \delta\right)^{1-\eta_{1} / 2}\left(a_{n}^{2 \beta \gamma} \delta^{\gamma-\alpha / 2}+\delta^{\gamma \gamma_{m}-\alpha / 2}\right)\right) .
\end{gathered}
$$

In the end set $q 9.7 .3:=c_{3} \vee c_{6}$ to finish the proof.

Proof of Propositon 9.4.18: Let $R=33 \eta_{1}^{-1}\left(1 \vee\left((2-\alpha)^{-1}\right)\right), \eta_{0} \in\left(R^{-1},(1 \wedge\right.$ $\left.(2-\alpha)) \eta_{1} / 32\right)$. Set

$$
\begin{aligned}
d & =d\left((t, x),\left(t^{\prime}, x^{\prime}\right)\right) \\
\bar{d}_{N} & =d \vee 2^{-N} \\
\bar{d}_{n, N} & =a_{n}^{\lambda / 2} \vee \bar{d}_{N} .
\end{aligned}
$$

Let $N_{2}=N \overline{9.7 .1} \vee N \overline{9.7 .2} \vee N \overline{9.7 .3}$ and $c_{1}\left(K, \eta_{1}\right)=q_{9.7 .1} \vee q_{9.7 .2} \vee q_{9.7 .3}$. By Lemmas 9.7.1. 9.7.2, 9.7.3 we get for $(t, x) \in Z(N, n, K, \beta), N \geq N_{2}$ that:

$$
\begin{gathered}
Q_{a_{n}^{\lambda}, \eta_{0}}\left(t, x, t^{\prime}, x^{\prime}\right)^{1 / 2} \leq c_{1}\left(K, \eta_{1}\right)\left(a_{n}^{-\varepsilon_{0}}+2^{2 N_{2}}\right)\left[d ^ { \frac { 2 - a } { 2 } ( 1 - \eta _ { 1 } / 2 ) } \overline { d } _ { N } ^ { \gamma } \left(a_{n}^{\beta \gamma}+\bar{d}_{n, N}^{\gamma\left(\gamma_{m}+1\right)}\right.\right. \\
\left.+d^{1-\eta_{1} / 2} a_{n}^{\frac{\lambda}{2}(\gamma-\alpha / 2)}\left(a_{n}^{\frac{\lambda}{2} \gamma\left(\gamma_{m}-1\right)}+a_{n}^{\beta \gamma}\right)\right]
\end{gathered}
$$

for $t \leq t^{\prime}$ and $\left|x^{\prime}\right| \leq K+1$. Therefore, define

$$
\Delta_{1}\left(m, n, \bar{d}_{N}\right)=2^{-100}(q+1)^{-3} c(\alpha)^{-1} a_{n}^{-\varepsilon_{0}} \bar{d}_{N}^{\gamma}\left(a_{n}^{\beta \gamma}+\bar{d}_{n, N}^{\gamma\left(\gamma_{m}+1\right)}\right)
$$

and

$$
\Delta_{2}(m, n, \lambda)=2^{-100}(q+1)^{-3} c(\alpha)^{-1} a_{n}^{-\varepsilon_{0}} a_{n}^{\frac{\lambda}{2}(\gamma-\alpha / 2)}\left(a_{n}^{\frac{\lambda}{2} \gamma\left(\gamma_{m}-1\right)}+a_{n}^{\beta \gamma}\right) .
$$


where $c(\alpha)=\left(1-2^{-(2-\alpha) / 4}\right)^{-1}$. Note that the $\Delta_{2}$ does not even depend on $\bar{d}_{N}$.

Choose $N_{3}=\frac{33}{(2-\alpha) \eta_{1}}\left[N_{2}+N_{4}\left(K, \eta_{1}\right)+3(\log (q+1)-\log c(\alpha))(\log 2)^{-1}\right]$, where $N_{4}$ is chosen in such a way that

$$
\begin{aligned}
c(\alpha)(q+1)^{3} R_{0}^{\gamma} c_{1}\left(K, \eta_{1}\right) & {\left[a_{n}^{-\varepsilon_{0}}+2^{2 N_{2}}\right]\left(2^{-\eta_{1} N_{3} / 4} \vee 2^{-\eta_{1}(2-\alpha) N_{3} / 8}\right) } \\
& \leq R_{0}^{\gamma} c_{1}\left(K, \eta_{1}\right)\left[a_{n}^{-\varepsilon_{0}}+2^{2 N_{2}}\right] 2^{-4 N_{2}-4 N_{4}} \\
& \leq a_{n}^{-\varepsilon_{0}} 2^{-104}
\end{aligned}
$$

i.e. $N_{4}=N_{4}\left(a_{n}, \varepsilon_{0}, N_{2}, c_{1}\left(K, \eta_{1}\right)\right)$ and hence, $N_{3}=N_{3}\left(n, \varepsilon_{0}, N_{2}, K, \eta_{1}\right)$. Assume that $d \leq 2^{-N}$. Then, it is true that on

$$
\begin{gathered}
\left\{\omega:(t, x) \in Z(N, n, K+1, \beta), N \geq N_{3}\right\} \\
Q_{a_{n}^{\lambda}, \eta_{0}}\left(t, x, t^{\prime}, x^{\prime}\right)^{1 / 2} \leq \frac{1}{4}\left[d^{\frac{2-a}{2}\left(1-\frac{3}{4} \eta_{1}\right)} \Delta_{1}\left(m, n, \bar{d}_{N}\right)+d^{1-\frac{3}{4} \eta_{1}} \Delta_{2}(m, n, \lambda)\right] \\
\forall t \leq t^{\prime} \leq T_{k},\left|x^{\prime}\right| \leq K+2 .
\end{gathered}
$$

Remembering the decomposition of the $u_{2, \delta}$-difference at the beginning of this chapter into the sum of 3 martingales and applying the Dubins-Schwarz-Theorem (Theorem 3.2.8), we can write as long as $t \leq t^{\prime}$ and $d \leq 2^{-N}$ :

$$
\begin{aligned}
& \mathbb{P}\left[\left|u_{2, a_{n}^{\lambda}}(t, x)-u_{2, a_{n}^{\lambda}}\left(t^{\prime}, x^{\prime}\right)\right| \geq d^{\frac{2-a}{2}\left(1-\eta_{1}\right)} \Delta_{1}\left(m, n, \bar{d}_{N}\right)+d^{1-\eta_{1}} \Delta_{2}(m, n, \lambda)\right. \\
& \left.\quad(t, x) \in Z(N, n, K+1, \beta), N^{\prime} \wedge N \geq N_{3}, t^{\prime} \leq T_{K}\right] \\
& \quad \leq 3 \mathbb{P}\left[\sup _{u \leq 1}|B(u)| \geq \frac{16}{3}\left(d^{-\frac{\eta_{1}}{4} \frac{2-a}{2}} \wedge d^{-\frac{\eta_{1}}{4}}\right)\right] \\
& \quad \leq c \int_{d^{-(2-\alpha) \eta_{1} / 4}}^{\infty} \exp \left(-y^{2} / 2\right) d y \\
& \quad \leq c_{0} \exp \left(-d^{-(2-\alpha) \eta_{1} / 2} / 2\right),
\end{aligned}
$$

where we used the Reflection Principle in the next to last inequality.

In the case where $t^{\prime} \leq t$ and $d=d\left((t, x),\left(t^{\prime}, x^{\prime}\right)\right)$, observe that $\left(t^{\prime}, x^{\prime}\right) \in Z(N-$ $1, n, K, \beta)$. The same calculations as above apply for $N-1 \geq N_{3}$ instead of $N$ So choose $N_{5}=N_{3}+1$.

Next apply Lemma 9.8.1, where we should make clear what the parameters are. 
Take

$$
\begin{aligned}
& q_{1}=0, q_{2}=1, q_{3}=q, r=3, E=\{0\} \times \mathbb{R}_{+} \times \mathbb{R}^{q}, \\
& \bar{q}=q+2, v_{1}=v_{2}=2, v_{3}=1, v_{0}=-\infty, \\
& \hat{n}=(m, n, \lambda, \beta), S=\mathbb{N}^{2} \times[0,1 / 2] \times(0,1), \\
& \Sigma(N, K, \hat{n})=Z(N, n, K, \beta), \Sigma^{\prime}(N)=\{0\} \times\left\{0 \leq t \leq T_{K}\right\} \times \mathbb{R}^{q}, \\
& s=2, \alpha_{1}=(2-\alpha) / 2, \alpha_{2}=1, \Delta_{1}\left(\hat{n}, 2^{-N}\right)=\Delta_{1}\left(m, n, 2^{-N}\right), \\
& \Delta_{2}\left(\hat{n}, 2^{-N}\right)=\Delta_{2}(m, n, \lambda), k_{1}=4, k_{2}=1, \\
& c\left(\alpha_{1}, \alpha_{2}\right)=c(\alpha)=\left(1-2^{-(2-\alpha) / 4}\right)^{-1}, \eta=\eta_{1}, \\
& Y_{\hat{n}}(y)=u_{2, a_{n}^{\lambda}}(t, x) \text { with } y=(0, t, x), N_{0}(\eta, K, \hat{n})=N_{5} .
\end{aligned}
$$

Note, that the $N_{0}$ is uniformly bounded in $\hat{n}=(m, n, \lambda, \beta)$. Then, we obtain for $N \geq N \overline{9.4 .18}:=N_{1}(\eta, K, \hat{n})$ and $(t, x) \in Z(N, n, K, \beta)(\omega), d=d\left((t, x),\left(t^{\prime}, x^{\prime}\right)\right) \leq$ $2^{-N}$ and $t \leq t^{\prime} \leq T_{K}$ :

$$
\begin{aligned}
\left|u_{2, a_{n}^{\lambda}}(t, x)-u_{2, a_{n}^{\lambda}}\left(t^{\prime}, x^{\prime}\right)\right| \leq & 2^{3}(q+1) 4^{3+\log (q+1)} c(\alpha) \\
& {\left[d^{\frac{2-a}{2}\left(1-\eta_{1}\right)} \Delta_{1}\left(m, n, \bar{d}_{N}\right)+d^{1-\eta_{1}} \Delta_{2}(m, n, \lambda)\right] . }
\end{aligned}
$$

Thus,

$$
\left|u_{2, a_{n}^{\lambda}}(t, x)-u_{2, a_{n}^{\lambda}}\left(t^{\prime}, x^{\prime}\right)\right| \leq 2^{-94}\left(d^{(1-\alpha / 2)\left(1-\eta_{1}\right)} \bar{\Delta}_{1, u_{2}}+d^{1-\eta_{1}} \bar{\Delta}_{2, u_{2}}\right) .
$$

\subsection{Lemma 5.7 from [MP11] in $q$ dimensions}

This section contains a result which is a result of Kolmogorov-Centsov type. A similar result was given in Theorem 1.4.1 of [Kun90, but it does not cover this abstract situation here, since we want to make use of properties of random fields which only hold on certain subsets $\Sigma$ of the domain.

Let $E \subset \mathbb{R}^{q_{1}} \times \cdots \times \mathbb{R}^{q_{r}}=\mathbb{R}^{\bar{q}}$ be closed, where $r \in \mathbb{N}, q_{i} \in \mathbb{N}_{0}, i=1 \ldots, r, q_{1} \leq q_{2}$ and for $x \in E$ write by obvious notation $x=\left(x_{1}, \ldots, x_{r}\right)$. Define a metric on $E$ as

$$
d(x, y)=\left\|x_{1}-y_{1}\right\|_{2}^{1 / v_{1}}+\cdots+\left\|x_{r}-y_{r}\right\|_{2}^{1 / v_{r}}, x, y \in E
$$

for certain $v_{i} \in \mathbb{N}, i=1, \ldots, r$, but with $v_{2} \geq v_{1}$. We will also want to express the one-dimensional coordinates $x_{i, k}=x_{\alpha(i, k)}$ for $\alpha(i, k)=q_{1}+\cdots+q_{i-1}+k$ and $k=1, \ldots, q_{i}$. The projection $\pi_{2 \rightarrow 1}: \mathbb{R}^{q_{2}} \rightarrow \mathbb{R}^{q_{1}}$ to the first $q_{1}$ coordinates will also be needed. If we write inequalities between vectors, then we mean them 
componentwise.

We set for $q=\left(q_{1}, \ldots, q_{r}\right)$

$$
c(q)=2+\log \left(q_{1}+\cdots+q_{r}\right) .
$$

Let $v_{0} \in \overline{\mathbb{Z}} \cap\left[-\infty, v_{1}\right], S$ be a set and for some parameters $N, K \in \mathbb{N}, \hat{n} \in S$ let $\Sigma(N, K, \hat{n}) \subset E$ be a possibly random set with certain properties:

$$
\begin{aligned}
\Sigma(N+1, K, \hat{n}) & \subset \Sigma(N, K, \hat{n}) \\
\Sigma(N, K, \hat{n}) & \subset[-K, K]^{q_{1}+\cdots+q_{r}} \cap \Sigma(N, K+1, \hat{n}) \\
x \in \Sigma(N, K, \hat{n}), x^{\prime} \in E, d\left(x, x^{\prime}\right) & +2^{-N} \leq 2^{-N+c(q)}, \pi_{2 \rightarrow 1} x_{2}^{\prime}-x_{1}^{\prime} \leq 2^{-v_{0}(N-c(q))} \\
& \Rightarrow x^{\prime} \in \Sigma(N-c(q), K+1, \hat{n}) \\
x \in \Sigma(N, K, \hat{n}) & \Rightarrow 0 \leq \pi_{2 \rightarrow 1} x_{2}-x_{1} \leq 2^{-v_{0} N}
\end{aligned}
$$

and another nonrandom set $\Sigma^{\prime}(N) \subset E$ with the property that for $x \in \Sigma^{\prime}(N)$ :

$$
0 \leq \pi_{2 \rightarrow 1} x_{2}-x_{1} \leq 2^{-v_{0} N} .
$$

Lemma 9.8.1. Let $c_{0}, c_{1}, c_{2}, \alpha_{j}$ and $k_{j}$ be positive constants, $\eta \in(0,1 / 2), \Delta_{j}$ : $S \times(0,1] \rightarrow \mathbb{R}_{+}$satisfy $\Delta_{j}\left(\hat{n}, 2^{-N+1}\right) \leq k_{j} \Delta_{j}\left(\hat{n}, 2^{-N}\right), \hat{n} \in S, N \in \mathbb{N}, j=1, \ldots, s$ for a given $s \in \mathbb{N}$. For $\hat{n} \in S$ assume that $\left\{Y_{\hat{n}}(x), x \in E\right\}$ is a real-valued continuous process. Assume for each $\hat{n} \in S, K \in \mathbb{N}$ there is an $N_{0}(\omega)=N_{0}(\eta, K, \hat{n})(\omega)$ almost surely stochastically bounded uniformly in $\hat{n}$ such that for any $N \in \mathbb{N},\|x\|_{1} \leq K$, if $d=d\left(x, x^{\prime}\right) \leq 2^{-N}$ :

$$
\begin{gathered}
\mathbb{P}\left(\left|Y_{\hat{n}}(x)-Y_{\hat{n}}\left(x^{\prime}\right)\right|>\sum_{j=1}^{s} d\left(x, x^{\prime}\right)^{(1-\eta) \alpha_{j}} \Delta_{j}\left(\hat{n}, 2^{-N}\right),\right. \\
\left.x \in \Sigma(N, K, \hat{n}), x^{\prime} \in \Sigma^{\prime}(N), N \geq N_{0}\right) \\
\leq c_{0} \exp \left(-c_{1} d^{-\eta c_{2}}\right) .
\end{gathered}
$$

Then there is a $N_{1}(K, \eta, \hat{n})$ almost surely stochastically uniformly bounded in $\hat{n}$ such that when $N \geq N_{1}$ for $x \in \Sigma(N, K, \hat{n})$ and $x^{\prime} \in \Sigma^{\prime}(N), d\left(x, x^{\prime}\right) \leq 2^{-N}$ we have almost surely

$$
\left|Y_{\hat{n}}(x)-Y_{\hat{n}}\left(x^{\prime}\right)\right| \leq 2^{3} \bar{q} \bar{c}\left(\alpha_{1}, \ldots, \alpha_{s}\right) \sum_{j=1}^{s} k_{j}^{c(q)+1} d\left(x, x^{\prime}\right)^{\alpha_{j}(1-\eta)} \Delta_{j}\left(\hat{n}, 2^{-N}\right) .
$$

with $\bar{c}\left(\alpha_{1}, \ldots, \alpha_{s}\right)=\left(1-2^{-\inf \alpha_{j} / 2}\right)^{-1}, \bar{q}=q_{1}+\cdots+q_{r}$.

Proof. Let for $\ell \in \mathbb{N}, K \in \mathbb{N}$

$$
\begin{aligned}
& \Theta(K, \ell)=\left\{x \in[-K, K]^{\bar{q}}: \exists m_{1}, \ldots, m_{r} \in \mathbb{N}: x=\left(m_{1} 2^{-v_{1} \ell}, \ldots, m_{r} 2^{-v_{r} \ell}\right)\right\}, \\
& \Theta^{\prime}(\ell)=\left\{y \in \mathbb{R}^{\bar{q}}: \exists e_{i} \in\left\{0,1, \ldots, 2^{v_{i}}\right\}^{q_{i}}, i=1, \ldots, r: y=\left(e_{1} 2^{-v_{1} \ell}, \ldots, e_{r} 2^{-v_{r} \ell}\right)\right\} .
\end{aligned}
$$


Assume additionally that $N \in \mathbb{N}, \hat{n} \in S$ and define

$$
\begin{aligned}
M_{\ell, N}:= & \max \left\{\left|Y_{\hat{n}}(x)-Y_{\hat{n}}(x+y)\right|\right. \\
& \left.x \in \Sigma(N, K, \hat{n}) \cap \Theta(K, \ell),(x+y) \in \Sigma^{\prime}(K), y \in \Theta^{\prime}(\ell)\right\}
\end{aligned}
$$

and

$$
A_{N}=\left\{\omega: \exists \ell \geq N+c(q) \text { s.t. } M_{\ell, N}>\sum_{i=1}^{s} 2^{-(\ell-c(q))(1-\eta) \alpha_{i}} \Delta_{i}\left(\hat{n}, 2^{-N}\right), N \geq N_{0}\right\}
$$

Estimating roughly, we observe that $\# \Theta(K, \ell) \leq \prod_{i=1}^{r}\left(2^{v_{i} \ell}(2 K+1)+1\right)^{q_{i}}$ and $\# \Theta(\ell) \leq \prod_{i=1}^{r}\left(2^{v_{i}}+1\right)^{q_{i}}$. Assume that $x \in \Theta(K, \ell), y \in \Theta^{\prime}(\ell)$ have the form as in the definition of the sets and $\ell \geq N^{\prime}+c(q), N^{\prime} \in \mathbb{N}$. Then

$$
\begin{gathered}
d:=d(x, y)=\left\|e_{1} 2^{-v_{1} \ell}\right\|_{2}^{1 / v_{1}}+\ldots\left\|e_{r} 2^{-v_{r} \ell}\right\|_{2}^{1 / v_{r}} \\
\quad \leq 2^{-\ell}\left(2 \sqrt{q_{1}}+\ldots 2 \sqrt{q_{r}}\right) \leq 2^{-(\ell-c(q))} \leq 2^{-N^{\prime}} .
\end{gathered}
$$

Using this (twice) and 9.149) we obtain

$$
\begin{aligned}
& \mathbb{P}\left(\bigcup_{N^{\prime}=N}^{\infty} A_{N^{\prime}}\right) \leq \sum_{N^{\prime}=N}^{\infty} \sum_{\ell=N^{\prime}+c(q)}^{\infty} \mathbb{P}\left(M_{\ell, N^{\prime}}>\sum_{i=1}^{s} 2^{-(\ell-c(q))(1-\eta) \alpha_{i}} \Delta_{i}\left(\hat{n}, 2^{-N^{\prime}}\right), N^{\prime} \geq N_{0}\right) \\
& \leq \sum_{N^{\prime}=N}^{\infty} \sum_{\ell=N^{\prime}+c(q)}^{\infty} \# \Theta(K, \ell) \# \Theta^{\prime}(\ell) \\
& \max _{x \in \Theta(K, \ell), y \in \Theta^{\prime}(\ell)} \mathbb{P}\left(\left|Y_{\hat{n}}(x)+Y_{\hat{n}}(x+y)\right|>\sum_{i=1}^{s} d^{(1-\eta) \alpha_{i}} \Delta_{i}\left(\hat{n}, 2^{-N^{\prime}}\right),\right. \\
& \left.\quad x \in \Sigma\left(N^{\prime}, K, \hat{n}\right),(x+y) \in \Sigma^{\prime}(K), N^{\prime} \geq N_{0}\right) \\
& \leq \sum_{N^{\prime}=N}^{\infty} \sum_{\ell=N^{\prime}+c(q)}^{\infty} \prod_{i=1}^{r}\left(2^{v_{i}}+1\right)^{q_{i}}\left(2^{v_{i} \ell}(K+1)+1\right)^{q_{i}} c_{0} \exp \left(-c_{1} 2^{-(\ell-c(q)) \eta c_{2}}\right) \\
& \leq c_{3}(K) \exp \left(-c_{1}^{\prime} 2^{N \eta c_{2}}\right)
\end{aligned}
$$

for certain constants $c_{3}(K)>0$ and $c_{1}^{\prime} \in\left(0, c_{1}\right)$. Let

$$
N_{2}=N_{2}(\hat{n}, K)=\min \left\{N: \omega \in \bigcap_{N^{\prime}=N}^{\infty} A_{N^{\prime}}^{c}\right\}<\infty \text { (by Borel-Cantelli). }
$$

Then we have by the previous calculation, that

$$
\mathbb{P}\left(N_{2}>N\right) \leq c_{3}(K) \exp \left(-c_{1}^{\prime} 2^{N \eta c_{2}}\right)
$$


which ensures that

$$
N_{0}^{\prime}:=\left(N_{0} \vee N_{2}\right)+c(q)
$$

is stochastically bounded uniformly in $(\hat{n})$.

Assume

$$
N \geq N_{0}^{\prime}, x \in \Sigma(N, K, \hat{n}), x^{\prime} \in \Sigma^{\prime}(N), d\left(x, x^{\prime}\right) \leq 2^{-N}
$$

Define dyadic approximations for $x, x^{\prime}$ and $\ell \in \mathbb{N}$ according to the metric $d$ componentwise:

$$
x_{i}^{(\ell)}:=\sum_{k=1}^{q_{i}}\left\lfloor 2^{v_{i} \ell} x_{i, k}\right\rfloor 2^{-v_{i} \ell} e_{i, k}, i=1, \ldots, r,
$$

where $u_{i, k}$ is the $\left(q_{1}+\cdots+q_{i-1}+k\right)$ th eigenvector in $E$ and $\lfloor m+\varepsilon\rfloor=m$ for $m \in \mathbb{Z}$, $\varepsilon \in[0,1)$ is the floor function. Similarly define $x^{\prime}$.

If $\ell \geq N$ :

$$
\begin{aligned}
d\left(x^{(\ell)}, x\right) & \leq d\left(x^{(\ell)}, x^{\prime}\right)+d\left(x^{\prime}, x\right) \\
& \leq \sum_{i=1}^{r} q_{i}^{1 / v_{i}} 2^{-\ell}+2^{-N} \\
& \leq 2^{-(N-c(q))-1}
\end{aligned}
$$

which then implies that $d\left(x^{(\ell)}, x\right)+2^{-N} \leq 2^{-(N-c(q))}$. Additionally for $j=1, \ldots, q_{1}$ we easily see the first inequality and then use triangle inequality:

$$
\begin{aligned}
0 \leq \pi_{2 \rightarrow 1} x_{2, j}^{(\ell)}-x_{1, j}^{(\ell)} & \leq\left|x_{2, j}^{(\ell)}-x_{2, j}^{\prime}\right|+\left|\pi_{2 \rightarrow 1} x_{2, j}^{\prime}-x_{1, j}^{\prime}\right|+\left|x_{1, j}^{\prime}-x_{1, j}^{(\ell)}\right| \\
& \leq 2^{-v_{1} \ell}+2^{-v_{0} N}+2^{-v_{2} \ell} \leq 2^{-v_{0}(N-c(q))} .
\end{aligned}
$$

Therefore,

$$
x^{\prime(\ell)} \in \Sigma(N-c(q), K+1, \hat{n}) \forall \ell \geq N .
$$

If we assume that $N \geq N_{0}^{\prime} \geq N_{2}+c(q)$, then both $\omega \in A_{N-c(q)}^{c}$ and $N-c(q) \geq N_{0}$. Therefore, for all $\ell \geq N-c(q)+c(q)=N$ :

$$
M_{\ell, N-c(q)} \leq \sum_{i=1}^{s} 2^{-(\ell-c(q))(1-\eta) \alpha_{i}} \Delta_{i}\left(\hat{n}, 2^{-(N-c(q))}\right) .
$$

If we choose $N^{\prime} \geq N$ s.t. $2^{-N^{\prime}-1}<d\left(x, x^{\prime}\right) \leq 2^{-N^{\prime}}$, then $x^{\prime\left(N^{\prime}\right)}=x_{i}^{\left(N^{\prime}\right)}+g_{i} 2^{-v_{i} N^{\prime}}$ for $g_{i} \in\{-1,0,1\}^{q_{i}}$ for $i=1, \ldots, r$. In addition, we have for $i=1, \ldots, r$ :

$$
\begin{aligned}
x_{i}^{(\ell)} & =x_{i}^{(\ell)}+f_{i} 2^{-v_{i} \ell} \text { for } f_{i} \in\left\{-2^{v_{i}}+1, \ldots, 0, \ldots, 2^{v_{i}}-1\right\}, \\
x^{\prime(\ell)} & =x^{\prime(\ell)}+f_{i}^{\prime} 2^{-v_{i} \ell} \text { for } f_{i}^{\prime} \in\left\{-2^{v_{i}}+1, \ldots, 0, \ldots, 2^{v_{i}}-1\right\} .
\end{aligned}
$$


By 9.152, 9.151), 9.153), the continuity of $Y_{\hat{n}}$ and the assumptions in 9.150 we get

$$
\begin{aligned}
\left|Y_{\hat{n}}(x)-Y_{\hat{n}}\left(x^{\prime}\right)\right| \leq \mid & Y_{\hat{n}}\left(x^{\left(N^{\prime}\right)}\right)-Y_{\hat{n}}\left(x^{\left(N^{\prime}\right)}\right) \mid \\
& +\sum_{\ell=N^{\prime}+1}^{\infty}\left|Y_{\hat{n}}\left(x^{(\ell)}\right)-Y_{\hat{n}}\left(x^{(\ell-1)}\right)\right|+\left|Y_{\hat{n}}\left(x^{(\ell)}\right)-Y_{\hat{n}}\left(x^{(\ell-1)}\right)\right| \\
\leq & M_{N^{\prime}, N-c(q)}+\sum_{\ell=N^{\prime}+1}^{\infty} 2 M_{\ell, N-c(q)} \leq 2 \sum_{\ell=N^{\prime}}^{\infty} M_{\ell, N-c(q)} \\
\leq & 2 \sum_{i=1}^{s}\left[\sum_{\ell=N^{\prime}+1}^{\infty} 2^{-(\ell-c(q))(1-\eta) \alpha_{i}} \Delta_{i}\left(\hat{n}, 2^{-(N-c(q))}\right)\right] \\
\leq & \sum_{j=1}^{s} 2^{c(q)+1} 2^{-N^{\prime}(1-\eta) \alpha_{j}}\left(1-2^{-(1-\eta) \alpha_{j}}\right)^{-1} k_{j}^{\lceil c(q))} \Delta_{j}\left(\hat{n}, 2^{-N}\right) \\
\leq & 2^{3} \bar{q} \bar{c}\left(\alpha_{1}, \ldots, \alpha_{s}\right) \sum_{j=1}^{s} k_{i}^{c(q)+1} d\left(x, x^{\prime}\right)^{\alpha_{j}(1-\eta)} \Delta_{j}\left(\hat{n}, 2^{-N}\right),
\end{aligned}
$$

with $\bar{c}\left(\alpha_{1}, \ldots, \alpha_{s}\right)=\left(1-2^{-\inf \alpha_{j} / 2}\right)^{-1}$.

\subsection{Proof of Theorem 5.3 .3}

Within this section we extend Mytnik, Perkins and Sturm's Theorem 4.1 to the case where a Lipschitz-drift is included and $\sigma$ may depend on $t$ and $x$. That means the SPDE has the form

$$
\frac{\partial u}{\partial t}(t, x)=\frac{1}{2} \Delta u+b(t, x, u(t, x))+\sigma(t, x, u(t, x)) \dot{W}(t, x),
$$

where the details on the coefficients can be found after (5.1).

We will show a simplified version of Theorem 4.1 of [MPS06].

Theorem 9.9.1. Let $\xi \in(0,1)$ satisfy

$$
\begin{aligned}
& \exists N_{\xi}=N_{\xi}(K, \omega) \in \mathbb{N} \text { a.s. such that } \forall N \geq N_{\xi},(t, x) \in Z_{N, K} \\
& d\left(\left(t^{\prime}, y\right),(t, x)\right) \leq 2^{-N}, \quad t, t^{\prime} \leq T_{K} \Rightarrow\left|u(t, x)-u\left(t^{\prime}, y\right)\right| \leq 2^{-N \xi} .
\end{aligned}
$$

Let $0<\xi_{1}<1 \wedge(\xi \gamma+1-\alpha / 2)$. Then there is an $N_{\xi_{1}}=N_{\xi_{1}}(K, \omega) \in \mathbb{N}$ a.s. such that, for any $N \geq N_{\xi_{1}}$ in $\mathbb{N}$ and any $(t, x) \in Z_{N, K}$,

$$
d\left(\left(t^{\prime}, y\right),(t, x)\right) \leq 2^{-N}, \quad t, t^{\prime} \leq T_{K} \Rightarrow\left|u(t, x)-u\left(t^{\prime}, y\right)\right| \leq 2^{-N \xi_{1}} .
$$


Moreover, there are strictly positive constants $R, \delta, c_{1}, c_{2}$ depending only on $\left(\xi, \xi_{1}\right)$ and $N(K) \in \mathbb{N}$, which also depends on $K$, such that

$$
\mathbb{P}\left(N_{\xi_{1}} \geq N\right) \leq c_{1}\left(\mathbb{P}\left(N_{\xi} \geq N / R\right)+K^{q+1} \exp \left(-c_{2} 2^{N \delta}\right)\right),
$$

provided that $N \geq N(K)$.

Using the same argument as in Corollary 4.2 of [MPS06] one can derive Theorem 5.3 .3 from this result.

Remember the formulation of a mild solution:

$$
\begin{aligned}
u(t, x)= & \int_{\mathbb{R}^{q}} p_{t}(x-y) u_{0}(y) d y+\int_{0}^{t} \int_{\mathbb{R}^{q}} p_{t-s}(x-y) b(s, y, u(s, y)) d y d s \\
& +\int_{0}^{t} \int_{\mathbb{R}^{q}} p_{t-s}(x-y) \sigma(s, y, u(s, y)) W(d s d y) .
\end{aligned}
$$

In fact, the extension is much simpler than their original proof, since the drift integrals are Riemann integrals. However, that does not mean that the proof can be compressed within one lemma. It needs to go along their proof and fill in the drift parts, where necessary.

We start directly on their page 1936 for the proof of their Theorem 4.2: Fix $(t, x),\left(t^{\prime}, y\right) \in \mathbb{R}_{+} \times \mathbb{R}^{q}$ with $d\left((t, x),\left(t^{\prime}, y\right)\right) \leq \varepsilon \equiv 2^{-N}$ for a certain $N \in \mathbb{N}$ and $t \leq t^{\prime}$. Their equation (58) stays unchanged for a certain $N_{1}=N_{1}\left(\omega, \xi, \xi_{1}\right)$ to be chosen later:

$$
\begin{aligned}
& \mathbb{P}\left(|u(t, x)-u(t, y)| \geq|x-y|^{1-\alpha / 2-\delta} \varepsilon^{p},(t, x) \in Z_{N, K, \xi}, N \geq N_{1}\right) \\
& +\mathbb{P}\left(\left|u\left(t^{\prime}, x\right)-u(t, x)\right| \geq\left|t^{\prime}-t\right|^{\frac{1}{2}(1-\alpha / 2-\delta)} \varepsilon^{p},(t, x) \in Z_{N, K}, t^{\prime} \leq T_{K}, N \geq N_{1}\right) .
\end{aligned}
$$

Let us define

$$
\begin{aligned}
B^{x, y, t, t^{\prime}}(w, s) & =B_{5.4}\left|p_{t-s}(x-w)-p_{t^{\prime}-s}(y-w)\right||u(s, w)|, \\
B^{x, t^{\prime}}(w, s) & =B_{5.4} p_{t^{\prime}-s}(x-w)|u(s, w)|
\end{aligned}
$$

additional to the quantities $D$ defined in [MPS06]. This allows to bound (9.154) by the sum of two expressions (itself sums): 


$$
\begin{aligned}
\mathbb{P} & \left(|u(t, x)-u(t, y)| \geq|x-y|^{1-\alpha / 2-\delta} \varepsilon^{p},(t, x) \in Z_{N, K}, N \geq N_{1},\right. \\
& \int_{0}^{t} \int_{\mathbb{R}^{2 q}} D^{x, y, t, t}(w, z, s) d w d z d s \leq|x-y|^{2-\alpha-2 \delta^{\prime}} \varepsilon^{2 p}, \\
& \left.\int_{0}^{t} \int_{\mathbb{R}^{q}} B^{x, y, t, t}(w, s) d w d s \leq \frac{1}{2}|x-y|^{1-\alpha / 2-\delta} \varepsilon^{p}\right) \\
+ & \mathbb{P}\left(\left|u\left(t^{\prime}, x\right)-u(t, x)\right| \geq\left|t^{\prime}-t\right|^{\frac{1}{2}(1-\alpha / 2-\delta)} \varepsilon^{p},(t, x) \in Z_{N, K}, t^{\prime} \leq T_{K}, N \geq N_{1},\right. \\
& \int_{0}^{t} \int_{\mathbb{R}^{2 q}} D^{x, x, t, t^{\prime}}(w, z, s) d w d z d s+\int_{t}^{t^{\prime}} \int_{\mathbb{R}^{2 q}} D^{x, t^{\prime}}(w, z, s) d w d z d s \leq\left|t^{\prime}-t\right|^{1-\alpha / 2-\delta} \varepsilon^{2 p}, \\
& \left.\int_{0}^{t} \int_{\mathbb{R}^{q}} B^{x, x, t, t^{\prime}}(w, s) d w d s+\int_{t}^{t^{\prime}} \int_{\mathbb{R}^{q}} B^{x, t^{\prime}}(w, s) d w d s \leq \frac{1}{2}\left|t^{\prime}-t\right|^{\frac{1}{2}(1-\alpha / 2-\delta)} \varepsilon^{p}\right) \\
= & P_{1}+P_{2}
\end{aligned}
$$

and

$$
\begin{aligned}
& \mathbb{P}\left(\int_{0}^{t} \int_{\mathbb{R}^{2 q}} D^{x, y, t, t}(w, z, s) d w d z d s>|x-y|^{2-\alpha-2 \delta^{\prime}} \varepsilon^{2 p},(t, x) \in Z_{N, K}, N \geq N_{1}\right) \\
& +\mathbb{P}\left(\int_{0}^{t} \int_{\mathbb{R}^{2 q}} D^{x, x, t, t^{\prime}}(w, z, s) d w d z d s+\int_{t}^{t^{\prime}} \int_{\mathbb{R}^{2 q}} D^{x, t^{\prime}}(w, z, s) d w d z d s\right. \\
& \left.\quad>\left|t^{\prime}-t\right|^{1-\alpha / 2-\delta} \varepsilon^{2 p},(t, x) \in Z_{N, K}, N \geq N_{1}\right) \\
& +\mathbb{P}\left(\int_{0}^{t} \int_{\mathbb{R}^{q}} B^{x, y, t, t}(w, s) d w d s>\frac{1}{2}|x-y|^{1-\alpha / 2-\delta} \varepsilon^{p},(t, x) \in Z_{N, K}, N \geq N_{1}\right) \\
& +\mathbb{P}\left(\int_{0}^{t} \int_{\mathbb{R}^{q}} B^{x, x, t, t^{\prime}}(w, s) d w d s+\int_{t}^{t^{\prime}} \int_{\mathbb{R}^{q}} B^{x, t^{\prime}}(w, s) d w d s>\frac{1}{2}\left|t^{\prime}-t\right|^{\frac{1}{2}(1-\alpha / 2-\delta)} \varepsilon^{p}\right. \\
& \left.\quad(t, x) \in Z_{N, K}, N \geq N_{1}\right) \\
& =P_{3}+P_{4}+P_{5}+P_{6} .
\end{aligned}
$$

The expressions $P_{1}, P_{2}$ can be bounded using Dubins-Schwarz (Theorem 3.2.8) and the reflection principle for the martingale as on page 1938 of [MPS06. Similarly, the expressions $P_{3}$ and $P_{4}$ lead to the estimates given in (102) and (103) of [MPS06]. So let us only focus on $P_{5}$ and $P_{6}$. We use the sets $A_{j}^{i}$ as in their page 1938 and 
consider the integral in $P_{5}$ :

$$
\begin{aligned}
& \int_{0}^{t-\varepsilon^{2}} \int_{\mathbb{R}^{q}}\left|p_{t-s}(x-w)-p_{t-s}(y-w)\right||u(s, w)| \mathbb{1}\left(|w-x| \leq 2 \sqrt{t-s} \varepsilon^{1-\delta_{1}}\right) d w d s \\
& +\int_{0}^{t-\varepsilon^{2}} \int_{\mathbb{R}^{q}}\left|p_{t-s}(x-w)-p_{t-s}(y-w)\right||u(s, w)| \mathbb{1}\left(|w-x|>2 \sqrt{t-s} \varepsilon^{1-\delta_{1}}\right) d w d s \\
& +\int_{t-\varepsilon^{2}}^{t} \int_{\mathbb{R}^{q}}\left|p_{t-s}(x-w)-p_{t-s}(y-w)\right||u(s, w)| \mathbb{1}\left(|w-x| \leq 2 \varepsilon^{1-\delta_{1}}\right) d w d s \\
& +\int_{t-\varepsilon^{2}}^{t} \int_{\mathbb{R}^{q}}\left|p_{t-s}(x-w)-p_{t-s}(y-w)\right||u(s, w)| \mathbb{1}\left(|w-x|>2 \varepsilon^{1-\delta_{1}}\right) d w d s \\
& \quad=R_{1}+R_{2}+R_{3}+R_{4} .
\end{aligned}
$$

A similar decomposition can be given for the epressions in $P_{6}$.

$$
\begin{aligned}
& \int_{0}^{t-\varepsilon^{2}} \int_{\mathbb{R}^{q}}\left|p_{t-s}(x-w)-p_{t^{\prime}-s}(x-w)\right||u(s, w)| \mathbb{1}\left(|x-w| \leq 2 \sqrt{t-s} \varepsilon^{\delta_{1}}\right) d w d s \\
& +\int_{0}^{t-\varepsilon^{2}} \int_{\mathbb{R}^{q}}\left|p_{t-s}(x-w)-p_{t^{\prime}-s}(x-w)\right||u(s, w)| \mathbb{1}\left(|x-w|>2 \sqrt{t-s} \varepsilon^{\delta_{1}}\right) d w d s \\
& +\int_{t-\varepsilon^{2}}^{t} \int_{\mathbb{R}^{q}}\left|p_{t-s}(x-w)-p_{t^{\prime}-s}(x-w)\right||u(s, w)| \mathbb{1}\left(|x-w| \leq 2 \varepsilon^{1-\delta_{1}}\right) d w d s \\
& +\int_{t-\varepsilon^{2}}^{t} \int_{\mathbb{R}^{q}}\left|p_{t-s}(x-w)-p_{t^{\prime}-s}(x-w)\right||u(s, w)| \mathbb{1}\left(|x-w|>2 \varepsilon^{1-\delta_{1}}\right) d w d s \\
& +\int_{t}^{t^{\prime}} \int_{\mathbb{R}^{q}} p_{t^{\prime}-s}(x-w)|u(s, w)| \mathbb{1}\left(|w-x| \leq 2 \varepsilon^{1-\delta_{1}}\right) d w d s \\
& +\int_{t}^{t^{\prime}} \int_{\mathbb{R}^{q}} p_{t^{\prime}-s}(x-w)|u(s, w)| \mathbb{1}\left(|w-x|>2 \varepsilon^{1-\delta_{1}}\right) d w d s \\
& =S_{1}+S_{2}+S_{3}+S_{4}+S_{5}+S_{6} .
\end{aligned}
$$

It will be helpful to use their Lemma 5.4, which we quote:

Lemma 9.9.2 (Lemma 5.4 of [MPS06]). Let $N \geq N_{1}$. Then on $\left\{\omega:(t, x) \in Z_{N, K}\right\}$, $|u(s, w)| \leq 10 \varepsilon^{\left(1-\delta_{1}\right) \xi} \quad$ for $s \in\left[t-\varepsilon^{2}, t^{\prime}\right],|w-x| \leq 2 \varepsilon^{1-\delta_{1}}$, $|u(s, w)| \leq\left(8+3 K 2^{N_{\xi} \xi}\right) e^{\lambda|w|}(t-s)^{\xi / 2} e^{-\delta_{1} \xi}$, for $s \in\left[0, t-\varepsilon^{2}\right],|w-x| \leq 2 \sqrt{t-s} \varepsilon^{1-\delta_{1}}$

and anywhere else the bound $|u(s, w)| \leq K e^{\lambda|w|}$ for $s \leq K, w \in \mathbb{R}^{q}$ (by (5.7)).

Lemma 9.9.3. Assume that $(t, x) \in Z_{N, K}$ and $N \geq N_{\xi}$. There is a constant 9.9.3 $(q, K)<\infty$ such that

$$
R_{1}+\cdots+R_{4} \leq q 9.9 .3|x-y|\left(\varepsilon^{1+\left(1-\delta_{1}\right) \xi}+K 2^{N_{\xi} \xi}\right) .
$$


Proof. We start with $R_{1}$. By Lemma 9.9.2 and Lemma 9.6.1 obtain

$$
\begin{aligned}
R_{1} & \leq\left(8+3 K 2^{N_{\xi} \xi}\right) \int_{0}^{t-\varepsilon^{2}} d s(t-s)^{(\xi-1) / 2} e^{-\delta_{1} \xi} \\
& \sum_{i=1}^{q} \int_{0}^{\left|y_{i}-x_{i}\right|} \int_{\mathbb{R}^{q}} d w e^{\lambda|w|} p_{2 t}\left(w-x+\hat{v}_{i-1}+r_{i} e_{i}\right) \\
& \leq\left(8+3 K 2^{N_{\xi} \xi}\right) \int_{0}^{t-\varepsilon^{2}} d s(t-s)^{(\xi-1) / 2} K e^{K} \sum_{i=1}^{q}\left|y_{i}-x_{i}\right| \\
& \leq C(K)\left(8+3 K 2^{N_{\xi} \xi}\right)|x-y|,
\end{aligned}
$$

where we also used the estimate (47) of [MPS06]. For $R_{2}$ observe that for such $y$ : $|y-w| \geq|x-w|+|x-y| \geq 2 \sqrt{t-s} \varepsilon^{-\delta_{1}}-\sqrt{t-s}>\sqrt{t-s} \varepsilon^{-\delta_{1}}$. Trivially bounding $u(s, w) \leq K e^{|w|}$ and using again Lemma 9.6.1 and (47),

$$
\begin{aligned}
R_{2} & \leq \int_{0}^{t-\varepsilon^{2}} d s(t-s)^{(\xi-1) / 2} \sum_{i=1}^{q} \int_{0}^{\left|y_{i}-x_{i}\right|} \\
& \quad \int_{\mathbb{R}^{q}} d w e^{\lambda|w|} p_{2 t}\left(w-x+\hat{v}_{i-1}+r_{i} e_{i}\right) \mathbb{1}\left(w-x+\hat{v}_{i-1}+r_{i} e_{i} \mid>\sqrt{t-s} \varepsilon^{-\delta_{1}}\right) \\
& \leq C(K) \exp \left(-\frac{1}{4} \varepsilon^{-\delta_{1}}\right)|x-y|
\end{aligned}
$$

Next, $R_{3}$ is bounded using Lemma 9.9.2 and [MPS06]'s Lemma 5.2(a) with $\beta=1$,

$$
R_{3} \leq 10 \varepsilon^{\left(1-\delta_{1}\right) \xi} C(K) \int_{t-\varepsilon^{2}}^{t} d s(t-s)^{-1 / 2}|x-y| \leq C(K) \varepsilon^{1+\left(1-\delta_{1}\right) \xi}|x-y| .
$$

And finally $R_{4}$ can be bounded just as $R_{2}$, since here $|y-w|>|x-w|-|y-x|>$ $2 \varepsilon^{1-\delta_{1}}-\varepsilon>\varepsilon^{1-\delta_{1}}>\sqrt{t-s} \varepsilon^{-\delta_{1}}$. Then,

$$
R_{4} \leq C(K) \exp \left(-\frac{1}{4} \varepsilon^{-\delta_{1}}\right)|x-y|
$$

Setting $c=11 C(K)$, we obtain the result.

Lemma 9.9.4. Assume that $(t, x) \in Z_{N, K}$ and $N \geq N_{\xi}$. There is a constant q9.9.4 $(q, K)<\infty$ such that

$$
S_{1}+\cdots+S_{6} \leq q .9 .94\left|t^{\prime}-t\right|^{\frac{1}{2}}\left(\varepsilon^{\left(1-\delta_{1}\right) \xi}+K 2^{N_{\xi} \xi}\right) .
$$


Proof. We extensively use (47) of [MPS06] and Lemma 9.6.1.

$$
\begin{aligned}
S_{1} \leq & C\left(8+3 K 2^{N_{\xi} \xi}\right)\left|t-t^{\prime}\right|^{1 / 2} \int_{0}^{t-\varepsilon^{2}} d s(t-s)^{-1 / 2} \\
& \int_{\mathbb{R}^{q}} d w p_{(t-s)}(w-x)+p_{4\left(t^{\prime}-s\right)}(w-x) e^{\lambda|w|}(t-s)^{\xi / 2} \\
\leq & C(K)\left(8+3 K 2^{N_{\xi} \xi}\right)\left|t-t^{\prime}\right|^{1 / 2} .
\end{aligned}
$$

For $S_{2}$ observe that for $\operatorname{such} w$ and $s$ :

$$
|w-x|>2 \sqrt{t-s} \varepsilon^{-\delta_{1}}>2 \frac{\sqrt{t^{\prime}-s}}{\sqrt{t^{\prime}-t}+\sqrt{t-s}} \sqrt{t-s} \varepsilon^{-\delta_{1}}>\sqrt{t^{\prime}-s} \varepsilon^{-\delta_{1}} .
$$

Then,

$$
\begin{aligned}
S_{2} \leq C\left|t^{\prime}-t\right|^{1 / 2} \exp \left(-\frac{1}{2} \varepsilon^{-\delta_{1}}\right) \int_{0}^{t-\varepsilon^{2}} d s(t-s)^{-1 / 2} \\
\quad \int_{\mathbb{R}^{q}} p_{2(t-s)}(w-x)+p_{4\left(t^{\prime}-s\right)}(w-x) e^{|w|} d w \\
\leq C\left|t^{\prime}-t\right|^{1 / 2} \exp \left(-\frac{1}{2} \varepsilon^{-\delta_{1}}\right) .
\end{aligned}
$$

Using Lemma 9.9 .2 , obtain

$$
S_{3} \leq C \varepsilon^{\left(1-\delta_{1}\right) \xi}\left|t^{\prime}-t\right|^{1 / 2} \int_{t-\varepsilon^{2}}^{t} d s(t-s)^{-1 / 2} \leq C \varepsilon^{\left(1-\delta_{1}\right) \xi}\left|t^{\prime}-t\right|^{1 / 2} .
$$

Next, observe that for $s$ and $w$ as in $S_{4}$ :

$$
\frac{|w-x|}{\sqrt{t^{\prime}-s}}>\frac{|w-x|}{\sqrt{t^{\prime}-t}+\sqrt{t-s}}>\frac{|w-x|}{\varepsilon+\varepsilon}>\varepsilon^{-1} \varepsilon^{1-\delta_{1}}=\varepsilon^{-\delta_{1}} .
$$

Thus,

$$
\begin{aligned}
S_{4} \leq C\left|t^{\prime}-t\right|^{1 / 2} \exp \left(-\frac{1}{2} \varepsilon^{-\delta_{1}}\right) \int_{0}^{t-\varepsilon^{2}}(t-s)^{-1 / 2} \\
\quad \int_{\mathbb{R}^{q}} p_{2(t-s)}(w-x)+p_{4\left(t^{\prime}-s\right)}(w-x) e^{|w|} d w \quad \leq C\left|t^{\prime}-t\right|^{1 / 2} \exp \left(-\frac{1}{2} \varepsilon^{-\delta_{1}}\right) .
\end{aligned}
$$

For $S_{5}$ we use Lemma 9.9 .2

$$
S_{5} \leq 10 \varepsilon^{\left(1-\delta_{1}\right) \xi}\left|t^{\prime}-t\right| C(K)
$$

and finally, using 9.157)

$$
S_{6} \leq C\left|t^{\prime}-t\right| \exp \left(-\varepsilon^{-\delta_{1}}\right) .
$$

If we add up the results and use that $\varepsilon=2^{-N}$ is small enough, such that $\exp \left(-\frac{1}{2} \varepsilon^{-\delta_{1}}\right)<$ $\varepsilon^{1-\delta_{1}}$, we get the lemma. 
Recall that $N_{0}\left(K, \xi, \delta_{1}, p\right)$ defined on page 1947 is a deterministic constant, which we will use. We give estimates for $P_{5}$ and $P_{6}$. Choose $\delta_{2} \in(0, \alpha / 2+\delta-p)$ (see (56) of [MPS06]).

Lemma 9.9.5. If $N \geq N_{5} \vee N_{0}^{\prime}$, where $N_{5}:=\frac{1}{\delta_{2}} N_{\xi}$ and $N_{0}^{\prime}=\delta_{2}^{-1}(\log q 9.9 .3+$ $\log K+1)$, then $P_{5}=0$.

Proof. Then,

$$
\begin{aligned}
& P_{5} \leq \mathbb{P}\left(R_{1}+R_{2}+R_{3}+R_{4}>\frac{1}{2}|x-y|^{1-\alpha / 2-\delta} \varepsilon^{p},(t, x) \in Z_{N, K, \xi}, N \geq N_{1}\right) \\
& \leq \mathbb{P}\left(q_{9.9 .3}|x-y|\left(K 2^{N_{\xi} \xi}+\varepsilon^{1+\left(1-\delta_{1}\right) \xi}\right)>\frac{1}{2}|x-y|^{1-\alpha / 2-\delta} \varepsilon^{p}\right) \\
& \leq \mathbb{P}\left(\widetilde{9.9 .3}\left(K 2^{N_{\xi} \xi}+1\right)>\frac{1}{2}|x-y|^{-\alpha / 2-\delta+p}\right) \\
& \leq \mathbb{P}\left(2 q 9.9 .3\left(K 2^{N_{\xi} \xi}+1\right)>2^{N(\alpha / 2+\delta-p)}\right) \\
& \leq \mathbb{P}\left(2 q 9.9 .3\left(K 2^{N_{\xi} \xi}+1\right)>2^{N \delta_{2}}\right) \\
& =0 \text {, }
\end{aligned}
$$

The same proof more or less holds for $P_{6}$ and we obtain:

Lemma 9.9.6. If $N \geq N_{6}$, where $N_{6}:=\frac{1}{\delta_{2}} N_{\xi}$ and $N_{0}^{\prime \prime}=\delta_{2}^{-1}(\log$ q9.9.4 $+\log K+1)$, then $P_{6}=0$.

Both lemmas allow us to neglect the expressions $P_{5}$ and $P_{6}$ if $N$ is chosen large enough. So we can do all the calculations of Mytnik, Perkins and Sturm and end with the slightly different new definition of $N_{\xi_{1}}$ on page 1949:

$$
N_{\xi_{1}}=\max \left(N_{3}(\omega)+m,\left[\left(5 \delta_{1}^{-1}+\delta_{2}^{-1}\right) N_{\xi}(\omega)\right],\left[C_{0}\left(\xi, \delta_{1}\right) N_{\xi}\right], N_{0} \vee N_{0}^{\prime} \vee N_{0}^{\prime \prime} \vee N_{4}\right)
$$

The bound on $N_{\xi_{1}}$ can be obtained in the same way as in MPS06, here using $R=\left(5 \delta_{1}^{-1}+\delta_{2}^{-1}\right) \vee C_{0}$. This finishes the proof of Theorem 9.9.1.

\subsection{Incorporation of drifts}

In this section we want to present the analogue of Section 8 in MP11 which incorporates the drift $b$ in the SPDE (5.1). Remember that in the beginning of Section 9.2 we assumed that $b \equiv 0$ and did all the calculations to do the proof of Proposition 9.1.2. As we will not repeat the whole argument, we will just indicate the places where one needs to change the calculations already made to incorporate 
the drift. Of course, what needs to be changed is also explained. If $B(s, y):=$ $b\left(s, y, X^{1}(s, y)\right)-b\left(s, y, X^{2}(s, y)\right)$, then equation 9.16 becomes

$$
\begin{aligned}
u(t, x) & =\int_{0}^{t} \int_{\mathbb{R}^{q}} p_{t-s}(y-x) D(s, y) W(d y d s)+\int_{0}^{t} \int_{\mathbb{R}^{q}} p_{t-s}(y-x) B(s, y) d y d s \\
& =: u_{D}(t, x)+u_{B}(t, x) \quad \text { a.s. } \forall t>0, x \in \mathbb{R}^{q}
\end{aligned}
$$

and the Lipschitz condition on $B$ gives:

$$
|B(s, y)| \leq B|u(s, y)| .
$$

We define $u_{1, \delta}$ and $u_{2, \delta}$ in the same way as before and obtain in Lemma (9.2.1) (here using ordinary Fubini theorem, additionally):

$$
\begin{aligned}
& u_{1, \delta}(t, x)=\int_{0}^{(t-\delta)^{+}} \int_{\mathbb{R}^{q}} p_{t-s}(x-y) D(s, y) W(d s d y)+\int_{0}^{(t-\delta)^{+}} \int_{\mathbb{R}^{q}} p_{t-s}(x-y) B(s, y) d y d s \\
& u_{2, \delta}(t, x)=\int_{(t-\delta)^{+}}^{t} \int_{\mathbb{R}^{q}} p_{t-s}(x-y) D(s, y) W(d s d y)+\int_{(t-\delta)^{+}}^{t} \int_{\mathbb{R}^{q}} p_{t-s}(x-y) B(s, y) d y d s .
\end{aligned}
$$

Clearly, also the functions $G$ and $F$ (defined as before) now consist of two parts:

$$
\begin{aligned}
-\nabla G_{\delta}(s, t, x)= & F_{\delta}(s, t, x)=\int_{0}^{(s-\delta)^{+}} \nabla p_{(t \vee s)-r}(y-x) D(r, y) W(d r d y)+ \\
& +\int_{0}^{(s-\delta)^{+}} \nabla p_{(t \vee s)-r}(y-x) B(r, y) W(d r d y) \\
= & : F_{D, \delta}(s, t, x)+F_{B, \delta}(s, t, x) .
\end{aligned}
$$

For the induction step in Section 9.4 and the estimates in Section 9.5, we needed the four Propositions 9.4.11, 9.4.14, 9.4.17 and 9.4.18. Given Theorem 5.3.3 the proofs for the $D$-parts were already done. To do the same estimates on the $B$-parts we also require Theorem 5.3 .3 for the drift. As for the $B$-parts we are not confronted with stochastic integrals, calculations get considerably easier. We show the four propositions subsequently:

Proposition 9.10.1. Let $0 \leq m \leq \bar{m}+1$ and assume that $\left(P_{m}\right)$ holds. Then for all $n \in \mathbb{N}, \eta_{1} \in(0,1 / 2], \varepsilon_{0} \in(0,1), K \in \mathbb{N} \geq K_{1}, \lambda \in[0,1], \beta \in[0,1 / 2]$ there is an $N_{9.4 .11}=N_{9.4 .11}\left(\eta_{1}, K\right)(\omega) \in \mathbb{N} \geq 2$ almost surely such that for all $N \geq N_{9.4 .11}$, $(t, x) \in Z(N, n, K, \beta), s \leq t \leq t^{\prime}, s^{\prime} \leq t^{\prime} \leq T_{K}$ and $d\left((t, x)\left(t^{\prime}, x^{\prime}\right)\right)<2^{-N}$ and $\left|s-s^{\prime}\right|<N^{-1}$ it holds that

$$
\begin{aligned}
\mid F_{a_{n}^{\lambda}, l}(s, t, x) & -F_{a_{n}^{\lambda}, l}\left(s^{\prime}, t^{\prime}, x^{\prime}\right) \mid \leq 2^{-86} q^{-4}\left[\left|s^{\prime}-s\right|^{1 / 2} 2^{-N\left(1-\eta_{1}\right)}+\left|s^{\prime}-s\right|^{1-\eta_{1} / 2}\right. \\
& \left.+d^{1-\eta_{1}}\left[1+a_{n}^{-\lambda / 2(1+\alpha / 2)} 2^{-N \gamma \gamma_{m}}+a_{n}^{\beta \gamma}\left(a_{n}^{\lambda / 2} \vee 2^{-N}\right)^{\gamma}\right]\right], \quad l=1, \ldots, q .
\end{aligned}
$$


The proof is similar to the proof of Proposition 8.1 in [MP11.

Proof. We refer to the proof of Proposition 8.1 in [MP11] and only do the part which differs, that is the estimate for the second part $T_{2,2}$ of $T_{2}$ there. Using Lemma 9.4.3 with the actual $m$ of the proposition (instead of $m=0$ there), we obtain:

$$
\begin{aligned}
& T_{2,2} \leq \int_{0}^{s-a_{n}^{\lambda}} \int_{\mathbb{R}^{q}}\left|p_{t-r, 1}(y-x)-p_{t^{\prime}-r}\left(y-x^{\prime}\right)\right| B \sqrt{C[9.4 .3} e^{|y-x|} \\
& {\left[(\sqrt{t-r}+|y-x|) \vee 2^{-N}\right]^{\xi}\left[\left(\sqrt{a_{n}} \vee 2^{-N} \vee(\sqrt{t-r}+|y-x|)\right)^{\bar{\gamma}_{m}-1}+a_{n}^{\beta}\right]} \\
& \mathbb{1}\left(|y-x|<\left(t^{\prime}-r\right)^{1 / 2-\eta_{1} / 4} \vee 2\left|x-x^{\prime}\right|\right) d y d r \\
& \leq 32 B \sqrt{C[\overline{9.4 .3}} \int_{0}^{s-a_{n}^{\lambda}}\left[(t-r)^{\xi / 2\left(1-\eta_{1} / 2\right)}+2^{-N \xi}\right] d r \\
& \int_{\mathbb{R}^{q}}\left|p_{t-r, 1}(y-x)-p_{t^{\prime}-r}\left(y-x^{\prime}\right)\right| e^{|y-x|} \mathbb{1}(|y-x|<2(2 K+1)) d y \\
& {\left[\left(\sqrt{a_{n}} \vee 2^{-N} \vee(t-r)^{1 / 2-\eta_{1} / 4}\right)^{\bar{\gamma}_{m}-1}+a_{n}^{\beta}\right] .}
\end{aligned}
$$

Next, use Hölder inequality for the spatial integral and use Lemma 4.4 in [MP11 to obtain

$$
\begin{aligned}
T_{2,2} \leq c_{1}(K) & \sqrt{C_{9.4 .3}} \int_{0}^{s-a_{n}^{\lambda}}(t-r)^{-3 / 4}\left(1 \wedge \frac{d^{2}}{t-r}\right)^{1 / 2}\left[(t-r)^{1 / 2-\eta_{1} / 2}+\right. \\
+ & \left.2^{-N \xi}\left(a_{n}^{\frac{1}{2}\left(\gamma_{m}-1\right)}+2^{-N\left(\gamma_{m}-1\right)}+a_{n}^{\beta}\right)+2^{-N \xi}(t-r)^{\frac{1}{2}\left(1-\eta_{1} / 2\right)\left(\gamma_{m}-1\right)}\right] \\
\leq c_{2}(K) & \sqrt{C_{9.4 .3}} \int_{0}^{s-a_{n}^{\lambda}} d r(t-r)^{-1 / 4-\eta_{1} / 2}\left(1 \wedge \frac{d^{2}}{t-r}\right)^{1 / 2} \\
& +(t-r)^{-3 / 4}\left(1 \wedge \frac{d^{2}}{t-r}\right)^{1 / 2} 2^{-N \xi}\left(a_{n}^{\frac{1}{2}\left(\gamma_{m}-1\right)}+2^{-N\left(\gamma_{m}-1\right)}+a_{n}^{\beta}\right) \\
& +(t-r)^{\frac{1}{2}\left(\gamma_{m}-5 / 2\right)-\eta_{1} / 4}\left(1 \wedge \frac{d^{2}}{t-r}\right)^{1 / 2} 2^{-N \xi} .
\end{aligned}
$$


Next, we apply Lemma 4.1 of [MP11] with using (4.1) and (4.3) there:

$$
\begin{gathered}
T_{2,2} \leq c_{3}(K) \sqrt{C_{9.4 .3}}\left[\left(d^{2} \wedge t\right)^{1 / 2} t+\left(d^{2}\right)^{1 / 2}\left(d^{2} \vee\left(t-\left(s-a_{n}^{\lambda}\right)\right)\right)^{-1 / 4} 2^{-N \xi}\right. \\
\left(a_{n}^{\frac{1}{2}\left(\gamma_{m}-1\right)}+2^{-N\left(\gamma_{m}-1\right)}+a_{n}^{\beta}\right) \\
\left.+\left[\left(d^{2} \wedge t\right)^{1 / 2} t+\left(d^{2}\right)^{1 / 2}\left(d^{2} \vee\left(t-\left(s-a_{n}^{\lambda}\right)\right)\right)^{\frac{1}{2}\left(\gamma_{m}-3 / 2\right)-\eta_{1} / 4}\right] 2^{-N \xi}\right] \\
\leq c_{4}(K) \sqrt{C_{9.4 .3}} d^{1-\eta_{1} / 4}\left[1+a_{n}^{-\lambda / 4} 2^{-N}\left(a_{n}^{\frac{1}{2}\left(\gamma_{m}-1\right)}+2^{-N\left(\gamma_{m}-1\right)}+a_{n}^{\beta}\right)\right. \\
\left.+\left(a_{n}^{\lambda / 2}\right)^{\gamma_{m}-3 / 2-\eta_{1} / 2} 2^{-N}\right] \\
\leq c_{4}(K) \sqrt{C_{9.4 .3}} d^{1-\eta_{1} / 4}\left[1+a_{n}^{-\lambda / 4} 2^{-N \gamma_{m}}+a_{n}^{-\lambda / 4+\beta} 2^{-N}\right. \\
\left.+a_{n}^{-\lambda / 4} 2^{-N} a_{n}^{\frac{1}{2}\left(\gamma_{m}-1\right)}+a_{n}^{\lambda / 2\left(\gamma_{m}-3 / 2-\eta_{1} / 2\right)} 2^{-N}\right]
\end{gathered}
$$

Then, bound this expression further by elementary computations,

$$
\begin{aligned}
& T_{2,2} \leq c_{4}(K) \sqrt{C_{9.4 .3}} d^{1-\eta_{1} / 4}\left[1+a_{n}^{-\lambda / 2(1+\alpha / 2)}\left(2^{-N \gamma_{m}}+a_{n}^{\beta} 2^{-N}\right)\right. \\
&\left.\left(\mathbb{1}\left(a_{n}^{\lambda / 2} \leq 2^{-N}\right)+\mathbb{1}\left(a_{n}^{\lambda / 2}>2^{-N}\right)\right)\left[a_{n}^{-\lambda / 4} 2^{-N} a_{n}^{\frac{1}{2}\left(\gamma_{m}-1\right)}+a_{n}^{\lambda / 2\left(\gamma_{m}-3 / 2-\eta_{1} / 2\right)} 2^{-N}\right]\right] \\
& \leq c_{4}(K) \sqrt{C_{9.4 .3}} d^{1-\eta_{1} / 4}\left[1+a_{n}^{-\lambda / 2(1+\alpha / 2)}\left(2^{-N \gamma \gamma_{m}}+a_{n}^{\beta \gamma}\left(a_{n}^{\lambda / 2} \vee 2^{-N}\right)^{\gamma}\right.\right. \\
&\left.\left(a_{n}^{-\lambda / 2(1+\alpha / 2)} 2^{-N \gamma_{m}}+a_{n}^{-\lambda / 4} a_{n}^{\lambda / 2} a_{n}^{0}+a_{n}^{-\lambda / 2(1+\alpha / 2)} 2^{-N \gamma_{m}}+a_{n}^{0}\right)\right] .
\end{aligned}
$$

Finally, choose $N \overline{9.10 .1}$ in the appropriate way.

We need to investigate whether these estimates imply Proposition 9.4.11 and Proposition 9.4.14. The first is obvious and for the latter, observe that $d=0$ and

$$
\begin{aligned}
(t-s)^{1-\eta_{1} / 2} & =(t-s)^{\frac{1}{2}\left(1-\eta_{1}\right)} \sqrt{t-s} \leq(t-s)^{\frac{1}{2}\left(1-\eta_{1}\right)}\left(\sqrt{t-s} \vee \sqrt{a_{n}}\right) \\
& \leq(t-s)^{\frac{1}{2}\left(1-\eta_{1}\right)}\left(\sqrt{t-s} \vee \sqrt{a_{n}}\right)^{\gamma \tilde{\gamma}_{m}-1-\alpha / 2}
\end{aligned}
$$

Next we consider the analogue of Proposition 9.4.17

Proposition 9.10.2. Let $0 \leq m \leq \bar{m}+1$ and assume that $\left(P_{m}\right)$ holds. For any $n \in \mathbb{N}, \eta_{1} \in(0,1 / 2), \varepsilon_{0} \in(0,1), K \in \mathbb{N} \geq K_{1}, \lambda \in(0,1], \beta \in[0,1 / 2]$ there is a $N \overline{\text { 9.10.2 }}\left(K, \eta_{1}\right) \in \mathbb{N}$ a.s. such that for all $N \geq N \overline{\text { 9.10.2, }}(t, x) \in Z(N, n, K, \beta), s \leq t$, $\sqrt{t-s} \leq 2^{-N}$ it holds that

$$
\left|G_{a_{n}^{\lambda}, B}(s, t, x)-G_{a_{n}^{\lambda}, B}(t, t, x)\right| \leq 2^{-90} a_{n}^{-\varepsilon_{0}}(t-s)^{\left(1-\eta_{1}\right) / 2}\left[t-s+(t-s)^{1 / 2} 2^{-N}\right] .
$$

Proof. Assume $\xi=1-\eta_{1} / 4$ and $N \geq 2{ }^{91} \eta_{1}^{-1} N$ 9.4.3. The claim is trivial, if $\delta:=$ $a_{n}^{\lambda} \geq t$, so we assume that this is not the case. Then, by Lemma 9.4 .3 for $m=0$ 
observe that

$$
\begin{aligned}
\left|G_{a_{n}^{\lambda}, B}(s, t, x)-G_{a_{n}^{\lambda}, B}(t, t, x)\right|=\int_{(s-\delta)^{+}}^{t-\delta} \int_{\mathbb{R}^{q}} p_{t-r}(y-x) B(r, y) d y d r \\
\quad \leq B \sqrt{C_{9.4 .3}} \int_{(s-\delta)^{+}}^{t-\delta} \int_{\mathbb{R}^{q}} p_{t-r} e^{|y-x|}\left((\sqrt{t-r}+|y-x|) \vee 2^{-N}\right)^{\xi} d y d r \\
\leq c_{1}(K) \sqrt{C_{9.4 .3}} \int_{(s-\delta)^{+}}^{t-\delta}\left((t-r)^{\xi / 2}+2^{-N \xi}\right) d r \\
\left.\leq c_{1}(K) \sqrt{C_{9.4 .3}}(t-s)^{1+\xi / 2}+(t-s) 2^{-N \xi}\right) \\
\leq c_{1}(K) 2^{2 N_{1}}(t-s)^{\left(1-\eta_{1}\right) / 2}\left[(t-s)^{1-\eta_{1} / 4}+(t-s)^{1 / 2+\eta_{1} / 4} 2^{-N \xi}\right] \\
\leq c_{1}(K) 2^{2 N_{1}} 2^{-N \eta_{1} / 4}(t-s)^{\left(1-\eta_{1}\right) / 2} \\
\quad\left[(t-s)^{1-\eta_{1} / 4} 2^{N \eta_{1} / 4}+(t-s)^{1 / 2+\eta_{1} / 4} 2^{-N\left(1-\eta_{1} / 2\right)}\right] .
\end{aligned}
$$

Next we choose $N_{9.10 .2}=720 \eta_{1}^{-1} c_{1}(K) N_{1}(0, K, \xi)$ to obtain the claim.

Next show the analogue of Proposition 9.4.18.

Proposition 9.10.3. Let $0 \leq m \leq \bar{m}+1$ and assume that $\left(P_{m}\right)$ holds. Then for all $n \in \mathbb{N}, \eta_{1} \in\left(0, \frac{\alpha}{4} \wedge \frac{2-\alpha}{2}\right), \varepsilon_{0} \in(0,1), K \in \mathbb{N} \geq K_{1}, \lambda \in(0,1], \beta \in[0,1 / 2]$ there is $N_{9.10 .3}=N_{9.10 .3}\left(K, \eta_{1}\right) \in \mathbb{N}$ a.s. such that $\forall N \geq N_{9.10 .3},(t, x) \in Z(N, n, K, \beta)$, $t^{\prime} \leq T_{K}$ and $d:=d\left((t, x),\left(t^{\prime}, x^{\prime}\right)\right) \leq 2^{-N}$ we have

$$
\begin{aligned}
\left|u_{2, a_{n}^{\lambda}, B}(t, x)-u_{2, a_{n}^{\lambda}, B}\left(t^{\prime}, x^{\prime}\right)\right| \leq 2^{-96}[ & d^{1-\eta_{1}} a_{n}^{\frac{\lambda}{2}\left(\gamma \bar{\gamma}_{m}-\alpha / 2\right)} \\
& \left.+d^{(1-\alpha / 2)\left(1-\eta_{1} / 2\right)} 2^{-N \gamma}\left(a_{n}^{1 / 2} \vee 2^{-N}\right)^{\gamma\left(\bar{\gamma}_{m}-1\right)}\right] .
\end{aligned}
$$

Proof. We decompose the integral into four parts:

$$
\begin{aligned}
& \left|u_{2, a_{n}^{\lambda}, B}(t, x)-u_{2, a_{n}^{\lambda}, B}\left(t^{\prime}, x^{\prime}\right)\right| \leq \int_{\left(t-a_{n}^{\lambda}\right)^{+}}^{t \wedge\left(t^{\prime}-a_{n}^{\lambda}\right)^{+}} \int_{\mathbb{R}^{q}} p_{t-s}(x-y) B u(s, y) d y d s \\
& \quad+\int_{t^{\prime}-\left(a_{n}^{\lambda} \wedge\left(t^{\prime}-t\right)\right)}^{t^{\prime}} \int_{\mathbb{R}^{q}} p_{t^{\prime}-s}\left(y-x^{\prime}\right) B u(s, y) d y d s \\
& +\mathbb{1}\left(t^{\prime}-t<a_{n}^{\lambda}\right) \int_{t^{\prime}-a_{n}^{\lambda}}^{t} \int_{\mathbb{R}^{q}}\left(p_{t^{\prime}-s}\left(y-x^{\prime}\right)-p_{t-s}(y-x)\right) u(s, y) \\
& +\mathbb{1}\left(t^{\prime}-t<a_{n}^{\lambda}\right) \int_{t^{\prime}-a_{n}^{\lambda}}^{t} \int_{\mathbb{R}^{q}}\left(p_{t^{\prime}-s}\left(y-x^{\prime}\right)-p_{t-s}(y-x)\right) u(s, y) \\
& \quad T_{1}+T_{2}+T_{3}+T_{4} .
\end{aligned}
$$


We start calculating $T_{3}$ using (5.7), Hölder inequality and Lemma 9.6.4.

$$
\begin{aligned}
& T_{3} \leq \mathbb{1}\left(t^{\prime}-t<a_{n}^{\lambda}\right) \int_{t^{\prime}-a_{n}^{\lambda}}^{t} \int_{\mathbb{R}^{q}}\left(p_{t^{\prime}-s}\left(y-x^{\prime}\right)-p_{t-s}(y-x)\right) K e^{|y-x|} e^{K} \\
& \mathbb{1}\left(|y-x|>\left(t^{\prime}-r\right)^{\frac{1}{2}-\frac{\eta_{1}}{4}} \vee\left|x-x^{\prime}\right|\right) d y d s \\
& \leq \mathbb{1}\left(t^{\prime}-t<a_{n}^{\lambda}\right) \int_{t^{\prime}-a_{n}^{\lambda}}^{t}\left(\int_{\mathbb{R}^{q}}\left(p_{t^{\prime}-s}\left(y-x^{\prime}\right)-p_{t-s}(y-x)\right)^{2} e^{4|y-x|}\right. \\
& \left.\mathbb{1}\left(|y-x|>\left(t^{\prime}-r\right)^{\frac{1}{2}-\frac{\eta_{1}}{4}} \vee\left|x-x^{\prime}\right|\right) d y\right)^{1 / 2}\left(\int_{\mathbb{R}^{q}} e^{-2|y-x|} d y\right)^{1 / 2} d s \\
& \leq c_{1}(K) \mathbb{1}\left(t^{\prime}-t<a_{n}^{\lambda}\right) \int_{t^{\prime}-a_{n}^{\lambda}}^{t}(t-s)^{-1 / 2} \exp \left(-\frac{\eta_{1}\left(t^{\prime}-s\right)^{-\eta_{0} / 2}}{256}\right)\left[1 \wedge \frac{d}{t-s}\right]^{1-\eta_{1} / 2} d s .
\end{aligned}
$$

Now, continuing as in the proof of Lemma 9.4.6 here, and applying Lemma 4.1 (eq. $(4.2))$ in [MP11], allows to bound this by

$$
T_{3} \leq c_{2}(K)\left(d^{2} \wedge a_{n}^{\lambda}\right)^{1-\alpha / 2} d^{2}+\left(d^{2} \wedge a_{n}^{\lambda}\right)^{1-\eta_{1} / 4} a_{n}^{\lambda\left(2-\alpha / 2+\eta_{1} / 4\right)} .
$$

If we choose $N \overline{9.10 .3}=100 \eta_{1}^{-1}+\log c_{2}(K)$ we can bound this by

$$
T_{3} \leq 2^{-100} d^{1-\alpha / 2} 2^{-N \gamma} 2^{-N \gamma\left(\bar{\gamma}_{m}-1\right)}+d^{1-\eta_{1} / 4} a_{n}^{\lambda / 2\left(\gamma \bar{\gamma}_{m}-\alpha / 2\right)} .
$$

Next consider $T_{4}$. By Lemma 9.4.3 for $m=0$ and later Lemma 5.2 (a) of [MPS06] with an obvious extension we bound $T_{4}$ by

$$
\begin{aligned}
T_{4} \leq & B \sqrt{G \overline{9.4 .3}} \int_{t^{\prime}-a_{n}^{\lambda}}^{t} \mathbb{1}\left(t^{\prime}-t<a_{n}^{\lambda}\right) \int_{\mathbb{R}^{q}}\left(p_{t^{\prime}-s}\left(x^{\prime}-y\right)-p_{t-s}(x-y)\right) e^{|y-x|} \\
& \left(2^{-N} \vee\left(|t-s|^{1 / 2}+|y-x|\right)\right)^{\xi} \mathbb{1}\left(|y-x| \leq\left(t^{\prime}-s\right)^{1 / 2-\eta_{1} / 4} \vee\left|x-x^{\prime}\right|\right) d y d s \\
\leq & 2 B \sqrt{G \overline{9.4 .3}} \int_{t^{\prime}-a_{n}^{\lambda}}^{t} \mathbb{1}\left(t^{\prime}-t<a_{n}^{\lambda}\right) \int_{\mathbb{R}^{q}}\left(p_{t^{\prime}-s}\left(x^{\prime}-y\right)-p_{t-s}(x-y)\right) e^{|y-x|} d y \\
& \left(2^{-N \xi}+(t-s)^{\xi / 2}\right)^{1-\eta_{1} / 2} d s \\
& \leq 2 B c_{1}(K) \sqrt{C_{9.4 .3}} \int_{t^{\prime}-a_{n}^{\lambda}}^{t} \mathbb{1}\left(t^{\prime}-t<a_{n}^{\lambda}\right)\left(1 \wedge \frac{d^{2}}{t-s}\right)^{1 / 2} \\
& \left(2^{-N\left(1-\eta_{1}\right)}+(t-s)^{\frac{1}{2}\left(1-\eta_{1}\right)}\right) d s \\
& c_{2}(K) B \sqrt{C_{9.4 .3}}\left[\left(d^{2} \wedge a_{n}^{\lambda}\right)^{1 / 2} a_{n}^{\lambda / 2} 2^{-N\left(1-\eta_{1}\right)}+\left(d^{2} \wedge a_{n}^{\lambda}\right) a_{n}^{\lambda\left(1-\eta_{1} / 2\right)}\right] .
\end{aligned}
$$

Here we used Lemma 4.1 (a) of [MP11] twice. The first term can be bounded considering the two cases $a_{n}^{\lambda / 2} \leq 2^{-N}$ and $a_{n}^{\lambda / 2}>2^{-N}$ separately:

- If $a_{n}^{\lambda / 2} \leq 2^{-N}$, then $\left(d^{2} \wedge a_{n}^{\lambda}\right)^{1 / 2} a_{n}^{\lambda / 2} 2^{-N\left(1-\eta_{1}\right)} \leq d 2^{-2 N} \leq d^{1-\eta_{1} / 2} 2^{-2 N+\eta_{1} / 2}$, 
- If $a_{n}^{\lambda / 2}>2^{-N}$, then $\left(d^{2} \wedge a_{n}^{\lambda}\right)^{1 / 2} a_{n}^{\lambda / 2} 2^{-N\left(1-\eta_{1}\right)} \leq d a_{n}^{\lambda / 2} a_{n}^{\lambda / 2} 2^{N \eta_{1}} \leq d^{1-\eta_{1}} a_{n}^{\lambda}$.

So we can bound

$$
\begin{aligned}
T_{4} & \leq c_{2}(K) B \sqrt{C_{9.4 .3}} d^{1-\eta_{1}}\left[2^{-2 N}+a_{n}^{\lambda}+d^{\eta_{1}} a_{n}^{2 \lambda-\eta_{1} / 2}\right] \\
& \leq 2 c_{2}(K) B \sqrt{C_{\overline{9.4 .3}}} d^{1-\eta_{1}}\left[2^{-N \gamma}\left(a_{n} \vee 2^{-N}\right)^{\gamma\left(\bar{\gamma}_{m}-1\right)}+a_{n}^{\frac{\lambda}{2}\left(\gamma \bar{\gamma}_{m}-1\right)}\right] \\
& \leq 2^{-100} d^{1-\eta_{1}}\left[2^{-N \gamma}\left(a_{n} \vee 2^{-N}\right)^{\gamma\left(\bar{\gamma}_{m}-1\right)}+a_{n}^{\frac{\lambda}{2}\left(\gamma \bar{\gamma}_{m}-\alpha / 2\right)}\right] .
\end{aligned}
$$

To treat $T_{2}$ we use again Lemma 9.4 .3 for $m=0$ :

$$
\begin{aligned}
T_{2} & \left.\leq \int_{t^{\prime}-\left(a_{n}^{\lambda} \wedge\left(t^{\prime}-t\right)\right)}^{t^{\prime}} \int_{\mathbb{R}^{q}} p_{t^{\prime}-s}\left(y-x^{\prime}\right) B e^{|y-x|} \sqrt{C_{9.4 .3}}\left(|y-x|+|t-s|^{1 / 2}\right) \vee 2^{-N}\right)^{\xi} d y d s \\
& \leq c_{1} \sqrt{C_{9.4 .3}} \int_{t^{\prime}-\left(a_{n}^{\lambda} \wedge\left(t^{\prime}-t\right)\right)}^{t^{\prime}}\left(\left(t^{\prime}-s\right)^{\xi / 2}+2^{-N \xi}\right) d s \\
& \leq c_{2} \sqrt{C_{9.4 .3}}\left(\left(a_{n}^{\lambda} \wedge d^{2}\right)^{1+\xi / 2}+2^{-N \xi}\left(a_{n}^{\lambda} \wedge d^{2}\right)\right] \\
& \leq c_{2} \sqrt{C_{\overline{9.4 .3}}} 2^{-N \eta_{1} / 4}\left[a_{n}^{\lambda} d^{1-\eta_{1} / 4} 2^{N \eta_{1} / 4}+2^{N \eta_{1} / 2} d 2^{-N \gamma}\left(a_{n}^{1 / 2} \vee 2^{-N}\right)^{\gamma(\bar{\gamma}-1)}\right. \\
& \leq 2^{-100}\left[d^{1-\eta_{1}} a_{n}^{\frac{\lambda}{2}\left(\gamma \bar{\gamma}_{m}-\alpha / 2\right)}+d^{(1-\alpha / 2)\left(1-\eta_{1} / 2\right)} 2^{-N \gamma}\left(a_{n}^{1 / 2} \vee 2^{-N}\right)^{\gamma\left(\bar{\gamma}_{m}-1\right)}\right],
\end{aligned}
$$

where we used Lemma 4.1 (a) of [MP11]. Finally, bound $T_{1}$ using Lemma 9.4 .3 for $m=0$ :

$$
\begin{aligned}
T_{1} & \leq \int_{\left(t-a_{n}^{\lambda}\right)^{+}}^{t \wedge\left(t^{\prime}-a_{n}^{\lambda}\right)^{+}} \int_{\mathbb{R}^{q}} p_{t-s}(x-y) e^{|x-y|} \sqrt{C_{\overline{9.4 .3}}}\left(\left(|y-x|+|t-s|^{1 / 2}\right) \vee 2^{-N}\right)^{\xi} d y d s \\
& \leq c_{1} \sqrt{C_{9.4 .3}} \int_{\left(t-a_{n}^{\lambda}\right)^{+}}^{t \wedge\left(t^{\prime}-a_{n}^{\lambda}\right)^{+}}\left((t-s)^{\xi / 2}+2^{-N \xi}\right) \\
& \leq c_{2} \sqrt{C_{9.4 .3}}\left[2^{-N \xi}\left(\left|t^{\prime}-t\right| \wedge a_{n}^{\lambda}\right)+\left(\left|t^{\prime}-t\right| \wedge a_{n}^{\lambda}\right)^{1+\xi / 2}\right],
\end{aligned}
$$

where we used Lemma 4.1 (a) of [MP11]. Now, proceed as for $T_{2}$ to finish the proof.

\subsection{An integral estimate}

There is another lemma. The proof can be found on page 1929 of [MPS06] but we include it for completeness.

Lemma 9.11.1. For $x \in \mathbb{R}^{q}, n \geq 2$ and functions $\Phi$ as introduced before (9.6), there is a constant $99.11 .1=9.11 .1(\alpha, q,\|\Phi\|)$ such that

$$
\int_{\mathbb{R}^{2 q}} d w d z \Phi_{x}^{m_{n+1}}(w) \Phi_{x}^{m_{n+1}}(z)\left(|w-z|^{-\alpha}+1\right) \leq \text { 9.11.1 } m_{n+1}^{\alpha} .
$$


Proof. Note that for fixed $x \in \mathbb{R}^{q}$, it holds by triangle inequality that

$$
\begin{aligned}
\Phi_{x}^{m_{n}}(w) \Phi_{x}^{m_{n}}(z) & \leq m_{n}^{q}\|\Phi\|_{\infty} \mathbb{1}_{B^{q}(0,1)}\left(m_{n}(x-w)\right) m_{n}^{q}\|\Phi\|_{\infty} \mathbb{1}_{B^{q}(0,1)}\left(m_{n}(x-z)\right) \\
& \leq m_{n}^{2 q}\|\Phi\|_{\infty}^{2} \mathbb{1}_{B^{q}(0,1)}\left(m_{n}(x-w)\right) \mathbb{1}_{B^{q}(0,1)}\left(\frac{1}{2} m_{n}(w-z)\right)
\end{aligned}
$$

Hence,

$$
\begin{aligned}
& \int_{\mathbb{R}^{2 q}} d w d z \Phi_{x}^{m_{n}}(z) \Phi_{x}^{m_{n}}(w)\left(|w-z|^{-\alpha}+1\right) \\
& \quad \leq c \int_{\mathbb{R}^{2 q}} d w d z m_{n}^{2 q} \mathbb{1}_{B^{q}(0,1)}\left(m_{n}(x-w)\right) \mathbb{1}_{B^{q}(0,1)}\left(\frac{1}{2} m_{n}(w-z)\right)\left(|w-z|^{-\alpha}+1\right) .
\end{aligned}
$$

Since for $|w-z|<2 m_{n}^{-1}:|w-z|^{-\alpha}>\left(2 m_{n}^{-1}\right)^{-\alpha}>1$ (for $n \geq 3$ ) and introducing $y:=w-z$ the integral gets bounded by

$$
\begin{aligned}
& \leq 2 c \int_{\mathbb{R}^{2 q}} d w d y m_{n}^{2 q} \mathbb{1}_{B^{q}(0,1)}\left(m_{n}(x-w)\right) \mathbb{1}_{B^{q}(0,1)}\left(\frac{1}{2} m_{n} y\right)|y|^{-\alpha} \\
& =2 c \int_{\mathbb{R}^{q}} d w m_{n}^{q} \mathbb{1}_{B^{q}(0,1)}\left(m_{n}(x-w)\right) \int_{\mathbb{R}^{q}} d \tilde{y} \mathbb{1}_{B^{q}(0,1)}(\tilde{y})\left|\frac{2}{m_{n}} \tilde{y}\right|^{-\alpha} \quad\left(\text { for } \tilde{y}=\frac{m_{n}}{2} y\right) \\
& \leq c\left(\alpha, q,\|\Phi\|_{\infty}\right) m_{n}^{\alpha},
\end{aligned}
$$

since $\alpha<q$. 


\section{The Compact Support Property}

In this chapter we show Theorem 5.4.1 and Proposition 5.4.2. In Section 10.1 we show the proof of Theorem 5.4.1 which relies on Proposition 10.1.1. The proof of this proposition is postponed to Section 10.2. In Section 10.3 we provide the proof of Proposition 5.4.2. Both of the preceding sections will rely on a lower bound on a quadratic variation term. This lower bound is given by a real analysis lemma in Section 10.4 .

We start recalling the setup and some definitions. Note that we are considering a weak non-negative solution of

$$
\partial_{t} u(t, x)=\frac{1}{2} \partial_{x}^{2}(t, x)+\sigma(u(t, x)) \dot{W}(t, x),
$$

where $\sigma(u) \geq \sigma_{0} u^{\gamma}$ for a certain $\gamma \in(0,1)$ and a colored noise $\dot{W}=\dot{W}^{k}$ with Rieszkernel $k(x, y)=|x-y|^{-\alpha}$. It is required to have $\alpha \in(0,1)$ and $\sigma(u) \leq c(1+|u|)$ to have existence of a weak solution, i.e. for any $\phi \in C_{c}^{\infty}(\mathbb{R})$ we almost surely have

$$
\begin{array}{r}
\int_{\mathbb{R}} \phi(x) u(t, x) d x=\int_{\mathbb{R}} \phi(x) u_{0}(x) d x+\int_{0}^{t} \int_{\mathbb{R}} \frac{1}{2} \phi^{\prime \prime}(x) u(s, x) d x d s \\
+\int_{0}^{t} \int_{\mathbb{R}} \phi(x) u(s, x) W(d s d y) .
\end{array}
$$

The existence of a weak solution implies

$$
u(t, x)=\int_{\mathbb{R}} u_{0}(y) p_{t}(x-y) d y+\int_{0}^{t} \int_{\mathbb{R}} p_{t-s}(x-y) \sigma(u(s, y)) W(d s d y),
$$

where $p_{r}(z)=(2 \pi r)^{-1 / 2} \exp \left(-z^{2} / 2 r\right), r>0, z \in \mathbb{R}$ is the one-dimensional heat kernel. We write

$$
S\left(u_{t}\right)=\sup \operatorname{supp}(u(t, \cdot)) \text {. }
$$

\subsection{Proof of Theorem 5.4 .1}

We give two results which we prove later. They allow to do the proof of Theorem 5.4.1. The first result tells us that if $y$ is chosen far enough to the right of the initial support, then in a short time interval the probability that mass can be transported 
to $y$ is small. This first result is the core of the argument. Recall the definition of $h(r)=\sqrt{r \log (1 / r)}, r \in\left(0, e^{-1}\right)$

Proposition 10.1.1. Assume $u_{0}(x)=0$ for all $x \geq x_{0}$. There is a constant $\overline{10.1 .1}<$ $\infty$ such that for $c=16+132(1-\gamma)^{-1}$, it holds for given $t \in\left(0, e^{-1}\right)$ and $y \geq \operatorname{ch}(t)$ that

$$
\mathbb{P}\left(\int_{0}^{t} u\left(s, x_{0}+y\right) d s>0\right)<q_{10.1 .1} t^{2} .
$$

The second result is some type of lower-semicontinuity of $s \mapsto S\left(u_{s}\right)$, the function defined in 10.4.

Lemma 10.1.2. Assume that $\operatorname{supp}\left(u_{0}\right)$ is compact. Almost surely it holds that $s \mapsto S\left(u_{s}\right)$ is lower-semicontinuous in the sense that almost surely

$$
\lim _{\varepsilon \rightarrow 0+} S\left(u_{s-\varepsilon}\right) \geq S\left(u_{s}\right) \forall s>0 .
$$

A proof of Proposition 10.1.1 and Lemma 10.1.2 is given in Section 10.2. We can follow standard methods combining these two results for the next proof.

Proof of Theorem 5.4.1. For $c=16+132(1-\gamma)^{-1}$ let for $n \in \mathbb{N}, n \geq 2$,

$$
B_{n, j}=\left\{S\left(u_{j 2^{-n}}\right)>S\left(u_{(j-1) 2^{-n}}\right)+c h\left(2^{-n}\right)\right\}, j \in \mathbb{N} .
$$

And set

$$
\begin{aligned}
A_{n} & =\bigcup_{j=1}^{n 2^{n}} B_{n, j} \\
& =\bigcup_{j=1}^{n 2^{n}}\left\{S\left(u_{j 2^{-n}}\right)>S\left(u_{(j-1) 2^{-n}}\right)+\operatorname{ch}\left(2^{-n}\right), S\left(u_{k 2^{-n}}\right) \leq S\left(u_{(k-1) 2^{-n}}\right)+\operatorname{ch}\left(2^{-n}\right)\right. \\
& \forall 1 \leq k \leq j-1\} .
\end{aligned}
$$

If $\omega$ is in $B_{n, j}$, then $S\left(u_{(j-1) 2^{-n}}\right)<S\left(u_{0}\right)+(j-1) \operatorname{ch}\left(2^{-n}\right)$. Then, by Proposition 10.1.1 we get for $x_{0}=S\left(u_{0}\right)+(j-1) h\left(2^{-n}\right), y=c h(t)$ and using the Markovproperty of the solution at time $(j-1) 2^{-n}$, that $\mathbb{P}\left(B_{n, j}\right) \leq q 10.1 .1\left(\gamma,\left\|u_{(j-1) 2^{-n}}\right\|\right) 2^{-2 n}$.

$$
\mathbb{P}\left(A_{n}\right) \leq \frac{q_{10.1 .1}}{n 2^{n}} \sum_{j=1}\left(2^{-n}\right)^{2} \leq q_{10.1 .1} n 2^{-n} .
$$

This is summable in $n \in \mathbb{N}$. So, by Borel-Cantelli we know that $\mathbb{P}$-a.s. there is a $N(\omega)<\infty$ such that

$$
\forall n \geq N(\omega): S\left(u_{j 2^{-n}}\right) \leq S\left(u_{(j-1) 2^{-n}}\right)+c h\left(2^{-n}\right) \forall 1 \leq j \leq n 2^{n} .
$$


Then, we can do the proof as in Per02] for his Theorem III.1.3: Let w.l.o.g. $N=$ $N(\omega) \geq\left(\log \frac{25}{18}\right)^{-1}$. For $0 \leq r<s<N$ and $0<s-r<2^{-N}$ choose $n \geq N$ s.t. $2^{-n-1}<s-r \leq 2^{-n}$ and define $s_{k}=\sum_{l=-\infty}^{k} j_{l} 2^{-l} \nearrow s$ and $r_{k}=\sum_{l=-\infty}^{k} i_{l} 2^{-l} \nearrow r$, dyadic approximations of $s$ and $r$. One easily shows that $j_{l}, i_{l} \in\{0,1\}, l \geq 1$ and so we obtain by Lemma 10.1.2 using 10.5:

$$
\begin{aligned}
S\left(u_{s}\right)-S\left(u_{s_{n}}\right) & \leq \sum_{k=n+1}^{\infty}\left|S\left(u_{s_{k}}\right)-S\left(u_{s_{k-1}}\right)\right| \\
& \leq \sum_{k=n+1}^{\infty} j_{k} \operatorname{ch}\left(2^{-k}\right) \leq c^{\prime} h\left(2^{-n}\right) \leq 2 c^{\prime} h(s-r) .
\end{aligned}
$$

where $c^{\prime}=c\left(1-\frac{1}{\sqrt{2}}\right)^{-2} \leq 6 c$. The same estimate holds for $S\left(u_{r}\right)$ :

$$
S\left(u_{r}\right)-S\left(u_{r_{n}}\right) \leq c^{\prime} h\left(2^{-n}\right) \leq 2 c^{\prime} h(s-r) .
$$

And similarly by 10.5 , we get

$$
S\left(u_{s_{n}}\right)-S\left(u_{r_{n}}\right) \leq \operatorname{ch}\left(2^{-n}\right) \leq 2 \operatorname{ch}(s-r) .
$$

Putting that all together we obtain

$$
S\left(u_{s}\right)-S\left(u_{r}\right) \leq 2(12 c+c) h(s-r)=26\left(16+132(1-\gamma)^{-1}\right) h(s-r) .
$$

\subsection{Proof of Proposition 10.1.1}

The proof of Proposition 10.1.1 is obtained in several steps. First we give Lemma 10.2.1 which is analogous to Lemma 2.1 of [Kry97. It will allow us to bound the accumulated mass in a certain point $x>0$ via the mass traversing the point $x_{0}=0$ plus a second expression. Then, a bound on the mass traversing the point $x_{0}$ is given in Lemma 10.2.3. Finally, an iterative procedure similar to the one described in the heuristics in Section 5.4 allows to derive Proposition 10.1.1.

Let $\beta=\frac{1}{3}$.

Lemma 10.2.1. Let $T \geq 0$ be a stopping time. Assume $u_{0}(x)=0$ for all $x \geq 0$.

(a) For $A_{T}=\left\{\omega: \int_{0}^{T} u(s, 0) d s=0\right\}$ and $B_{T}=\{\omega: u(s, x)=0$ for all $0 \leq s \leq$ $T, x>0\}$, it holds that $\mathbb{P}\left(A_{T} \cap B_{T}^{c}\right)=0$. 
(b) If $\gamma \in(1 / 2,1):$ For any $K>\mathbb{E}\left[H^{16 / \beta}\right], r \in(0,1), M=(2 \beta \gamma+2) /(\beta+1)$ and $\nu=M / 3$, there is a constant $410.2 .1=110.2 .1\left(c_{k}, K\right)$ such that:

$$
\begin{array}{r}
\frac{1}{r} \int_{r}^{2 r} d x \mathbb{P}\left(\int_{0}^{T} u(s, x) d s \geq p\right) \leq \mathbb{P}\left(\int_{0}^{T} u(s, 0) d s \geq q\right)+ \\
\\
110.2 .1 r^{-5 \nu}\left(\frac{q^{2 / M}}{p}\right)^{\nu} .
\end{array}
$$

(c) If $\gamma \in(0,1 / 2]:$ For any $K>\mathbb{E}\left[H^{16 / \beta}\right], r \in(0,1), M=(2 \beta \gamma+2) /(\beta+1)$, there is a constant $q_{10.2 .1}=q_{10.2 .1}\left(c_{k}, K\right)$ such that:

$$
\begin{array}{r}
\frac{1}{r} \int_{r}^{2 r} d x \mathbb{P}\left(\int_{0}^{T} u(s, x) d s \geq p\right) \leq \mathbb{P}\left(\int_{0}^{T} u(s, 0) d s \geq q\right)+ \\
410.2 .1 r^{-5 / 2}\left(\frac{q^{2 / M}}{p}\right)^{\frac{1}{2}} .
\end{array}
$$

In order to prove the result we use Theorem 5.2.1 and the definition of the Hölder constant $H=H_{K}$ in that theorem which is in $L^{p}$ for any $p>0, K>0$. Since $1-\alpha / 2>1 / 3$ we can assume that we have Hölder continuity of order $\beta=\frac{1}{3}$. Hölder continuity is meant in the sense of (5.8). Additionally, we also require an integral estimate which is given in Section 10.4. Before starting with the proof we will give the following Lemma 3.1 from Kry97:

Lemma 10.2.2. The weak formulation (10.2) holds for any continuous function $\phi$ with compact support such that the generalised function $\phi^{\prime \prime}$ is a finite measure on the Borel $\sigma$-field on $\mathbb{R}$.

In Kry97 it is proven in the white noise setting, however its proof also applies to the colored noise setting. It requires an application of dominated convergence similar to the first steps of Proposition 5.4.2, which we will see later. Now we can do the proof of Lemma 10.2 .1 .

Proof. We first show (b) and (a) in the case $\gamma>\frac{1}{2}$. Let

$$
T_{K}=T \wedge \inf \left\{t>0: \int_{0}^{\infty} u(t, x) d x>K\right\} .
$$

Since $u \in C\left(\mathbb{R}_{+}, C_{\text {rap }}\right)$, we have that $T_{K} \nearrow T$ almost surely for $K \rightarrow \infty$. So, if we show (b) for the stopping time $T_{K}$, then it follows for $T$ by monotone convergence. 
If we show the claim in (a) for $T_{K}$, then it also follows for $T$, since

$$
\begin{aligned}
\left\{\int_{0}^{T} u(s, 0) d s=0,\right. & u(s, x)=0,0 \leq s \leq T, x>0\} \\
& =\bigcap_{K}\left\{\int_{0}^{T_{K}} u(s, 0) d s=0, u(s, x)=0,0 \leq s \leq T_{K}, x>0\right\} .
\end{aligned}
$$

Then, let us omit this dependence on $K$ and write $T$ in the proof, but assuming the bound on the integral of $u$. Let $\psi \in C_{c}^{\infty}(\mathbb{R})$ be non-negative with $\psi(0)=1$ and let $\psi_{n}(x)=\psi(x / n), x \in \mathbb{R}, n \in \mathbb{N}$. Apply Lemma 10.2 .2 to the function $\zeta(x)=$ $(x \vee 0) \psi_{n}(x)$ to obtain

$$
\int_{0}^{\infty} x \psi_{n}(x) u(t, x) d x=\int_{0}^{t} u(s, 0) d s+A_{t}^{n}+m_{t}^{n},
$$

for $t \leq T$, since $u_{0}(x)=0$ for all $x>0$. Here,

$$
A_{t}^{n}=n^{-1} \int_{0}^{t} \int_{0}^{\infty}\left(2 \psi^{\prime}(x)+n^{-1} x \psi^{\prime \prime}(x)\right) u(s, x) d x d s
$$

and $m^{n}$ is a continuous local martingale with quadratic variation

$$
\left\langle m^{n}\right\rangle_{t}=\int_{0}^{t} \int_{0}^{\infty} x \psi_{n}(x) y \psi_{n}(y) u(s, x)^{\gamma} u(s, y)^{\gamma} k(x, y) d x d y d s .
$$

Since by Theorem 5.2 .1 we have $\mathbb{E}\left[\sup _{t \leq T} \int_{\mathbb{R}}(1+x) u(t, x) d x\right]<\infty$, we have

$$
\begin{aligned}
& \lim _{n \rightarrow \infty} \sup _{t \leq T}\left|A_{t}^{n}\right| \\
& \quad \leq \lim _{n \rightarrow \infty} \sup _{t \leq T} t\left[n^{-1} 2\left\|\psi^{\prime}\right\| \int_{0}^{\infty} u(t, x) d x+\left\|\psi^{\prime \prime}\right\| \int_{0}^{\infty} x u(t, x) d x\right]=0,
\end{aligned}
$$

in $L^{1}$ and almost surely. By non-negativity of the LHS in 10.6), we obtain for $\xi_{t}^{n}=\int_{0}^{t} u(s, 0) d s+A_{t}^{n}$ :

$$
0 \leq \xi_{t}^{n}+m_{t}^{n}
$$

Consider the negative part $\left(m_{t}^{n}\right)_{-}=-\left(m_{t}^{n} \wedge 0\right)$ of $m_{t}^{n}$. Since $\xi^{n}$ is increasing, $\left(m_{s}^{n}\right)_{-} \leq \xi_{t}^{n}$, for any $s \leq t$, so also for any stopping times $\sigma, \tau \leq T$ replacing $s$ and $t$. Taking expectations, we obtain

$$
\mathbb{E}\left[\left(m_{\sigma \wedge \tau}^{n}\right)_{-}\right] \leq \mathbb{E}\left[\xi_{\tau}^{n}\right] .
$$

As $m^{n}$ are local martingales on $[0, T]$, there must be a sequence of stopping times $\sigma_{i} \nearrow \infty$, such that $\left(m_{t \wedge \sigma_{i}}^{n}\right)$ are martingales. By the optional sampling theorem (see 
Proposition 3.2.5, we know that also $\left(m_{t \wedge \sigma_{i} \wedge \tau}^{n}\right)$ are continuous martingales and hence,

$$
\mathbb{E}\left[\left(m_{\sigma_{i} \wedge \tau}^{n}\right)_{+}\right]=\mathbb{E}\left[\left(m_{\sigma_{i} \wedge \tau}^{n}\right)_{-}\right] \leq \mathbb{E}\left[\xi_{\tau}^{n}\right],
$$

the last inequality by 10.8$)$. So, $\mathbb{E}\left[\left|m_{\sigma_{i} \wedge \tau}^{n}\right|\right] \leq 2 \mathbb{E}\left[\xi_{\tau}^{n}\right]$. Letting $i \rightarrow \infty$, Fatou's lemma allows to get rid of the dependence on $\sigma_{i}$ to obtain:

$$
\mathbb{E}\left[\left|m_{\tau}^{n}\right|\right] \leq 2 \mathbb{E}\left[\xi_{\tau}^{n}\right] .
$$

Let us apply [RY91]'s Lemma IV.4.7 with $\nu^{\prime} \in(0,1)$ for the processes stopped at $\tau$ to get:

$$
\mathbb{E}\left[\sup _{t \leq \tau}\left|m_{t}^{n}\right|^{\nu^{\prime}}\right] \leq c\left(\nu^{\prime}\right) \mathbb{E}\left[\sup _{t \leq \tau}\left|\xi_{t}^{n}\right|^{\nu^{\prime}}\right] .
$$

Using the lower bound of the BDG-inequality (Theorem 3.2.9) for the continuous local martingale $m^{n}$ we obtain for a constant $c$ depending on $\nu^{\prime}$ :

$$
\mathbb{E}\left(\int_{0}^{\tau} \int_{0}^{\infty} \int_{0}^{\infty} x \psi_{n}(x) y \psi_{n}(y) u(s, x)^{\gamma} u(s, y)^{\gamma} k(x, y) d x d y d s\right)^{\nu^{\prime} / 2} \leq c \mathbb{E}\left[\sup _{t \leq \tau}\left(\xi_{t}^{n}\right)^{\nu^{\prime}}\right] .
$$

Letting $n \rightarrow \infty$, obtain by 10.7) and the monotonicity of $\xi_{t}=\int_{0}^{t} u(s, 0) d s$,

$$
\mathbb{E}\left(\int_{0}^{\tau} \int_{0}^{\infty} \int_{0}^{\infty} x y u(s, x)^{\gamma} u(s, y)^{\gamma} k(x, y) d x d y d s\right)^{\nu^{\prime} / 2} \leq c \mathbb{E}\left[\xi_{\tau}^{\nu^{\prime}}\right]
$$

This shows (a) of Lemma 10.2.1, since if the right hand side of 10.10$)$ is zero, the left hand side also is and the non-negative values of $u$ need to be zero. Set

$$
\kappa=T \wedge \inf \left\{r>0: \xi_{r} \geq q\right\} .
$$

By the inequalities of Markov and Jensen (twice) with $M \in(1,2)$ and $\nu=M / 3$,

$$
\begin{aligned}
& \frac{1}{r} \int_{r}^{2 r} d x \mathbb{P}\left(\int_{0}^{\kappa} u(s, x) d s>p\right) \leq p^{-\nu} \mathbb{E}\left[\frac{1}{r} \int_{r}^{2 r}\left(\int_{0}^{\kappa} u(s, x) d s\right)^{\nu} d x\right] \\
& \quad \leq p^{-\nu} \mathbb{E}\left[\left(r^{-1} \int_{r}^{2 r} \int_{0}^{\kappa} u(s, x) d s d x\right)^{\nu}\right] \\
& \quad \leq p^{-\nu} K^{\nu(1-1 / M)} \mathbb{E}\left[\left(\int_{0}^{\kappa} d s\left(r^{-1} \int_{r}^{2 r} d x u(s, x)\right)^{M}\right)^{\nu / M}\right]
\end{aligned}
$$

where we used $\kappa \leq K$. Here is the trick of the whole argument: Decompose $\Omega=$ $\{\kappa \leq t\} \cup\{\kappa>t\}$. Then, we have for $q>0$ :

$$
\begin{aligned}
& \frac{1}{r} \int_{r}^{2 r} d x \mathbb{P}\left(\int_{0}^{t} u(s, x) d s>p\right) \leq \mathbb{P}\left(\int_{0}^{t} u(s, 0) d s \geq q\right) \\
& +p^{-\nu} r^{-\nu} K^{\nu(1-1 / M)} \mathbb{E}\left[\left(\int_{0}^{\kappa} d s\left(\int_{r}^{2 r} d x u(s, x)\right)^{M}\right)^{\nu / M}\right]
\end{aligned}
$$


where we trivially estimated the first probability by $\mathbb{P}(\kappa \leq t)$. If we choose $M=$ $(2 \beta \gamma+2) /(\beta+1)<2$ and apply Lemma 10.4 .2 (with $q=1$, see the remark after the lemma), we can write

$$
\begin{gathered}
\left(\int_{r}^{2 r} d x u(s, x)\right)^{M} \leq c^{-1}\left(c_{k}\right) K^{2} r^{\alpha}\left[H^{1 / \beta} \vee r^{-1} \int_{r<x<2 r} u(s, x) d x\right]^{2} \\
\int_{\mathbb{R}^{2}} u(s, w)^{\gamma} u(s, z)^{\gamma} k(w, z) \mathbb{1}_{\{r<w, z<2 r\}} d w d z \\
\leq c K^{4} r^{\alpha-4} H^{2 / \beta} \\
\int_{\mathbb{R}^{2}} w u(s, w)^{\gamma} z u(s, z)^{\gamma} k(w, z) \mathbb{1}_{\{r<w, z<2 r\}} d w d z \\
\leq c K^{4} r^{\alpha-4} H^{2 / \beta} \int_{0}^{\infty} \int_{0}^{\infty} x y \sigma(u(s, x)) \sigma(u(s, y)) k(x, y) d x d y
\end{gathered}
$$

where we use $t \leq T_{K}$ and $\sigma(u) \geq \sigma_{0} u^{\gamma}$. Put this back in 10.11); with the Hölder inequality for $a=1 / 2+M / 4 \nu$, we obtain

$$
\begin{aligned}
\frac{1}{r} \int_{r}^{2 r} d x & \mathbb{P}\left(\int_{0}^{t} u(s, x) d s \geq p\right) \leq \mathbb{P}\left(\int_{0}^{t} u(s, 0) d s \geq q\right)+c K^{5} p^{-\nu} r^{-\nu(1-\alpha / M+4 / M)} \\
& \mathbb{E}\left[H^{2 \nu / \beta M}\left(\int_{0}^{\kappa} \int_{0}^{\infty} \int_{0}^{\infty} x y \sigma(u(s, x)) \sigma(u(s, y)) k(x, y) d x d y d s\right)^{\nu / M}\right] \\
\leq & \mathbb{P}\left(\int_{0}^{t} u(s, 0) d s \geq q\right)+c K^{5} p^{-\nu} r^{-\nu(1-\alpha / M+4 / M)}\left(\mathbb{E}\left[H^{2 a \nu / \beta M(a-1)}\right]\right)^{(a-1) / a} \\
\leq & \mathbb{P}\left(\int_{0}^{t} u(s, 0) d s \geq q\right)+c K^{6} p^{-\nu} r^{-5 \nu}\left(\mathbb{E}\left[\xi_{\kappa}^{2 a \nu / M}\right]\right)^{1 / a} \quad\left(\nu^{\prime}=a \nu / M\right) \\
\leq & \mathbb{P}\left(\int_{0}^{t} u(s, 0) d s \geq q\right)+c K^{6} r^{-5 \nu}\left(\frac{q^{2 / M}}{p}\right)^{\nu},
\end{aligned}
$$

where we used the bound $\mathbb{E}\left(H^{16 / \beta}\right) \leq K$ and the definitions of $\xi$ and $\kappa$.

The proof in the case $\gamma \leq \frac{1}{2}$ is even easier. We use the same $M$, but change $\nu:=\frac{1}{2}$.

There is an estimate on the first expression of Lemma 10.2.1

Lemma 10.2.3. Assume that $u_{0}(x)=0$ for all $x>0, t \in\left(0, e^{-1}\right)$ and $\delta \in(0,1)$. If for $c>0$ we have $y>c \sqrt{t \log (1 / t)}$, then

$$
\mathbb{P}\left(\int_{0}^{t} u(s, y) d s \geq \xi\right) \leq \delta^{-1 / 2}\left\|u_{0}\right\|_{\infty} \xi^{-1} t^{1+(1-\delta) c^{2} / 2}
$$


Proof. By Markov's inequality and Fubini's theorem, we obtain

$$
\mathbb{P}\left(\int_{0}^{t} u(s, y) d s \geq \xi\right) \leq \xi^{-1} \int_{0}^{t} \mathbb{E}[u(s, y)] d s .
$$

Let us note that for $y$ as in the lemma and $z \leq 0,0 \leq s \leq t$, we have

$$
\frac{|y-z|^{2}}{s} \geq \frac{y^{2}}{t}>c^{2} \log (1 / t) \text {. }
$$

Using the formulation of a mild solution in $(10.3)$ note that the expectation of the martingale term vanishes. Use the previous line, write for $0 \leq s \leq t$ and $\delta \in(0,1)$ :

$$
\begin{aligned}
\mathbb{E}[u(s, y)] & =\int_{-\infty}^{\infty} p_{s}(y-z) u_{0}(z) d z \\
& \leq\left\|u_{0}\right\|_{\infty} \int_{-\infty}^{0} p_{s}(y-z) d z \\
& =\left\|u_{0}\right\|_{\infty} \int_{-\infty}^{0}(2 \pi s)^{-1 / 2} \exp \left(-\delta \frac{|y-z|^{2}}{2 s}\right) \exp \left(-(1-\delta) \frac{|y-z|^{2}}{2 s}\right) d z \\
& \leq\left\|u_{0}\right\|_{\infty} \delta^{-1 / 2} \int(2 \pi s / \delta)^{-1 / 2} \exp \left(-\delta \frac{|y-z|^{2}}{2 s}\right) d z t^{(1-\delta) c^{2} / 2} \\
& =\left\|u_{0}\right\|_{\infty} \delta^{-1 / 2} t^{(1-\delta) c^{2} / 2} .
\end{aligned}
$$

Put this back into 10.12 and bound the constant of integration w.r.t. $s$ by 1 .

Combining the two previous ideas allow us do the proof of Proposition 10.1.1

Proof of Proposition 10.1.1. We will do the case $\gamma \in\left(\frac{1}{2}, 1\right)$ first and comment on the other case later. Let $y \geq \operatorname{ch}(t)$ as in the proposition for $t \in\left(0, e^{-1}\right)$.

Fix $\xi \in(0,1]$. Take $x_{0}=0$ and let $r_{i} \searrow 0$ (specified later), $p_{i}=\xi e^{-i}, i=$ $0,1,2, \ldots$ By Lemma 10.2 .1 (b) find $x_{1} \in\left[r_{0}, 2 r_{0}\right]$ s.t. for $i=1$ :

$$
\mathbb{P}\left(\int_{0}^{t} u\left(s, x_{i}\right) d s \geq p_{i}\right) \leq \mathbb{P}\left(\int_{0}^{t} u\left(s, x_{i-1}\right) d s \geq p_{i-1}\right)+\left[10.2 .1 r_{i-1}^{-5 \nu}\left(\frac{p_{i-1}^{2 / M}}{p_{i}}\right)^{\nu} .\right.
$$

After this, use $x_{1}$ as the new origin, apply Lemma 10.2.1 (b) and find an $x_{2} \in$ $\left[x_{1}+r_{1}, x_{1}+2 r_{1}\right] \subset\left[0,2\left(r_{1}+r_{2}\right)\right]$, s.t. 10.13$)$ holds for $i=2$. Iteratively construct a sequence of $x_{i}$, such that 10.13 holds. Using $p_{i} \searrow 0$ and assuming $R=\sum_{i} r_{i}<\infty$, there is a $x_{\infty} \in[0,2 R]$ with

$$
\mathbb{P}\left(\int_{0}^{t} u\left(s, x_{\infty}\right) d s>0\right) \leq \mathbb{P}\left(\int_{0}^{t} u(s, 0) d s \geq \xi\right)+\frac{10.2 .1}{\sum_{i \geq 1}} r_{i-1}^{-5 \nu}\left(\frac{p_{i-1}^{2 / M}}{p_{i}}\right)^{\nu} .
$$


By transfering the origin from 0 to $y$ and using Lemma 10.2 .3 , for $\delta=\frac{1}{2}$ we obtain

$$
\begin{aligned}
& \mathbb{P}\left(\int_{0}^{t} u\left(s, y+x_{\infty}\right) d s>0\right) \\
& \quad \leq \sqrt{2} \xi^{-1} t^{1+c^{2} / 4}\left\|u_{0}\right\|_{\infty}+\xi^{\nu(2 / M-1)} e^{2 \nu / M} \frac{10.2 .1}{\sum_{i \geq 1}} r_{i}^{-5 \nu} \exp (-i \nu(2 / M-1)) .
\end{aligned}
$$

This required $y \geq \operatorname{ch}(t)$. Choose $\eta=y\left(\sum_{i \geq 1} \exp \left(-\frac{i}{10}\left(\frac{2}{M}-1\right)\right)\right)^{-1}$ and now specify

$$
r_{i}:=\frac{\eta}{2} \exp \left(-\frac{i}{10}\left(\frac{2}{M}-1\right)\right), i=1,2, \ldots
$$

This ensures $y=2 \sum_{i} r_{i} \geq x_{\infty}$. Lemma 10.2.1 (a) implies that with probability 1

$$
\left\{\int_{0}^{t} u\left(s, y+x_{\infty}\right) d s=0\right\} \subset\left\{\int_{0}^{t} u(s, 2 y) d s=0\right\} .
$$

Thus, we have

$$
\mathbb{P}\left(\int_{0}^{t} u(s, 2 y) d s>0\right) \leq \mathbb{P}\left(\int_{0}^{t} u\left(s, y+x_{\infty}\right) d s>0\right) .
$$

Observe that for the values of $r_{i}, i \in \mathbb{N}$ we have:

$$
\sum_{i \geq 1} r_{i}^{-5 \nu} \exp (-i \nu(2 / M-1)) \leq \eta^{-5 \nu}\left(1-\exp \left(-\frac{\nu}{2}(2 / M-1)\right)\right)^{-1}<\infty .
$$

Defining

$c_{1}=\frac{10.2 .1}{e^{2 \nu / M}}\left(1-\exp \left(-\frac{\nu}{2}\left(\frac{2}{M}-1\right)\right)\right)^{-1}$ and $c_{2}=c_{1}\left(\sum_{i} \exp \left(-\frac{i}{10}\left(\frac{2}{M}-1\right)\right)\right)^{5 \nu}$

we can write

$$
\begin{aligned}
\mathbb{P}\left(\int_{0}^{t} u(s, 2 y) d s>0\right) & \leq \sqrt{2} \xi^{-1} t^{1+c^{2} / 2}\left\|u_{0}\right\|_{\infty}+c_{1} \xi^{\nu(2 / M-1)} \eta^{-5 \nu} \\
& =\sqrt{2}\left\|u_{0}\right\|_{\infty} \xi^{-1} t^{1+c^{2} / 4}+c_{2} \xi^{\nu(2 / M-1)} y^{-5 \nu} \\
& \leq \sqrt{2}\left\|u_{0}\right\|_{\infty} \xi^{-1} t^{1+c^{2} / 4}+c^{-5 \nu} c_{2} t^{-5 \nu / 2} \xi^{\nu(2 / M-1)}
\end{aligned}
$$

by the bound $y>c \sqrt{t}$. Choose $\xi=t^{c^{2} / 4-1} \in(0,1]$ (the lower bound on $c$ to be imposed in a moment assures that) and observe that

$$
t^{-5 \nu / 2} \xi^{\nu(2 / M-1)} \leq t^{2}
$$


if $c \geq 2+[(8+10 \nu) M /(2-M) \nu]^{1 / 2} \in\left(1,8+66(1-\gamma)^{-1}\right.$ ) (obtain that estimate by using $\beta=\frac{1}{3}$ ). We do do not give a further estimate on a possibly better (i.e. smaller) choice of $c$. If $c>8+66(1-\gamma)^{-1}$ surely all of the above holds and we conclude for $y \geq \operatorname{ch}(t)$ :

$$
\mathbb{P}\left(\int_{0}^{t} u(s, 2 y) d s>0\right) \leq\left(\sqrt{2}\left\|u_{0}\right\|_{\infty}+c_{2}\right) t^{2} \equiv q 10.1 .1 t^{2} .
$$

In the case $\gamma \leq \frac{1}{2}$ all the steps go through replacing $\nu$ with $\frac{1}{2}$, but in fact it is even easier then.

Remark 10.2.4. It might be worth noting that $q 10.1 .1 \nearrow \nearrow \infty$ for $\gamma \nearrow 1$. This is, since $c_{1}=c_{1}(M(\gamma)) \leq q_{10.2 .1} e^{\frac{2}{3}}\left(1-\exp \left(\frac{1}{36}(1-\gamma)\right)\right)^{-1} \rightarrow \infty$ as $\gamma \rightarrow 1$. Additionally, we also have $\sum_{i} r_{i}=\infty$ in that case. One can remedy one of these problems, but not both.

Finally, we can do the proof of Lemma 10.1.2.

Proof of Lemma 10.1.2. The proof is very simple. We know that $u: \mathbb{R}_{+} \times \mathbb{R} \rightarrow \mathbb{R}_{+}$ is continuous almost surely. Denote $d_{s}:=S\left(u_{s}\right)$, for a fixed value $s \geq 0$.

Assume that there exists $\bar{\varepsilon}>0$ such that for all $\varepsilon \in(0, \bar{\varepsilon}]: u\left(s, d_{s}-\varepsilon\right)=0$. Then either $u(s, \cdot)$ is not continuous at $d$ (which cannot be), or $S\left(u_{s}\right) \leq d_{s}-\bar{\varepsilon}$, which is also a contradiction to the definition of $d$. Thus, we know that

$$
\forall \bar{\varepsilon}>0 \exists \varepsilon \in(0, \bar{\varepsilon}], \delta(\varepsilon)>0: u_{s}\left(d_{s}-\varepsilon\right)>\delta(\varepsilon) .
$$

Let $\bar{\varepsilon}>0$ be arbitrary and let $\varepsilon=\varepsilon(\bar{\varepsilon})$ and $\delta=\delta(\varepsilon)$ according to the preceding line. Then, by continuity of $u(\cdot, \cdot)$ at $\left(s, d_{s}-\varepsilon\right)$ we know that there is a $\bar{\mu}>0$ such that

$$
\forall \mu \in[0, \bar{\mu}]: u\left(s-\mu, d_{s}-\varepsilon\right)>\frac{1}{2} \delta(\varepsilon) .
$$

Thus, for all $\mu \in(0, \bar{\mu}]$ :

$$
d_{s-\mu} \geq d_{s}-\varepsilon \geq d-\bar{\varepsilon}
$$

As $\bar{\varepsilon}$ was arbitrary, this shows the claim.

\subsection{Extinction of the process}

In this section we provide the proof of Proposition 5.4.2. The proof is similar to that of Proposition 3.10 in MP92 with an adaption of their equation (3.25). Their result stated the extinction property of solutions to 10.1 where $\dot{W}$ is colored noise.

Proof of Proposition 5.4.2. Let $\phi(x)=\phi^{(a)}(x)=\cosh (a x)$ and using the functions $\psi_{n}$ defined just before (10.6), set $\phi_{n}(x)=\phi(x) \psi_{n}(x), x \in \mathbb{R}$. First, note that 
since $u \in C\left(\mathbb{R}_{+}, C_{\text {rap }}\right)$ the integral $\left\langle u_{t}, \phi\right\rangle$ is integrable over a finite time interval. Additionally, $\phi_{n} \in C_{c}^{\infty}$ for any $n \in \mathbb{N}$. By $(10.3)$, we can write

$$
\left\langle u_{t}, \phi_{n}\right\rangle=\left\langle u_{0}, \phi_{n}\right\rangle+\int_{0}^{t} \int_{\mathbb{R}} u(r, x) \frac{1}{2} \phi_{n}^{\prime \prime}(x) d x d r+\int_{0}^{t} \int_{\mathbb{R}} \sigma(u(r, x)) \phi_{n}(x) W(d r d x) .
$$

Observe that $\left|\phi_{n}^{\prime \prime}\right|+\left|\phi_{n}\right| \leq C|\phi|$ allows us to use dominated convergence theorem for $n \rightarrow \infty$ in all terms except the martingale term. For the martingale term, we observe

$$
\begin{aligned}
& \int_{0}^{t} \int_{\mathbb{R}^{2}} \sigma(u(r, x)) \sigma(u(r, y))\left(\phi_{n}(x)-\phi(x)\right)\left(\phi_{n}(y)-\phi(y)\right) k(x, y) d x d y d r \\
& \leq \int_{0}^{t} \int_{\mathbb{R}^{2}} c^{2}(1+u(r, x))(1+u(r, y))\left(\phi_{n}(x)-\phi(x)\right)\left(\phi_{n}(y)-\phi(y)\right) k(x, y) d x d y d r .
\end{aligned}
$$

And this expression tends to zero by the dominated convergence theorem, since solutions are integrable. As $\phi^{\prime \prime}=a^{2} \phi$, we have letting $n \rightarrow \infty$ in the above

$$
\begin{aligned}
\left\langle u_{t}, \phi\right\rangle & =\left\langle u_{0}, \phi\right\rangle+\int_{0}^{t} \int_{\mathbb{R}} u(r, x) \frac{1}{2} \phi^{\prime \prime}(x) d x d r+\int_{0}^{t} \int_{\mathbb{R}} \sigma(u(r, x)) \phi(x) W(d r d x) \\
& =:\left\langle u_{0}, \phi\right\rangle+\left(a^{2} / 2\right) \int_{0}^{t}\left\langle u_{r}, \phi^{(a)}\right\rangle d r+M_{t}=: X_{t}^{(a)} .
\end{aligned}
$$

Note that $X^{(a)}$ gets extinct at the same time as $\langle u(\cdot, \cdot), 1\rangle$. Clearly, $M_{t}$ is a continuous martingale and an application of Corollary 10.4 .4 gives

$$
\begin{aligned}
\frac{d}{d t}\langle M\rangle_{t} & \geq \int_{\mathbb{R}^{2}} \sigma_{0}^{2} u(r, x)^{\gamma} u(r, y)^{\gamma} \phi(x) \phi(y) k(x, y) d x d y \\
& =\sigma_{0}^{2} \int_{\mathbb{R}^{2}} c^{2}\left(u(r, x) \phi(x)^{1 / \gamma}\right)^{\gamma}\left(u(r, y) \phi(y)^{1 / \gamma}\right)^{\gamma} k(x, y) d x d y \\
& \geq \sigma_{0}^{2} c H^{-2 / \beta}\left(\int_{\mathbb{R}} u(t, x) \phi(x)^{1 / \gamma} d x\right)^{\frac{2 \beta \gamma+2}{\beta+1}} \\
& \geq c \sigma_{0}^{2} H^{-2 / \beta}\left(\int_{\mathbb{R}} u(t, x) \phi(x) d x\right)^{\frac{2 \beta \gamma+2}{\beta+1}} \quad(\phi \geq 1) \\
& =: c \sigma_{0}^{2} H^{-2 / \beta} b(u, t) .
\end{aligned}
$$

Remember that $U_{0}$ was the extinction time of $u$. Let

$$
\begin{aligned}
C_{t} & =\int_{0}^{t} b(u, s)^{-1} d\langle M\rangle_{s} \quad \text { for } t<U_{0}, \\
\tau_{t} & =\inf \left\{u: C_{u}>t\right\} \quad \text { for } u<C_{U_{0}} .
\end{aligned}
$$


Then, for $t<C_{U_{0}}$ :

$$
\frac{d \tau}{d t}(t)=\left(\frac{d C}{d u}\left(\tau_{t}\right)\right)^{-1}=b\left(u, \tau_{t}\right)\left(\frac{d\langle M\rangle}{d u}\left(\tau_{t}\right)\right)^{-1} \leq c \sigma_{0}^{2} H^{2 / \beta} .
$$

So we can define $\tau\left(C_{U_{0}}\right)=\tau\left(C_{U_{0}-}\right)<\infty$ if $C_{U_{0}}<\infty$. Let $\tilde{X}^{(a)}(t):=\tilde{X}(t):=$ $\left\langle u\left(\tau_{t \wedge C_{U_{0}}}, \cdot\right), \phi^{(a)}\right\rangle$. Call $U_{\tilde{X}}$ the extinction time of the process $\tilde{X}$. Call $U_{\tilde{X}}\left(U_{X}\right)$ the extinction time of the process $\tilde{X}$ (X, respectively). Then $U_{\tilde{X}}=C_{U_{0}}$ and because of 10.17) and the finiteness of $H$ :

$$
\left\{U_{\tilde{X}^{(a)}}<\infty\right\} \subset\left\{U_{X^{(a)}}<\infty\right\}
$$

almost surely. By the optional stopping theorem $(\tau(t)$ is stochastically bounded) the process

$$
\tilde{M}_{t}:=M\left(\tau\left(t \wedge U_{\tilde{X}}\right)\right) \text { is a continuous local martingale. }
$$

For $t<U_{\tilde{X}}$ observe by 10.17 :

$$
\frac{d}{d t}\langle\tilde{M}\rangle_{t}=\frac{d\langle M\rangle}{d u}\left(\tau_{t}\right) \frac{d \tau}{d t}(t)=b\left(u, \tau_{t}\right) .
$$

Hence, using Theorem 3.2.8 we may assume that there is a Brownian motion $\left(B_{t}\right)_{t \geq 0}$ such that

$$
\tilde{M}_{t}=\int_{0}^{t} b\left(u, \tau_{r}\right)^{1 / 2} d B_{r}
$$

Then, 10.16 implies for $t<U_{\tilde{X}}$,

$$
\begin{aligned}
\tilde{X}_{t} & =\left\langle u_{\tau_{t}}, \phi\right\rangle=\left\langle u_{0}, \phi\right\rangle+\int_{0}^{t} b\left(u, \tau_{r}\right)^{1 / 2} d B_{r}+\left(a^{2} / 2\right) \int_{0}^{\tau\left(t \wedge U_{\tilde{X}}\right)}\left\langle u_{r}, \phi\right\rangle d r \\
& =\left\langle u_{0}, \phi\right\rangle+\int_{0}^{t}\left(\left\langle u_{\tau_{r}}, \phi\right\rangle\right)^{\frac{\beta \gamma+1}{\beta+1}} d B_{r}+\left(a^{2} / 2\right) \int_{0}^{t}\left\langle u_{\tau_{r}}, \phi\right\rangle \tau^{\prime}(r) d r \\
& =\left\langle u_{0}, \phi\right\rangle+\int_{0}^{t}\left(\tilde{X}_{r}\right)^{\frac{\beta \gamma+1}{\beta+1}} d B_{r}+\left(a^{2} / 2\right) \int_{0}^{t} \tilde{X}_{r} \tau^{\prime}(r) d r .
\end{aligned}
$$

Let $\bar{H}>0$ and $Y_{t}^{(a)}$ be the pathwise unique solution (see Theorem 4.3.1 of

$$
Y_{t}=\left\langle u_{0}, \phi\right\rangle+\int_{0}^{t} Y_{r}^{\frac{\beta \gamma+1}{\beta+1}} d B_{r}+a^{2} \frac{\bar{H}}{2 c \sigma_{0}^{2}} \int_{0}^{t} Y_{r} d r .
$$

By Theorem 5.5.6 in [KS00 we know that on the probability space chosen:

$$
\tilde{X}_{t}^{(a)} \mathbb{1}_{\left\{H^{2 / \beta} \leq \bar{H}\right\}} \leq Y_{t}^{(a)} \mathbb{1}_{\left\{H^{2 / \beta} \leq \bar{H}\right\}} \text { for all } t \geq 0 \text { almost surely. }
$$


Using Theorem 51.2 (ii) of [RW87] one can show that 0 is an accessible boundary point for the diffusion $Y^{(a)}$ with scale function

$$
s_{a}(x)=\int_{1}^{x} \exp \left(-\frac{a^{2} \bar{H}}{2 c \sigma_{0}^{2}} \frac{\beta+1}{\beta \gamma+1}\left(y^{2 \frac{\beta-\beta \gamma}{\beta+1}}-1\right)\right) d y .
$$

For the latter, one can use the explicit formula in Exercise VII.3.20 of [RY91]. We want to apply Proposition VII.3.1 in [RY91. Note that

$$
\left\{U_{\tilde{X}^{(a)}}<\infty\right\} \supset\left\{U_{Y}<\infty, H \leq \bar{H}\right\}=\left\{U_{Y}<\infty\right\} \backslash\{H>\bar{H}\} .
$$

Using this, 10.18 and $\lim _{a \rightarrow 0} \lim _{x \rightarrow \infty} s_{a}(x)=\infty$, we have

$$
\begin{aligned}
\mathbb{P}\left(U_{0}<\infty\right) & \geq \lim _{a \rightarrow 0} \mathbb{P}\left(U_{\tilde{X}^{(a)}}<\infty\right) \\
& \geq \lim _{a \rightarrow 0} \mathbb{P}\left(U_{Y^{(a)}}<\infty\right)-\lim _{\bar{H} \rightarrow \infty} \mathbb{P}\left(H^{2 / \beta}>\bar{H}\right) \quad(\tilde{X} \leq Y) \\
& \geq \lim _{a \rightarrow 0} \frac{s_{a}(\infty)-s_{a}\left(\left\langle u_{0}, \phi^{(a)}\right\rangle\right)}{s_{a}(\infty)-s_{a}(0)}-\lim _{\bar{H} \rightarrow \infty} \bar{H}^{-1} \mathbb{E}\left(H^{2 / \beta}\right) \\
& =1,
\end{aligned}
$$

since $\mathbb{E}\left(H^{2 / \beta}\right)<\infty$.

Note that if the continuous nonnegative martingale $X_{t}=\left\langle u_{t}, 1\right\rangle$ with $\sigma(0)=0$, attains the value 0 for a certain $t>0$, then $X$ stays in zero after time $t$ (Problem 1.5.12 of [KS00]). This shows the second claim.

\subsection{Integral estimates}

Denote the open ball in $\mathbb{R}^{q}$ centered in the origin with radius $r>0$ by

$$
B(0, r)=\left\{x \in \mathbb{R}^{q}:|x|<r\right\} .
$$

Lemma 10.4.1. For $0 \leq a<b$ let $x_{0} \in B(0, b) \backslash B(0, a)$ and $c>0$. Then there is a constant $C=C(q)$, s.t.

$$
\int_{\mathbb{R}^{q}} \mathbb{1}_{\left\{a<|x|<b,\left|x-x_{0}\right|<c\right\}} d x \geq C(q)((b-a) \wedge c)^{q} .
$$

Proof. Call the set in the integral $A$. Observe that at least two disjoint cone-like ball segments with angle $\pi / 2$, top $x_{0}$ and centered around $x_{0}+\mathbb{R}_{+} x_{0}$ and $x_{0}+\mathbb{R}_{-} x_{0}$ are contained in $A$. Call them $A_{+}$and $A_{-}$. Note that $A_{+}$is a cone with height at least $h_{+}=2^{-1 / 2}\left[\left(b-\left|x_{0}\right|\right) \wedge c\right]$ and ground area $c_{q-1} h_{+}^{q-1}\left(c_{q-1}\right.$ the volume of the 
unit ball in $\left.\mathbb{R}^{q-1}\right)$. Likewise the same holds for $A_{-}$with $h_{-}=2^{-1 / 2}\left[\left(\left|x_{0}-a\right|\right) \wedge c\right]$. Using the formula for the $q$-dimensional cone and summing the two parts obtain:

$$
\begin{aligned}
|A| & =\left|A_{+}\right|+\left|A_{-}\right| \geq c(q)\left[\left(\left(b-\left|x_{0}\right|\right) \wedge c\right)^{q}+\left(\left(\left|x_{0}\right|-a\right) \wedge c\right)^{q}\right] \\
& \geq c(q)\left[\left(\left(b-\left|x_{0}\right|\right)+\left(\left|x_{0}\right|-a\right)\right) \wedge c\right]^{q} \\
& \geq c(q)((b-a) \wedge c)^{q} .
\end{aligned}
$$

Let $k: \mathbb{R}^{2 q} \rightarrow \mathbb{R}_{+}$with $k(b x, b y)=b^{-\alpha} \tilde{k}(x-y)$ for a certain $\alpha>0$. The function $\tilde{k}: \mathbb{R}^{q} \rightarrow \mathbb{R}_{+}$is bounded from below near the origin in the sense that $\tilde{k}(z) \geq c_{k}>0$ for all $z \in \mathbb{R}^{q}$ with $|z| \leq 2$.

Lemma 10.4.2. Let $0<\beta, \gamma \leq 1$ and let $f: \mathbb{R}^{q} \rightarrow \mathbb{R}_{+}$be Hölder continuous of $\operatorname{order} \beta$ :

$$
|f(x)-f(y)| \leq H|x-y|^{\beta}
$$

for any $x, y \in \mathbb{R}^{q}$, where $H>0$ is a uniform constant. Then for $k(x, y)=\mid x-$ $\left.y\right|^{-\alpha}, \alpha \in(0,1), a \in[0,1], r>0$ there is a constant $c\left(q, c_{k}\right)$

$$
\begin{aligned}
& \int_{\mathbb{R}^{q}} \int_{\mathbb{R}^{q}} f^{\gamma}(x) f^{\gamma}(y) k(x, y) \mathbb{1}_{\{a r<|x|<2 r, a r<|y|<2 r\}} d x d y \\
& \quad \geq c\left(q, c_{k}\right)\left(r^{-1} F^{1 /(\beta+q)} \vee 1\right)^{2 q}\left[H^{-1 / \beta} \wedge \frac{r}{F^{1 /(\beta+q)}}\right]^{2 q} r^{-\alpha} F^{\frac{2 / \beta \gamma+2 q}{\beta+q}},
\end{aligned}
$$

where $F=\int_{a r<|x|<2 r} f(x) d x$.

The proof will closely follow the proof in BMP11, Theorem 4.

Proof. First, let us show the claim for a Hölder continuous function $g: \mathbb{R}^{q} \rightarrow \mathbb{R}_{+}$ with the Hölder constant $H$ as in 10.20 satisfying

$$
\int \mathbb{1}_{\{a r<|x|<2 r\}} g(y) d y=1 .
$$

We consider two cases, the first one being that $\sup _{|y| \in(a r, 2 r)} g(y)<1$. Clearly, $g^{\gamma}(y)>g(y)$ for any $|y| \in(a r, 2 r)$ and so

$$
\begin{aligned}
\int_{\mathbb{R}^{q}} \int_{\mathbb{R}^{q}} g^{\gamma}(x) g^{\gamma}(y) k(x, y) \mathbb{1}_{\{a r<|x|<2 r, a r<|y|<2 r\}} d x d y \\
\quad=\int_{\mathbb{R}^{q}} \int_{\mathbb{R}^{q}} g^{\gamma}(r \tilde{x}) g^{\gamma}(r \tilde{y}) \tilde{k}(\tilde{x}-\tilde{y}) r^{2 q-\alpha} \mathbb{1}_{\{a<|\tilde{x}|,|\tilde{y}|<2\}} d \tilde{x} d \tilde{y} \\
\geq c_{k} r^{2 q-\alpha} \int_{\mathbb{R}^{q}} \mathbb{1}_{\{a<|\tilde{x}|<2\}} g(r \tilde{x}) d \tilde{x} \int_{\mathbb{R}^{q}} \mathbb{1}_{\{1<|\tilde{y}|<2\}} g(r \tilde{y}) d \tilde{y} \\
=c_{k} r^{-\alpha}\left(\int_{\mathbb{R}^{q}} \mathbb{1}_{\{a r<|x|<2 r\}} g(x) d x\right)^{2}=c_{k} r^{-\alpha}
\end{aligned}
$$


since the integral equals 1.

The second case to consider for $g$ is that of $\sup _{|y| \in(a r, 2 r)} g(y) \geq 1$. Then, there is a $y_{0} \in \mathbb{R}^{q}$ with ar $<\left|y_{0}\right|<2 r$, s.t. $g\left(y_{0}\right) \geq 1$. Then by the Hölder continuity of $g$, we observe

$$
g(y) \geq g\left(y_{0}\right)-\left|g(y)-g\left(y_{0}\right)\right| \geq 1-H(2 H)^{-1}=\frac{1}{2}
$$

for any $y \in \mathbb{R}^{q}$ with $\left|y-y_{0}\right| \leq(2 H)^{-1 / \beta}$. Therefore, using Lemma 10.4 .1 obtain

$$
\begin{aligned}
& \int_{\mathbb{R}^{q}} \int_{\mathbb{R}^{q}} g^{\gamma}(x) g^{\gamma}(y) k(x, y) \mathbb{1}_{\{a r<|x|,|y|<2 r\}} d x d y \\
& \geq \iint g^{\gamma}(x) g^{\gamma}(y) k(x, y) \mathbb{1}_{\left\{a r<|x|<2 r,\left|x-y_{0}\right|<(2 H)^{-1 / \beta}\right\}} \mathbb{1}_{\left\{a r<|y|<2 r,\left|y-y_{0}\right|<(2 H)^{-1 / \beta}\right\}} d x d y \\
& \geq 2^{-2 \gamma} \iint r^{2 q-\alpha} \tilde{k}(\tilde{x}-\tilde{y}) \mathbb{1}_{\left\{a<|\tilde{x}|<2,\left|\tilde{x}-y_{0} / r\right|<\frac{1}{r}(2 H)^{-1 / \beta}\right\}} \\
& \quad \mathbb{1}_{\left\{a<|\tilde{y}|<2,\left|\tilde{y}-y_{0} / r\right|<\frac{1}{r}(2 H)^{-1 / \beta\}}\right.} d \tilde{x} d \tilde{y} \\
& \geq 2^{-2 \gamma} r^{2 q-\alpha} c_{k}\left[C(q)\left(1 \wedge r^{-1}(2 H)^{-1 / \beta}\right)\right]^{2 q} \\
& \geq 2^{-2 \gamma-2 q / \beta} c_{k} C(q)^{2 q} r^{-\alpha}\left(r \wedge H^{-1 / \beta}\right)^{2 q} .
\end{aligned}
$$

Putting the results together for the two cases, we observe that for $g$ such that $\int \mathbb{1}_{\{a r<|x|<2 r\}} g(y) d y=1$, we have

$$
\begin{aligned}
& \int_{\mathbb{R}^{q}} \int_{\mathbb{R}^{q}} g^{\gamma}(x) g^{\gamma}(y) k(x, y) \mathbb{1}_{\{a r<|x|<2 r, a r<|y|<2 r\}} d x d y \\
& \geq C\left(q, c_{2}\right) r^{-\alpha}\left(1 \wedge r^{2 q}\right)\left(1 \wedge \frac{H^{-1 / \beta}}{r}\right)^{2 q} \\
&=C\left(q, c_{2}\right) r^{-\alpha}\left(r^{-2 q} \wedge 1\right)\left(r \wedge H^{-1 / \beta}\right)^{2 q} .
\end{aligned}
$$

Now, if $f$ is any non-negative function, then define for $b>0$ :

$$
g(y)=b^{-\beta} f(b y)
$$

Observe that $g$ satisfies 10.20 and obtain

$$
\begin{aligned}
\int_{\mathbb{R}^{q}} g(y) \mathbb{1}_{\{a r / b<|y|<2 r / b\}} d y & =b^{-\beta} \int_{a r<|b y|<2 r} f(b y) b^{-q} d(b y) \\
& =b^{-q-\beta} \int_{a r<|x|<2 r} f(x) d x=1,
\end{aligned}
$$


provided that

$$
b=F^{\frac{1}{\beta+q}}=\left(\int_{a r<|x|<2 r} f(x) d x\right)^{\frac{1}{\beta+q}} .
$$

Using the result for the function $g$ with $r$ replaced by $r / b$, we obtain

$$
\begin{aligned}
C\left(q, c_{k}\right) & \left(\frac{r}{b}\right)^{-\alpha}\left(\frac{r}{b}^{-2 q} \wedge 1\right)\left(\frac{r}{b} \wedge H^{-1 / \beta}\right)^{2 q} \leq \\
& \leq \iint g^{\gamma}(x) g^{\gamma}(y) k(x, y) \mathbb{1}_{\left\{\frac{a r}{b}<|x|<\frac{2 r}{b}, \frac{a r}{b}<|y|<\frac{2 r}{b}\right\}} d x d y \\
& =\iint\left(b^{-\beta} f(b x)\right)^{\gamma}\left(b^{-\beta} f(b y)\right)^{\gamma} k(b x, b y) b^{\alpha} \mathbb{1}_{\{a r<|b x|<2 r, a r<|b y|<2 r\}} d x d y \\
& =b^{-2 \beta \gamma+\alpha-2 q} \iint f(w)^{\gamma} f(z)^{\gamma} k(w, z) \mathbb{1}_{\{a r<|w|<2 r, a r<|z|<2 r\}} d w d z .
\end{aligned}
$$

So, altogether we obtain

$$
\begin{aligned}
\int_{\mathbb{R}^{q}} \int_{\mathbb{R}^{q}} f^{\gamma}(x) f^{\gamma}(y) k(x, y) \mathbb{1}_{\{a r<|x|<2 r, a r<|y|<2 r\}} d x d y \\
\quad \geq c\left(q, c_{k}\right)\left(r^{-1} F^{1 /(\beta+q)} \vee 1\right)^{2 q}\left[H^{-1 / \beta} \wedge \frac{r}{F^{1 /(\beta+q)}}\right]^{2 q} r^{-\alpha} F^{\frac{2 \beta \gamma+2 q}{\beta+q}},
\end{aligned}
$$

that is what we needed to show.

Remark 10.4.3.

(a) Note that we can leave out the first factor including the $(\cdot \vee 1)^{2 q}$ since it is bounded below by 1 .

(b) In the one-dimensional case, it is also possible to restrict to $\mathbb{1}_{a r<x<2 r}$ instead of $\mathbb{1}_{a r<|x|<2 r}$ and the same proof goes through.

Similarly we can obtain the following result neglecting all the restrictions on the variables:

Corollary 10.4.4. In the setting of Lemma 10.4.2:

$$
\begin{aligned}
\int_{\mathbb{R}^{q}} \int_{\mathbb{R}^{q}} f^{\gamma}(x) f^{\gamma}(y) k(x, y) d x d y \\
\quad \geq c\left(q, c_{k}\right) H^{-2 q / \beta} r^{-\alpha}\left(\int_{\mathbb{R}^{q}} f(x) d x\right)^{\frac{2 \beta \gamma+2 q}{\beta+q}} .
\end{aligned}
$$

Remark 10.4.5. The condition $\tilde{k}(z) \geq c_{2}$ for all $|z|<2$ is in some sense illusory. By definition $k(x, y)=p^{-\alpha}|x-y|^{-\alpha} \tilde{k}((x-y) / p|x-y|)$ for any $p>0$. That means the lower bound on $k$ would only change by a factor $p^{-\alpha}$ if we required $\tilde{k}$ to be lower-bounded on $B(0,1 / p)$ instead of $B(0,2)$. 


\section{Particle system}

\subsection{Offspring distributions}

Consider a continuous time branching process $B=\left(B_{t}\right)_{t \geq 0}$ with values in $\mathbb{N}_{0}$ and started with $B_{0}=x \in \mathbb{N}$ individuals. The offspring law $\tilde{\nu}$ to be considered shall be critical binary, i.e. $\tilde{\nu}=\left(\delta_{0}+\delta_{2}\right) / 2$ and the reproduction rate per individual shall be $\lambda>0$. We can equivalently say that $B$ is a Feller process in $\mathbb{N}_{0}$ started in $x$ with generator $A$ :

$$
A f(y)=\frac{\lambda y}{2}(f(y+1)-2 f(y)+f(y-1)), y \in \mathbb{N}, A f(0)=f(0)
$$

for a bounded function $f: \mathbb{N}_{0} \rightarrow \mathbb{R}$. The solution measure for this Feller process is denoted by $\mathbb{P}^{x}$ and its expectation $\mathbb{E}^{x}$. Let us define the generating function $u: \mathbb{N} \times[0,1] \times \mathbb{R}_{+}$of that process

$$
u(x, a, t):=\mathbb{E}^{x}\left[a^{B_{t}}\right], x \in \mathbb{N}, a \in[0,1], t \geq 0 .
$$

As the mapping $x \mapsto a^{x}$ is bounded for our choice of $a$ we easily obtain using Kolmogorov's backward equation

$$
\begin{aligned}
\partial_{t} u(x, a, t) & =\mathbb{E}^{x}\left[\left.A\left(y \mapsto a^{y}\right)\right|_{y=B_{t}}\right]=\mathbb{E}^{x}\left[\frac{\lambda B_{t}}{2}\left(a^{B_{t}+1}-2 a^{B_{t}}+a^{B_{t}-1}\right]\right. \\
& =\frac{\lambda}{2}(a-1)^{2} \partial_{a} u(x, a, t) .
\end{aligned}
$$

It is good to note that the expression $(a-1)^{2} / 2$ is the generating function of our offspring law $\tilde{\nu}$ and one can check that this holds similarly for general offspring laws. The partial differential equation above is a so-called transport equation and can be solved applying standard methods. We obtain

$$
u(x, a, t)=\left(1-\frac{1}{\lambda t}+\frac{1}{\lambda t}(1+\lambda t-\lambda s t)^{-1}\right)^{x} .
$$

The exponent $x$ should not surprise the reader as it is due to the fact of the independence of the $x$ initial individuals. Standard calculations for generating functions 
allow us to get the following results for $x=1$ :

$$
\begin{aligned}
p_{n}(t):=P^{1}\left(B_{t}=n\right) & =\frac{(\lambda t)^{n-1}}{(1+\lambda t)^{n+1}}, n \in \mathbb{N}, p_{0}(t)=P^{1}\left(B_{t}=0\right)=\frac{\lambda t}{1+\lambda t}, \\
\mathbb{E}^{1}\left[B_{t}\right] & =1 \\
\operatorname{Var}^{1}\left[B_{t}\right] & =\lambda t
\end{aligned}
$$

and by independence of individuals' branching

$$
\mathbb{E}^{x}\left[B_{t}\right]=x, \operatorname{Var}^{x}\left[B_{t}\right]=\lambda x t .
$$

We are interested in taking the distribution $\nu^{x, t}=\mathcal{L}\left[B_{t} \mid B_{0}=x\right]$ at a fixed time $t$, e.g. $t=1$. Consider now a discrete time branching process $\left(Y_{n}\right)_{n \in \mathbb{N}_{0}}$ with individual offspring law $\nu_{0}=\left(\nu^{1, x}\right)^{* 1 / x}=\nu^{1,1}$. It is well-known, that the laws $\mathcal{L}\left[N^{-1} B_{N t} \mid B_{0}=\right.$ $N x]$ and $\mathcal{L}\left[N^{-1} Y_{N t} \mid Y_{0}=N x\right]$, both converge to the law of the Feller diffusion in an appropriate sense.

Observe that for $t=1$, the law of $B_{t}$ has linear variance in $x$. We would like to construct an offspring law for the discrete time process $Y$, which models density dependent branching. Density will be called the total mass of individiduals alive (here $x$ initially), but we will be more specific later. A way to achieve that is choosing not $t=1$, but rather $t=x^{\gamma^{\prime}}$ for a certain $\gamma^{\prime} \in(-\infty, \infty)$. So, let us define a new offspring law on $\mathbb{N}_{0}$ :

$$
\nu_{\gamma^{\prime}}(x, \cdot)=\left(\mathcal{L}\left[B_{x \gamma^{\prime}} \mid B_{0}=1\right]\right) .
$$

From the three lines above we easily observe that

$$
\begin{aligned}
\nu_{\gamma^{\prime}}(x, n) & =\frac{\left(\lambda x^{\gamma^{\prime}}\right)^{n-1}}{\left(1+\lambda x^{\gamma^{\prime}}\right)^{n+1}}, n \in \mathbb{N}, \nu_{\gamma^{\prime}}(x, 0)=\frac{\lambda x^{\gamma^{\prime}}}{1+\lambda x^{\gamma^{\prime}}}, \\
\nu_{\gamma^{\prime}}(x,(n \mapsto n)) & =1, \\
\nu_{\gamma^{\prime}}\left(x,\left(n \mapsto(n-x)^{2}\right)\right) & =\lambda x^{\gamma^{\prime}} .
\end{aligned}
$$

If we consider an $x$-fold convolution of $\nu_{\gamma^{\prime}}(x, \cdot)$, then we obtain for $Y \stackrel{d}{=} \mathcal{L}\left[\nu_{\gamma^{\prime}}(x, \cdot)\right]^{* x}$ :

$$
\mathbb{E}(Y)=x, \operatorname{Var}^{x}(Y)=\lambda x^{1+\gamma^{\prime}},
$$

which should be seen in contrast to (11.1): the individual offspring law $\nu_{\gamma^{\prime}}(x, \cdot)$ is critical and leads to nonlinear variance $x^{1+\gamma^{\prime}}$ (of course, only if $\gamma^{\prime} \neq 0$ ).

Consider now the case where each individual is assigned mass $N^{-1}$ for a certain $N \in \mathbb{N}$ and we start with $N x$ individual for a given $x \in N^{-1} \mathbb{N}$. Since density of individuals is $N^{-1} \cdot N x=x$, we assume that each individual has offspring law 
$\nu_{\gamma^{\prime}}(x, \cdot)$. Thus, the summed offspring of all individuals after one timestep is given by $Y_{1} \stackrel{d}{=} \mathcal{L}\left[B_{x^{\gamma^{\prime}}}\right]^{N x}$ and

$$
\mathbb{E}^{x}\left[N^{-1} Y_{1}\right]=x, \operatorname{Var}^{x}\left(N^{-1} Y_{1}\right)=N^{-1} x^{1+\gamma^{\prime}} .
$$

The superscript here refers to the fact that initially total mass $N^{-1} Y_{0}=x$.

\subsection{Proofs and proof ideas}

Remember the model given in Section 6.2. There we had the measure-valued process $\left(X_{t}^{N}\right)_{t \geq 0}$ and the density process $\left(u^{N}(t, x)\right)_{t \geq 0, x \in \mathbb{R}}$. The motion of particles was governed by the Feller-generator $A=\frac{\Delta}{2}$. For the proof of Conjecture 4 it is necessary to show tightness and give an identification of the limit. We start with arguments concerning tightness and conjecture:

Conjecture 6. The sequence $u^{N}$ is tight in $D\left(\mathbb{R}_{+}, C_{\text {tem }}\right)$.

It is easy to note that $u^{N}$ in fact has paths in this space. We give some indication, why the conjecture should hold true and how to make a proof:

A helpful criterion is that of Theorem 3.1 in [Jak86]. Let $F=\left\{H_{\phi}: \phi \in C_{c}^{\infty}\left(\mathbb{R}^{q}\right)\right\}$ be the family of mappings $H_{\phi}: C_{\text {tem }} \rightarrow \mathbb{R}, f \mapsto \int_{\mathbb{R}^{q}} f(x) \phi(x) d x$. In order to show tightness for a process with càdlàgpaths and values in $C_{\text {tem }}$ we use the following proposition:

Proposition 11.2.1. A family of probability measures $\left\{\mu_{i}: i \in J\right\}$ on $\mathcal{B}\left(D\left(\mathbb{R}_{+}, C_{\text {tem }}\right)\right)$ is D-tight, if the following two conditions hold

(a) For all $\epsilon>0, T>0$ there is a compact set $K_{\epsilon, T} \subset C_{\text {tem }}$ s.t.

$$
\inf _{i \in J} \mu_{i}\left(x \in D: x(t) \in K_{\epsilon, T} \forall t \leq T\right)>1-\epsilon .
$$

(b) The family $\left\{\mu_{i}: i \in J\right\}$ is F-weakly tight, i.e. for each $\phi \in C_{c}^{\infty}\left(\mathbb{R}^{q}\right)$ the family $\left\{\mu_{i} \circ \tilde{H}_{\phi}: i \in J\right\}$ of probability measures on $D\left(\mathbb{R}_{+}, \mathbb{R}\right)$ is tight. Here

$$
\tilde{H}_{\phi}: D\left(\mathbb{R}_{+}, C_{\text {tem }}\right) \rightarrow D\left(\mathbb{R}_{+}, \mathbb{R}\right),\left[H_{\phi}(f)\right](t)=\int_{\mathbb{R}^{q}} f(t, x) \phi(x) d x, t \in \mathbb{R}_{+} .
$$

Proof. We apply Theorem 3.1 from [Jak86] to the case $E=C_{\text {tem }}$. This only requires to show three conditions for the family $F$, which are easily checked: clearly $F$ separates points in $C_{\text {tem }}$ and is closed under addition. Finally one can also show that any mapping $H_{g}$ is continuous, even Lipschitz-continuous. 
First, give an idea, how to verify Condition (b) of Proposition 11.2.1. Let $\phi \in$ $C_{c}^{\infty}(\mathbb{R})$ be fixed throughout this section. We need to show that

\section{Lemma 11.2.2.}

$$
\left\{\left(\int_{\mathbb{R}^{q}} u^{N}(t, y) \phi(y) d y\right)_{t \geq 0}: N \in \mathbb{N}\right\}
$$

is tight in $D\left(\mathbb{R}_{+}, \mathbb{R}\right)$.

In order to show that lemma decompose

$$
\begin{aligned}
\int_{\mathbb{R}^{q}} u^{N}(t, y) \phi & (y) d y=\frac{1}{N} \int_{\mathbb{R}^{q}} \sum_{\beta \sim_{N} t} p_{1 / N}\left(Y^{\beta, N}(t)-y\right) \phi(y) d y \\
= & X_{t}^{N}(\phi)+\frac{1}{N} \sum_{\beta \sim_{N} t}\left(\int_{\mathbb{R}^{q}} p_{1 / N}\left(Y^{\beta, N}(t)-y\right) \phi(y) d y-\phi\left(Y^{\beta, N}(t)\right)\right) \\
= & X_{t}^{N}(\phi)+E_{t}^{N}(\phi) .
\end{aligned}
$$

\section{Conjecture 7.}

$$
\lim _{N \rightarrow \infty} \mathbb{E}\left[\sup _{t \leq T}\left|E_{t}^{N}(\phi)\right|\right]=0 .
$$

Proof Sketch. There are two things to do: Taylor-expansion of $\phi(y)=\phi\left(Y^{\beta, N}(t)\right)+$ $\phi^{\prime}()\left(y-Y^{\beta, N}(t)\right)+\frac{1}{2} \phi^{\prime \prime}(\xi)\left(y-Y^{\beta, N}(t)\right)^{2}$ and secondly, application of Hölder inequality.

Then, tightness of $\left\langle u^{N}, \phi\right\rangle$ will hold, if $X^{N}(\phi)$ is tight. So, we continue to deal with $X_{t}^{N}(\phi)$. To prove its tightness we closely follow the arguments from Perkins [Per02], pages 149-156, where we borrow again some notation:

$$
\underline{t}=N^{-1}\lfloor N t\rfloor, \sim \text { is used for } \sim_{N} \text { if there are no disambiguities. }
$$

Define for each $\alpha \in I==\mathbb{N}_{0}^{*}$ and $\underline{s} \in N^{-1} \mathbb{N}$ s.t. $|\alpha|=N \underline{s}$ :

$$
M_{s}^{\alpha}(\phi)=\phi\left(Y_{s}^{\alpha}\right)-\phi\left(Y_{\underline{s}}^{\alpha}\right)-\int_{\underline{s}}^{t} A \phi\left(Y_{r}^{\alpha}\right) d r, s \in\left[\underline{s}, \underline{s}+N^{-1}\right] .
$$

Note that by construction, $\left\{M_{s}^{\alpha}(\phi): N^{-1}|\alpha| \leq s \leq N^{-1}(|\alpha|+1)\right\}$ is a martingale started in zero with respect to the filtration

$$
\mathcal{F}_{s}=\sigma\left(Y^{\beta, N}(r): r \leq s,|\beta| \leq N \underline{s}+1\right) .
$$

Let us also observe that

$$
X_{\underline{s}+N^{-1}}^{N}(\phi)=\frac{1}{N} \sum_{\alpha \sim N \underline{s}} \phi\left(Y_{\underline{s}+N^{-1}}^{\alpha}\right) N^{\alpha, N},
$$


which easily leads to

$$
\begin{aligned}
& X_{\underline{s}+N^{-1}}^{N}(\phi)-X_{\underline{s}}^{N}(\phi)=\frac{1}{N} \sum_{\alpha \sim_{N \underline{s}}}\left[\phi\left(Y_{\underline{s}+N^{-1}}^{\alpha}\right) N^{\alpha, N}-\phi\left(Y_{\underline{s}}^{\alpha}\right)\right] \\
&=\frac{1}{N} \sum_{\alpha \sim_{N} \underline{s}}\left[\phi\left(Y_{\underline{s}+N^{-1}}^{\alpha}\right) N^{\alpha, N}\right. \\
&\left.-\left(\phi\left(Y_{\underline{s}+N^{-1}}^{\alpha}\right)-M_{\underline{s}+N^{-1}}^{\alpha}(\phi)-\int_{\underline{s}}^{\underline{s}+N^{-1}} A \phi\left(Y_{r}^{\alpha}\right) d r\right)\right] \\
&= \frac{1}{N} \sum_{\alpha \sim N^{\underline{s}}} \phi\left(Y_{\underline{s}+N^{-1}}^{\alpha}\right)\left(N^{\alpha}-1\right)+M_{\underline{s}+N^{-1}}^{\alpha}(\phi) \\
&+\int_{\underline{s}}^{\underline{s}+N^{-1}} \frac{1}{N} \sum_{\alpha \sim_{N} r} A \phi\left(Y_{r}^{\alpha}\right) d r .
\end{aligned}
$$

Much easier, we obtain for $t>0$ :

$$
\begin{aligned}
X_{t}^{N}(\phi)-X_{\underline{t}}^{N}(\phi)= & \frac{1}{N} \sum_{\alpha \sim_{N} t} M_{t}^{\alpha}(\phi) \\
& +\int_{\underline{t}}^{t} \frac{1}{N} \sum_{\alpha \sim_{N} t} A \phi\left(Y_{r}^{\alpha}\right) d r .
\end{aligned}
$$

Adding up both calculations for $\underline{s}<\underline{t}$, one gets for $t>0$ :

$$
\begin{aligned}
X_{t}^{N}(\phi)=X_{0}^{N}(\phi) & +\frac{1}{N} \sum_{\underline{s}<\underline{t}} \sum_{\alpha \sim_{N} \underline{s}} \phi\left(Y_{\underline{s}+N^{-1}}^{\alpha}\right)\left(N^{\alpha}-1\right) \\
& +\frac{1}{N} \sum_{\underline{s}<\underline{t}} \sum_{\alpha \sim_{N} \underline{s}} M_{\underline{s}+N^{-1}}^{\alpha}(\phi)+\frac{1}{N} \sum_{\alpha \sim_{N} t} M_{t}^{\alpha}(\phi) \\
& +\int_{0}^{t} d r \frac{1}{N} \sum_{\alpha \sim_{N} r} A \phi\left(Y_{r}^{\alpha}\right) \\
=: X_{0}^{N}(\phi) & +M_{\underline{t}}^{b, N}(\phi)+M_{t}^{s, N}(\phi)+\int_{0}^{t} X_{s}^{N}(A \phi) d s
\end{aligned}
$$

Since $\left(M_{t}^{\alpha}, \mathcal{F}_{t}\right)_{t \in\left[\underline{t}, \underline{t}+N^{-1}\right]}$ is a martingale for each $\alpha \in I$, the fact that $\{\alpha \sim \underline{t}\} \in \mathcal{F}_{\underline{t}}$ easily imply the fact that $\left(M_{t}^{s, N}(\phi), \mathcal{F}_{t}\right)_{t \geq 0}$ is a martingale (integrability follows from Lemma II.3.3 (a) in [Per02]).

One easily checks the following using $\mathbb{E}\left[N^{\alpha}-1\right]=0$ :

Lemma 11.2.3. $M^{b, N}$ and $M^{s, N}$ are martingales. 
The same argument as that of [Per02] Lemma II.4.4 leads to:

Lemma 11.2.4. $\sup _{t \leq K}\left|M_{t}^{s, N}(\phi)\right| \stackrel{L^{2}}{\rightarrow} 0$ as $N \rightarrow \infty$ for all $K>0$.

We wanted to show tightness of $X^{N}(\phi)$. By the preceding lemma, we are left to show tightness of $X_{0}^{N}(\phi), M^{b, N}$ and the integral $\int_{0}^{t} X_{s}^{N}(A \phi) d s$. To show tightness of $M^{b, N}$ we would like to use Lemma II.4.5 in Per02, which we quote here

Lemma 11.2.5 (Lemma II.4.5 ([Per02])). Let $\left(M_{t}^{N}, \overline{\mathcal{F}}_{t}^{N}\right)$ be martingales with $M_{0}^{N}=$ 0 . Let $\left\langle M^{N}\right\rangle_{\underline{t}}=\sum_{0 \leq \underline{s}<\underline{\underline{t}}} \mathbb{E}\left[\left(M_{\underline{s}+N^{-1}}^{N}-M_{\underline{s}}^{N}\right)^{2} \mid \overline{\mathcal{F}}_{\underline{s}}^{N}\right]$, and extend $M^{N}$ and $\left\langle M^{N}\right\rangle$. to $\mathbb{R}_{+}$as right-continuous step functions.

(a) If $\left\{\left\langle M^{N}\right\rangle\right.$.: $\left.N \in \mathbb{N}\right\}$ is C-relatively compact in $D\left(\mathbb{R}_{+}, \mathbb{R}\right)$ and

$$
\sup _{0 \leq \underline{t} \leq K}\left|M^{N}\left(\underline{t}+N^{-1}\right)-M^{N}(\underline{t})\right| \stackrel{P}{\rightarrow} 0 \text { as } N \rightarrow \infty \forall K>0,
$$

then $\left\{M^{N}\right\}$ is $C$-relatively compact in $D\left(\mathbb{R}_{+}, \mathbb{R}\right)$.

(b) If, in addition,

$$
\left\{\left(M_{\underline{t}}^{N}\right)^{2}+\left\langle M^{N}\right\rangle_{\underline{t}}: N \in \mathbb{N}\right\} \text { is uniformly integrable } \forall \underline{t} \in T,
$$

then $M^{N_{k}} \stackrel{w}{\Rightarrow} M$ in $D(\mathbb{R})$ implies $M$ is a continuous $L^{2}$ martingale and $\left(M^{N_{k}},\left\langle M^{N_{k}}\right) \stackrel{w}{\Rightarrow}(M,\langle M\rangle)\right.$ in $D(\mathbb{R})^{2}$.

(c) Under the statement of uniform integrability in (b) the converse to (a) holds.

We do not know, if the following holds, which is remarked in the measure-valued setting in Per02] for measure-valued processes:

Open Question 1. Does $C$-tightness in $D\left(\mathbb{R}_{+}, \mathbb{R}\right)$ transfer to $C$-tightness in $D\left(\mathbb{R}_{+}, C_{\text {tem }}\right)$ in the previous statement?

In Section 6.2 we gave the calculation of the quadratic variation of $M^{b, N}$. There appeared the terms $\varepsilon_{\underline{t}, 1}^{N}(\phi)$ and $\varepsilon_{\underline{t}, 2}^{N}(\phi)$ and we obtained:

$$
\left\langle M^{b, N}(\phi)\right\rangle_{\underline{t}}=\int_{0}^{\underline{t}} d s \int_{\mathbb{R}^{q}} d y u^{N}(s, y)^{1+\gamma^{\prime}} \lambda \phi(y)^{2}+\varepsilon_{\underline{t}, 1}^{N}(\phi)+\varepsilon_{\underline{t}, 2}^{N}(\phi) .
$$

Proposition II.4.8 in Per02] suggests that $\varepsilon_{t, 1}^{N}(\phi)$ goes to zero and there are indications that also $\varepsilon_{t, 2}^{N}(\phi)$ goes to zero. Additionally following further his proof strategy it should be possible to obtain establish Condition (b) of Proposition 11.2.1. To 
show the compact containment condition (a) in that proposition we can use Proposition 3.13 of [Zäh04]: We need to show that for any $\varepsilon>0, T>0$ there is a compact set $\Gamma_{\varepsilon, T} \in C_{\text {tem }}$ s.t.

$$
\sup _{N \in \mathbb{N}} \mathbb{P}\left(u^{N}(t, \cdot) \in \Gamma_{\varepsilon, T}^{c} \text { for some } t \leq T\right)<\varepsilon .
$$

Lemma 11.2.6 (Proposition 3.13 in [Zäh04]). It is sufficient to show that for any $\varepsilon^{\prime}>0, T>0, \eta, \lambda, K>0$ there are $h(\varepsilon, \eta, K, T), H(\lambda, \varepsilon)>0$ such that

$$
\begin{aligned}
& \mathbb{P}\left(\sup _{\left|x-x^{\prime}\right| \leq h, x, x^{\prime} \in[-K, K]^{q}}\left|u^{N}(t, x)-u^{N}(t, y)\right|>\eta \text { for some } t \leq T\right)<\varepsilon^{\prime} \\
& \mathbb{P}\left(\sup _{x \in \mathbb{R}^{q}}\left|u^{N}(t, x)\right| e^{-\lambda|x|}>H \text { for some } t \leq T\right)<\varepsilon^{\prime} .
\end{aligned}
$$

Remembering (11.4), the argumentation as in Proposition II.5.7 of [Per02], combined with that of [MT94] should allow to establish that claim in our case.

We should give more indication, why the resulting SPDE is the stochastic heat equation as given in Conjecture 4. Combining (11.4) and Conjecture 7 it should be possible to obtain

$$
\left\langle u^{N}(t, \cdot), \phi\right\rangle \approx\left\langle u_{0}^{N}(\cdot), \phi\right\rangle+M_{\underline{t}}^{b, N}(\phi)+\int_{0}^{t}\left\langle u^{N}(s, \cdot), A \phi\right\rangle d s .
$$

Here $M^{b, N}$ is a martingale with approximate quadratic variation given by

$$
\left\langle M^{b, N}(\phi)\right\rangle_{\underline{t}} \approx \int_{0}^{\underline{t}} d s \int_{\mathbb{R}^{q}} d y u^{N}(s, y)^{1+\gamma} \lambda \phi(y)^{2} .
$$

Assuming the tightness (where indications were given before) it remains to identify the limit. For any convergent subsequence $u^{N_{k}}, k \in \mathbb{N}$, first use Skorohod representation just as before (8.6). Then, apply the usual technique for introducing a noise $\dot{W}$ (see Step 4 of [MT94] for SPDE, Theorem 5.3.3 of [EK86] for SDE) will allow to describe the martingale in the limit:

$$
M_{t}^{b}(\phi)=\int_{0}^{t} \int_{\mathbb{R}} \sqrt{\lambda} u(s, y)^{\frac{1}{2}(1+\gamma)}(s, y) \phi(y) d y d s .
$$

Remembering $A=\frac{\Delta}{2}$, then the limiting SPDE reads like

$$
\partial_{t} u(t, x)=\frac{1}{2} u^{\prime \prime}(t, x)+u^{\frac{1}{2}(1+\gamma)} \dot{W}(t, x),
$$

where $\dot{W}$ is white noise on $\mathbb{R}_{+} \times \mathbb{R}$. This is the limiting SPDE we wanted to obtain, if we set $\gamma^{\prime}=2 \gamma-1$. 


\section{Index}

\begin{tabular}{|c|c|}
\hline$L_{\mathrm{loc}}^{p}$ & locally $p$-integrable functions, $p \geq 1$. \\
\hline$\delta_{x}$ & Dirac- $\delta$-distribution at $x \in \mathbb{R}^{q}$ \\
\hline$a \wedge b, a \vee b$ & $\min (a, b), \max (a, b)$, respectively \\
\hline $\mathcal{M}^{2, c}$ & space of square-integrable continuous martingales \\
\hline$\partial^{\alpha}$ & partial derivative in $\mathbb{R}^{q}$ of order $\left(\alpha_{1}, \ldots, \alpha_{q}\right)$ \\
\hline$\Delta$ & Laplace operator \\
\hline $\mathcal{S}\left(\mathbb{R}^{q}\right), \mathcal{S}^{\prime}\left(\mathbb{R}^{q}\right)$ & tempered functions, tempered distributions \\
\hline $\mathcal{D}\left(\mathbb{R}^{q}\right), \mathcal{D}^{\prime}\left(\mathbb{R}^{q}\right)$ & Schwartz space, Schwartz distributions \\
\hline $\mathcal{F} f$ & Fourier-transform of $f$ \\
\hline$H^{s}\left(\mathbb{R}^{q}\right)$ & Sobolev space of order $s \in \mathbb{R}$ \\
\hline$[[a, b]]$ & \\
\hline$\langle f, g\rangle$ & $L^{2}$ inner-product \\
\hline$\|\cdot\|_{0, t},\|\cdot\|_{0}$ & norm for integrable random variables \\
\hline$\dot{W}, \dot{W}^{k}, \dot{W}^{\delta}$ & noise, colored noise, white noise \\
\hline$S(f)$ & supremum of support \\
\hline$U_{0}$ & extinction time of $u$ \\
\hline$A^{\nu}$ & $\nu / 2$-th power of the Laplacian \\
\hline$I$ & set of labels \\
\hline$\alpha_{i},|\alpha|$ & $i+1$-th entry of the vector $\alpha$, length of $\alpha$ \\
\hline$p_{t}(\cdot)$ & heat kernel \\
\hline$p_{t, l}(\cdot)$ & spatial derivative of the heat kernel in direction $x_{l}$ \\
\hline$T_{K}$ & a stopping time used in Chapter 9 \\
\hline$J_{n, i}, \tilde{J}_{n, i}$ & sets used in Chapter 9 \\
\hline$\varepsilon_{0}, \varepsilon_{1}, \beta_{i}$ & \\
\hline$u_{1, \delta}, u_{2, \delta}$ & \\
\hline$G_{\delta}, F_{\delta, l}$ & \\
\hline$A^{*}$ & adjoint operator of $A$ \\
\hline$L_{k}$ & covariance functional \\
\hline$C_{\text {tem }}, C_{\text {rap }}$ & tempered growing and rapdidly growing functions \\
\hline
\end{tabular}




\section{Bibliography}

[BCR84] Christian Berg, Jens Peter Reus Christensen, and Paul Ressel, Harmonic analysis on semigroups, Graduate Texts in Mathematics, vol. 100, Springer-Verlag, New York, 1984, Theory of positive definite and related functions. MR 747302 (86b:43001)

[Ber99] Jean Bertoin, Subordinators: examples and applications, Lectures on probability theory and statistics (Saint-Flour, 1997), Lecture Notes in Math., vol. 1717, Springer, Berlin, 1999, pp. 1-91. MR 1746300 (2002a:60001)

[Blo96] Douglas Blount, Diffusion limits for a nonlinear density dependent space-time population model, Ann. Probab. 24 (1996), no. 2, 639-659. MR 1404523 (98c:60138)

[BMP11] Krzysztof Burdzy, Carl Mueller, and Edwin Perkins, Non-uniqueness for non-negative solutions of parabolic stochastic partial differential equations, preprint (2011), arXiv:1008.2126v4 [math.PR].

[Bre68] Leo Breiman, Probability, first ed., Addison Wesley Publishing, Reading, Massachusetts, 1968.

[BTA04] Alain Berlinet and Christine Thomas-Agnan, Reproducing kernel hilbert spaces in probability and statistics, Kluwer Academic Publishers, Boston, Dordrecht, London, 2004.

[CM94] René A. Carmona and S. A. Molchanov, Parabolic Anderson problem and intermittency, Mem. Amer. Math. Soc. 108 (1994), no. 518, viii+125. MR 1185878 (94h:35080)

[Da199] Robert C. Dalang, Extending the martingale measure stochastic integral with applications to spatially homogeneous s.p.d.e.'s, Electron. J. Probab. 4 (1999), no. 6, 29 pp. (electronic). MR 1684157 (2000b:60132)

[Daw75] Donald A. Dawson, Stochastic evolution equations and related measure processes, J. Multivariate Anal. 5 (1975), 1-52. MR 0388539 (52 \#9375) 
[DIP89] Donald A. Dawson, I. Iscoe, and Edwin A. Perkins, Super-Brownian motion: path properties and hitting probabilities, Probab. Theory Related Fields 83 (1989), no. 1-2, 135-205. MR 1012498 (90k:60073)

[DKRA09] R.C. Dalang, D. Khoshnevisan, and F. Rassoul-Agha, A minicourse on stochastic partial differential equations, Lecture Notes in Mathematics, no. Bd. 1962, Springer, 2009.

[DMS93] D. A. Dawson, B. Maisonneuve, and J. Spencer, École d'Été de Probabilités de Saint-Flour XXI-1991, Lecture Notes in Mathematics, vol. 1541, Springer-Verlag, Berlin, 1993, Papers from the school held in Saint-Flour, August 18-September 4, 1991, Edited by P. L. Hennequin. MR 1242574 (94d:60001)

[Doo01] Joseph L. Doob, Classical potential theory and its probabilistic counterpart, Springer-Verlag, 2001.

[DP91] Donald A. Dawson and Edwin A. Perkins, Historical processes, Mem. Amer. Math. Soc. 93 (1991), no. 454, iv+179. MR 1079034 (92a:60145)

[DPZ08] G. Da Prato and J. Zabczyk, Stochastic equations in infinite dimensions, Encyclopedia of Mathematics and Its Applications, Cambridge University Press, 2008.

[EK86] Stewart N. Ethier and Thomas G. Kurtz, Markov processes - characterization and convergence, Wiley Series in Probability and Mathematical Statistics: Probability and Mathematical Statistics, John Wiley \& Sons Inc., New York, 1986. MR MR838085 (88a:60130)

[Eng07] János Engländer, Branching diffusions, superdiffusions and random media, Probab. Surv. 4 (2007), 303-364. MR 2368953 (2009b:60249)

[Eva10] Lawrence C. Evans, Partial differential equations, Graduate Studies in Mathematics, American Mathematical Society, 2010.

[FK10] Mohammud Foondun and Davar Khoshnevisan, On the global maximum of the solution to a stochastic heat equation with compact-support initial data, Ann. Inst. Henri Poincaré Probab. Stat. 46 (2010), no. 4, 895-907. MR 2744876 (2012c:35493)

[FMW10] Klaus Fleischmann, Leonid Mytnik, and Vitali Wachtel, Optimal local Hölder index for density states of superprocesses with $(1+\beta)$-branching mechanism, Ann. Probab. 38 (2010), no. 3, 1180-1220. MR 2674997 (2011e:60195) 
[Fri76] Avner Friedman, Partial differential equations, Krieger Publishing Company, Malabar, 1976.

[FSS06] Marco Ferrante and Marta Sanz-Solé, SPDEs with coloured noise: analytic and stochastic approaches, ESAIM Probab. Stat. 10 (2006), 380405 (electronic). MR 2263072 (2007m:60176)

[GK05] Jürgen Gärtner and Wolfgang König, The parabolic Anderson model, Interacting stochastic systems, Springer, Berlin, 2005, pp. 153-179. MR 2118574 (2005k:82042)

[Haw71] John Hawkes, A lower Lipschitz condition for the stable subordinator, Z. Wahrscheinlichkeitstheorie und Verw. Gebiete 17 (1971), 23-32. MR 0282413 (43 \#8125)

[HKPS93] Takeyuki Hida, Hui-Hsiung Kuo, Jürgen Potthoff, and Ludwig Streit, White noise, Mathematics and its Applications, vol. 253, Kluwer Academic Publishers Group, Dordrecht, 1993, An infinite-dimensional calculus. MR 1244577 (95f:60046)

[Hör85] Lars Hörmander, The Analysis of Linear Partial Differential Operators. III: Pseudo-Differential Operators, Springer, Berlin, 1985.

[HS08] Takeyuki Hida and $\mathrm{Si} \mathrm{Si}$, Lecutures on white noise functionals, World Scientific publishing, Singapore, 2008.

[Isc88] I. Iscoe, On the supports of measure-valued critical branching Brownian motion, Ann. Probab. 16 (1988), no. 1, 200-221. MR 920265 (88j:60097)

[Jac80] Jean Jacod, Weak and strong solutions of stochastic differential equations, Stochastics 3 (1980), no. 3, 171-191. MR 573202 (82a:60086)

[Jac05] Niels Jacob, Pseudo-differential operators and markov processes vol. 3: Markov processes and applications, Imperial College Press, London, 2005.

[Jak86] Adam Jakubowski, On the Skorokhod topology, Ann. Inst. H. Poincaré Probab. Statist. 22 (1986), no. 3, 263-285. MR 871083 (89a:60008)

[Jan97] Svante Janson, Gaussian hilbert spaces, Cambridge University Press, Cambridge, 1997.

[Kal02] Olav Kallenberg, Foundations of modern probability, second ed., Probability and its Applications (New York), Springer-Verlag, New York, 2002. MR 1876169 (2002m:60002) 
[Kle08] Achim Klenke, Wahrscheinlichkeitstheorie, second ed., Springer, Berlin, 2008 (deutsch).

[Kli11] Sandra Kliem, Convergence of rescaled competing species processes to a class of SPDEs, Electron. J. Probab. 16 (2011), no. 22, 618-657. MR 2786644 (2012f:60095)

[Kot92] Peter Kotelenez, Comparison methods for a class of function valued stochastic partial differential equations, Probab. Theory Related Fields 93 (1992), no. 1, 1-19. MR 1172936 (93i:60116)

[Kry96] Nicolai V. Krylov, On $L_{p}$-theory of stochastic partial differential equations in the whole space, SIAM J. Math. Anal. 27 (1996), no. 2, 313-340. MR 1377477 (97b:60107)

[Kry97]_, On a result of C. Mueller and E. Perkins, Probab. Theory Related Fields 108 (1997), no. 4, 543-557.

[KS88] Nuri Konno and Tokuzo Shiga, Stochastic partial differential equations for some measure-valued diffusions, Probability Theory and Related Fields 79 (1988), 201-225, 10.1007/BF00320919.

[KS00] I. Karatzas and S. Shreve, Brownian motion and stochastic calculus, second ed., Springer, New York, 2000 (english).

[Kun90] Hiroshi Kunita, Stochastic flows and stochastic differential equations, Cambridge Studies in Advanced Mathematics, vol. 24, Cambridge University Press, Cambridge, 1990. MR 1070361 (91m:60107)

[Kur07] Thomas G. Kurtz, The Yamada-Watanabe-Engelbert theorem for general stochastic equations and inequalities, Electron. J. Probab. 12 (2007), 951-965. MR 2336594 (2008h:60291)

[Mey66] Paul A. Meyer, Probability and potentials, Blaisdell book in pure and applied mathematics, Blaisdell Pub. Co, 1966.

[MMP12] Carl Mueller, Leonid Mytnik, and Edwin Perkins, Nonuniqueness for a parabolic spde with $\frac{3}{4}-\epsilon$-hölder diffusion coefficients, preprint (2012), arXiv:1201.2767v1 [math.PR].

[MP92] Carl Mueller and Edwin A. Perkins, The compact support property for solutions to the heat equation with noise, Probab. Theory Related Fields 93 (1992), no. 3, 325-358. MR 1180704 (93k:60156) 
[MP11] Leonid Mytnik and Edwin Perkins, Pathwise uniqueness for stochastic heat equations with Hölder continuous coefficients: the white noise case, Probab. Theory Related Fields 149 (2011), no. 1-2, 1-96. MR 2773025

[MPS06] Leonid Mytnik, Edwin Perkins, and Anja Sturm, On pathwise uniqueness for stochastic heat equations with non-Lipschitz coefficients, Ann. Probab. 34 (2006), no. 5, 1910-1959. MR 2271487 (2007k:60185)

[MT94] Carl Mueller and Roger Tribe, A phase transition for a stochastic PDE related to the contact process, Probab. Theory Related Fields 100 (1994), no. 2, 131-156. MR 1296425 (95k:60151)

[Mue91] Carl Mueller, On the support of solutions to the heat equation with noise, Stochastics Stochastics Rep. 37 (1991), no. 4, 225-245. MR 1149348 (93e:60122)

[Myt96] Leonid Mytnik, Superprocesses in random environments, Ann. Probab. 24 (1996), no. 4, 1953-1978. MR 1415235 (97h:60046)

[Myt98] - Weak uniqueness for the heat equation with noise, Ann. Probab. 26 (1998), no. 3, 968-984. MR 1634410 (99i:60124)

[Par72] Étienne Pardoux, Sur des équations aux dérivées partielles stochastiques monotones, C. R. Acad. Sci. Paris Sér. A-B 275 (1972), A101-A103. MR 0312572 (47 \#1129)

[Paz83] Amnon Pazy, Semigroups of linear operators, Springer, New-York, 1983.

[Per02] Edwin Perkins, Dawson-Watanabe superprocesses and measure-valued diffusions, Lectures on probability theory and statistics (Saint-Flour, 1999), Lecture Notes in Math., vol. 1781, Springer, Berlin, 2002, pp. 125-324. MR 1915445 (2003k:60104)

[PR07] Claudia Prévôt and Michael Röckner, A concise course on stochastic partial differential equations, Lecture Notes in Mathematics, Springer, 2007.

[PZ00] Szymon Peszat and Jerzy Zabczyk, Nonlinear stochastic wave and heat equations, Probab. Theory Related Fields 116 (2000), no. 3, 421-443. MR 1749283 (2001f:60071)

[Rau91] Jeffrey Rauch, Partial differential equations, Graduate Texts in Mathematics, Springer-Verlag, 1991. 
[Rei89] Mark Reimers, One dimensional stochastic partial differential equations and the branching measure diffusion, Probability Theory and Related Fields 81 (1989), 319-340, 10.1007/BF00340057.

[RW87] L. C. G. Rogers and David Williams, Diffusions, Markov processes, and martingales. Vol. 2, Wiley Series in Probability and Mathematical Statistics: Probability and Mathematical Statistics, John Wiley \& Sons Inc., New York, 1987, Itô calculus. MR 921238 (89k:60117)

[RY91] Daniel Revuz and Marc Yor, Continuous martingales and brownian motion, first ed., Springer, New York, 1991 (english).

[Shi94] Tokuzo Shiga, Two contrasting properties of solutions for onedimensional stochastic partial differential equations, Canad. J. Math. 46 (1994), no. 2, 415-437. MR 1271224 (95h:60099)

[SSS02] M. Sanz-Solé and M. Sarrà, Hölder continuity for the stochastic heat equation with spatially correlated noise, Seminar on Stochastic Analysis, Random Fields and Applications, III (Ascona, 1999), Progr. Probab., vol. 52, Birkhäuser, Basel, 2002, pp. 259-268. MR 1958822 (2003k:60159)

[Ste67] E. M. Stein, Singular integrals, harmonic functions, and differentiability properties of functions of several variables, Singular integrals (Proc. Sympos. Pure Math., Chicago, Ill., 1966), Amer. Math. Soc., Providence, R.I., 1967, pp. 316-335. MR 0482394 (58 \#2467)

[Stu03] Anja Sturm, On convergence of population processes in random environments to the stochastic heat equation with colored noise, Electron. J. Probab. 8 (2003), no. 6, 1-39.

[Vio76] Michel Viot, Solutions faibles d'équations aux dérivées partielles non linéaires, Thése, Université Pierre et Marie Curie, Paris, 1976.

[Wal86] John B. Walsh, An introduction to stochastic partial differential equations, École d'été de probabilités de Saint-Flour, XIV—1984, Lecture Notes in Math., vol. 1180, Springer, Berlin, 1986, pp. 265-439. MR 876085 (88a:60114)

[Xio12] Jie Xiong, Super-brownian motion as the unique strong solution to an spde, preprint (2012), arXiv:1203.4873v1 [math.PR].

[YW71] Toshio Yamada and Shinzo Watanabe, On the uniqueness of solutions of stochastic differential equations., J. Math. Kyoto Univ. 11 (1971), 155-167. MR 0278420 (43 \#4150) 
[Zäh04] Henryk Zähle, Stochastic heat equation and catalytic super-brownian motion, Technical University of Berlin, 2004. 


\section{Danksagung}

$\mathrm{Zu}$ allererst möchte ich mich bei meiner Betreuerin Prof. Dr. Anja Sturm bedanken. Sie brachte mich zum Thema stochastische partielle Differentialgleichungen, insbesondere der Fragestellung der pfadweisen Eindeutigkeit. Während der Promotion gab sie mir in zahllosen Gesprächen wertvolle Hinweise und Unterstützung zu vielen fachlichen Fragen. Zudem möchte ich mich bei ihr auch für die Möglichkeiten bedanken an verschiedenen Veranstaltungen teilzunehmen und Kontakte zu knüpfen. Insbesondere der Besuch bei Prof. Leonid Mytnik, dem ich für interessante Diskussionen und die Idee zu Kapitel 10 danke und die Treffen mit Prof. Dr. Anita Winter zählen dazu.

Vielen Dank gebührt auch allen Mitarbeitern am Institut für Mathematische Stochastik. Für ideelle Unterstützung möchte ich mich bei Prof. Dr. Axel Munk bedanken. Ein großer Dank geht an diejenigen, die Teile dieser Arbeit gelesen haben und mir Verbesserungsvorschläge machten: Rebekka Brink-Spalink, Sebastian Engelke, Benjamin Heuer, Kirstin Strokorb und Simone Maxand.

Zuguterletzt gilt der größte Dank meiner Familie und meinen Freunden, die mich in den letzten Jahren auf verschiedene Weisen unterstützt haben. Ohne alle Namen aufzuzählen, möchte ich mich bei all denen bedanken, die ich besuchen durfte, die mit mir auf Reisen gingen, durch Göttingen spazierten, Sport trieben, Arabisch sprachen, Karten spielten, tanzten, zelteten oder wohnten. 


\section{Lebenslauf Thomas Rippl}

14. Mai 1984 geboren in Bayreuth.

Juni 2003 Abitur, Gymnasium Eschenbach in der Oberpfalz.

2003 - 2009 Studium der Mathematik, Universität Erlangen-Nürnberg.

2005 - 2006 Austauschstudent, University of Bath, United Kingdom.

Juli 2009 Diplom in Mathematik, Universität Erlangen-Nürnberg. Diplomarbeit bei Prof. Dr. A. Greven zum Thema:

"Asymptotic Genealogy of Catalytic Branching Models."

2009 - 2012 Promotionsstudent, Universität Göttingen.

$2009-2011$ Wissenschaftlicher Mitarbeiter, Institut für Math. Stochastik Göttingen.

2011 - 2012 Wissenschaftlicher Koordinator, DFG/SNF-Forschergruppe FOR 916. 